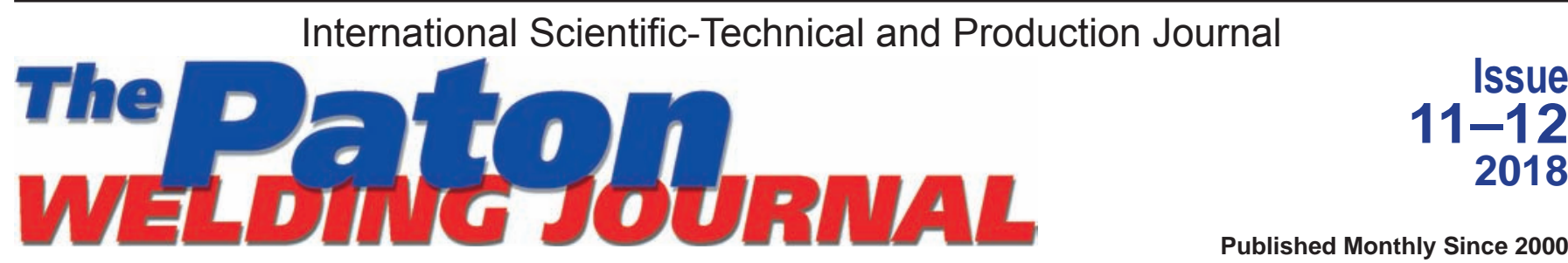

English translation of the monthly «Avtomaticheskaya Svarka» (Automatic Welding) journal published in Russian since 1948

\section{EDITORIAL BOARD \\ Editor-in-Chief B.E. Paton}

Scientists of PWI, Kyiv S.I. Kuchuk-Yatsenko (vice-chief ed.), V.N. Lipodaev (vice-chief ed.)

Yu.S. Borisov, G.M. Grigorenko, A.T. Zelnichenko, V.V. Knysh,

I.V. Krivtsun, Yu.N. Lankin, L.M. Lobanov, V.D. Poznyakov, I.A. Ryabtsev, K.A. Yushchenko

Scientists of Ukrainian Universities V.V. Dmitrik, NTU «KhPI», Kharkov V.V. Kvasnitsky, NTUU «KP|», Kyiv E.P. Chvertko, NTUU «KPI», Kyiv

Foreign Scientists N.P. Alyoshin

N.E. Bauman MSTU, Moscow, Russia Guan Qiao

Beijing Aeronautical Institute, China M. Zinigrad

Ariel University, Israel V.I. Lysak

Volgograd STU, Russia Ya. Pilarczyk

Welding Institute, Gliwice, Poland U. Reisgen

Welding and Joining Institute, Aachen, Germany G.A. Turichin

St. Petersburg SPU, Russia

\section{Founders}

E.O. Paton Electric Welding Institute, NASU International Association «Welding» Publisher

International Association «Welding» Translators

A.A. Fomin, O.S. Kurochko, I.N. Kutianova Editor

N.G. Khomenko

Electron galley

D.I. Sereda, T.Yu. Snegiryova Address

E.O. Paton Electric Welding Institute, International Association «Welding» 11 Kazimir Malevich Str. (former Bozhenko Str.), 03150, Kyiv, Ukraine

Tel.: (38044) 20060 16, 2008277

Fax: (38044) 2008277,2008145

E-mail: journal@paton.kiev.ua

www.patonpublishinghouse.com

State Registration Certificate

KV 4790 of 09.01 .2001

ISSN 0957-798X

DOI: http://dx.doi.org/10.15407/tpwj Subscriptions

$\$ 348,12$ issues per year

air postage and packaging included. Back issues available.

All rights reserved.

This publication and each of the articles contained herein are protected by copyright.

Permission to reproduce material contained in this

journal must be obtained in writing from the Publisher.

\section{CONTENTS}

\section{Plenary Reports of the International Conference «Welding and Related Technologies - \\ Present and Future», December 5-6, 2018, Kyiv, Ukraine}

Borys Evgenovych Paton is 100 2

Paton B.E. Advanced studies and developments of the E.O. Paton Electric Welding Institute in the field of welding and related technologies

Pietras A., Wegglowska A. and Rams B. The FSW technology of non-ferrous metals - process conditions and examples of application

Kuchuk-Yatsenko S.I. Technologies and equipment for flash-butt welding of rails: 60 years of continuous innovations

Lobanov L.M. and Volkov V.S. Thin-walled welded transformable-volume structures of space purpose

Qiao Guan. Low stress no distortion welding based on thermal tensioning effects

Ilyushchenko A.F., Manojlo E.D., Lovygin S.I., Letsko A.I. and Radchenko A.A. Promising directions of works in the field of welding and related technologies at SSPA «Powder Metallurgy»

Kvasnytskyi V.V., Kvasnytskyi V.F., Chen Hexing, Matvienko M.V. and Yermolayev G.V. Diffusion welding and brazing of dissimilar materials with controlled stress-strain state

Ipekoğlu G., Akçam Ö. and Çam G. Effect of plate thickness on weld speed in friction stir welding of AA6061-T6 Al-alloy plates

Yushchenko K.A., Zadery B.A., Gakh I.S. and Zvyagintseva A.V. Prospects of development of welded single-crystal structures of heat-temperature nickel alloys

Krivtsun I.V. Anode processes in welding arcs

Nazarchuk Z.T. and Skalsky V.R. Magnetoacoustic diagnostics of hydrogen damage of ferromagnetics

Beloev M. and Lolov N. Some technological peculiarities of performance of assembly-welding works in construction of isothermal tanks

Andreev M.A. and Markova L.V. Structure and properties of wear-resistant ion-beam vacuum coatings

Nesterenkov V.M., Khripko K.S. and Matviichuk V.A. Electron beam technologies of welding, surfacing, prototyping: Results and prospects

Akhonin S.V. Tendencies of development of special electrometallurgy of titanium in Ukraine

Küçükömeroğlu T., Aktarer S.M., Ipekoğlu G. and Çam G. Effect of multi-pass friction stir processing on the microstructure and mechanical properties of dual phase steel 


\section{Borys Evgenovych Paton is 100}

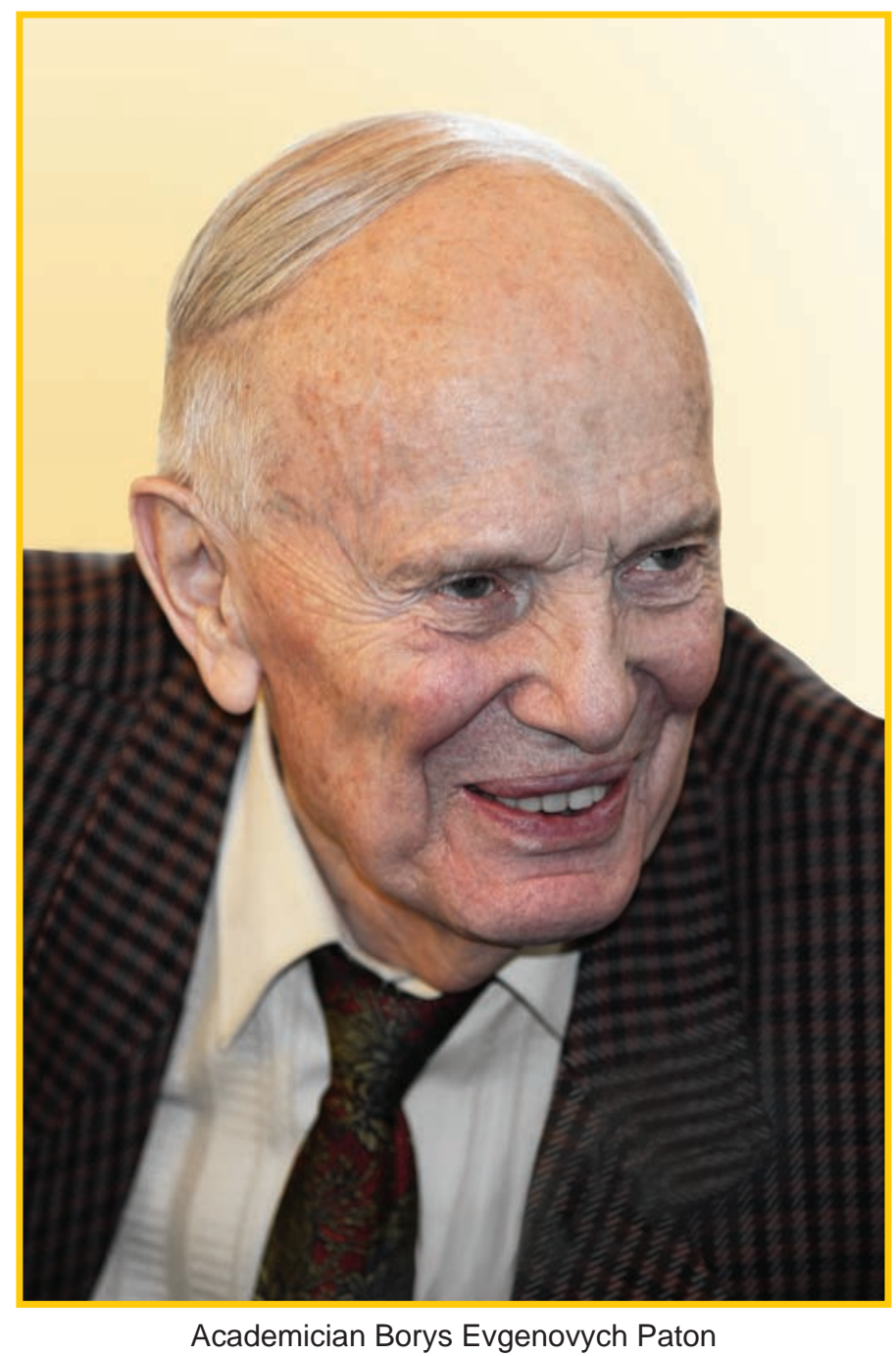

We have a great honor to work together with academician Borys Evgenovych Paton, who by his selfless labor made great contributions to the restructuring of the Academy of Sciences of Ukraine, founded in 1918 by the initiative of Volodymyr Ivanovych Vernadsky, the famous scientist and public figure.

During the years of its activity the Academy attained great achievements in many fields of science and technology. Numerous discoveries and inventions were made, many theories and conceptions were put forward, scientific and technical directions were started, the world-known scientific schools were organized, to which the Paton school belongs, which educated and united the scientists in the field of electric welding and related technologies, materials science and metallurgy.

All the labor life of Borys Paton is inseparably connected with the Institute of Electric Welding. This is the call of «technical nature», family tradition and son's duty to continue the father's activity.

The talent of the famous scientist with a wide sphere of interests was especially pronounced in Borys E. Paton at the post of the Institute director. To define clearly the purpose of investigation, to forecast the most important link in it, to attract interest of a large team of scientists to the new direction are the main tasks of the scientific leader, which were successfully realized by him showing the self ability to work with the team, readiness in supporting of any interesting idea, to assess worthy the fulfilled work. His inexhaustible enthusiasm, unique working ability and respect of each staff member provided the healthy creative atmosphere in the working staff.

Over 65 years the work of the Institute under the leadership of Borys E. Paton is the wonderful continuation and implementation of profound and challenging principles of scientific-technical activity of Evgen Oscarovych Paton, the founder of our Institute. The scientific-technical directions, started by Borys Paton, widened greatly the subjects of studies and developments. The purposeful fundamental studies, started at the Institute, became the theoretical bases of welding science, transforming it into powerful means of technical progress, that led to the revolutionary changes in many branches of industry, such as: machine-, ship- and aircraft building, aerospace industry, power engineering, railway transport, mining complexes, metallurgy and chemical production, promoted the creation of pipeline transport, construction industry. Many scientific developments predetermined the world development of welding technologies and went down in history of the science and technology progress.

The world authority of B.E. Paton came by comprehensive and fruitful scientific and engineering activity, desire to direct the fundamental research to the solution of actual problems of the scientific-technical progress. By his contributions he made grounds of arc welding, namely the theory of the automatic machines for arc welding, which further was improved by many specialists in the field of the automatic control of welding processes. Under the supervision of Borys Paton the studies were made in the field of welding power sources; the processes of arc, electroslag, resistance, electron beam and many other processes of welding and allied technologies were developed; a large complex of fundamental and applied studies in the field of static and cyclic strength of welded joints, their resistance to brittle and fatigue fracture, serviceability under conditions 
of low temperatures was carried out. For the first time in Ukraine the systems of diagnostics of welded structures, to which the high requirements of safety are specified, were developed. Borys E. Paton supervised the investigations with use of the electroslag process for quality improvement of metals and alloys, which resulted in appearance of the radically new direction in the metallurgy: electroslag remelting and casting. The technologies of the electroslag remelting, developed at the Institute, found the wide application and were worldly recognized.

Borys E. Paton is the scientist-innovator. The technologies, developed under his supervision, are successfully operating on the Earth, under the water and in space. The E.O. Paton Electric Welding Institute of the National Academy of Sciences of Ukraine, headed by B.E. Paton, is the powerful scientific institution, where many generations of scientists-welders were born and formed. Borys Paton brought up hundreds of talented scientists and engineers.

The thorough understanding of science role in the society, its aims and tasks, high international authority of the scientist, devotion to the science, inexhaustible energy and high moral qualities, active social-political activity, experience in management of the large scientific staff became a decisive arguments in election of Borys E. Paton on the post of the President of the Academy of Sciences of the Ukrainian SSR in 1962 (now the National Academy of Sciences of Ukraine). Since that time he is heading this leading scientific organization in Ukraine over 56 years.

His talent of the eminent organizer was greatly displayed on the responsible post of the President of the National Academy of Sciences of Ukraine. With his participation the new structure of the Academy of Sciences and its new status were worked out, directed to the most rational application of scientific efforts and means, their concentration in the solution of the most important fundamental and applied scientific problems, which have the great importance for the country economy, dozens of new institutes and organizations were founded, which developed and intensified investigations in the most important scientific directions.

The distinguished abilities of B.E. Paton as a leader, scientist and organizer were revealed in a dramatic Chernobyl tragedy. The associates of many institutes of the Academy of Sciences, its Presidium were included from the first days into the work for liquidation of the catastrophe consequences.

Borys E. Paton made a lot of efforts for the development of the international scientific cooperation of Ukraine, the implementation of national science in the European and world scientific community. By his initiative the International Association of Academies of Sciences was organized in 1993, which united the National Academies of 15 countries of Europe and Asia. During almost 25 years Borys E. Paton was permanent President of this Association.

Borys E. Paton is the well-known state and public figure. Many years he was elected to the structure of government bodies, he was and is now a leader and member of different committees and commissions. By occupying high posts, he is working fruitfully with a high sense of personal responsibility to the state, people, own conscience.

Academician Borys E. Paton is a creative person, clever, honest and responsive man, he possesses fantastic energy, ability to work, keen analytic intellect. His life experience, deep knowledge in many branches of science and technology, international authority in science and society played a great role in saving the national scientific potential in the first hard decades of the Ukraine independence.

It is symbolic that Borys E. Paton was born on 27 November, 1918, the day of foundation of the National Academy of Sciences of Ukraine. During celebration of the $80^{\text {th }}$ anniversary of the Academy and its President in 1998 the large hall of Palace «Ukraina» applauded the news about awarding the title of Hero of Ukraine to B.E. Paton, being the first one awarded in the state. Nevertheless, Borys E. Paton continue to direct the efforts of scientists of the National Academy of Sciences of Ukraine, all the national scientific community to the development of science, education, culture, providing the dynamic progress of Homeland.

The inborn talent of Borys E. Paton, strong will and tireless, selfless labor guaranteed the success at all the stages of life. And now, in the year of his celebrated jubilee he preserves usual working state, supervises the investigations, initiates the research, orients to the new results and wide implementation of the Academy into the world scientific community.

We cordially wish a strong health and new successive achievements to the hero of the anniversary in inspired labor on the scientific arena.

Editorial Board of the Journal 


\title{
ADVANCED STUDIES AND DEVELOPMENTS OF THE E.O. PATON ELECTRIC WELDING INSTITUTE IN THE FIELD OF WELDING AND RELATED TECHNOLOGIES
}

\author{
Academician B.E. PATON \\ E.O. Paton Electric Welding Institute of the NAS of Ukraine \\ 11 Kazimir Malevich Str., 03150, Kyiv, Ukraine. E-mail: office@paton.kiev.ua
}

\begin{abstract}
A number of recent new developments of the E.O. Paton Electric Welding Institute is presented, namely the technologies and equipment for welding with applying the highly-concentrated power sources: plasma, laser and electron ones. Technologies were developed for welding of pipes, thick titanium, aluminium-lithium alloys, high-strength steels. The vapor-phase technologies were developed for producing nanostructured materials for welding composite materials and intermetallics. Technologies and equipment for underwater welding and cutting, new electron beam tool for welding in open space were developed. To increase the life and safety of the weld, the postweld treatment was suggested by using the high-density electric pulses and high-frequency mechanical peening. To control the quality of welded structures, the designed digital equipment, based on high-sensitive solid-body converters and an industrial robot with a technical vision system for products of intricate geometry were developed. The new method was developed for growing refractory metal single crystals. New equipment is presented for welding of live tissues. 28 Ref., 2 Tables, 25 Figures.
\end{abstract}

Keywords : plasma, laser, electron beam and resistance welding, titanium, aluminium-lithium alloys, strength, quality control, surfacing, single crystals, welding of live tissues

Welding and related technologies are now constantly and stably progressing. With their applying the industrially developed countries of the world produce over a half of their gross national product. Having a confidential advancing, the welding entered all the spheres of the mankind life. It is widely used not only in the industry and construction, but also in manufacture of home appliance, sports equipment, in creation of art masterpieces and even in medicine. All this allows the world's welding community to state that the present welding technologies not only take part in the material production of different products, but also have a significant influence on the improvement of quality of people life and promote the continuous progress of the nowadays society.

Analysis of tendencies in development of a global market of welding equipment shows that the technologies of welding will be further widened in future. The demand for energy-saving welding technologies, which are based on application of the highly-concentrated energy: plasma, laser, electron beam, as well of hybrid power sources, will significantly grow. Automation and robotization of the welding processes will continue its widening and become useful in those cases where they are mostly effective.

The application of new designs will allow updating the welded structures, and decrease in their metal consumption will provide applying of steels and alloys of the higher strength. But it is not necessary to (C) B.E. PATON, 2018 forget the requirements for safety, life and quality of the welded structures.

The challenging are the studies and developments of the Electric Welding Institute, carried out during the recent years with account for the tendencies in the development of present directions of the welding science and technology.

Plasma, as a highly-concentrated power source, finds ever more application in welding and related technologies. A number of developments were made at the Institute with its application, giving good results. The developed technology of high-speed plasma welding of alloys of up to $12 \mathrm{~mm}$ thickness per one pass and welding equipment: welding plasmatrons of unique design, plasma module, control system by means of a programmed logic controller with a feasibility of integration with a welding robot, allowed increasing the technological and technical-economic characteristics of the thick metal welding process. The equipment provides the feasibility of operation with variable-polarity pulses of current at a preset wave shape, at a smooth control and discrete control of duration of pulses and pauses between them within the wide range, as well as the feasibility of operation at direct and pulsed current of a straight and reverse polarity. In comparison with the traditional process of arc TIG welding, the weld, produced by the plasma welding, has by $40 \%$ smaller width and mass of metal being deposited, and also more fine-dispersed and homogeneous structure of the fusion zone. More- 
over, the value of energy input is decreased by $2.5-3.0$ times, and zone of weakening - by 1.5 times.

The significant achievement of the recent years is the development of a hybrid plasma-arc welding [1, 2]. The combination of two welding power sources provides the higher base metal penetration. The technology of welding steels and aluminium alloys of 5-12 $\mathrm{mm}$ thickness has been developed which allows increasing the welding speed by $25-40 \%$ and decreasing the consumption of welding wire by $40 \%$, as compared with a pulsed-arc consumable electrode welding. To realize this technology, the plasmatron of a unique design (Figure 1) and basic technological processes were developed.

To provide the high physicomechanical properties of aluminium alloy welds is possible by the developed technology for the spot plasma welding at a pulse of a special shape and stabilization of its length and equipment for its realization. In comparison with the pressure spot resistance welding, this technology can be used at a single-sided access to the site of welding. The cathode cleaning of aluminium alloy surfaces welded; high efficiency and lower power consumption allow its integration into the robotic welding lines.

With application of plasma power sources the high-efficient process of a supersonic plasma spraying of coatings from powders of metal, alloys, ceramic materials and their mixtures was developed at the Institute. For its realization the equipment of a new generation has been designed (Figure 2), which envisages the feasibility of a separate feeding of components of inexpensive plasma-forming gas on air base

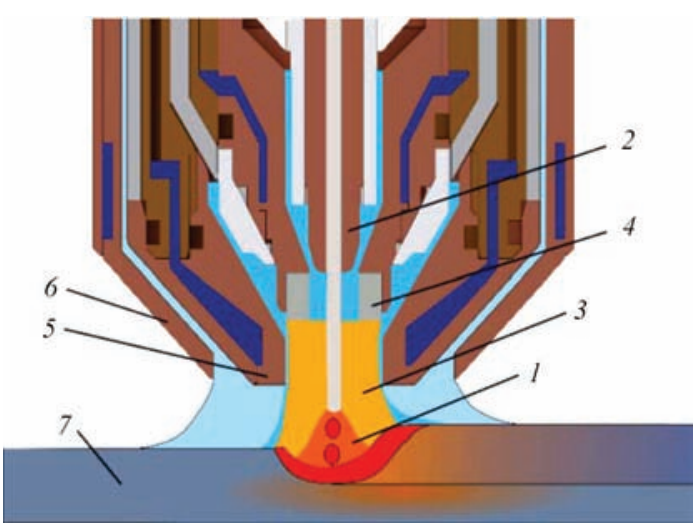

Figure 1. Scheme of hybrid welding plasmatron: 1 - consumable electrode arc; 2 - consumable electrode nozzle; 3 - non-transferred constricted arc; 4 - plasmatron tubular electrode; 5 plasma-forming nozzle; 6 - protective nozzle; 7 - specimen being welded

(air and additions of methane or propane in amount of 5-10\%). The plasmatron, generating the supersonic jet, increases the kinetic energy of particles being sprayed by 9-16 times, and, as a consequence, provides the significant increase in all the service properties of coatings. In particular, the adhesion strength is 1.5-2.0 times increased as compared with coatings, which were produced by a plasma spraying at subsonic modes.

Traditionally at the Institute, the investigations and developments are continued by using the laser and electron beam power sources. On the basis of modern fiber disc and diode high-safety lasers the technology and automatic equipment have been developed for the laser welding of high-strength and stainless steels, aluminium and titanium alloys, which are used

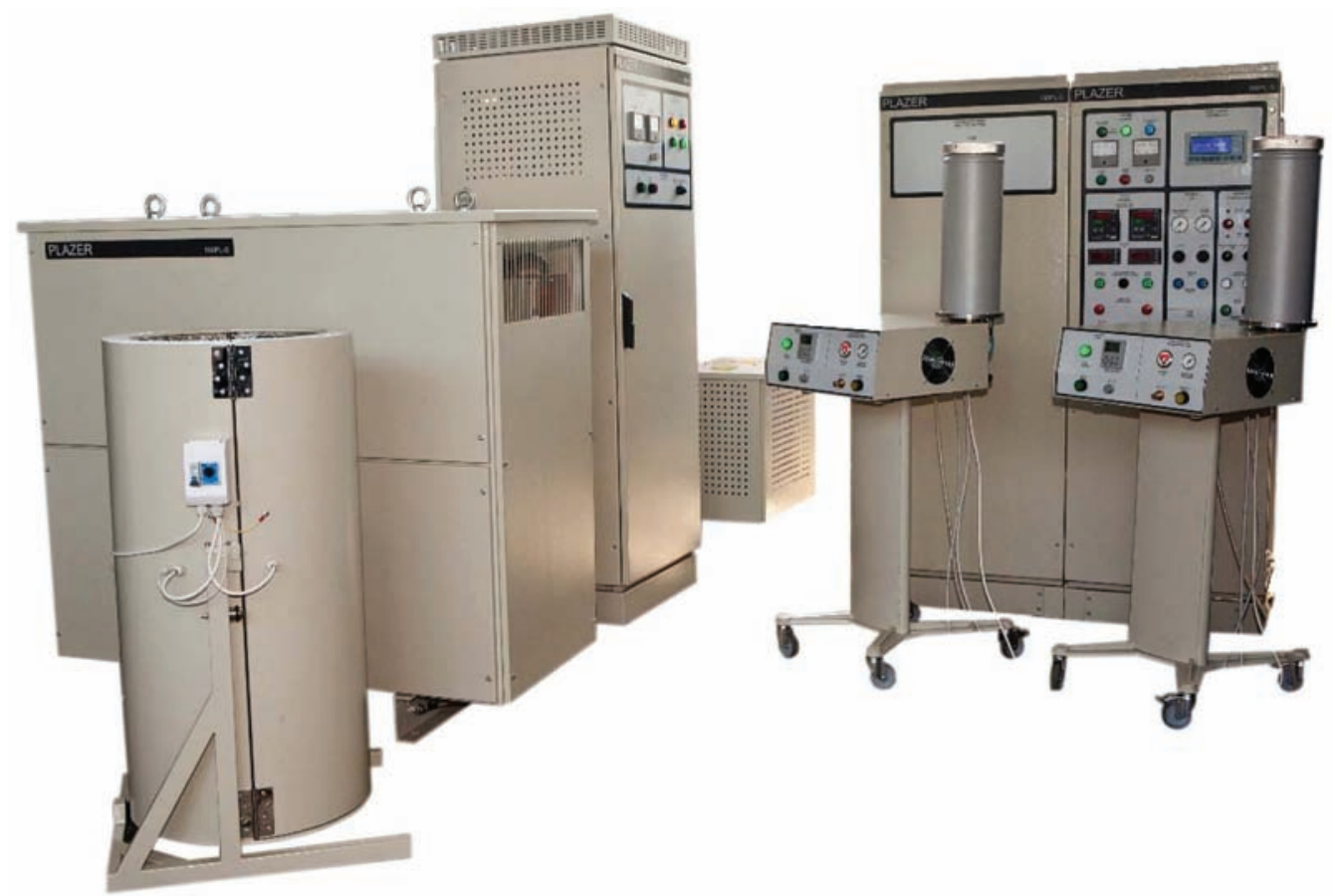

Figure 2. Equipment for high-efficient supersonic plasma spraying 


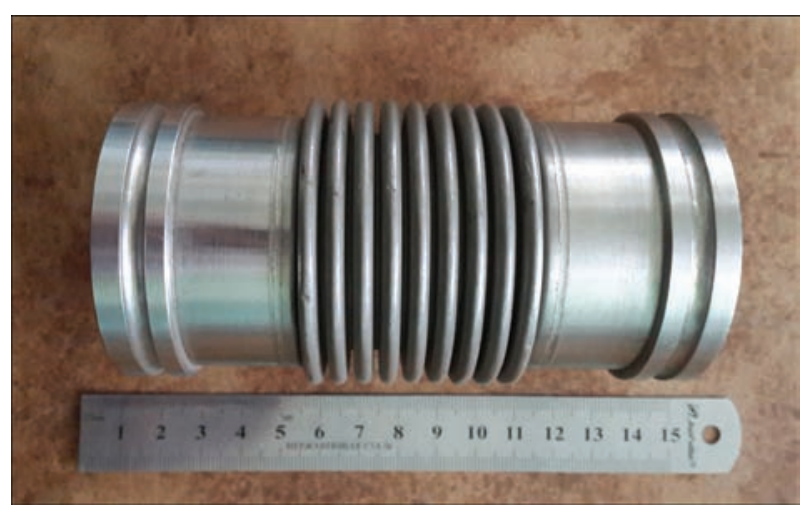

Figure 3. Multilayer bellow, made of tubes, welded by laser radiator

for manufacture of a large spectrum of products. For example, they are used for welding of thin-walled various-diameter pipes of stainless steels for manufacture of multilayer bellows (Figure 3). The designed high-power laser welding equipment is successfully applied in railway car, automobile and ship building, and for site works in these industries the semiautomatic manual laser tool of up to $2 \mathrm{~kW}$ power has been designed (Figure 4).

At the Ukrainian and foreign enterprises of aircraft and rocket construction the developments of the Institute in the field of laser welding, in particular for welding of dissimilar metals, stringer panels, nozzles of liquid-propellant rocket engines, aerospace control surface elements, thin-walled hull structures and other elements of flying vehicles passed the industrial trials.

The Electric Welding Institute occupies one of the leading positions in the world in design and manufacture of equipment for the electron beam welding. Its products are exported to many countries of the world. Thus, the equipment for electron beam welding (EBW) has been designed and manufactured at

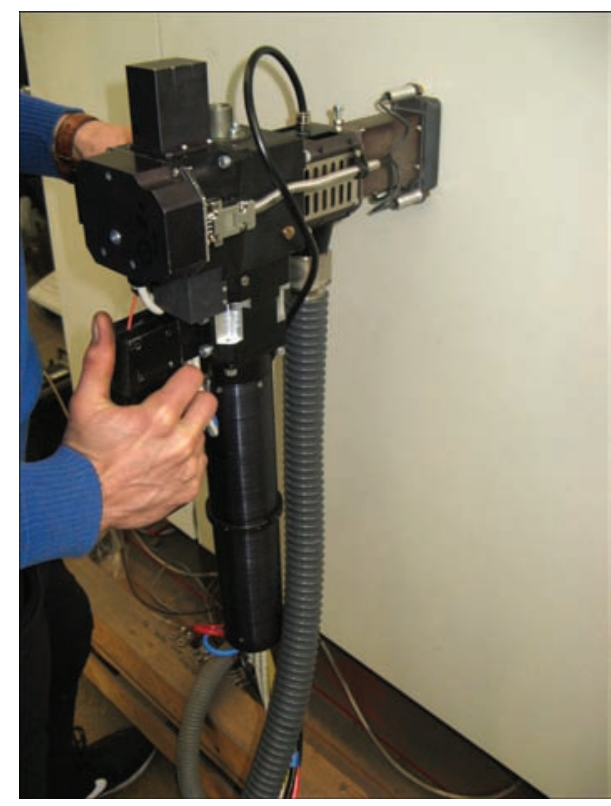

Figure 4. Semi-automatic manual laser tool

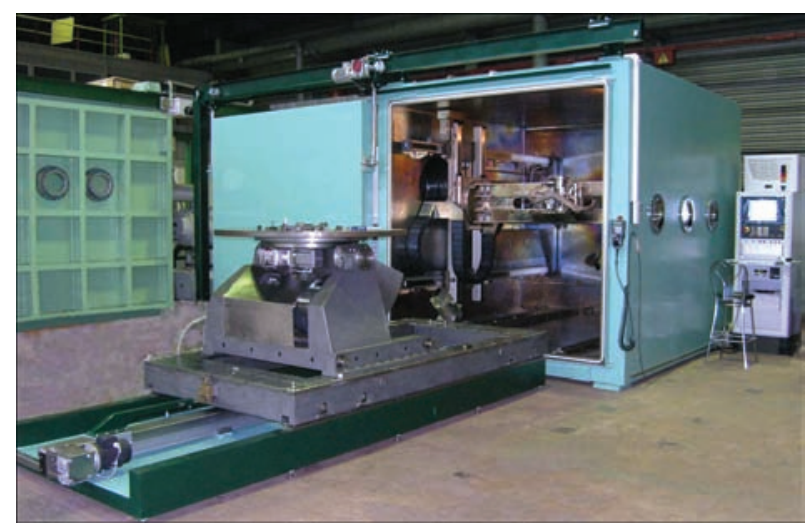

Figure 5. Typical medium-sized chamber with a mobile gun and wheeled-out table

the Institute, which can be divided conditionally into several types by the chambers dimensions, namely «small» $\left(0.26-5.7 \mathrm{~m}^{3}\right)$, «medium» $\left(19-42 \mathrm{~m}^{3}\right)$ and «large» (80-100 $\left.\mathrm{m}^{3}\right)$ (Figure 5). Coming from a definite task of the Customer the chamber type is defined and the appropriate technology of welding is developed [3]. The chambers have the mechanical equipment with a mobile electron beam welding gun on a precision multiaxial mechanism of movement. This mechanism with a digital programmed control provides a controlled linear movement of the gun along three Cartesian coordinates of axes, as well the gun rotation by $0-90^{\circ}$ in plane $Z-X$ (from vertical orientation to horizontal one). The rotation of a part welded is provided by the precision welding manipulators with horizontal and vertical axes of rotation. The most technological flexibility is provided by the manipulator with an inclined axis of rotation, which performs the electron beam welding, for example, of intricate concentric sections of aircraft engines (Figure 6) or aircraft components with a variable geometry.

Depending on a definite application the chamber is completed with high-voltage invertor power sourc-

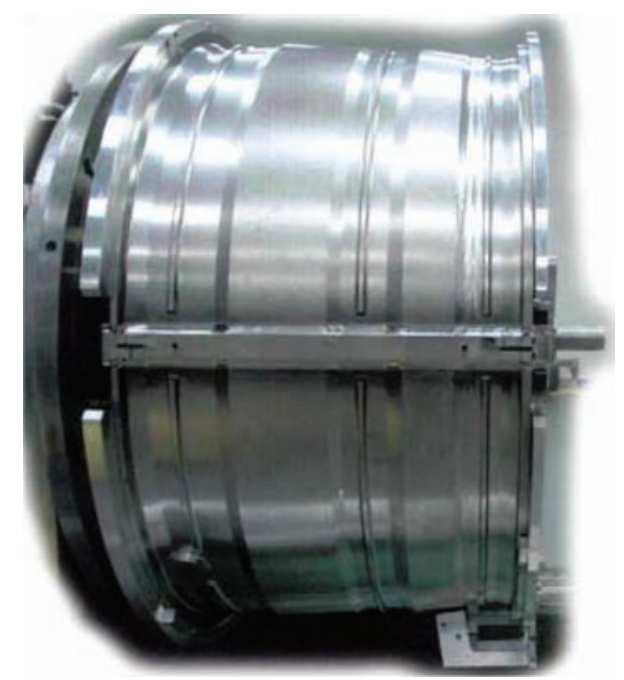

Figure 6. Welded billet of aircraft engine section of titanium alloy VT-6 


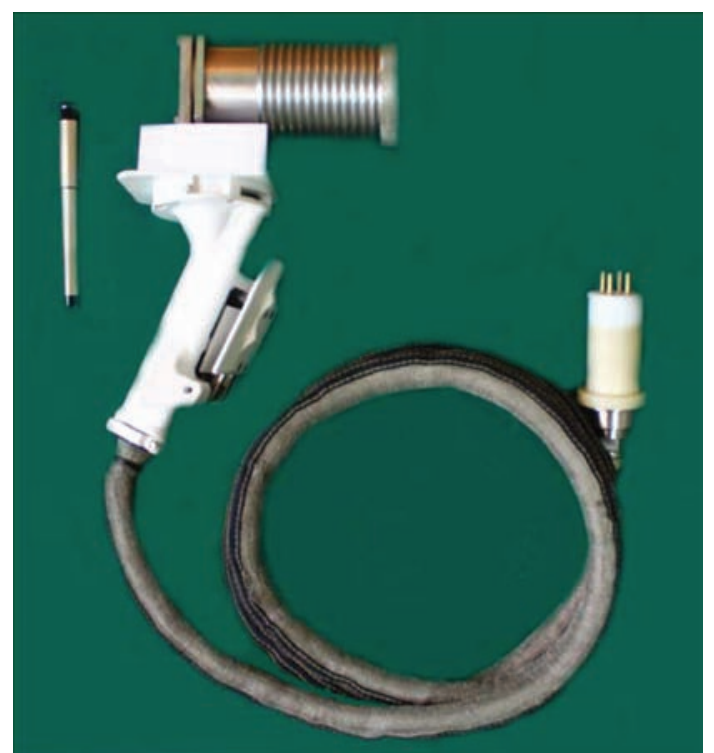

Figure 7. Manual electron beam gun with a high-voltage connector

es of 15,30 and $60 \mathrm{~kW}$ capacity and the system of secondary-emission electron visualization RASTR, which forms the welding zone image both before, during and after finishing of welding. The EBW equipment has a sophisticated system of control, allowing the user communication with equipment through Windows - oriented graphical interface [4].

The Institute has a successful large experience in design and manufacture of electron beam equipment and technologies for the space purpose. The works were carried out for design of the new generation of an electron beam welding tool for making site and repair-restoration works in the open space (Figure 7). The tool is equipped with a triode electron beam gun of up to $25 \mathrm{~kW}$ capacity, separated from the high-voltage power source. Such design solution and application of a flexible high-voltage cable with a compact high-voltage connector for supply allowed decreasing greatly the dimensions and mass of the tool, as well as increasing its manoeuvrability for realizing the technological processes. The service life of continuous operation and safety of the tool was also increased. There is a possibility to obtain a sharply focused beam of $\leq 0.6 \mathrm{~mm}$ diameter. The gun weight is $3 \mathrm{~kg}$. The tool

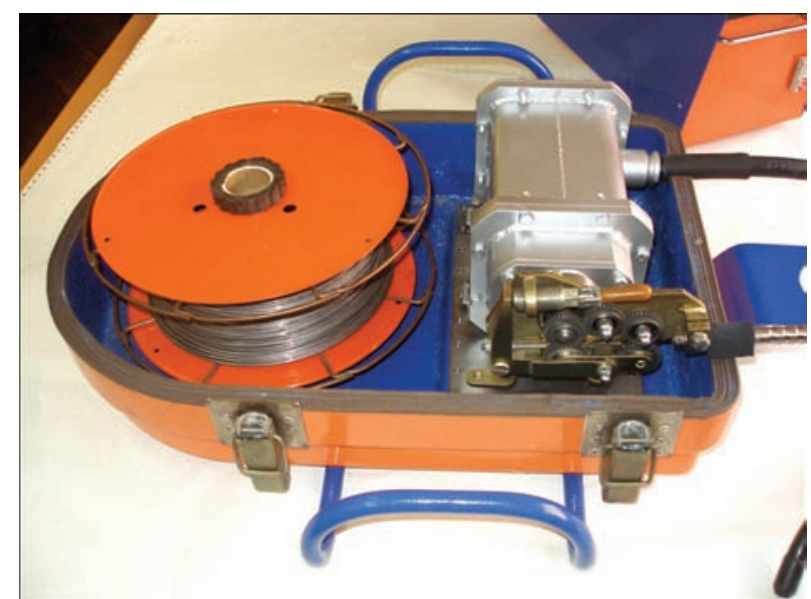

Figure 8. Semi-automatic machine for underwater welding and cutting by flux-cored wire at up to $200 \mathrm{~m}$ depth

can operate in manual and automatic modes with use of the robotic equipment or manipulators.

Traditionally, the Institute pays a lot of attention to the pipe subject. Over the recent years the investigations were carried out and technologies and equipment were developed for the press magnetically-impelled arc butt welding (MIAB) of position welds of high-strength steel pipes with wall thickness of up to $10 \mathrm{~mm}$ and diameter of up to $200 \mathrm{~mm}$. The physical principle of MIAB welding process is characterized by that the arc is moved in the gap between pipe edges being welded under the effect of external magnetic field, generated by the magnetic systems. The welded joint is formed under pressure and simultaneous plastic deformation of pipe edges. The dominating factor, which makes joint, is the presence of a melt layer at the beginning of the upsetting period.

Table 1 presents the mechanical properties of welded joints of pipes of different sizes and steel grades. They meet the requirements of the International standards for gas pipelines. The developed technologies and equipment found the wide application in industry, providing the quality welding both in field and stationary conditions.

The underwater welding is one of the directions of studies and developments of the Institute, where our scientists made important scientific-technical

Таблиця 1. Mechanical properties of pipe welded joints

\begin{tabular}{|c|c|c|c|c|c|c|c|c|}
\hline Steel grade & Pipe size, $\mathrm{mm}$ & $\begin{array}{c}\text { Base metal, } \sigma_{\mathrm{t}} \\
\mathrm{MPa}\end{array}$ & $\begin{array}{c}\text { Welded joint, } \sigma_{\mathrm{t}} \\
\mathrm{MPa}\end{array}$ & $\begin{array}{c}\text { Base metal, } \\
K C V_{+20}, \mathrm{~J} / \mathrm{cm}^{2}\end{array}$ & $\begin{array}{c}\text { Welded joint, } \\
K C V_{+20}, \mathrm{~J} / \mathrm{cm}^{2}\end{array}$ & $\begin{array}{c}\text { Base metal } \\
K C V_{-20}, \mathrm{~J} / \mathrm{cm}^{2}\end{array}$ & $\begin{array}{c}\text { Welded joint } \\
K C V_{-20}, \mathrm{~J} / \mathrm{cm}^{2}\end{array}$ & $\begin{array}{c}\text { Welded joint } \\
K C V_{-40}, \mathrm{~J} / \mathrm{cm}^{2}\end{array}$ \\
\hline $09 \mathrm{G} 2 \mathrm{~S}$ & $42(\delta=5)$ & $\frac{460-478}{469}$ & $\frac{453-478}{465}$ & $\frac{57-5}{58}$ & $\frac{59.0-78.1}{68.5}$ & $\frac{57.8-58.0}{57.9}$ & $\frac{64.0-74.5}{69.3}$ & - \\
\hline 35 & $89(\delta=10)$ & $\frac{538-565}{551}$ & $\frac{528-554}{541}$ & $\frac{56-64}{60}$ & $\frac{52-965}{70}$ & - & - & - \\
\hline 01 Star520 & $191(\delta=7)$ & $\frac{638-665}{651}$ & $\frac{618-674}{656}$ & $\frac{116-154}{135}$ & $\frac{87-152}{119}$ & - & - & - \\
\hline STRG410 & $\begin{array}{c}60.5 \\
(\delta=5.5)\end{array}$ & $\frac{452-464}{458}$ & $\frac{450-462}{456}$ & $\frac{90-98}{94}$ & $\frac{86-92}{89}$ & $\frac{102-104}{98}$ & $\frac{87-94}{91}$ & $\frac{88-94}{92}$ \\
\hline
\end{tabular}



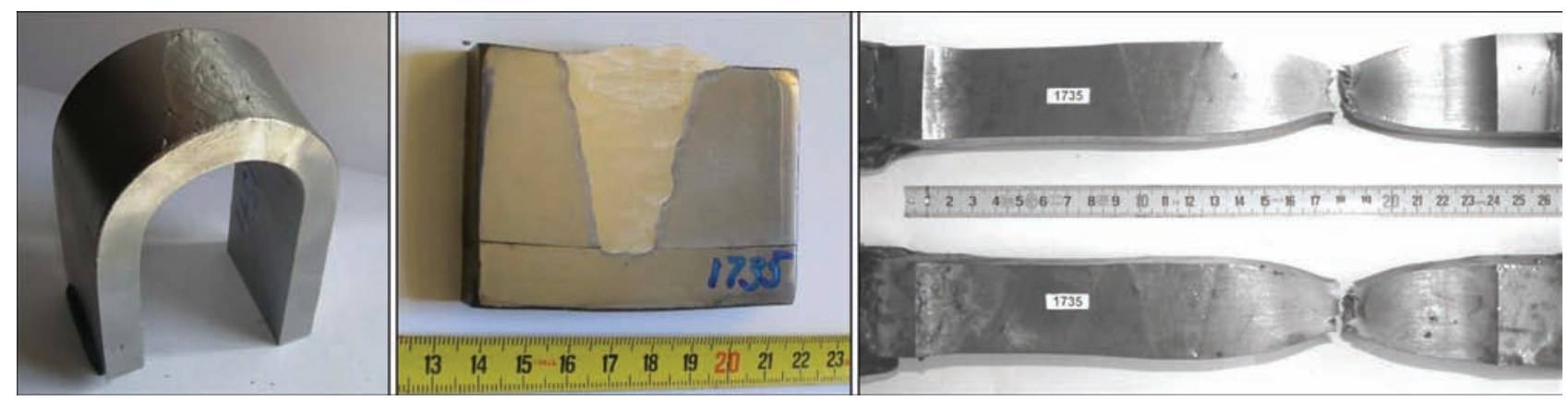

Figure 9. Microsection and specimens after mechanical tests (metal thickness is $40 \mathrm{~mm}$ )

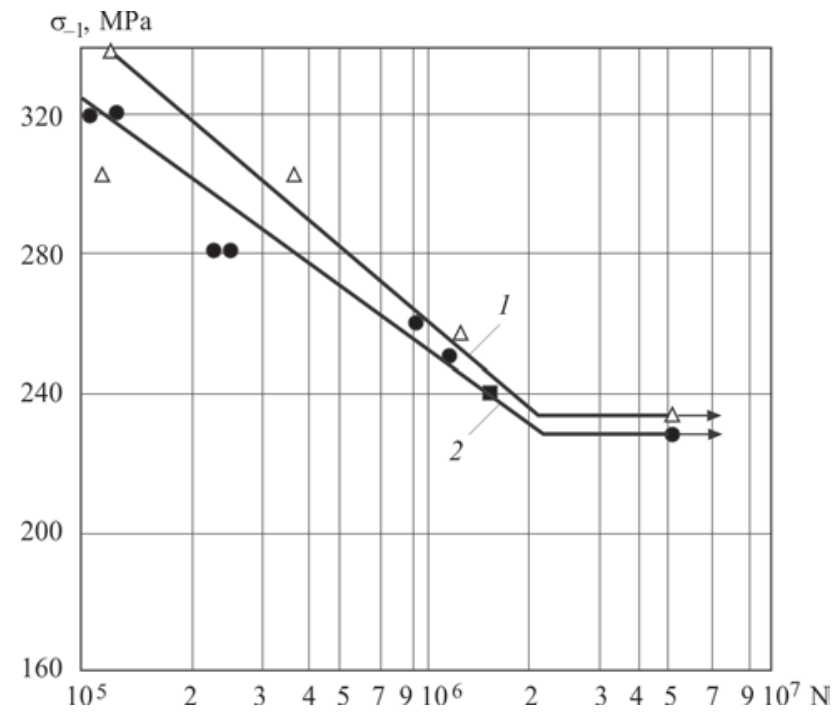

Figure 10. Fattigue strength at alternating-sign bending: 1 - underwater welding; 2 - welding in air

achievements, in particular in the field of welding materials. The carried out theoretical and experimental studies of peculiarities of arc burning under the water and conditions of guarantee of the continuous arc process at different hydrostatic pressure allowed developing the new flux-cored wires and electrodes for the so-called wet welding of low-carbon, low-alloy steels and those of the higher strength [5].

For the arc cutting of steels and alloys under water at the depth of up to $200 \mathrm{~m}$ the electrodes and fluxcored wire, as well as the new type of the semi-au-
Table 2. Mechanical properties of weld metal, produced under water (ANSI/AWSD3.6, class A)

\begin{tabular}{|c|c|c|c|c|c|}
\hline Material & $\begin{array}{c}\sigma_{0.2}, \\
\mathrm{MPa}\end{array}$ & $\begin{array}{c}\sigma_{\mathrm{t}}, \\
\mathrm{MPa}\end{array}$ & $\begin{array}{c}\delta, \\
\%\end{array}$ & $\begin{array}{c}\psi, \\
\%\end{array}$ & $\begin{array}{c}K C V_{-20}, \\
\mathrm{~J} / \mathrm{cm}^{2}\end{array}$ \\
\hline Electrodes & $\geq 460$ & $\geq 600$ & $\geq 29$ & $\geq 47$ & $\geq 100$ \\
\hline Flux-cored wire & $\geq 350$ & $\geq 550$ & $\geq 30$ & $\geq 60$ & $\geq 80$ \\
\hline Steel X60 & 435 & 580 & 18 & - & 60 \\
\hline
\end{tabular}

tomatic machine, which feeding mechanism, located under water beside the diver-welder, were developed (Figure 8). The carried out investigations of mechanical properties of weld metals (Figure 9), the results of which are given in Table 2, proved the high quality of works made by the proposed technology of the underwater welding, because the metal structures safely operate by dozens of years. Studies of results of strength values of welded joints at a cyclic load showed that they are not inferior to joints made under the normal conditions (Figure 10).

Titanium is one of the basic nowadays structural materials, which are used in many branches of industry in manufacture of critical structures. By starting from the development of the technology of welding of a sheet titanium of small thickness at the beginning of the $50 \mathrm{~s}$ of the last century the Institute is continuously and comprehensively dealing with studies of the titanium welding problems. Thus, at the Institute the technology of welding the titanium products with medium and large thicknesses by tungsten electrode into

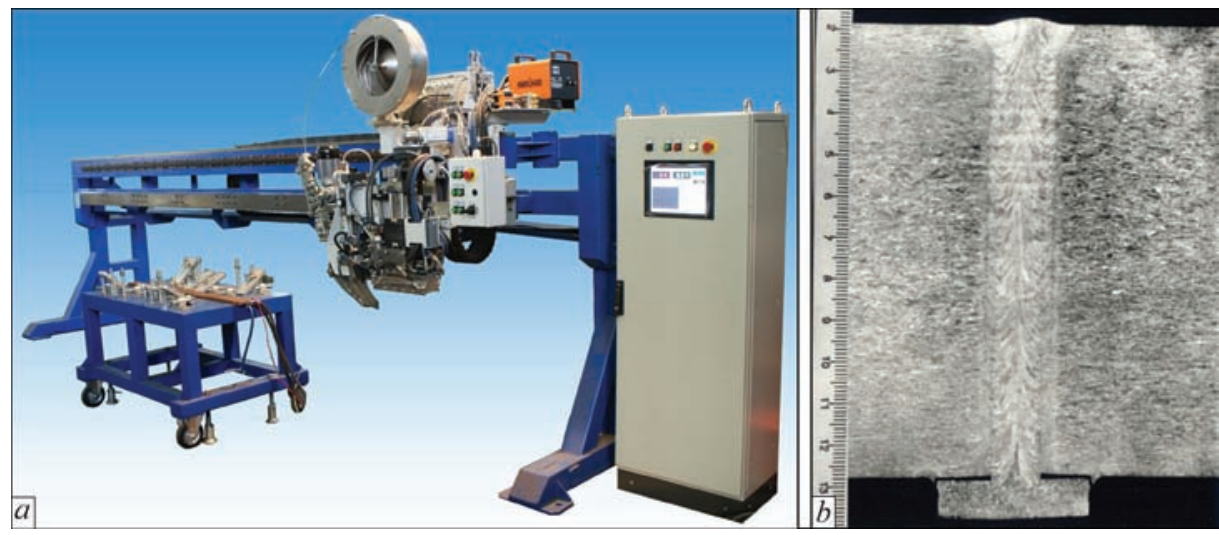

Figure 11. Welding equipment UD 682 for welding and surfacing of parts of up to $110 \mathrm{~mm}$ thickness and up to $4 \mathrm{~m}$ length (a) and macrosection of welded joints $(b)$ 


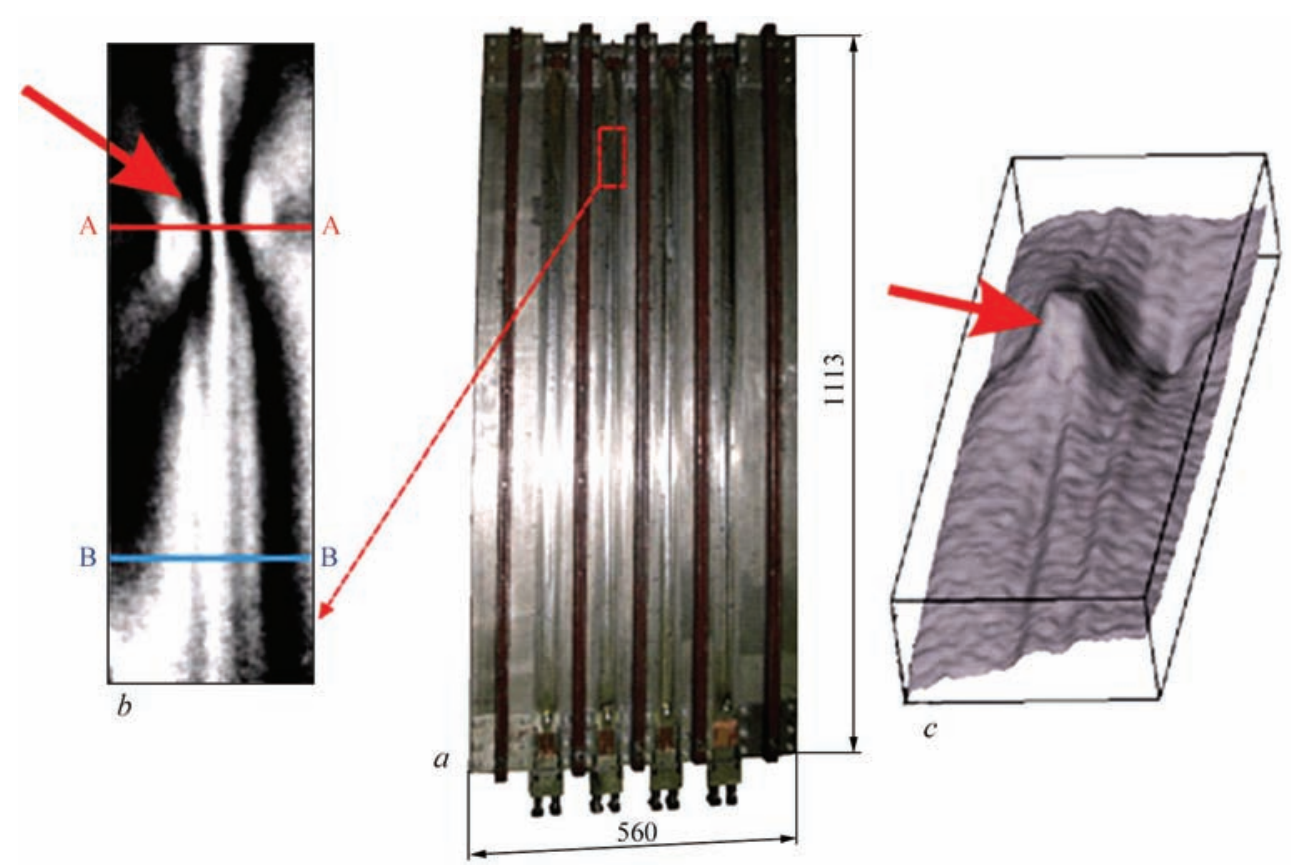

Figure 12. Nondestructive quality testing of stringer panel of alloy VT-20 by electron shearography method: $a$ - panel in mechanical fixture after welding; $b$ - pattern of interference fringes in the examined region; $c$ — three-dimensional pattern of deformed surface

a narrow gap was successfully developed. This technology is distinguished by such advantages as a low consumption of welding wire, small width of weld and heat-affected zone, decrease in angular deformations and residual welding stresses. To guarantee the fusion of side walls with weld, the controllable variable magnetic field was used. The installation UD 682 was designed (Figure 11) for welding and surfacing of joints of up to $110 \mathrm{~mm}$ thickness and length of up to $4 \mathrm{~m}$. By using this equipment the titanium alloys PT3V, VT6, VT20 of various thickness were welded. The investigations of welded joints confirmed their high quality. The strength level of welded joints of titanium VT6 in applying the welding filler titanium wire SPT2 is $95 \%$ of the base metal strength, and the impact toughness $K C U$ of weld metal is $85 \mathrm{~J} / \mathrm{cm}^{2}$. Content of gas impurities in weld metal is at the level of their content in filler wire, which proves the high quality of the gas shielding.

The titanium alloys found wide spreading in the manufacture of structures in aircraft and aerospace industries. At the Institute a complex of investigations was carried out for the development of technology of the deformation-free welding of stringer panels of high-strength titanium alloy VT-20 with a guarantee of their high precision and strength at cyclic loads. It was proved that the making of high-penetration welds by the argon arc nonconsumable electrode welding over the layer of activating flux with applying the preliminary elastic deformation and high-frequency mechanical peening of welds guarantees the higher values of fatigue life of these panels as compared with electron beam and argon arc nonconsumable electrode welding with an immersed arc. Applying before welding the preliminary elastic deformation of a sheet and stiffeners at the level of $(0.3-0.4) \sigma_{y}$ provides relieving the welding deformations and creates the necessary conditions for making the welding process in the automatic mode. It was found, that the effective method of nondestructive testing of panel welds quality is the electron shearography. Figure 12 shows the result of this testing of panel in tension state directly after panel welding. At the general background of deformation of the test region a local distortion is observed, that indicates the defective zone. This is also confirmed by the three-dimensional pattern of the deformed surface. $\mathrm{X}$-ray control confirmed the presence of pores clustering in the anomalous zone. The developed technologies of deformation-free welding and nondestructive testing were recommended for industrial manufacture of the aircraft panels.

One of the main requirements to the structural materials for the aerospace engineering is a specific strength. The aluminium-lithium alloys of different systems of alloying, which have a low density and higher specific strength meet this requirement. However, the application of these alloys in welded structures was limited by the insufficient study of their weldability. At the Electric Welding Institute a complex of works was carried out for investigation of weldability of the aluminium-lithium alloys. As a result, the effective methods of welding, filler material, modified by scandium, were suggested. The effect of welding methods on strength and crack resistance of 

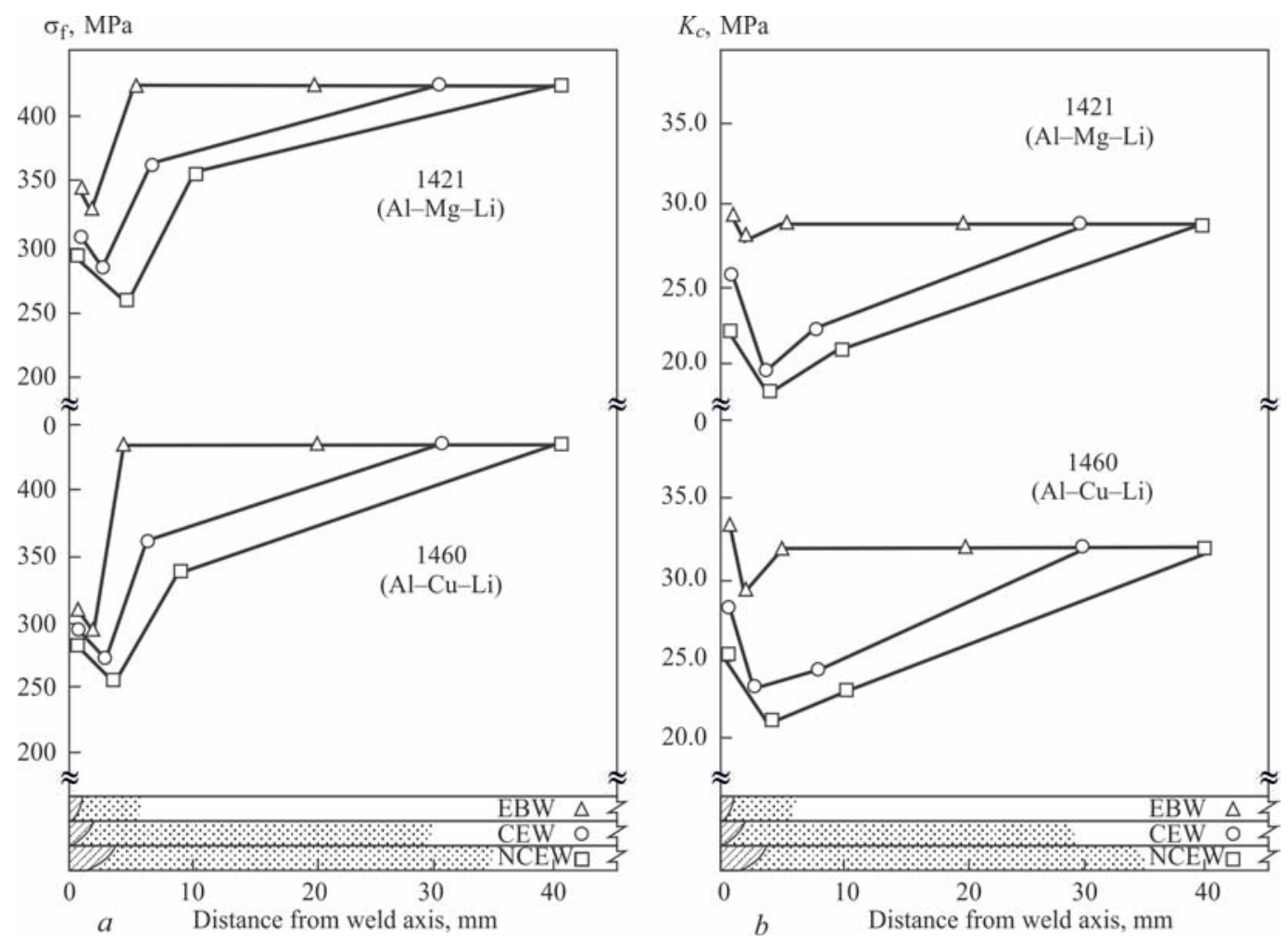

Figure 13. Effect of methods of welding by electron beam (EBW), consumable (CEW) and non-consumable electrodes (NCEW) on fracture strength $\sigma_{\mathrm{f}}(a)$ and crack resistance $K_{c}(b)$ of different zones of joints of aluminium-lithium alloys 1421 and 1460

different zones of joints, made of aluminium-lithium alloys, was investigated (Figure 13).

To solve the problems of geometric and technological adaptation during robotic welding of critical structures (Figure 14), the specialized systems of technical vision were developed at the Institute [10 11]. The welding robots with technical vision systems find automatically a joint and correct in a real time scale the trajectory and welding mode parameters for compensation of errors in assembly and mounting of semi-products. Thus, they perform the welding oper-

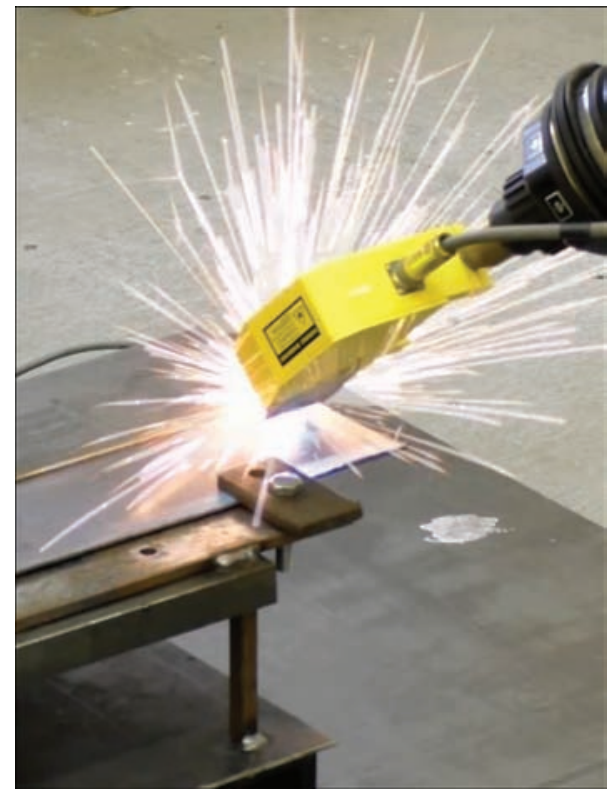

Figure 14. Welding robot with a technical vision system [7] ations completely in the automatic mode. The technical vision systems, developed at the Institute, are successfully applied with robots of such well-known manufacturers as ABB, FANUC, KUKA.

Composite materials and intermetallics find the more demands in many branches of industry, construction, medicine, etc. due to their unique properties. However, their application is restricted by a lack of reliable technologies of producing the permanent joints of structures of dissimilar or new nanostructured materials. The application of traditional methods of welding and brazing do not guarantee the strength characteristics in practice.

To solve this problem, the vapor-phase technologies were developed at the Institute for producing nanostructured materials (NM) with a large-length boundaries of grains, close to materials welded by the chemical composition $[8,9]$. Figure 15 presents structures of some produced nanomaterials on the base of single-phase and heterophase systems. The nanostructured materials have a high plasticity during heating and a low energy of activation of diffusion mobility of alloys. The application of such NM as intermediate layers in the form of foils (Figure 15,a) solves the problem of welding of alloys, based on intermetallics and composites $[10,11]$. Figure $15, b$ presents structure of joints, made of alloys, based on intermetallics $\gamma$-Ti-Al and high-temperature alloy based on nickel (Figure 15, c). The high reaction ability of the nanolayer foils and 


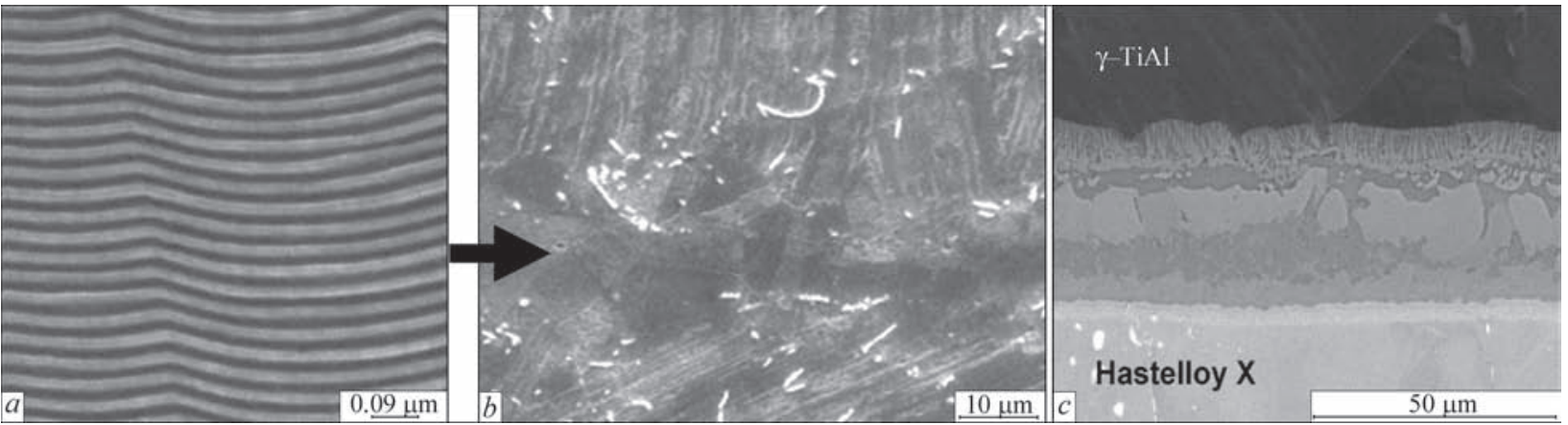

Figure 15. Structure of nanolayer foil and welded joints by applying of nanostructured materials: $a$ - microstructure of cross-section of the nanolayer foil from layers of titanium (dark) and aluminium (light); $b$ - microstructure of joining zone of alloy on $\gamma$-Ti-Al base; $c$ - microstructure of joining zone of alloy on $\gamma$-Ti-Al base and high-temperature $\mathrm{Ni}$

their superplasticity during heating under conditions of external loading give opportunity to realize the reaction brazing processes for a short-time heating of the joining zone at a low pressure. This technological scheme can be used for repair works under conditions of a local heating of the joint zone at a restricted access to power sources and possibility of applying the intensive radiation beams, for example, in the space conditions.

Today, in the production of metal structures many advanced materials are used, but steel is the main structural material. At the Institute, a complex of investigations was carried out to determine the optimum parameters of welding of high-strength steels $10 \mathrm{G} 2 \mathrm{FB}$, microalloyed by vanadium and niobium, as well as steels 12GN2MFAYu, 12GN3MFAYuDR [12, 13]. It was found, that the probability of cold crack formation in welded joints of high-strength steels can be reduced to minimum, if to apply the technology of welding, which provides cooling of joints at the rate $w_{6 / 5}$ of not more than $10{ }^{\circ} \mathrm{C} / \mathrm{s}$, content of diffusive hydrogen in deposited metal of not more than $4 \mathrm{~cm}^{3} / 100 \mathrm{~g}$ and level of residual stresses in steel joints of less than 0,5 of yield limit. Owing to these investigations the reliable and efficient technologies of welding of high-strength low-carbon alloyed steels with yield limit of $1000 \mathrm{MPa}$ and higher were developed. They were used in manufacture of metal roof- ing structures of NSC «Olimpiysky» in Kiev, modern high-capacity tanks for oil storage and other objects.

Reliability is one of the most important qualities, which is characteristic for the present advanced welded structures. To guarantee it, many advanced technologies, developed at the Institute of Electric Welding, were directed. It should be noted among them the technological processes of electrodynamic treatment (EDT) and high-frequency mechanical peening (HMP).

The new technological process, which is used after producing the welded joints, is the electrodynamic treatment by pulses of high-density electric current. It promotes the increase in toughness and refinement of the metal structure, allows decreasing greatly the residual stresses (Figure 16,a) and increasing the fatigue resistance (Figure 16, $b$ ) of welded joints. The designed equipment allows removing the residual welding distortions in thin-walled structure elements (Figure 17). The developed technology and equipment provided the treatment of critical welded joints of ship hull and aircraft structures, which contributes to increase in their life and reliability $[14,15]$.

The studies, carried out at the Institute, proved that the effective decrease in intensity of corrosion-fatigue fracture of welded joints of steel metal structures is guaranteed by applying the technology of high-frequency mechanical peening [16-18]. Influence of in-
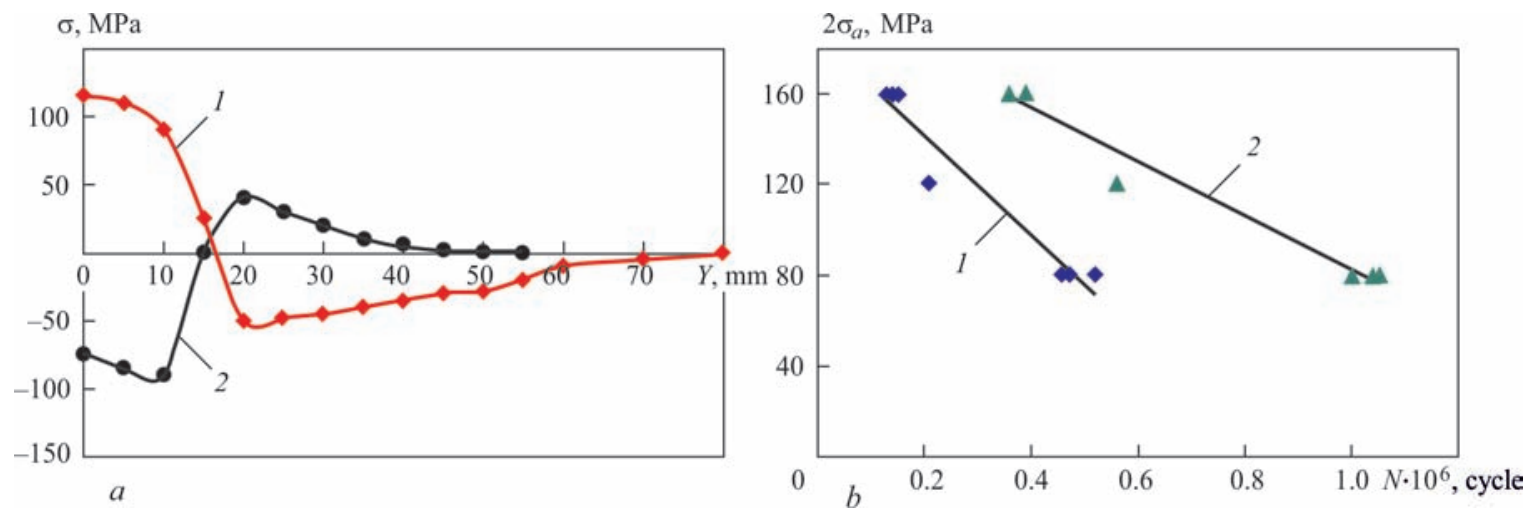

Figure 16. Effect of EDT on residual stresses and life of welded joints of alloy AMg6, made by TIG welding: $a$ - residual stresses before (1) and after (2) treatment; $b$ - results of fatigue tests of welded specimens without (1) and with (2) treatment 


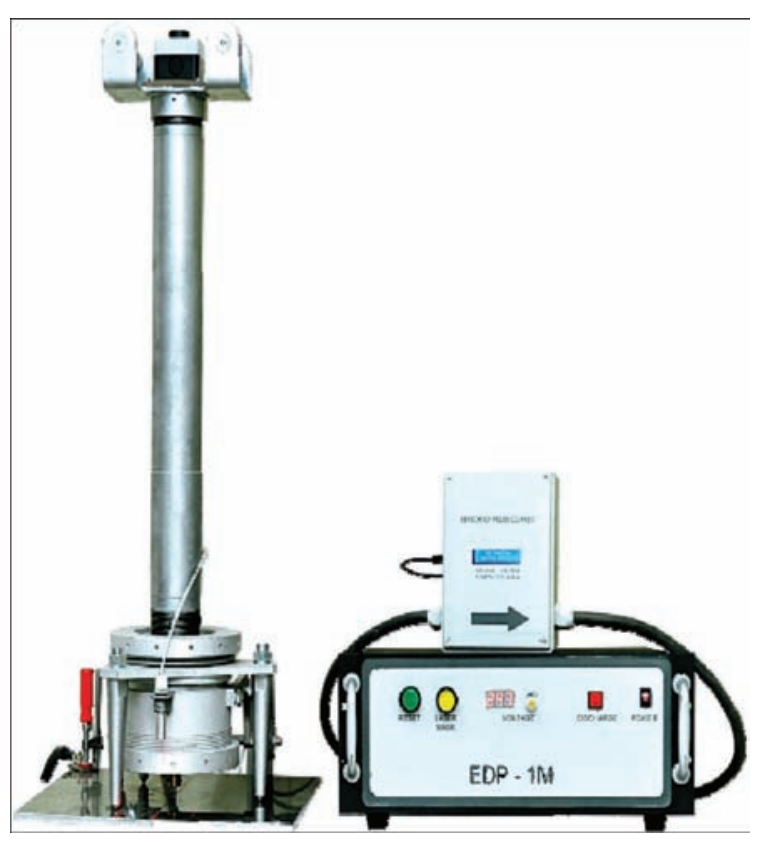

Figure 17. Manual tool and mobile power source for EDT

dustrial atmosphere of moderate climate was modeled by holding of specimens of T-type and butt welded joints of $350 \times 70 \times 12 \mathrm{~mm}$ size of steel $15 \mathrm{KhSND}$ in moisture chamber during $1200 \mathrm{~h}$ at $40{ }^{\circ} \mathrm{C}$ temperature and $98 \%$ air humidity. Fatigue tests of specimens were carried out at the from-zero change in tension at frequency of $5 \mathrm{~Hz}$. The obtained fatigue curves of test joints are given in Figure 18. The test results show that the life limits on the base of 2 million cycles of $\mathrm{T}$ - and butt joints are increased by 47 and $39 \%$, respectively; cyclic life of welded joints is increased up to 7 times depending on levels of applied loads.

Development of reliable and efficient technologies and equipment for improving the wear resistance of

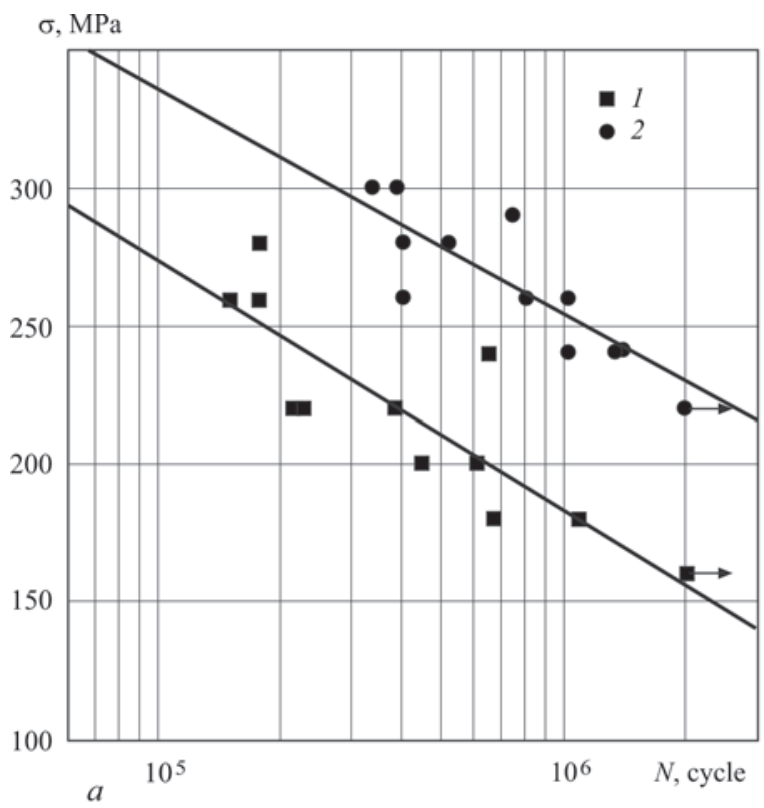

parts occupies traditionally the important place in the research subjects of the Institute. They are demanded for metallurgical industry, power engineering, agricultural machine building, etc. The carried out integrated studies of main regularities of effect of technological parameters of arc surfacing on the principles of structure formation and change in physical-mechanical properties of deposits by layers depending on content of carbon in wheel steels, which varies in the range from 0.55 up to $0.75 \%$, promoted the development of the new technology of arc surfacing for restoration of wheel pairs of cargo railway cars [19], which contains the method and modes of surfacing, requirements for wheels preparation, chemical composition of materials being surfaced, preheating, the temperature of which depends on the carbon content in the wheel steel. The important feature of this technology is a slow cooling after surfacing at a rate $\leq 35-40{ }^{\circ} \mathrm{C}$ during $4-5 \mathrm{~h}$. The application of the new technology allowed increasing the impact toughness of HAZ metal and resistance of deposited metal to brittle fracture by up to $2-3$ times. The safe service life of the railway wheels was increased by two times.

The works of the Institute in the field of brazing, which started at the beginning of the $60 \mathrm{~s}$ of last century, contributed greatly to this trend of works. The developed scientific fundamentals of vacuum brazing of thin-walled structures from stainless steels of different classes were used for manufacture of critical-purpose products, such as honeycomb panels, antennas, etc. Nowadays, the study of physical-metallurgical processes, occurring during high-temperature vacuum brazing of high-temperature dispersion-hardened nickel alloys, the regularities of structure formation of

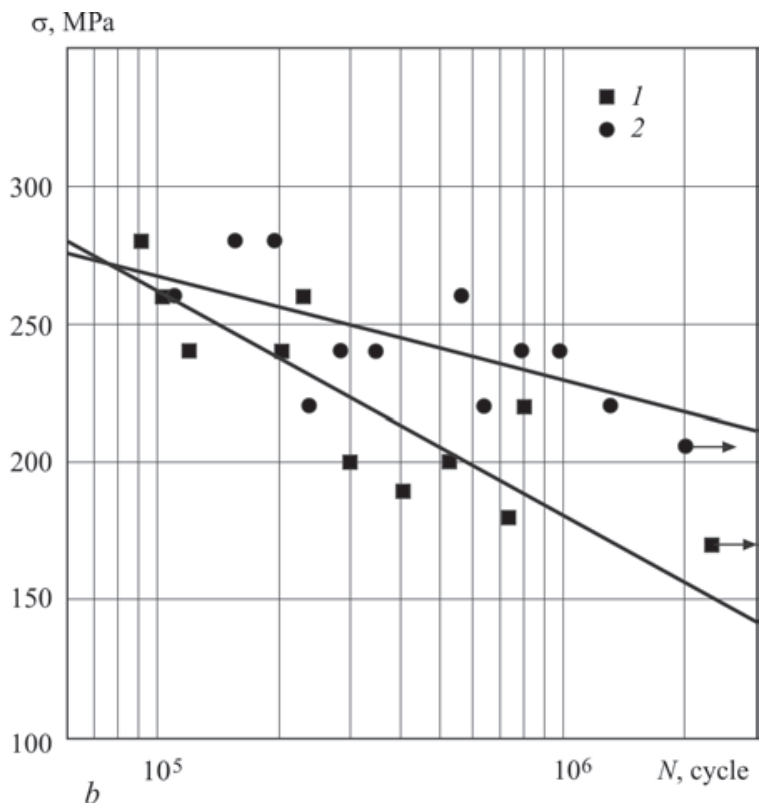

Figure 18. Fatigue curves of T- $(a)$ and butt $(b)$ welded joints of steel 15KhSND under effect at industrial atmosphere of moderate climate for $1200 \mathrm{~h}:: 1$ — in initial state; 2 — after treatment by HMP method 


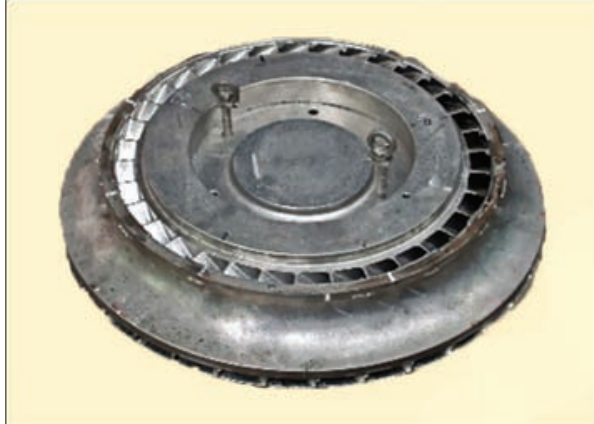

Figure 19. Gas turbine engine compressor centrifugal wheel, manufactured by applying the new brazing alloy

brazed joints allowed developing the $\mathrm{Ni}-\mathrm{Pd}-\mathrm{Cr}-1 \mathrm{Ge}$ systems. As a depressant the germanium was used, which provides the structure of solid solution, based on palladium, in brazed welds. The brazed joints have a stable static strength of 1230-1290 MPa at room temperature and $1000-1030 \mathrm{MPa}$ at $550{ }^{\circ} \mathrm{C}$ temperature, that is two times higher in comparison with an industrial brazing alloy. With the application of the developed brazing alloy the centered wheels of axial-flow compressor of gas turbine engine of dispersion-hardened nickel alloy (Figure 19), etc. are manufactured.

The successful progress of welding technologies and manufacture of long-life reliable structures of critical purpose is impossible without application of flaw detection and advanced methods of nondestructive testing of the welded joints quality.

The great achievement of the recent years is the design of a portable digital X-ray-TV equipment (Figure 20) on the base of high-sensitive solid-body converters. The portability, digital processing of images, low cost open up the new opportunities for carrying out the radiographic control in the field and shop conditions of different objects, which have no today possibilities of nondestructive testing. The application of the portable X-ray-TV complex helps to solve the problems of control of numerous gas-, oil- and hydro-distributing pipelines of small diameter, technological pipelines of petrochemical production.

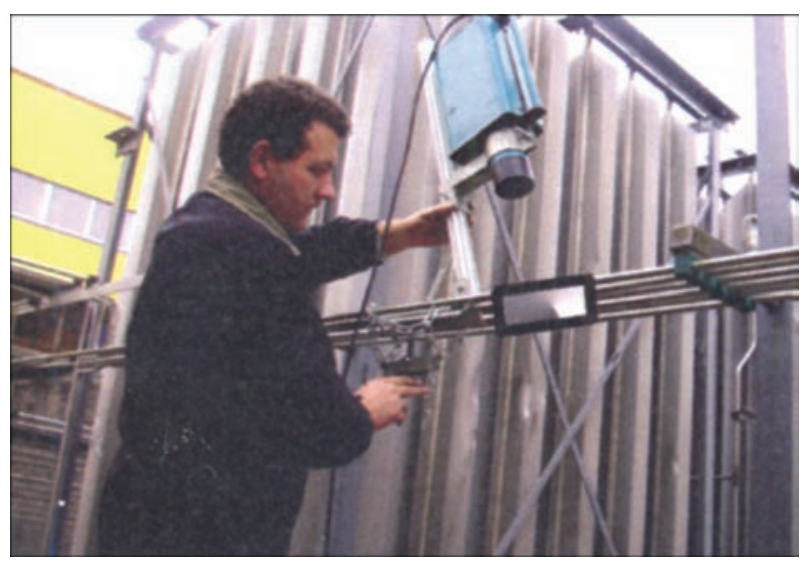

Figure 20. Portable digital X-ray-TV equipment arranged on object

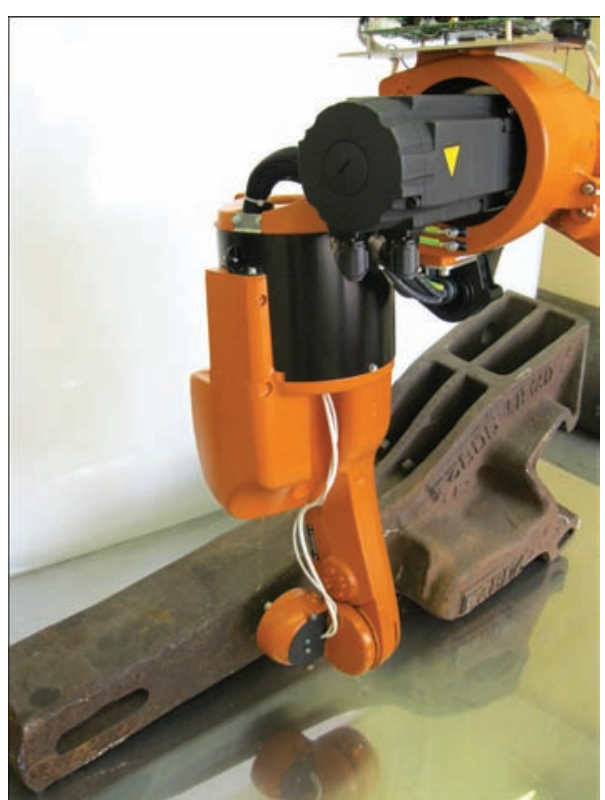

Figure 21. Robot with technical vision system for nondestructive testing of intricate geometry products [20]

Automation and robotization of the processes of nondestructive testing give an opportunity of increasing greatly the validity of taken decision about the imperfectness of products and excluding the human factor influence. A complex was designed for the nondestructive testing of products of intricate geometry, which included an industrial robot and technical vision system (Figure 21). Identification of geometry of the control object $(\mathrm{CO})$ and scanning of its surface by eddy-current sensors are made by the complex Kaskad without human participation. It is capable: to define the position of $\mathrm{CO}$ by means of the technical vision system; to keep automatically the fixed interval between the sensors and $\mathrm{CO}$; to stabilize the movement rate of the eddy-current converter on the $\mathrm{CO}$ surface; to form the certificate of object imperfection

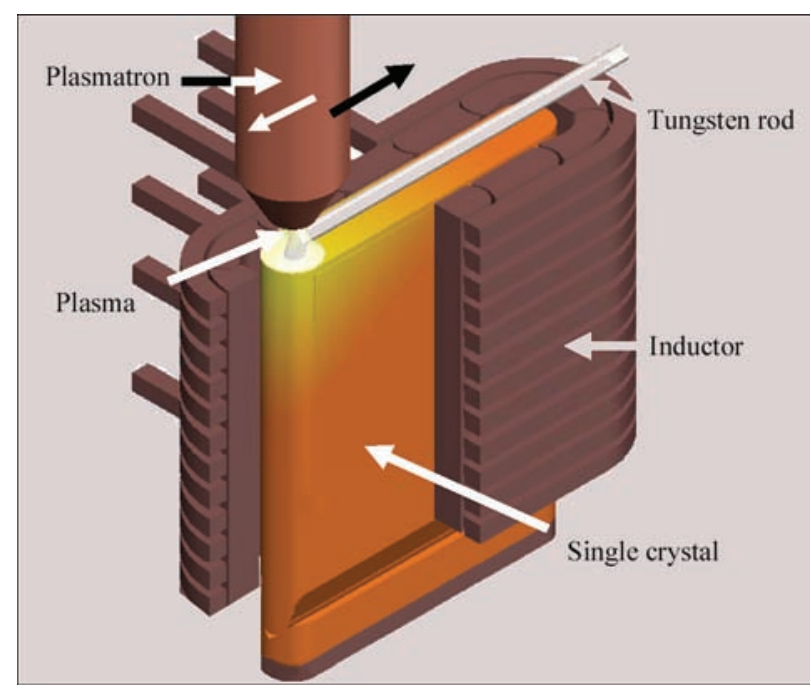

Figure 22. Scheme of equipment for growing single crystals of refractory metals 


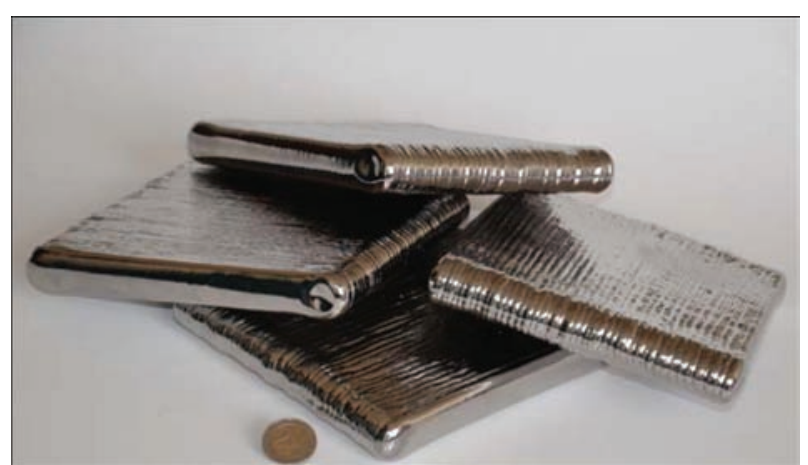

Figure 23. Single crystals of tungsten

(defects, defining the space coordinates of defects; to guarantee the high efficiency of control.

At the Institute, the metallurgical direction: special electrometallurgy continues the successful development, guaranteeing the high-quality steel production. However, its capabilities are no exhausted by this. It was occurred that by using the special electrometallurgy it is possible to grow single crystals of large sizes, which cannot be made by the traditional technology. The radically new methods of growing single crystals of refractory metals, developed at the Institute, are successfully realized in practice, where two different sources of electric heating, i.e. plasma-arc and induction [21], are used simultaneously (Figure 22). The plasma-arc source realizes the remelting of consumable material (rod) and forms a single-crystal body of a preset configuration, the induction source maintains a local metal pool from flowing out and creates a necessary temperature field in crystal, the crystal is growing at high temperature of preheating $(0.5-0.6) T_{\mathrm{m}}[22]$. As a result the stresses and density of dislocations are decreased (less than $10^{6} \mathrm{~cm}^{-2}$ ) in the growing crystal, that promotes the formation of more perfect single crystal structure. The single crystal preheating up to preset temperature is one of the key elements of this technology. The unique installation for production of large single crystals of tungsten and molybdenum in the form of plates of $20 \times 170 \times 160 \mathrm{~mm}$ size was designed (Figure 23). This technology is referred to the high-level additive technologies [23].

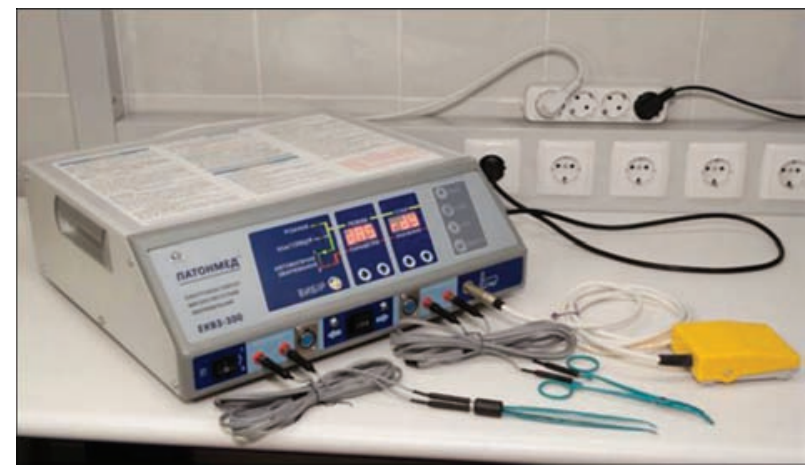

Figure 24. Apparatus EKVZ -300 Patonmed for welding of live tissues

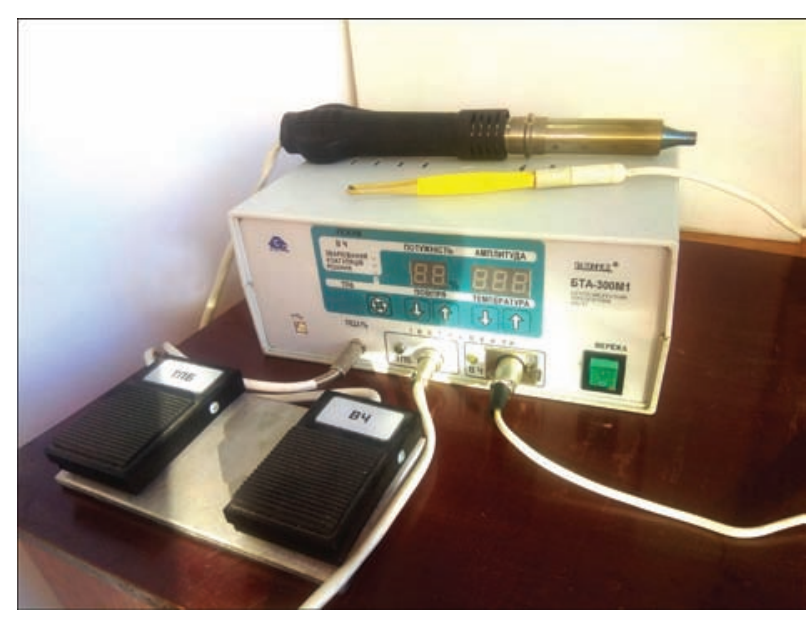

Figure 25. Multifunctional thermosurgical apparatus EK-300 M1 Patonmed and surgical instruments

Welding, remaining as one of the main technological processes in different branches of industry, intruded into the new for it sphere: medicine. And now we can state that the dream of surgeons about quick and bloodless cutting and joining of live tissues without use of a sewing material was realized.

The Institute in the creative cooperation with leading medical establishments of Ukraine developed the technology and equipment (Figure 24) of high-frequency welding of soft live tissues [24-28]. At the given moment of time 200 different surgical procedures were developed and implemented, by which annually 35-40 thou operations are carried out in such fields as abdominal and thoracic surgery, traumatology, pulmonology, proctology, urology, mamaliology, ophthalmology, neurosurgery, etc. The developed apparatuses and instruments for the high-frequency welding of live tissues continue their improvement. It should be noted that many countries in the world showed interest to this technology.

And by this we did not stop. At the Institute a new process of nocontact convective-infrared treatment of live tissues has been developed. It passed the comprehensive checking and displayed its effectiveness. By its application both the emergency aid and also specialized surgical aid are given. It gives opportunity to stop bleeding from parenchimatous organs, spongy bones and small diameter vessels, sanation of infected and chronic purulent wounds in traumata, coagulation of tissue for making bloodless cutting, thermoablation of tumors and metastases. Each of mentioned technologies has its advantages and its applications. Their combination in one apparatus allows increasing the opportunities of the surgeons (Figure 25).

This far for the complete review of our works shows that on the base of the problem-oriented fundamental studies the new scientific technologies and equipment are successfully developed at the E.O. Pa- 
ton Electric Welding Institute. We will continue also in future to work actively for such studies and developments which will be challenging and demanded at the world welding market.

1. Korzhik, V.N., Pashchin, N.A., Mikhoduj, O.L. et al. (2017) Comparative evaluation of methods of arc and hybrid plasmaarc welding of aluminum alloy 1561 using consumable electrode. The Paton Welding J., 4, 30-34.

2. Korzhik, V.N., Sydorets, V.N., Shanguo Han, Babich, A.A. (2017) Development of a robotic complex for hybrid plasmaarc welding of thin-walled structures. Ibid., 5, 62-70.

3. Hamm, R.W. (2008) Reviews of accelerator science and technology. Industrial Accelerators, 1, 163-184.

4. Paton, B.E., Nazarenko, O.K., Nesterenkov, V.M. et al. (2004) Computer control of electron beam welding with multicoordinate displacements of the gun and workpiece. The Paton Welding J., 5, 2-5.

5. Maksimov, S. (2017) E.O. Paton Electric Welding Institute activity in the field of underwater welding and cutting. Pidvodni Tekhnologii, 6, 37-45.

6. Paton, B.E., Lebedev, V.A., Maksimov, S.Yu. et al. (2011) Improvement of equipment for underwater mechanized and automated welding and cutting with flux-cored wire. Svarka $i$ Diagnostika, 5, 54-59 [in Russian].

7. Shapovalov, E.V., Dolinenko, V.V., Kolyada, V.A. et al. (2016) Application of robotic and mechanized welding under disturbing factor conditions. The Paton Welding J., 7, 42-46.

8. Ustinov, A.I., Polishchuk, S.S., Demchenkov, S.A., Petrushinets, L.V. (2015) Effect of microstructure of vacuumdeposited Fe100- $x \operatorname{Nix}(30<x<39)$ foils with FCC structure on their mechanical properties. J. Alloys and Compounds, 622, 54-61.

9. Ustinov, A. I. (2008) Dissipative properties of nanostructured materials. Strength of Materials, 40, 571-576.

10. Ustinov, A., Falchenko, Yu., Ishchenko, A. (2008) Diffusion welding of $\gamma$-TiAl alloys through nano-layered foil of Ti/Al system. Intermetallics, 16, 1043-1045.

11. Ustinov, A., Falchenko, Yu., Melnichenko, T. (2013) Diffusion welding of aluminum alloy strengthened by $\mathrm{Al} 2 \mathrm{O} 3$ particles through an $\mathrm{Al} / \mathrm{Cu}$ multilayer foil. J. of Materials Processing Technology, 213(4), 543-552.

12. Zhdanov, S.L., Poznyakov, V.D., Maksimenko, A.A. et al. (2010) Structure and properties of arc-welded joints on steel 10G2FB. The Paton Welding J., 11, 8-12.

13. Poznyakov, V.D., Zhdanov, S.L., Sineok, A.G. et al. (2011) Experience of application of S355J2 steel in metal structures of the roofing over NSC «Olimpijsky» (Kiev). Ibid., 6, 45-46.

14. Lobanov, L.M., Paschin, N.A., Mihoduy, O.L. (2014) Repair the AMg6 aluminum alloy welded structure by the electric processing method. Weld Research and Application, 1, 55-62.
15. Lobanov, L.M., Pashchin, N.A., Savitsky, V.V., Mikhoduj, O.L. (2014) Investigation of residual stresses in welded joints of heat-resistant alloy ML10 using electrodynamic treatment. Problemy Prochnosti, 6, 33-41 [in Russian].

16. Knysh, V.V., Solovei, S.A., Kadyshev, A.A., Nyrkova, L.I., Osadchuk, S.A. (2017) Influence of high-frequency peening on the corrosion fatigue of welded joints. Materials Sci., 53, $7-13$.

17. Daavary, M., Sadough Vanini, S.A. (2015) Corrosion fatigue enhancement of welded steel pipes by ultrasonic impact treatment. Materials Letter, 139, 462-466.

18. Fan, Y., Zhao, X., Liu, Y. (2016) Research on fatigue behavior of the flash welded joint enhanced by ultrasonic peening treatment. Materials \& Design, 94, 515-522.

19. Gajvoronsky, O.A., Poznyakov, V.D., Klapatyuk, A.V. (2014) Method of restoration of high-carbon steel products. Pat. 107301, Ukraine [in Ukrainian].

20. Dolinenko, V.V., Shapovalov, E.V., Skuba, T.G. et al. (2017) Robotic system of non-destructive eddy-current testing of complex geometry products. The Paton Welding J., 5-6, $51-57$.

21. Paton, B.E., Shapovalov, V.A., Grigorenko, G.M. et al. (2016) Plasma-induction growing of profiled single crystals of refractory metals. Kiev, Naukova Dumka [in Russian].

22. Shapovalov, V.A., Yakusha, V.V., Nikitenko, Yu.A. et al. (2014) Studying the temperature field of profiled tungsten single-crystals produced by plasma-induction process. Sovrem. Elektrometall., 3, 31-35 [in Russian].

23. Shapovalov, V.A., Yakusha, V.V., Gnizdylo, A.N., Nikitenko, Yu.A. (2016) Application of additive technologies for growing large profiled single crystals of tungsten and molybdenum. The Paton Welding J., 5-6, 134-136.

24. (2009) Tissue-saving high-frequency electric welding surgery. Ed. by B.E. Paton, O.N. Ivanova. Kiev, PWI, IAW [in Russian].

25. Paton, B.E., Krivtsun, I.V., Marinsky, G.S. et al. (2013) High-frequency welding and thermal treatment of live tissues in surgery. Nauka i Praktyka, 1, 25-39 [in Russian].

26. Paton, B.E., Marinsky, G.S., Podpryatov, S.E. et al. (2012) Welding high-frequency electrocoagulator EKVZ-300. Pat. 72577U, Ukraine, Int. Cl. A61 B 18/12 [in Ukrainian].

27. Paton, B.E., Krivtsun, I.V., Marinsky, G.S. et al. (2013) Welding, cutting and heat treatment of live tissues. The Paton Welding J., 10-11, 142-153.

28. Paton, B.E., Tkachenko, V.A., Marinsky, G.S., Matviichuk, G.M. (2014) Method of joining human and animal biological tissues using high-frequency current. Pat. 106513, Ukraine [in Ukrainian]. 


\title{
THE FSW TECHNOLOGY OF NON-FERROUS METALS - PROCESS CONDITIONS AND EXAMPLES OF APPLICATION
}

\author{
A. PIETRAS, A. WEGLOWSKA and B. RAMS \\ Poland Institute of Welding \\ 44-100, Gliwice, Str. Bl. Czeslawa 16-18, Poland. E-mail: adam.pietras@is.gliwice.pl
}

\begin{abstract}
The paper presents welding conditions and the process of creating a FSW weld during welding elements made of wrought aluminium alloys and cast aluminium alloy. Investigations were conducted at Institute of Welding, Gliwice (Instytut Spawalnictwa), on the FSW stands equipped with vertical milling machine adapted for friction stir welding process. Influence of the tool's dimension and shapes on the plasticization process of welded material and formation of a weld behind the tool, at different welding set of parameters were studied. During the investigations it was noticed, that forces and torque occuring during FSW and stiffness of the clamping system influence the temperature of the welding area, the plasticity of the material, the particles fragmentation and the quality of the joints. On the basis of research correct welding conditions were selected and process of welding was conduct using specially designed instrumentation. The views of exemplary friction stir welded elements and macrosections from the welding areas are shown. 14 Ref., 4 Tables, 16 Figures.
\end{abstract}

Ke ywords : Friction stir welding, wrought and cast aluminium alloys, copper tool dimension

Introduction. FSW (Friction Stir Welding) is the method that makes it possible to successfully join aluminium alloys, including casting alloys and copper alloys, which are difficult to weld using known welding techniques. This method is increasingly used in the world mainly in the shipbuilding, rail, automotive and building industries.

In FSW process the stirring and rotating tool penetrating material along the line of welding is applied for the friction heating and softening of the material. After the tool is put into rotation and frictional heating is softening the material around the probe, the tool is traversed along the joint line. The heated and plasticised materials of the components being welded are extruded around the tool probe backwards, where before cooling down they are stirred and upset by the shoulder. In the centre of the weld the zone called «nugget» is formed behind the tool in the result of the stirring of the softened metals of both parts being welded $[1,2]$.
The shape and dimensions of the weld depend on the shape and size of the stirring tool and applied welding parameters. The tool penetrating welding zone has three basic functions: heating metal in the area of joining, stirring of materials and upsetting of plasticised metal behind the tool in the phase of weld forming. Basic parameters of FSW process comprise:

- tool rotation speed $-\omega\left(\mathrm{mm}^{-1}\right)$;

- welding speed $-V_{\mathrm{zg}}(\mathrm{mm} / \mathrm{min})$;

- tilt angle $-\alpha\left[^{\circ}\right]$;

- tool type and size of the tool: probe diameter $-d(\mathrm{~mm})$, shoulder diameter $-D(\mathrm{~mm})$, probe length $-1(\mathrm{~mm})$.

During FSW process, material of the workpiece subjected to mechanical stirring and intensive plastic deformation at higher temperature, spreads out also outside the stirring zone (thermomechanicaly affected zone). Additionally, the significant temperature gradient occurs across the weld outside the thermomechanicaly affected zone (in the heat affected zone). As the

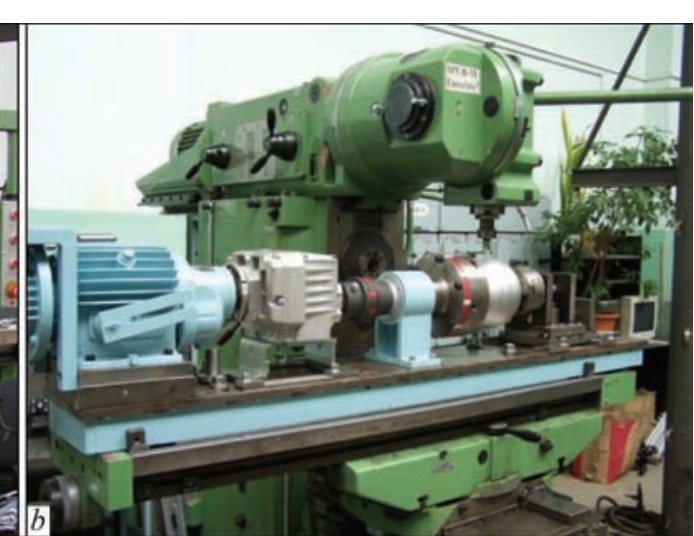

Figure 1. Station for FSW welding process: $a$ - tooling for plate welding; $b$ - tooling for cylindrical elements 
Table 1. Tools used during studies

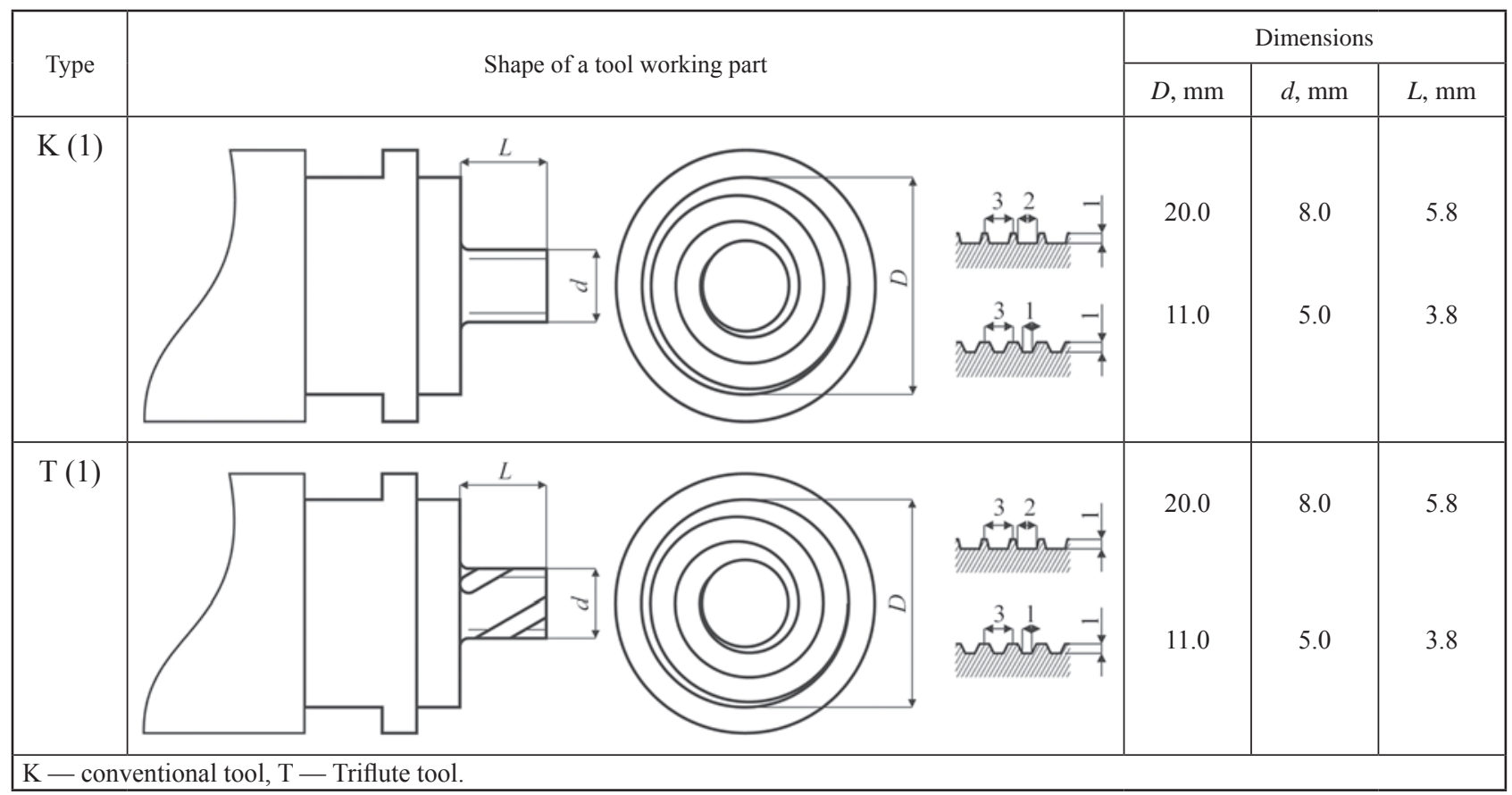

result of those factors more complex structure is being formed in the cross-section of the weld, which influences directly its mechanical properties [3-5]. In case of aluminium casting alloys welding, the changes in the microstructure are associated both with processes of deforming, recovery and recrystallization and also with microstructure component phase changes, e.g. with precipitation or dissolving of intermetallic phases [6]. The aim of this research was finding the relation between welding conditions: welding parameters, a tool shape, the process of forming of friction welded joint and welding quality.

Research station. The investigation into FSW process was conducted at Instytut Spawalnictwa on the vertical milling machine FYF32JU2 equipped with special clamps fixing the welded parts and special ISstir head constructed at Instytut Spawalnictwa for the measuring of torque and friction force. The station for testing of linear welding of plates is shown in Figure 1, $a$, while Figure $1 \mathrm{~b}$ presents the station for welding of cylindrical or spherical elements, equipped with a special positioner. Some of the testing was con- ducted on the station build on the basis of numerical milling machine FNC 50NC.

Tools. During research, the impact of several tools having different shapes and dimensions of working parts, on the process and welding quality was tested for various configuration of components being welded. The tools were made of high-speed steel S 6-5-2 [7]. The shapes and dimensions of the tools used in research are shown in Table 1.

Tested material. During FSW, forces and weld forming process were tested for wrought aluminium alloys, casting alloy and copper M1. Chemical composition of aluminium alloys used in the research is presented in Table 2.

Research into FSW process. Testing of the tool effect on welding quality. Research into the influence of tool shape and dimensions on FSW quality was conducted using tools for various welding parameters, enumerated in Table 1. Tests were conducted for wrought aluminium alloys, casting alloys, copper M1 as well as wrought alloy welded together with casting alloy. Obtainment of the correct weld is difficult, in

Table 2. Chemical composition of aluminium alloys used during studies $[8,9]$

\begin{tabular}{|c|c|c|c|c|c|c|c|c|c|c|c|}
\hline \multirow{2}{*}{ No. } & \multicolumn{2}{|c|}{ Alloy denotation } & \multicolumn{9}{|c|}{ Content of chemical elements, $\%$} \\
\hline & Numerical & Chemical symbol & $\mathrm{Si}$ & $\mathrm{Cu}$ & $\mathrm{Mg}$ & Mn & $\mathrm{Fe}$ & $\mathrm{Ti}$ & $\mathrm{Zn}$ & $\mathrm{Ni}$ & $\mathrm{Al}$ \\
\hline 1 & $\begin{array}{c}\text { EN AC-43200 } \\
\left(\text { AK9) }{ }^{*}\right.\end{array}$ & EN AC-Al Si10Mg(Cu) & $9.0-11.0$ & 0.35 & $0.20-0.45$ & 0.55 & 0.65 & 0.20 & 0.35 & 0.15 & Rest \\
\hline 2 & $\begin{array}{l}\text { EN AW-2017A } \\
\left(\text { PA6) }{ }^{* *}\right.\end{array}$ & EN AW-Al Cu4MgSi & $0.2-0.8$ & $3.5-4.5$ & $0.40-1.00$ & $0.40-1.00$ & 0.70 & 0.15 & 0.25 & - & Same \\
\hline 3 & $\begin{array}{c}\text { EN AW-6082 } \\
\text { (PA4) }\end{array}$ & EN AW-Al SiMgMn & $0.7-1.3$ & 0.1 & $0.60-1.20$ & $0.40-1.00$ & 0.50 & 0.10 & 0.20 & - & $\gg$ \\
\hline
\end{tabular}


Table 3. Welding parameters

\begin{tabular}{|c|c|c|c|l|}
\hline \multirow{2}{*}{ Parameter No. } & \multirow{2}{*}{$\begin{array}{c}\text { Material from advanc- } \\
\text { ing side }\end{array}$} & \multicolumn{2}{|c|}{ Tool movement parameters } & \multirow{2}{*}{ Notes } \\
\cline { 3 - 4 } & & $\omega, \mathrm{min}^{-1}$ & \multirow{2}{*}{$V_{\mathrm{zg}}, \mathrm{mm} / \mathrm{min}$} & \\
\hline 1 & EN AC-43200 & 560 & 280 & Correct weld face. Linear discontinuity \\
\hline 3 & EN AC-43200 & 900 & 180 & Correct weld face and structure \\
\hline 6 & EN AW-2017A & 560 & 280 & Correct weld face. Linear discontinuity \\
\hline 9 & EN AW-2017A & 450 & 560 & Correct weld face and structure. Very small imperfections \\
\hline 10 & EN AW-2017A & 900 & 560 & Correct weld face. Linear discontinuity \\
\hline 11 & EN AW-2017A & 560 & 280 & Uneven weld surface. Correct weld structure \\
\hline 14 & EN AW-2017A & 450 & 180 & Correct weld face and structure \\
\hline 15 & EN AW-2017A & 900 & 560 & Correct weld face. Linear discontinuity \\
\hline 16 & EN AC-43200 & 900 & 180 & Correct weld face. Linear discontinuity \\
\hline 19 & EN AC-43200 & 900 & 560 & Incorrect weld material stirring. Large wormhole cavity \\
\hline
\end{tabular}

particular in case of welding of dissimilar materials. Selected sets of parameters used while welding of wrought alloy EN AW-2017A + casting alloy EN AC43200 are shown in Table 3.

During testing the process of weld face forming, material subjected to the strains and deformations, quality of the surface and weld structure were analysed. The example of a weld surface is shown in Figure 2.

Testing of friction forces. In FSW process the change of mechanical energy into friction heat occurs, what makes it possible to create the conditions for welding in solid state. In this case the coefficient of friction between the tool metal and the welded plates surface is essential.

During the first stage of research into the friction phenomenon in the FSW process the impact of friction speed on friction coefficient was tested. Aluminium plates were initially preheated up to the temperature of: $350,450,500$ and $550^{\circ} \mathrm{C}$, i.e. up to the temperature occurring in the FSW process. While measuring, the tool having a pipe shape in the working part was pressed down to the plate surface and the down force and friction torque for different tool rates of rotation were measured. The tool was made of the same tool steel as FSW tools. For the known geometrical shapes of the tool and rotational speed, it is possible to calculate the distribution of friction coefficient depending on frictional speed for various temperatures of preheated plates. The tests were conducted on the numerically controlled station (build on the basis of milling machine FNC 50NC) at the same times of approach-

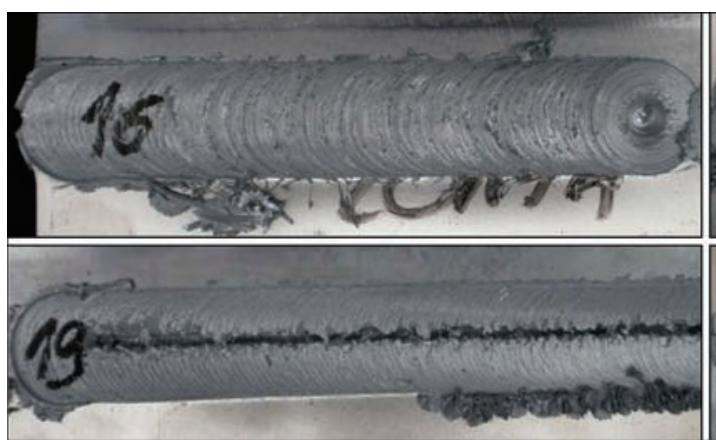

EN AC -43200 from advancing side ing and retracting the tool $\left(t_{\mathrm{wst}}=3.0 \mathrm{~s}\right)$ and the same friction time $t_{\mathrm{t}}=1.0 \mathrm{~s}$.

The test results for EN AW-2017Aalloy are presented in Figure 3. For the remaining alloys being tested, the dependence of friction coefficient on friction speed was similar.

Similar research was conducted for FSW process in which a conventional tool, having a tool shoulder $\varnothing 11.0 \mathrm{~mm}$ and a probe $\varnothing 5.0 \mathrm{~mm}$, was used on $4 \mathrm{~mm}$ thick plates of EN AW-2017A alloy, and a tool rotational speed $\omega$ was being changed while welding speed $V_{z g}$ remained on the same level of $200 \mathrm{~mm} /$ min. During making of a single longitudinal weld, the sequence of three different rotational speeds was changed twice, as in Figure $4(\omega=350,550$ and $750 \mathrm{~min}^{-1}$ ), while measuring down force and friction torque using ISstir head. After the production of the first weld, the plates have been heated to the temperature up to $150{ }^{\circ} \mathrm{C}$ and the second welding cycle was performed with changing the rotational speed of a tool and then the plates were heated up to the temperature of $250{ }^{\circ} \mathrm{C}$ and the third welding cycle was done. The results of the measured friction torque and down force are presented in Table 4.

Testing of metal structure in the area of tool friction during FSW process. During this testing, friction torque (down force), that resulted from summarized frictional resistance coming from two sources was recorded. One of the source was external friction, i.e. from force of shearing micro-joints and irregularities in local friction points and second source was internal

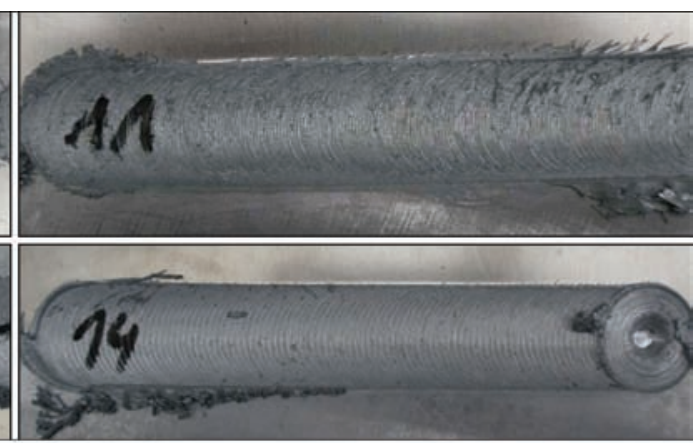

EN AW-2017A from advancing side

Figure 2. View of the surfaces of the selected welds EN AC-43200 + EN AW-2017A obtained for various process parameters 

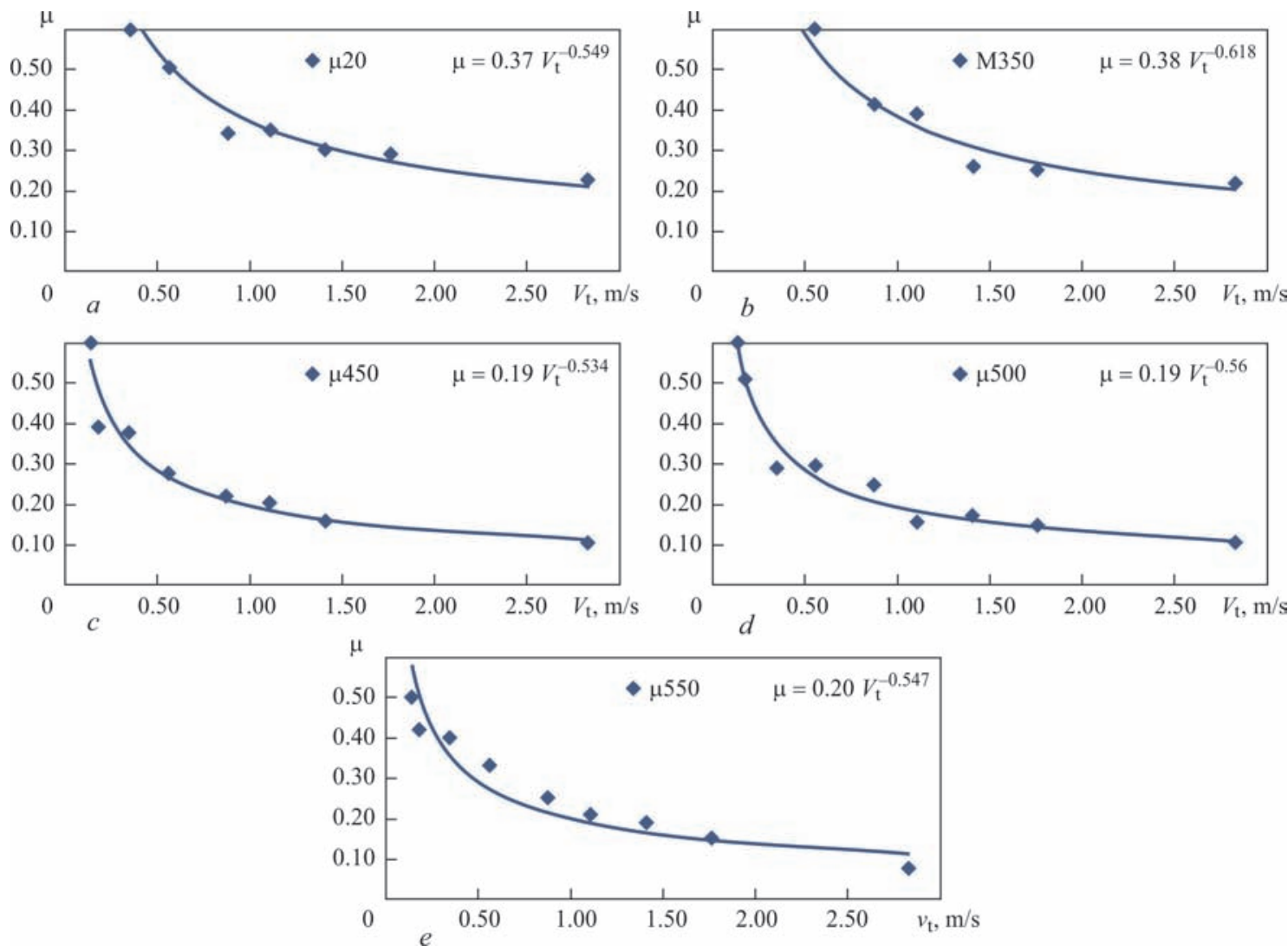

Figure 3. The change of friction coefficient depending on friction speed at various temperatures: $a-20{ }^{\circ} \mathrm{C} ; b-350{ }^{\circ} \mathrm{C} ; c-450{ }^{\circ} \mathrm{C}$; $d-500{ }^{\circ} \mathrm{C} ; e-550{ }^{\circ} \mathrm{C}[10]$

friction, i.e. from forces connected with plastic strain of the metal layer up to the certain depth.

For samples undergoing friction both phenomena are clearly visible. During testing of friction coefficient and FSW process, it can be noticed that both friction forces and thickness of material layers undergoing plastic straining depend on friction speed. The structure of a layer being modified using friction is different from that of parent material. Figures 5 and 6 shows metal deformed during the process in the area of a tool action. The area under observation includes a modified by friction layer of EN AC 43200 alloy in the cross-section parallel to the plane of welded plates, on the depth of $1 \mathrm{~mm}$, in the area of tool action after its retracting. Welded plates were of EN AC-43200 + EN AW-6082, $6.0 \mathrm{~mm}$ in thickness.

The image of the process of metal movement around a tool and creating a weld behind a tool observed from above (from a welding machine spindle) is shown in Figure 7. Those images present cross-section of a weld made parallel to the surface of welded plates on the depth of 1.5, 3.0 and $4.5 \mathrm{~mm}$ below a tool shoulder, i.e. in the location of primary action of a tool probe. The shown image of a welding process has been obtained after adequate preparation of the samples in the place of rapid stoppage of a tool movement and retracting it from a weld region. The direction of rotation and translation movement of a tool is marked schematically in its position.

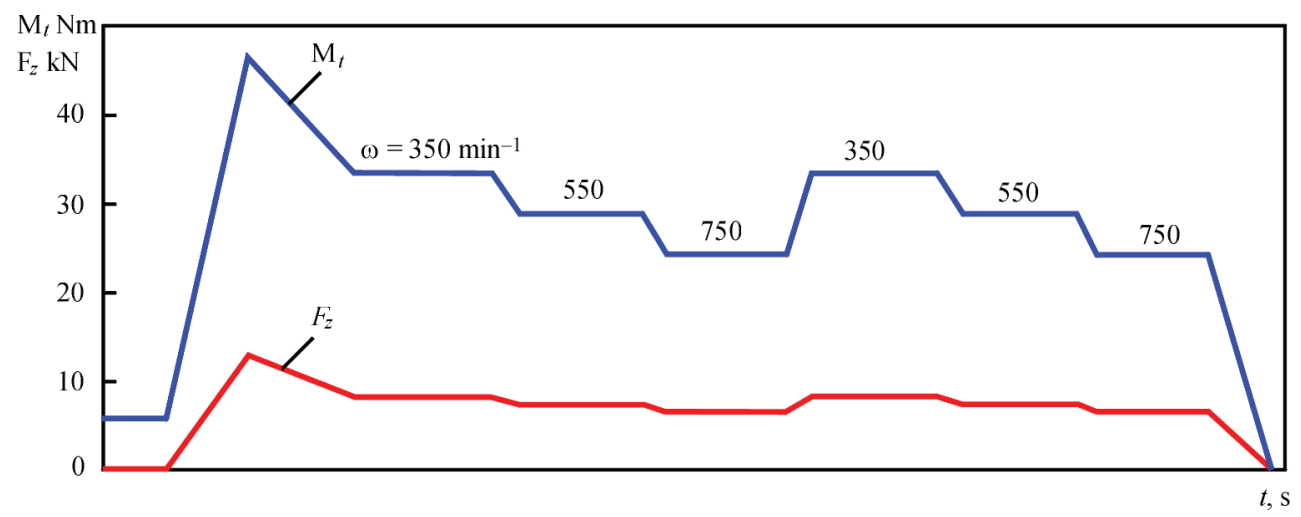

Figure 4. Diagram of changes of friction torque $\mathrm{M}_{\mathrm{t}}$ and down force $\mathrm{F}_{\mathrm{z}}$ during testing of welding process with changing of frictional rotation speed of a tool [10] 


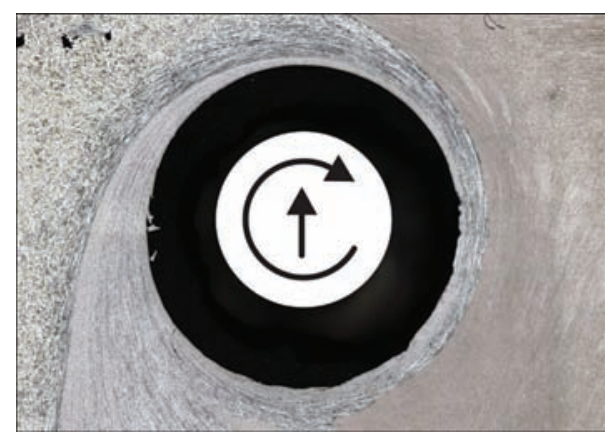

Figure 5. Metal layers around a tool. Friction modified layer located in the direct vicinity of a probe. EN AW 6082 alloy from a retreating side (right side), EN AC 43200 alloy from advancing side (left side) extruded around a tool probe during FSW. The direction of rotation and translation movement of the tool is marked schematically

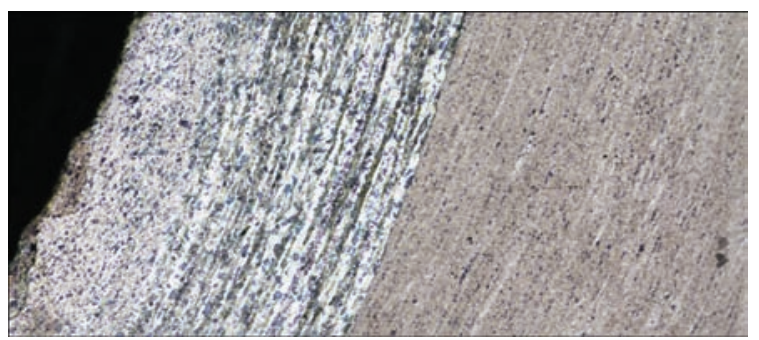

Figure 6. Fragment of the modified layer of Figure 5 on retreating side

As can be seen in the above images, material is extruded around a tool on the retreating side, in accordance with the tool probe rotation. Along the tool probe the material from retreating side penetrates into the material from the advancing side on various depth. The observed image of the structure being created is obtained after stoppage of a translation movement and rapid retraction of a tool from the welding area.

Around the tool, on the retreating side, the material is moved backwards through a relatively wide area of plasticised layer towards the weld being created. This phenomenon is clearly visible at low tool rotational speeds (low friction speeds) which is accompanied by high friction forces. In the case of high tool rotational
Table 4. Results of measuring friction torque and down force recorded while testing of FSW using preheated plates [10]

\begin{tabular}{|c|c|c|c|c|}
\hline $\begin{array}{c}\text { Measure } \\
\text { No. }\end{array}$ & $\begin{array}{c}\text { Temperature } \\
\text { of plates } \\
\text { prior } \\
\text { to the } \\
\text { process, } \\
{ }^{\circ} \mathrm{C}\end{array}$ & $\begin{array}{c}\text { Tool rotation } \\
\text { speed } \omega, \\
\mathrm{min}^{-1}\end{array}$ & $\begin{array}{c}\text { Friction } \\
\text { torque } \\
\mathrm{M}_{\mathrm{t}}, \mathrm{Nm}\end{array}$ & $\begin{array}{c}\text { Down force } \\
\mathrm{F}_{\mathrm{z}}, \mathrm{kN}\end{array}$ \\
\hline 1 & 20 & 350 & 35.4 & 8.7 \\
\hline 2 & 20 & 550 & 30.5 & 7.8 \\
\hline 3 & 20 & 750 & 25.7 & 6.9 \\
\hline 4 & 20 & 350 & 37.1 & 9.0 \\
\hline 5 & 20 & 550 & 29.2 & 7.3 \\
\hline 6 & 20 & 750 & 25.1 & 7.1 \\
\hline 7 & 150 & 350 & 33.8 & 8.0 \\
\hline 8 & 150 & 550 & 29.0 & 6.9 \\
\hline 9 & 150 & 750 & 23.4 & 6.6 \\
\hline 10 & 150 & 350 & 33.7 & 7.7 \\
\hline 11 & 150 & 550 & 28.0 & 6.8 \\
\hline 12 & 150 & 750 & 24.8 & 6.7 \\
\hline 13 & 250 & 350 & 35.1 & 8.3 \\
\hline 14 & 250 & 550 & 27.4 & 6.9 \\
\hline 15 & 250 & 750 & 22.8 & 6.3 \\
\hline 16 & 250 & 350 & 33.8 & 7.7 \\
\hline 17 & 250 & 550 & 26.9 & 6.7 \\
\hline 18 & 250 & 750 & 23.1 & 6.6 \\
\hline & & & & \\
\hline
\end{tabular}

speeds (high friction speeds) the forces are noticeably lower and the thickness of a layer being modified is also lower (Figure 8). Material is extruded backwards on retreating side, however is not deformed that strongly in large volume. Practically, at those high friction speeds only material from the front of a specimen is being moved backwards (in this case EN AC 43200). This phenomenon was clearly revealed while testing of FSW process using EN AC 43200 material of tool probe width, being implemented into AW 6082 .

As can be seen in Figures 7 and 8, the thickness of the modified and plasticised layer during friction depends on the friction conditions and is of great significance during generating of friction heat. This friction modified layer takes part in the creation of a weld of specific structure and properties.

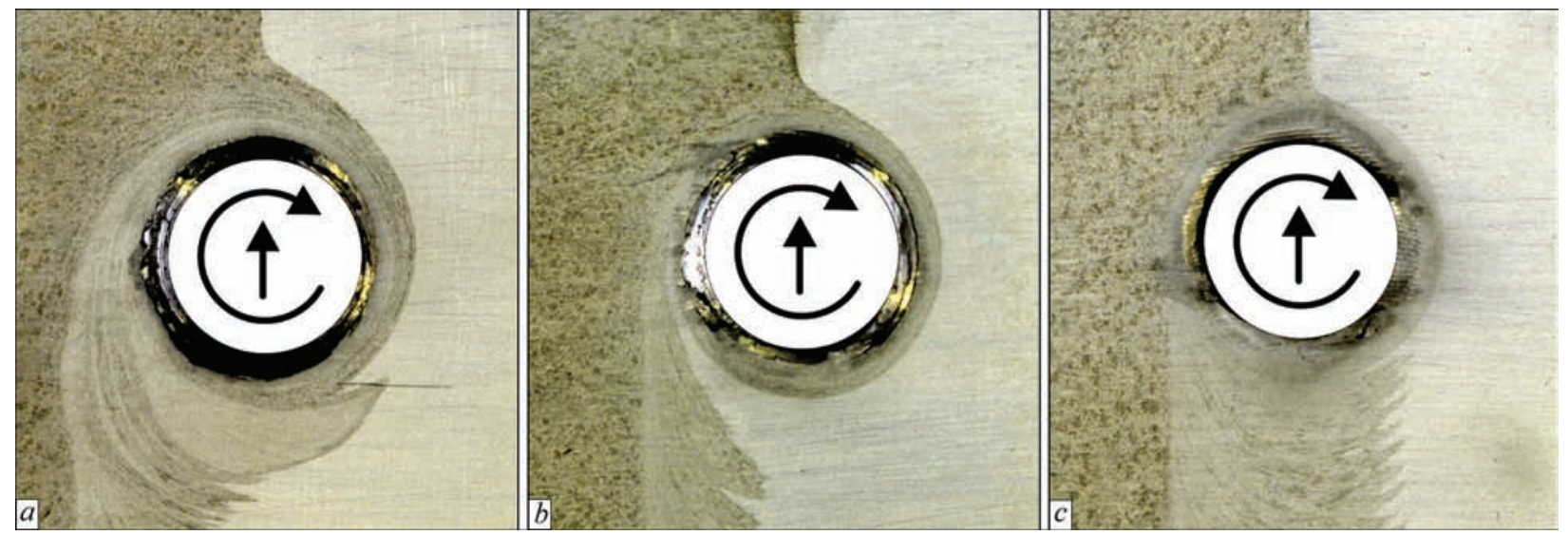

Figure 7. The image of a weld formed around a tool, observed on the depth of: $a-1.5 \mathrm{~mm} ; b-3.0 \mathrm{~mm} ; c-4.5 \mathrm{~mm}$ from weld face surface. From advancing side (left side) is located EN AC 43200 alloy, while from retreating side (right side) EN AW 6082 alloy. Welding parameters: $\omega=450 \mathrm{~min}^{-1} / V_{\mathrm{zg}}=224 \mathrm{~mm} / \mathrm{min}$ 


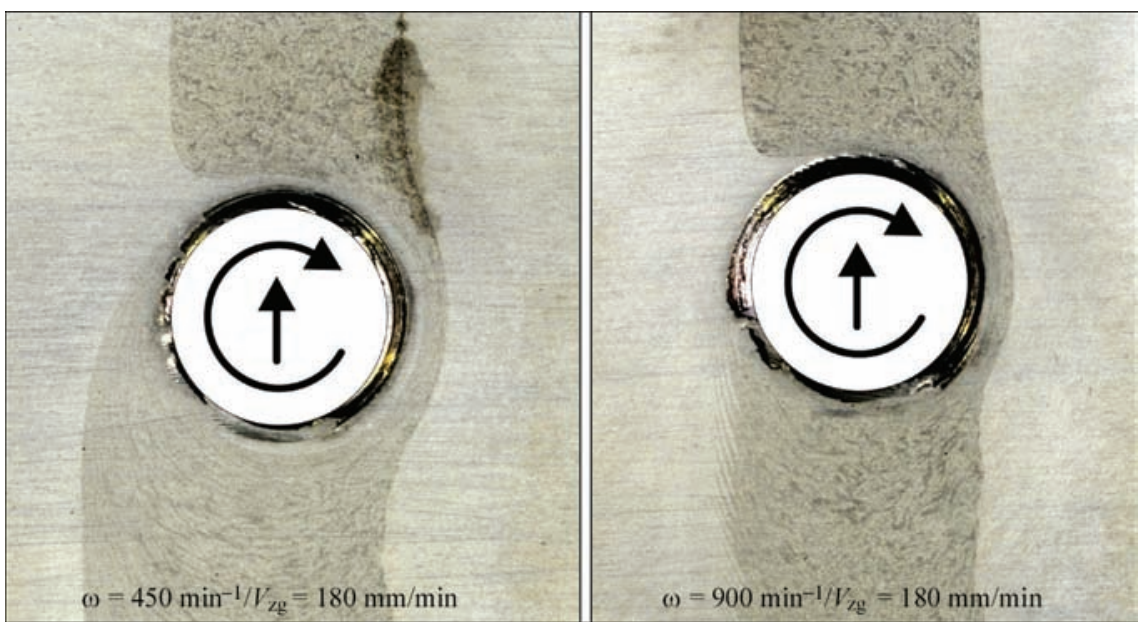

Figure 8. A weld being created around the tool with implemented EN AC 43200 material of a tool probe width. Rotation of a tool and direction of the movement is marked in the photographs. On the right side: retracting, on the left side: advancing

While welding material is extruded around a weld backwards, not changing its position behind the tool relative to the weld line. This phenomenon is clearly visible in the testing of FSW process using so called marker made of casting alloy. A rod of EN AC 43200 casting alloy having a dimensions of $3.0 \times 3.0 \mathrm{~mm}$ was included into the material of EN AW 6082 alloy, $6 \mathrm{~mm}$ thick, as in Figure 9.

After welding, the location of the rod of EN AC 43200 is visible on the weld cross-sections in the separate welding areas. In case of location of a rod A on the advancing side before welding (Figure 9, a), during the process its material is moved around the tool and after welding is situated in a weld, as in Figure 10.

As can be seen in the weld structure, despite extruding material through the narrow area around the tool, the EN AC 43200 alloy (material A) is located approximately in the same weld area as before the process completion.

In case of location of a rod on the retreating side before welding (Figure 9, b), during the process its material is moved around the tool and after welding is situated in a weld, as in Figure 11. In case of location of a rod in the root area on the advancing side before welding (Figure 9,c) after welding the weld structure is obtained as shown in Figure 12.

In spite of the fact that in welding process the material being implemented was strongly heated and deformed while extruding around the tool (as in Figures 5 and 7) it successfully recreated its previous lo-
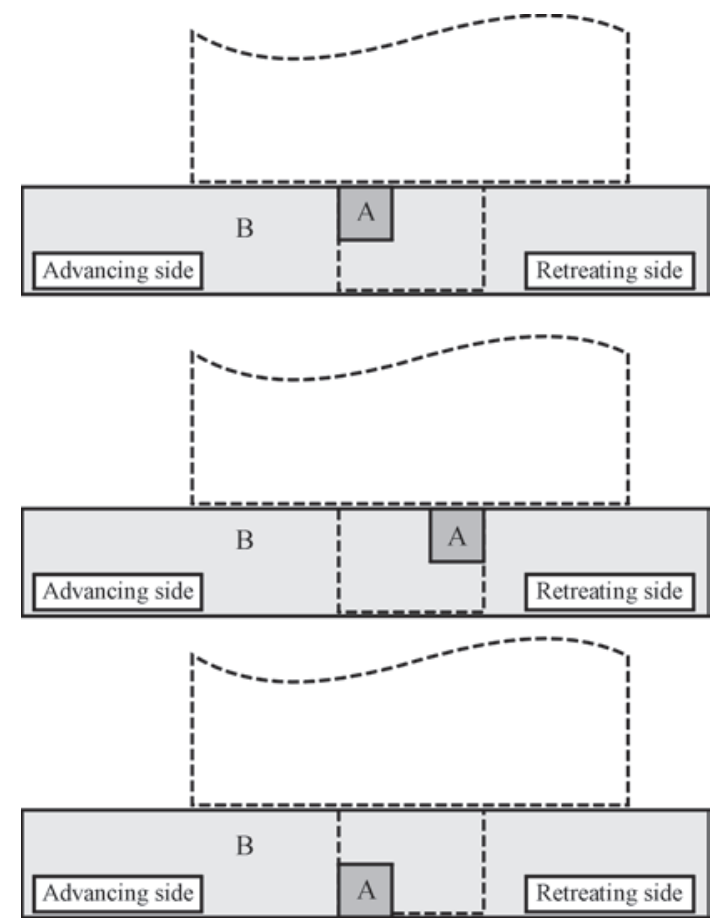

Figure 9. Diagram of the distribution of a «marker» of EN AC 43200 alloy (A) in the material of EN AW 6082 alloy (B) before welding using FSW process

calisation. Dissimilar physical properties of materials do not prevent localisation of material is such a way after extruding around the tool.

Some molecules coming from the material being included can be moved in a random way and injected into

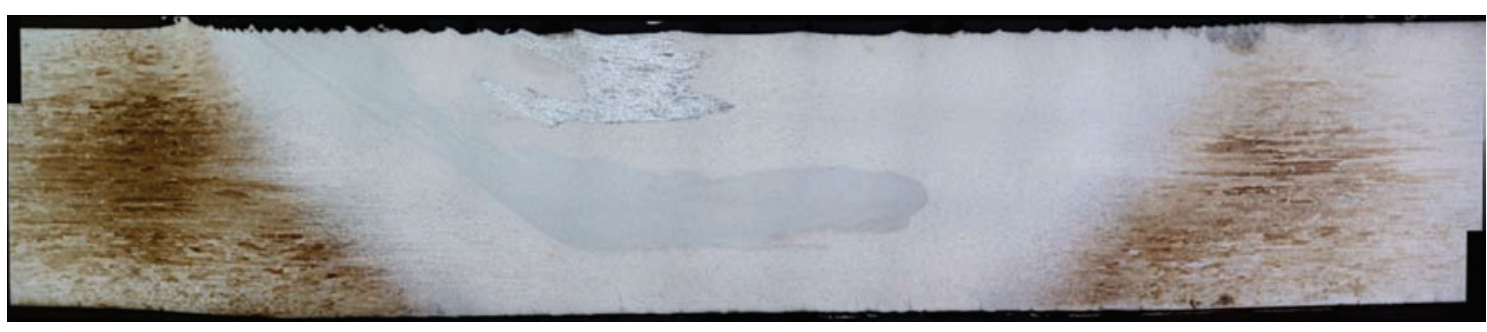

Figure 10. Structure of FSW weld in case of location EN AC 43200 rod (A) on the advancing side (as in Figure 9, a). Welding parameters: $\omega=450 \mathrm{~min}^{-1}, V=224 \mathrm{~mm} / \mathrm{min}$. Triflute tool. Keller etching 


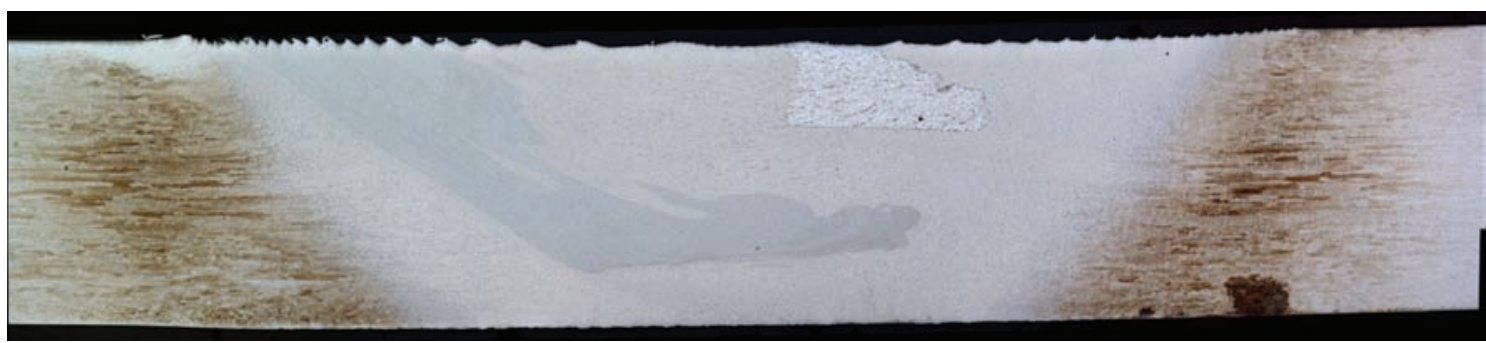

Figure 11. Structure of a weld in case of location of a rod of EN AC 43200 on retreating side (as in Figure 9, b). Welding parameters: $\omega=450 \mathrm{~min}^{-1}, V=224 \mathrm{~mm} / \mathrm{min}$. Keller etching

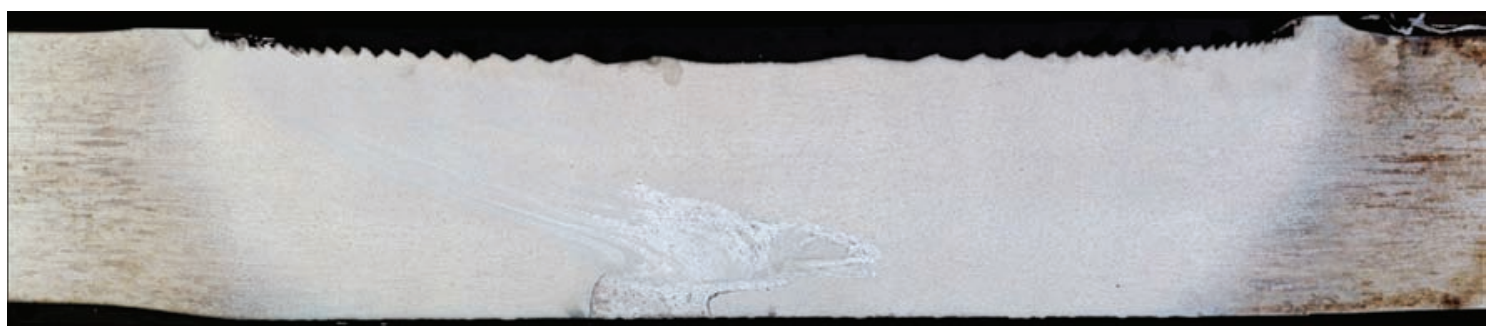

Figure 12. Structure of a weld in case of location of a rod of EN AC 43200 in the root on the advancing side (as in Figure 9, c). Welding parameters: $\omega=450 \mathrm{~min}^{-1}, V_{\text {zg }}=224 \mathrm{~mm} / \mathrm{min}$. Keller etching

the metal matrix, however in the whole mass the substantial part of the included material do not change its location after FSW completion. Such phenomenon occurs during welding with relatively low frictional speeds of a tool. For high rotational speeds, especially while using a tool with screwed probe the process of metal extruding is more complex [3, 6, 11, 12].

The structure (Figure 13) reveals a good metallic bonding between EN AC 43200 alloy layer (from the top) and material matrix of EN AW 6082 alloy. For many places in the weld structure from Figs. 10-12 the SEMEDS analysis was conducted. The exemplary results of the analysis are shown in Figure 14.

The area subjected to the significant strains and deformations was tested from the viewpoint of the average size of the particle and shape factor. In Figure 15 histograms of the particles occurrence frequency in separate intervals of shape factor are presented. In parent material (Figure 15, a) very elongated particles dominate $-12.8 \%$ particles have shape factor below 0.1 and $54 \%$ particles below 0.3 . For the tested area

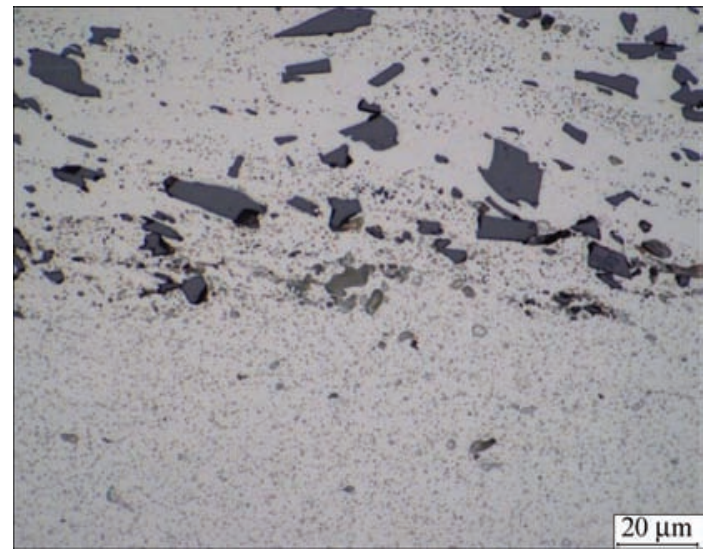

Figure 13. Fragment of a structure from Figure 9. Bonding of metal layer of EN AC 43200 with EN AW 6082 alloy of a weld (Figure 15) only 13.5\% particles have the shape factor below 0.3 , whereas the particles of shape factor between $0.3-0.8$ are the dominant $(68.4 \%)$. The research results have indicated that plastic deformation occurring while welding caused very high refinement of particles and changed their shape towards particles being more equiaxial.

The results of conducted research into the process of friction welded joints forming together with testing of their mechanical properties were used while developing FSW process conditions for welding components in the industrial practice. Such elements, for which FSW technology was developed at Instytut Spawalnictwa, basing among other things on the research mentioned above, are the following (Figure 16): components of pneumatic cylinder of EN AW-2017A alloy, heat exchangers of EN AC 43200 casting alloy

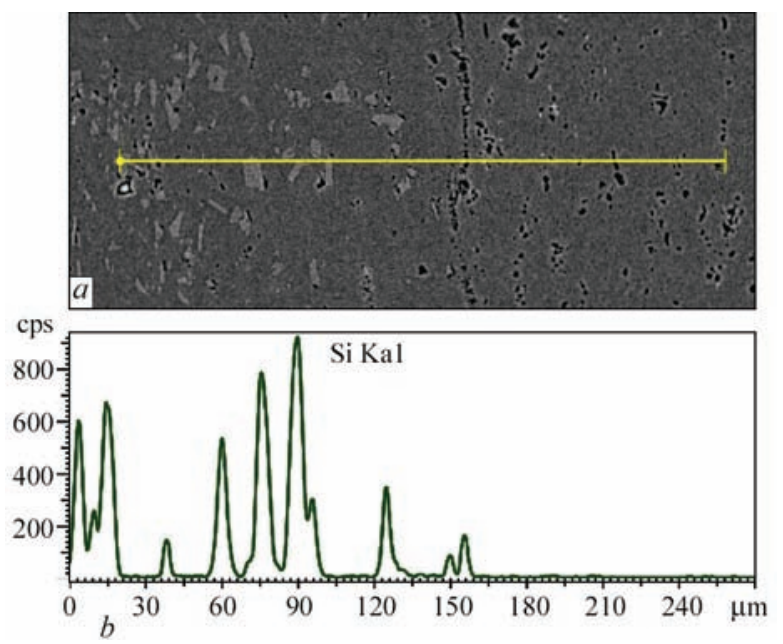

Figure 14. Boundary between EN AC 43200 alloy (on the left side), and EN AW 6082 alloy (on the right side); $a$ - line, along which the analysis of chemical content SEM-EDS was conducted; $b-$ Si concentration along measuring line 

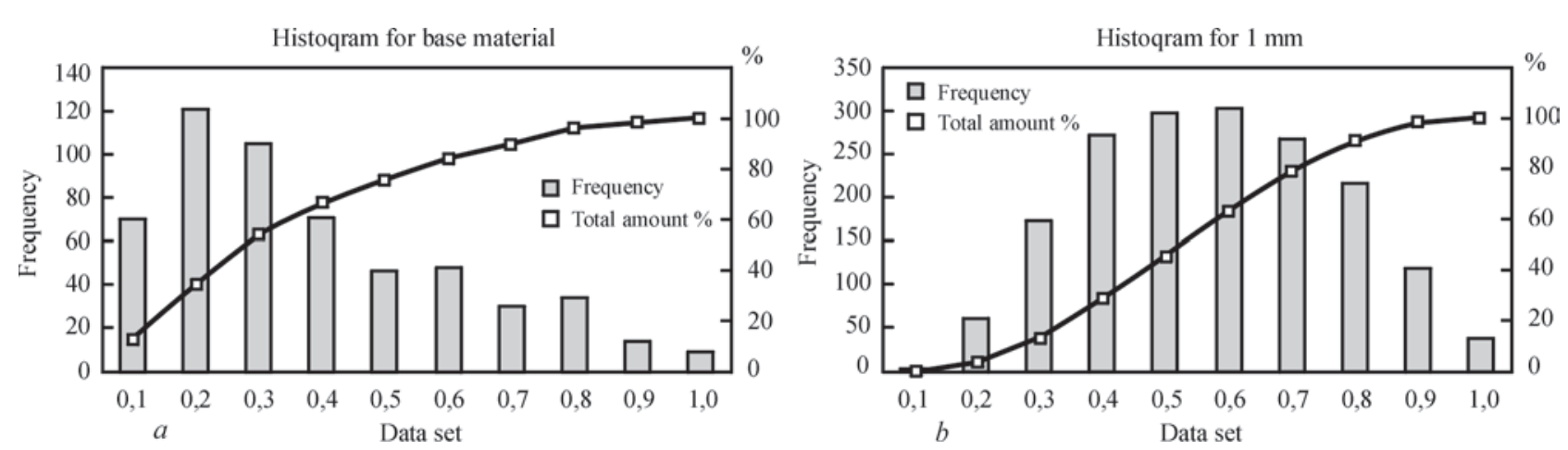

Figure 15. The measurement of grain size in parent material of casting alloy $(a)$ and in material subjected to the strains and deformations $(b)$

and EN AW-6082 alloy as well as copper M1, balls for check valves of EN AC 43200 casting alloy, M1 copper contact rails and others [13].

Summary. While using FSW process it is possible to join various materials, including materials of significantly different physical properties. During testing, application of two alloys being able to be joined but differing in chemical composition and properties, like EN AC 43200 casting alloy and wrought alloy EN AW 6082, proved useful. Placing materials from the retreating or advancing side enables observation of the process of heating and creation of the weld structures. Casting alloy used as a marker made it possible to observe the process of metal movement around the tool and the structure of the FSW joint.

As it was revealed [6] during the FSW process the largest amount of heat is produced as the result of tool shoulder action, however the proper structure of a weld in the whole thickness of the components being joined, in case of elements thicker that approximate-
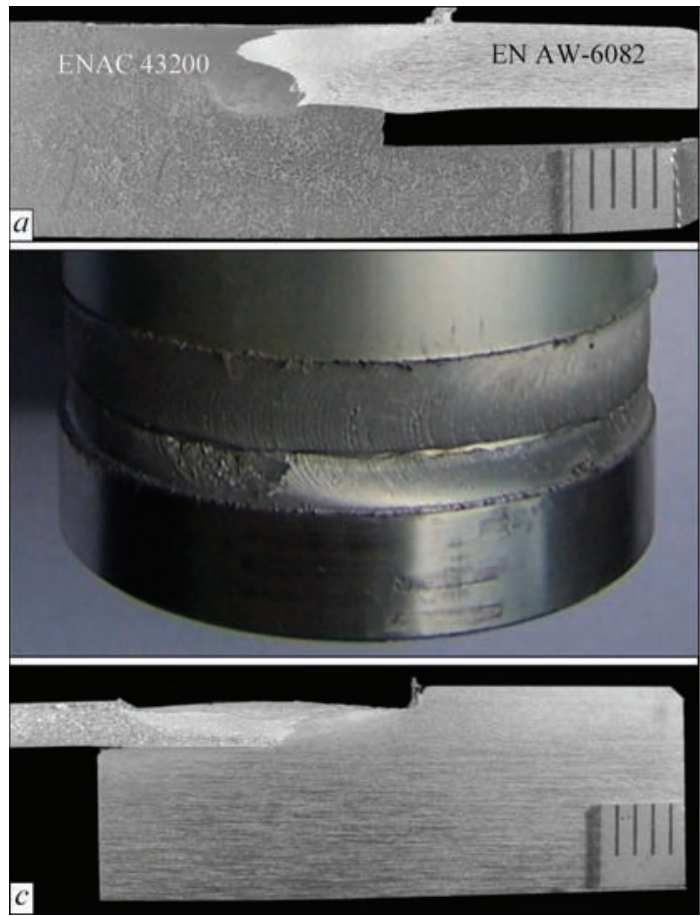

ly $2.0 \mathrm{~mm}$, depends on the shapes and dimensions of the tool probe and its movement. For thin elements the probe is not playing the crucial role in the process of the weld formation. The tool moving and plunging into the material starts to heat and plasticise various material zones. Heat in the welding process, as a result of good conduction is heating also material before a tool, what facilitates plunging a tool into the material preheated to the certain temperature.

The research revealed that with increasing the friction speed the friction factor $\mu$ is reduced during working of a tool on each tested material and in all temperature conditions.

At temperature higher than $400{ }^{\circ} \mathrm{C}$, i.e. in the temperature range observed in FSW process, for higher frictional speed the change of friction factor value along with changing temperature does not practically occur. This results in the fact that at higher frictional speeds such a friction conditions are stabilised in the friction interface, that the temperature of the plate (base) does not

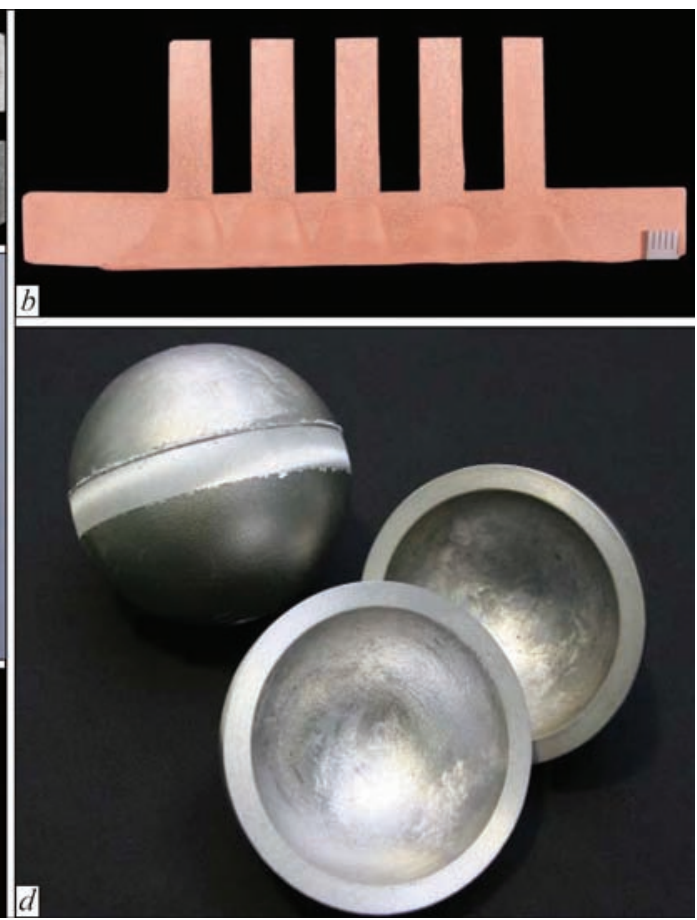

Figure 16. Examples of the application of FSW process [12]: $a$ - heat exchanger cross-section; $b$ - cross-section of an electrical connector made of M1 copper; $c$ — components and cross-section of a weld in pneumatic cylinder of EN AW-2017A alloy; $d$ - components of balls for check valves of EN AC 43200 alloy 
influence significantly the friction process and associated friction forces. At high temperature of the plates, friction forces are relatively low. For low frictional speeds, temperature has an impact on friction force value.

The above presented research revealed that during conducting typical FSW process the temperature of plates being welded has little influence on the friction torque. In turn, rotational speed of a tool influences it significantly. It can be however noticed, while analysing the research results, that tool rotational speed has an impact on the down force. During testing, it was not assumed that the process would be conducted at the same, constant down force. Down force value in this case is a resulting value and is associated with a tool leading in such a way, that the weld face is formed correctly.

As can be noticed, for growing frictional speeds of a tool, down force is being reduced. It is associated, as can be concluded, with heating of the material in the friction zone, which for higher frictional speeds is being heated more intensively, causing the reduction of mechanical properties of metal and consequently, the reduction of down force recorded by measuring head.

Thus, frictional speed has an impact on friction processes, it is associated with temperature of metal in friction zone and this metal temperature, in turn, influences in a certain way the down force of a tool (in a tool axis). However, temperature of plates fails to influence clearly the value of friction force (friction torque).

Beside temperature, which undoubtedly influences friction processes, the second factor which should be taken into consideration in the analysis of the processes occurring while forming of the FSW joint, is a layer of metal being modified using friction processes and undergoing plastic distortion.

By the action of a tool in the conditions of friction heating and upsetting, the metal layer in the direct vicinity of a tool undergoes thorough modification. Research revealed that it is a region of very high refinement of grains having a shape factor 0.4-0.7 (Figure 15) and undergoing dynamic recrystallisation and/or healing.

The analysis of friction force courses (friction coefficient), structures of material layers being modified and temperature in the friction area has revealed that overall friction forces, recorded during testing (e.g. friction torque), more depend on the thickness of a layer undergoing modification and distortion, (i.e. internal friction) and in lower extend on external friction, that is on conditions in the friction interface: tool - welded metal. In turn, the thickness of a layer undergoing plastic deformation in which internal friction occurs, depends on friction speed. The higher friction speed the thinner layer [10].

The research into friction welding quality in a straight majority of cases shows, that metals coming from retreating and advancing side, modified with friction process and upset against each other on the retreat- ing side, while movement backwards are joined correctly. Depending on the welding process parameters as well as applied tool and thickness of the plates being welded, the area of limited metal coherence can be located behind the tool on the advancing side $[10,12,14]$.

The manner of the metal transfer around the tool significantly influences the structure of FSW joint. As can be seen, at the specimens with included casting alloy, strongly heated and deformed material during extruding around the tool effectively recreated its previous localisation. Dissimilar physical properties of materials on both sides of a tool (advancing and retreating sides) proved not to prevent such a systematic placement of material after the process of extruding backwards.

Research into average size of the particles and the shape factor of the material subjected to the strains and deformations shown that FSW process caused very high refinement of particles and changed their shape towards particles being more equiaxial.

1. Thomas, W.M. Friction Stir Butt Welding. Int. Patent Application. PCT/GB92/02203.1991.

2. (2010) Friction Stir Welding. From basics to applications. Woodhead Publishing Limited.

3. Mroczka, K., Dutkiewicz, J., Pietras, A. (2010) Characterization of friction stir welds of 6013 and 6013/2017A aluminium alloy sheets. Inżynieria Materiałowa, 31(3).

4. Guohong Luan, Guang Li, Weibing Wang, Ju Kang (2010) The fundamental research of the friction flow welding. In: Proc. of 8th Int. Symp. on Friction Stir Welding (Germany, 18-20 May 2010).

5. Kumar, N, Yuan, W, Mishra, R.S. (2015) Friction stir welding of dissimilar alloys and materials. Butterworth-Heinemann (Elsevier), Oxford.

6. Hamilton, C., Kopyściański, M., Dymek, S. et al. (2017) Microstructure of friction stir welded AlSi9Mg cast with 5083 and 2017A wrought aluminium alloys. J. of Mater. Eng. and Performance, 26(1).

7. PN-EN 10027-1:2007P: Systemy oznaczania stali-Część 1: Znaki stali.

8. PN-EN 1706:2010: Aluminium i stopy aluminium Odlewy — Skład chemiczny i własności mechaniczne.

9. PN-EN 573-3:2010P: Aluminium i stopy aluminium — Skład chemiczny i rodzaje wyrobów przerobionych plastycznie Część 3: Skład chemiczny i rodzaje wyrobów.

10. Pietras, A. (2014) Praca Badawcza ST 330. In: Materials of Welding Research, Institute of Welding, Gliwice.

11. Mroczka, K., Pietras, A., Jura, J. (2016) Features of 2017A and AlSi9Mg aluminum alloys friction stir welded with rootside heating. Metall. Found. Eng., 42(2).

12. Mroczka, K. (2014) Charakterystyka mikrostruktury I właściwości zgrzein FSW wybranych stopów aluminiu (Characterisation of the microstructure and properties of FSW welds of selected aluminium alloys). Wydawnictwo Naukowe UP, Kraków.

13. Pietras, A. et al. (2010-2015) Works of the Institute of Welding. Gliwice for industrial partners.

14. Węglowski, M.S., Pietras, A., Węglowska, A. (2009) Effect of welding parameters on mechanical and microstructural properties of Al 2024 joints produced by friction stir welding. $J$. of Kones Powertrain and Transport, 19(1). 


\title{
TECHNOLOGIES AND EQUIPMENT FOR FLASH-BUTT WELDING OF RAILS: 60 YEARS OF CONTINUOUS INNOVATIONS
}

\author{
S.I. KUCHUK-YATSENKO \\ E.O. Paton Electric Welding Institute of the NAS of Ukraine \\ 11 Kazimir Malevich Str., 03150, Kyiv, Ukraine. E-mail: office@paton.kiev.ua
}

\begin{abstract}
The article summarizes the 60-year experience of the E.O. Paton Electric Welding Institute in innovative solutions in the field of development of technologies and equipment for flash-butt welding of rails. Many of them are pioneering, which is confirmed by a number of licensing agreements with leading countries of the world. Today, the stock of flashbutt welding machines and complexes, operating throughout the world, designed at the PWI and manufactured by the Kakhovka Plant of Electric Welding Equipment, amounts to more than 2,500 units, which is about $60 \%$ of the global stock of machines. 13 Ref., 4 Tables, 22 Figures.
\end{abstract}

Keywords : flash-butt welding, rails, eutectoid and hypereutectoid steels, technologies and equipment, continuous flashing, pulsating flashing, heating simulation, long-length rail sections, welding with tension, quality monitoring

In the postwar period, in order to restore tens of thousands of kilometers of railway tracks which were destroyed or worn out during the war, it was urgently necessary to perform a large amount of works on rail welding. Some existing rail welding workshops in different regions of the country, equipped with imported machines for resistance welding of rails, could not cope with this task, since they did not have a sufficient power base and the required power to supply the resistance welding machines of $400-500 \mathrm{kV} \cdot \mathrm{A}$. Moreover, the main task was not solved: welding of rails on the track during construction of seamless tracks. The use of other well-known technologies of rail welding: electric arc and thermit ones did not provide the required level of mechanical properties of welded joints in seamless tracks. In addition, they were significantly inferior to resistance welding by the efficiency and cost of works. In the early 1960s, the task was put forward by the government to the team of the PWI to create technology and equipment for welding rails directly on the track when laying the seamless tracks. For this purpose, it was necessary to solve the priority tasks:

- to find the possibility of a significant reduction in the power of welding machine and, accordingly, the necessary source of power supply;

- to significantly reduce the mass of welding equipment, which would allow maneuvering of welding machine in welding rails laid on the track;

- to fully automate the process of welding and eliminate the influence of the accuracy of preparation of rail ends before welding on the quality of joints;
- to develop welding technology, which provides equal strength of welded joints with the base metal and high resistance to cyclic loads.

The development of the technology for resistance welding of rails was based on the method of metal heating using continuous flashing (CF). The CF process is applied in industry for flash-butt welding of parts with a wall thickness of up to $10-12 \mathrm{~mm}$ [1]. It is characterized by stable energy input and high quality of joints in welding of thin-walled parts. In welding parts with larger thickness of cross-section elements, including rails, CF is not used in the world practice, because it does not provide the required heating of ends of the materials to be joined. In addition, for exciting of $\mathrm{CF}$ a significant power is required.

The PWI has developed methods and devices for significant reducing the power required to excite $\mathrm{CF}$ [2], as well as for intensifying heating during flashing due to the increase in its thermal efficiency. The main of them is the search for processes of stable flashing at low specific powers due to simultaneous control of instantaneous values of voltage and speed during flashing using feedbacks by the value of welding current.

It was established [3] that one of the main conditions for stable reproduction of a stable flashing process is the reduction of short circuit resistance of the welding circuit in flash butt-welding machines $\left(Z_{\text {sh.c }}\right)$. Due to the original design of welding circuit, where as its current-conducting elements, the power units of flash-butt welding machines are used, it was possible to significantly reduce $Z_{\text {sh.-c. }}$. As a result of using these innovations, the power consumed during welding and, accordingly, the power of supply sources was 


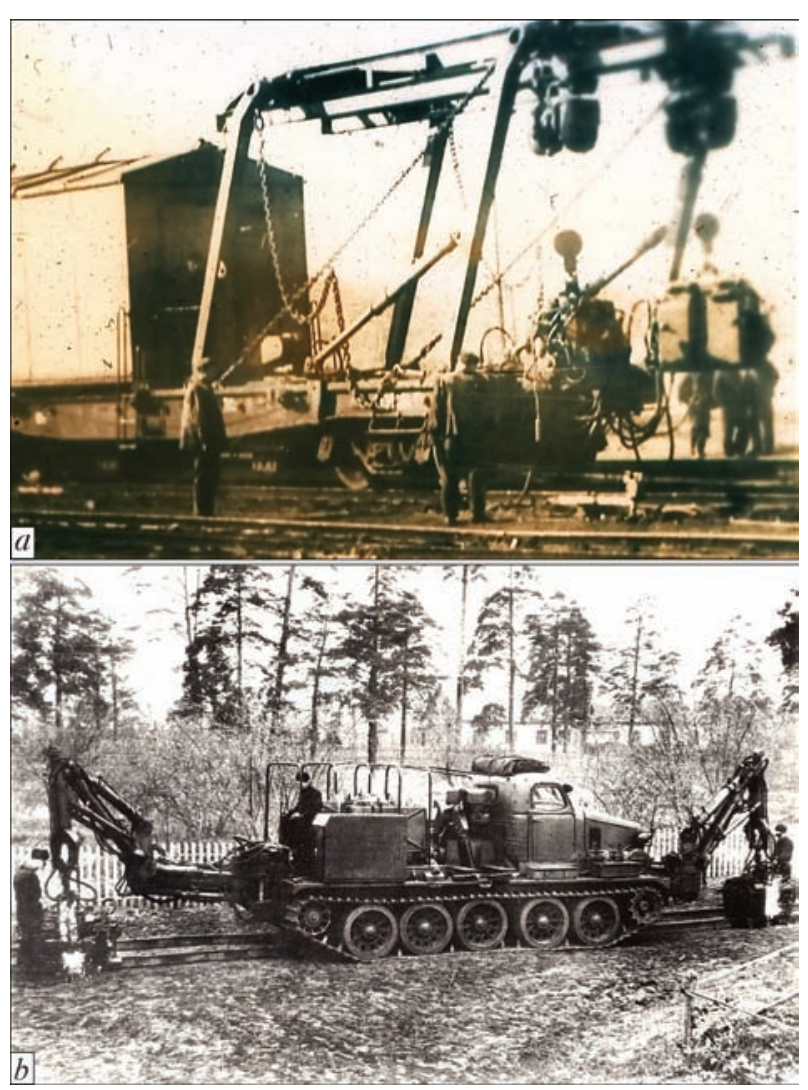

Figure 1. Mobile rail welding complexes with the machine K355: $a$ - PRSM; $b$ - RSA

significantly (2.5-3.0 times) reduced. The welding process was fully automated, providing high and stable quality of welded joints. Based on this technology, the first mobile machines for rail welding on the track were developed. As a result of these works, the fundamentally new technologies of welding using CF with the programmed control of basic parameters were developed [4]. Already in the early 1960s at the PWI the mobile and rail welding machines of the type K155 were designed and manufactured. The machines were distinguished by a small mass ( 2 tons instead of 15-20 tons) and lower power (150 kW instead of $350-500 \mathrm{~kW}$ ) as compared to the best stationary machines for flash-butt welding of rails. The control system of the machines was based on the developments of the PWI [5]. The first model of the industrial rail welding machine (K355) was manufactured at the PWI and tested on the railways of Ukraine. On the base of this machine, the world's first mobile complexes for flash-butt welding of rails on the track were created. According to the documentation developed at the PWI, the industrial production of machines K355 since 1960s was mastered by the Kakhovka Plant of Electric Welding Equipment (KZESO). By the end of the 1960s on the railways of the post-Soviet space, already about 100 machines K355 were in operation, the implementation of which was carried out by the PWI specialists. Taking into account the experience of the production operation of the first batches of welding machines, their design was improved, different mobile rail welding complexes (Figure 1) were created, which included, except of welding machines, self-propelled installations with lifting mechanisms on the base of mobile platforms (see Figure 1, a), as well as on the base of all-terrain vehicles (see Figure $1, b$ ). In mobile complexes, standard power plants of $200-250 \mathrm{~kW}$ power were used. To increase efficiency, in mobile rail welding complexes of the type PRSM - track rail welding self-propelled machine (see Figure 1,a) used in the railways of the former Soviet republics, two welding machines were installed, each of which is oriented to a single rail section. Such a scheme of works was accepted during reconstruction of tracks with a complete replacement of rail sections. For repair works, the complexes with one machine were used. The complexes based on all-terrain vehicles proved to be very efficient (see Figure 1, $b)$. In this case, there is no need in power plants for the power supply of welding machines. Both welding machines, operating simultaneously, receive power supply from one generator connected to the diesel of an all-terrain vehicle. Since the early 1970s, mobile rail welding machines produced by the KZESO began to be exported to different countries of the world. The first batches of machines were delivered to France (Matiza), Austria (Placer), USA (Holland). At the same time, different mobile complexes on the automobile and railway travel were used, developed on the base of the machine K355, taking into account different forms of works organization during reconstruction and repair of railways. During implementation and operation of tens and hundreds of machines in different countries, the PWI continued its works on the development of the technology of welding rails using CF, taking into account the different types of sizes and chemical composition of rail steels and optimized welding modes and typical programs for control of welding process. One of such typical programs is shown in Figure 2. At the same time, algorithms for assessing the quality of the joints by deviations of the recorded values from those, preset by the programs, were determined. The common recommendations for assessing the quality of rail joints in real-time mode were developed immediately after performing welding according to the results of in-process control. The in-process control became an integral part of the technologies of flash-butt welding of rails. Such control was particularly relevant during works at remote areas of railway tracks. The in-process control during welding of rails on railroads was introduced by normative documents from the 1980s as an obligatory operation during welding of rails. Basing on it from 
the beginning of the 1990s the computerized control systems for the control of basic welding parameters were developed, with which the new generation of rail welding machines of the type K900 was equipped (Figure 3). In the CIS countries, such machines replaced the machines K355. From the beginning of the 1990s the machines K900 began to be exported to Europe, the USA and China.

Technologies and equipment for flash-butt welding of high-strength rails of eutectoid and hypereutectoid structure. The increase in the freight and speed traffic on the railways in the last decade caused the necessity in increasing mechanical properties of the rail, their wear resistance and service life. The majority of high-strength rails of modern production have a guaranteed service life of 1.5-2.0 times higher than in the rails of previous generations. This is achieved due to the application of new technologies for production of rail steel using a converter process in combination with continuous rolling and vacuum control. At the same time, a volumetric or differentiated thermal hardening of rail rolled metal is used. The production of rails of eutectoid and hypereutectoid class was mastered, which allowed a significant increase in the hardness and wear resistance of the rail steel. The chemical composition and mechanical properties of such rails are given in Table 1. In flashbutt welding of such rails by the technologies, used for joining rails with carbon content at the level of hypereutectoid steels, the required parameters of mechanical properties were not provided.

One of the causes for lowering the mechanical properties is the metal softening in the welding zone as a result of the formation of a coarse-grained structure in the central part of the weld, reducing the joint strength.. Decreasing the energy input by shortening the duration of flashing process has a positive effect on the improvement of the structure, but leads to occurring defects in the zone of a joint [6], which significantly reduces the strength and ductility of welded joints. The relationship was established between the occurrence of defects in the plane of a joint with the formation of a spark gap, in particular, during flashing, in the sections with the maximum value of a spark gap $\Delta_{\mathrm{g} \max }$ and the thickness of the melt on the flashing surface $\delta_{1}$, as well as the temperature gradient in the near-contact layer of the part ends [7]. The formation of defects occurs if a melt has a time to crystallize before the beginning of ends deformation. For producing qualitative joints it is necessary to fulfill the condition: the duration of melt crystallization $t_{\mathrm{m}}$ on the ends of the flashed parts:

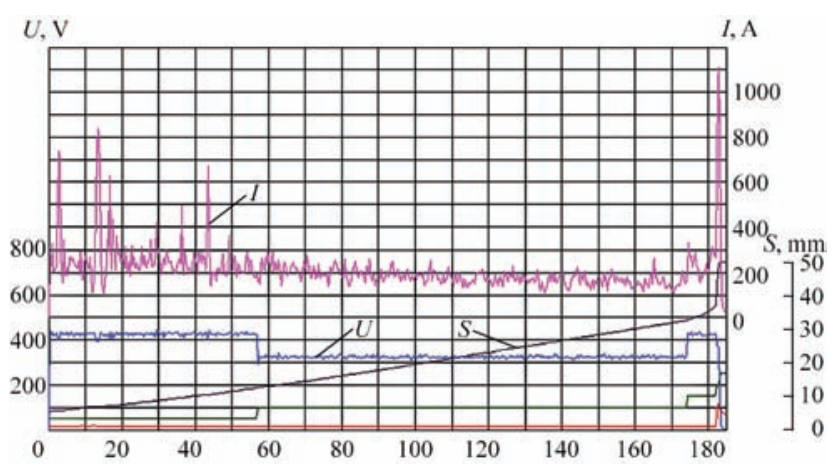

Figure 2. Typical program for changing basic parameters during welding rails using continuous flashing

$$
t_{\mathrm{m}} \geq \frac{\Delta_{\mathrm{g} \max }}{v_{\mathrm{f}}},
$$

where $t_{\mathrm{m}}$ is the duration of crystallization of the liquid layer of the melt of thickness $\delta_{1 ;} \Delta_{\mathrm{g} \text { max }}$ is the maximum value of the spark gap; $v_{\mathrm{f}}$ is the final flashing rate before upsetting.

$$
t_{\mathrm{m}} \rightarrow f\left(\delta_{1}, \theta_{1}, A \lambda \frac{d \theta}{d x}\right),
$$

where $\theta$ is the average melt temperature; $\mathrm{A}$ is the dimensionless parameter, determined by the thermophysical steel properties; $\lambda \frac{d \theta}{d x}$, is the gradient of temperature field in the near-contact layer.

With an increase in the temperature field gradient, the time of crystallization decreases and the probability of defects formation increases. In order to provide the required crystallization conditions, it is necessary to increase the final rate of flashing before upsetting $v_{\mathrm{f}}$, or to reduce the value of $\Delta_{\mathrm{g} \text { max }}$. The increase in the flashing rate $v_{\mathrm{f}}$ is provided by the program of changing the voltage at $\mathrm{CF}$, but the possibilities of this measure are largely exhausted, since a further increase in the $v_{f}$ is associated with the need in increasing the voltage $U_{2}$ and is accompanied by an increase in the craters depth. At the same time the value $\delta_{1}$ is decreased. In the course of investigations carried out at

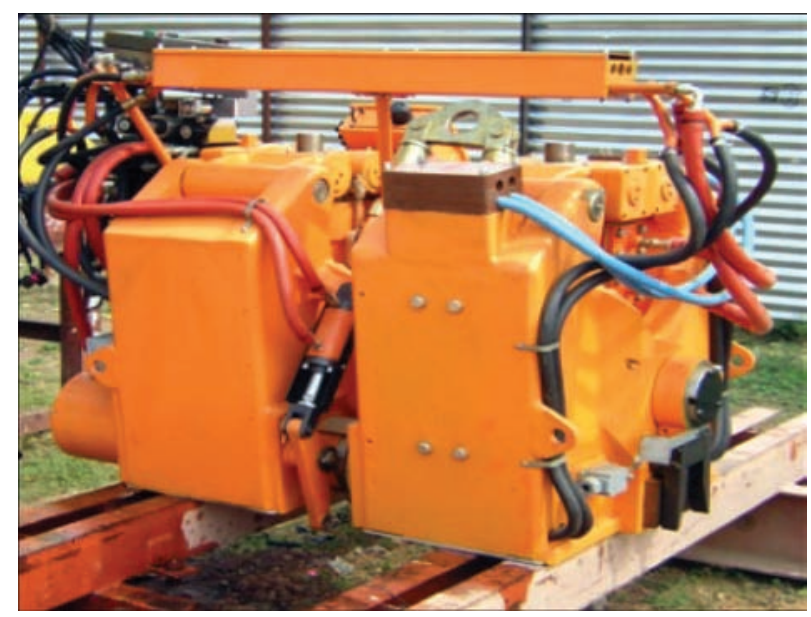

Figure 3. Mobile rail welding machine K900 
Table 1. Chemical composition and mechanical properties of high-strength rails of modern production

\begin{tabular}{|c|c|c|c|c|c|c|}
\hline \multirow{2}{*}{ Steel grade } & \multicolumn{6}{|c|}{ Chemical composition. \% } \\
\hline & $\mathrm{C}$ & Mn & $\mathrm{Si}$ & V & $\mathrm{Ti}$ & $\mathrm{Cr}$ \\
\hline M76 & $0.71-0.82$ & $0.80-1.30$ & $0.25-0.45$ & - & - & - \\
\hline K76F & $0.71-0.82$ & $0.80-1.30$ & $0.25-0.45$ & $0.03-0.07$ & - & - \\
\hline E76F; K76F & $0.71-0.82$ & $0.75-1.05$ & $0.25-0.45$ & $0.03-0.15$ & - & - \\
\hline R260 & $0.62-0.82$ & $0.70-1.20$ & $0.15-0.58$ & 0.03 & - & $\leq 0.15$ \\
\hline R350NT & $0.72-0.82$ & $0.70-1.20$ & $0.15-0.58$ & 0.03 & - & $\leq 0.15$ \\
\hline R350NT & $0.72-0.82$ & $0.15-0.60$ & $0.65-0.75$ & 0.03 & - & 0.15 \\
\hline VS-350Ya; 350LTD & $0.72-0.82$ & $0.7-1.2$ & $0.35-1.0$ & 0.01 & 0.025 & $0.3-0.7$ \\
\hline AREAL $13610 \mathrm{SP}$ & $0.81-0.82$ & $1.0-1.15$ & $0.50-0.54$ & 0.005 & 0.002 & $1.3-1.22$ \\
\hline AREAL 136 HE370 & $0.99-1.00$ & $0.69-0.71$ & $0.50-0.52$ & 0.002 & 0.001 & $0.21-0.22$ \\
\hline
\end{tabular}

Table 1 (cont.)

\begin{tabular}{|c|c|c|c|c|c|c|}
\hline Steel grade & Hardness HV & $\begin{array}{c}\text { Tensile strength } \sigma_{t}, \\
\text { MPa }\end{array}$ & $\begin{array}{l}\text { Yield strength } \sigma_{y}, \\
\mathrm{MPa}\end{array}$ & $\begin{array}{c}\text { Relative elongation } \\
\delta, \%\end{array}$ & $\begin{array}{c}\text { Relative reduction } \\
\text { in area } \psi \%\end{array}$ & Plant-manufacturer \\
\hline M76 & $260-280$ & $800-1100$ & $500-700$ & $\geq 6$ & $\geq 20$ & \multirow{2}{*}{ Ukraine } \\
\hline K76F & $341-388$ & $1300-1380$ & $950-1050$ & $10-15$ & $25-35$ & \\
\hline E76F; K76F & $370-410$ & 1180 & 800 & 8 & 25 & Russia \\
\hline R260 & $260-300$ & $942-980$ & $498-540$ & $10-15$ & $15-25$ & Poland \\
\hline R350NT & $350-380$ & $1240-1300$ & - & $9-12$ & - & France \\
\hline R350NT & $650-380$ & $1240-1300$ & - & $9-12$ & - & Austria \\
\hline VS-350Ya; 350LTD & $362-400$ & $>1240$ & - & $>9$ & - & \multirow{3}{*}{ Japan } \\
\hline AREAL $13610 \mathrm{SP}$ & $388-420$ & $1350-1400$ & $840-950$ & $12-15$ & - & \\
\hline AREAL 136 HE370 & $380-430$ & $1320-1350$ & $850-950$ & $12-15$ & $22-25$ & \\
\hline
\end{tabular}

the PWI, it was found that adjusting the spark gap by changing the instantaneous feed rate in combination with a voltage change, allows suppressing the explosive-like process of its flashing (at which the relief of the flashing surface is formed), increasing its thermal efficiency and intensity of heating. At the same time, the value of $\Delta_{g \max }$ decreases. The process was called pulsating flashing (PF) [8]. Its application allows changing the intensity of heating during melting in wide ranges, at the same time with the high gradients of temperature field, the heating to a high temperature of the near-contact metal layer is provided, the spark gap decreases, and favorable conditions for the formation of joints at a decreased energy input are created. Figure 4 shows the dependences characterizing the change in thermal efficiency in the process of $\mathrm{PF}$, as well as in CF of R65 rails for comparison. The calculation of basic parameters, characterizing heat-

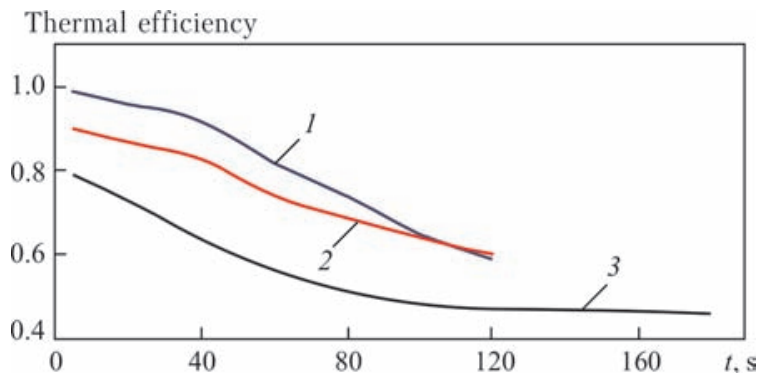

Figure 4. Dependence of thermal efficiency on flashing duration at PF $(1,2)$ and $\mathrm{CF}(3): 1-120 \mathrm{~s}, I-450 \mathrm{~A} ; 2-120 \mathrm{~s}, I-$ $370 \mathrm{~A} ; 3-180 \mathrm{~s}, I-170 \mathrm{~A}$ ing, was performed using mathematical modeling of heating at $\mathrm{CF}$. The calculation is based on the model of heating a single contact at the change in density of current, passing through the contact, heating temperature of the ends of parts and degree of overheating. As is seen from the abovementioned diagram, the efficiency at $\mathrm{CF}$ is quite high in the initial period of flashing -0.7 and it decreases as the temperature of the ends of parts grows to 0.45 [9]. Reducing the voltage in the welding process allows increasing it to 0.5 . This is the maximum possible value achieved at CF. From the analysis of dependence, it can be seen, that at $\mathrm{CF}$ there is a significant reserve for increasing the intensity of heating due to an increase in thermal efficiency, especially in the second period provided for by the program (see Figure 4). Applying PF, there is a possibility of increasing the mean current $I_{\mathrm{w}}$ passing through the specimens by increasing the area of simultaneously existing contacts, not exceeding the average speed of parts shortening, by adjusting the instantaneous values of feeding rates and voltage $U_{2}$. Figure 5 shows the records of current values at $\mathrm{PF}$ and $\mathrm{CF}$. In both cases, the same programs for changing the voltage $U_{2}$ of the average feeding rate $v_{\text {feed }}$ were established. By adjusting the instantaneous feed rates, the mean value of current and voltage is maintained at a preset level, and the value of the mean current increases significantly (by $35 \%$ in the first flashing period and by $30 \%$ in the second one) during flash- 


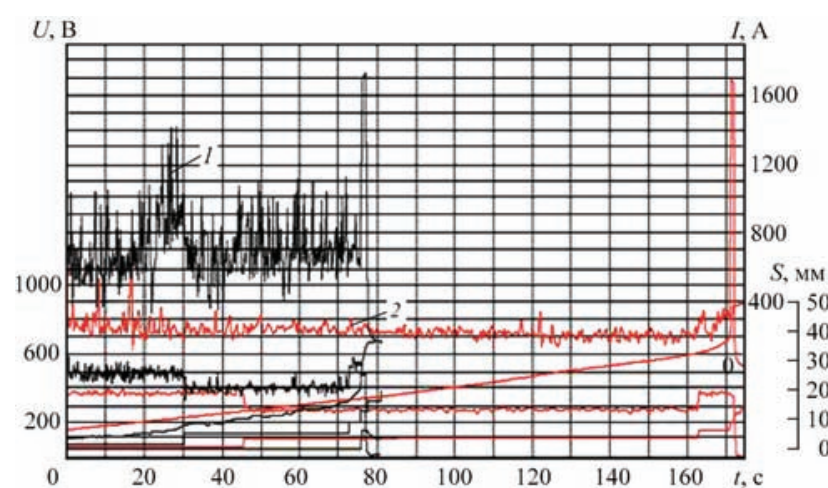

Figure 5. Record of basic welding parameters at PF (1) and CF (2)

ing. During PF the heating process quickly reaches a quasistationary state and heating is stabilized at this level. The distribution of temperature in the HAZ metal changes significantly. The temperature of the near-contact metal layers despite the reduction in energy input, increases and the width of the HAZ decreases (Figure 6). In this case, the ratio of the energy, generated in single contacts and lost at their fracture, is changed. The suppression of the explosive-like phase of heating the contact, when the metal is brought to boiling, reduces the fraction of lost energy and leads to increase in the efficiency at all the stages of contact heating.

Figure 7 shows the temperature fields obtained by calculation and experimental way at different values of welding current $\left(I_{\mathrm{w}}\right)$ in the process of PF of rails R65 made of steel M76 and K76F. The program for changing the basic parameters of $U_{2}, v_{\text {feed }}$ is accepted as the same, and the duration of flashing corresponds to the established quasistationary temperature state for each heating mode. The current value at PF was accepted to be higher relative to that accepted at CF. As can be seen from the comparison of curves, the transition to pulsating flashing leads to the increase in the mean value of welding current $\left(\mathrm{I}_{\mathrm{w}}\right)$ and reduction in the duration of the process. With increasing current,

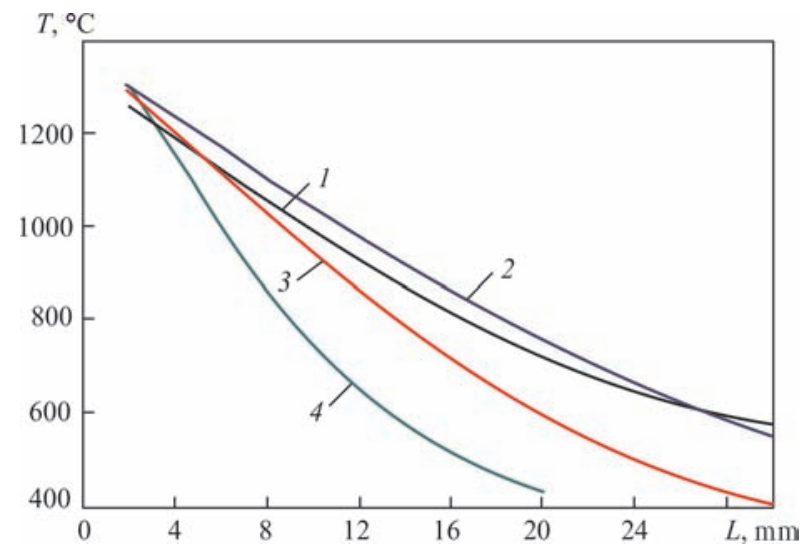

Figure 6. Temperature distribution in the HAZ before upsetting during welding of $\mathrm{R} 65$ rails at different modes: 1 - with preheating $\left(t_{\mathrm{w}}=200-250 \mathrm{~s}\right) ; 2-\mathrm{CF}$ with programmed voltage drop $\left(t_{\mathrm{w}}=180-220 \mathrm{~s}\right) ; 3-\mathrm{PF}\left(t_{\mathrm{w}}=110-120 \mathrm{~s}\right) ; 4-\mathrm{PF}\left(t_{\mathrm{w}}=60-70 \mathrm{~s}\right)$

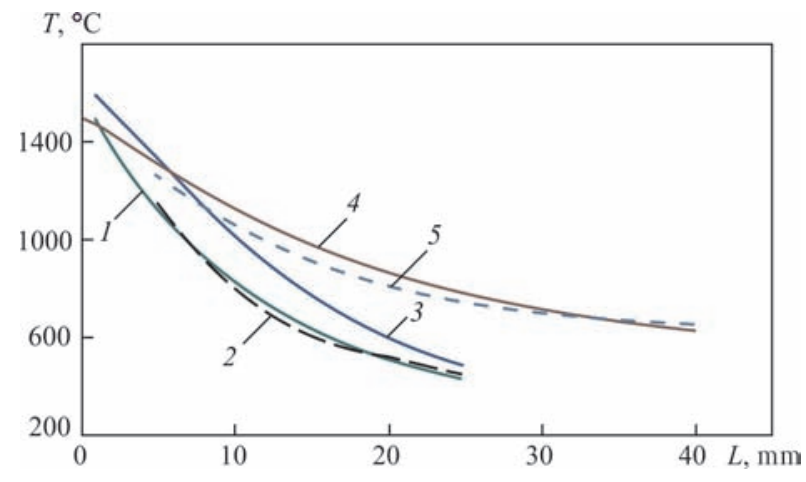

Figure 7. Temperature distribution in the HAZ before upsetting during welding R65 rails with different values of current and flashing duration: $1-\mathrm{PF}$ at $T=60 \mathrm{~s}, I=370 \mathrm{~A}$ (calculated); 2 $\mathrm{PF}$ at $T=60 \mathrm{~s}, I=370 \mathrm{~A}$ (experimental); $3-\mathrm{PF}$ at $T=60 \mathrm{~s}, I=$ $=450 \mathrm{~A}$ (calculated); $4-\mathrm{CF}$ at $T=180 \mathrm{~s}, I=170 \mathrm{~A}$ (calculated); $5-\mathrm{CF}$ at $T=180 \mathrm{~s}, I=170 \mathrm{~A}$ (experimental)

the gradient of the temperature field increases, and the temperature of the near-contact layers adjacent to the flashing surface increases. The flashing surface is smoother as compared to that of CF (Figure 8). Even at 3 times shortened duration of heating as compared to $\mathrm{CF}$, the temperature of near-contact layers undergoing intensive deformation remains higher than that at CF (see Figure 7). This creates necessary conditions for the formation of quality joints at high gradients of the temperature field.

To test the possibilities of producing quality joints during welding of rails with a minimum energy input, a batch of high-strength rails M76, manufactured by the NKMZ, as well as rails of Azovstal, was welded. For comparison, the batches of rails M76 and KF were also welded at the modes accepted for CF. The accepted modes for welding of these batches using PF provided a lower energy input, characterized by temperature fields (see Figure 6). For comparison, the temperature fields are also given, corresponding to the accepted modes of heating using CF. The total width of the zone of structural transformations was smaller than at the optimal mode of welding accepted at $\mathrm{CF}$, and the duration of flashing process was reduced by $2-3$ times. All the batches of M76 and KF, weld-

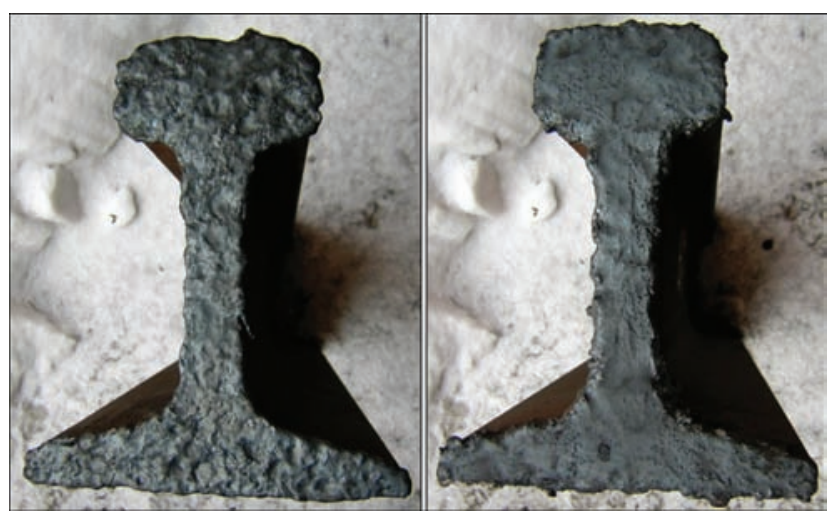

Figure 8. Surface of flashing rail ends before upsetting 
Table 2. Results of tests of M76 and K76F steel rails on static bending in welding using CF and PF with different energy input ${ }^{*}$

\begin{tabular}{|c|c|c|c|c|}
\hline Values of mechanical tests & 1 & 2 & 3 & 4 \\
\hline \multicolumn{5}{|c|}{ E76F «Evraz» (Russia) } \\
\hline Fracture load, t & $\frac{1700-2200}{2000}$ & $\frac{1750-1900}{1850}$ & $\frac{2200-2400}{2250}$ & $\frac{1950-2100}{1900}$ \\
\hline Deflection, mm & $\frac{10-30}{20}$ & $\frac{12-26}{18}$ & $\frac{32-40}{35}$ & $\frac{20-28}{24}$ \\
\hline \multicolumn{5}{|c|}{ K76F (Azovstal Iron \& Steel Works (Ukraine)) } \\
\hline Fracture load, t & $\frac{1600-2000}{1800}$ & $\frac{1750-1950}{1850}$ & $\frac{2100-2400}{2250}$ & $\frac{1850-2050}{1900}$ \\
\hline Deflection, mm & $\frac{18-30}{25}$ & $\frac{21-30}{22}$ & $\frac{34-50}{42}$ & $\frac{25-32}{28}$ \\
\hline
\end{tabular}

ed with different energy input, were tested for static bending in accordance with the accepted method, and the structure of welded joints was also examinated. The test results are shown in Table 2. During tests of batches of rails of steel grade M76 and K76F, welded at $\mathrm{CF}$, the test results were distinguished by unstable and low values. Reduction in the flashing duration from 200 to $160 \mathrm{~s}$ allowed improving the structure and increasing the values of ductility during tests of single specimens, but a number of specimens with defects in the welding plane significantly increased. The values of mechanical tests of batches, welded at PF met the regulatory requirements, but differed depending on energy input. The highest and stable values were observed in the batch welded at $\mathrm{PF}$ with a heating duration of $60 \mathrm{~s}$ for the rails M76 and $80 \mathrm{~s}$ for $\mathrm{K} 76 \mathrm{~F}$. The main result of these investigations was the conclusion that at PF it is possible to vary the energy input during welding of high-strength rails in a wide range without fear of tdefects occurrence in the joint

Table 3. Results of tests on static bending in welding using CF (period II)

\begin{tabular}{|l|c|c|}
\hline \multicolumn{1}{|c|}{ Steel grade } & Fracture loading, $\mathrm{t}$ & Deflection, mm \\
\hline M76 & $\frac{1900-2200}{2150}$ & $\frac{34-65}{48}$ \\
\hline K76F & $\frac{2100-2400}{2250}$ & $\frac{34-50}{42}$ \\
\hline E76F & $\frac{2300-2600}{2400}$ & $\frac{32-42}{35}$ \\
\hline R260 & $\frac{2250-2500}{2400}$ & $\frac{37-50}{48}$ \\
\hline R350NT & $\frac{2000-2200}{2100}$ & $\frac{34-50}{40}$ \\
\hline R350NT & $\frac{2870-3100}{3000}$ & $\frac{58-66}{62}$ \\
\hline VS-350Ya & $\frac{2300-2700}{2500}$ & $\frac{30-50}{40}$ \\
\hline AREAL 136 10 SP & $\frac{2200-2400}{2300}$ & $\frac{35-45}{40}$ \\
\hline AREAL 136 HE370 & $\frac{2200-2550}{2300}$ & $\frac{34-45}{38}$ \\
\hline
\end{tabular}

plane. In fractures of the test batches of rails, during tests with fracture of rails in the joint plane, no defects such as oxide films were detected, even at a minimum welding duration. The absence of defects in welding with high gradients of temperature fields proves the fact, that at PF more favorable conditions for the formation of joints are created.

The carried out investigations established that at PF the maximum value of $\Delta_{\text {curv }}$ decreases by $1.5-2.0$ times, and the thickness of the melt $\delta_{1}$ is more stable and its minimum values are higher than at $\mathrm{CF}$. It is also necessary to take into account the fact that even at minimum energy input, the temperature of the near-contact layers with thickness of up to $3 \mathrm{~mm}$ is close to $1300{ }^{\circ} \mathrm{C}$, which provides their high degree of deformation during welding, contributing to the removal of oxide structures. The microstructure in different areas of the HAZ differed depending on the value of energy input. Metallographic examinations of the microstructure of welded joints of rail batches allowed identifying common features of their formation. The total width of the heat-affected zone is more than 2 times decreased with a decrease in energy input as compared to that accepted at CF (Figure 9, a). In the welding zone, an increase in hardness with its local decrease in the weld center and along the zone boundaries is observed. This is predetermined by a change in the metal structure in the tempering zone at its HAZ boundaries and a decrease in the carbon content in the joint plane (Figure 9,b). The width of these sections is negligible and does not affect the wear resistance of the surface of a rail contact head. In the areas with increased hardness, on separate areas the structure of sorbite-like pearlite is transformed into a bainitic structure; such structures are formed on welding modes of less than $40 \mathrm{~s}$. At the maximum duration of flashing the weld center in the joint plane, the formation of free ferrite inclusions along the grain boundaries is observed (Figure 10, $b$ ). The absence of 

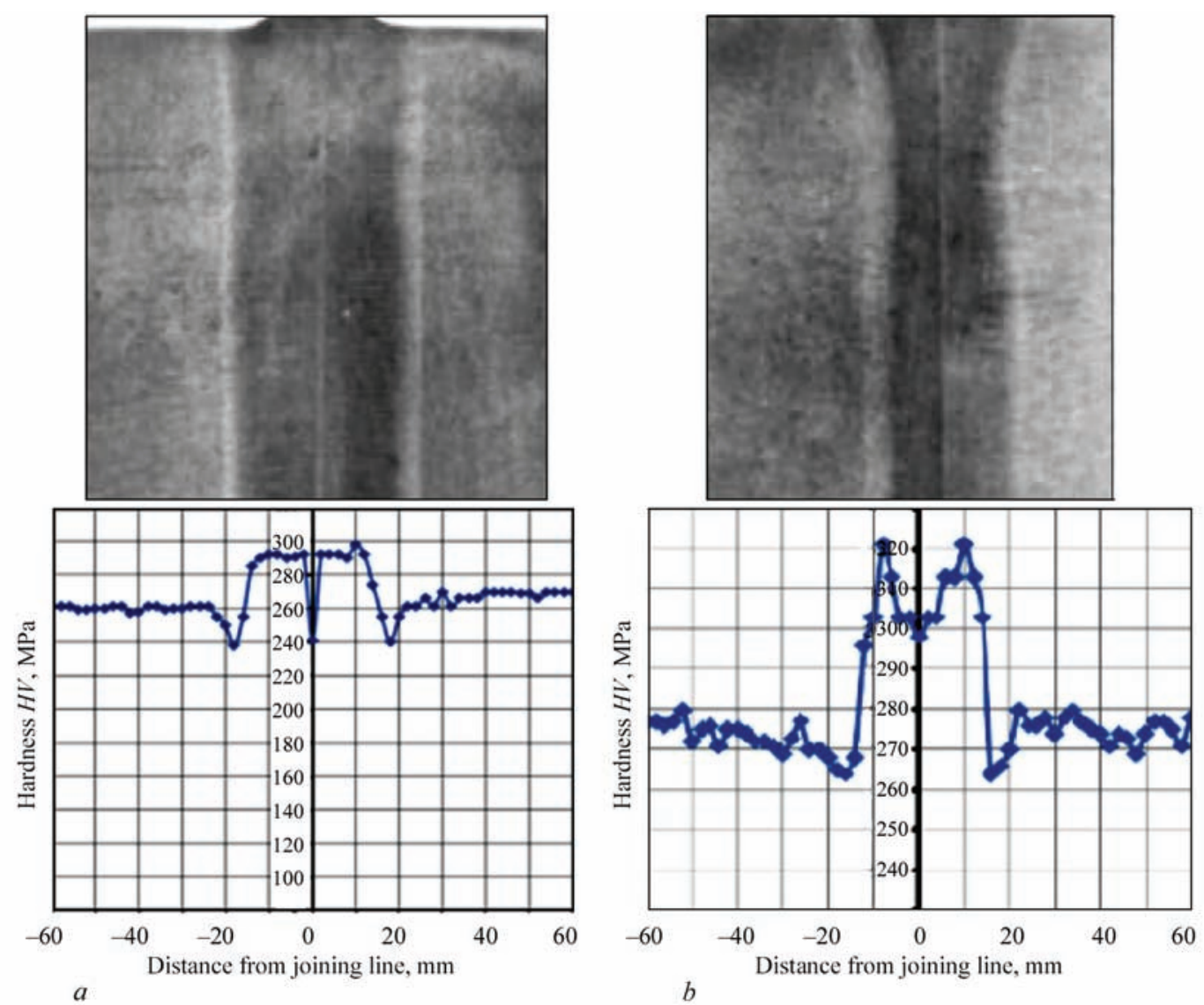

Figure 9. Macrosections during flashing of welded joints of rails M76, using CF (a) and PF (b)

defects in welding with high gradients of temperature fields indicates the fact that at PF, the choice of the optimal value of energy input can be determined based on the conditions for obtaining an optimal structure in the HAZ.

In the last decade, in the railways of many countries of the world, including Ukraine, the use of high-strength rails of eutectoid and hypereutectoid composition began. The flash-butt welding of rails is performed applying stationary and mobile machines of PWI design, which are exported to many countries of the world. The PWI team took part in the developments of technologies for welding rails of different production, including high-strength ones. The considerable experience was gained in welding of rails of different manufacturers.

For welding of high-strength rails with eutectoid and hypereutectoid carbon content, the programs of welding using PF are used shown in Figure 11. The programs for changing the basic parameters are taken as basic and are certified. The control of energy input is carried out by changing the flashing duration and the welding current value. Since the programs are focused on using the design of the PWI in the machines, their adaptation to the capabilities of welding equipment (resistance to $Z_{\text {sh.c }}$ and power of the welding circuit, quick-response of the drive) was minimized. The basic elements, which determine the mechanical properties of metal in all the batches of rails, are iron, carbon, manganese and silicon. As the alloying elements vanadium, titanium, niobium, nitrogen and chromium were used. The mentioned elements strengthen the metal, including carbides and carbonitrides. The basic microstructure for all the investigated batches of rails is the quenching sorbite, which differs only by the degree of dispersion in different rails. The steels R260 and R350NT are distinguished by an increased content of manganese, which improves their calcination ability. When changing the energy input in sufficiently wide ranges $\left(t_{\mathrm{w}}=40-80 \mathrm{~s}\right)$, the formation of defects in the welding plane was not observed, but a significant effect of change in energy input on the structural transformations in the HAZ was revealed. In welding of steels R350NT of the investigated batches of rails on the boundary of the heat-affected zone and in the central part of the joint zone, a decrease in hardness is observed. This is predetermined by the heating of the HAZ metal to a high tempering temperature and a decrease in the carbon content in the central part of the weld as a result of heating the metal to the melting temperature. With a decrease in the energy input, the reduction in hardness is manifested to a lesser extent, and the width of the softening regions decreases. This effect is manifested in all the investigated rails, hav- 

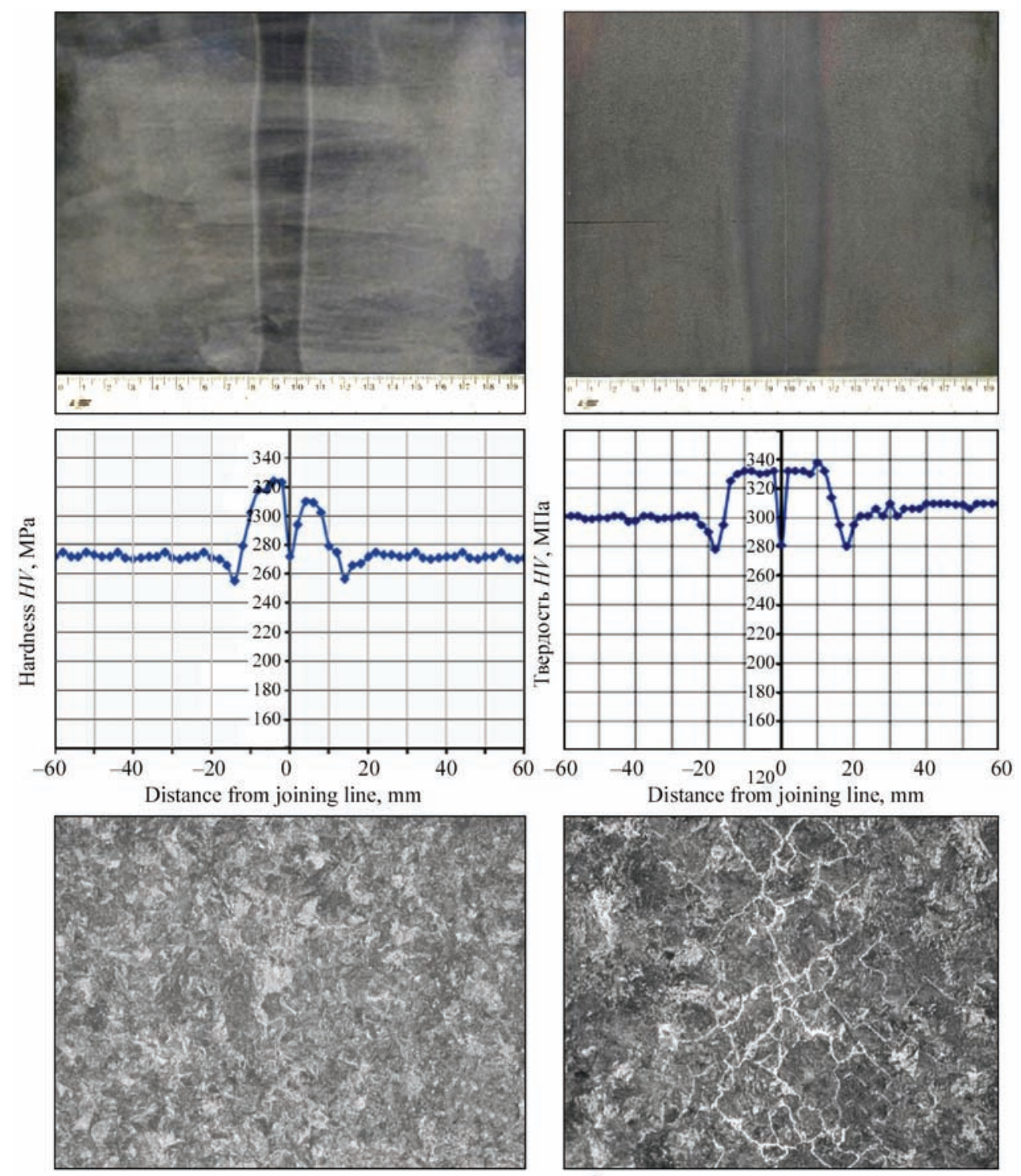

$a$

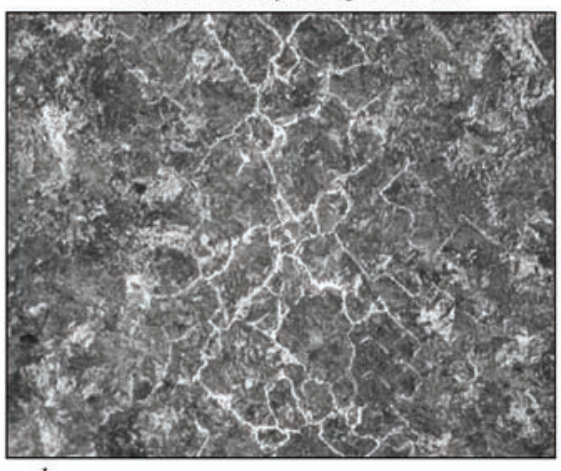

$b$

Figure 10. Macro-, microstructure $(\times 100)$ of hardness distribution $(H V)$ of welded joints of R260 rails with different heat input: $a-$ $T_{\mathrm{w}}=65-75 \mathrm{~s} ; b-90-100 \mathrm{~s}$

ing a hardness of 380-400 MPa. Completely different is the influence of reduction in energy input on the HAZ structure of steels R260. The hardness at a decrease in the energy input increases sharply over the entire width of the HAZ, and at its boundary and in the center the softening is manifested, but to a lesser extent. This is explained by the fact that steel R260

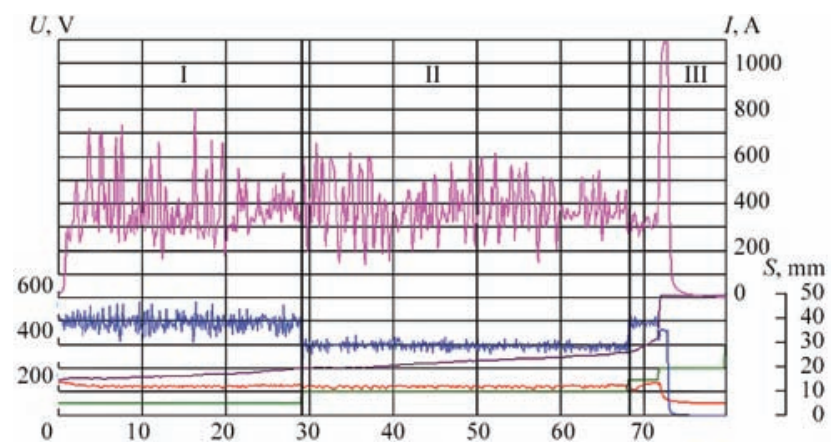

Figure 11. Basic program for changing basic parameters in welding using PF of different rails (I, II, III — periods of program) with eutectoid carbon content is strengthened (during manufacture) to a lesser extent, not reaching the maximum possible value for obtaining higher values of plasticity. During welding, a repeated quenching with increased strength and hardness occurs. According to the technical conditions regulating the requirements to welded joints, it is necessary that during welding the steels of the specified class, the values of hardness deviations in the HAZ should not be higher than $+60 \mathrm{HV}$ and below $-30 \mathrm{HV}$ of the base metal hardness. This complicates the determination of optimal welding conditions, especially for the rails R260. The distribution of hardness in the HAZ during welding the rails R260 at different energy input is shown in Figure 10. To obtain the required hardness, it is necessary to dose the energy input during welding more accurately (see Figure 10, a). The distribution of hardness in the welds of steel R260 at changing the energy 

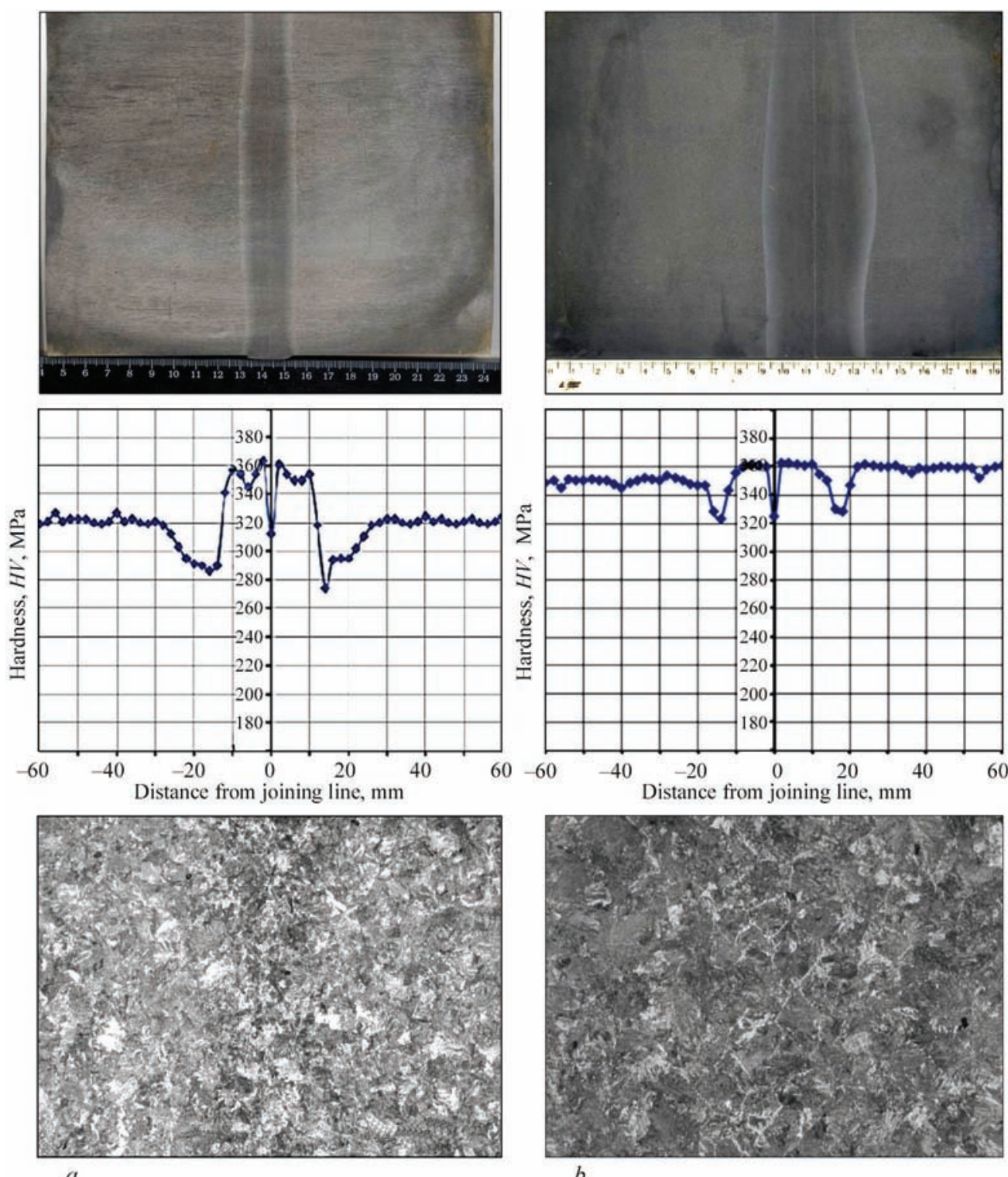

Figure 12. Macro-, microstructure $(\times 100)$ and hardness distribution $(H V)$ in the welded joint of rails of grade R350NT with different heat input: $a-T_{\mathrm{w}}=65-75 \mathrm{~s} ; b-90-100 \mathrm{~s}$

input differs from the optimum value specified by the program. In the specimens of steel R260, the width of the HAZ is determined by the distance between the boundaries of the high tempering areas. The structure of these areas represents a tempering sorbite, in the weld center the structure of primary austenite has a grain size number 3-4, along the boundaries of these grains, the areas of free ferrite are detected. With an increase in the energy input, their thickness increases, which is accompanied by a decrease in hardness at the weld center and mechanical properties during testing. A change in the energy input duration within relatively small limits $(10 \mathrm{~s})$ leads to noticeable changes in hardness in the HAZ (see Figure 10). In the specimens of the steel R350NT (Figure 12), the width of the HAZ is greater. At its boundaries and in the center, a decrease in hardness is observed, which is predetermined by the softening at the boundaries and the formation of the pearlite-sorbite structure in the center with precipitations of free ferrite along the grain boundaries of the primary austenite. Unlike the steel R260, with the change in energy input, the hardness in the HAZ increases slightly and remains at the level of the base metal. In the center, the degree of hardness reduction is determined by the amount of free ferrite. In the considered specimens, the ferrite is not formed in the form of a solid network around the grains of primary austenite, which guarantees a high level of value during bending tests.

The steels ARIEL13610SP and ARIEL136HE370 are distinguished by their high mechanical properties and wear resistance [10].

In the base metal of these steels (Figure 13), at the boundary of the primary austenitic grains of the pre-eutectoid ferrite, the precipitations of the carbide phase are present. At the size of the colonies of sorbite 

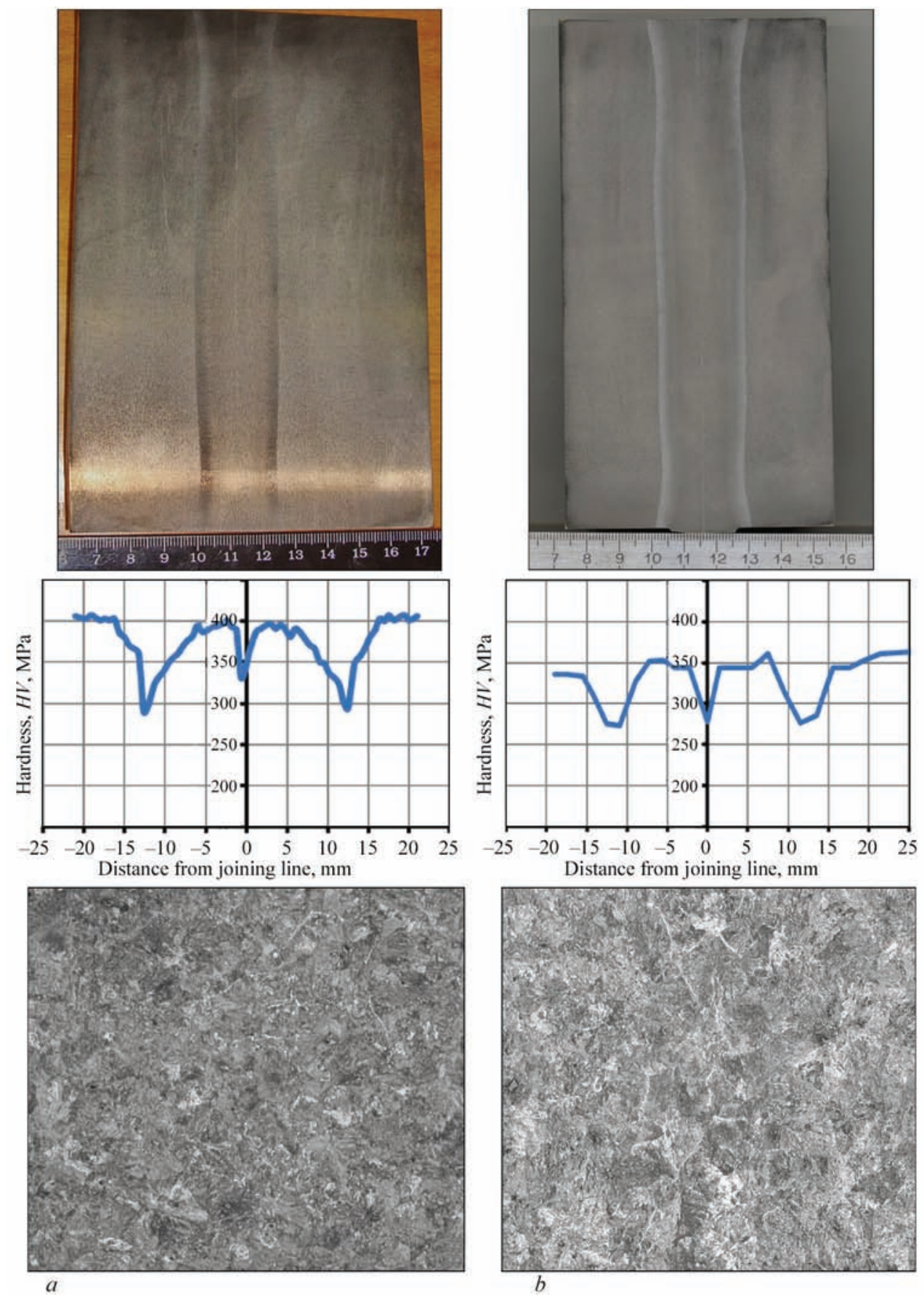

Figure 13. Macro-, microstructure $(\times 100)$ of the joint zone and hardness distribution $(H V)$ in the welded joint of rails of grade AREAL $13610 \mathrm{SP}(a)$ and AREAL $136 \mathrm{HE} 370(b)$

of $10-15 \mathrm{~mm}$, the thickness of precipitations of the carbide phase amounts to tenths fractions of a micron. The presence of this phase increases the wear resistance [11]. In welded joints of these steels, produced at optimal conditions, the carbide phase is absent, and along the joining line, as in the other given examples of high-strength steels, the hardness decreases as compared to the level of the base metal. The degree of reduction is determined by the amount of energy input. During welding of such steels at the accepted mode, the width of the heating zone is larger than in the steel R350NT and amounts to (about $30 \mathrm{~mm}$ ), which allows withstanding the required tolerances for deviations of hardness values in welded joints.

The welding of rails of the mentioned steels was carried out in the mobile machines K922 of the PWI design, which are used in the railways of Ukraine and other countries. The technology of welding using PF was based on the program of energy input (see Figure 6). Its basic parameters are the following: the value of open-circuit voltage $U_{2}$, upsetting forces were taken the same for the rails of all the batches. The energy input was regulated by changing the flashing duration and the value of welding current $I_{w}$ in the pe- 


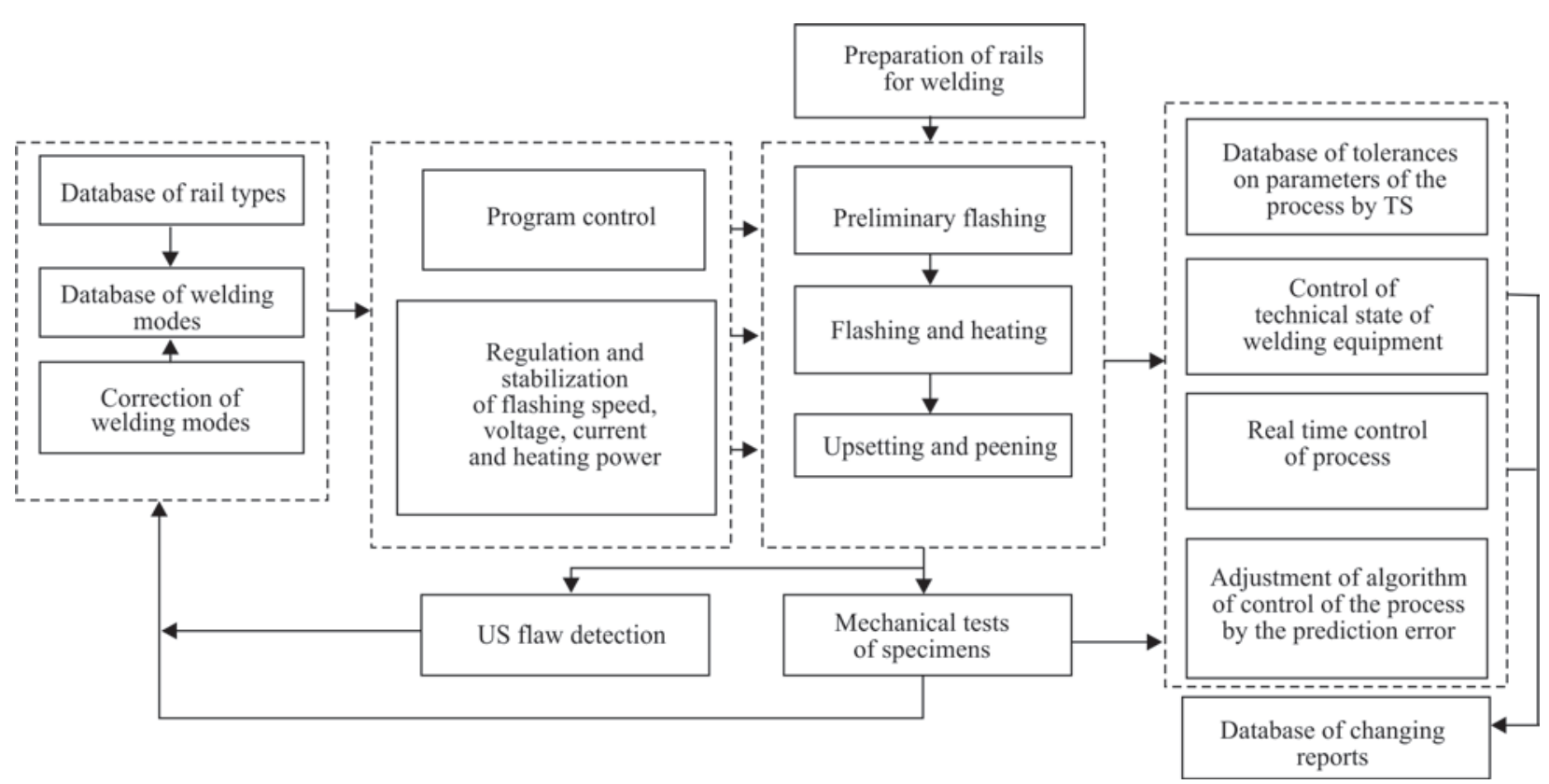

Figure 14. System of automatic multifactor control of parameters of welding process in welding of high-strength rails $\mathrm{K} 76 \mathrm{~F}$

riod II, specified by the program. The optimal modes for welding each rail batch were established and the reference batches in the amount of 10 butt joints were welded, which were subjected to tests in accordance with the requirements of the EU and Ukrainian standard. The test results are given in Table 3. All the welded joints of reference batches satisfy the requirements of the mentioned standards not only by the results of mechanical tests, but also during testing the quality of joints using nondestructive methods of testing. The rails of the type R260 and R350 were tested for fatigue and withstood $5 \mathrm{mln}$ cycles without fracture. It should be noted that all the welded joints of reference batches were not subjected to heat treatment after welding. In the process of investigations and gained experience of the production use of the developed technologies, it was established that during welding at the modes characterized by a low energy input, a more strict limitation of the admissible deviations of the preset welding parameters is necessary, especially those affecting the energy input. Therefore, the implementation of $\mathrm{PF}$ welding technology in production conditions became possible after the development of the new systems and algorithms for automatic control of the welding process at the PWI. Instead of using rigid programs for changing the basic parameters accepted during welding of standard rails, a self-adjusting system for control of parameters was developed (Figure 14). The system allows maintaining the optimum mode of stable flashing and heating in real industrial conditions, largely regardless the changes in operating conditions (voltage mains fluctuations, changes in ambient temperature as compared to the calculated one, being $20^{\circ} \mathrm{C}$ ).
A distinctive feature of the developed technologies is the presence of feedback by the basic parameters in the control systems, automatically correcting their value preset by the program during deviation from the preset values. In the process of flashing, short-term corrections of the preset values are carried out in order to stabilize their preset mean values. The algorithms for control of this process were developed for each of the parameters, for example, the value of welding current and energy are regulated by the feed speed so, that their mean values maintained at a preset level. At the same time, the level of the voltage is taken into account, supplied to the parts to be welded, resistance of the welding circuit and the force, generated by the hydraulic drive of the machine (during welding with tension). The similar multifactor algorithms for regulation are also used by the other flashing parameters. In some cases, the accumulation of short-term changes of the values preset by the program, can lead to correction of the program itself, for example, to increase in flashing duration in each of its periods. If these changes do not exceed the admissible ones, then they are acceptable. The algorithms for assessing the effect of changes on the quality of joints were determined. They allow expanding the range of admissible deviations and provide a high reproducibility of the preset welding programs.

The capabilities of the automatic system for control of welding parameters are not infinite and cannot prevent rough violations of the operating conditions of welding equipment.

The registration of programs for changing basic parameters during welding of a butt joint provides an effective use of the developed algorithms for evalua- 
tion of the joint quality according to the value of deviations in a real time of works. For each welded butt joint, the computer system for control of the welding machine issues a certificate, where the change in the basic parameters, as well as their actual deviation from the optimal values is shown in textual and graphical form. The algorithms of control were developed, on the basis of which the system provides a quality evaluation of a welded butt joint in real time and includes it to the certificate. The test results are issued simultaneously after the end of welding on the machine display for information to the operator and simultaneously to the diagnostic center, where a more thorough analysis is carried out taking into account the results of destructive testing of butt joints and ultrasonic inspection. The specialists of the PWI together with the PrJSC «Ukrzaliznytsia» performed a large amount of works (several tens of thousands of welded butt joints). On the basis of this information, the algorithms for assessing the quality of joints during in-process control and the normative documents regulating the quality assessment [11] were specified.

The multifactor system for control of welding machines (see Figure 14) is used in stationary and mobile machines of the PWI design. In the design version for mobile machines, it is combined with a drive, providing welding of rails with tension, where additional feedbacks are used, providing the fulfillment of programs for moving welded rails combined with their tension.

These operations are controlled by a common computer, which simultaneously performs welding programs, in-process control and rail tension by the mobile machines.

Welding of long-length rails with tension. During repair of seamless railroad tracks after cutting out of defective or worn-out sections of rails, as well as during reconstruction of tracks, it is necessary to weld long-length rail sections attached to the crossties between them. For this purpose, instead of a cutout section of rails between the ends of the joined sections, a rail-insert is installed, which is welded-on to the ends of the rail sections [12]. During welding of the second closing butt, in accordance with the standards, it is necessary to restore the temperature-stress state on the section where welding was performed. This is achieved by adjusting the length of the rail-insert due to its elongation or shortening by the value $L_{t}=f(\Delta T)$, where $\Delta T=\theta_{\text {fix }}-\theta_{\mathrm{w}}\left(\theta_{\text {fix }}\right.$ is temperature of the fixing welded plates, $\theta_{\mathrm{w}}$ is the temperature at the moment of welding rails). For railways of Ukraine, the temperature of fixing the rails is $\theta_{\text {fix }}=30^{\circ} \mathrm{C}$. Most of such works are carried out at the ambient temperatures $\theta_{\text {fix w }}\left(\theta_{\text {fix w }}<\theta_{\text {fix }}\right)$, respectively, it is at the same time necessary to shorten the length of the insert-rail.
In order to obtain the required increment of $L_{\mathrm{t}}$ and also to provide allowances for welding, the insert and a part of the rail track of the released section are bent. After welding the section is returned to its initial position. This operation is very labor-intensive. It is performed using a set of mechanisms (lifting, tension) and a large number of auxiliary workers. The railway traffic in the period of repair is stopped, which is associated with great material losses. The PWI specialists together with the USA companies, engaged in reconstruction and repair of railways, developed the technology and equipment which allowed combining welding with restoration of temperature-stress state of the track. It is based on the idea of creating such a level of tensile stresses in the process of welding in the joined rail sections, at which, at a preset temperature range $\left(-40-+50^{\circ} \mathrm{C}\right)$, the possibility of the formation of compression stresses is excluded. The carried out calculations showed that to solve this problem, it is necessary, that the level of tensile stresses does not reach the limit values, exceeding $16 \%$ of the yield strength of rail steel, which is acceptable. To perform the tension, it is proposed to use a hydraulic drive for flash-butt welding machines of the PWI design, performing flashing and upsetting. The process of flashbutt welding was proposed to be carried out using programs involving two stages. At the first stage, the high-speed movement of the end of the welded insert is provided to the size of the preset gap $\Delta_{\mathrm{g}}$. The duration of this stage does not exceed a few seconds. After contact of the ends of the rails and the exciting of flashing, the program of welding rails accepted for the rails of this type with their simultaneous tension is performed. During welding, the rails are shortened and their tension is continuing. After the end of welding, the required shortening is provided $L_{t}=L_{w t}+\Delta_{g}$, where $L_{\mathrm{w}}$ is the shortening of the rail during welding $\left(l_{\mathrm{w}}=l_{\text {flash }}+l_{\text {upsett }}\right) \Delta_{\mathrm{g}}$ is the gap between the ends of rails, preset before welding. The regulation of the tension value is carried out by setting the gap $\Delta_{\mathrm{g}}$. In the considered variant, the rails adjoining the welded joint are not stretched and the whole tension $\mathrm{L}_{\mathrm{t}}$ is carried out due to the elongation of a rail-insert. In many cases, the forces required for tension of a rail-insert of the standard length of $24 \mathrm{~m}$ cannot be provided. In this case, the required value of tension is provided due to additional jointing of rail ends from the crossties adjacent to the weld to the value $L_{\text {cr.t. }}, T$. The total force $L_{t}$ is formed due to the choice of the value $L_{\text {cr.t }}$ and $\Delta_{\mathrm{g}}$, i.e. $L_{\mathrm{t}}=L_{\text {cr.t }}+\Delta_{\mathrm{g}}+L_{\mathrm{w}}$.

In welding of rails with tension, the technology of welding rails with $\mathrm{PF}$ is used. The programs for control of welding process are stored the same as during welding of separate rails without tension us- 
ing common systems for automatic control of flashing parameters and introduction of additional feedbacks of parameters controlling energy input. The change of stress forces during welding affects the operation of the hydraulic drive of welding machines. Except of the data determining the parameters of welding modes, the development of additional systems of automatic control, stabilizing the energy input, was required.

In welding with a tension, the data on the temperature of rails, at which their welding is performed, are entered into the computerized control system: $\theta_{w}$, the total length of sections areas released from the fasteners $\left(L_{\text {cr.t }}\right)$, the length of the rail-insert, as well as the size of the gap between rail section and the insert $\Delta_{\mathrm{g}}$. In addition, the value of the fixing temperature on the welded area $\theta_{\text {fix }}$ is separately entered.

In welding with tension after the end of welding, the weld metal is heated to the temperature of 1100 $1200{ }^{\circ} \mathrm{C}$ and is maintained in a compressed state by the drive of the welding machine. At the same time, the control systems, mounted into the machine, control the duration of cooling the weld to the temperature of $100-200^{\circ} \mathrm{C}$. Simultaneously, the flash, formed during welding, is cut out automatically. After performing these operations, the rails are unclamped and the welding machine is removed from the butt. The duration of rail welding does not exceed $2 \mathrm{~min}$, and the auxiliary operations largely depend on the chosen scheme of works organization during welding. Performing the tension according to the variant, when preparation of a rail-insert of a preset length is used without jointing the adjacent areas of the sections, the maximum efficiency of rail welding complexes is provided, and using the variant, requiring the jointing of a certain section of the track, the duration of auxiliary operations increases and depends on the length of the section, released from the cross-ties.

Figure 15 shows the dependence of the force (1) and the value of the travel of the movable clamp of the welding machine (2), necessary for the implementation of the rails tension, corresponding to $\Delta T=30^{\circ} \mathrm{C}$ at different length of their section, released from the cross-ties. If the tension is carried out only with the help of the rail-insert of $24 \mathrm{~m}$ length, a tension force of $100 \mathrm{t}$ and the travel of the movable clamp of about $150 \mathrm{~mm}$ are required. At the condition that the ends of rails are released from fastenings at different length, the force is significantly reduced. An increase in the travel of the movable clamping machine has a much smaller effect on the weight of the rail welding machine than the effect of the upsetting force. Therefore, the rational relationship of these parameters of the machines determines their capabilities during

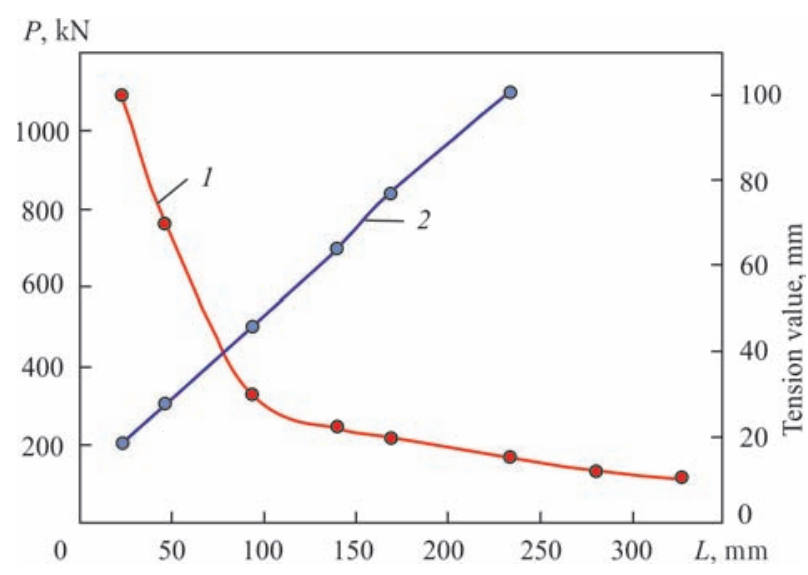

Figure 15. Dependence of tension forces (1) and tension value (2) of travel of moving clamp of the machine on the length of the jointing section of the rail fastening on the cross-ties at $\Delta T=30^{\circ} \mathrm{C}$

operation in different conditions. The rail welding machines of previous generations of the type K355, K900, available at the railways, cannot be used for welding with tension, primarily due to the insufficient upsetting force and the limited travel of the movable clamps. In addition, during development of new generations of mobile rail welding machines for welding with tension, a significant change in their basic units, systems of hydroelectric control, design of the drive for welding circuit and flash remover were required.

In recent years, the PWI developed several generations of machines for flash-butt welding with tension. Unlike the machines of previous generations, a number of new solutions was applied in them, namely:

- machines have upsetting forces and, accordingly, clamping forces which are 2-3 times higher. The upsetting forces and clamps are increased with an increase in the movement travel of the movable clamping;

- machines have a multifactor system for the control of basic parameters of the welding process, providing a stable reproduction of preset welding programs regardless of the change in the tension forces;

- welding circuit of the machines despite the increase in the sizes of the force drive for feed the mechanical part, provides the required minimum level of $Z_{\text {sh.-c; }}$;

- cutting of flash during welding is carried out by the mechanism of flash remover, built-in to the body, which is controlled in the clamped state up to the full cooling of the butt joint. This eliminates the probability of rupture of a heated butt joint during unclamping of the machine.

The developed new generation of rail welding machines allows welding long-length rail sections, combined with their tension. The technical characteristics of the machines are given in Table 4. The machines are distinguished by an increased upsetting force, exceeding the values of previous generations of machines by $2-3$ times. In addition, the movement drive 
Table 4. Technical characteristics of stationary and mobile rail welding machines developed by the E.O. Paton Electric Welding Institute and manufactured by the PJSC «KZESO»

\begin{tabular}{|c|c|c|c|c|c|c|c|c|c|c|}
\hline \multirow{2}{*}{ Technical parameters } & \multicolumn{10}{|c|}{ Types of machines of the OJSC «KZESO» } \\
\hline & K900 & K920 & K921 & K922-1 & K930 & K950 & K945 & K960-1 & K1045 & K1000 \\
\hline Rated voltage of mains, $\mathrm{V}$ & 380 & 380 & 380 & 380 & 380 & 380 & 380 & 380 & 380 & 380 \\
\hline Highest secondary current, not lower than, kA & 60 & 67 & 67 & 67 & 67 & 67 & 67 & 67 & 60 & 84 \\
\hline Rated power, (DC $50 \%$ ), $\mathrm{kV} \cdot \mathrm{A}$ & 150 & 211 & 236 & 210 & 210 & 210 & 210 & 210 & 150 & 300 \\
\hline Upsetting force, $\mathrm{kN}$ & 450 & 1000 & 1500 & 1200 & 1200 & 1200 & 1200 & 2000 & 600 & 900 \\
\hline Clamping force, $\mathrm{kN}$ & 1350 & 2500 & 2900 & 2900 & 2900 & 2900 & 2900 & 4650 & 1500 & 2000 \\
\hline Travel of moving column of the machine, $\mathrm{mm}$ & 70 & 100 & 150 & 100 & 200 & 250 & 400 & 280 & 100 & 100 \\
\hline Weight of the machine, $\mathrm{kg}$ & 2700 & 3000 & 4100 & 3450 & 3600 & 3650 & 3700 & 5670 & 3500 & 8800 \\
\hline
\end{tabular}

has a 2-4 times increased travel of the movable part of the machine, taking into account the different values of tension during welding.

The machines provide different $\mathrm{PF}$ processes to a full extent, adapted to the specifics of flashing drive loads while performing a pulsating process of moving welded parts in combination with their tension. The drive is characterized by a high quick-response, providing a change in the value of spark gap with an accuracy of tenths of millimeters. With an increase in the upsetting and clamping force, the weight of the machines increases, which defines the design of the auxiliary equipment used in flash-butt welding of rails. The power part of mobile complexes is determined by the power of diesel generators. It remains the same as for previous generations of machines of $250-300 \mathrm{kV} \cdot \mathrm{A}$. With an increase in the weight of welding machines, more powerful lifting devices of the complexes are required, which reduces their mobility. During the use of the machines K900 of previous generations, a large number of such complexes were manufactured in different countries. Therefore, the demand for flash-butt welding machines with tension appeared, having a lower weight and adapted to the existing mobile rail welding complexes of previous production.

The advantages of flash-butt welding with tension are fully realized in the machines K921 (Figure 16) designed by the PWI. The upsetting force of $150 \mathrm{t}$, developed by the drive of the machine, allows car-

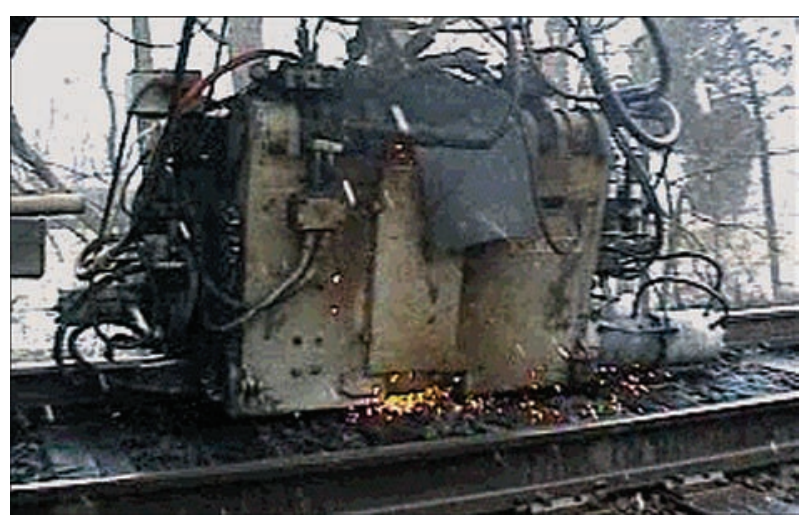

Figure 16. Appearance of the machine K921 rying out welding with tension during reconstruction of railway tracks with their transfer to the high-speed mode of operation. During welding, the section regions, adjacent to a welded butt for not more than 15$20 \mathrm{~m}$, are released. The machines K921 are used in the USA, where more than 10 thou of high-speed racks are welded using such machines. The machines K922 (Figure 17), designed at the PWI in accordance with license agreements, which are adapted to the already operated complexes in many countries of the world, are used in different mobile complexes equipped with flash-butt welding machines K922 of the KZESO production. About 20 of such machines operate at the railways of Ukraine. A successful combination of upsetting force (up to $120 \mathrm{t}$ ) and the travel of the moving clamp $(150 \mathrm{~m})$ provides minimization of the length of the section, released from the cross-ties, and allows using these machines in different conditions. At the same time, the machines K920, K930 with upsetting force of $100 \mathrm{t}$ are being developed and they are adapted to the mobile rail welding complexes of the machines (K900) of previous generation, available in many countries of the world.

In accordance with the license agreement with the Holland Company, the PWI developed the machines K930 and K945 (Figure 18), which have an increased travel of the mobile clamping of up to $450 \mathrm{~m}$ with an upsetting force of 120 tons. This allows welding long length rail sections during reconstruction of railway

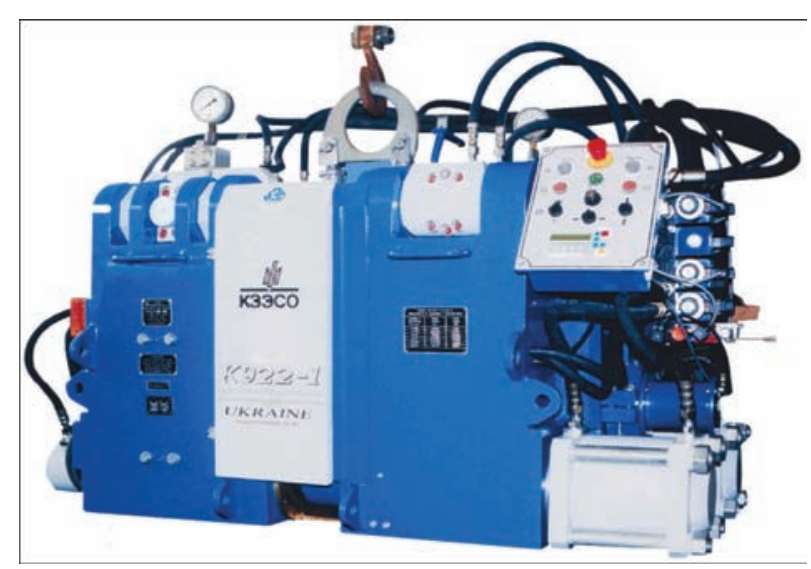

Figure 17. Appearance of the machine K922 


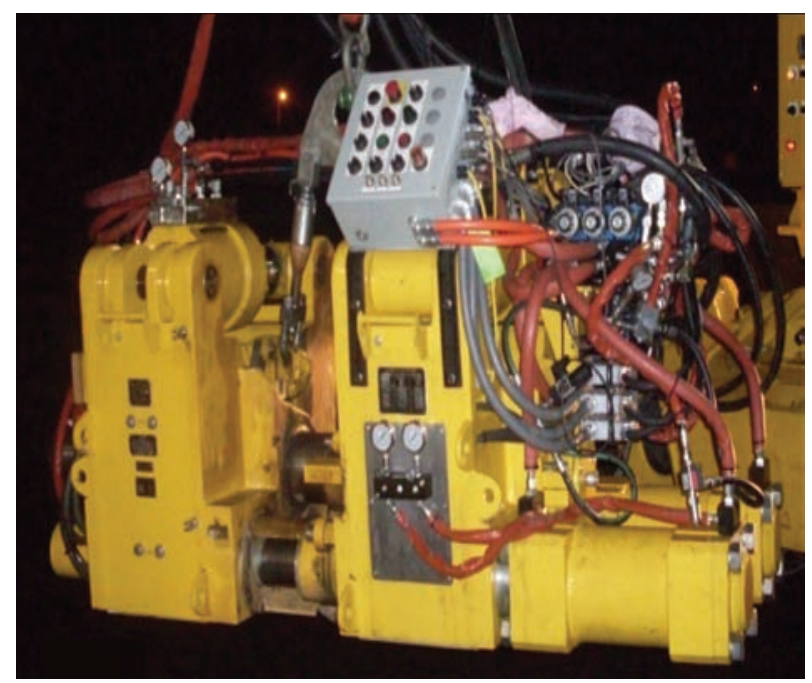

Figure 18. Appearance of the machine K945

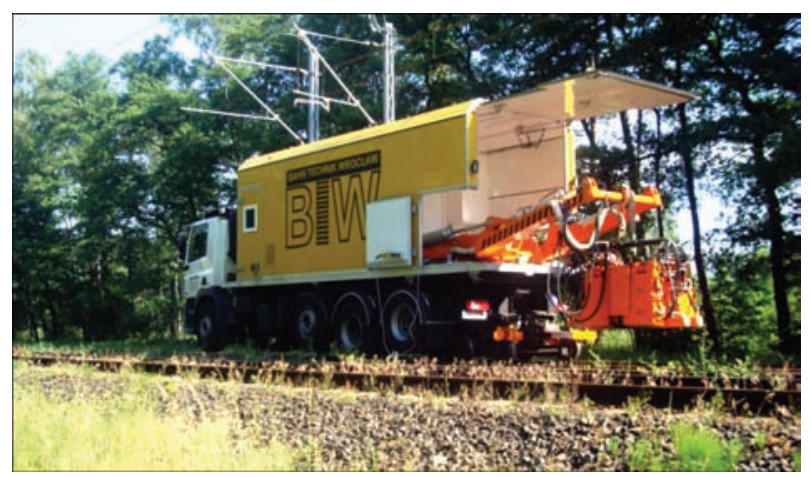

Figure 19. Mobile rail welding complex KSM 007 with welding head K922-1 based on a Volvo machine

tracks. Accordingly, the mobile complexes for operation with such machines were developed. The minimization of weight and expanded capabilities of upsetting drive allowed creating highly-manouevrable complexes using the machines K945 (Figures 19-22). Ten such complexes have been operating since 2014 at the railways of the Great Britain. They use the machines K945, developed at the PWI and manufactured at the KZESO.

\section{Conclusion}

The many-year developments of the PWI technologies and equipment for flash-butt welding of rails made a significant contribution to the development of railway roads in the countries of the former CIS and the world. According to the data of the plant KZESO, at present more than 2,500 machines is operating, developed by the PWI, manufactured and supplied by the plant in accordance with license agreements and contracts to different countries of the world. Only in the last 5 years, such rail welding machines were exported to the USA, Canada, China, France, Iran, Malaysia, Austria, Morocco, Poland and other countries, which accounts for $60 \%$ of the world stock of

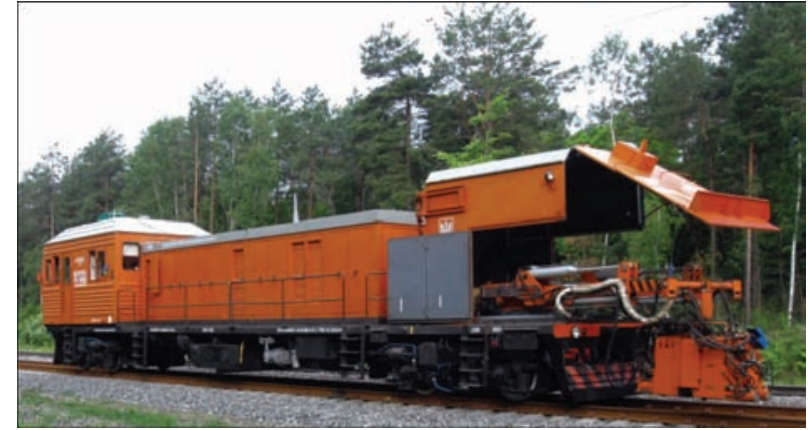

Figure 20. Mobile rail welding complex KRS-1 with welding head K922-1

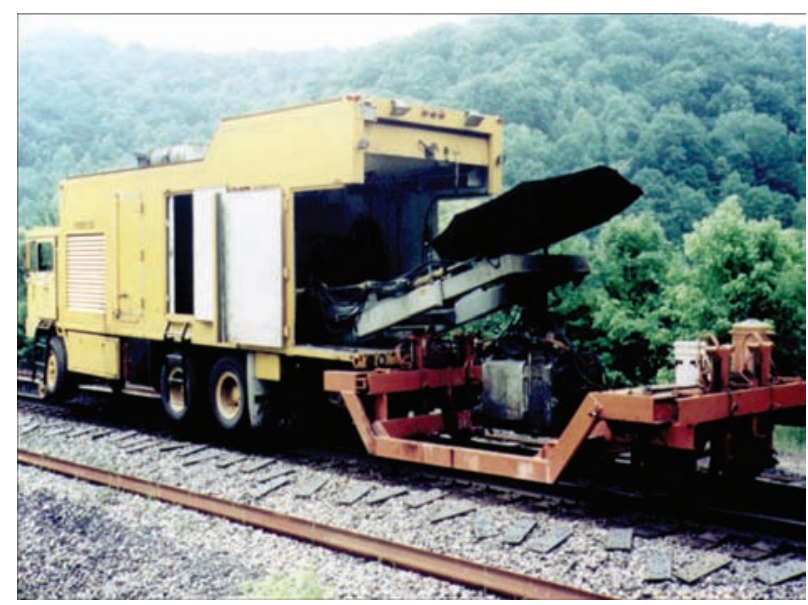

Figure 21. Mobile rail welding complex (USA)

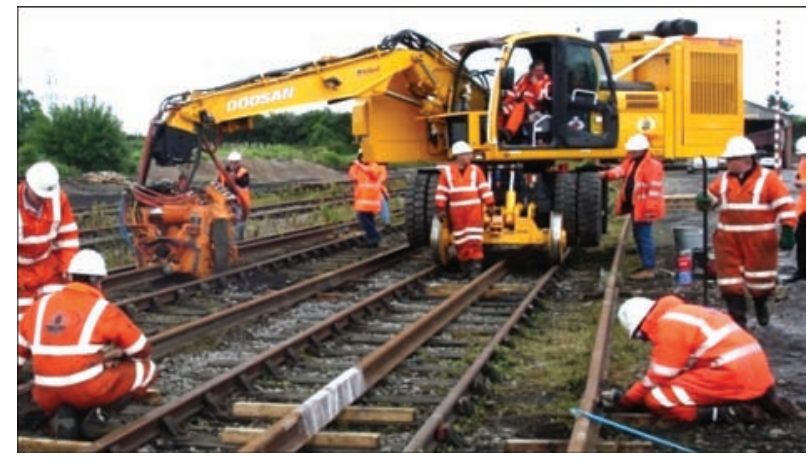

Figure 22. Mobile rail welding complex (UK)

flash-butt rail welding machines and $90 \%$ of mobile rail welding machines [13]. Their use contributed to the acceleration of construction and reconstruction of railways, including high-speed railroads. Only at the railways of China more than 100 machines K922 are used, which contributed to the acceleration of the construction of high-speed railroads there. At the USA railways, the machines K900, K920, K930, K922, K921 are used.

The PWI continues to develop the technologies and equipment for flash-butt welding of high-strength rails, the demands for which are continuously sent to the Institute. This is predetermined by the increase in the traffic volume of railroads and the need in in- 
creasing the life of rails in operation. The metallurgical industry begins to master the production of highstrength rails with a guaranteed service life, which exceeds the average level of achieved wear resistance by $2-3$ times. The existing experience shows, that the rails of such quality are difficult to weld. The new technologies of their welding and automatic system for the control of welding process are needed.

Along with the developments of new technologies, the PWI continues to develop new equipment for welding of rails on the track and in stationary conditions.

In accordance with a licensing agreement with the Progress Rail, USA, an experimental model of the machine K960 with an upsetting force of 200 tons was designed and manufactured, which is being tested, as well as the machine K963 for welding rails with rail frogs. The machine K960 is characterized by unique capabilities for the further development of technologies of welding with tension, and the new machine K963 allows for the first time to weld-in the rails with the ends of railway frogs on the track.

The further improvement of the system of remote quality monitoring of rail joints in real time is very promising. The use of modern means of information technology opens up broad opportunities for the further development of an effective type of quality control of welded rail joints. The development of new technologies, systems for automated control of the process of flash-butt welding of rails continues.

1. (1980) Welding handbook. Vol. 3: Flash welding. $7^{\text {th }}$ Ed. Sect., AWS, 60-65.
2. Kuchuk-Yatsenko, S.I., Lebedev, V.K. (1969) Continuous flash-butt welding. USSR author's cert. 226745, Int. Cl B23K 11/04. Pat. GB11623073, Great Britain; Pat. FR1517114, France; Pat. SE322003, Switzerland; Pat. IT796912, Italy.

3. Paton, B.E., Lebedev, V.K. (1969) Electric equipment for resistance welding. Moscow, Mashinostroenie, 40-68 [in Russian].

4. Kuchuk-Yatsenko, S.I., Krivenko, V.G., Bogorsky, M.V. (1974) Method of control of flash-butt welding process. USSR author's cert. 542604, Int. Cl. B23K 11 [in Russian].

5. Paton, B.E., Sakharnov, V.A., Lebedev, V.K., Kuchuk-Yatsenko, S.I. (1967) Machine for flash-butt welding. USSR author's cert. 201561, Int.Cl. B23K 11, Pat. US3349216, USA; Pat. GB1056812, Great Britain; Pat. FR1384943, France; Pat. DE1465942, FRG; Pat. F149917, Finland.

6. Kuchuk-Yatsenko, S.I., Shvets, V.I., Didkovsky, A.V et al. (2013) Defects of joints of high-strength rails produced using flash-butt welding. The Paton Welding J., 9, 2-8.

7. Kuchuk-Yatsenko, S.I. (1992) Flash-butt welding. Ed. by V.K. Lebedev. Kiev, Naukova Dumka [in Russian].

8. Kuchuk-Yatsenko, S.I., Didkovsky, O.V., Bogorsky, M.V. et al. (2002) Flash-butt welding method. Pat. 46820, Ukraine, Int.Cl. 6B23K11/04, C2; Pat. 2222415, RF (2003); Pat. 6.294.752; USA (20.06.01); Pat. ZL001016772/5 (2004), PRC.

9. Kuchuk-Yatsenko, S.I., Milenin, A.S., Velikoivanenko, E.A. et al. (2018) Mathematical modeling of metal heating process in continuous flash-butt welding. The Paton Welding J., 10.

10. Kimura Tatsumi, Takemasa Mineyasu, Honjo Minoru (2011) Development of SP3 rail with high wear resistance and rolling contact fatigue resistance for heavy haul railways. Jfe Technical Report, 16.

11. (2016) TU U 27.1-40081293-002:2016: New welded rails for railways. Dnipropetrovske NKTB KG, Ukrzaliznytsya [in Ukrainian].

12. Kuchuk-Yatsenko, S.I., Velikoivanenko, E.A., Rozynka, G.F. et al. (2004) Investigation of residual stresses in welded joints of rails produced by flash-butt welding. The Paton Welding J., 9, 32-35.

13. Mazur, M.A. (2018) Historical outlines of Paton scientific school. Kharkov [in Russian]. 


\title{
THIN-WALLED WELDED TRANSFORMABLE-VOLUME STRUCTURES OF SPACE PURPOSE
}

\author{
L.M. LOBANOV and V.S. VOLKOV \\ E.O. Paton Electric Welding Institute of the NAS of Ukraine \\ 11 Kazimir Malevich Str., 03150, Kyiv, Ukraine. E-mail: office@paton.kiev.ua
}

\begin{abstract}
The rational methods of construction of metal transformable shells with the aim of creation of the space-purpose structures were investigated. The mathematical modeling of the neutral shell surface movement of the transformable-volume structure (TVS) was performed, the result of which was used to set the reference surface at the kinematic modeling of its stress-strain states during a compact folding. The parameters of the process of welding thin shells of stainless steel were determined, guaranteeing the maximum approaching of physicomechanical properties of welded joints to similar properties of the base metal, thus providing their vacuum density. The methods of surface modification of thin metal shells of stainless steel, which allow improving the safety margin of TVS of space purpose without changing their mass and compactness were developed. The possibility was experimentally confirmed and the conditions of stability of complete reverse transformation of a multisectional conical-type TVS were formulated. 7 Ref., 12 Figures.
\end{abstract}

K e y w o r d s : deployable structures, thin shells, microplasma welding, microstructure and weld metal, surface engineering

The relevance of works on creation of transformable-volume structures (TVS) is predetermined by the contradiction between the need in creating shelltype structures of the necessary parameters and the possibility of their further delivery to the site of operation under the conditions, which exclude the feasibility of realizing their long-time and labor-intensive manufacturing process at the intended site. The most typical example of solving such problems can be the delivery of elements of aerospace engineering, based on shell-type structures, to the near-earth orbit. At the same time, the engineering progress has a demand for shells of ever-increasing volume, the range of application of which is restricted, first of all, by the lack of appropriate means of transportation.

The deployable shell structures allow simplifying the delivery of a payload to the near-earth orbit and represent a one of the areas of space technologies being actively developing. They are divided into three main classes: soft load-carrying; those, constructed on the base of a transformable frame and rigid ones. In the world practice, among the first TVS the transformable load-carrying soft shells found industrial application, which were used in building industry, during construction of aircrafts and spacecrafts. In the last decade, the attempts were made to test prototypes of inhabited transformable shell-type structures outside the earth's atmosphere. For today, the project of NASA and «Bigelow Aerospace» «BEAM» (Bigelow expandable activity module) was realized, which implements the task of creating a segment of the International Space

(C) L.M. LOBANOV and V.S. VOLKOV, 2018
Station (ISS) with a soft shell. In 2016, the module BEAM was delivered to the orbit in a nonsealed cargo container of the «Dragon» spacecraft; after docking the capsule to the ISS, the module was deployable by the inner pressure of the station until reaching the design dimensions $-4 \mathrm{~m}$ length and $3.2 \mathrm{~m}$ diameter, in this case the initial length increased by 1.86 times [1].

The modern materials with new properties allow creating space deployable structures [2], the ratio of linear transformation $K_{\mathrm{t}}$ of which can reach 10; however, the problem of combining these parameters with a sufficient strength, air-tightness and service life of shells remains unsolved. It is obvious that technologically acceptable characteristics with a simultaneous air-tightness are achievable only in rigid load-carrying shells, whose surface transformation into a more compact form requires extraordinary solutions in the field of technologies of cold volumetric deformation.

At the E.O. Paton Electric Welding Institute the possibility of transforming the volume of closed all-welded metal shells, which were called TVS, was theoretically grounded, and in empirical way the technological solutions for its implementation were found $([3,4])$. The use of existing modifications at the present stage required the selection of a type of load-carrying TVS acceptable for using in space technology, the working out of new design and technological solutions for load-carrying TVS and the creation of a universal computational model, which allows determining geometric and technological parameters during designing and manufacturing of a wide range of TVS using different structural materials in relation 
to the extreme conditions of operation in the open space. Thus, the general aim of the mentioned works was to develop the scientific fundamentals for the theory of construction of deployable shell structures of space purposes. Their practical embodiment is the applied development and the further investigations of all-welded thin-walled load-carrying TVS, which are optimized for applying in the open space and can be used during implementation of existing and future aerospace projects.

The fulfillment of the specified task required definition of the following:

- type of transformable surface, optimal for designing and manufacturing the metal long-length TVS of space purpose, as well as the designing scheme of TVS, which requires the creation of a computational methodology, which determines the relationship between its geometric, technological parameters and space environmental factors (SEF), under the action of which the structure is in the process of operation;

- universal algorithm for transformation of a surface of a selected type to a compact form, which allows structure deploying without losing its functional properties, and the technology of volumetric deformation, in which the specified algorithm should be implemented for real shell materials.

In creating of transformable shells a class of surfaces, theoretically capable of deploying into a plane is of the greatest interest. These are linear surfaces of zero Gaussian curvature: cylindrical, conical, and developable surfaces. The first two classes can serve as the base for construction of shell structures and are widely used in engineering. However, isometric transformation of a cylinder by the method of mirror reflection cannot be realized through the equalities of any its horizontal intersections, and transformation by movement in all the cases is reduced to partition a cylindrical surface into many adjacent flat polygons, similar to the «origami folding» transformation schemes [2]. The movement to the plane of a cylindrical shell of real sheet materials in such a case represents a bending with non-zero radii along the lines of their joining, which leads to the formation of stress concentrators, most pronounced at the assemblies of shaping fins, and a significant reduction in the compactness of a transformable shell. In particular, the simplest principle of transformation of TVS of cylindrical type with the use of the so-called hyperboloid fold (Figure 1, $a, b$ ) allows achieving transformation ratios $K_{\mathrm{t}}$, i.e. the ratio of lengths of the structure in the deployed and compact state, which do not exceed the values $K_{\mathrm{t}}=4.0-4.5$.

The use of thin-sheet metals as a material for shells and the application of the considered methods of their transformation impose restrictions on the ratio of their height and bases diameters, which, ultimately, requires the sectioning of a long-length structural shell. Thus, the bases profiles of cylindrical TVS sections (Figure 1,a) are not flat curves at any moment of deformation, therefore, the adjacent sections cannot be joined with each other without rigid fixing on a flat circumferential contour. The influence of the edge effect near the shell rigid contour boundary leads to the characteristic distortion of rectilinear shell folds along the lines of generatrices (Figure 1, b, E), which are the main cause of a low compactness of the cylindrical-type transformable shells.

TVS of the conical type (Figure 1,e) is characterized by higher deformability, however, it is capable to compensate higher values of the load without losing stability. Except of high values of the linear transformation coefficient, it can reach the value of $K_{t}=150$,
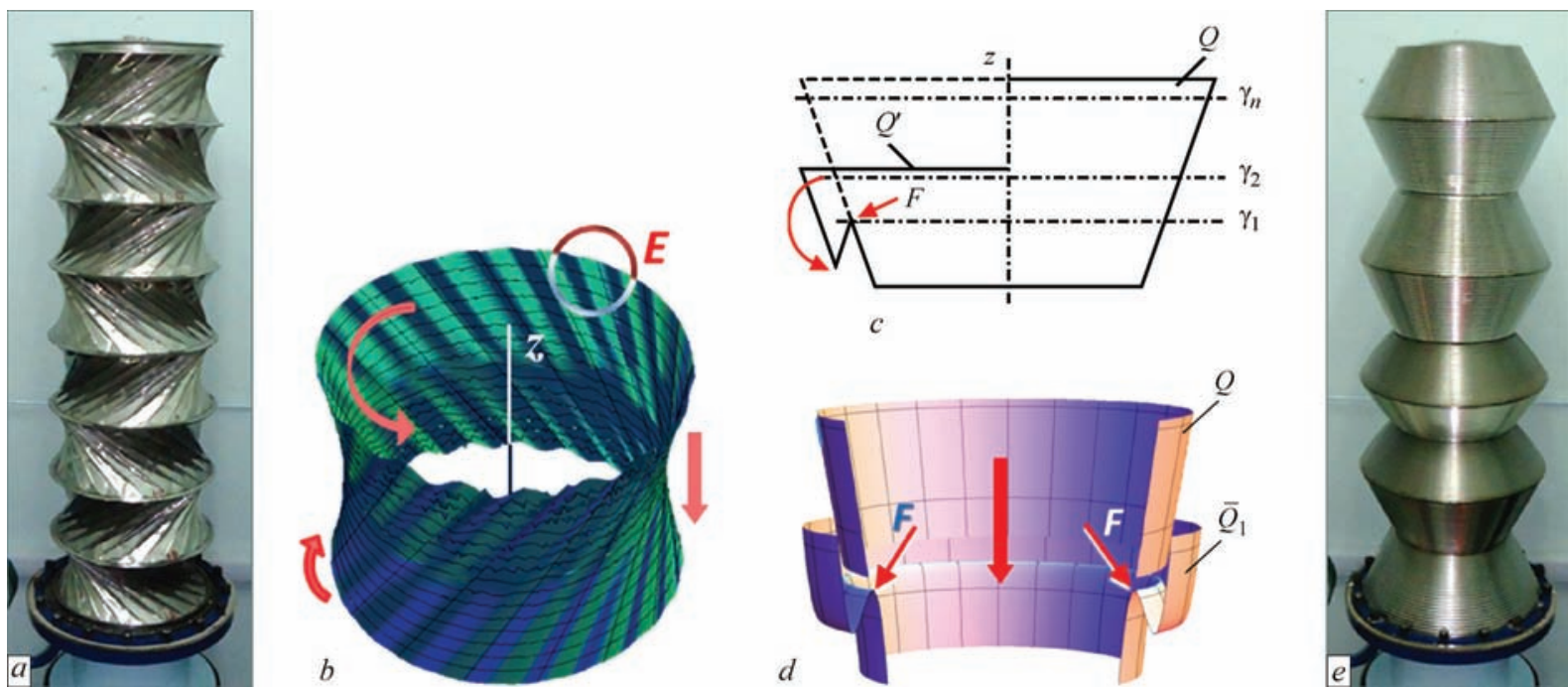

Figure 1. Cylindrical TVS (a) and topological model of transformation of neutral surface of cylindrical shell into hyperbolic folds (b); transformation of neutral surface of conical shell into circumferential folds: theoretical model of isometric transformation (c) and graphical interpretation of mathematical model of movement during formation of the fold $F$ with non-zero bending radii $(d)$; conical TVS (e) 
the conical shell is the only one of the linear surfaces, the compact transformation of which can be implemented almost without tensions and compressions (Figure 1, $d$ ), and, so, with a practical approximation to the theoretical model of isometric transformation of surfaces $Q \rightarrow Q^{\prime}$ by successive mirror reflection with respect to the cutting planes $\gamma_{1}-\gamma_{n}$ (Figure 1,c). The totality of these advantages allows confirming the rationality of using TVS of the conical type when solving the problem of creating long-length TVS.

The considered task of creating a long-length load-carrying transformable structure was oriented to the solution of the actual problem of removing the payload (PL), i.e the research equipment of $40 \mathrm{kN}$ weight beyond the limits of the own outer atmosphere of the ISS, the negative effect of which on the performance of PL devices is pronounced at the distance of $5 \mathrm{~m}$ from the space station outer surface. Some of the basic geometric parameters of TVS are predetermined by the characteristics of the transport compartment of the rocket-carrier (the maximum diameter of structure shell, its size in the transport state and the inner volume after deployment). In addition, TVS of the specified length in the conditions of the action of regulated inertial and temperature loads should have a double safety margin and provide the absence of deviations of a free end with a PL for more than $150 \mathrm{~mm}$.

To construct a computational model of a multisectional conical shell, the ratios of the main geometric parameters of a long-length conical TVS were determined. Thus, the task of using the structure also predetermined its total length $L=5 \mathrm{~m}$ and the maximum diameter $D=400 \mathrm{~mm}$, which allows presetting the radius of the larger base of the conical sections. The compactness of a multisectional structure is regulated by the value of its height in a compact state $\left(h_{\text {pack }}\right)$ and the inner volume in the state of deployment $\left(V_{\mathrm{TVS}}\right)$. The acceptable angles of the shell conicity $\alpha$ were determined, at which its bending with compliance with the admissible values of relative circumferential deformations in structural materials is accepted. For metallic materials widely used in the aerospace industry with a ductility margin within the range of $\sigma_{y} /$ $\sigma_{t}=0.3-0.8$, admissible for realization of the task of volumetric deformation of the test shells, the values of relative deformations should not exceed the values $\varepsilon_{\mathrm{c} . \mathrm{d}}$ $\leq 2.0-2.5 \%$. According to the scheme of deformation of a thin metallic shell, corresponding to Figure 1,d, the obtained values of relative circumferential deformations correspond to the values of the conicity angle $\alpha=25-27^{\circ}$.

Further, an auxiliary parameter was introduced: the coefficient of volumetric transformation $K_{V}=V_{1}$; $V_{2}$, where $V_{1}$ is the volume of a truncated conical shell; $V_{2}$ is the volume of the shell in a compact state. At the preset values of the conicity angle $\alpha$ and the length $L$, as well as the known values of $R, K_{v}, h_{\text {pack }}$ and $V_{\text {TVS, }}$, the determination of the basic geometric characteristics of TVS is reduced to finding the radius of the smaller base $r$; the height of the conical section in the compact state $h$, which is accepted to be equal to the depth of the formed corrugation $a$; required, in accordance with the specified characteristics, compactness of the number of folds (corrugations) $n_{1}$, and, accordingly, to the number of TVS sections $n$ (Figure 2).

Having expressed the coefficient of volumetric transformation $K_{V}$ through the main geometric parameters of the transformable conical section

$$
K_{V}=\frac{(R-r) \operatorname{ctg} \alpha}{3 h}\left(1+\frac{r}{R}+\frac{r^{2}}{R^{2}}\right),
$$

let us write the expression for $r$ :

$$
r=-\frac{R}{2}+\sqrt{\frac{3 V_{\mathrm{TVS}}}{\pi L}-\frac{3}{4} R^{2}} .
$$

Then, it is possible to determine the parameters:

$$
n=\frac{L}{\operatorname{ctg} \alpha(R-r)} ; \quad h=\frac{h_{\text {pack }}}{n} ; \quad n_{1}=\frac{L}{2 h n} .
$$

Thus, after determining the thickness of shell material within the range $\delta=0.1-0.2 \mathrm{~mm}$, predetermined by the desire to limited decrease in the mass of the structure at a simultaneous providing the integrity of welded joints after extreme deformation, the task of construction of a complete calculation scheme of multiconical TVS was limited only by determining the optimal width or step of the fold (corrugation) $b$.

The fundamental difference in the transformation scheme for real materials (Figure 1, d) from the scheme of isometric transformation in Figure 1, $c$ is

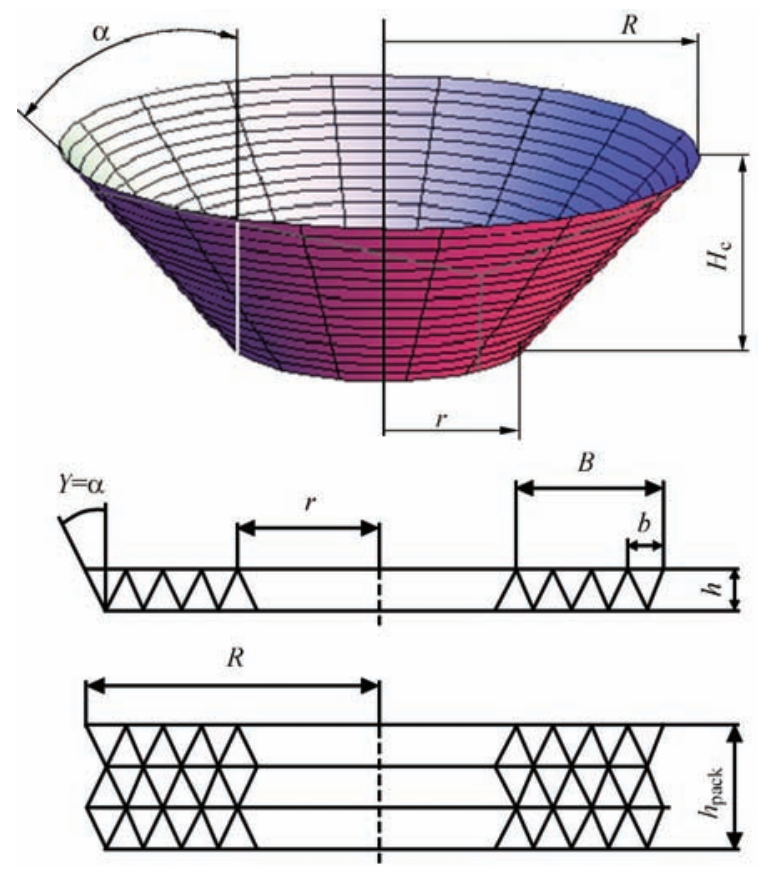

Figure 2. Calculation scheme of multisectional conical shell 
the introduction of nonzero radii of bending $r_{\mathrm{c}}$ of the fold $F$, which are determined, first of all, by the TVS shell thickness. To determine the geometric parameters of the conical shell corrugation of metallic materials of a preset thickness, an adaptation of its finite profile to the known requirements of not exceeding the minimum bending radius for thin plates and shells was carried out. The determination of the minimum bending radii of the fold (4) was carried out by the admissible value of thinning at the apexes of corrugations formed:

$$
\Delta \delta=\frac{\delta^{3}}{4\left(2 r_{\text {cirv. }}+\delta\right)^{2}},
$$

where $\Delta \delta$ is the size of thinning; $\delta$ is the workpiece thickness; $r_{\text {curv. }}$ is the radius of the curvature of middle surface, $r_{\text {curv. }}=r_{\text {c }}+\delta / 2$.

From the formula (4) it follows that the maximum reduction in thickness (at $r_{\mathrm{c}}=0$ ) amounts to approximately $\delta / 4$ and at $r_{\text {curv. }} \geq 5 \delta$ the thickness at bending remains almost unchanged (Figure $3, a$ ).

The relative radius of rounding the support fins $r_{c}$ can be found as a circle radius using the semi-graphical method in the medium Wolfram Mathematica, being maximum approximated to the profile of corrugation flat apex (Figure 3, b), and corresponds to the ratio:

$$
r_{\mathrm{c}}=0.15 b \text {, }
$$

where $b$ is the corrugation pitch.

Having chosen the mean value of material thickness $\delta=0.15 \mathrm{~mm}$ from the specified range for preliminary calculations, according to (5) and the ratio $r_{c} / \delta=5$, it is possible to determine the value $r_{\mathrm{c}}=0.75 \mathrm{~mm}$, which corresponds to the corrugation pitch $b=5 \mathrm{~mm}$. It is obvious,
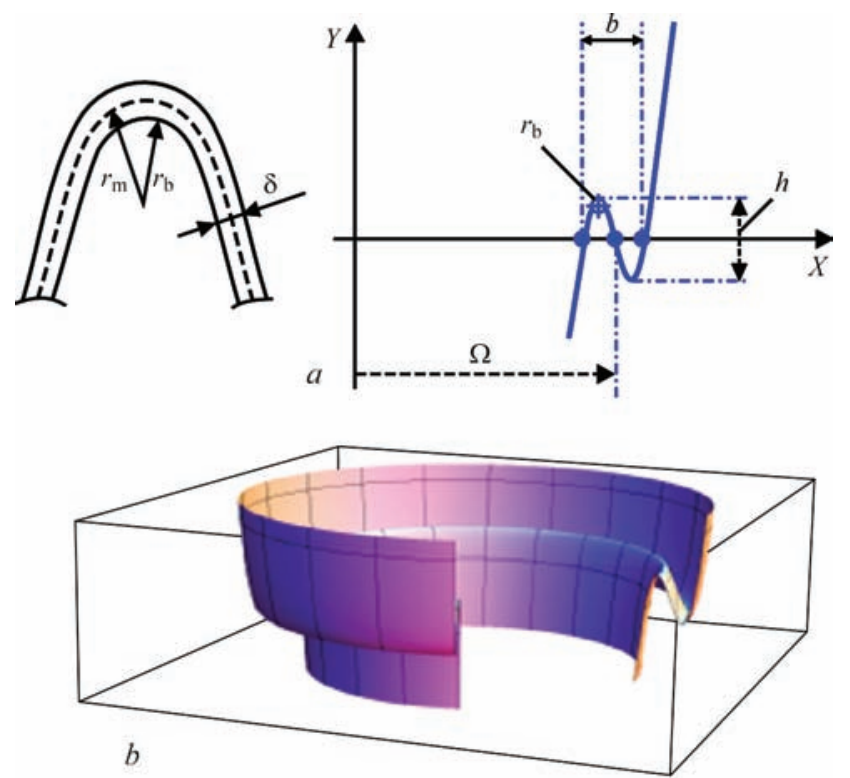

Figure 3. Determination of minimum bending radii of fold according to the admissible value of thinning near the apexes of corrugations $(a)$ and graphic interpretation of mathematical model of shape transformation of the truncated conical surface with the formation of a circumferential fold $(b)$ that the obtained geometrical ratios of a corrugation of a metal shell correspond to its highest compactness, i.e. the smallest radius of rounding at the fold apexes.

For quantitative evaluation of stress-strain states (SSS) in the process of formation of corrugations, the numerical modeling applying the finite element method was used, realized with the help of universal software systems of finite element analysis. For generalization of the obtained results, the equivalent deformations $\varepsilon_{\mathrm{e}}$ and equivalent or total stresses $\sigma_{\mathrm{e}}$ (von Mises) were used according to Mises-Guber's theory of strength (ductility), or the theory of the highest specific potential energy of shaping. As a criterion of strength during calculations of a transformable shell, the achievements of values of yield strength in its material by equivalent stresses were accepted, i.e $\sigma_{\mathrm{e}}<\sigma_{02}$.

The comparison of kinematic finite element model of formation of folds of conical shell with the theoretical (Figure 3,b) model of bending the neutral surface while using different sheet materials: steel, titanium and aluminium, and also the plotting of fields of stresses distribution (Figure 4) in the folds being formed allowed in each case to determine the conformity of the shell material with the accepted strength criterion. Thus, the influence of physical and mechanical properties of different structural materials of the shell on geometric parameters of circumferential folds and on the technological process of compacting of the conical surface was determined. As was noted above, for calculations as a shell material, the metals with a characteristic ratio of the yield strength $\sigma_{y}$ to the tensile strength $\sigma_{t}$ were selected, which are widely used in the processes of cold volumetric deformation and can theoretically provide the necessary characteristics of structural strength.

Deformation of the shell is accompanied by occurrence of stresses in it, the values and nature of distribution of which is substantially changed in the process of loading. In all the variants of calculations, the highest stresses cover insignificant geometric regions and sharply reduce their values at their removing from local stress concentrators: the lines of the shell bends. The highest values of equivalent stresses $\sigma_{e}$ on the face surface of folds are noted on the shell of steel AISI $321(171 \mathrm{MPa})$, for the shell of titanium VT1-0 and aluminium AMg-5 they amount to 125 and $55 \mathrm{MPa}$, respectively, which illustrates the conformity to the accepted criterion of strength of each of the deformed shells with the selected ratios of geometric parameters of corrugations. The highest values of residual equivalent stresses $\sigma_{e}$ on the neutral surface are noted in the shell of titanium VT1-0 (64.2 MPa). The corresponding values $\sigma_{e}$ for the shell of steel AISI 321 are $50.4 \mathrm{MPa}$ and have the smallest values in the shell of aluminium AMg-5 (15.8 MPa). The given result indicates the presence of smaller, as compared with 

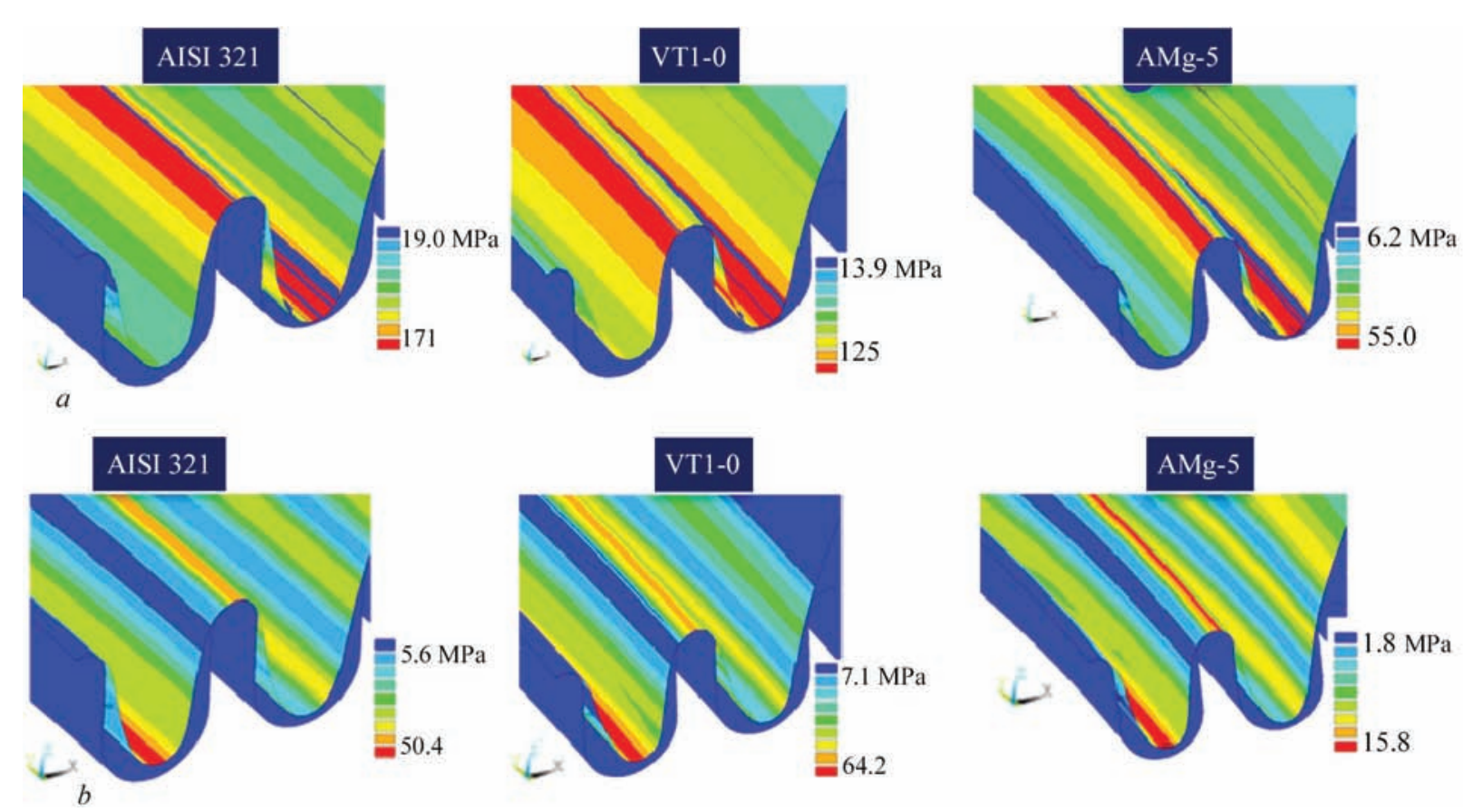

Figure 4. Equivalent stresses in the upper face surface of folds of conical shell of TVS from different materials being formed (a) and equivalent stresses at the neutral surface of the formed folds $(b)$

titanium, tensions of the neutral surface of steel shell during the formation of corrugations, i.e., its closer approximation to the desired isometric transformation. Further, the values of elasticity modulus for titanium and, especially, aluminium, lowered as compared to steel, show the high deformability of structures, made of these materials, during operating conditions under the action of SEF. An additional argument in favor of choosing the steel AISI32 as a material, is the best manufacturability of its welding process and repeatability of the joints quality, absence of need in special preparation of semi-products and complete protection of the inert gas welding zone.

In the process of shaping the each successive corrugation of the depth $h$, the height of the conical shell $H_{c}$ decreases by a value, equal to $2 h$. Hence, the coefficient of linear transformation of the shell $K_{t}$ equals to $2 n_{1}$, where $n_{1}$ is the number of corrugations; at other equal conditions, a structure made of discs with a larger number of corrugations will have smaller dimensions in the transportation state. At the known value $K_{\mathrm{t}}=L / h_{\text {pack }}=30$ and $D=400 \mathrm{~mm}$, the length of the transformable area of conical shell is $B=\left(K_{t} / 2\right)$ $b=75 \mathrm{~mm}$. Thus, the radius of the small base of the conical shell of TVS is $r=125 \mathrm{~mm}$, the height of the conical section at $\alpha=25^{\circ}$ is $H_{c} \approx 160 \mathrm{~mm}$, respectively, the total number of shells at the maximum test length of TVS $L=5000 \mathrm{~mm}$ is $n_{1}=31$. The design scheme of a multiconical TVS, whose geometric parameters can be specified by the results of a complex of calculations of the structure for strength and stability under the conditions of action of specific factors of space environment, is shown in Figure 5.
In general, the optimization of geometric characteristics of the conical TVS during designing is reduced to finding the most advantageous combination of shell strength and its compactness, or the transformation coefficient $K_{\mathrm{t}}$ at the least possible mass. Reducing the angle of conicity and the approach of structural sections to the configuration of the cylinder, which is the most advantageous in terms of resistance to the action of non-axisymmetric loads, leads to the decrease in the coefficient of TVS transformation. The opposite approach (increase in $\alpha$ ) significantly decreases the spatial rigidity of the structure but allows increasing $K_{\mathrm{t}}$ and simplifying the process of compact folding.

It is obvious that welding is almost the only method for construction of metallic air-tight shell structures. One of the main problems in creation of TVS shells is the achievement of combination of high physico-mechanical characteristics at the simultaneous vacuum tightness of rectilinear welds which undergo a complex of mechanical loads and specific aggressive external factors (SEF). The scheme of the shell

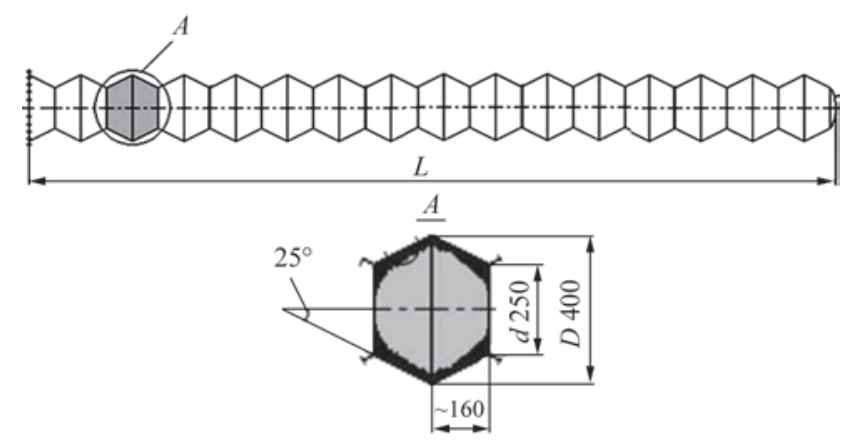

Figure 5. Designed scheme of multiconical load-carrying TVS of space purpose 
isometric transformation requires the isotropy of the structural material; therefore, a weld should have the physical and mechanical properties almost equivalent with those of the structure base metal. While choosing a method for welding butt joints of stainless steel with a thickness $\delta=0.1-0.2 \mathrm{~mm}$, the advantage was given to microplasma welding. This method allowed a significant simplifying the preparation of edges of the deployable conical semi-product for welding, thereby increasing the efficiency of the TVS manufacture, and reducing the thermal deformations of a weld using the previous flanging of edges welded. During welding the semi-products of stainless steel, a flanging of edges at a value, equal to two thicknesses of the material welded, was used. In addition, the microplasma method allowed providing almost a complete repeatability of the fixed result of welding.

During experimental welding of specimens of steel of grade AISI $321 \mathrm{~N}$ with a thickness $\delta=0.175 \mathrm{~mm}$ in pulsed mode at the value $I_{\mathrm{w}}=6 \mathrm{~A}, U_{w}=12 \mathrm{~V}$ and welding speed $V_{\mathrm{w}}=3.1 \mathrm{~mm} / \mathrm{s}$, the quality formation of welded joints was achieved at the value of energy input $Q_{\text {input }}=17.59 \mathrm{~J} / \mathrm{mm}$. In the technologically accepted range of pulsed current frequencies, the given value $Q_{\text {input }}$ corresponds to the combinations of parameters $\tau_{\mathrm{i}}=4 \mathrm{~ms} ; \tau_{\mathrm{p}}=1 \mathrm{~ms}$ at the pulsed welding current frequency $f_{\mathrm{w}} \stackrel{\mathrm{p}}{=} 200 \mathrm{~Hz}$ and $\tau_{\mathrm{i}}=30 \mathrm{~ms} ; \tau_{\mathrm{p}}=$ $=10 \mathrm{~ms}$ at the frequency $f_{\mathrm{w}}=25 \mathrm{~Hz}$, where $\tau_{\mathrm{i}}$ is the duration of the current pulse, $\mathrm{ms}, \tau_{\mathrm{p}}$ is the duration of the pause, ms. At the same time, the change $f_{\mathrm{w}}$, and, consequently, the character of the dynamic effect of the plasma column on the area of the molten pool, which is in the state of solidification, also contributes to the improvement of its structure, reduction in the size of the grains (crystallites), breaking the cells (nuclei) of their formation [5]. Therefore, the task of the investigation was to determine a certain range of combinations of frequency and duty factor of the welding pulsed current, corresponding to the optimal macroand microstructure of the vacuum-tight welded joint.
The results of metallographic examinations of characteristic specimens from the series of welded joints, produced at different combinations of welding process parameters, are presented in Figure 6. The nature of the heterogeneity of the weld metal, near-weld zone and the base metal of the TVS shell was determined. The microstructure of specimens produced at a frequency $f_{\mathrm{w}}=200 \mathrm{~Hz}$ (Figure 6,a), measured by Vickers in the microhardness meter Leco M-400, is characterized by a considerably higher homogeneity and coaxiality of the cells at a simultaneous reducing in microhardness deviations along the horizontal and vertical axes of the weld. The size of the equiaxial cells corresponds to a range of 5-7 $\mu \mathrm{m}$ in the entire weld section, the deviations of the measured value of microhardness in any of the directions of the intersection does not exceed $5 \%$ of its value for the base metal.

Comparing the presented results with the results of tests of a series of similar specimens for static tension and bending, which simulates the real operating conditions, the approximation of the elastic-plastic properties for the base metal and welded joints is noted with increasing the pulsed current frequency. Thus, for a typical specimen of the strip AISI 321 of $0.175 \mathrm{~mm}$ thickness with a weld produced at the pulsed current $f_{\mathrm{w}}=200 \mathrm{~Hz}$, the ultimate rupture strength $\sigma_{\mathrm{t} . \mathrm{w}}=489 \mathrm{MPa}$, which amounts to $90 \% \sigma_{\mathrm{tb} . \mathrm{m}}$, the conditional yield strength $\sigma_{0.2 \mathrm{w}}=256 \mathrm{MPa}$ (Figure $7, b$ ). For a similar specimen of the base metal, $\sigma_{t_{b, m}, \mathrm{~m}}=543 \mathrm{MPa}, \sigma_{0.2 \mathrm{~b} . \mathrm{m}}=256 \mathrm{MPa}$ (Figure 7,a).

Determination of nature of the transformation of the middle surface of a truncated conical shell allowed designating the criteria for approaching to the isometricity in the process of its movement and, accordingly, to provide the absence of a local loss of stability in the deployable state. However, the values of stresses and displacements of the deformed shell are subjected to an accurate evaluation. The plotting of distribution fields of the mentioned values allows concluding about the possibility of realization of the calculated parameters
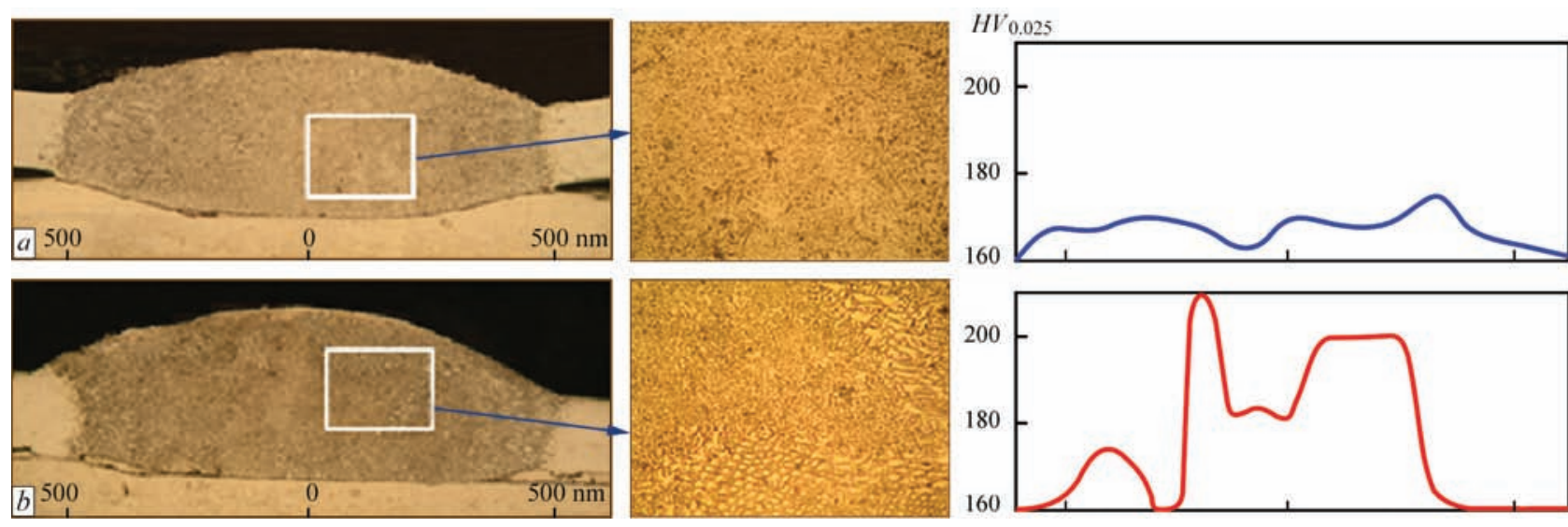

Figure 6. Results of metallographic examinations of a series of specimens of welded joints of foil with a thickness of $0.175 \mathrm{~mm}$ from steel AISI 321, produced at equal values of energy input by microplasma welding method at a pulsed current of 200 (a) and $25 \mathrm{~Hz}$ (b) frequency 
of the process of isometric transformation without local loss in the stability of the TVS shell and to confirm the validity of the selected calculation scheme. In the course of the work, numerical calculations of stressstrain state of TVS with a rigid fixation around the contour of the base, corresponding to Figure 5, with the influence of characteristic factors of space environment on it and their combinations was carried out.

The finite element model of the multisectional structure was performed in a three-dimensional statement using shell finite elements of general statement. As the calculation values, the values of loads were taken into account, to the effect of which the structure could be subjected in the deployed state, during arrangement on the outer surface of the basic spacecraft (ISS). The characteristic effects at the stage of transportation to the orbit (vibration, pressure drops, acoustic effects) were excluded from the consideration, since the structure in the transport state is characterized by a significant safety margin and is equipped with devices for recording the displacements.

For the accepted designing scheme of TVS, the temperature loads in the ranges, given below, were modeled The static problem of radiant heat exchange with the Sun was considered, in which at the same time the maximum and minimum temperatures of the corresponding range at the opposite generatrices lines of the structure shell are reached. In all of the considered variants, the TVS shaping was characterized by the deviation of free end of the shell to the side with the smallest values of the applied temperature.

\section{Dependence of maximum displacements} of free end of TVS (cm) on $\Delta T,{ }^{\circ} \mathrm{C}$

$\Delta T_{1}=100--125$

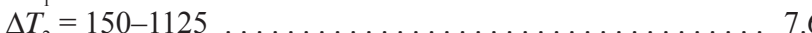

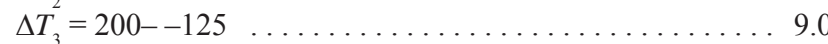

The regions with the highest values of stresses are located on the surface which is very limited in area, their values are sharply reduced with the removal from the fixation zone. The results of modeling allow concluding about the conformity of maximum displacements to the allowable values of deviation of the free end, which are accepted for this type of structure (up to $150 \mathrm{~mm}$ ). The schematic presentation of the TVS with a PL, rigidly fixed on a universal workplace on the outer surface of the ISS service module, is shown in Figure 8.

During general calculation of the effect of accelerations in the presence of PL, the unit of scientific equipment with a mass of $40 \mathrm{kN}$, rigidly fixed at the free end of the TVS in six degrees of freedom, and also thermocyclicity, depending on the own frequency $(\omega)$ of the structure, the following values of acting accelerations were taken. According to the results of calculation, the frequency of the natural oscillations of the structure was $\omega=22.43 \mathrm{~Hz}$, taking into account the load concentrated
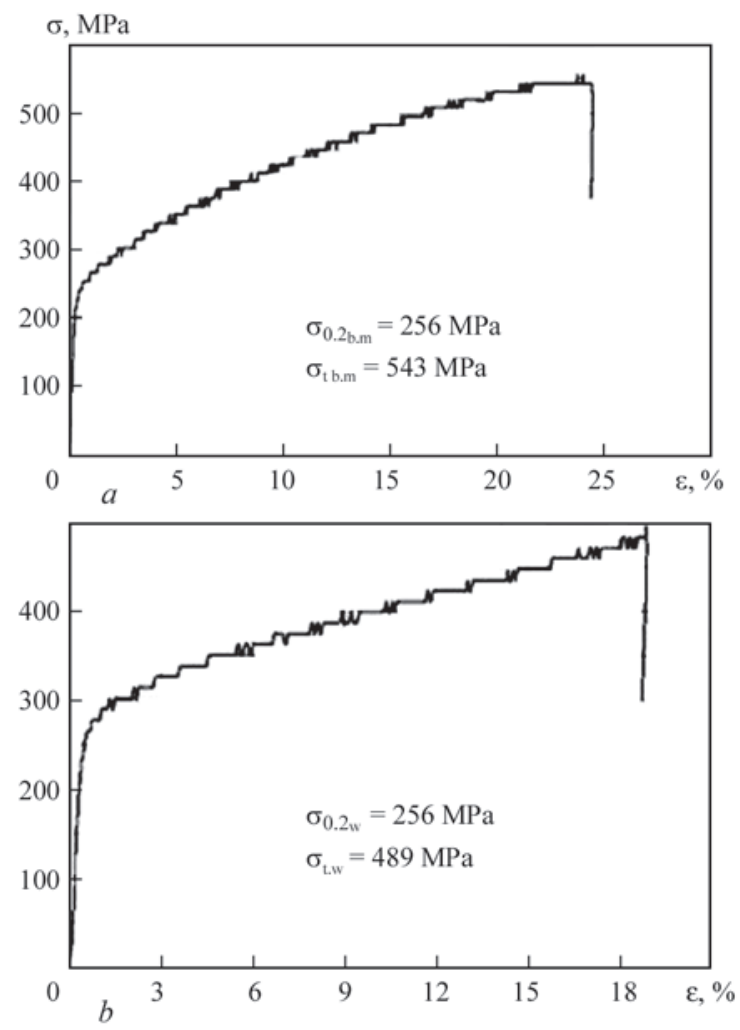

Figure 7. Diagrams of tension of base metal (a) and welded joint, produced at a pulsed current frequency of $200 \mathrm{~Hz}(b)$

from the mass of PL, which undergoes linear and angular accelerations and is fixed at the ends of the TVS, leads to a decrease in the frequency of the natural oscillations of the structure to $\omega=20.45 \mathrm{~Hz}$. For the values $\omega \geq$ $\geq 20 \mathrm{~Hz}: a_{x} \pm 4.5 \mathrm{~m} / \mathrm{s}^{2} ; a_{y}=a_{z}= \pm 6.0 \mathrm{~m} / \mathrm{s}^{2} ; \varepsilon_{x}= \pm 0.2 \mathrm{rad} /$ $\mathrm{s}^{2} ; \varepsilon_{y}=\varepsilon_{z}= \pm 0.7 \mathrm{rad} / \mathrm{s}^{2} ;$ the directions of accelerations are preset in the right coordinate system $O X Y Z$, where axis $Z$ coincides with the longitudinal axis (axis of symmetry) of TVS, the axes $Y$ and $X$ are perpendicular to it. Respectively, $a_{x}$ is the acceleration in the direction of the axis $X, a_{y}$ and $a_{z}$ are the accelerations in any transverse plane parallel to the plane $O Y Z, \varepsilon_{x}$ is the angular acceleration around the axis $X$, and $\varepsilon_{y}$ and $\varepsilon_{z}$ are the angular accelerations around any transverse axis, which lies in the plane parallel to the plane $O Y Z$ and passes through the axis $X$.

The joint effect of accelerations and temperature loads leads to increase in the values of equivalent stresses, the maximum value of which reaches $\sigma_{\mathrm{e}}=$ $=226 \mathrm{MPa}$. It should be noted that insignificant influence of inertial loads on the general character of stress distribution in the shell of TVS is associated with the peculiarity of mutual orientation of vectors of accelerations and temperature gradients, which almost eliminates their superposition. In the area of joining the support conical section with the further elements, the maximum values of equivalent stresses do not exceed the admissible stresses for the applied steel (the yield strength $\sigma_{0.2}=250-260 \mathrm{MPa}$ ) and do not cause necessity in correction of the accepted designed scheme. The maximum total values of linear 


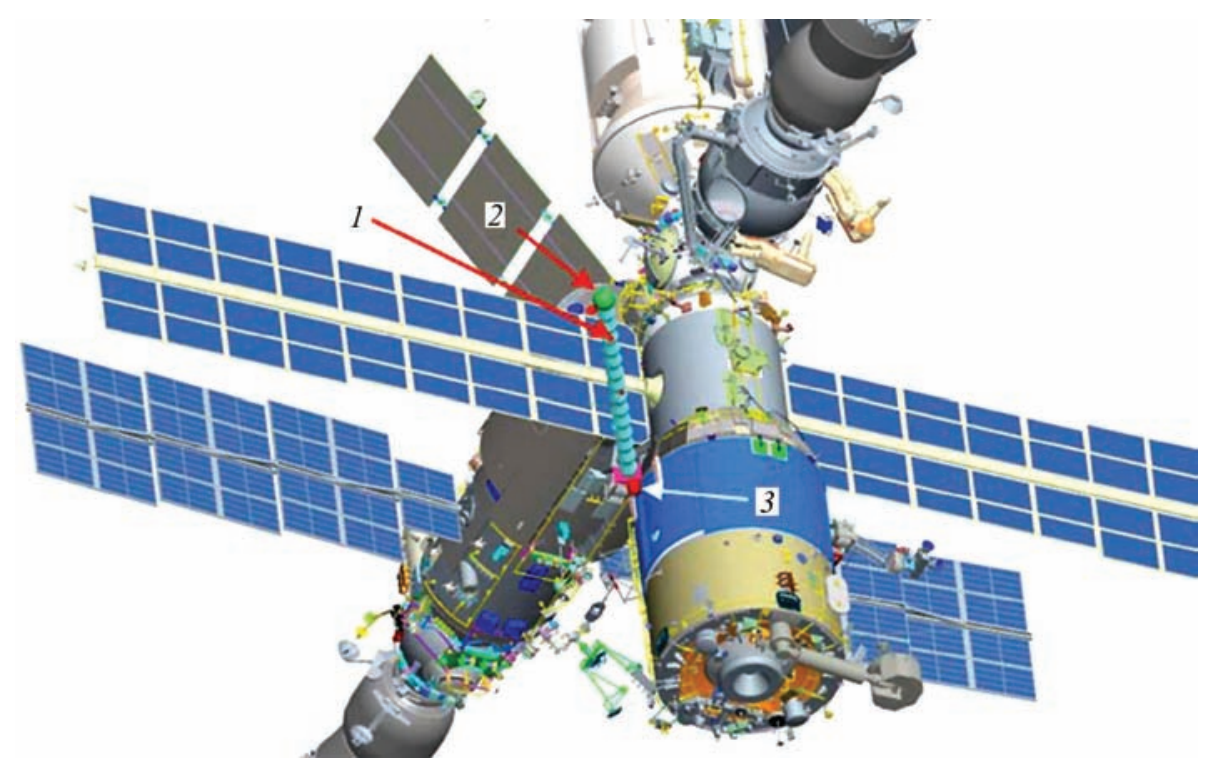

Figure 8. Layout of the TVS (1) with the payload (2) on the universal working place (3) of the ISS service module

displacements of a structure free end at the combined action of accelerations and temperature ranges reach values $U_{\mathrm{a}+\mathrm{t}}=0.1499(\mathrm{~m})$. The fields of distribution of equivalent stresses, as well as maximum total values of linear displacements in the structure shell are shown in Figure 9. The obtained result leads to the need in the accurate keeping with the thermal mode of structure, in which the critical values of temperatures are determined at the stage of choosing thermo-optical characteristics of the surface. As the largest contribution to the general deformation scheme of the structure is made by the components of linear displacements, which are predetermined by the cyclic radiation heating of its shell, the search for methods of surface engineering of the TVS for changing its thermo-optical properties, is most relevant to determine the structure deformability in the conditions of orbital flight. Respectively, the methods were developed to modify the surface of thin-walled stainless steel shells, which allow reducing the deformability of the TVS of space purpose without changing their mass and compactness. It is shown that the modification of

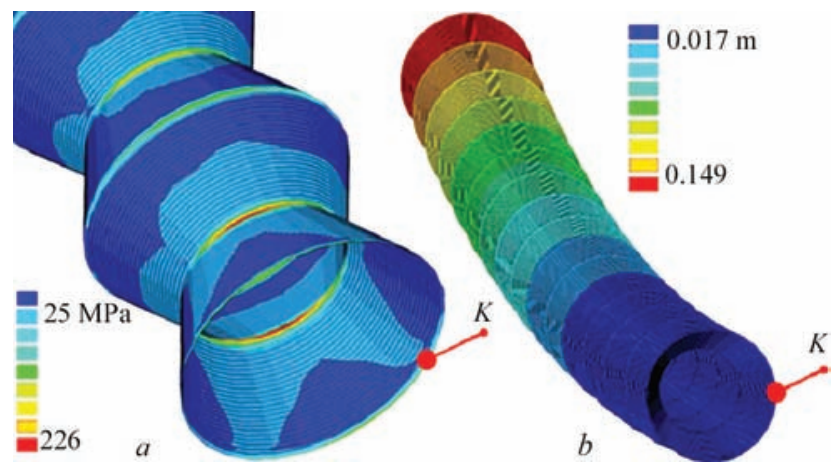

Figure 9. Fields of distribution of equivalent stresses $\sigma_{\mathrm{e}}$, (MPa) (a) and total values of linear displacements $U_{\mathrm{a}+\mathrm{t}}(\mathrm{m})$ at the combined action of accelerations and temperatures $(b)$ on the neutral surfaces of the TVS in the conditions of acting SEF; $K$ - contour of rigid fixation of the TVS (according to Figure 5) thermo-optical properties of the surface by depositing multilayer coatings using the method of electron beam deposition reduces the temperature load to the regulated conditions of the space experiment in the range of $-43-63{ }^{\circ} \mathrm{C}$, which leads to 1.45 times decrease in the deformability considered in the operation of the multisectional TVS at the 1.17 times reduction in the maximum equivalent stresses [6].

Figure 10 illustrates the dependence of the minimum $\left(T_{\min }\right)$ and maximum $\left(T_{\max }\right)$ calculation values of temperatures at the surface of the TVS conical type on the ratio $A_{s} / \varepsilon$, where $A_{s}$ is the absorption coefficient of solar radiation; $\varepsilon$ is the coefficient of radiation of the optical surface of the TVS, during operation of the structure at an arbitrary operating point of the ISS outer surface. The marked area $T_{\text {opt }}$ limits the abovementioned optimal temperature range $\left(-43-63{ }^{\circ} \mathrm{C}\right)$, which allows carrying out the necessary operations in case of open-space spacecraft's activity, and corresponds to the range of ratios $A_{S} / \varepsilon=0.26-0.54$. The object of investigation was the correction of the ratio $A_{s} / \varepsilon$ of the definite material of the shell by depositing different combinations of materials and their compounds on its surface, performing the functions of selective-radiating coatings. It should be noted that the coatings known in the space technologies of this purpose do not correspond to the conditions of extreme deformation of the investigated structure either because of the lack of flexibility (enamel), or sufficient strength and compactness (screen-vacuum thermal insulation). Moreover, the selection of the required coating is determined not only by its thermo-optical properties, but also by adhesion to the surface of metallic shell, taking into account the considerable deformations of its surface during deployment, as well as different rates of sub- 
limation of materials under the vacuum conditions of space environment. Preparation of optical surfaces of TVS after correction of roughness can be realized by means of electron beam spraying of thin coatings of metals and their compounds with the necessary ratios of $A_{s} / \varepsilon$; this method allows providing a sufficient adhesive strength of coatings and effectively control their thickness. The necessary result was obtained applying the aluminum coating of $480 \mu \mathrm{m}$ thickness on the surface of a shell of steel AISI321 of $0.175 \mathrm{~mm}$ thickness, followed by spraying $\mathrm{Al}_{2} \mathrm{O}_{3}$ layer of $45 \mu \mathrm{m}$ thickness on aluminum, which imitates the formation of oxide film, significantly increasing the absorption coefficient $A_{S}$. The measurement of integral hemispherical thermo-radiation characteristics of the mentioned coatings, carried out using calorimetric methods of investigations, confirmed their conformity with the above-mentioned calculated range of ratios $A_{S} / \varepsilon$.

The challenging tasks of using multiconical TVS as telescopic-type systems and hull structures of orbital space modules are related with the need in their recycling, which requires the development of a reverse transformation mechanism with the preservation of an original geometry. The ability of rigid shells of a transformable volume to multiple reproduction of stable geometric parameters is coordinated with the principles of a regular isometric transformation method. However, the full-scale metallic shell after the first repeated cycle of transformation acquires wavy deformations in the intercorrugated gaps, which indicate a local loss of stability. The analysis of the mathematical model of movement and the results of the carried out experiments on the transformation of TVS elements allowed concluding about the causes for the loss of stability of conical shells subjected to reverse transformation by creating a vacuum in the interior space. Therefore, the direct transformation of the shell takes place gradually, starting from the circumferential corrugation of the largest diameter, which undergoes the greatest load under the condition of the constant transformation pressure during the whole process. On the contrary, during reverse transformation, any of the elements of the surface of the deployed shell of TVS undergoes a load which is approximately equal to normal atmospheric pressure, moreover, all the elements of the surface begin to move simultaneously. During the process of transformation, local deformations in the vicinity of the apexes of the circumferential corrugations are increasing, the areas of their localization are united, which leads to a general loss of structure stability and significant distortions of its surface.

The conclusion on the local nature of bending deformation at the boundary of buckling and the appropriate deformations of the middle surface for the test thin shell is confirmed by finite element modeling of

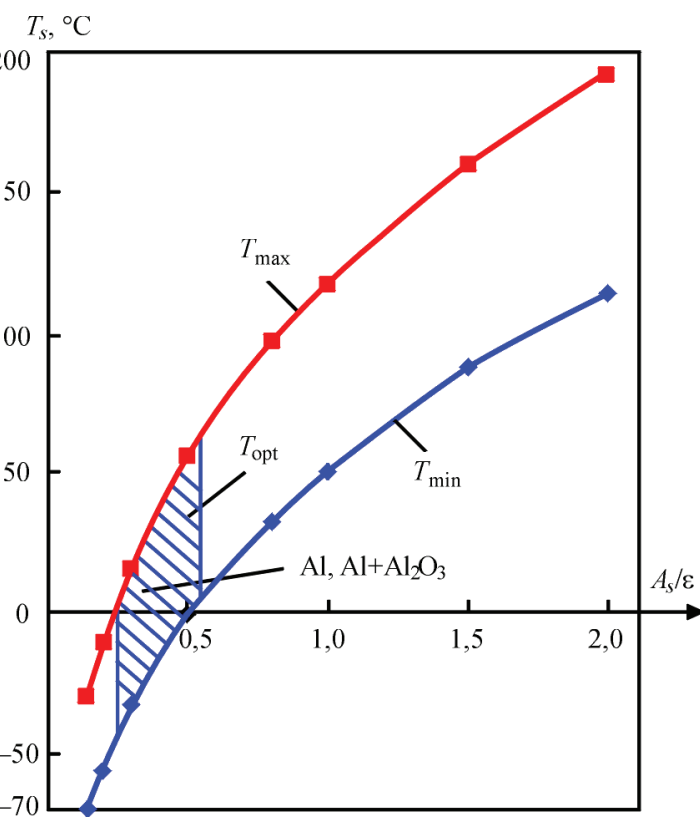

Figure 10. Dependence of minimum $\left(T_{\min }\right)$ and maximum $\left(T_{\max }\right)$ calculated values of temperatures on the surface of the TVS of conical type on the ratio $A_{\mathrm{s}} / \varepsilon$

stresses and deformations in the process of volumetric deformation. Thus, the equivalent stresses on the face surfaces are located in the near-boundary elastic-plastic zone, and on the neutral surface of the corrugation their values do not exceed 10-20 MPa. On the basis of the mentioned considerations it can be assumed that one of the possible variants of the transformation technology change for realization of multiple shaping of the shell may be the decrease in the radius of rounding at the apexes of the matrix fins. Moreover, the areas of maximum elastic-plastic deformations are localized in the vicinity of the corrugation apexes, the number of transition zones of equivalent stresses decreases, and the sinusoidal profile of the conical generatrix approaches to the form of a piecewise broken curve, corresponding to the respective mathematical model of mirror reflection of the truncated conical surface (see Figure 1,c).

For experimental investigation of behavior of the TVS shell of $D=400 \mathrm{~mm}, d=250 \mathrm{~mm}$ diameters, the angle of conicity $\alpha=25^{\circ}$ and the steel AISI321 shell thickness of $\delta=0.175 \mathrm{~mm}$ at the reverse transformation, a test specimen of the structure with the length $L=1190 \mathrm{~mm}$ of 7 sections with a ratio of $r_{\mathrm{c}} / \delta=3.0$ was manufactured. At the same time, the basic criterion of approaching to the isometric transformation, which consists in the equality of the length of the initial and the transformed area of the conic shell meridian, as well as the ratio of the thickness of its material $\delta$ to the step size $b$, did not change.

The process of direct and also reverse transformation, realized during creating vacuum in the inner volume of the experimental structure, is presented in 

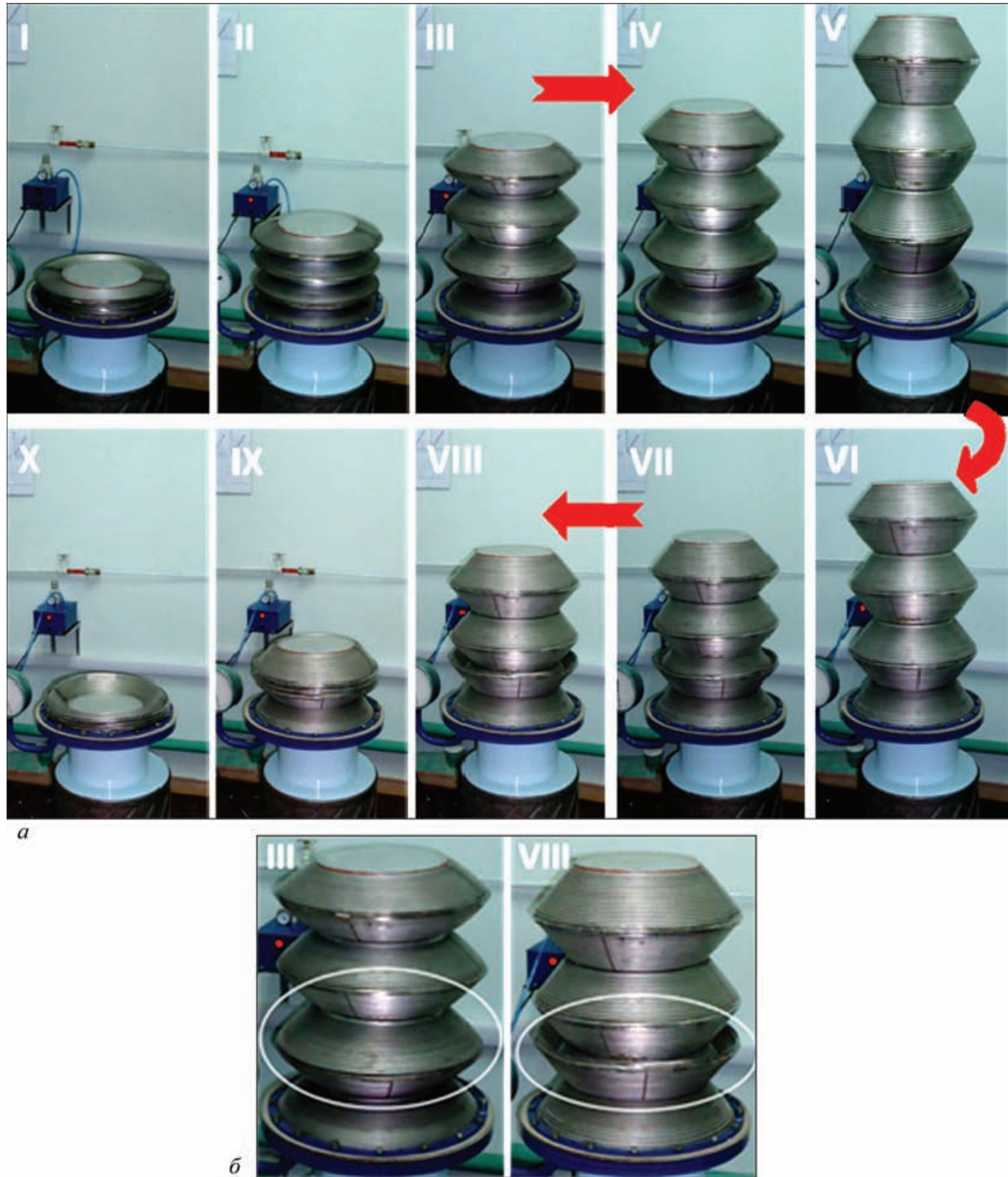

Figure 11. Experiment of direct and reverse transformation of the TVS with the length $L=1190 \mathrm{~mm}$ and diameter $D=400 \mathrm{~mm}(a)$ and magnified stages III and VIII $(b)$

Figure 11, the pressure curve for the stages (I-X) is in Figure 12. The experiment confirmed the possibility of repeated coaxial folding of the multisectional TVS to the state, which allows performing its necessary disas-

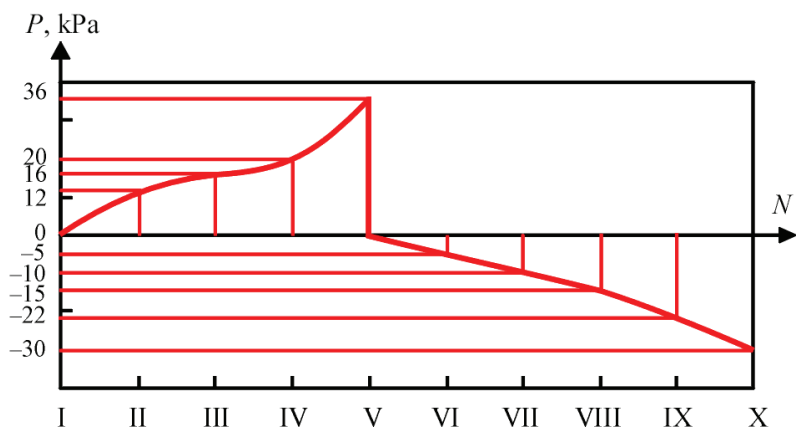

Figure 12. Curve of pressure for stages $N(\mathrm{I}-\mathrm{X})$ (as is shown in Figure 11) sembly and recycling after termination of its exposure period in the near-earth orbit. At the same time, it was noted that the increased circumferential rigidity of corrugations of a larger radius in the area of the rigid fixation contour leads to the effect of «snap buckling» of the shell (see the stage of deploying «III» and the folding stage «VIII» corresponding to it, Figure 11, b) at which the reverse transformation can occur not always successively, starting with the fold of a larger radius, but from some arbitrary corrugation with the smallest circumferential rigidity, which can be predetermined by the initial geometric imperfections of the initial conical semi-product or deviations of the technological process of corrugation. As a result, the shell fragment between the largest and the first «folded» corrugation remains untransformed, which has some negative effect on the 
finite compactness of the TVS (see Figure 11, stages «I» and «X»). It is possible to avoid arising of the similar effect by a successive changing the radius of generatrix of corrugations in slight ranges by reducing their total radius. The determination of correlations between geometric, strength and rigid characteristics of TVS, which arise in this case, can be a subject of challenging investigations.

To determine critical values of external loads of single shells, in particular, under the action of axial compressive forces, there are some well-known analytical solutions. However, for the problems of stability of conical shells with a complex shape of generatrix in the composition of multilevel systems at an arbitrary direction of applying loads, such solutions are absent. In the modern works dealing with the problems of nonlinear mechanics of multifolded structures, the numerical methods of calculation are used based on the principle of minimizing the potential energy of the system for solving the stability problems (determination of bifurcation points, critical loads, and forms of stability loss, accompanied by the so-called snap-through buckling [7], Similarly, the stability of the TVS equilibrium can be determined as $J \equiv\left(\partial^{2} \Pi\right) /\left(\partial[D]^{2}\right)$, where $J$ is the determinant of the Jacobi matrix (Jacobian), $\Pi$ is the general potential energy of the model, $[D]$ is the matrix of assembly displacements [6]. At the condition of $J>0$, the structure is in a stable equilibrium, and at $J=0$ it is unstable. In the latter case, the critical loading results in the appearance of a bifurcation point, i.e., to the possibility of forming several forms of stability loss. As for the investigated TVS, during repeated bending of a piecewise-broken profile, the possibility of creating new forms of stability loss is much lower than in the shell with a sinusoidal profile. This is explained by the fact that appearance of a local stability loss during folding takes place in the areas of thinning the shell material at the apexes of corrugations, which have a more local character at a smaller bending radius.

The mentioned results of the development illustrate the possibility of creating metallic load-carrying shell structures, which can be delivered to the near-earth orbit in a compact state and transformed to the designed dimensions with a minimal energy losses. The use of the described structures in space technologies allows transferring to a new qualitative level by carrying out the scientific experiments related to the open-space spacecraft activities, during manufacture of hull structures of orbital stations, and in future, it can be applied in the creation of long-term erections on the Moon surface.

\section{Conclusions}

1. An algorithm for calculating the designing scheme of a welded multisectional TVS of a space purpose is presented, which allows determining its geometric parameters. A calculation-analytical evaluation of the correlation between the physicomechanical characteristics of the TVS material and corrugation parameters was carried out and the conclusion about the advantage of using stainless steel AISI321 was obtained.

2.The parameters of the process of pulsed microplasma welding of conical shells of stainless steel (energy input $Q_{\mathrm{c}}=17.5 \mathrm{~J} / \mathrm{mm}$, pulsed current frequency $f_{\mathrm{pc}}=200 \mathrm{~Hz}$ and the ratio of the current pulse duration to the cycle duration 0.75 ) were determined, which allow providing the strength of welds at the level of 0.9 of the strength of the base metal ensuring their vacuum tightness.

3 . It is shown, that modification of the surface of thin-walled shells of stainless steel allows increasing the stability of the TVS of space purpose without changing their mass and compactness. The modification of the thermo-optical properties of the surface by applying multilayer coatings $\mathrm{Al} / \mathrm{Al}_{2} \mathrm{O}_{3}$ with a total thickness of $525 \mathrm{~nm}$ by the method of electron beam deposition allows 1.45 times reducing the deformability of the multisectional TVS from radiation heating.

4. The possibility of a complete reverse transformation of the multisectional TVS without a local stability loss was confirmed.

5. The developed technologies can be used for creation of load-carrying rod and shell structures of space purpose.

1. (2017) Bigelow Aerospace. The Bigelow Expandable Activity Module (BEAM). https://bigelowaerospace/com/pages/beam/

2. Viquerat, A., Schenk, M., Sanders, B., Lappas, V.J. (2014) Inflatable rigidisable mast for end-of-life deorbiting system. In: Proc. of Europ. Conf. on Spacecraft Structures, Materials and Environmental Testing (SSMET) 2014 (April 1-4, 2014, Braunschweig, Germany).

3. Paton, B.E., Samilov, V.N., Gonchar, O.Yu. et al. (1999) Transformable all-welded metal structures. Avtomatich. Svarka, 10, 81-85 [in Russian].

4. Paton, B.E., Lobanov, L.M., Samilov, V.N. et al. (2006) Design and features of fabrication technology of a large sized transformable shell structure. The Paton Welding J., 7, 2-10.

5. Lobanov, L.M., Volkov, V.S. (2015) Peculiarities in manufacture of thin-walled welded transformable-volume structures for space application. Ibid., 1, 29-34.

6. Lobanov, L.M., Volkov, V.S., Yakimkin, A.V., Savitsky, V.V. (2016) Functional characteristics improvement of metal transformable-volume structures for space applications. J. of Aerospace Technol. and Management, 8, 55-62. doi:10.5028/jatm. v8il.529

7. Ario, I., Watson, A. (2009) Structural stability of multifolding structures with contact problem. J. of Sound and Vibration, 1-2, 263-282. doi:10.1016/j.jsv.2009.01.057 


\title{
LOW STRESS NO DISTORTION WELDING BASED ON THERMAL TENSIONING EFFECTS
}

\author{
QIAO GUAN \\ Chinese Academy of Engineering, \\ Beijing Aeronautical Manufacturing Technology Research Institute (BAMTRI) \\ PO Box 863, 100024, Beijing, China
}

\begin{abstract}
In manufacturing sheet metal formed plates, panels and shells by welding, buckling distortions become substantial especially for aerospace structures with material thickness less than $4 \mathrm{~mm}$. To prevent buckling, Low Stress No Distortion (LSND) welding techniques have been pioneered and developed at the Beijing Aeronautical Manufacturing Technology Research Institute. These innovative techniques have been applied successfully in manufacturing aerospace structural components. In this paper, the mechanisms of LSND welding techniques using either the whole cross-sectional thermal tensioning effect or the localized thermal tensioning effect are described and summarized in detail. The basic idea of LSND welding techniques is to perform active in-process control of inherent plastic (incompatible) strains and stresses formation during welding to achieve distortion-free results so that no costly post weld reworking operations for distortion correction is required. Emphasis is given to the finite element analysis to predict and optimize the localized thermal tensioning technique with a trailing spot heat sink coupled to the welding heat source. Selection of parameters for engineering solution are recommended. 21 Ref., 1 Table, 15 Figures.
\end{abstract}

Keywords : welding residual stress, low stress no distortion welding, buckling distortion, thermal tensioning, temperature gradient stretching, finite element analysis

Introduction. Buckling distortions are more pronounced than any other form of welding distortion in manufacturing thin-walled structures, and they are the main troublesome problem in sheet metal fabrication where fusion welding is applied, especially for aerospace structures such as sheet metal formed airframe panels, fuel tanks, shells of engine cases, etc., where thin sheet materials of less than $4 \mathrm{~mm}$ thickness are widely used. Buckling distortions affect the performance of welded structures in a great many ways. During the past decades efforts have been made and progress has been achieved in solving buckling problems by experts in the welding science and technology field world-wide. Many effective methods for removal, mitigation and prevention of buckling distortions adopted before welding, during welding or after welding have been successfully developed and widely applied in industries $[1,2]$. Over the past 25 years, authors at the Beijing Aeronautical Manufacturing Technology Research Institute (BAMTRI) have devoted their efforts to achieve distortion-free results in manufacturing thin-walled aerospace structural components by implementing active in-process control of inherent residual plastic strain formation during welding without having to undertake costly reworking operations for distortion correction after welding [3]. Extensive research and development

(c) QIAO GUAN, 2018 studies to explore Low Stress No Distortion (LSND) welding techniques were carried out at BAMTRI.

Two innovative methods of LSND welding techniques have been developed for industrial application: one is based on the whole cross-section thermal tensioning effect [4], the other is based on the localized thermal tensioning effect [5].

Buckling distortions. The nature of buckling is mostly a phenomenon of loss of stability of thin elements under compressive stresses. Buckling distortions caused by longitudinal welds either in plates, panels or in shells are mainly dominated by longitudinal compressive residual stresses induced in areas away from the weld. Figure 1, $a, b$ show the typical patterns of buckled components. The mechanism of buckling in weldments lies in the action of inherent residual plastic (incompatible) strains formed during welding.

Losing stability, the buckled plate (Figure 1,a) is released from an unstable flat position of high potential energy with the maximum level of residual stress distribution after conventional welding (Figure 1,c) and takes a stable warped shape. Losing stability, the buckled plate reaches a state of minimum potential energy. In other words, any forced change of the stable curvature of the buckled plate will cause increase in potential energy and once the force is removed, the buckled plate will be restored to its stable position minimizing the potential energy. 

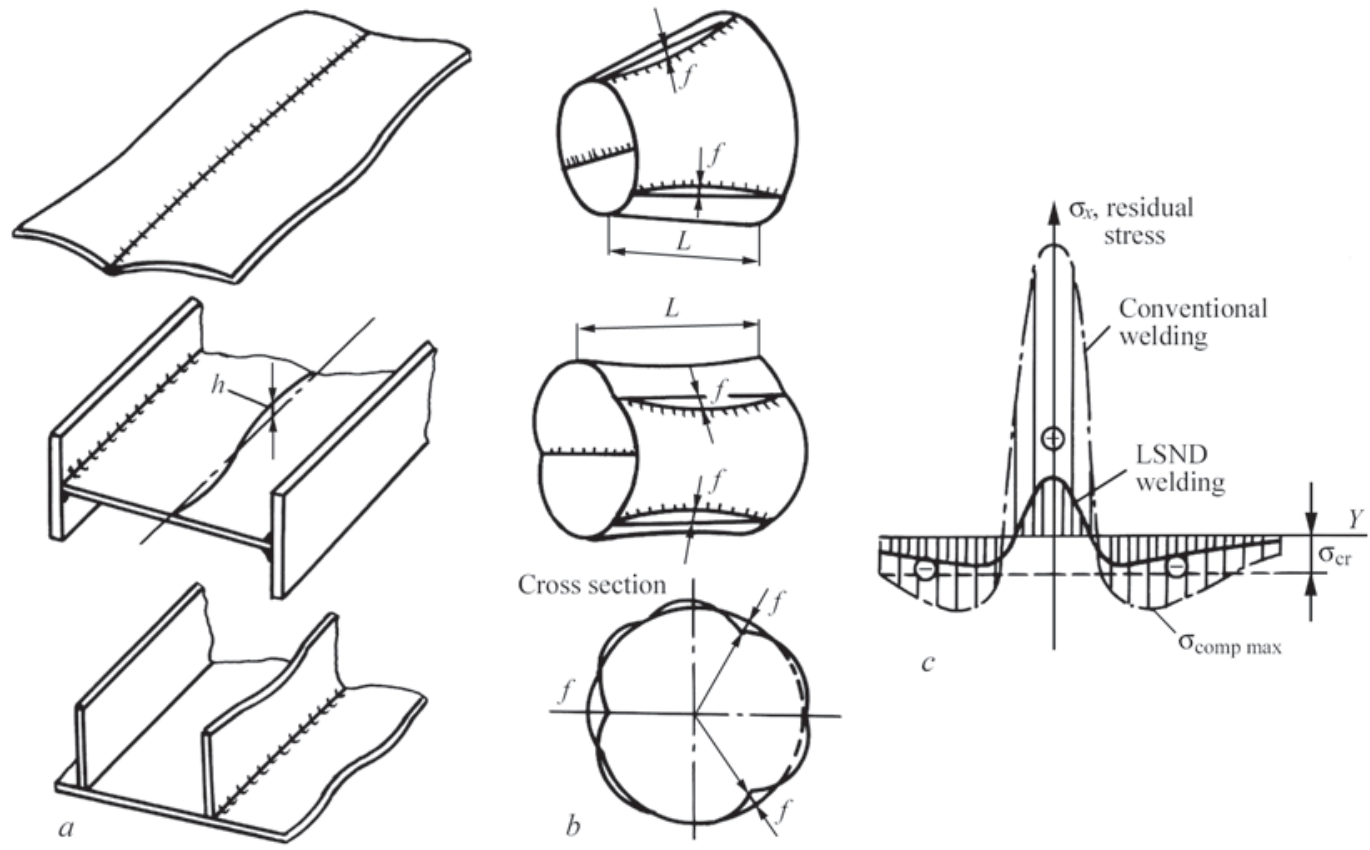

Figure 1. Typical buckling patterns of plates, panels $(a)$ and shells $(b)$ with longitudinal welds; to prevent buckling, reduce $\sigma_{\text {comp max }}$ to a value lower than $\sigma_{\mathrm{cr}}(c)$, thus achieving the low stress no distortion result

For plates of thickness less than $4 \mathrm{~mm}$ as widely used in aerospace and modern vehicle welded structures, the value of $\sigma_{\mathrm{cr}}$ is much lower than the peak value of compressive stress $\sigma_{\text {comp max }}$ after conventional gas tungsten arc welding (GTAW) (Figure 1, c). However, the actual value $\sigma_{\mathrm{cr}}$ for a welded element is difficult to be solely determined either by the linear stability theory of small deformations or by the non-linear theory of large deformations in theory of plates and shells. These problems are very complex [6].

In principle, all efforts either «passive» post-weld correction measures or «active» in-process control methods of LSND welding to eliminating buckling aim at adjusting the compressive residual stresses to achieve $\sigma_{\text {comp max }}<\sigma_{\text {cr }}$ at which buckling occurs (Figure $1, c)$ by means of reduction and redistribution of the inherent residual plastic strains.

In the past decade, welding simulation and prediction by computational method has been increasingly applied in addition to classic analytical and conventional empirical procedures. Finite element method was adopted by Michaleris, Deo et al [7, 8] for analyzing buckling distortions of stiffened rectangular welded plates for shipbuilding. Shrinkage forces were obtained from a thermal elastic-plastic cross-sectional model analysis. Based on the finite element analysis for large displacements, and using an inherent shrinkage strain method, Tsai et al [9] investigate the buckling phenomena of a rectangular plate of aluminum alloy with longitudinal $T$ stiffeners.

Buckling can be controlled by a variety of methods applied before welding, during welding, and after welding for its removal, mitigation or prevention.
Pre-tensioning can be classified in either the category of methods applied before or during welding [10]. For each particular structural design of panels, a device for mechanical tensile loading is required. Owing to their complexity and low efficiency in practical execution, application of these methods is limited. In this respect, the thermal tensioning technique is more flexible in stiffened panel fabrication.

LSND results could be achieved during the welding process based on the thermal tensioning (temperature gradient stretching ) effect which is produced by establishing a specific temperature gradient either in whole cross section of the plate to be welded or in a localized area in the near-arc zone. Simultaneously, restraining transient out-of-plane warpage movements of the workpiece is necessary. Differing from the «passive» methods which have to be applied after welding once buckling is in existance, LSND welding techniques can be classified as «active» methods for in-process control of buckling distortions with no need of reworking operations after welding.

Thermal tensioning effects. The method for low temperature stress relieving [11] is well-known in shipbuilding and vessel manufacturing industries. This technique is practiced with flame heating combined with water cooling of thicker plate sections of thickness $20-40 \mathrm{~mm}$ for mitigation of longitudinal residual stresses after welding. It is based on temperature gradient stretching effect induced by local linear heating and cooling parallel to the weld-line on plates. This technique is not applicable for either stress relieving or buckling removal after welding of thinwalled elements of less than $4 \mathrm{~mm}$ thickness where 


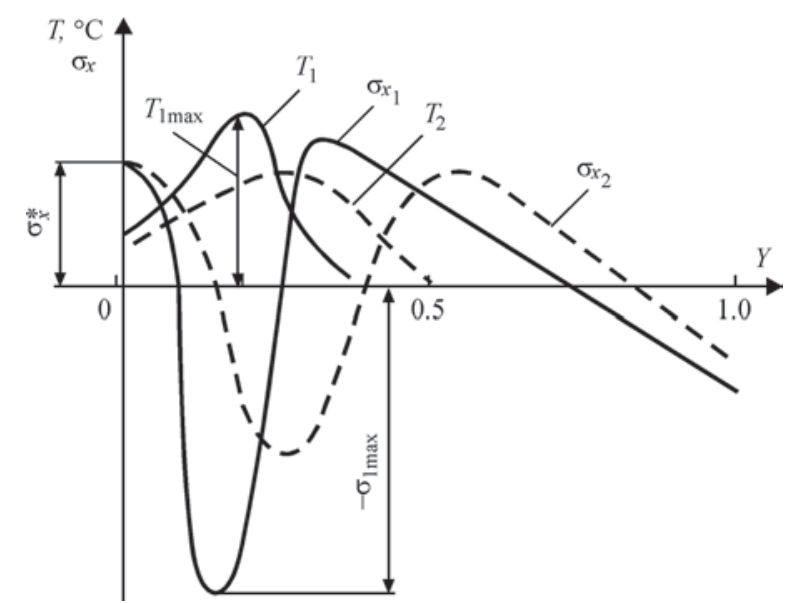

Figure 2. Basic principle of whole cross-section thermal tensioning effect

the metal sheets are not stiff enough to resist the transient out-of-plane displacement during local heating and forced cooling. But the idea of the temperature gradient stretching effect (or commonly termed thermal tensioning effect) is logically feasible for avoiding buckling of plates and shells during welding. Efforts in this direction were made during last decades [2-4, 12-14].

The basic principle of the whole cross-section thermal tensioning effect is shown in Figure 2. Two curves $\left(\sigma_{x_{1}}\right.$ and $\left.\sigma_{x_{2}}\right)$ of thermal stress distributions are created by a preset heating with the temperature profiles $\left(T_{1}\right.$ and $\left.T_{2}\right)$ correspondingly on the thin plate. In this case, the thermal tensioning effect is defined as the value of $\sigma_{x}^{*}$ in the plate edge of $Y=0$ where the weld bead will be applied. For a given $\sigma_{x}^{*}$, the greater temperature gradient $\left(\partial T_{1} / \partial Y\right)>\left(\partial T_{2} / \partial Y\right)$, the higher

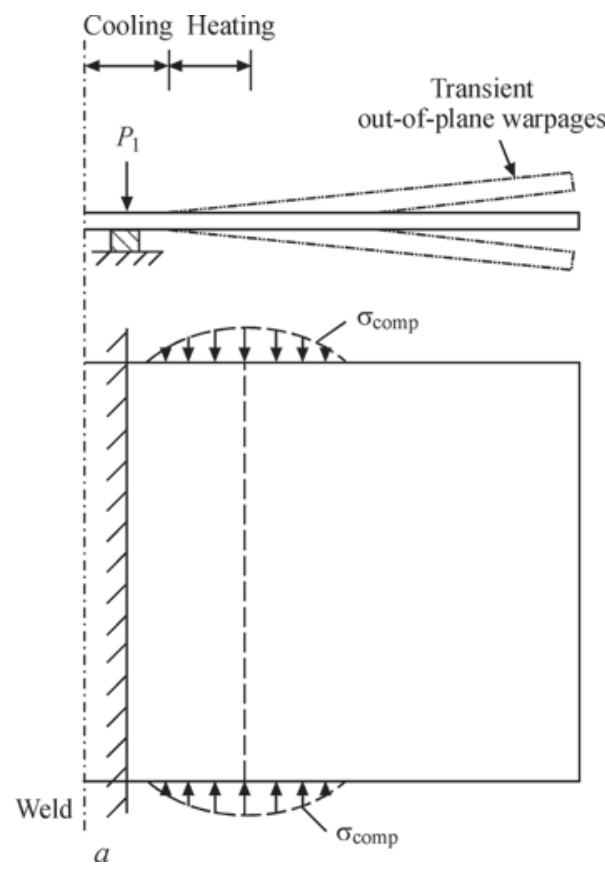

will be the induced maximum value of compressive stress $-\sigma_{1 x_{\max }}$. An optimized temperature curve can be calculated mathematically for an estimated value $\sigma_{x}^{*}$ while the value $-\sigma_{x_{\max }}$ is kept below the yield stress.

Based on the results of mathematical analysis for the thermal tensioning effect, Burak et al $[13,14]$ conducted an experiment to control longitudinal plastic strains in weld on aluminum plate of $4 \mathrm{~mm}$ thickness.

Early in the 1980's, to apply the thermal tensioning effect to avoid buckling in aerospace structures of less than $4 \mathrm{~mm}$ thickness, a series of experiment was carried out by Guan et al $[4,12]$. It has been proved by the results of repeated experiments that the Burak's scheme for the plates thicker than $4 \mathrm{~mm}$ is not applicable to eliminating buckling in elements of less than $4 \mathrm{~mm}$ thickness. The reason is that owing to the susceptibility to losing stability of the thinner elements, transient out-of-plane displacements occur in areas away from the weld zone (Figure 3,a). The transient out-of-plane displacements outside the clamping fingers (indicated by $P$ in Figure 3, $a$ ) release the potential energy of the thermal plane stresses distribution. In the lost stability position, the expected preset thermal tensioning stress $\sigma^{*}$ (Figure 2) ceases to exist.

Progress was made in solving the above mentioned problem to improve the thermal tensioning technique and make it applicable to elements of less than 4 $\mathrm{mm}$ thickness especially in manufacturing aerospace structures [4]. Figure 3, b shows the improvement in clamping systems. In conventional clamping system with 'one-point' finger fixture (indicated by $P_{1}$ in Figure 3,a), the transient out-of-plane warpages

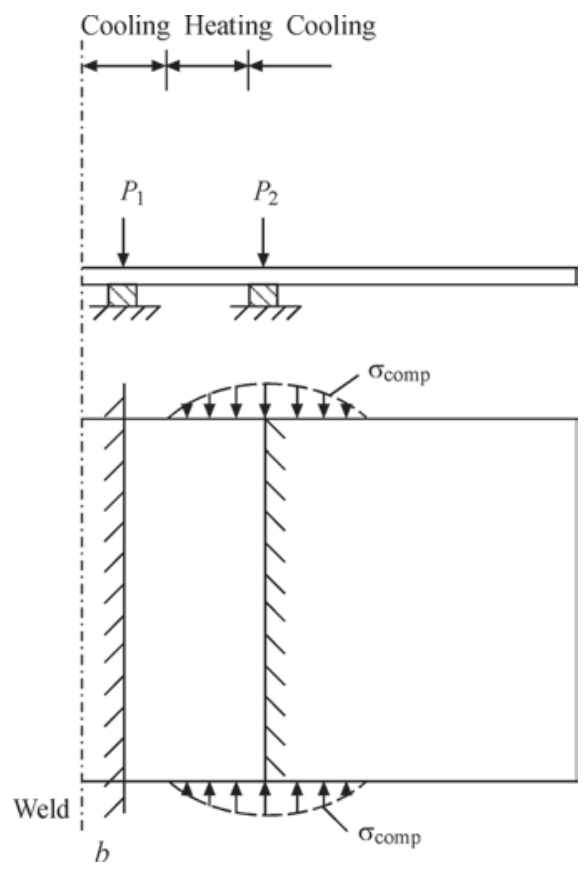

Figure 3. Transient out-of-plane warpage displacement of workpiece in conventional clamping system $(a)$ and its prevention in the newly improved 'two-point' fingers clamping system $(b)[4]$ 
Two categories of thermal tensioning effects

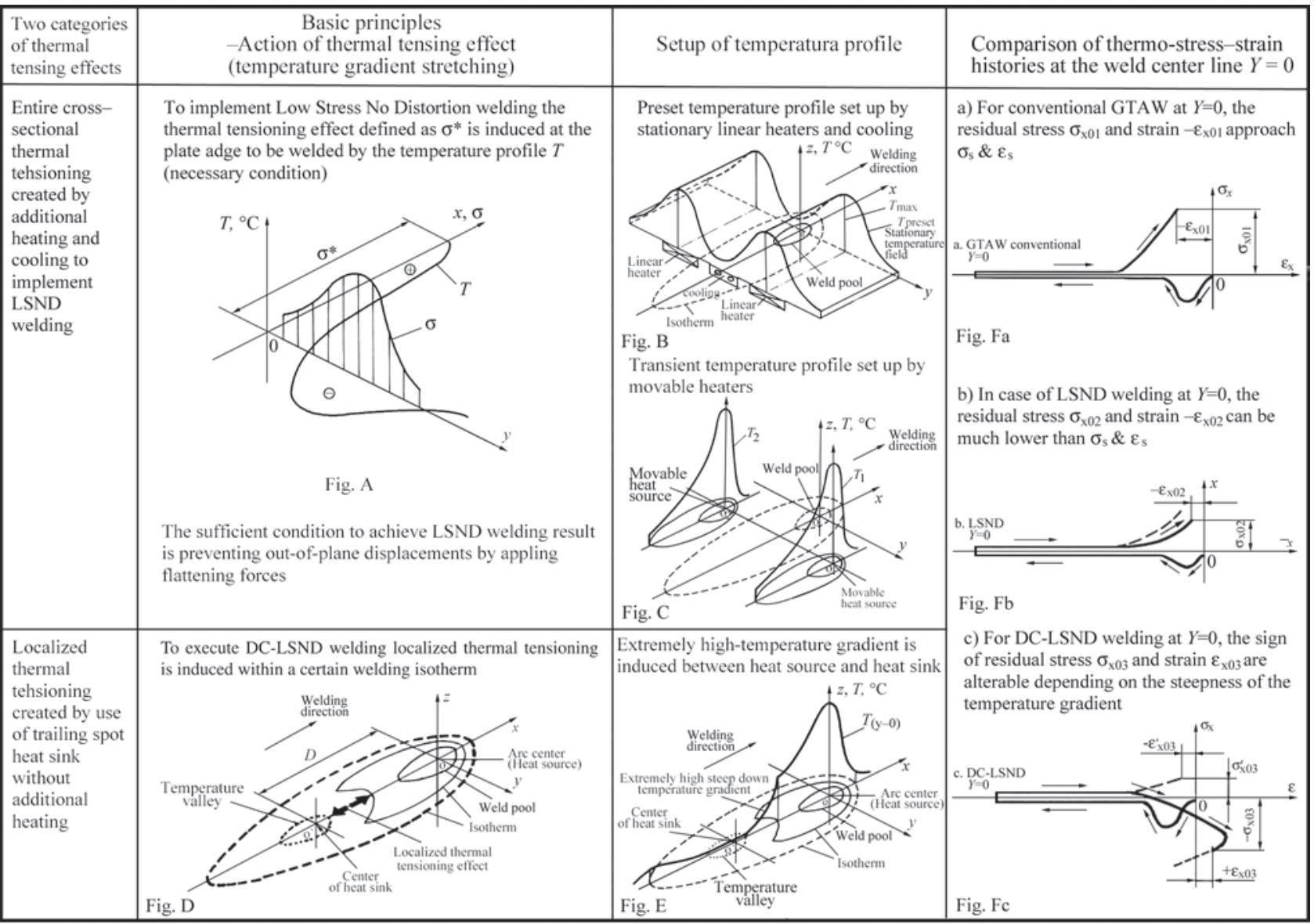

of the workpiece are inevitable, whereas, using the improved 'two-point' finger clamping system (indicated by $P_{1}$ and $P_{2}$ in Figure $3, b$ ) the desirable thermal tensioning effect in terms of $\sigma_{x}^{*}$ (Figure 2) can be established without transient out-of-plane warpage displacements.

As an active in-process control method, this improved technique is more widely acknowledged as LSND welding method for thin materials $[3,4]$. It is worthwhile to note, that the LSND welding technique as an active in-process control method is replacing the formerly adopted passive measures for buckling removal after welding in most cases in aerospace engineering in China.

To create the whole cross-section thermal tensioning effect along the plate edges to be welded, the temperature profile can be built up either statically as a preset temperature field by stationary linear heaters arranged underneath the workpiece parallel to the weld direction or as a transient temperature field built up by two movable heating devices on both sides of the weld and synchronously traveling with the welding torch [15]. The LSND welding techniques can be implemented in either of the two ways.

In a broad sense of the term «thermal tensioning», the effect can be created not only in the longitudinal direction of the weld to control the longitudinal plastic strains in weld zone, but the effect in mitigating the transverse shrinkage of the weld could also be utilized for hot-cracking prevention [16]. Furthermore, manipulating the combination of heat sources and heat sinks, the thermal tensioning effect as well as the thermal compressing effect could also be established properly for specific purposes. Mitigating residual stresses in Al-Li repair welds [17] is an example in applying the alternative options of thermal tensioning effect.

The thermal tensioning effects can be classified into two categories: one is created in an entire cross-section of plate ( whole cross-section thermal

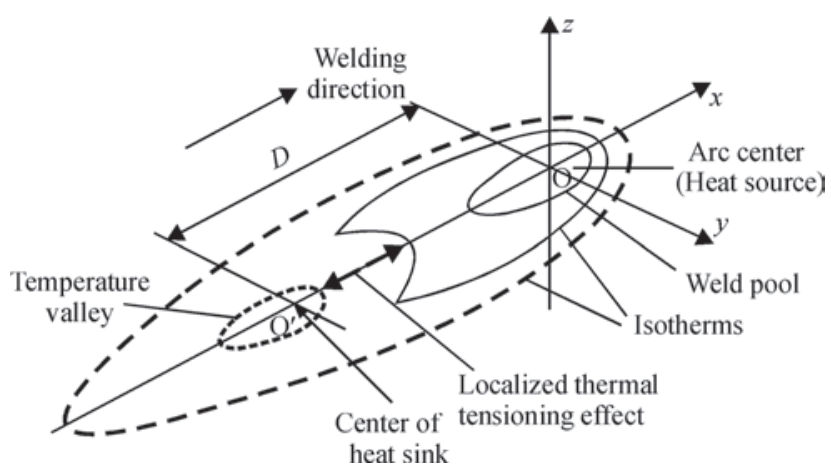

Figure 4. Localized thermal tensioning effect (shown by heavy arrows) induced by a trailing spot heat sink coupled to the welding arc in a distance $D$ behind 

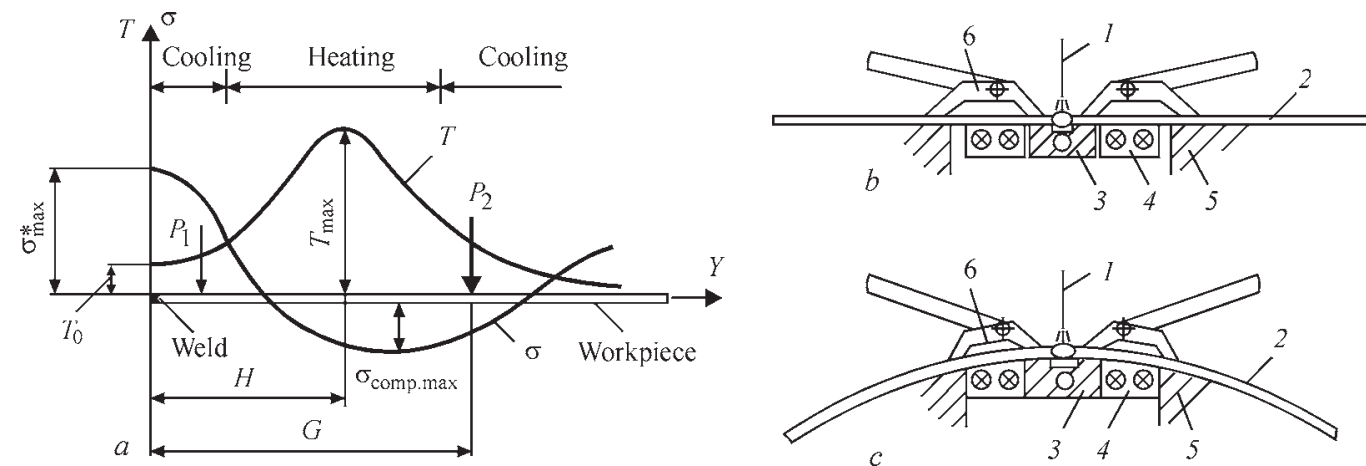

Figure 5. Basic principle for implementation of LSND welding (a), clamping jigs for longitudinal weld in plates $(b)$ and cylindrical shells (c) [4]: 1 - arc; 2 - workpiece; 3 - water-cooling backing bar; 4 - linear heaters; 5 - supporting mandrel; 6 - «two-point» finger clamping system

tensioning ) using additional heating and cooling as mentioned above, the other is created in a localized zone limited to a near arc high temperature area within a certain isotherm induced solely by welding arc without any additional heating ( localized thermal tensioning ), as classified in the table.

For the localized thermal tensioning a sourcesink system - a heat sink coupled with welding heat source, could be utilized (Figure 4).

Whole cross-sectional thermal tensioning LSND welding. To satisfy the stringent geometrical integrity requirements and ensure dimensionally consistent fabrication of aerospace structures, LSND welding technique for thin materials, mainly for metal sheets of less than $4 \mathrm{~mm}$ thickness, was pioneered and developed early in 1980's at the Beijing Aeronautical Manufacturing Technology Research Institute $[4,12]$. This technique was aimed to provide an in-process active control method to avoid buckling distortions based on the whole cross-section thermal tensioning effect.
Figure 5. shows schematically the basic principle for practical implementation of LSND welding [4]. The thermal tensioning effect with the maximum tensile stress $\sigma_{\max }^{*}$ in the weld zone (Figure 5,a) is formed due to the cooling contraction of the zone 1 by water-cooling backing bar underneath the weld and the heating expansion of zone 2 on both sides adjacent to the weld by linear heaters. Both the curve $T$ and curve $\sigma$ are symmetrical to the weld centerline. The higher $\sigma_{\text {max }}^{*}$, the better will be the results of controlling buckling distortions.

It is proved by experiments and engineering applications, that the thermal tensioning effect is the necessary condition for LSND welding of materials of less than $4 \mathrm{~mm}$ thickness, whereas the sufficient condition is the prevention of transient out-of-plane displacements by applying flattening forces in «twopoint» finger clamping systems shown by $P_{1}$ and $P_{2}$ in Figure 5, $a$. The selected curve $T$ is mainly deter-

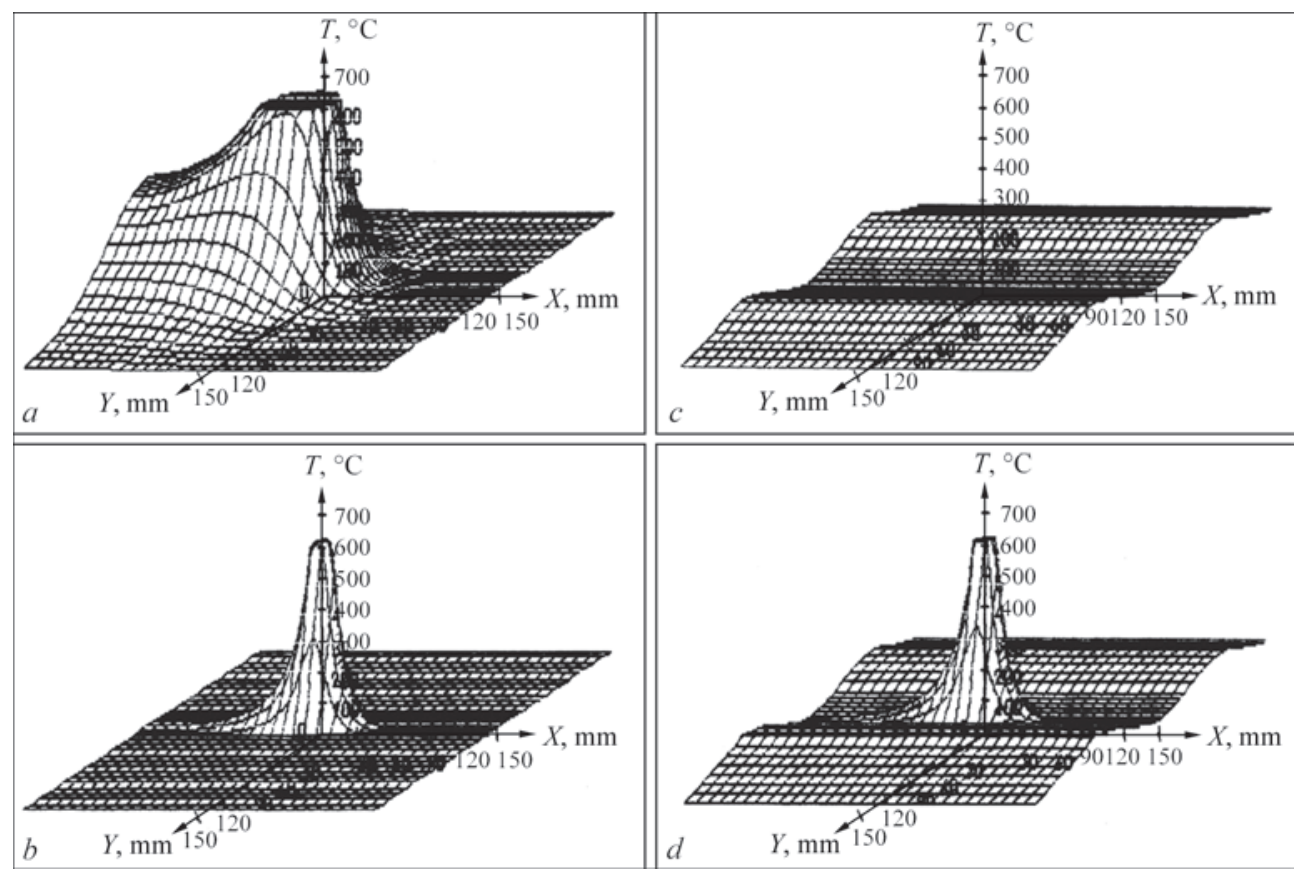

Figure 6. Temperature fields on thin plate of conventional GTAW (a), GTAW on copper backing bar with intensive heat transfer (b), preset temperature field $(c)$ and temperature field for LSND welding $(d)$ 

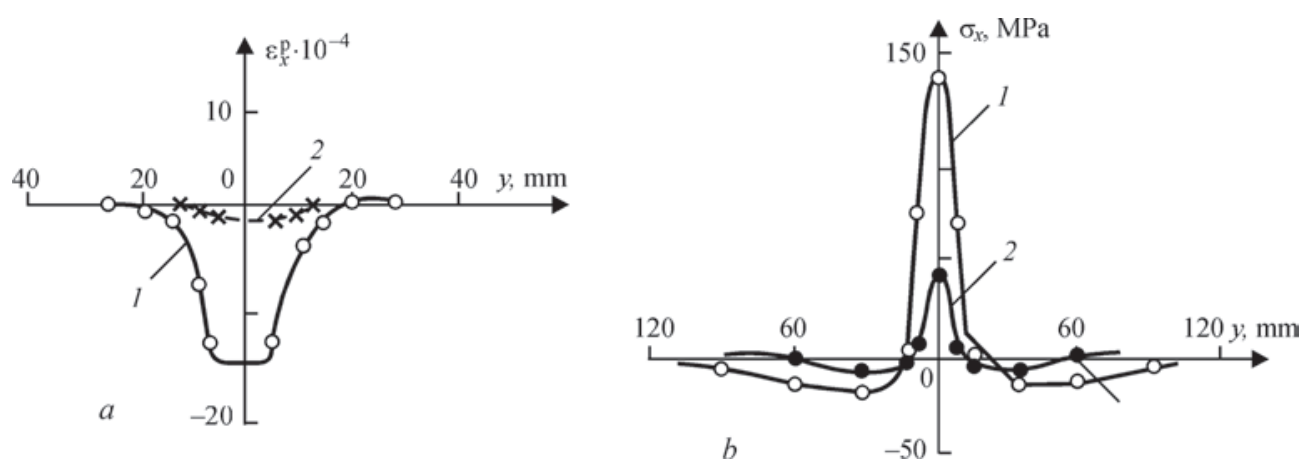

Figure 7. Comparisons between experimentally measured inherent strains $\varepsilon_{x}^{p}(a)$ and residual stresses $\sigma_{x}(b)$ distributions after conventional GTAW (curve 1) and LSND welding (curve 2) of aluminum plate of $1.5 \mathrm{~mm}$ thick $[3,12]$

mined by: $T_{\max }, T_{0}$ and $H$ - distance of $T_{\max }$ to the weld centerline. The thermal tensioning effect $\sigma_{\max }^{*}$ becomes stronger as the temperature gradient $\left(T_{\max }-\right.$ $T_{\mathrm{o}}$ ) increases while $H$ decreases. The optimization of $\sigma_{\max }^{*}$ and technological parameters such as $H$ etc. can be implemented computationally using FEA and verified experimentally. Figure 5 shows schematic views of practical implementation of LSND welding method and apparatus for longitudinal joints in flat plates (Figure 5,b) and cylindrical shells (Figure 5,c).

The typical temperature field in GTA welding of thin plate is shown schematically in Figure 6, a. Actually, in engineering practice, the GTAW of longitudinal weld on thin plate is performed in a longitudinal seam welder. Workpieces are rigidly fixed in a pneumatic finger-clamping system with copper backing bar on mandrel support. Owing to the intensive heat transfer from workpiece to copper backing bar, the temperature field is different from the normal shape and takes a narrowed distribution as shown in Figure 6, b. To implement LSND welding, additional preset temperature field as shown in Figure 6, $c$ is formed by heating and cooling.
Therefore, the LSND welding temperature field shown in Figure 6, $d$ results by superposition of the temperature fields of Figure 6, $b, c$.

For clearer quantitative assessment of LSND welding technique, a systematic investigation was carried out $[3,12]$. Figure 7 shows comparisons between the experimentally measured inherent strain $\varepsilon_{x}^{p}$ distributions (Figure 7,a) and residual stress $\sigma_{x}$ distributions (Figure 7, b) after conventional GTAW (curve 1) and LSND welding (curve 2) of aluminum plate of $1.5 \mathrm{~mm}$ thick. Reductions of either $\varepsilon_{x}^{p}$ or $\sigma_{x}$ are obvious (as indicated by curve 2 in comparison with curve 1).

The photographs in Figure 8 show that the specimens of either stainless steel (Figure 8,a) or aluminum alloy (Figure 8,b)welded conventionally (upper photo) are severely buckled in all cases. But the specimens welded by use of LSND welding (lower photo) are completely buckle-free and as flat as before welding.

Comparisons are also given in Figure $8, c, d$ between the results of measured deflections $f$ on specimens of $1.6 \mathrm{~mm}$ thick welded conventionally using GTAW
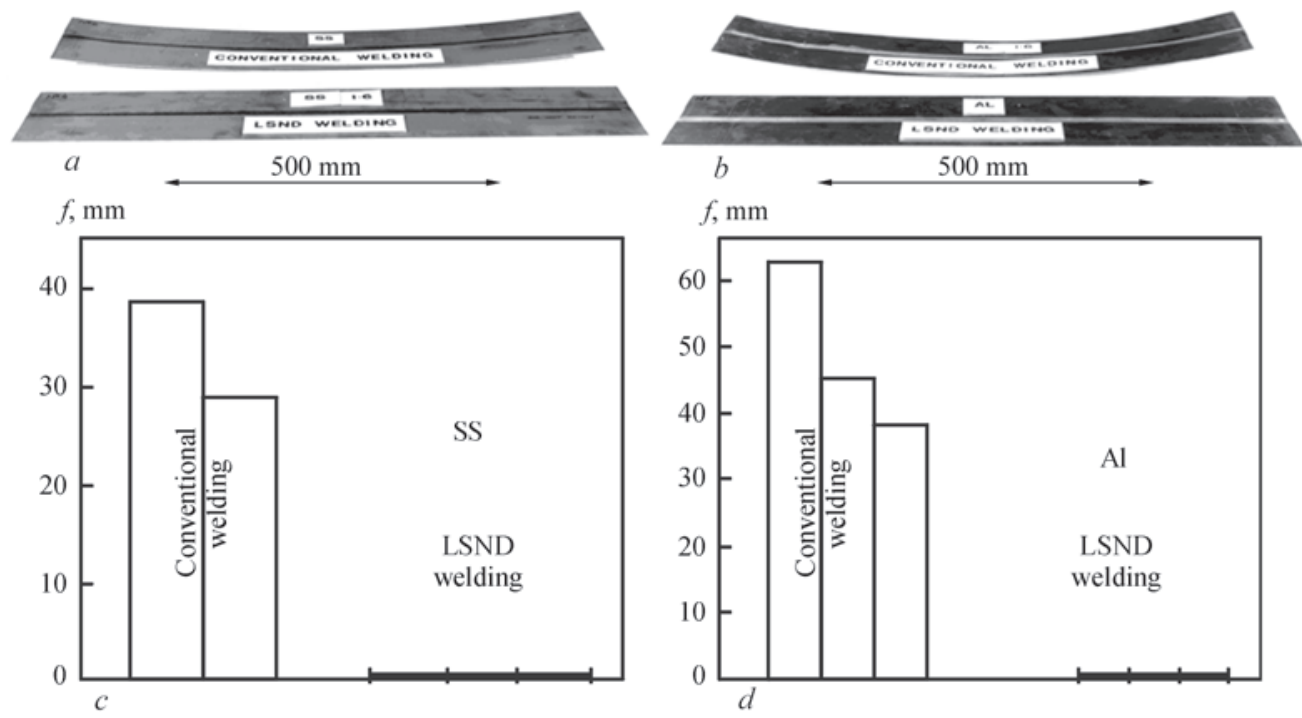

Figure 8. Specimens of $1.6 \mathrm{~mm}$ thick, $1000 \mathrm{~mm}$ long, of stainless steel (a) and aluminum alloy (b) welded by conventional GTAW, severely buckled (upper), and welded by LSND welding, buckle-free (lower). Completely buckle-free results $(f=0)$ can be achieved using optimized LSND welding technique on both stainless steel (c) and aluminum alloy (d) specimens of $1.6 \mathrm{~mm}$ thick 


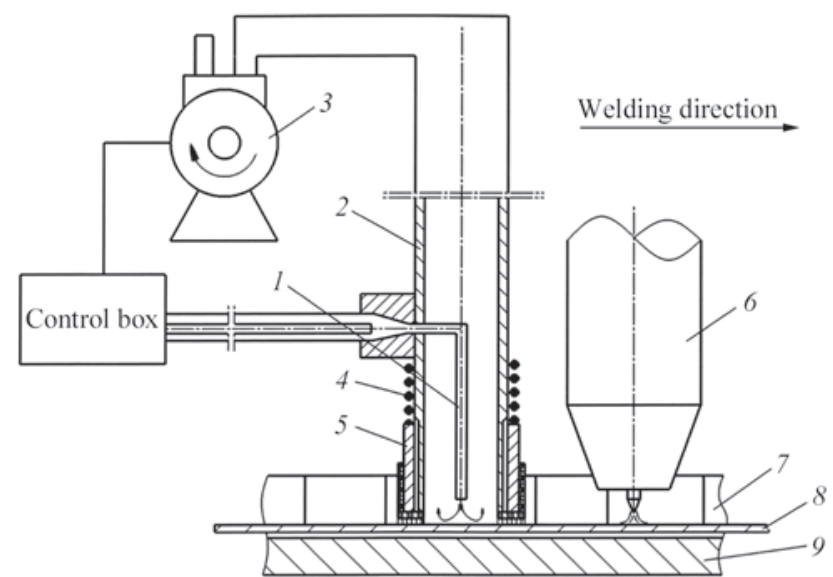

Figure 9. Specially designed device for buckle-free DC-LSND welding of thin-walled elements [5]: 1 - nozzle for atomized cooling jet of liquid media; 2 - co-axial tube to draw the vaporized coolant; 3 - vacuum pump, 4 - spring; 5 - axle oversleeve tube; 6 - GTA welding torch; 7 - clamping fingers; 8 workpiece; 9 - beneath weld backing bar

and those welded using LSND welding technique for stainless steel (Figure 8,c) and aluminum alloy (Figure $8, d)$. Completely buckle-free $(f=0)$ results were achieved while the optimized technological parameters for LSND welding techniques were selected.

As demonstrated above, designers and manufacturers who suffer from problems of buckling could now adopt a new idea that buckling is no longer inevitable with LSND welding technique. Buckling can be prevented completely and residual stresses can be reduced significantly or controlled to a level lower than $\sigma_{\mathrm{cr}}$ at which buckling occurs.

Successful results in preventing buckling distortions were achieved in manufacturing thin-walled jet engine cases of nickel base alloys, stainless steels as well as rocket fuel tanks of aluminum alloys where the acceptable allowance of residual buckling deflections $f$ at a weld length of $L$ should be limited to the ratio of $f / L<0.001$ [18].

Localized thermal tensioning - LSND welding with a trailing spot heat sink. Over the past
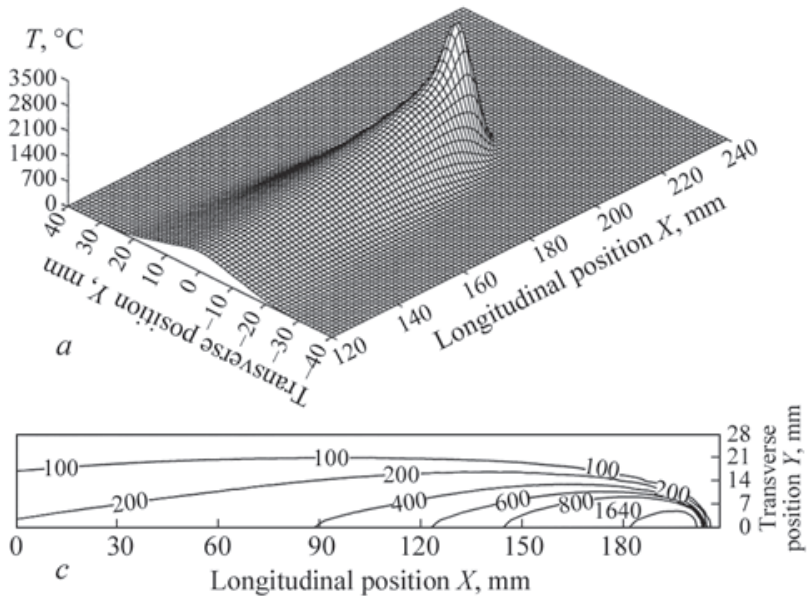

10 years, progress has been made in seeking active in-process control of welding buckling to exploit a localized thermal tensioning technique using a trailing spot heat sink. The heat sink moving synchronously with the welding arc creates an extremely high temperature gradient along the weld bead within a limited area of high temperature zone close to the weld pool (Figure 4). This technique was entitled «Dynamically Controlled Low Stress No Distortion welding method» (DC-LSND) $[5,18,19]$. In this innovative method, the preset heating (as shown in Figure 5,a) is no longer necessary. The formation of specific inverse plastically stretched inherent strains $\varepsilon_{x}^{p}$ in the near arc zone behind the welding pool is dynamically controlled by a localized trailing thermal tensioning effect induced between the welding heat source and the spot heat sink along the weld bead (Figure 4).

Device for engineering implementation of the DCLSND welding technique was designed and further developed at BAMTRI as shown schematically in Figure 9 [5].

With this device attached to the welding torch, an atomized cooling jet of the trailing spot heat sink impinges directly on the just solidified weld bead. Liquid coolant, such as $\mathrm{CO}_{2}, \mathrm{Ar}, \mathrm{N}_{2}$ or water, could be selected for atomized cooling jet. Atomizing the liquid coolant is essential to improve the efficiency of intensive cooling rather than using liquid jet directly impinging the weld bead. To protect the arc from the possible interference of the cooling media, there is a co-axial tube to draw the vaporized media out of the zone nearby the arc. The technological parameters for the trailing spot heat sink and all the welding procedures are automatically synchronously-controlled with the GTAW process. The dominating factors: the distance between the heat source and the heat sink, the intensity of the cooling jet can be selected properly to reach a buckle-free result.
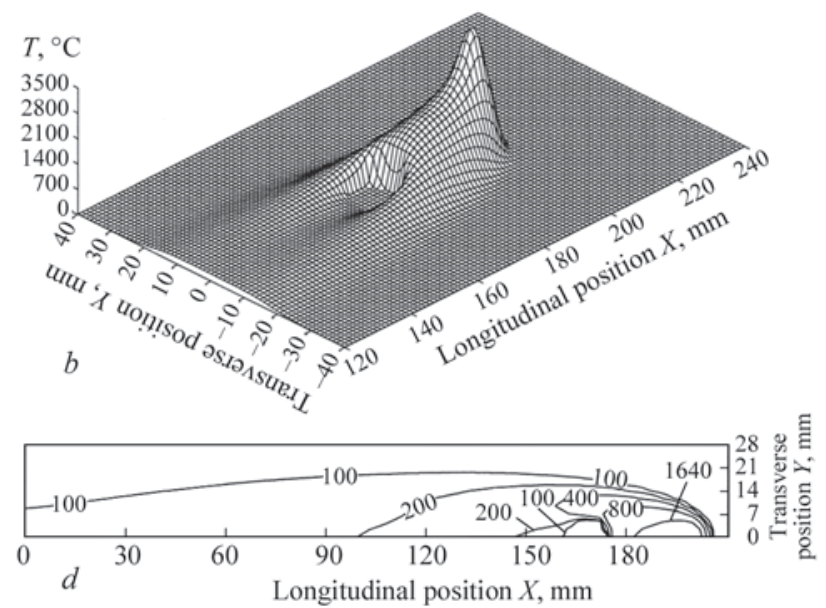

Figure 10. Temperature fields and isotherms on Ti-6Al-4V (2.5 mm thick) plate [20], welding parameters: $200 \mathrm{~A}, 12 \mathrm{~V}, 12 \mathrm{~m} / \mathrm{h} ; a, c-$ conventional GTA welding on copper backing bar; $b, d$-DC-LSND welding 

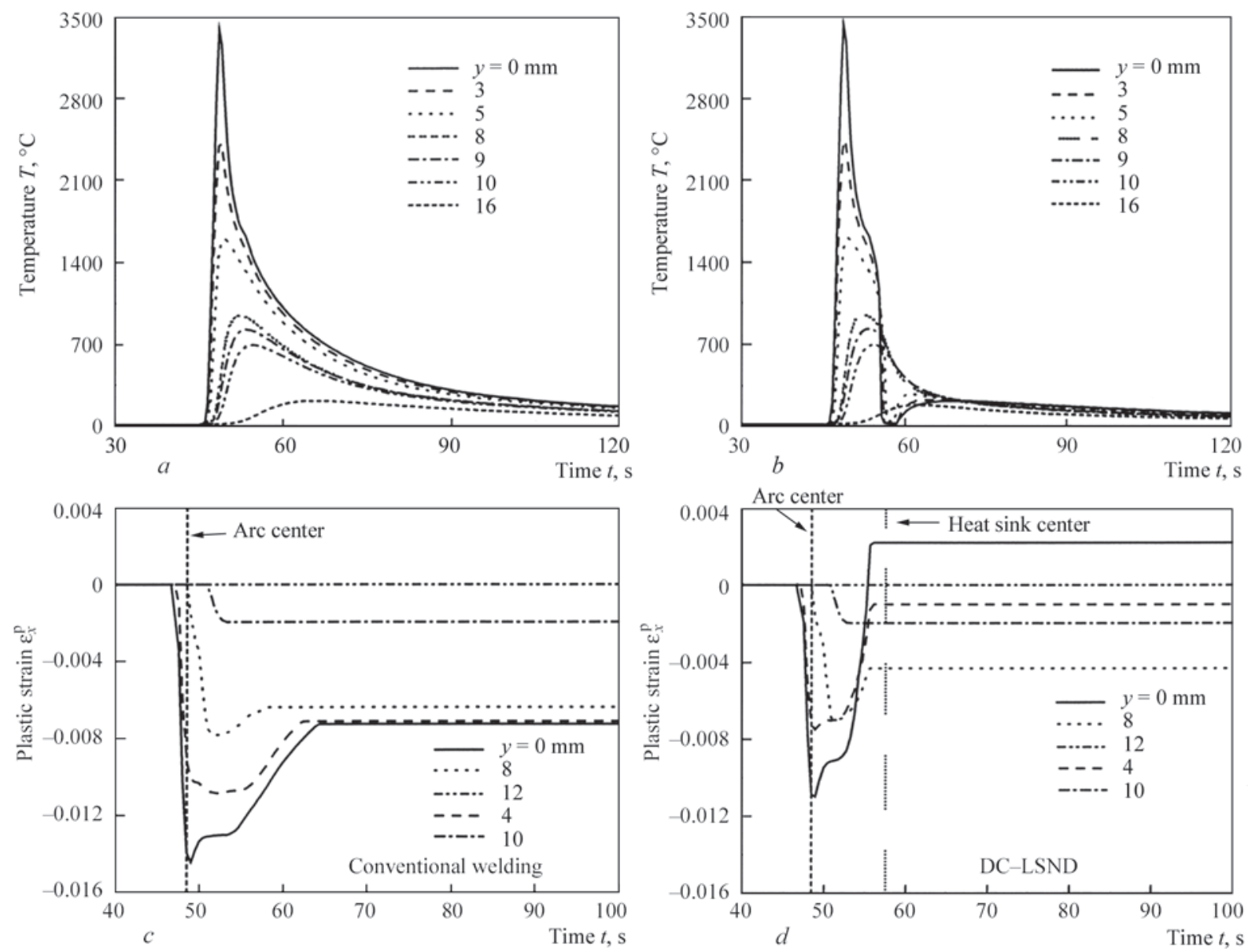

Figure 11. Comparisons of thermal cycles $(a, b)$ and transient plastic strain cycles $(c, d)$ between conventional GTAW $(a, c)$ and DC-LSND welding $(b, d)$ [20]

In systematic investigations, finite element analysis with a model of cooling jet impinging the weld bead surface is combined with a series of experimental studies [19-21]. Comparisons between the tem-

perature fields on conventional GTA welded titanium plate and on plate welded using DC-LSND technique are given in Figure 10.
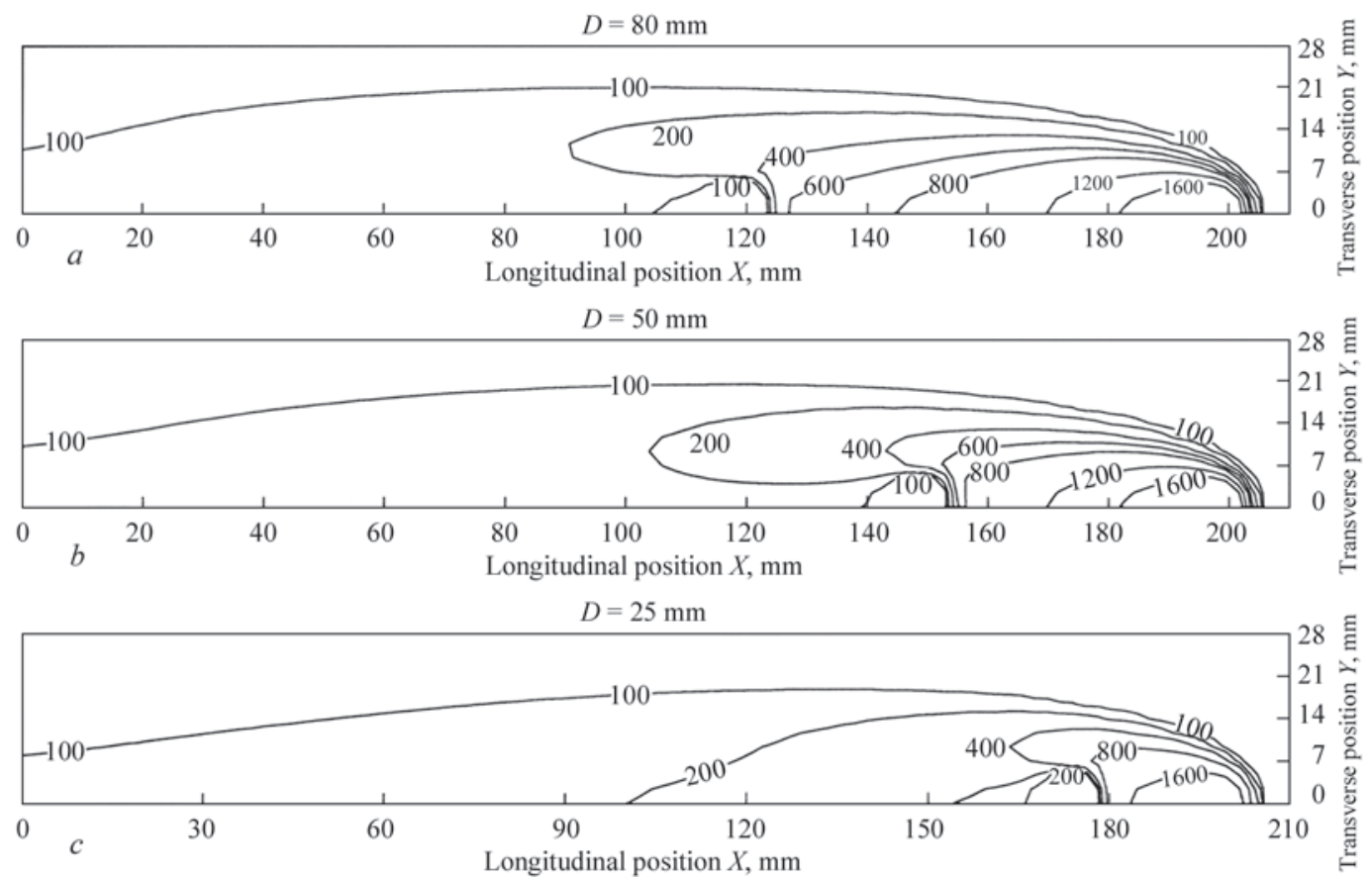

Figure 12. Isotherms on titanium plate with different distance $D$ between the arc center and the cooling jet center [20] 

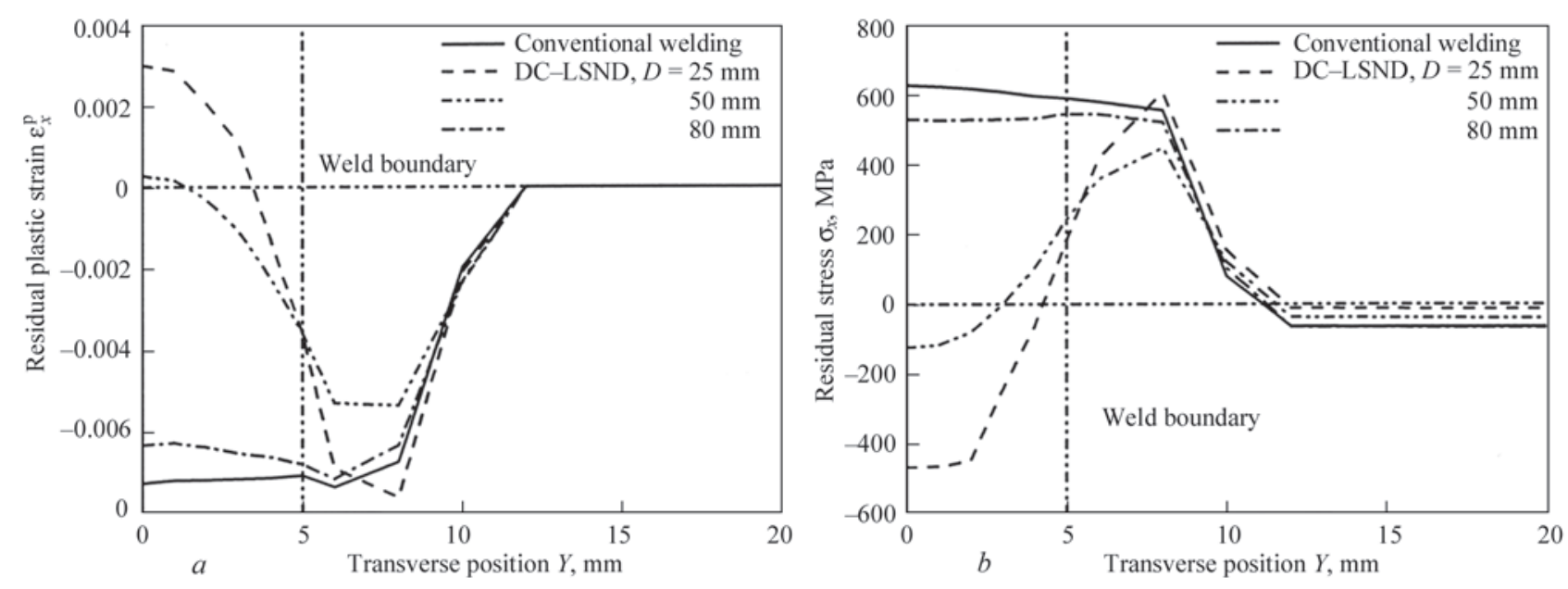

Figure 13. Residual strain $\varepsilon_{x}^{p}(a)$ and stress $\sigma_{x}(b)$ distributions in cross-section of the weld on titanium plate welded conventionally and using DC-LSND welding technique [21]
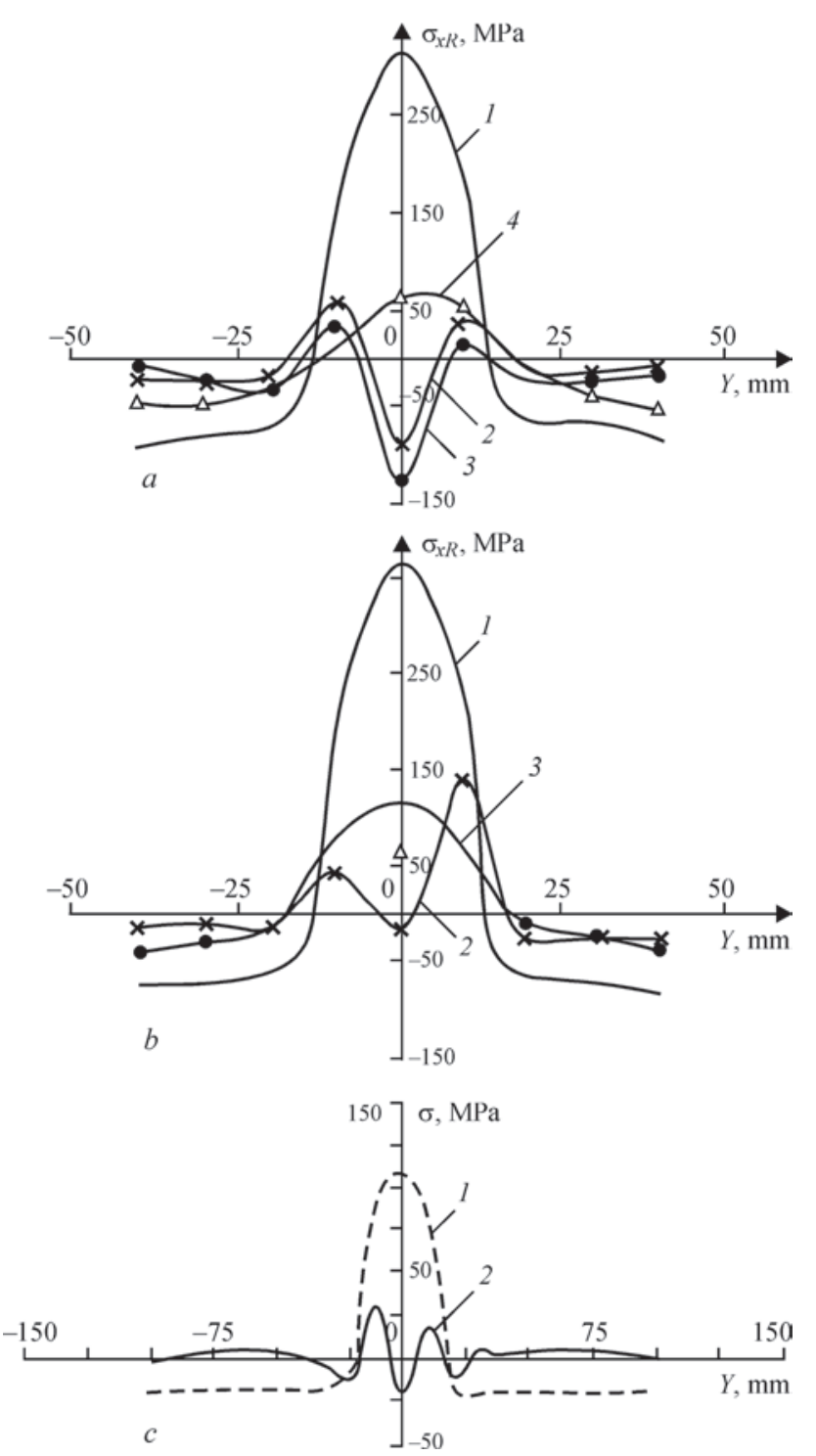

Figure 14. Measured residual stress distributions on plates $1 \mathrm{~mm}$ thick mild steel $(a)$, stainless steel $(b)$ and $2 \mathrm{~mm}$ thick aluminum alloy $(c)$ welded using conventional GTAW (curve 1 ) and by use of DC-LSND welding technique $(2-D=25 \mathrm{~mm}, 3-50 \mathrm{~mm}$, $4-80 \mathrm{~mm}$ ) [19]
In this case, DC-LSND welding was carried out using the same parameters as in conventional GTA welding. The flow rate of cooling medium (atomized water) was selected at $2.5 \mathrm{ml} / \mathrm{s}$. The distance between the arc and cooling jet were regulated from $80 \mathrm{~mm}$ to $25 \mathrm{~mm}$. It can be seen clearly (Figure 10, $b, d$ ) that in DC-LSND welding there is a deep temperature valley formed by the cooling jet behind the weld pool. An extremely high temperature gradient from the peak to the valley is created. The $800{ }^{\circ} \mathrm{C}$ and $400^{\circ} \mathrm{C}$ isotherms in front of the heat sink are severely distorted pushing forward closer to the weld pool (Figure 10,d).

The abnormal thermal cycles by DC-LSND welding (Figure 11, b) produce correspondingly the abnormal thermo-elastic-plastic stress and strain cycles (Figure 11, d) in comparison with the cycles formed by conventional GTAW (Figure 11, a, c). Obviously, the localized thermal tensioning effect is acting only within a limited zone behind the weld pool.

It can be seen also from Figure 11, $d$, that behind the arc, the compressive plastic strains formed before in the just solidified weld zone can be compensated properly by the inherent tensile plastic strains in the area of temperature valley (Figure 11, $d$ ).

In DC-LSND welding, both the value of inherent plastic strains and the width of its distribution can be controlled quantitatively by selecting the proper technological parameters: the distance $D$ between the welding heat source and the heat sink (Figure 12) as well as the intensity of the heat sink.

Figure 13 shows the residual strain (a) and stress (b) distributions in cross-section of the weld on titanium plate. Comparisons are given between conventional welding (shown by solid line) and DC-LSND welding with different distance $D=25 \mathrm{~mm}, 50 \mathrm{~mm}$, $80 \mathrm{~mm}$ (Figure 12). For a selected intensity of heat sink, the closer the heat sink to the heat source (the 

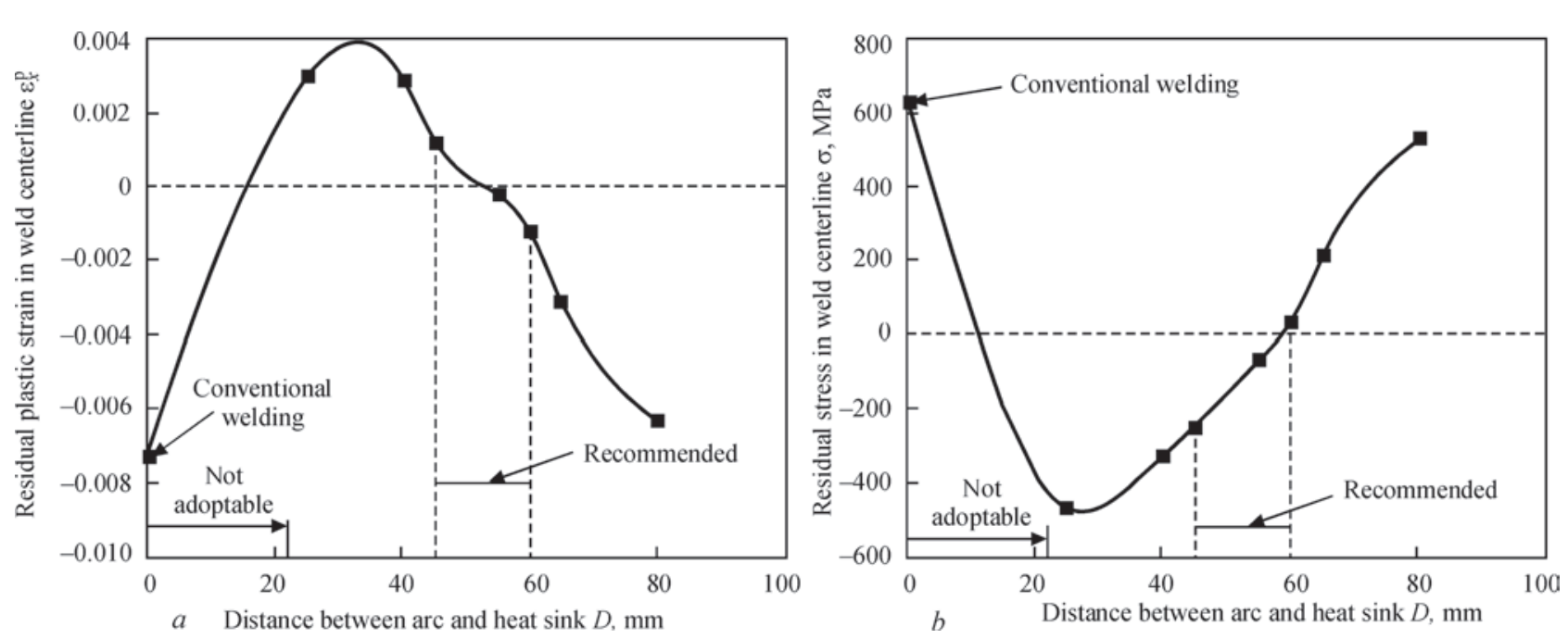

Figure 15. The peak value of residual plastic strain $\varepsilon_{x}^{p}$ in weld centerline $(a)$ and residual stress $\sigma_{x}$ in weld centerline $(b)$ as function of distance $D$ (according to Figure 13)

shorter the distance $D$ ), the stronger is the localized thermal tensioning effect. For example, at the distance $D=25$, the residual plastic inherent strain $\varepsilon_{x}^{p}$ on the weld centerline even changes its sign from negative to positive (Figure 13,a), and the residual stress on the weld centerline changes from tensile to compressive correspondingly (Figure13,b).

Figure 14 gives some typical examples from the systematic investigation program. As shown in Figure $14, a$, the peak tensile stress in weld on mild steel plate welded using conventional GTAW reaches $300 \mathrm{MPa}$ (curve 1) and the maximum compressive stress in the peripheral area is about $90 \mathrm{MPa}$ which causes buckling with deflections more than $20 \mathrm{~mm}$ in the center of specimen of $500 \mathrm{~mm}$ long. In the case of DC-LSND welding the patterns of residual stress distribution (curves 2, 3, 4) alter dramatically with different technological parameters, even with the compressive residual stresses in the centerline of the weld. The reason is that the shrinkage induced by the great temperature gradient between the arc and the cooling jet tends not only to compensate the welding compressive plastic strains but also to alter the sign of residual strain to its opposite. Results show that the distance $D$ has more significant influence on both $\varepsilon_{x}^{p}$ and $\sigma_{x}$ in controlling buckling on thin materials. After DC-LSND welding, the specimens are completely buckle-free and as flat as original before welding. Similar results were obtained as shown in Figure 14, $b, c$ on stainless steel and aluminum plates. Based on the experimental investigations and FEA results, the recommended parameters for engineering application of DC-LSND welding are given in Figure 15 (for the case of titanium plate examined according to Figure 10) to achieve buckle-free results.
Metallurgical and mechanical examinations show that the cooling jet medium gives no noticeable influence on the titanium weld joint properties. Actually the cooling jet is impinging directly on the solidified weld bead at a temperature less than $400{ }^{\circ} \mathrm{C}$ as shown by the distorted abnormal isotherm of $400{ }^{\circ} \mathrm{C}$ in front of the heat sink.

Recent progress in numerical simulation of welding phenomena offers researchers powerful tools for studying in more detail of welding thermal and mechanical behaviors. These tools allow for the prediction of precise control of the abnormal temperature fields and therefore the abnormal thermal elastic-plastic cycles created by the possible variable combinations of the heat source-heat sink welding techniques. It is expected that a variety of coupled heat sourceheat sink processes are feasible for not only welding distortion controlling but also defect-free welds. For example, the device for trailing spot heat sink can be attached not only to the GTAW torch but also could be coupled to other heat sources like laser beam or friction stir welding tool to control distortion, and to improve joint performances as well.

\section{Conclusions}

1. LSND welding techniques for thin materials can be implemented using either the whole cross-sectional thermal tensioning effect or the localized thermal tensioning effect.

2. Basic principles and mechanism of LSND welding techniques are clarified through experimental studies and theoretical analyses with FEA.

3. For LSND welding using the whole cross-sectional thermal tensioning, the necessary condition is to create an adequate temperature profile coupled to the welding temperature field whereas its sufficient condition is to 
keep the thin plate elements in a plane position without any transient loss of stability during welding.

4. In executing DC-LSND welding technique using localized thermal tensioning, the dominating technological parameters are: the distance between the heat source and the heat sink and the intensity of the heat sink. For engineering solution and industrial application, optimized technological parameters are recommended based on FEA results.

5. Both LSND welding techniques have been applied successfully in sheet metal industries to satisfy the stringent geometrical integrity requirements especially to ensure dimensional consistent fabrication of aerospace components.

Acknowledgments. This paper summarizes the main results of a series of research projects supported by the National Natural Science Foundation of China under Contract No. IX-85343 and the Foundation for Aerospace Science and Technology of China under Contracts No. 87625003, No. 98H25002. The author would like to express his gratitude to BAMTRI for the constant support to develop the LSND welding techniques and promote their industrial applications.

1. Terai, K. (1978) Study on prevention of welding deformation in thin-skin plate structures. Kawasaki Technical Review, 61, 61-66.

2. Masubuchi, K. (1980) Analysis of welded structures. Oxford, Pergamon Press.

3. Guan, Q. (1999) A survey of development in welding stress and distortion control in aerospace manufacturing engineering in China. Welding in the World, 43(1), 14-24.

4. Guan, Q., Guo, D.L. et al. (1987) Method and apparatus for low stress no-distortion welding of thin-walled structural elements. Original Chinese patent 87100959.5.1988. International patent specification No PCT/GB88/00136.

5. Guan, Q., Zhang, C.X. et al. (1993) Dynamically controlled low stress no-distortion welding method and its facility. Chinese patent 93101690.8 .
6. Zhong, X.M., Murakawa, H. and Ueda, Y. (1995) Buckling behavior of plates under idealized inherent strain. Transact. of JWRI. 24(2), 87-91.

7. Michaleris, P. et al. (1999) Minimization of welding residual stress and distortion in large structures. Welding J., 78(11), 361-366.

8. Deo, M.V., Michaleris, P. (2003) Mitigating of welding induced buckling distortion using transient thermal tensioning. Science and Technology of Welding and Joining, 8(1), 49-54.

9. Tsai, C.L. et al. (1999) Welding distortion of a thin-plate panel structure. Welding J., 78(5), 156-165.

10. Paton, B.E. et al. (1989) Fabrication of thin-walled welded large panels of high strength aluminum alloys. Avtomaticheskaya Svarka, 10 [in Russian].

11. Radaj, D. (1992) Heat effects of welding: temperature field, residual stress, distortion. Berlin, Springer-Verlag.

12. Guan, Q. et al. (1990) Low stress no-distortion (LSND) welding - a new technique for thin materials. Transactions of Chinese Welding Society, 11(4), 231-237 [in Chinese].

13. Burak, Ya.I. et al. (1977) Controlling the longitudenal plastic shrinkage of metal during welding. Avtomaticheskaya Svarka 3, 27-29.

14. Burak, Ya. I. et al. (1979) Selection of the optimum for preheating plates before welding. Ibid., 5, 5-9.

15. Mechaleris, P. et al. (1995) Analysis and optimization of weakly coupled thermo-elasto-plastic systems with application to weldment design. Int. J. for Numerical Methods in Engineering, 38, 1259-1285.

16. Yang, Y.P., Dong, P., Zhang, J. and Tian, X.T. (2000) A hot-cracking mitigation technique for welding high-strength aluminum alloy. Welding J., 79(1), 9-17.

17. Dong, P. et al. (1998) Analysis of residual stresses in Al-Li repair welds and mitigation techniques. Ibid., 77(11), 439-445.

18. Guan, Q. et al. (1996) Low stress no-distortion welding for aerospace shell structures. China Welding., 5(1), 1-9.

19. Guan, Q., Zhang, C.X. et al. (1994) Dynamic control of welding distortion by moving spot heat sink. Welding in the World, 33(4), 308-313.

20. Li, J., Guan, Q., Shi, Y.W. et al. (2004) Studies on characteristics of temperature field during GTAW with a trailing heat sink for titanium sheet. Journal of Materials Processing Technology, 147(3), 328-335.

21. Li, J., Guan, Q., Shi, Y.W. and Guo, D.L. (2004) Stress and Distortion mitigation technique for welding titanium alloy thin sheet. Science and Technology of Welding and Joining, 9(5), 451-458. 


\title{
PROMISING DIRECTIONS OF WORKS IN THE FIELD OF WELDING AND RELATED TECHNOLOGIES AT SSPA «POWDER METALLURGY»
}

\author{
A.F. ILYUSHCHENKO, E.D. MANOJLO, S.I. LOVYGIN, A.I. LETSKO and A.A. RADCHENKO \\ SSI «Powder Metallurgy Institute» \\ 41 Platonov Str., 220005, Minsk, Belarus. E-mail: alexil@mail.belpak.by
}

\begin{abstract}
The paper presents the promising directions and obtained results in the field of welding and related technologies, pursued in Belarus at SSPA «Powder Metallurgy», including friction stir welding, additive technologies, flame spraying of powder materials and electrospark coatings. Comprehensive development of works in the field of additive technologies is achieved by application of equipment, which ensures polymer and metal 3D-printing, production of metal powders and additional compaction operations. Alongside research performance, this equipment is used for product manufacturing. A unit with a system of recording the welding mode parameters was developed for conducting experimental studies and practical application of friction stir welding. Computer modeling programs (Deform, Sysweld, Ansys, etc.) are used during performance of investigations and development of technological processes and tool designs. Tool manufacture is performed in experimental production, in particular by the methods of powder metallurgy and additive technologies. New scientific and practical results were obtained on development of processes and equipment for flame deposition of coatings from powder materials on parts of various functional purposes, using high power jets (up to $125 \mathrm{~kW}$ ). Investigations were performed and equipment was developed for forming thick-layer (up to 5000-7000 $\mu \mathrm{m}$ ) electrospark coatings with application of electrode-anode vibration of $22 \mathrm{kHz}$ frequency. 18 Ref., 8 Figures.
\end{abstract}

Keywords: friction stir welding, additive technologies, flame spraying, computer modeling, tool manufacture, equipment for electrospark coating deposition

Research area devoted to study of high-energy processes of surface treatment and forming powder coatings has been developing at SSPA of the NAS of Belarus (SSPA PM) for the last twenty years [1-3]. Search works have been performed to study the influence of pulse-periodic laser impact on metal powder particles, as well as on these particles and metal surface, on which they were located [4]. These studies resulted in development (2005) together with the Institute of Physics of the NAS of Belarus of a promising sample of powder laser stereolithography unit $[4,5]$, which can be regarded $[5,6]$ as an analog of modern metallurgical 3D-printer. 3D-printing in this analog was based on selective laser sintering, as it was then believed, of metal powder particles. The developed analog of metallurgical 3D-printer, the characteristics of which are given below, allowed shifting the focus of the performed above-mentioned research towards studying the processes of producing advanced samples of porous permeable materials, primarily, for medical purposes (Figure 1). Results of these studies showed $[7,8]$ that there exist such modes of pulse-periodic laser radiation, at which permanent joints can form, both between the metal powder particles, and between these particles and metal base in the contact zones. The main mechanism of such joint formation is liquid-phase sintering.

\section{Service parameters of the analog of metallurgical 3D-printer}

Solid-state laser . . . . . . . . . . $P=150 \mathrm{~W} ; \lambda=1064 \mathrm{~nm}$, microprocessor control and monitoring system Layer deposition system ........ Adjustable microprocessor regulation system

Working print area, $\mathrm{mm} \ldots \ldots \ldots \ldots \ldots \ldots \ldots \ldots+250 \times 250 \times 100$ Minimum construction zone, $\mu \mathrm{m} \ldots \ldots x=100 ; y=100 ; z=20$ Repeatability, $\mu \mathrm{m} \ldots \ldots \ldots \ldots \ldots \ldots=20 ; y=20 ; z=20$ Loading system $\ldots \ldots \ldots \ldots \ldots \ldots \ldots \ldots$ Manual Powder collection and recovery system ........... Manual Consumable materials ............... Titanium alloys, nonferrous metal alloys

It should be noted that not only selective laser fusion used at 3D-printing by individual types of metallurgical 3D-printers can be considered as a variety of processes related to welding. Also regarded as such $[6,9$, $10]$ can be the following processes used at 3D-printing: Fused Deposition Modeling, or as they are also called, Fused Filament Fabrication. They are based on model layer-by-layer deposition of molten polymer filament. Alongside them, the processes of Direct Metal Deposition, based on direct layer-by-layer deposition of metal molten under the impact of laser radiation or electric current, as well as Electron Beam Melting, based on layer-by-layer selective electron beam penetration of the powder layer, should be also considered as a variety of processes related to welding. 


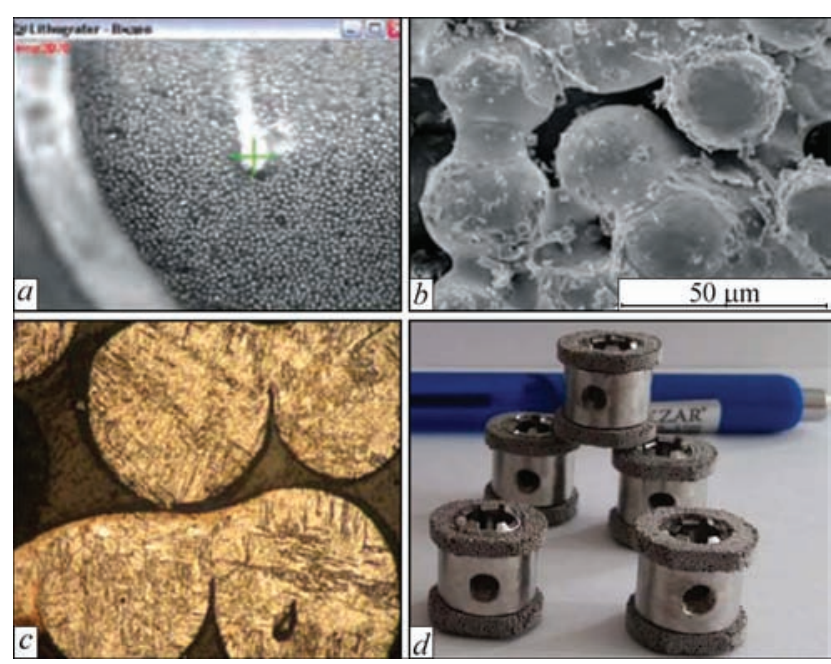

Figure 1. Process of pulse-periodic laser impact followed by the monitoring system of 3D-printer analog $(a)$, produced structure of porous material $(b)$ and particle contact $(c)$ of a spinal fusion implant $(d)$

Additive technologies, which appeared in the second half of 1980s in the form of rapid prototyping, using laser systems of selective photopolymerization, by the end of the first decade of XXIst century became the basis of the forming sixth wave of innovations, together with nanoelectronics, optoinformatics, photonics, artificial intelligence systems, as well as CALS technologies, which determines the present and future development of science and technology all over the world $[6,11]$. Starting from 2015, SSPA PM has conducted purpose-oriented activities for establishing our own additive manufacturing. Here, we take into consideration not only the earlier obtained practical experience, related to development and manufacture of an analog of metallurgical 3D-printer, as well as producing in it promising, primarily, for medical applications, porous permeable materials, but also the acquired experience of our own production of metal powders with spherical shape of particles, using the processes of melting and gas atomization of metal melts $[1,3]$. At present, additive manufacturing organized at SSPA PM is based on the following process equipment:

- assembled in France metallurgical 3D-printer ProX DMP 300 of 3D-Systems International Company (Headquarters in the USA), in which the process of $3 \mathrm{D}$ printing is performed by layer-by-layer selective laser fusion of metal powders;

- manufactured in China vacuum-induction unit JT-QWH-25KG;

- manufactured by Ultimaker B.V. Company (The Netherlands) 3D-printer Ultimaker 3 Extended, in which the process of 3D-printing is performed by layer-by-layer deposition of molten polymer filament;

- manufactured by GOM Company (Germany) 3D-scanner ATOS Triple Scan 16M.

Characteristics of the above process equipment are given below.

\section{Service parameters of 3D-printer ProX DMP 300}

Fiber laser ..............P $=500 \mathrm{~W}, \lambda=1070 \mathrm{~nm}$, microprocessor control and monitoring system

Layer deposition system Adjustable microprocessor regulation system Working print area, mm . . . . . . . . . . . 250×250×300 Minimum construction zone, $\mu \mathrm{m} \ldots \ldots . \quad x=100 ; y=100 ; z=20$ Repeatability, $\mu \mathrm{m} \ldots \ldots \ldots \ldots \ldots \ldots \ldots \ldots$ Loading system .......... Automatic pneumomechanical with microprocessor control system

Powder collection and recovery system ......... Automatic pneumomechanical with microprocessor control system Consumable materials .......... Stainless steel, tool steel, nonferrous metal alloys, high-temperature alloys, aluminium and titanium oxides, metal ceramics

\section{Service parameters \\ of vacuum induction unit JT-QWH-25KG}

Maximum load, $\mathrm{kg} \ldots \ldots \ldots \ldots \ldots \ldots \ldots$. . . . . . . . . 25 . Power of induction medium-frequency

heater, $\mathrm{kW} \ldots \ldots \ldots \ldots \ldots \ldots .100$ (at $4 \mathrm{KHz}$ frequency) Maximum temperature, ${ }^{\circ} \mathrm{C}$

in dispensing crucible . . . . . . . . . . . . $\leq 2200$

in melting crucible $\ldots \ldots \ldots \ldots \ldots \ldots \ldots \ldots \leq 1850$ Vacuum working pressure, $\mathrm{Pa} \ldots \ldots \ldots \ldots \ldots \ldots \leq .66$ Maximum pressure in spray chamber, $\mathrm{MPa} \ldots \ldots \ldots \ldots 6$ Gases used for spraying $\ldots \ldots \ldots \ldots \ldots \ldots \ldots \ldots \ldots \ldots \ldots, \mathrm{Ar}_{2}$

\section{Service parameters of 3D-printer Ultimaker 3 Extended}

Printing technology ...... Fused deposition modeling (FDM) Number of extruders $\ldots \ldots \ldots \ldots \ldots \ldots \ldots \ldots 2$ Working print area, mm . . . . . 215 $215 \times 315$ (for one extruder) $197 \times 215 \times 315$ (for two extruders) Consumable materials ..... ABS, PLA, PVA, HIPS, WATSON, nylon, polycarbonate

\section{Performance of 3D-scanner ATOS Triple Scan 16M}

Number of surface points from

selected zone captured in one scan ........ Up to 16 MP Scan field parameters in the selected

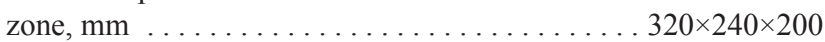
$535 \times 400 \times 320$

Scanning accuracy in the selected zone for

the scan field, mm:

$320 \times 240 \times 200 \ldots \ldots \ldots \ldots \ldots$. . . . . . . . worse than $7 \mu \mathrm{m}$

$535 \times 400 \times 320 \ldots \ldots \ldots \ldots \ldots$. Not worse than $19 \mu \mathrm{m}$

Work on development of technologies of producing both promising for mechanical engineering and medicine samples of products (Figure 2, $a, b$ ) from titanium and high-temperature metal alloys by 3D-printing, and of powders proper (Figure 2, d) from high-temperature alloys is performed in additive production. It is planned to use these powders as consumable material not only for metal 3D-printing, but also for traditional flame and plasma surfacing.

It should be also noted that studying the structure (Figure 2,c) of promising for mechanical engineering samples of products produced by 3D-printing in 
metallurgical 3D-printer ProX DMP 300 leads to the conclusion that the main mechanism of pulse-periodic laser fusion is microwelding, at which a molten pool can form, its dimensions exceeding the diameter of the initial powder particles several times.

Work on mastering the production of domestic medical implants (Figure 3, $a, b$ ) and endoprostheses (Figure $3, c, d$ ), the most accurately allowing for anatomical features of the patients, is also performed in additive production for the needs of Belarus health care.

In addition to perspective improvement of the developed additive technologies, it is also planned to develop and improve in additive production the technologies based on 3D-scanner application, both for automated quality control as to accuracy of product manufacturing, and reengineering. At development and improvement of reengineering technologies, it is planned to focus on eventual lowering of the cost of products manufactured in additive production. Within the framework of development of this production, it is also planned to develop and improve the technologies of rapid prototyping and producing molten polymer filament using the processes of model layer-by-layer deposition, as well as improve the already available at SSPA PM technologies of heat treatment and hot isostatic pressing of powder materials, including the technologies of laser and electroerosion cutting. Application of the above technologies in additive production will promote greater effectiveness of its functioning and at the same time improvement of physicomechanical and strength properties of the manufactured products.

Friction stir welding (FSW) has been one of the most dynamically developing areas in the field of welding over the last decades.

Starting from 1991, after patenting of this solid-phase friction welding process (Friction Stir Welding - FSW) by The Welding Institute (TWI), research in this field has been intensively conducted, and equipment and tools have been developed. Technical and economic advantages of this process enable its active introduction into production, ousting the traditional fusion welding methods. The main FSW advantages include:

- high stability of weld quality, strength and endurance of welded joints (impact toughness, ultimate strength, bend angle, cyclic strength and some other parameters) in absence of distortions or thermal deformations, without pores, inclusions or cracks;

- shortening of production cycle by 50-70\%, compared to regular welding processes, for instance, arc welding;

- possibility of welding parts from dissimilar materials, unweldable by the traditional methods;

- possibility of automation and application of real-time in-process quality control (with availability of

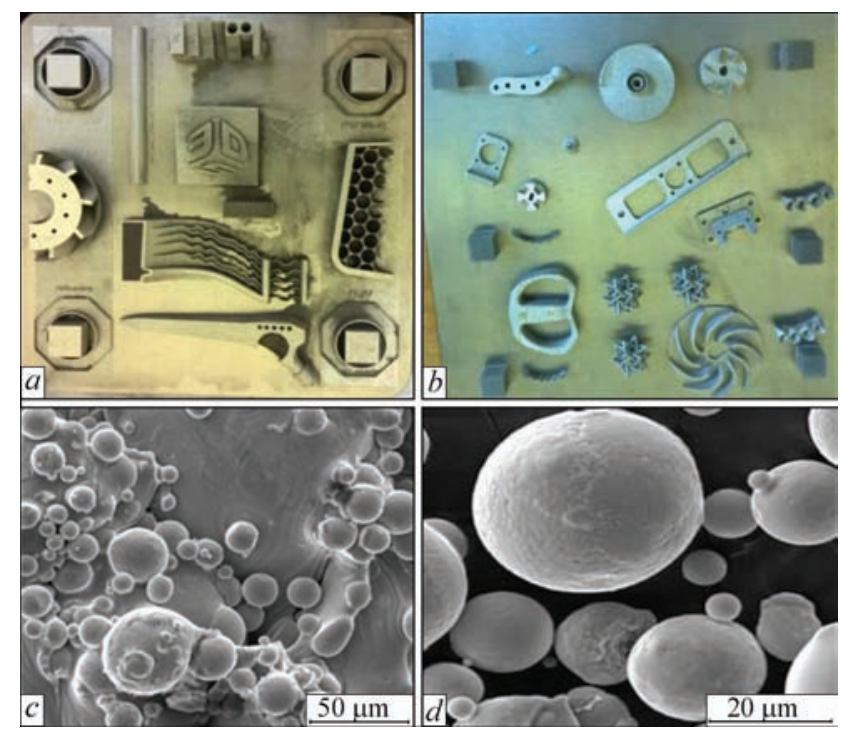

Figure 2. Test (a) and advanced (b) samples of products, their structure $(c)$ and proposed powders for 3D-printing $(d)$

special fixtures and tools it can be performed in standard metal-cutting equipment) in all-purpose milling machines and CNC machines, as well as with application of robotic systems;

- low energy consumption $(2.5 \%$ of energy consumed in laser welding; $10 \%$ of energy consumed in arc welding);

- fast payback due to low energy consumption and no need for consumable materials, or for envisaging the sanitary and ecological measures;

- high welding hygiene, as there is no hot metal spatter, evolution of harmful substances (welding aerosols and gases), no ultraviolet radiation, or electromagnetic fields.

The following should be regarded as the process disadvantages:

- need for large capital expenditures for introduction of sophisticated modern high-tech equipment with maximum complex automation and robotization;

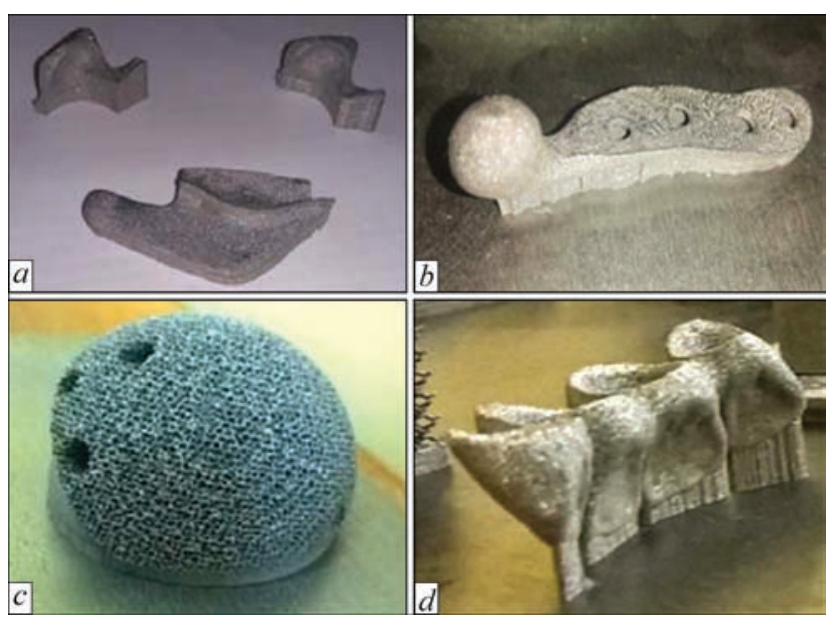

Figure 3. Samples of domestic implants in the form of: $a$ - temporomandibular joint; $b$ - mandibular miniplate; endoprotheses in the form of: $c-$ hip cup; $d-$ dental crown 


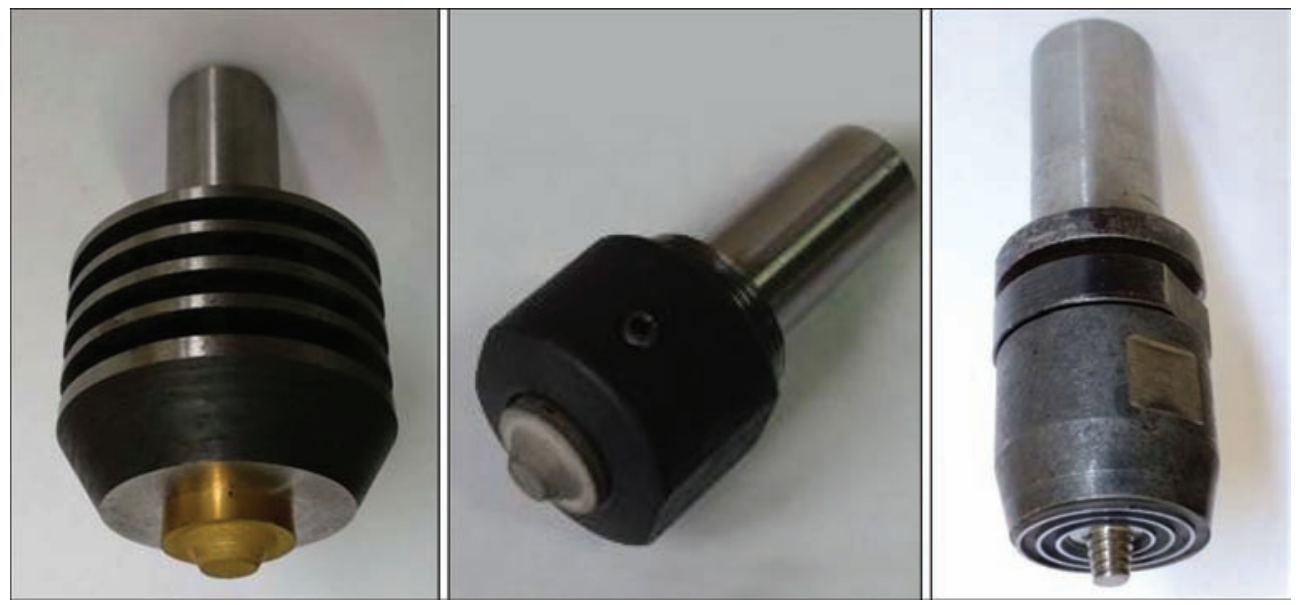

Figure 4. Examples of tools produced by the methods of powder metallurgy and 3D-printing

- need for specialized fixtures for basing and rigid fastening of parts before welding, which is several times greater than in fusion welding;

- need to apply runoff tabs or multiple complication of the structure design.

Successful application of FSW requires an integrated approach, including performance of research, development of technology, equipment, tools and their manufacture. Starting from 2014, SSPA PM has conducted investigations of FSW processes, developed and manufactured the tools. Technologies of welding similar and dissimilar aluminium, iron and copper based alloys 0.5 to $10 \mathrm{~mm}$ thick are studied and developed, and work on 3D-surfacing is performed.

In order to conduct experimental studies and for practical application of FSW, a laboratory set-up with system of programmable automatic control (CNC) of the technological process and system of recording the welding mode parameters was developed.

Investigation and development of technologies and tool designs are performed with application of computer modeling programs (Deform, Sysweld, Ansys, etc.).

Pilot production manufactures tools by the methods of powder metallurgy and 3D-printing. Tools from tool steels for welding aluminium alloys of 0.3 to $10 \mathrm{~mm}$ thickness, and from hard alloys and WRe for welding materials from 0.8 to $6.0 \mathrm{~mm}$ thick are currently produced. The manufactured tools are shown in Figure 4.
Work is in progress on development and setting up manufacturing of FSW equipment in the Republic of Belarus. One of the directions of work in the field of welding and related technologies, which was further developed at SSPA PM in scientific and practical terms, is the technology of flame spraying of coatings.

Flame spraying of coatings from self-fluxing alloys, developed by Wall Colmony Corporation (USA) in 1945, is still widely applied at surface strengthening of parts for various purposes, usually, of small overall dimensions, for instance, exhaust valves, levers and pushers of the gas-distributing mechanism of internal combustion engines, and parts of various machines. With increase of overall dimensions, and product weight, respectively, the amount of heat, evolving at operation of the currently available standard machines is insufficient for coating deposition.

Taking into account the disadvantages of the available methods, we developed a new process - continuous flame spraying of coatings from self-fluxing nickel alloys (CFSC) and equipment for its performance. In this process preheating of the sprayed surface of the part up to the required temperature, deposition of a layer of coating material, heating it and part surface up to the coating material melting temperature - surface melting, is performed without interruptions between the operations, by one high-power gas-flame spraying system TENA-GNpm (Figure 5).

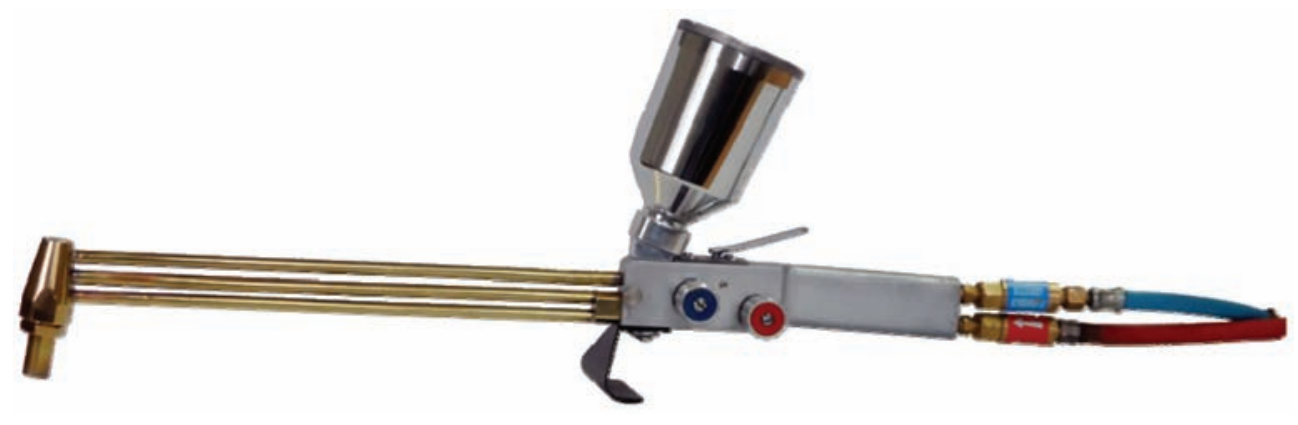

Figure 5. TENA-GNpm system 
An important feature of TENA-GNpm system is ensuring safe operation at coating deposition, that is achieved due to an ingenious system of gas mixing and gas nozzles. Used as the combustible gas is synthetic gas MAF to TU 38.102.1267-89 (methylacetylene-allen fraction), production of which is carried out in the Republic of Belarus. Work on gas-flame spraying of coatings is performed at its flow rate from 1.0 up to $3.5 \mathrm{~m}^{3} / \mathrm{h}$ (maximum thermal power of the flame is more than $100 \mathrm{~kW}$ ). Wide ranges of regulation of power of TENA-GNpm system allow deposition of coatings on parts of various dimensions and weight with the efficiency from 1 up to $8 \mathrm{~kg} / \mathrm{h}$ [12].

Amorphous flame coatings are currently applied for reconditioning and strengthening of various parts: crankshaft main and connecting rods, camshaft bearing journals, crankshafts and eccentric refrigeration shafts that allowed their service life to be extended 1.3 to 1.6 times, compared to new uncoated parts. Deposition of amorphized coating on cast iron piston rings allowed their wear resistance to be increased 1.6 to 2.5 times, compared to electroplating chromium. Here, lowering of friction coefficient 1.5 to 1.6 times and reduction of sleeve wear 1.4 to 2.4 times is observed [13].

We developed a new process of powder flame spraying and TPpm-18 unit for spray deposition of high-quality coatings from a wide range of materials, including metal alloys, oxide ceramics, amorphous materials, special composites, etc. The unit is fitted with a new system of gas mixing and special multinozzle tips, ensuring safe operation (without backfire) at a high power (up to $100 \mathrm{~kW}$ and more). An important distinctive feature of TENA-Ppm18 unit is its fitting with air nozzles, forming the combustion chamber (about $15 \mathrm{~mm}$ diameter, length from 25 to $120 \mathrm{~mm}$ ), as well as air ring distributor mounted on air nozzle, that allowed concentrating (compressing) the two-phase jet along the axis, raising its pressure and temperature, increasing the length of the jet high-temperature zone more than 3 times, and effective efficiency of powder heating, and lowering the thermal impact on the product.

The new process was used for repair of worn working surface of flexographic printing drum (1250$1600 \mathrm{~mm}$ diameter, $250-350 \mathrm{~mm}$ width) by spraying the coating layer with powder of amorphous alloy BKhM [14]. Spraying distance was equal to $250 \mathrm{~mm}$, time of spraying $0.6 \mathrm{~mm}$ coating was $90 \mathrm{mi}$, powder consumption was $11200 \mathrm{~g}$; and efficiency was $7.5 \mathrm{~kg} / \mathrm{h}$.

\section{Unit operating mode}

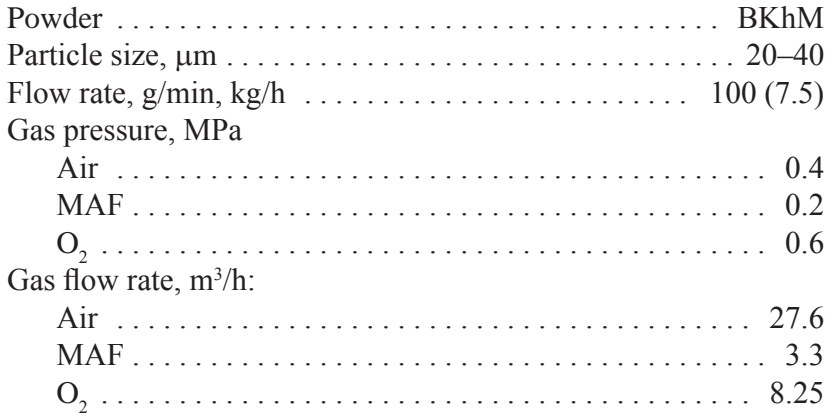

Power $N$, kW ..................... 81.36

Service tests showed that amorphous coating of the drum working surface with BKhM powder, when working three-shifts, ensures normal operation of the drum (without traces of wear) for two years at a high quality of printing. Flame spraying of a coating from BKhM amorphous material was also used for strengthening of the aluminium housing of Wankel engine. For comparison, Figure 6 shows the processes of spray deposition of an amorphous coating with BKhM powder by TPpm-18 unit on the drum of flexographic printing machine and of $\mathrm{WC}-12 \mathrm{Co}$ coating by Jet Kote supersonic spraying machine of Deloro Company on a shaft. One can see from Figure 6 that the jets are similar in both the processes. Hardness of amorphous coating deposited by TENA-TPpm-18 unit is equal to $H R C 70-74$, and that of $\mathrm{WC}-12 \mathrm{Co}$ coating deposited by Jet Kote equipment, is HRC 6268. Spraying efficiency in the first case is three times higher than in the second.
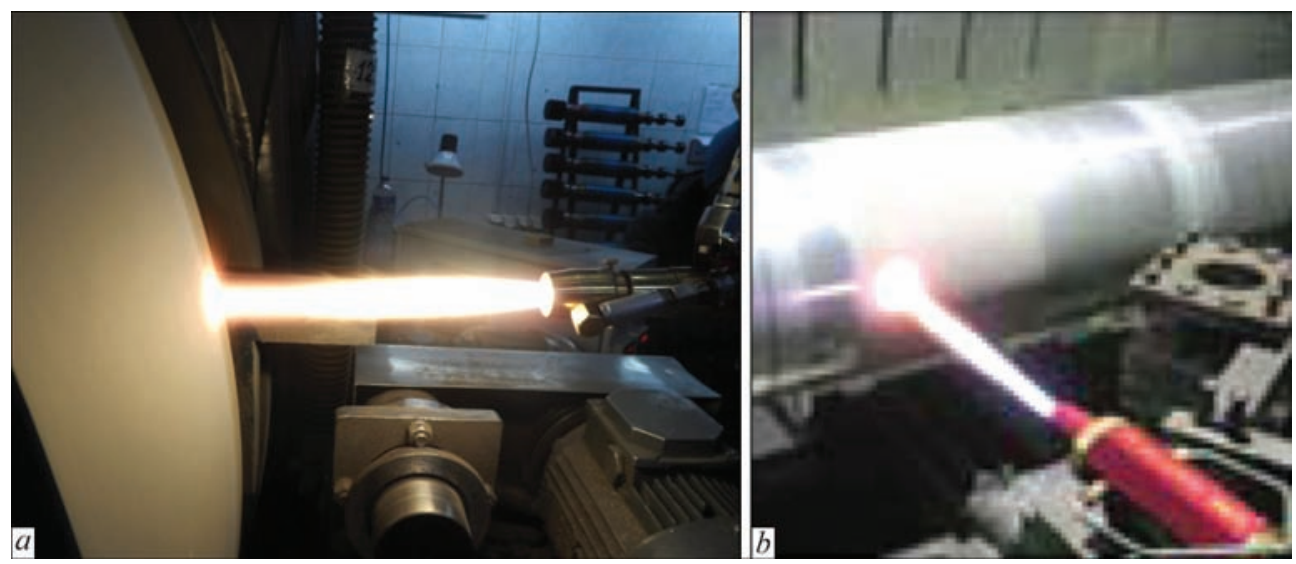

Figure 6. Spraying processes: $a$ - amorphous coating deposition with BKhM powder by TPpm-18 unit; $b$ - WC-12Co coating deposition by Jet Kote unit of Deloro Company 


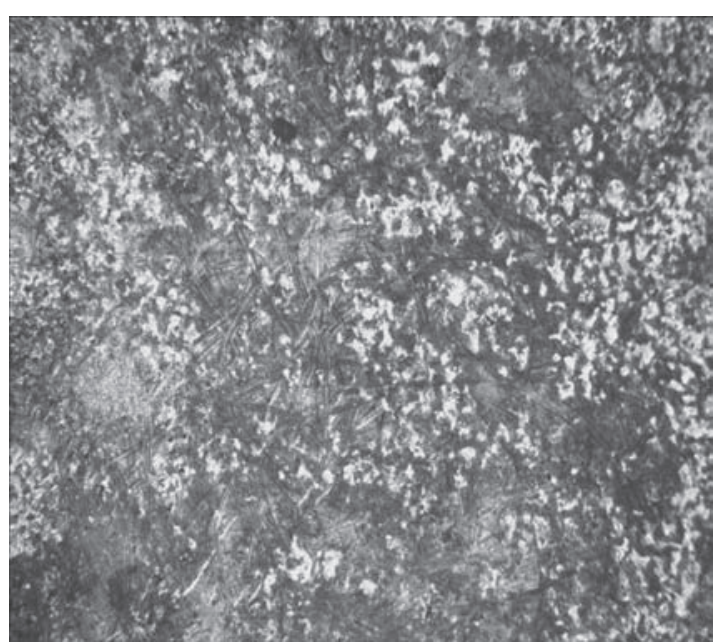

Figure 7. Coating formed at $22 \mathrm{kHz}$ frequency of anode oscillations $(\times 20)$

A new technology of gas-flame spraying of thick-layered $(2-4 \mathrm{~mm})$ coatings from nanostructured ferritic powders with a special complex of properties, in particular, ensuring wide-range microwave absorption, was developed, and process equipment for its implementation was manufactured [15]. The essence of the proposed technology consists in that the powdered ferromagnetic material with particle sizes of $(-53.0+5.0) \mu \mathrm{m}$ is fed into the jet of gas-flame sprayer TENA-Ppm, moving at a speed of $300-750 \mathrm{~m} / \mathrm{s}$, the energy of which is used to transport and deposit the powder particles on substrates fixed in the mandrel Flow rate of MAF combustible gas during unit operation is $3500 \mathrm{l} / \mathrm{h}$, and that of oxygen is $8750 \mathrm{l} / \mathrm{h}$. The process and equipment were transferred to the customer under a contract.

Saving energy resources and reducing labor consumption in production are in the first place in the activity of each mechanical engineering or repair enterprise. Under these conditions, methods with appli-

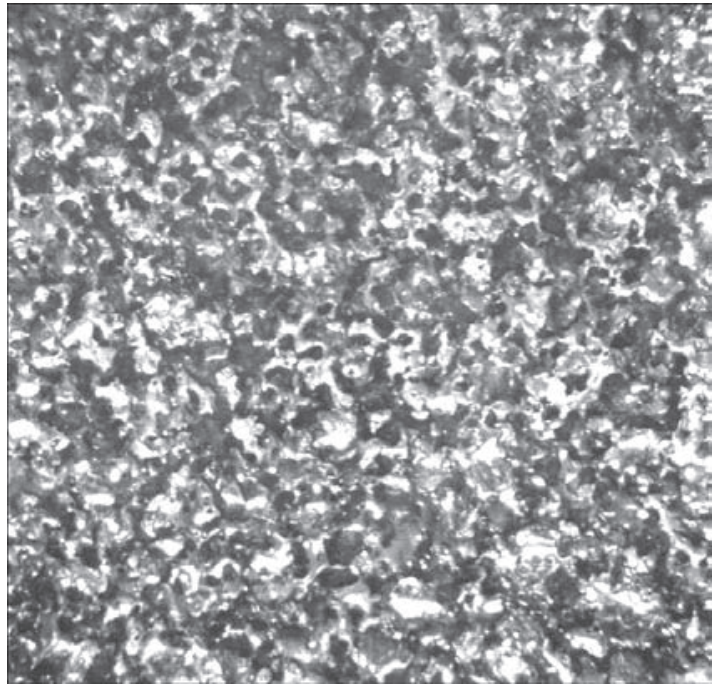

Figure 8. Coating produced at $600 \mathrm{~Hz}$ frequency of anode oscillations $(\times 20)$ cation of concentrated energy flows, which include electrospark alloying (ESA), are used for restoration of service properties of parts of machines and mechanisms lost in operation, improvement of service properties, and extension of service life. ESA method allows coating deposition on the treated surface by a compact electrode from any current-conducting material, and is characterized by low energy consumption. The formed spark coatings feature high strength of adhesion with base material. One of the drawbacks of the method, however, is the small thickness of the applied coating (up to $300-500 \mu \mathrm{m}$ ), that limits its application $[16,17]$.

The problem of increasing the thickness of spark coatings is being solved now. Thick-layer coatings of higher continuity were obtained at Federal State Budgetary Institution «All-Russian Scientific-Research Technological Institute of Repair and Maintenance of Machine and Tractor Park» (FGBNU GOSNITI, Russia) by the method of application of alternating cycles of deposition of coarse coatings with great roughness of surface profile and surface melting cycles for leveling the surface profile with reduction of the height of these irregularities by not less than $50 \%$. Here, surface melting of profile unevenness was performed with application of electrode materials with higher heat conductivity and erosion resistance relative to electrode material, forming the coating. Thick-layer coatings were formed in modes of low-frequency electrospark alloying with maximum frequency of alloying electrode vibration of $600 \mathrm{~Hz}$ [18].

SSPA PM performed a package of research work with application of experimental equipment for high-frequency electrospark alloying with the purpose of determination of the influence of ultrasonic impact (USI) on the process of forming electrospark coatings of greater thickness and continuity. Proceeding from the results of earlier studies, it was found that ultrasonic impact with $20.4-23.6 \mathrm{kHz}$ frequency applied before the start and after the end of the main electrospark treatment actively influences the increase of the coating mass and thickness. Increase of coating mass in the first case is three times greater, and in the second case it is two times greater than in the case, when additional USI is not applied [19].

Investigations were performed with application of Alier-55 unit and ultrasonic device developed in the Sector of Electrophysical Coatings of SSU IWPC, consisting of a generator, power unit and ultrasonic piezoelectric converter.

The following energy modes of Alier-55 unit were selected for experimental studies: coatings were deposited with pulse energy of $4.3 \mathrm{~J}$ and their repetition rate of $100 \mathrm{~Hz}$; surface melting of the coating was conducted by a combined method - working pulses 
with $10 \mathrm{~J}$ energy and $50 \mathrm{~Hz}$ repetition rate were obtained from Alier-55 unit, and the frequency of oscillations of electrode-anode of $20.4-23.6 \mathrm{kHz}$ was set by ultrasonic device generator. Periodical contact of «surface melted» electrode with the deposited coarse coating layer performed at ultrasonic frequency of $20.4-23.6 \mathrm{kHz}$, resulted in high dispersion of protrusions of the coating coarse layer with displacement of dispersed particles to the relief depressions, ensuring leveling of the coating and increase of its continuity up to $85-90 \%$ and of the thickness up to $5000 \mu \mathrm{m}$.

Figure 7 shows the topography of cathode surface (steel 45) after treatment with electrode-anode from titanium-tungsten-cobalt alloy T15K6 with vibroexciter frequency of $22 \mathrm{kHz}$. The formed coating has the structure with element size up to $10 \mu \mathrm{m}$. The coating after treatment by electrode-anode with vibroexciter frequency of $600 \mathrm{~Hz}$, is shown in Figure 8. The formed coating has the structure with element size up to $100 \mu \mathrm{m}$.

Thus, it is experimentally established that application of ultrasonic converter with vibration frequency of electrode-anode of $22 \mathrm{kHz}$ in surface melting operations allows performance of meting and refinement of the material of coating protrusions to values by an order of magnitude smaller than at application of vibroexciter with standard vibration frequency of $600 \mathrm{~Hz}$. Here, more complete movement of molten material of the protrusions into the depressions occurs, and coating continuity is increased.

It is determined that energy modes of Alier-55 unit of 4.3 and $10 \mathrm{~J}$ power ensuring the liquid-drop nature of mass transfer and melting of profile irregularities, as well as ultrasonic impact with the frequency of 20.4-23.6 kHz, during coating deposition and melting of the tops of the protrusions, create thermodynamic phenomena, similar to local annealing as to the nature of their impact on cathode material.

This promotes reduction of the values of tensile (residual) stresses, arising in the coating alloyed layer during electrospark alloying, and shifts the brittle fracture threshold of the material, thus creating favourable conditions for monotonic and continuous growth of the thickness of electrospark coatings up to values greater by an order of magnitude that at application of standard ESA techniques.

Investigation results are currently used to develop equipment for forming thick-layer (up to 5000 $7000 \mu \mathrm{m}$ ) electrospark coatings with application of electrode-anode vibration of $20.4-23.6 \mathrm{kHz}$ frequency.
1. (2000) 40 years of powder metallurgy in Belarus Republic. Ed. by E.A. Doroshkevich. Minsk, Kovcheg [in Russian].

2. Ilyushchenko, A.F. et al. (2007) High-energy treatment of plasma coatings. Minsk, Bestprint [in Russian].

3. Ilyushchenko, A.F. et al. (2010) 50 years of powder metallurgy in Belarus: History, achievements, perspectives. Minsk, Diateks [in Russian].

4. Belyavin, K.E. et al. (2008) On sintering of titanium spherical powders under action of solid-state laser. Poroshk. Metallurgiya, 7-8, 155-160 [in Russian].

5. Ilyushchenko, A.F. (2016) Efficient tool of modern mechanical engineering. Nauka i Innovatsii, 156(2), 16-20 [in Russian].

6. Dovbysh, V.M., Zabednov, P.V., Zlenko, M.A. (2014) Additive technologies and products from metal. Moscow, NAMI [in Russian].

7. Belyavin, K.E. et al. (2006) Examination of effect of power of pulse-periodic laser radiation on stability of liquidmetal contacts between particles powder in selective laser sintering. Poroshk. Metallurgiya, 29, 268-272 [in Russian].

8. Bykov, R.P. et al. (2010) Examination of laser selective sintering process of spherical titanium powders. Litio i Metallurgiya, 58(4), 137-140 [in Russian].

9. Ilyushchenko, A.F., Savich, V.V. (2017) History and current state of additive tecgnologies in Belarus, powders of metals and alloys for them. Kosmichna Nauka i Tekhnologiya, 23(4), 33-45 [in Russian].

10. Stepanova, E.Yu. Additive technologies as break through innovations of resource saving of 21st century. http://oreluniver. $\mathrm{ru} / \mathrm{file} / \mathrm{science} / \mathrm{confs} / 2015 / \mathrm{ee} / \mathrm{publ} / \mathrm{s}$ _ 7 Stepanova_E_YU.

11. Ilushchenko, A.F., Manyolo, E.D., Andreev, M.A., Onashchenko, F.E. (2017) Flame spraying of coatings of self-fluxing alloys. Welding Intern., 31(11), 887-891.

12. Borisov, Yu.S., Korzhik, V.N. (1995) Amorphous gasthermal coatings. Theory and practice (Review). Avtomaticheskaya Svarka, 4, 3-11.

13. Manoylo, E.D., Onashchenko, F.E., Susha, G.K. (2018) To problem of flame spraying of amorphous coatings. In: Proc. of Symp. on Technologies. Equipment. Quality in frame of Belpromforum (30 May - 1 June 2018, Minsk) [in Russian].

14. Ilushchenko, A.F., Manyolo, E.D., Onashchenko, F.E. Method of coating material on metallic substrate. Pat. 15869, RB, 2012.0630, Int. Cl. C23C 24/08 (2006.01). Application a 20101456, 08.10.2010 [in Russian].

15. Gitlevich, A.E., Perkansky, N.Ya., Ignatkov, D.A. (1981) On limitation of thickness of layers formed during spark alloying process. Elektronnaya Obrab. Materialov, 3, 25-29 [in Russian].

16. Ivanov, V.I., Burumkulov, F.Kh., Verkhoturov, A.D. et al. (2012) Formation of surface layer of low-alloy steel in spark treatment. Svarochn. Proizvodstvo, 11, 36-40 [in Russian].

17. Ivanov, V.I., Burumkulov, F.Kh. (2014) About spark deposition of three-layer coatings of higher density. Elektron. Obrabotka Materialov, 50(5), 7-12 [in Russian].

18. Lovygin, S.I. (2017) Influence of sequence of ultrasonic action during spark treatment on kinetics of mass transfer of alloying anodes and dimensional characteristics of coatings. Poroshk. Metallurgiya, 40, 201-206 [in Russian]. 


\title{
DIFFUSION WELDING AND BRAZING OF DISSIMILAR MATERIALS WITH CONTROLLED STRESS-STRAIN STATE
}

\author{
V.V. KVASNYTSKYI ${ }^{1}$, V.F. KVASNYTSKYI ${ }^{2}$, CHEN HEXING ${ }^{3}$, \\ M.V. MATVIENKO ${ }^{2}$ and G.V. YERMOLAYEV ${ }^{2}$ \\ ${ }^{1}$ NTTU «Igor Sikorskii Kyiv Polytechnic Institute» \\ 37 Prosp. Peremohy, 03056, Kyiv, Ukraine. E-mail: kvas69@ukr.net \\ ${ }^{2} \mathrm{Adm}$. Makarov National Shipbuilding University \\ 9 Heroiv Ukrainy Prosp., 54025, Mykolaiv, Ukraine. E-mail: welding@nuos.edu.ua \\ ${ }^{3}$ Guangdong Academy of Sciences \\ No.9, Building, 100 Xianlie Rd. Guangzhou, P. R. China. E-mail: chenhexing@gdas.gd.cn
}

\begin{abstract}
Stress-strain state in vacuum diffusion welding and brazing of dissimilar materials was studied, allowing for plastic deformations of instantaneous plasticity and creep. The role of plastic deformations in joint formation and residual stresses in vacuum diffusion welding of dissimilar metals, in particular of metals with nonmetals, as well as similar materials with interlayers is considered. Application of temperature loading simultaneously with external load at vacuum diffusion welding of dissimilar metals allows creating a bulk stressed state in the butt joint, with formation of axial, radial, circumferential, tangential stresses, promoting localization of plastic deformations in the butt joint zone and development of shear deformations, and intensification of the processes of formation of physical contact, activation of the surfaces and development of diffusion processes, respectively. Control of stress-strain state at joining of metals with nonmetals allows regulation of relaxation processes at cooling and prevention of joint fracture. 12 Ref., 9 Figures.
\end{abstract}

Keywords : diffusion welding, brazing, stresses, strains, modeling, structure, mechanical properties, diffusion

Manufacturing parts and components from dissimilar materials allows improvement of the performance of machines, devices and new equipment, as this enables combining the different properties of materials, which cannot be achieved in structures from similar materials. The range of materials used in combined structures, is quite diverse. New materials have been developed, for instance, composite materials, produced by powder metallurgy methods, high-temperature nickel casting superalloys, intermetallic materials and other, which cannot be heated up to melting temperature. In such cases solid-state welding, for instance, vacuum diffusion welding (VDW), friction welding, etc. are used [1]. Brazing is often applied, when joining metals to nonmetals, but problems related to the difference in physicomechanical properties (PMP) of the materials being joined and occurrence of residual stresses (RS) still remain $[2,3]$.

A problem in VDW of dissimilar materials is the nonuniformity of distribution of plastic deformations over the butt area, and, accordingly, also the processes of formation of physical contact, activation of the surfaces and bulk interaction (diffusion, recrystallization). In [3] we showed that VDW with thermal cycling is one of the promising methods for controlling the stress-strain state (SSS) and distribution of plastic deformations.
The objective of this work was improvement of the technology of VDW and brazing of dissimilar materials by controlling SSS formation, allowing for plastic deformations. Mechanics of joining in diffusion welding, brazing and spraying of dissimilar materials under the conditions of elasticity is considered in [4].

In order to establish the fundamental possibility of controlling the plastic deformations appearance in the zone of the butt joint of dissimilar materials and its regularities at VDW with thermal cycling, modeling of SSS in the most widely used assemblies of the type of cylinder-cylinder $(\mathrm{C}-\mathrm{C})$, bushing-bushing (B-B) and bushing-flange (B-F) was performed at heating and cooling for $100{ }^{\circ} \mathrm{C}$. Finite element method and ANSYS software package were used for SSS modeling. Adequacy of modeling results was confirmed by analytical solutions and data derived by experimental method of speckle-interferometry, described in [5]. General view of the section of $\mathrm{FE}$ models of $\mathrm{C}-\mathrm{C}$, $\mathrm{B}-\mathrm{B}$ and B-F assemblies is given in Figure 1.

Modeling of SSS of the assemblies allowing for plastic deformations of instantaneous plasticity showed that principal regularities of formation of SSS of cylindrical assemblies, established in $[3,4]$ for the elastic state, are preserved, but the stress level decreases considerably. At modeling, the moduli 


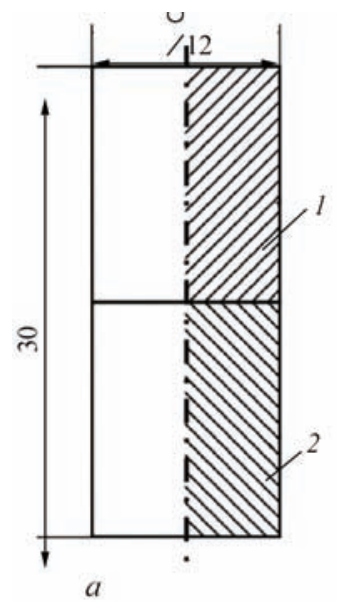

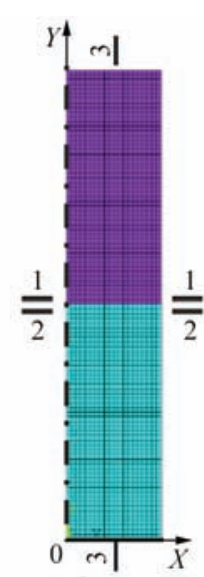

b

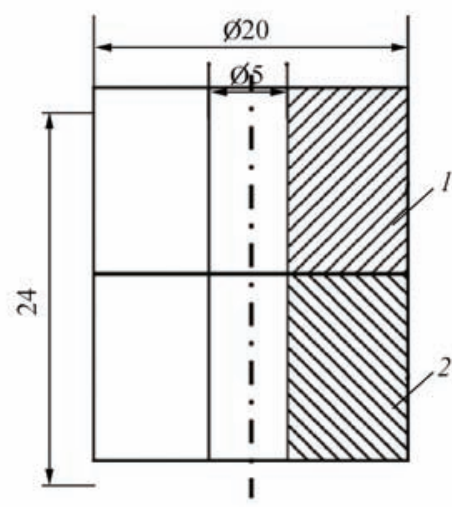

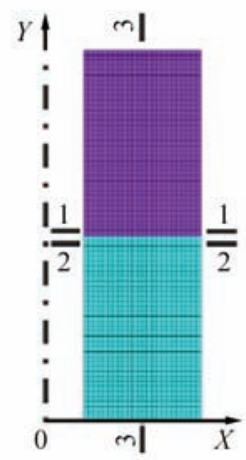

$d$
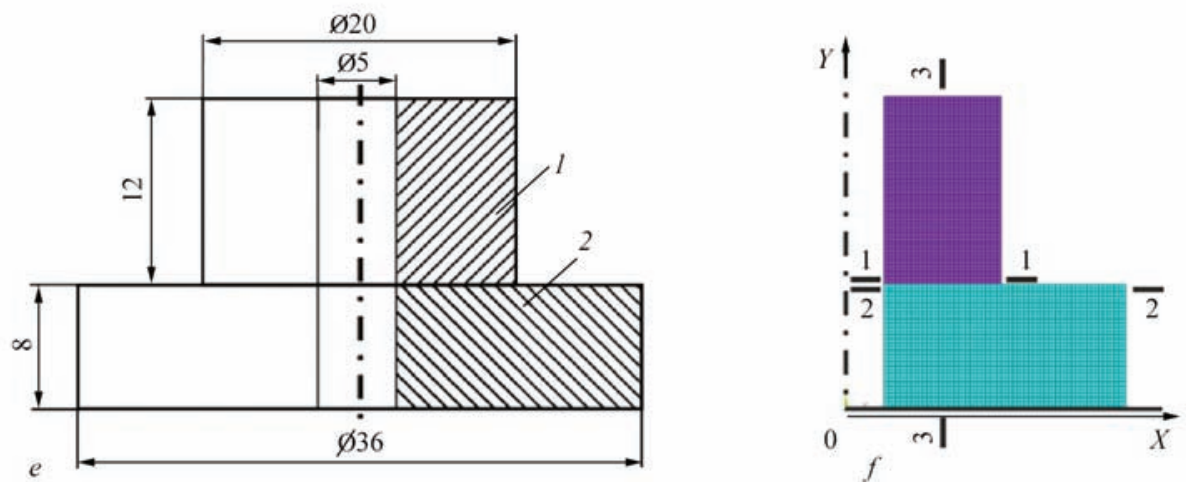

Figure 1. General view $(a-c)$ in the section of $\mathrm{FE}$ models $(b, e, f)$ of $\mathrm{C}-\mathrm{C}, \mathrm{B}-\mathrm{B}, \mathrm{B}-\mathrm{F}$ assemblies, respectively

of elasticity were taken to be the same, yield limit in $\mathrm{C}-\mathrm{C}$ and $\mathrm{B}-\mathrm{B}$ assemblies was smaller in part 1 , in B-F assembly the yield limits are both the same, and smaller in the bushing or in the flange. Temperature coefficients of linear expansion (TCLE) were taken to be different two times, except for the model for VDW by the traditional schematic (at constant temperature) at different yield limits.

It is established that with the traditional VDW scheme for $\mathrm{C}-\mathrm{C}$ and $\mathrm{B}-\mathrm{B}$ assemblies equivalent stresses in the butt joint zone decrease in the material with lower yield limit at the moment of appearance of plastic deformations, and at further loading the portion of the component removed from the butt joint is plastically deformed (Figure 2, $a, b$ ). The butt joint in Figure 2, $a, b$ is shown by an arrow. In $\mathrm{B}-\mathrm{F}$ assembly at the same yield limits and smaller limit in the bushing, the deformation fields are similar in $\mathrm{C}-\mathrm{C}$ and $\mathrm{B}-\mathrm{B}$ assemblies with noticeable increase at the stress raiser (Figure 2, c, d). At a smaller yield limit of flange material, plastic deformations in it develop in a very narrow zone near the stress raiser (point $A$, Figure 1,e).

Modeling allowing for plastic creep deformations showed that the pattern of deformation fields does not change. With the traditional VDW scheme, plastic deformations are also absent on the greater part of the butt joint. At VDW with thermal cycling (heat-

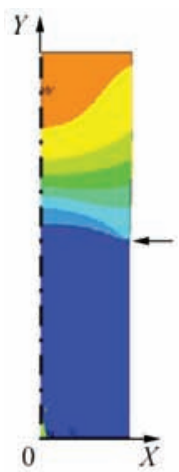

$a$
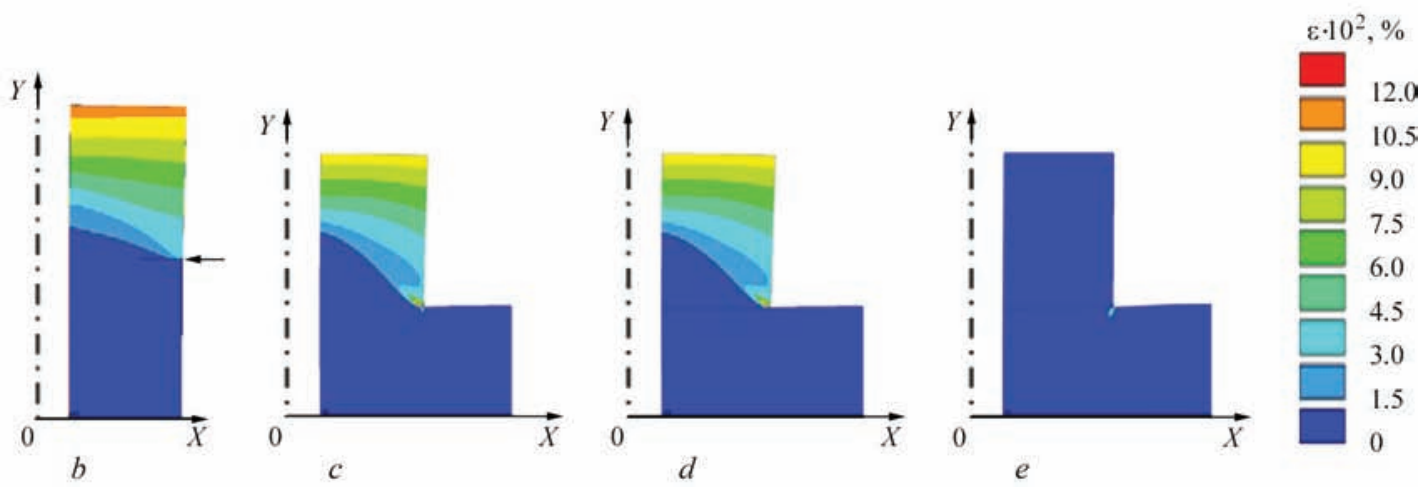

Figure 2. Fields of instantaneous plastic deformations of C-C (a), B-B (b) assemblies, at lower yield limit of part 1, B-F assembly at equal yield limits $(c)$, smaller one in the bushing $(d)$ and smaller one in the flange $(e)$ at the traditional scheme of VDW, allowing for instantaneous plasticity deformation 


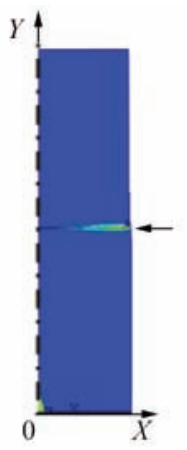

a

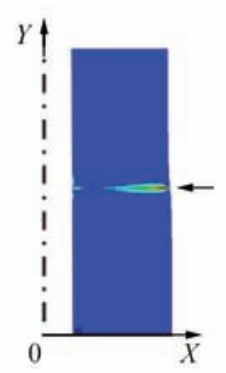

b

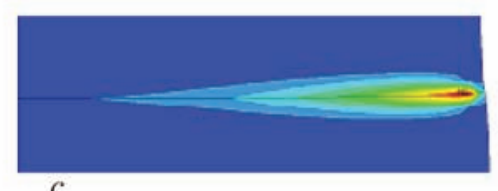

$c$

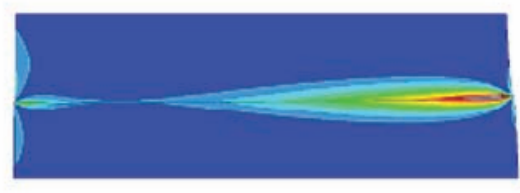

$d$

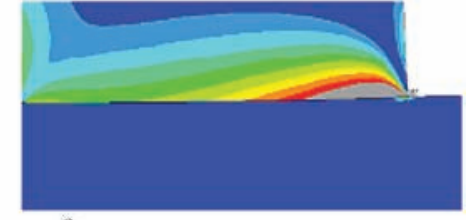

$e$

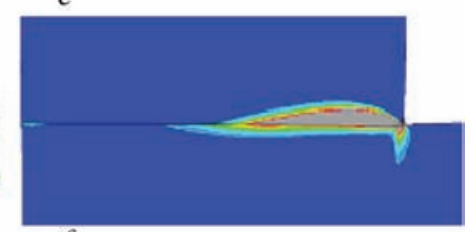

$f$

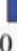

0

3.

4.5

Figure 3. Fields of equivalent instantaneous plastic deformations in $\mathrm{C}-\mathrm{C}(a, c), \mathrm{B}-\mathrm{B}(b, d)$ and $\mathrm{B}-\mathrm{F}(e, f)$ assemblies with the same yield limit and smaller TCLE of parts 1 and bushing through the entire assembly $(a, b)$ and near butt joint $(c-f)$ at thermal loading without external pressure after adhesion of the surfaces

ing or cooling by $100{ }^{\circ} \mathrm{C}$ ) without external loading by compression, after adhesion of the surfaces of $\mathrm{C}-\mathrm{C}$ and $\mathrm{B}-\mathrm{B}$ assemblies from materials with equal yield limits and different TCLE, ideal localizing of plastic deformations in the joint zone is achieved, covering both the materials being joined symmetrically relative to the butt joint (Figure 3, $a-d$ ). In B-F assembly at the same yield limits of bushing and flange materials the deformation fields are not symmetrical (Figure 3, $d, f)$. Both at heating and at cooling they are concentrated near the butt joint, to a greater degree from the side of the bushing. Plastic deformations zone from the flange side much smaller. Appearance of plastic deformations reduces the peaks of all the stresses in the concentration point.

At simultaneous force (compression) and thermal (heating-cooling) loading and equal yield limits, plastic deformations increase in all the components and are more uniformly distributed in B-F assemblies. In $\mathrm{C}-\mathrm{C}$ and $\mathrm{B}-\mathrm{B}$ assemblies they appear alternatively in the material with smaller TCLE at heating and material with greater TCLE at cooling. If the yield limits are different, then under the modeling conditions just the material with a lower yield limit and smaller TCLE was deformed at heating.

A feature of the fields and epures of plastic deformation distribution at thermal cycling, both without external pressure, and with pressure is presence of a zone in the butt joint, where equivalent stresses and plastic deformations have minimum value, and tangential stresses and shear deformations pass through zero. It is proposed to call the point with zero tangential stresses and the surrounding zone near it with minimum equivalent stresses and deformations, the point and zone of deformation stagnation. Absence of shear deformations complicates the possibility of activation of the surfaces being joined. In $\mathrm{C}-\mathrm{C}$ assembly this zone is located in its center, in B-B and $\mathrm{B}-\mathrm{F}$ assemblies it is on the inner surface at the distance of approximately $0.23-0.25$ of bushing thickness. Plastic deformations gradually increase from this zone to the inner and outer surface.

Effect of plastic creep deformations was studied to enhance the possibilities for SSS regulation. This was the third stage of SSS modeling (first stage for the elastic state, second stage allowing for instantaneous plasticity deformations). Here, three cycles of heating and cooling by $100{ }^{\circ} \mathrm{C}$ during $60 \mathrm{~s}$ with soaking for $60 \mathrm{~s}$ in each cycle at compressive pressure of $15 \mathrm{MPa}$ were considered. Magnitude and distribution of plastic deformations after each cycle are shown in Figure 4.

During thermal cycling the stresses and plastic deformations vary by their magnitude, but the nature of their distribution along the butt joint remains the same. Material creep increases the value of plastic deformations that promotes formation of physical contact, activation of the surfaces being joined and development of recrystallization and diffusion processes, unlike the traditional VDW scheme, under the conditions of which slowly running diffusion processes have the main role [6].

VDW under the conditions of simultaneous compression and superposition of thermal cycles is more favourable for distribution of plastic deformations, ensuring their localization in the butt zone, more uniform distribution over the joint area and intensive development of the processes of joint formation.

SSS modeling was used at development of the technology of VDW of solenoid valve bodies, in which four butt joints of $12 \mathrm{Kh} 18 \mathrm{~N} 10 \mathrm{~T}$ ( 3 parts) and 10895 (2 parts) steels are welded simultaneously. Welded billet of a valve is shown in Figure 5.

Welding was conducted at total heating by high-frequency currents at three cycles of heating and cooling. As it was established by preliminary research on models, deformation occurred only at heating in the zone of butt 

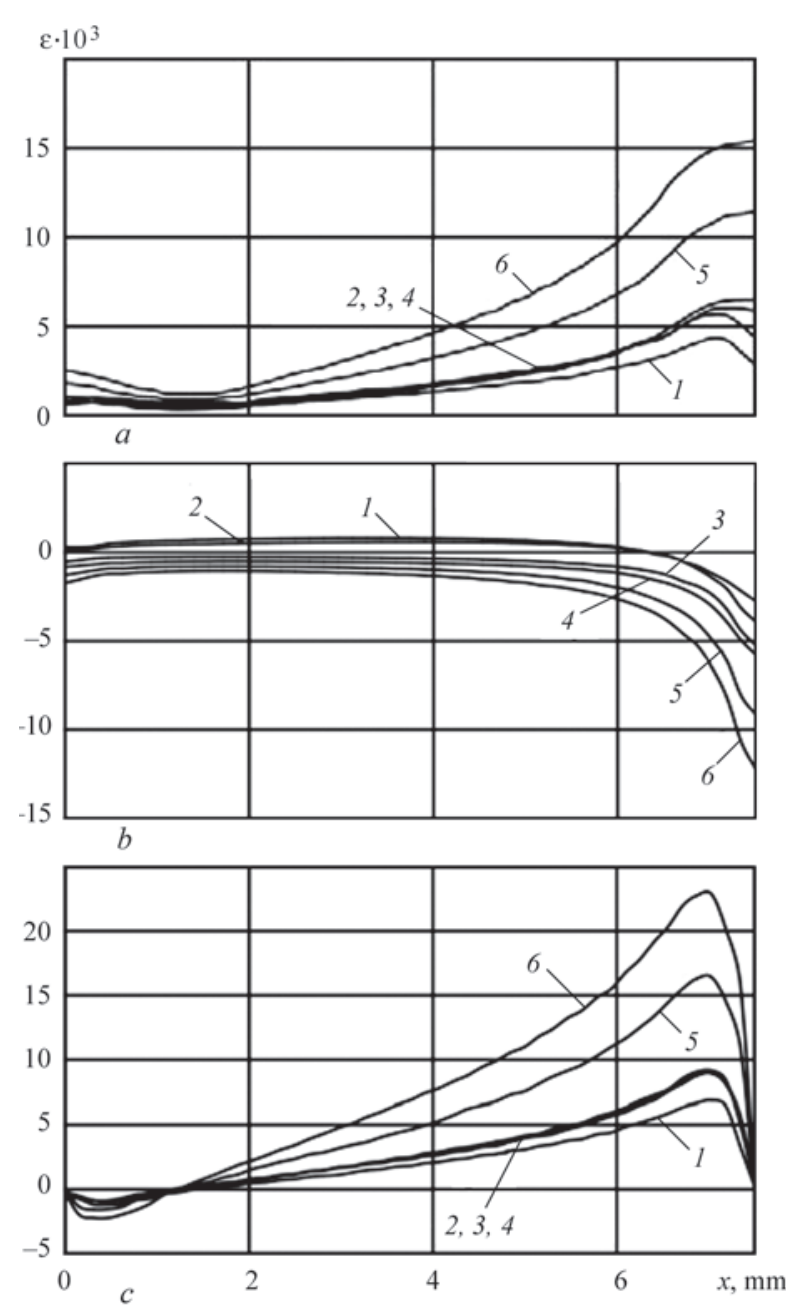

Figure 4. Epures of equivalent $(a)$, axial $(b)$ and shear $(c)$ deformations at VDW 60 (1), 120 (2), 180 (3), 240 (4), 480 (5) and $720 \mathrm{~s}(6)$ after the start of thermal cycling of B-B assembly

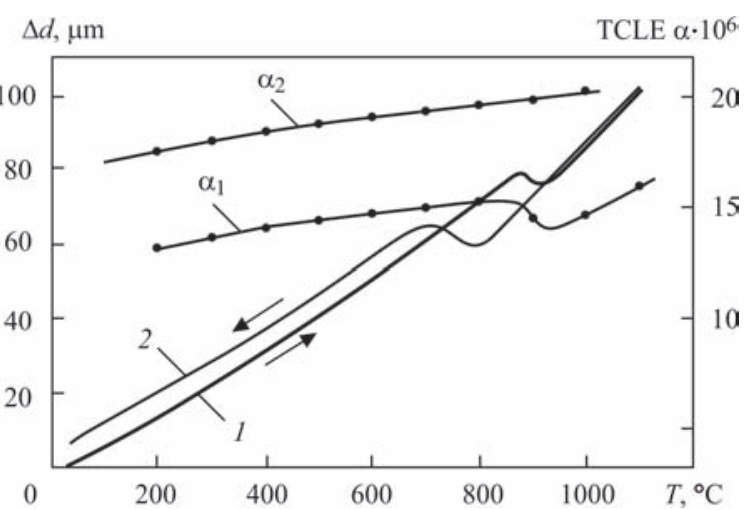

Figure 6. Dilatograms of heating (1) and cooling (2) of 10895 steel and TCLE of the same steel $\left(\alpha_{1}\right)$, and 12Kh18N10T steel $\left(\alpha_{2}\right)$

joint of less strong 10895 steel. Measurements and result analysis were conducted after VDW.

Moduli of elasticity, yield limits, Poisson's ratios, dilatograms of heating and cooling, curves, parameters and equations of creep of the material being joined were determined as a function of temperature, in order to perform SSS modeling.

At heating 10895 steel goes from $\alpha$-phase into $\gamma$-phase with the change of physicomechanical properties (PMP), in particular, with the change of TCLE, that is shown by dilatograms of heating and cooling of 10895 steel, as well as TCLE of the same steel (Figure 6). This allowed using not only temperature, but also structural deformations during thermal cycling.

Dependence of the yield limit on temperature, used at modeling, and creep curves of 10895 steel are given in Figure 7. In the thermal cycling range of $750-1050{ }^{\circ} \mathrm{C}$ the creep rate of $12 \mathrm{Kh} 18 \mathrm{~N} 10 \mathrm{~T}$ steel is
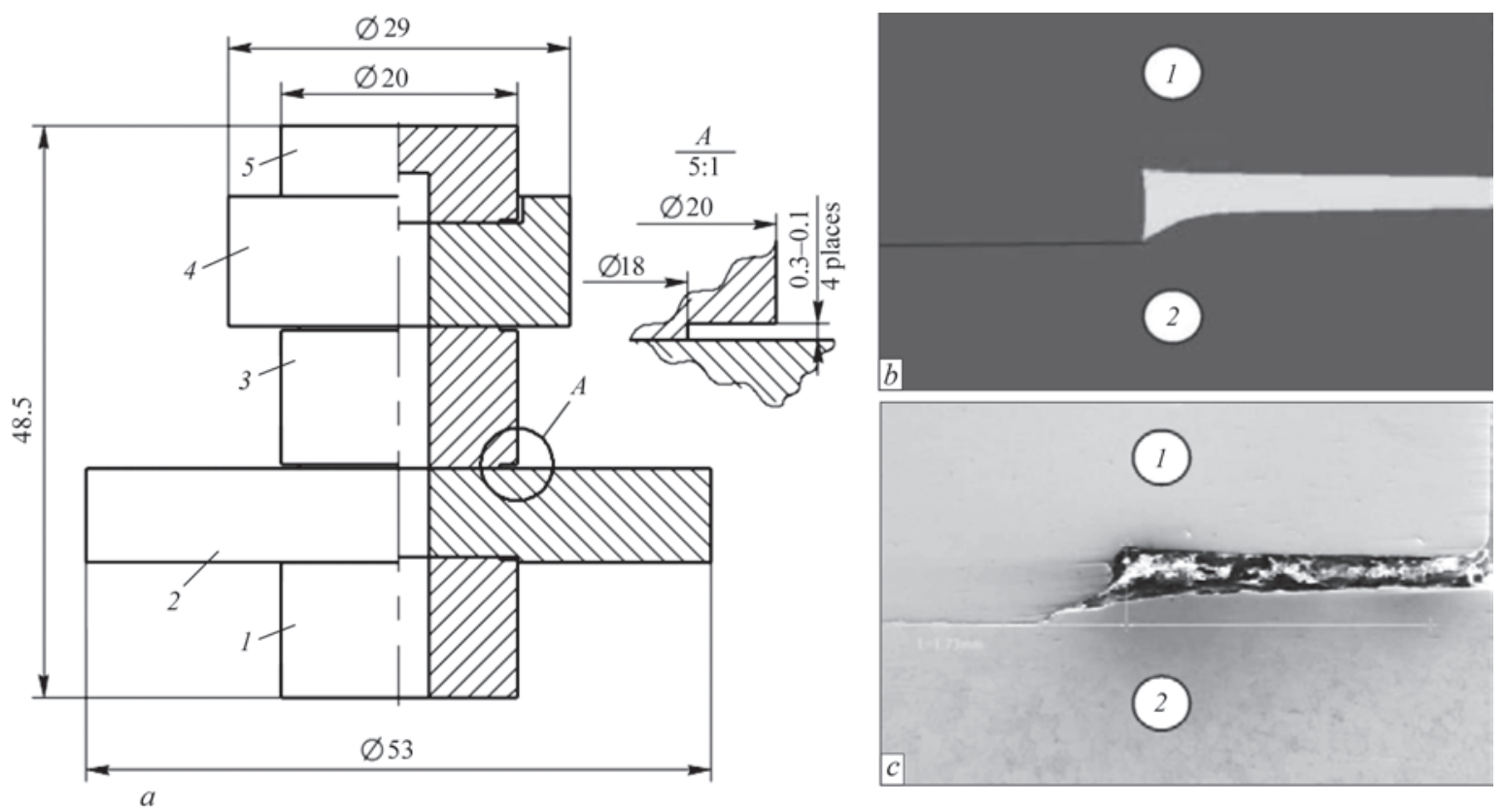

Figure 5. Welded billet of the body of the solenoid valve $(a)$ and nature of billet deformation after VDW in assembly A of the joint of bushing 1 and flange 2 by the results of modeling $(b)$ and actually $(c): 1,3,5-12 \mathrm{Kh} 18 \mathrm{~N} 10 \mathrm{~T}$ steel; 2, $4-10895$ steel 

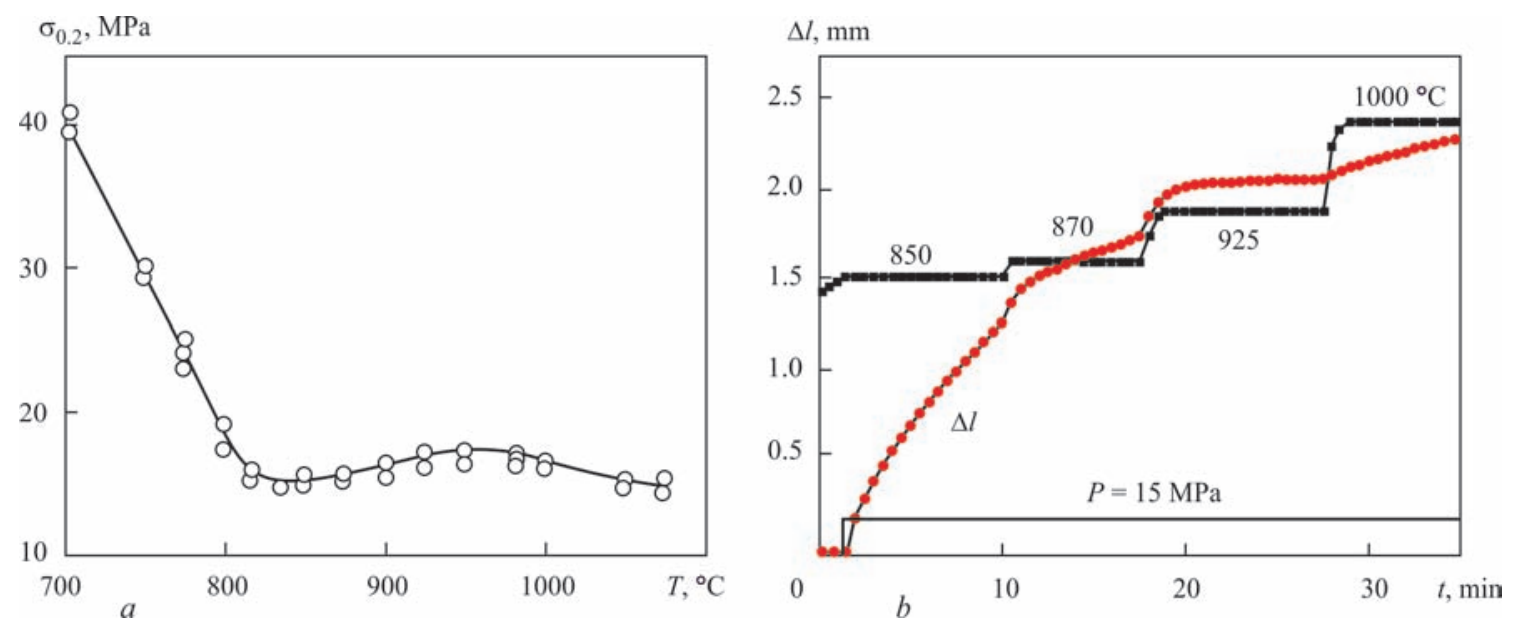

Figure 7. Influence of temperature on the yield limit $(a)$ and cyclogram of creep testing of 10895 steel with the change of sample length $\Delta l$ at temperatures of $850,870,925,1000{ }^{\circ} \mathrm{C}$ and pressure of $15 \mathrm{MPa}(b)$

very small, compared to 10895 steel, and its deformation can be neglected.

Creep parameters of 10895 steel were determined experimentally with application of Gleeble-3800 unit and upgraded unit for vacuum diffusion welding. Results were processed with application of an equation describing creep at the first and second stages:

$$
\varepsilon=C_{1} p^{C_{2}} \frac{t^{C_{3}+1}}{C_{3}+1} \exp \left(-\frac{C_{4}}{T}\right)+C_{5} p^{C_{6}} \exp \left(-\frac{C_{7}}{T}\right),
$$

where $\varepsilon$ is the deformation value; $C_{1}-C_{7}$ are the coefficients determined by experimental creep curves, here $C_{1}>0 ; C_{3}<0 ; C_{5}>0 ; C_{4}=\left(\Delta H_{n(1)} / R\right) ; C_{7}=\left(\Delta H_{n(2)} / R\right)$; $\Delta H_{n(1)}$ and $\Delta H_{n(2)}$ is the creep activation energy at the first stage; $\Delta H_{n(2)}$ is the energy of creep activation at the second stage; $C_{2}$ and $C_{6}$ are the constants for the first and second stages, respectively.

The coefficients are determined from the series of curves, obtained for different temperatures and pressures: $C_{1}=4.99 \cdot 10^{-24} ; C_{2}=4.59-4.65 ; C_{3}=-0.32$ $-0.52 ; C_{4}=32700-39800 ; C_{5}=1.641 \cdot 10^{-25} ; C_{6}=$ $=4.49-4.95 ; C_{7}=32700-39800$.

Comparison of modeling and experimental results confirmed modeling adequacy. For instance, Figure 5, $a$ shows the billet configuration in the zone of the joint of bushing 3 with flange 2 in assembly $A$ (see Figure 5, $b, c)$. The nature of billet deformation at modeling and in the real part is the same, and quantitative values of deformations have satisfactory convergence.

Mechanical testing was also performed for experimental assessment of the effect of thermal cycling on welded joint quality. It is found that in welding without thermal cycling the samples at tensile testing failed at up to $255 \mathrm{MPa}$ stresses. Fracture surface had up to $40 \%$ of «adhesion» area, as well as the shape of a cone, that is indicative of the start of fracture in the sample center. In welding with thermal cycling three thermal cycles are sufficient to ensure equal strength of the joints with 10895 steel (more than $360 \mathrm{MPa}$ ). At impact bend testing cylindrical samples of $15 \mathrm{~mm}$ diameter did not fail at bend angle of $90^{\circ}$.

At testing of samples simulating the item, fracture ran in 10895 steel at stresses of (363-398)/383 MPa (mean value is given in the denominator).

To study the structure of metal in the butt zone, the method of electronic foil translucence at up to 50000 magnifications and local X-ray spectral microanalysis were used for determination of fine structure, diffusion processes and distribution of dislocation density over the butt joint area, which is due to the value and intensity of plastic deformations. Results of these studies are given in works $[1,7,8]$. The width of the diffusion zone even in the center of the cylinder, determined by distribution of chromium, nickel and iron, was equal to about $10 \mu \mathrm{m}$, that is significantly higher than the accepted in literature criterion of strength by the width of the diffusion zone of 3-5 $\mu \mathrm{m}$ [6]. At bench testing, the items failed with multiple margin of normative strength. Results of experimental studies confirmed the effectiveness of using not just the temperature, but also structural deformations for controlling the formation of SSS during welding.

Structural deformations were also used at VDW of dispersion-hardening high-temperature nickel alloys for joining parts, one of which was in the austenitized, and the other - in the aged condition, while thermal cycling was performed in the temperature range of $1000-1175^{\circ} \mathrm{C}$, in which the strengthening phase precipitation and dissolution occurs, and, consequently, change of the specific volume (structural deformations), that promotes development of plastic deformations in the butt joint.

The main problem of development of metal-ceramic assemblies is formation of residual SSS, associated with the difference in PMP of metal and ceramics. Here, the determinant role is played by assembly design 
and processes of relaxation of stresses, arising even in matched junctions in brazing. SSS modeling allowed creating metal-ceramic assemblies without ceramics metallization with application of brazing (Figure 8).

Optimum thickness of kovar, the most critical zones of the joint, influence of deformations of instantaneous plasticity and creep on lowering of the level of residual stresses to the safe one and cooling mode were determined. Similar problems were solved at development of metal-graphite structures $[9,10]$.

In brazing metals to nonmetals, high-temperature nickel alloys or other similar materials, we have a joint with an interlayer, the properties and thickness of which also affect the SSS and performance of the assembly.

It is known that SSS influences mechanical properties of the material, its strength (yield limit), ductility (relative elongation) [11]. To assess this effect on the properties of the materials being joined and the interlayer in the joint zone, it is convenient to use the coefficient of stiffness of the stressed state, $K_{\mathrm{st}}$, equal to the ratio of maximum modulo principal stresses to equivalent ones, $K_{\mathrm{st}}=\sigma_{1(3)} / \sigma_{\mathrm{eq}}$. Value of coefficient $K_{\mathrm{st}}$ determines the degree of softening $\left(K_{\mathrm{st}}<1\right)$ or strengthening $\left(K_{\mathrm{st}}>1\right)$ of the material in a specific zone of the assembly, compared to the linear stressed state, under the conditions of which standard strength testing of materials is performed.

The interlayers are divided into «stiff» and «soft». At the plastic stage of work interlayers with lower yield limit $\sigma_{\text {int lim }}$, than that of the base metal $\left(\sigma_{y \text { int }}<\sigma_{y b . m}\right)$ belong to the soft ones, and those with greater $\sigma_{\mathrm{y} \text { int }}$ $\left(\sigma_{y \text { int }}>\sigma_{y \text { b.m }}\right)$ are regarded as stiff. When working in the elastic stage, the stiffness criteria are the moduli of elasticity: soft at $E_{\mathrm{int}}<E_{\mathrm{b} . \mathrm{m}}$ and stiff at $E_{\mathrm{int}}>E_{\mathrm{b} . \mathrm{m}}$.

Computer modeling of SSS of cylindrical assemblies with interlayers for tension and compression showed that both in the case of stiff and soft interlayer a bulk SSS with radial, hoop, axial and tangential stresses forms in a small zone of their joint with the base metal near the surface in the interlayer and adjacent regions of base metal of the width of about two interlayer thicknesses.

At tension tensile radial, hoop and axial stresses begin acting in the soft interlayer which starts deforming earlier than the base metal, while tensile axial and compressive radial and hoop stresses act in the stronger base metal. Here, the level of equivalent stresses in the interlayer decreases compared to applied load, and rises in the base metal, i.e. effect of unloading or strengthening of the interlayer and additional loading or softening of base metal is manifested [12].

In assemblies with stiff interlayer, axial tension in it is combined with compression in the radial and circumferential directions. Here, equivalent stresses in the
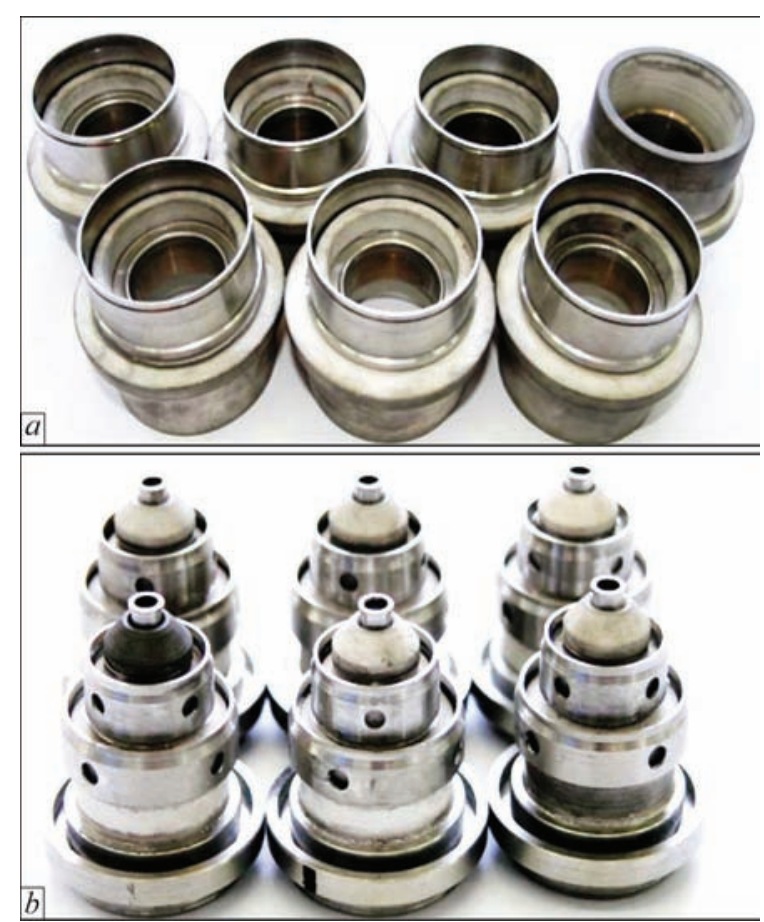

Figure 8. Metal-ceramic assemblies produced by brazing without ceramics metallization

interlayer increase, i.e. effect of additional loading of the interlayer or its softening is manifested [12]. That is why brittle interlayers are not allowed in brazed joints.

Computer modeling showed that at the stage of elastic deformation of the soft interlayer the coefficients of stiffness of the stressed state and strengthening of the soft interlayer and softening of the stronger base metal, respectively, depend only on the ratio of the moduli of elasticity and Poisson's ratios of the base metal and the interlayer, and do not depend on the magnitude of the applied axial load. At the plastic stage they also depend on the degree of interlayer overload, i.e. the ratio of the applied stress to the interlayer material yield limit: $K_{\mathrm{ov}}=\sigma_{\mathrm{l}} / \sigma_{\mathrm{y}}$, where $K_{\mathrm{ov}}$ is the coefficient of interlayer overload, $\sigma_{1}$ is the acting load on the assembly, $\sigma_{y}$ is the interlayer material yield limit.

The uniformity of stress distribution is abruptly violated near the assembly generatrix. The epures of axial stresses along the outer surface in the zone of the butt joint of the interlayer and base metal at different axial loads at the elastic stage are shown in Figure 9, $a$, and at the elastoplastic stage - in Figure 9, $b$.

Axial stresses are nonuniformly distributed near the butt joint. At the elastic stage of the work they vary from 70 up to $120 \mathrm{MPa}$ in the interlayer and from 100 up to $175 \mathrm{MPa}$ in the base metal, and at the elastoplastic stage - from 90 up to $120 \mathrm{MPa}$ in the interlayer and from 140 up to $255 \mathrm{MPa}$ in the base metal. Maximum axial stresses essentially exceed the level of the applied external load and are present in the base metal near the butt joint. As a result, fracture can run in the stronger base metal at the junction with the in- 

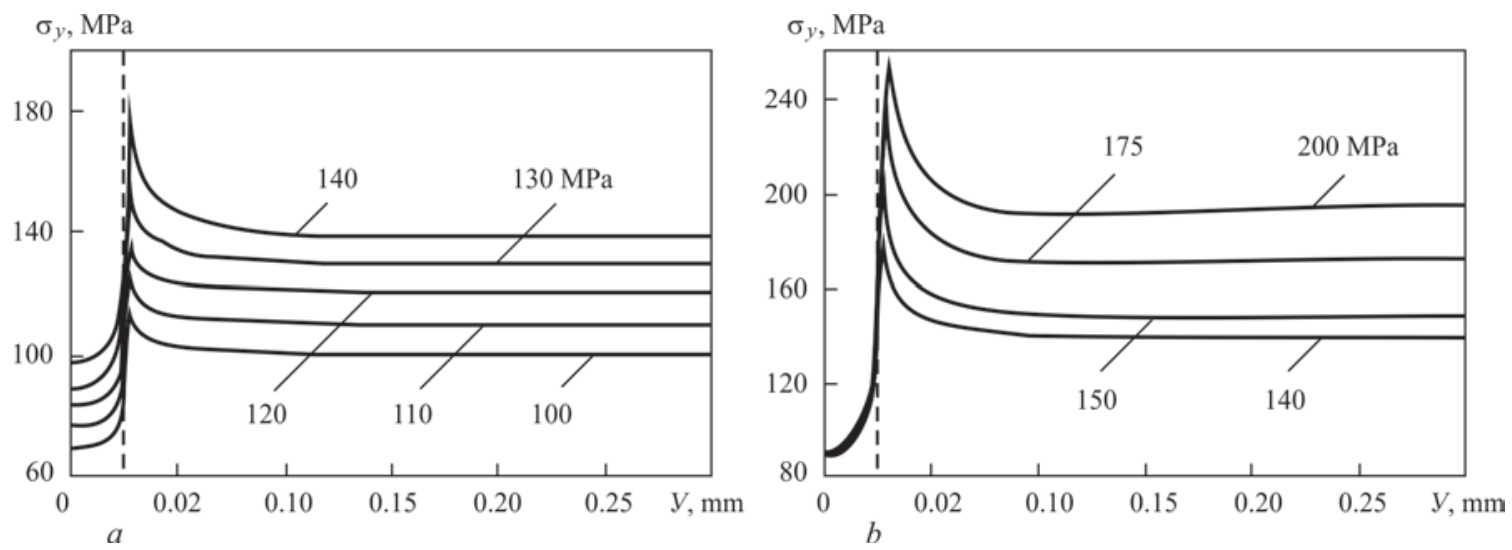

Figure 9. Epures of axial stresses along the generatrix in the zone of the butt joint of the interlayer and base metal at axial loads of $100-150 \mathrm{MPa}(a)$ and $140-200(b)$

terlayer at sufficient strengthening of the soft interlayer and softening of the base metal that is confirmed by the known experiments on brazing armco-iron with copper with about $0.05 \mathrm{~mm}$ thickness of the copper interlayer [2].

\section{Conclusions}

1. At VDW of dissimilar materials with thermal cycling appearance of radial, axial, hoop and tangential stresses leads to formation of bulk stressed state with development of shear deformation that provides localization of plastic deformations in the butt joint zone, activation of the surfaces being joined and their active interaction over the entire area. The nature and level of SSS are to the greatest extent determined by the external compressive pressure, difference in TCLE, temperature variation range and structural deformations. For instance, plastic deformation is localized in the zone of the butt joint at VDW of dispersion-hardened high-temperature nickel alloys, which are in the aged and austenitized states, $\alpha$ - to $\gamma$-phase transitions, etc.

2. At high-temperature brazing of nonmetallized ceramics with kovar deformations of instantaneous plasticity in the high-temperature and creep range at lower temperatures allow decreasing the residual stresses in metal-ceramic assemblies below the ultimate tensile strength of the ceramics. The mode of cooling under pressure promotes stress relaxation.

3. In the joints of similar metals with a soft interlayer, a bulk SSS forms in the butt joint zone at application of axial load. It changes the characteristics of strength and ductility of the metal, obtained at testing under the conditions of linear stressed state that promotes appearance of the effect of interlayer material strengthening and softening of the stronger base metal. Maximum axial stresses develop in the base metal

near the butt joint that, in combination with softening, can lead to joint fracture in the stronger base metal.

1. Krivtsun, I.V., Kvasnytskyi, V.V., Maksymov, S.Yu., Ermolayev, H.V. (2017) Special methods of welding. Ed. by B.E. Paton. Mykolaiv, NUK [in Ukrainian].

2. Ermolayev, H.V., Kvasnytskyi, V.V., Kvasnytskyi,V.F. et al. (2015) Brazing of materials. Ed. by V.F. Khorunov et al. Mykolaiv, NUK [in Ukrainian].

3. Makhnenko, V.I., Kvasnitsky, V.F. (2009) Peculiarities of formation of stress-strain state in diffusion bonds between dissimilar materials. The Paton Welding J., 8, 7-11.

4. Kvasnytskyi, V.V., Ermolayev, H.V., Matvienko, M.V. (2017) Mechanics of joints in diffusion welding, brazing and spraying of dissimilar materials under elasticity conditions. Nikolaev, NUK [in Russian].

5. Kvasnitsky, V.V., Zolotoj, Yu.G., Labartkava, A.B. et al. (2008) Experimental investigation of deformation of welded assembly of bush-bush type from dissimilar materials: Transact. NUK, Mykolaiv, NUK, 4, 65-73 [in Russian].

6. Kazakov, N.F. (1976) Diffusion welding of materials. Moscow, Mashinostroenie [in Russian].

7. Lobanov, L.M., Ermolayev, H.V., Kvasnytskyi, V.V. et al. (2016) Stresses and strains in welding and brazing. Ed. by L.M. Lobanov. Mykolaiv, NUK [in Ukrainian].

8. Kvasnitsky, V.V., Kvasnitsky,V.F., Markashova, L.I., Matvienko, M.V. (2014) Effect of stress-strain state on structure and properties of joints in diffusion welding of dissimilar metals. The Paton Welding J., 8, 8-14.

9. Emelyanov, V.M., Kvasnytskyi, V.V., Ermolayev, H.V. et al. (2009) Optimization of structure of metal-graphite slidebearings on the base of residual stresses analysis in brazed assemblies. Vestnik Khersonsk. NTU, 3, 42-46 [in Russian].

10. Ermolaev, G.B., Martynenko, V.A., Olekseenko, S.V. et al. (2017) Effect of the rigid interlayer thickness on the stressstrain of metal-grafite assemblies under thermal loading. Strength of Materials, May, 49(3) 422-428.

11. Kopelman, L.A. (2010) Principles of theory of strength of welded structures. St.-Petersburg, Lan [in Russian].

12. Kvasnytskyi, V.V., Kvasnytskyi,V.F., Dong Chunlin, Matvienko, M.V. et al. (2018) Stressed state of welded and brazed assemblies from similar materials with a soft interlayer under axial loading. The Paton Welding J., 4, 6-10.

Received 30.03.2018 


\title{
EFFECT OF PLATE THICKNESS ON WELD SPEED IN FRICTION STIR WELDING OF AA6061-T6 AL-ALLOY PLATES
}

\author{
G. İPEKOĞLU ${ }^{1}$, Ö. AKÇAM ${ }^{2}$ and G. ÇAM ${ }^{1}$ \\ ${ }^{1}$ Iskenderun Technical University \\ 31200 Iskenderun-Hatay, Turkey. E-mail: guven.ipekoglu@iste.edu.tr, gurel.cam@iste.edu.tr \\ ${ }^{2}$ GSI SLV-TR Kaynak Teknolojisi Test ve Mesleki Gelişim Merkezi Ltd. Şti., Çiğdem Mah., Gökkuşağı Sitesi, \\ Mavi Blok No:37-38, 06520 Balgat, Çankaya, Ankara, Turkey. E-mail: ozgur.akcam@gsi.com.tr
}

\begin{abstract}
In this study, it was aimed to determine the weld speeds required in order to obtain defect-free joints in AA6061-T6 alloy plates with two different thicknesses (namely 3.0 and $6.2 \mathrm{~mm}$ ) by friction stir welding at a constant rotational rate of $1000 \mathrm{rev} . \mathrm{min}^{-1}$. For this purpose, two different stirring tools (one tool for each plate thickness) have been obtained and used to determine suitable weld speed for each plate thickness. The microstructures of the joints obtained were investigated in both macro- and micro-scale, and their mechanical properties were determined by conducting microhardness measurements and tensile testing. The current study clearly indicated that AA6061-T6 alloy plates with different thicknesses can be successfully FS welded provided that suitable stirring tool and weld parameters are used. Moreover, it was also observed that the joint performance value of the thicker plates was somewhat lower than that of the thinner plates. This can be attributed to higher heat inputs required in order to obtain defect-free joints in thicker plates and lower cooling rates after the joining in the case of thicker plates. 42 Ref., 2 Tables, 7 Figures.
\end{abstract}

Ke yw ords : friction stir welding, AA6061-T6, plate thickness, microstructure, mechanical properties

Introduction. Friction stir welding (FSW) is a novel solid state joining technique used for welding low melting materials, particularly Al-alloys. In this joining method, the heat generated from the resistance of the material to plastic deformation is utilized to produce the joint. The workpieces to be joined are held against each other and a stirring tool rotating at a high rate is plunged into the workpieces and moved along the joint line. The workpieces does not melt but plasticizes by the heat generated from the resistance between the shoulder as well as the surface of the tip of the stirring tool. The plasticized workpieces are mixed together by the stirring tool as it travels along the joint line producing the joint [1-8].

This novel welding technique is at present widely employed for joining Al-alloys in industrial applications [6]. Furthermore, the method is also capable of being used in joining of $\mathrm{Cu}$-alloys [9-11], Mg-alloys [12-14], Pure $\mathrm{Pb}$ [15], and even for higher temperature structural materials such as steels [16-19] and Ti-alloys [4] provided that the limitations on suitable stirring tools that can withstand temperatures above $1000{ }^{\circ} \mathrm{C}$. The problems originating from the melting are not encountered in this joining technique since the workpieces do not melt during welding. These difficulties encountered in fusion joining include porosity formation and cracking in weld zone [20-26]. Moreover, it is desirable to obtain comparable mechanical properties in weld zone of a joint to those of the base plate [1]. However, the loss of strength in the weld region, both in weld nugget in the HAZ, takes place in

(C) G. İPEKOĞLU, Ö. AKÇAM and G. ÇAM, 2018 this joining method due to dissolution and coarsening of strengthening precipitates, respectively. Nevertheless, the loss of strength is much lower in FSW than that occurring in the fusion joining due to the lower heat inputs involved in FSW.

AA6061 Al-alloy exhibits a very good corrosion resistance. Owing to its good corrosion performance coupled with its high strength and light weight, this alloy is widely used in industrial applications. Thus, numerous studies have been conducted on friction stir weldability of these alloys [27-31]. In this study, AA6061-T6 alloy plates with two different thicknesses, namely 3.0 and $6.2 \mathrm{~mm}$ thick, have been friction stir welded using a different stirring tool for each. In these weld trials, the rotational rate (i.e., 1000 rev. $\mathrm{min}^{-1}$ ) chosen from the literature has been employed for both plate thickness. Thus, it was aimed to find out the influence of plate thickness on weld speed at a constant rotational rate of 1000 rev. $\mathrm{min}^{-1}$.

Experimental procedure. AA6061-T6 plates with two different thicknesses, namely 3.0 and $6.2 \mathrm{~mm}$, were used in this study. In order to conduct the weld trials, a welding fixture was designed and produced. This fixture is needed to hold the work pieces to be welded in a fixed position on CNC table throughout the joining process. Figure 1 illustrates the designed welding fixture, which was used in friction stir welding of the plates. Moreover, two FSW stirring tools with different geometries and tip lengths (namely 2.8 and $6.0 \mathrm{~mm}$ ) were purchased and used in the welding of plates, Figure 2. 


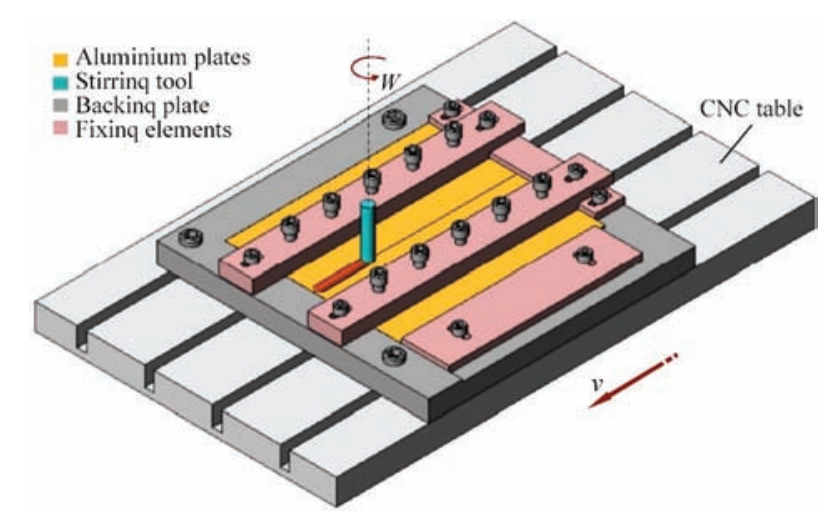

Figure 1. The fixture designed and produced to be used in weld trials and the welding procedure

The rotational rate, i.e. $1000 \mathrm{rev} \cdot \mathrm{min}^{-1}$, used in the FSW of the AA6061-T6 plates in this study is determined based on the reports existing in the literature [32-39]. This rotational rate was chosen as it was reported that defect-free joints produced at this rotational rate by FSW for both AA6061 and AA7075 Al-alloys plates. Furthermore, two different weld speeds were used for each plate thickness, namely 100 and $150 \mathrm{~mm} / \mathrm{min}$ for the plate thickness of $3.0 \mathrm{~mm}$ and 50 and $75 \mathrm{~mm} / \mathrm{min}$ for the plate thickness of $6.2 \mathrm{~mm}$. Thus, it was aimed to determine the optimum traverse speed at the constant rotational rate of $1000 \mathrm{rev} \cdot \mathrm{min}^{-1}$ for each plate thickness. A tilting of stirring tool was used for both plate thickness, i.e., $0,7^{\circ}$ for $3.0 \mathrm{~mm}$ thick plates and $0.5^{\circ}$ for $6.2 \mathrm{~mm}$ thick plates. The weld parameters used were given in detail in Table 1.

A metallography specimen and three tensile test specimens were extracted from each joint produced in order to evaluate microstructural and hardness variations within the weld region and to determine tensile

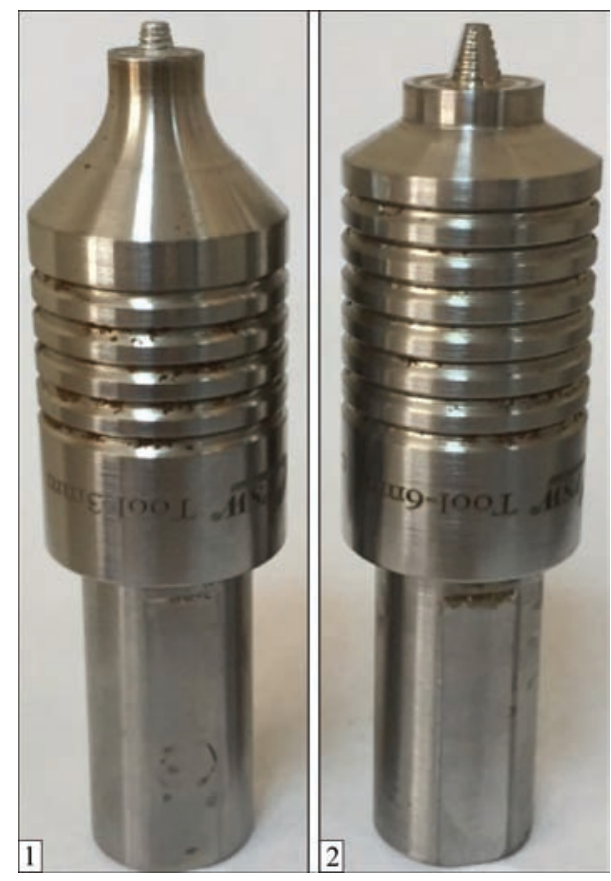

Figure 2. The FSW stirring tools used in this study $(1-$ pin length is $2.8 \mathrm{~mm}$ and shoulder diameter is $10 \mathrm{~mm} ; 2$ - pin length is $6.0 \mathrm{~mm}$ and shoulder diameter is $15 \mathrm{~mm}$ ) [38]
Table 1. The weld parameters used for FSW of AA6061-T6 alloy plates with two different thicknesses

\begin{tabular}{|c|c|c|c|c|}
\hline $\begin{array}{l}\text { Plate thick- } \\
\text { ness } t \text {, mm }\end{array}$ & $\begin{array}{c}\text { Shoulder } \\
\text { diameter } \\
d, \mathrm{~mm}\end{array}$ & $\begin{array}{l}\text { Rotational } \\
\text { rate, } \\
\text { rev. } \text { min }^{-1}\end{array}$ & $\begin{array}{l}\text { Weld speed, } \\
\mathrm{mm} / \mathrm{min}\end{array}$ & $\begin{array}{c}\text { Tool tilting, } \\
\text { deg }\end{array}$ \\
\hline \multirow{2}{*}{3.0} & \multirow{2}{*}{10} & \multirow{4}{*}{1000} & 100 & \multirow{2}{*}{0,7} \\
\hline & & & 150 & \\
\hline \multirow{2}{*}{6.2} & \multirow{2}{*}{15} & & 50 & \multirow{2}{*}{0,5} \\
\hline & & & 75 & \\
\hline
\end{tabular}

properties, i.e. weld performance values. Metallography specimens were ground and polished and then etched for $120 \mathrm{~s}$ using Keller's reagent for microstructural investigations and microhardness measurements. A detailed optical microscopy was conducted along the cross-sections of the joints produced in order to evaluate the microstructural alterations taking place within the weld regions. Several macro- and micrographs were taken from different regions of each joint and used to correlate the microstructure and the mechanical properties of the joints. Thus, it was aimed to determine the effect of weld speed on the microstructure and in turn on the mechanical behavior of the joints. Vickers microhardness measurements were conducted across the joint cross-sections using a load of $200 \mathrm{~g}$, loading time being $10 \mathrm{~s}$. The geometry of the tensile specimens tested was given in a previous publication [32]. All tensile tests were conducted using a loading rate of $0,1 \mathrm{~mm} / \mathrm{min}$.

Results and discussion. Microstructural aspects. Macrographs taken from the welded joints produced (3.0 and $6.2 \mathrm{~mm}$ thick plates) are given in Figures 3, 4, respectively. As seen from Figure 3, both joints produced on $3.0 \mathrm{~mm}$ thick plates using different weld speeds, namely 100 and $150 \mathrm{~mm} / \mathrm{min}$, did not exhibit any weld defects, such as porosity or tunnel-like void. Similarly, all the joints produced on $6.2 \mathrm{~mm}$ thick plates with different weld speeds (i.e., 50 and $75 \mathrm{~mm} /$ min) also did not display any weld defects, Figure 4. These results indicate that defect-free joints were successfully produced for both plates with different thickness using suitable tools and weld parameters.

Moreover, it was also clearly observed that the weld regions of the joints obtained on thicker plates were wider than those exhibited by the joints produced on thinner plates. These results were not surprising since the shoulder and pin diameters of the stirring tool used in FSW of thicker plates are larger. These results also indicate that the rotational rate of 1000 rev. $\mathrm{min}^{-1}$ can be used for both plate thicknesses.

The microstructures of both AA6061-T6 base plates with two different thicknesses (i.e., 3.0 and $6.2 \mathrm{~mm}$ ) used in this study are shown in Figures 5, $a$ and 6, $a$, respectively. As seen from these micrographs, both base plates exhibit a microstructure consisting of alpha grains containing randomly distributed large particles which are readily visible in optical microscopy. As reported in earlier publications $[33,34]$, the particles randomly ori- 


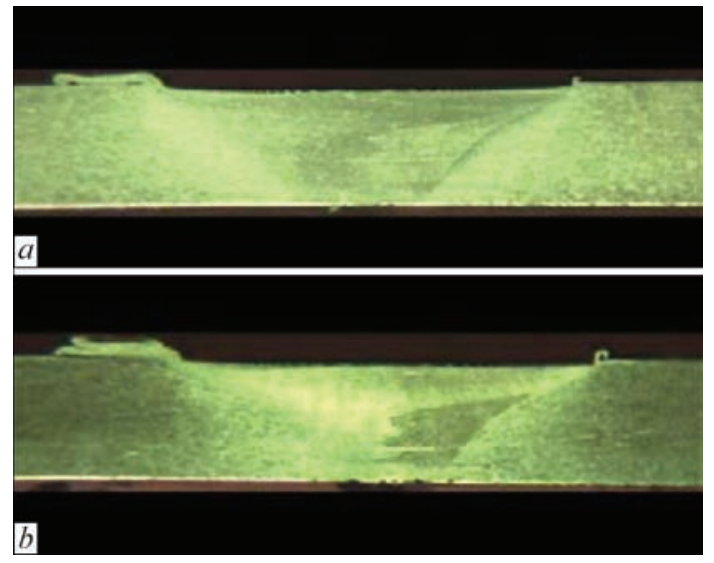

Figure 3. Macrographs taken from the FSWed joints produced on $3,0 \mathrm{~mm}$ thick plates using a rotational rate of $1000 \mathrm{rev} . \mathrm{min}^{-1}$ : $a-100 \mathrm{~mm} / \mathrm{min} ; b-150 \mathrm{~mm} / \mathrm{min}$

ented in alpha matrix are script-like $\mathrm{Fe}_{3} \mathrm{SiAl}_{12}$ particles and round $\mathrm{Mg}_{2} \mathrm{Si}$ particles. Alpha grains existing in the microstructures of both base plates are coarse-grained and no clear difference was observed in the alpha grain size between the microstructures of the plates with different plate thicknesses. The microstructure of AA6061 alloy also contains very fine grained $\mathrm{Mg}_{2} \mathrm{Si}$ precipitates homogeneously distributed within the alpha grains, which result in strengthening in T6 temper condition of this alloy. However, these strengthening precipitates are extremely fine, so that they are not visible under optical microscope, and even in scanning electron microscopy.

As clearly seen from Figure 5, grain refinement took place within the dynamically recrystallized zones (DXZs) of the joints produced on $3.0 \mathrm{~mm}$ thick AA6061-T6 plates using two different weld speeds, namely 100 and $150 \mathrm{~mm} / \mathrm{min}$. Moreover, it was also observed that the joint obtained using slower weld speed (i.e., $100 \mathrm{~mm} / \mathrm{min}$ ) exhibited coarser grains in the microstructure of the DXZ (Figure 5,b) than those of the joint produced at higher weld speed (i.e., $150 \mathrm{~mm} /$ $\mathrm{min}$ ), Figure 5, c. This indicates that the extent of grain refinement was lower in the joint obtained at slower weld speed, which is attributed to the higher heat input involved in this case leading to grain coarsening.

Similar results were also observed in the joints obtained on thicker plates, i.e. $6.2 \mathrm{~mm}$, using two different weld speeds (namely 50 and $75 \mathrm{~mm} / \mathrm{min}$ ), Figure 6. Grain refinement also occurred within the DXZs of these joints as well and finer alpha grains were observed in the microstructures of the DXZs than those in the microstructure of the base plate. When the grain sizes of the microstructures of these joints are compared it can be seen that the joint produced at higher welding speed displayed finer grains than those of the joint obtained at lower weld speed, Figure $6, b, c$. This result is again attributed to the lower heat input involved in the case of higher weld speed resulting in finer grains within the DXZ.

Furthermore, the extent of the grain refinement taking place in the DXZs of the joints produced on thin-

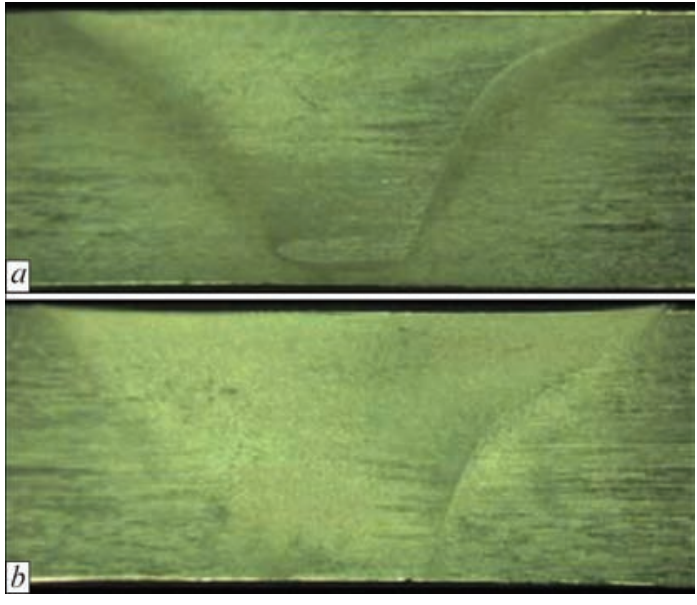

Figure 4. Macrographs taken from the FSWed joints produced on $6.2 \mathrm{~mm}$ thick plates using a rotational rate of $1000 \mathrm{rev} . \mathrm{min}^{-1}$ : $a-50 \mathrm{~mm} / \mathrm{min} ; b-75 \mathrm{~mm} / \mathrm{min}$

ner plates (i.e., $3.0 \mathrm{~mm}$ ) is higher than that occurring in the DXZ of the joints obtained on thicker plates (i.e., $6.2 \mathrm{~mm}$ ). In other words, the grains in the DXZs of the thinner joints are finer than those in DXZs of thicker joints, Figures 5, 6. This is believed to be due to the fact that heat input involved in FSW of thinner plates are in general much lower than those involved in FSW of thicker plates resulting from the use of larger stirring

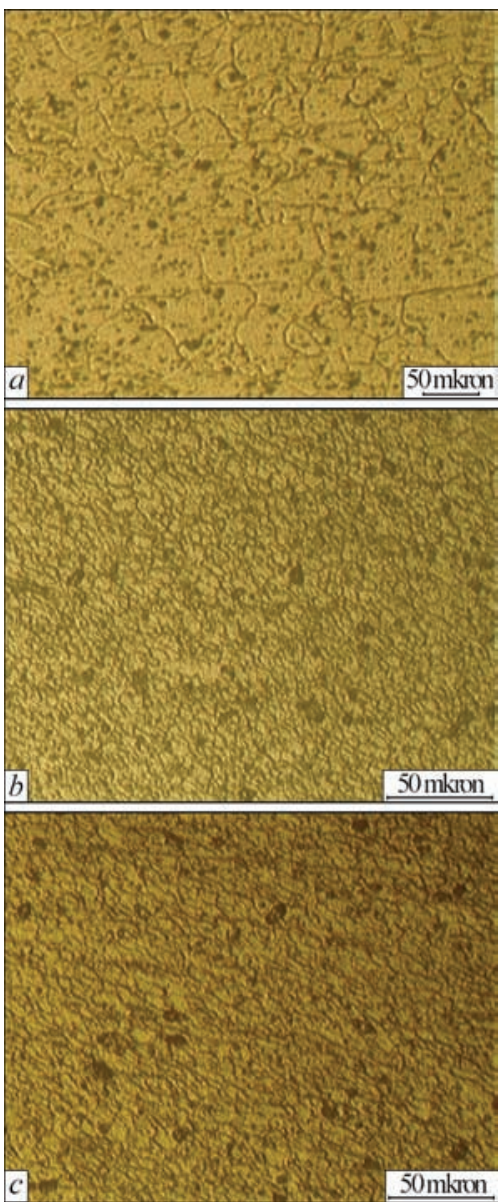

Figure 5. Optical micrographs of $3.0 \mathrm{~mm}$ thick base plate and DXZs of the joints produced on this plate: $a-$ base plate; $b-$ DXZ of the joint produced using a weld speed of $100 \mathrm{~mm} / \mathrm{min}$; $c-\mathrm{DXZ}$ of the joint produced using a weld speed of $150 \mathrm{~mm} / \mathrm{min}$ 
tools for thicker plates, as well as lower cooling rates involved in thicker plates after the welding.

Mechanical properties. Vickers microhardness measurements $\left(H V_{0.2}\right)$ were taken across the weld cross-section from numerous locations on a line in the mid-thickness of the welded joint using a load of 200 $\mathrm{g}$ in order to determine the hardness profiles of all the joints produced in this study. Figure 7 illustrates the obtained hardness profiles from both joints with different plate thicknesses, namely 3.0 and $6.2 \mathrm{~mm}$, produced using different weld speeds. These hardness profiles clearly indicate that a hardness loss took place in all the joints. On the other hand, metallographic investigations displayed that grain refinement occurred within

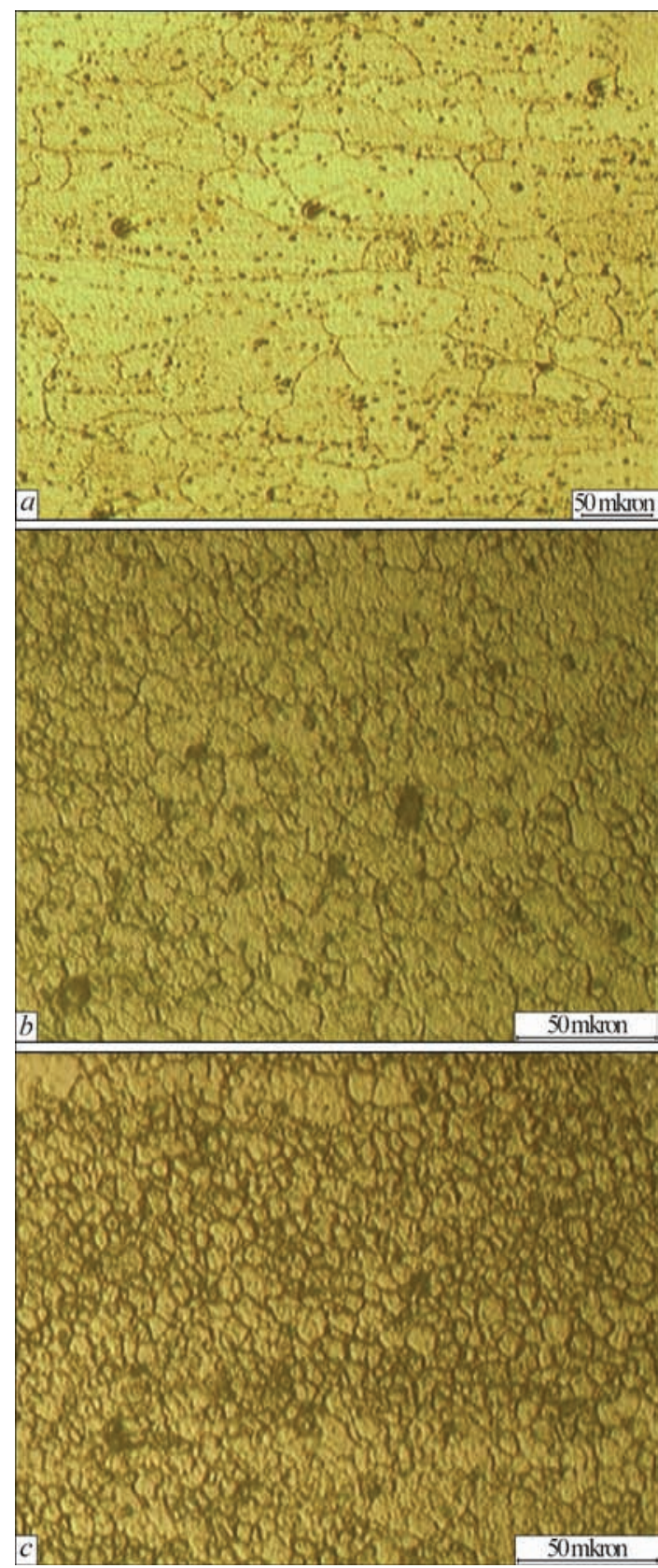

Figure 6. Optical micrographs of $6.2 \mathrm{~mm}$ thick base plate and DXZs of the joints produced on this plate: $a$ - base plate; $b-$ DXZ of the joint produced using a weld speed of $50 \mathrm{~mm} / \mathrm{min}$ $c-\mathrm{DXZ}$ of the joint produced using a weld speed of $75 \mathrm{~mm} / \mathrm{min}$ the weld nugget of all the joints, Figures 5, 6. It was expected that this grain refinement would increase the hardness in the weld region. However, the base plate used in this study is in the artificially aged temper condition (i.e., T6) and the strength in this temper condition originates mainly from very fine homogeneously distributed strengthening precipitates within the alpha grains. When this alloy is exposed to heat after aging, such as welding, dissolution or coarsening of the strengthening precipitates takes place depending on the level of temperature involved, thus resulting in a loss in hardness. This hardness loss occurs both in heat affected zone (HAZ) and in the weld nugget. The reason of the hardness loss in the weld nugget is the dissolution of the strengthening precipitates whereas the hardness loss in the HAZ is due to the coarsening of the precipitates as a result of overaging [40].

The hardness minimum lies within the overaged HAZ regions on both sides of the weld nugget since hardness loss in the weld nugget is partly recovered by the grain refinement taking place there, giving rise to a $\mathrm{W}$-shaped hardness profile (Figure 7). This type of hardness profile is very typical of this alloy as the case in fusion welding [5, 6, 20, 22, 23]. Moreover, a less significant hardness loss in the weld region was displayed by the joints produced at higher weld speeds than those obtained at lower speeds for both plate thickness as seen from Figure 7. This is attributed to lower heat inputs involved in the welds conducted at higher weld speeds.

It was also observed that the joints produced on $6.2 \mathrm{~mm}$ thick plates exhibited a more significant hardness loss within the weld region than the joints obtained on thinner plates, particularly the joint procuded using a weld speed of $50 \mathrm{~mm} / \mathrm{min}$, Figure $7, b$. The lowest hard-
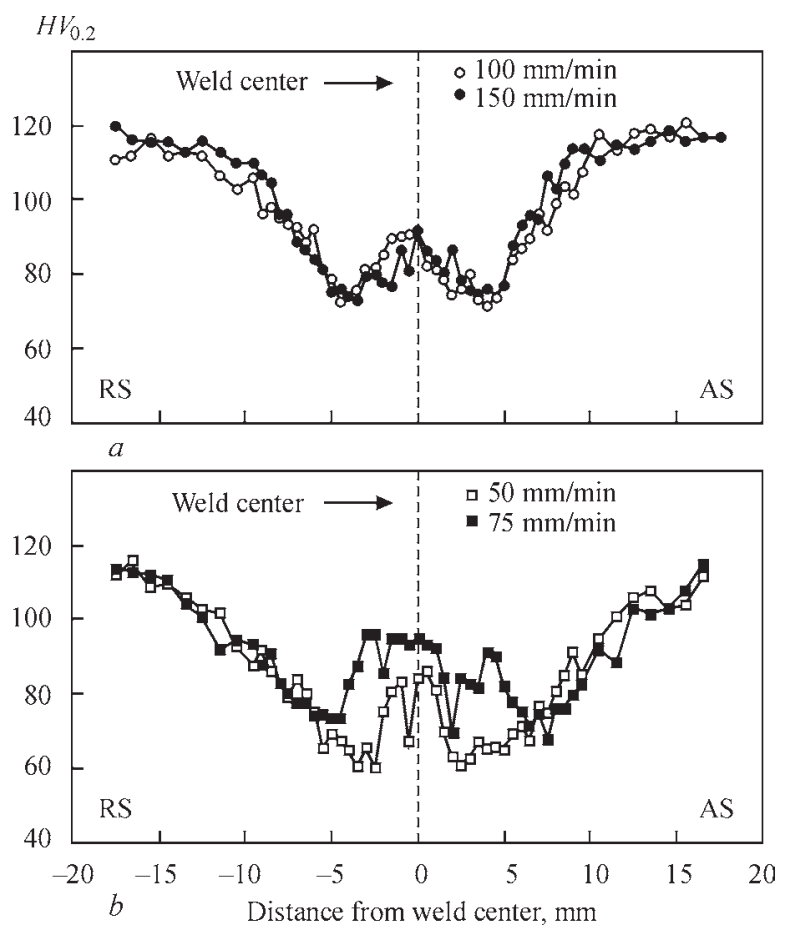

Figure 7. Hardness profiles of the joints produced: $a-3.0 \mathrm{~mm}$ thick joint; $b-6.2 \mathrm{~mm}$ thick joint 
Table 2. Tensile test results

\begin{tabular}{|c|c|c|c|c|c|c|c|}
\hline Specimen & $\begin{array}{l}\text { Weld speed, } \\
\mathrm{mm} / \mathrm{min}\end{array}$ & $\begin{array}{l}\% 0.2 \text { proof } \\
\text { stress, } \mathrm{MPa}\end{array}$ & $\begin{array}{c}\text { Tensile strength, } \\
\mathrm{MPa}\end{array}$ & Elongation, \% & $\begin{array}{c}\text { Proof stress } \\
\text { performance, } \\
\%\end{array}$ & $\begin{array}{c}\text { Tensile strength } \\
\text { performance, } \\
\%\end{array}$ & $\begin{array}{c}\text { Elongation } \\
\text { performance, } \\
\%\end{array}$ \\
\hline $\mathrm{BM}$ & - & $276^{*}$ & $310^{*}$ & $12 *$ & - & - & - \\
\hline \multirow{2}{*}{$\begin{array}{l}\text { FSWed joint } \\
\quad(3.0 \mathrm{~mm})\end{array}$} & 100 & $\begin{array}{l}170 ; 161 ; \\
162(164)\end{array}$ & $\begin{array}{l}220 ; 220 ; \\
221(220)\end{array}$ & $\begin{array}{l}4.2 ; 4.3 ; \\
4.1(4.2)\end{array}$ & 59 & 71 & 35 \\
\hline & 150 & $\begin{array}{l}178 ; 183 ; \\
181(181)\end{array}$ & $\begin{array}{l}224 ; 233 ; \\
230(229)\end{array}$ & $\begin{array}{l}4.5 ; 4.2 ; \\
4.4(4.4)\end{array}$ & 66 & 74 & 37 \\
\hline \multirow{2}{*}{$\begin{array}{l}\text { FSWed joint } \\
\quad(6.2 \mathrm{~mm})\end{array}$} & 50 & $\begin{array}{l}140 ; 146 ; \\
147(144)\end{array}$ & $\begin{array}{l}200 ; 211 ; \\
210(207)\end{array}$ & $\begin{array}{l}3.9 ; 4.1 ; \\
4.1(4.0)\end{array}$ & 52 & 67 & 33 \\
\hline & 75 & $\begin{array}{l}148 ; 148 ; \\
150(149)\end{array}$ & $\begin{array}{l}211 ; 210 ; \\
212(211)\end{array}$ & $\begin{array}{l}4.9 ; 5.3 ; \\
4.6(4.9) \\
\end{array}$ & 54 & 68 & 41 \\
\hline
\end{tabular}

ness observed within the weld region of the $6.2 \mathrm{~mm}$ thick joint produced at a welding speed of $50 \mathrm{~mm} / \mathrm{min}$ was about $60 \mathrm{HV}$, while the minimum hardness in the $3.0 \mathrm{~mm}$ thick joints was $72 \mathrm{HV}$. This result is in good agreement with the metallographic invsetigations which indicated a more significant grain refinement within the weld nugget of the $3,0 \mathrm{~mm}$ thick joints than the joints produced on thicker plates, Figures 5, 6. As discussed in the microstructural aspects section earlier, the reason for this is the higher shoulder and tip dimeter of the stirring tool used for joining thicker plates, thus leading to higher heat input during welding, as well as lower cooling rates involved in the thicker plates after joining. Moreover, the witdh of the weld region where a hardness loss occurred is larger in the case of the thick plate joints (approximately $15 \mathrm{~mm}$ wide, Figure $7, b$ ) than thin plate joints (being about $10 \mathrm{~mm}$ wide, Figure 7, a). This result is also in good agreement with the macrographs taken from the joints, Figures 3, 4.

The tensile test results of the joints are summarized in Table 2. Three tensile test were conducted for each joint and the average of these three test results were calculated, and the average values calculated are given in Table in bold and parenthesis. These average values were used in the calculations of the joint performance values. Three joint performance values, namely proof stress performance, tensile strength performance and ductility performance, were determined for all the joints produced. The minimum tensile properties were taken from the tensile data existing in the literature for AA6061-T6 plates and used in the calculation of performance values. Three performance values were determined as explained below:

- proof stress performance $(\%)=(\% 0.2$ proof stress of welded joint $/ \% 0.2$ proof stress of base plate) $\times 100$;

- tensile strength performance $(\%)=($ tensile strength of welded joint/tensile strength of base plate) $\times 100$;

- elongation performance $(\%)=(\%$ elongation of welded joint $/ \%$ elongation of base plate $) \times 100$.

As seen from Table 2, high weld performance values (i.e., proof stress and tensile strength performance values) have been obtained from all the joints produced on both plate thicknesses at a rotational rate of
1000 rev. min $^{-1}$ using tools with different geometries (Figure 2) and two different weld speeds. However, the joint produced with higher weld speed at each plate thickness exhibited higher weld strength performance values. The maximum proof stress and tensile strength performance values obtained from the specimens extracted from the $3.0 \mathrm{~mm}$ thick joints were displayed by the joint produced at a welding speed of $150 \mathrm{~mm} / \mathrm{min}$, which are 66 and $74 \%$, respectively.

On the other hand, the maximum strength performance values were exhibited by the $6.2 \mathrm{~mm}$ thick joints produced at a welding speed of $75 \mathrm{~mm} / \mathrm{min}$, which are 54 and $68 \%$, respectively. These values are lower than those obtained from the $3.0 \mathrm{~mm}$ thick joints. These results are in good agreement with the hardness profiles of the joints, Figure 7. As seen from Figure 7, the hardness loss in the weld regions of the $6.2 \mathrm{~mm}$ thick joints were more significant than those observed in the $3.0 \mathrm{~mm}$ thick joints. Moreover, the ductility performance values exhibited by all the joints are relatively low, ranging from 33 to $41 \%$. This can be attributed to the strength undermatching in the weld region. Since the strength is much lower within the weld region than that of the base plate the elongation takes place only within the weld region section of the transverse tensile test specimens and the base plate sections do not yield (it is in the elastic region throughout the test) and thus do not contribute to the total elongation. In the case of strength undermatching joints, the stress concentration and, thus, fracture take place in the lower strength weld metal region (confined plasticity), leading to an increase of constraint within the weld region and, thus, significantly lower ductility levels. This confined plasticity is also quite common in fusion welded or diffusion bonded joints with a strength undermatching weld region [20-26, 41, 42].

\section{Conclusions}

In this study, AA6061-T6 Al-alloy plates with two different thicknesses, i.e., 3.0 and $6.2 \mathrm{~mm}$, have been successfully friction stir welded at a rotation rate of $1000 \mathrm{rev} . \mathrm{min}^{-1}$. The following conclusions have been withdrawn from this experimental work: 
- it was observed that the tool geometry plays an important role on the heat input the workpieces experience during FSW. The shoulder and tip diameters of the tool used for FSW thicker plates are larger. Thus, the total surface area of the tool is larger which in turn results in higher frictional heat. As a result of this, a higher heat input is applied to the workpieces to be welded, leading to wider weld width in the thicker joints;

- a grain refinement was observed in the weld nuggets of all the joints produced due to dynamic recrystallization. The grains in the weld nugget are finer in general in thinner joints than those of the thicker joints due to the lower heat input involved in FSW of thinner plates. For each plate thickness, the joint produced using higher weld speed exhibited finer grains due the lower heat input involved at higher weld speeds;

- a more significant hardness decrease took place within the weld regions of the thicker joints. This can also be attributed to the higher heat inputs involved;

- due to the lower heat involved, the specimens extracted from the thinner joints displayed in general higher proof stress and tensile strength performances;

- on the other hand, ductility performances of all the joints are low, the maximum value being $41 \%$. This is not surprising since lower ductility levels are obtained from the transverse tensile specimens extracted from the joints with strength undermatching weld zone due to confined plasticity.

1. Thomas, W.M., Nicholas, E.D., Needham, J.C., Murch, M.G., Temple-Smith, P., and Dawes, C.J., December 1991. International Patent Application No. PCT/GB92/02203

2. Mishra, R.S., Ma, Z.Y. (2005) Materials Science and Engineering. Reports, 50, 1-78.

3. Nandan, R., DebRoy, T., Bhadeshia, H.K.D.H. (2008) Progress in Materials Science, 53(6), 980-1023.

4. Çam, G. (2011) International Materials Reviews, 56, 1-48.

5. Çam, G., Mistıkoğlu, S. (2014) Journal of Materials Engineering and Performance, 23(6), 1936-1953.

6. Çam, G., İpekoğlu, G. (2017) The International Journal of Advanced Manufacturing Technology, 91(5-8), 1851-1866.

7. Çam, G., İpekoğlu, G., Küçükömeroğlu, T., Aktarer, S.M. (2017) Journal of Achievements in Materials and Manufacturing Engineering, 80(2), 65-85.

8. Kashaev, N., Ventzke, V., Çam, G. (2018) To be published in J. Manuf. Proc.

9. Çam, G., Mistikoglu, S., Pakdil, M. (2009) Welding Journal, 88(11), 225-232.

10. Çam, G., Serindağ, H.T., Çakan, A. et al. (2008) Materialwissenschaft und Werkstofftechnik, 39(6), 394-399.

11. Küçükömeroğlu, T., Şentürk, E., Kara, L. et al. (2016) Journal of Materials Engineering and Performance, 25(1), 320-326.

12. Yang, J., Xiao, B.L., Wang, D., Ma, Z.Y. (2010) Materials Science and Engineering A, A527(3), 708-714.

13. Zhang, D., Suzuki, M., Maruyama, K. (2005) Scripta Materialia, 52(9), 899-903.

14. Lim, S.G., Kim, S.S., Lee, C.G. et al. (2005) Metallurgical and Materials Transactions A, 36A(6), 1609-1612.

15. Günen, A., Kanca, E., Demir, M. et al. (2018) Indian Journal of Engineering \& Materials Sciences, 25(1), 26-32.
16. Thomas, W.M., Threadgill, P.L., Nicholas, E.D. (1999) Science and Technology of Welding and Joining, 4(6), 365-372.

17. Ueji, R., Fujii, H., Cui, L. et al. (2006) Materials Science and Engineering A, A423, 324-330.

18. Miyazawa, T., İwamoto, Y., Maruko, T., Fujii, H. (2012) Science and Technology of Welding and Joining, 17(3), 213-218.

19. Küçükömeroğlu, T., Aktarer, S.M., İpekoğlu, G., Çam, G. (2018) To be published in International Journal of Minerals, Metallurgy and Materials.

20. Pakdil, M., Çam, G., Koçak, M., Erim, S. (2011) Mater. Sci. Eng. A, 528(24), 7350-7356

21. Çam, G. Koçak, M.J. (2007) Mater. Sci., 42(17), 7154-7161.

22. Çam, G., Ventzke, V., dos Santos, J.F. et al. (1999) Technol. Weld. Join., 4(5), 317-323.

23. Çam, G., Ventzke, V., dos Santos, J.F. et al. (1999) Prakt. Metallogr., 36(2), 59-89.

24. Çam, G., Güçlüer, S., Çakan, A., Serindağ, H.T. (2009) Mat.Wiss. u. Werkstofftech., 40(8), 638-642.

25. dos Santos, J., Çam, G., Torster, F. et al. (2000) Welding in the World, 44(6), 42-64.

26. von Strombeck, A., Çam, G., dos Santos et al. (2001) A comparison between microstructure, properties, and toughness behavior of power beam and friction stir welds in Al-alloys. In: Proc. of the TMS 2001 Annual Meeting Aluminum, Automotive and Joining (New Orleans, Louisiana, USA, February 12-14, 2001), eds: S.K. Das, J.G. Kaufman, and T.J. Lienert, pub. TMS, Warrendale, PA, USA, 249-264.

27. Trueba, L., Heredia, G., Rybicki, D., Johannes L.B. (2015) Journal of Materials Processing Technology, 219, 271-277.

28. Cao, J.Y., Wang, M., Kong, L. et al. (2017) Materials Characterization, 128, 54-62.

29. Liu, H.J., Hou, J.C., Guo H. (2013) Materials \& Design, 50, 872-878.

30. Elangovan, K., Balasubramanian, V. (2008) Materials Characterization, 59(9), 1168-1177.

31. Feng, A.H., Chen, D.L., Ma, Z.Y. (2010) Metallurgical and Materials Transact. A, 41A, 2626-2641.

32. İpekoğlu, G., Kıral, B.G., Erim, S., Çam, G. (2012) Materiali in Tehnologije, 46(6), 627-632.

33. İpekoğlu, G., Erim, S., Kıral, B.G., Çam, G. (2013) Kovove Materialy, 51(3), 155-163.

34. İpekoğlu, G., Erim, S., Çam, G. (2014) Metallurgical and Materials Transact. A, 45A(2), 864-877.

35. İpekoğlu, G., Erim, S., Çam, G. (2014) The International Journal of Advanced Manufacturing Technology, 70(1), 201213.

36. İpekoğlu, G., Çam, G. (2014) Metallurgical and Materials Transactions A, 45A(7), 3074-3087.

37. Çam, G., İpekoğlu, G., Serindağ, H.T. (2014) Science and Technology of Welding and Joining, 19(8), 715-720.

38. İpekoğlu, G., Akçam, Ö., Çam, G. (2017) Effect of plate thickness on weld speed in friction stir welding of AA6061-T6 Al-alloys plates. In: Proc. of the 10th Welding Technology National Congress and Exhibition (17-18 November 2017, Ankara, Turkey), 63-75 [in Turkish].

39. Woo, W., Choo, H., Brown, D.W., Feng, Z. (2007) Metallurgical and Materials Transact. A, 38A, 69-76.

40. İpekoglu G., Akçam Ö., Çam G. (2018) Afyon Kocatepe University Journal of Science and Engineering, 18(1), 015901, 324-335 [in Turkish].

41. Koçak, M., Pakdil, M., Çam, G. (2002) Sci. Technol. Weld. Join., 7(4), 187-196.

42. Çam, G., Koçak, M., Dobi, D. et al. (1997) Ibid., 2(3), 95-101 


\title{
PROSPECTS OF DEVELOPMENT OF WELDED SINGLE-CRYSTAL STRUCTURES OF HEAT-TEMPERATURE NICKEL ALLOYS
}

\author{
K.A. YUSHCHENKO, B.A. ZADERY, I.S. GAKH and A.V. ZVYAGINTSEVA \\ E.O. Paton Electric Welding Institute of the NAS of Ukraine \\ 11 Kazimir Malevich Str., 03150, Kyiv, Ukraine. E-mail: office@paton.kiev.ua
}

\begin{abstract}
Heat-temperature nickel alloys with single-crystal structure are used in such branches of production as turbine manufacture, aerospace engineering and power engineering. However, their further mastering is restrained by complexity, and sometimes impossibility of production of structure elements of large sizes and developed geometry. Production as well as repair using traditional methods of single-crystal products with developed geometry such as, for example, long or cooled gas turbine blades etc., represents a complex technological problem. Manufacture of assemblies, parts, structures of such type by means of their welding from separate elements or building-up often seems to be more reasonable and allows developing products with single-crystal structure on virtually new basis. The aim of the presented work is development of new approaches applicable to manufacture of the single-crystal welded structures of critical designation with increased mechanical characteristics and service parameters. The results of investigations and examples of pilot welded structures of such type, produced at the E.O. Paton Electric Welding Institute of the NAS of Ukraine, are presented. 30 Ref., 9 Figures.
\end{abstract}

Ke y w or d s: hight-temperature nickel alloys, single-crystals, welded structures of complex geometry, electron beam welding, gas turbine blades, conditions of formation of single-crystal structure

Development of aerospace, power-generating and others branches of industry require application of materials, which can withstand a complex of high service loads with increase of the terms and reliability of operation. The particularly high requirements are made to the materials of parts of a hot gas path of gas turbine engines (GTE) and systems on heat resistance, ductility, high thermal and low-cycle fatigue as well as increased resistance to effect of operating medium $[1,2]$.

To some extent, heat-temperature nickel alloys (HNA) with poly- and single-crystal structure [3] meet these requirements. However, substantial improvement of mechanical and service characteristics due to multicomponent alloying and formation of single-crystal structure result in deterioration of materials' workability, including to weldability reduction [4-8].

HNA has the widest application in manufacture of the elements of GTE hot gas path, i.e. rotor and nozzle turbine blades, to some extent combustion chambers, vectoring nozzle petals and others. Particularly critical and thermostressed of them are high-pressure turbine blades. Application of HNA with single-crystal structure for their manufacture allows significant increase of capability to keep the whole complex of loads, service parameters of GTE, their workability and reliability. At that, there is more than $15 \%$ rise of main characteristic of alloy, namely heat resistance $[9,10]$.
Regardless the successes of a technology of single-crystal growing by investment casting, manufacture of such products present itself not very simple technical task. The problems of manufacture of such products as cooled single-crystal blades with complex system of inner channels and their reaching the surface as well as long and different-thickness blades are of particular importance. A technology of manufacture of products of such type using the method of directed crystallization is characterized with significant complexity, labor and energy intensity, low percent of product yield.

It becomes obvious that manufacture of such products by welding or building-up of their separate constituents is more reasonable that allows developing fundamentally new structures. Thus, for example, RollsRoyse Company produces hollow welded polycrystal GTE titanium blades [12] that allows reducing weight of the blade, increasing its aerodynamic characteristics and thermodynamic efficiency of the turbine in whole.

Replacement of GTE rotors with lock retention of the blades to all-welded of «blisk» type allows up to $25 \%$ reduction of structure weight from the initial and increase of product life [13].

A problem is raised on development of a technology for manufacture, growing and formation of complex-geometry structures from HNA with single-crystal structure using the methods of welding, surfacing, 


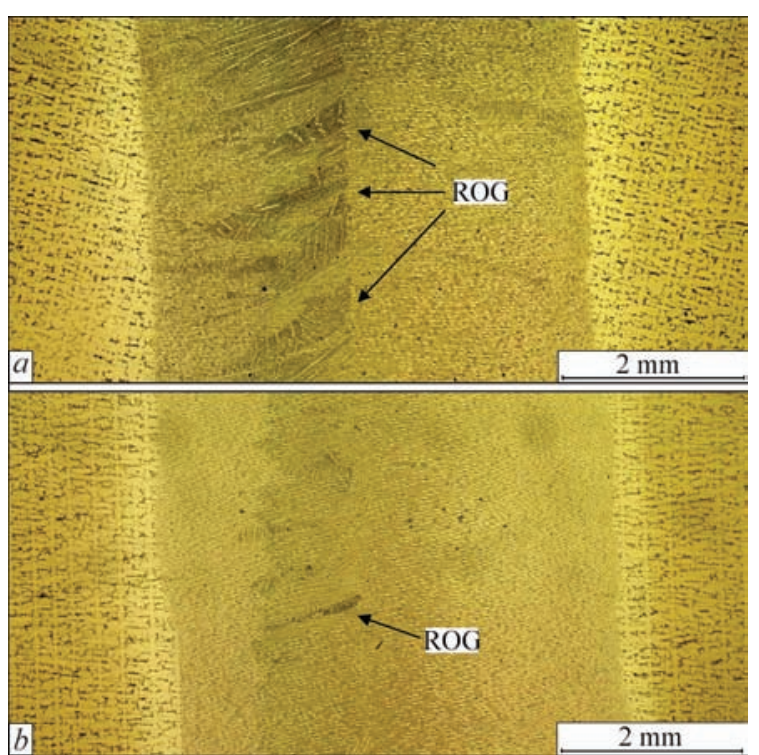

Figure 1. Structure of welded joints with different deviation of crystallographic orientation of joint edge surface from $\{001\}$ : $a-20^{\circ}$ (multiple formation of ROG); $b-5^{\circ}$ (single ROG)

coating deposition as the most mastered in the technological sense.

However, up to the moment there are neither theoretical (formation and preservation of single-crystal structure), nor practical (technology, structure) developments and recommendations on welded structures of particular such type.

E.O. Paton Electric Welding Institute actively has been carrying out the works in this direction [14-24]. The complex investigations of weldability of commercial cast alloys ZhS26, ZhS32, ZhS36, TsNK8 with single-crystal structure were performed.

The main criteria of assessment of welded joint quality were:

- orientation homogeneity of weld metal and heat affected zone with base metal;

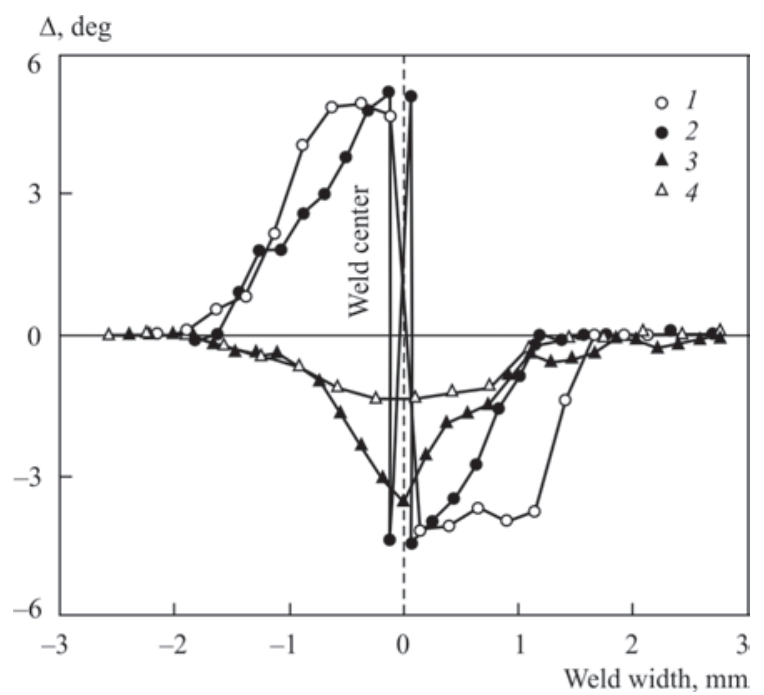

Figure 2. Deviation of orientation on weld section depending on crystallographic orientation of joint edg: 1 - joint edge $\{111\}$; $2-\{112\} ; 3-\{011\} ; 4-\{001\}$. Width of welds is approximately $3.5-3.8 \mathrm{~mm}$
- absence of cracks and defects of weld formation;

- absence of random orientation grains (ROG);

- level of mechanical properties of welded joints.

Evaluation of the main crystallization parameters at a weld solidification front, namely temperature gradient $(G)$ and front displacement rate $(R)$, responsible for perfection of the single-crystal structure, was carried out using a procedure of local thermometry of weld pool melt and analysis of thermokinetic curves [14].

Examination of peculiarities of structure of the welded joints depending on temperature-time conditions of weld formation was carried out using micro $\mathrm{X}$-ray spectrum analysis, metallography, X-ray diffractometry and EBSD analysis.

Initial crystallographic parameters of the welded joint assembly were set by orienting using X-ray method. Crystallographic orientation of the weld metal, presence and amount of ROG and their relative area were judged following the analysis of pole figures. Dislocations' density and their distribution were evaluated on width, shape and homogeneity of intensity distribution of X-ray reflections $I_{q \perp}$.

As a result of complex investigations there were determined the dependencies of formation of the single-crystal structure in welding of HNA single-crystals of 0.5-5.0 mm thickness. Effect of technological factors on crystallographic and temperature-time parameters of process of the weld metal structure formation were examined. They determine perfection of the single-crystal structure.

Figures 1-3 illustrate the results of effect of the technological factors on the crystallographic parameters of the process of weld metal structure formation. Figure 1 shows formation of ROG and change of crystallographic orientation of the weld metal (Figure 2) in variance of the crystallographic orientation of welded edges of high symmetry. It is determined that appearance of up to $10 \%$ of the random orientation grains is possible even at strict conformance to the indicated conditions of the weld symmetry. Such violations of single-crystallinity of the weld can be related with the fact that the weld pool has a curvature and, therefore, direction of the maximum temperature gradient $(G)$ changes on the solidification front in relation to direction of primary growth of crystals $<001>$ (Figure 3 ). This leads to violation of one of the main conditions of directed crystallization, namely, orientation effect of a substrate on single-crystal growth. Violation of the crystallographic orientation and single-crystal structure is mainly concentrated in that areas of weld metal, where the direction of maximum temperature gradient at the weld pool solidification front deviates from the orientation of primary growth by the angles more than $15^{\circ}$ (zone B) (Figure 3). This permitted to 


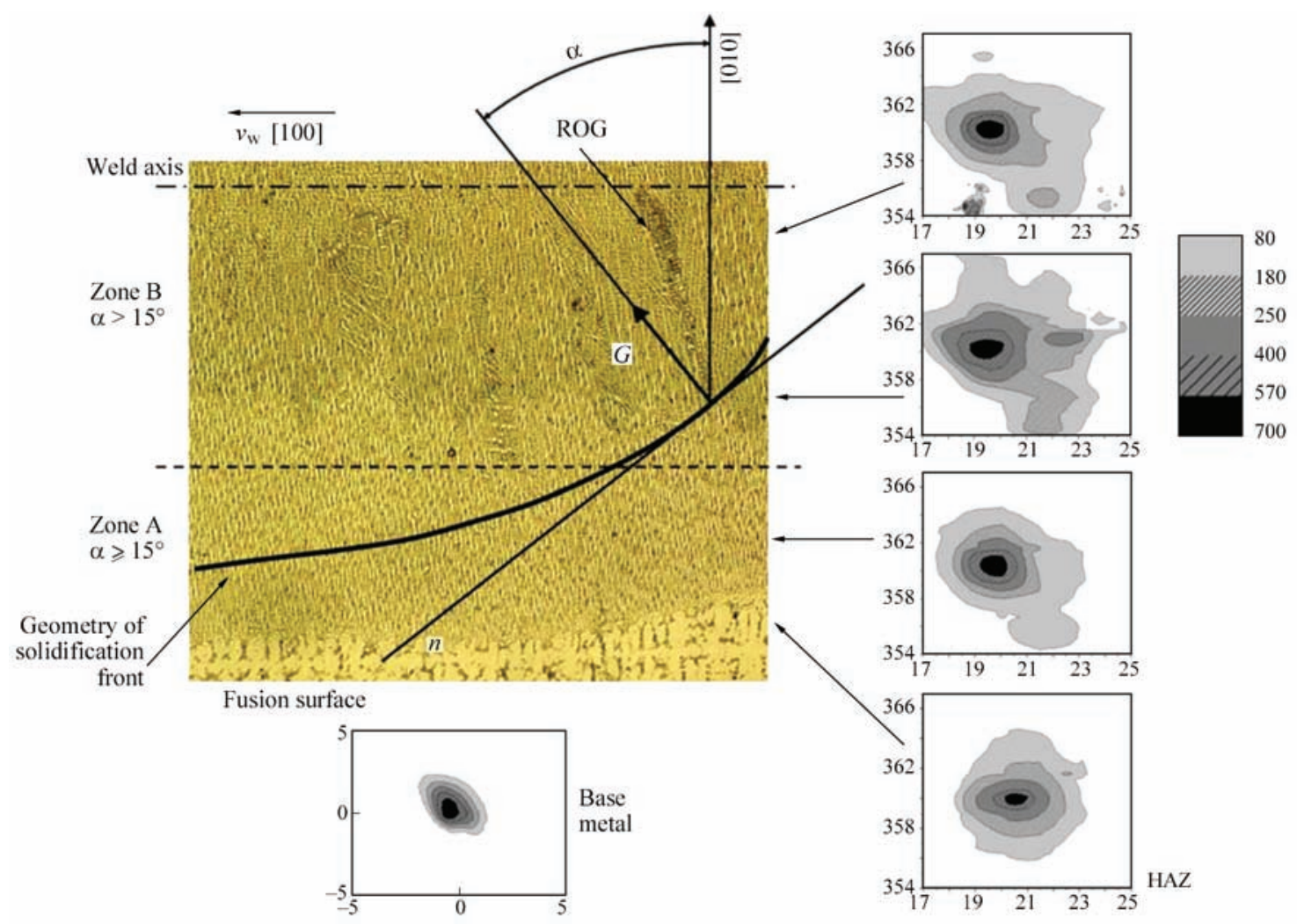

Figure 3. Microstructure $(\times 100)$ and isointense lines of distribution $I_{\mathrm{q} \perp}$ of separate weld metal sections, corresponding to different deviation of the maximum temperature gradient at weld pool solidification front: $\alpha$ - angle of deviation of maximum temperature gradient $\mathrm{G}$ on direction of primary crystallization

determine allowable disorientation, which provides formation of the weld with single-crystal structure.

Figure 4 is a generalization of a complex of investigations of temperature-time conditions of the weld metal structure formation. A calculation-experimental way [14] was used to determine a dependence between the main temperature-time parameters of $G / R$ process at the liquid pool solidification front, nature of forming structure and value of deviation of the welded joint crystallographic orientation from the crystal high symmetry. It is shown that formation of directed cellular-dendritic and dendritic structure limiting appearance of the grains of other orientation in welding of HNA single-crystals is possible in deviation of crystallographic orientation, exceeding the set limits. The complexity of technical realization of such approach lies in the need to limit the welding stresses, strains and fulfillment of the general requirements made to the quality of formation of sound joints. This is, in particular, obvious in welding of the complex geometry structures.

Thus, it is shown that production of the welds with perfect single-crystal structure and crystallographic orientation, corresponding to the initial metal is reached due to:

- providing initial crystallographic orientation in the joint area in the range of deviation from direction of axes of single-crystal high symmetry for not more than $5^{\circ}$ (Figures 1,2);
- keeping the relationship of direction of maximum heat sink at the weld pool solidification front and direction $<100>$ (light increase) with deviation for not more than $15^{\circ}$ (Figure 3);

- development of temperature-time conditions $(G / R)$ of the directed crystallization according to Figure 4 for different initial crystallographic orientation and welding modes. There is control of $G$ - temperature gradient at the weld pool solidification front and $R$ - solidification rate.

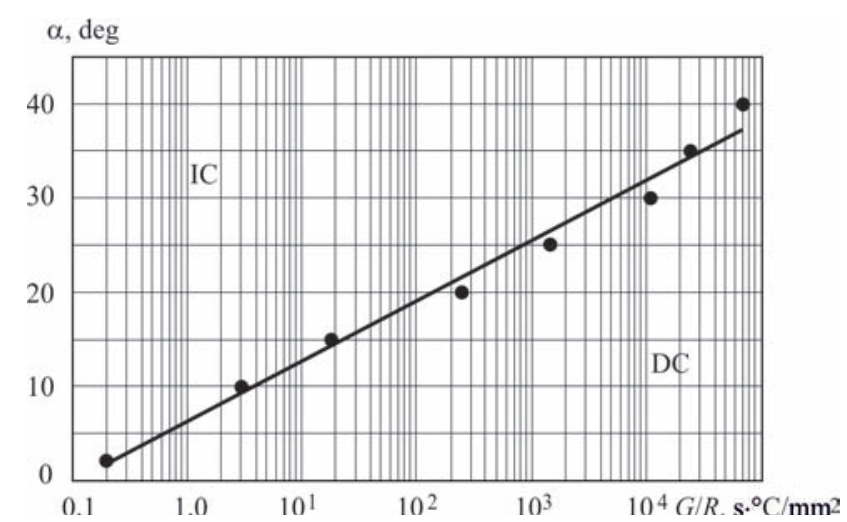

Figure 4. Calculation-experimental dependence of allowed deviations $\alpha$ of direction of maximum temperature gradient $\mathrm{G}$ on orientation of crystal primary growth $<100>$ at weld pool solidification front in EBW of ZhS26 alloy on $G / R$ value: DC - area of directed crystallization; IC — area of irregularity of directed crystallization 
Violation of one of these conditions promotes formation of weld structural defect, i.e. random orientation grains (ROG) and, as a result, cracks. Figures 1-3 in general form demonstrate irregularity of perfection of the single-crystal structure as a result of deviation of each from indicated conditions in production of common butt joints of small thickness plates.

Analysis of HNA industrial structures indicate that transfer to their production from the single-crystal billets using welding will provoke problems with fulfillment of indicated conditions, connected with different geometry, random crystallographic orientation, alloys of different kind and others.

Area of investigations necessary for development of such structures was determined based on the analysis of structure peculiarities of products from HNA single-crystals and experiments on welding of the reference samples as well as taking into account the main theoretical and fundamental technological conditions for the perfect single-crystal weld structure. Main of them lied in investigation of effect of technological factors on temperature-time and crystallographic-orientation characteristics of weld formation process, and, respectively, on quality of the single-crystal structure. The method, scheme, modes and procedures of welding were selected based on the investigation results.

The main attention was given to power and technological peculiarities of welding. Taking into account high specific power, precision, mobility of heat source, the most perspective in this sense are such methods of welding as electron beam and laser. Welding with electron beam in vacuum provides reliable protection of weld metal from contamination by impurities, which not only provoke deterioration of HNA main properties, but can initiate irregularity of perfection of the single-crystal structure.

Realizing the set physical conditions of the single-crystal structure formation and preservation of the initial crystallographic orientation are carried out by means of regulation of mode parameters, application of corresponding schemes and welding procedures. At that, regulation of the weld pool metal temperature and its gradient at the solidification front, rate of crystallization and cooling, time of melt existence, geometry of macrofront of the weld pool solidification are performed. As a result the better indices of HNA structure are obtained, namely refinement of dendrites; $\gamma^{\prime}$-phase, carbides, eutectic size and other phases, their chemical inhomogeneity decreases. This promotes improvement of mechanical properties as well as service characteristics $[1,3]$.

It should be noted that the problems of development of complex geometry structures, including from HNA using welding technologies in the recent years are actively solved by the methods of $3 \mathrm{D}$ printing (3D) or additive manufacturing (AM) [25-29].

It is informed that Siemens Company has successfully carried out the bench tests of the GTE experimental blades, produced on 3D printer. They were tested in the engine under conditions of complete loading at temperature more than $1250{ }^{\circ} \mathrm{C}$. Blades material is polycrystal nickel superalloy. General Electric Company started using additive technologies in manufacture of the elements of gas turbines [30] from polycrystal HNA.

Regardless the advantages of the indicated technologies, it is necessary to pay attention on a series of disadvantages, which complicate their application in manufacture of the complex geometry single-crystal structures from HNA.

First of all, production of the single-crystal product requires presence of an initial single-crystal billet, absence of which is one of the advantages of 3D-technology. Secondly, it is obvious, that during the whole process it is difficult to keep the conditions necessary for formation of single-crystal HNA structure, including $G / R$ relationship. The powders of corresponding composition are necessary. They have very high requirements on grain size and chemical composition, yield, production and storage conditions. Even insignificant deviations from AM technological process result in change of object geometry, nonuniformity of structure, including deterioration of single-crystallinity, unsatisfactory surface quality, partial lack of fusions and overlaps, that all together complicate production of structures of complex geometry by $3 \mathrm{D}$ printing method.

An alternative to the $3 \mathrm{D}$ printing method in manufacture of the single-crystal structures of complex geometry can be a technology based on discrete and successive melting of billet of specific composition by concentrated power source. Up to the moment the technology is updated in a part of determination of heat, deformation and orientation conditions and dependencies of structure formation.

In development of the single-crystal complex geometry welded structures there were proposed and tested the technological schemes based on:

- dosed heat input in welding of structure elements of different thickness or different chemical composition, realized due to discrete asymmetry scanning with regulation of frequency, amplitude, time of beam delay;

- regulated heat input on depth of weld pool in welding of structure elements up to $10 \mathrm{~mm}$, performed, mainly, due to discrete beam scanning in combination with high welding rate;

- development of temperature range in the area of successive local melting and quasistationary thermal 


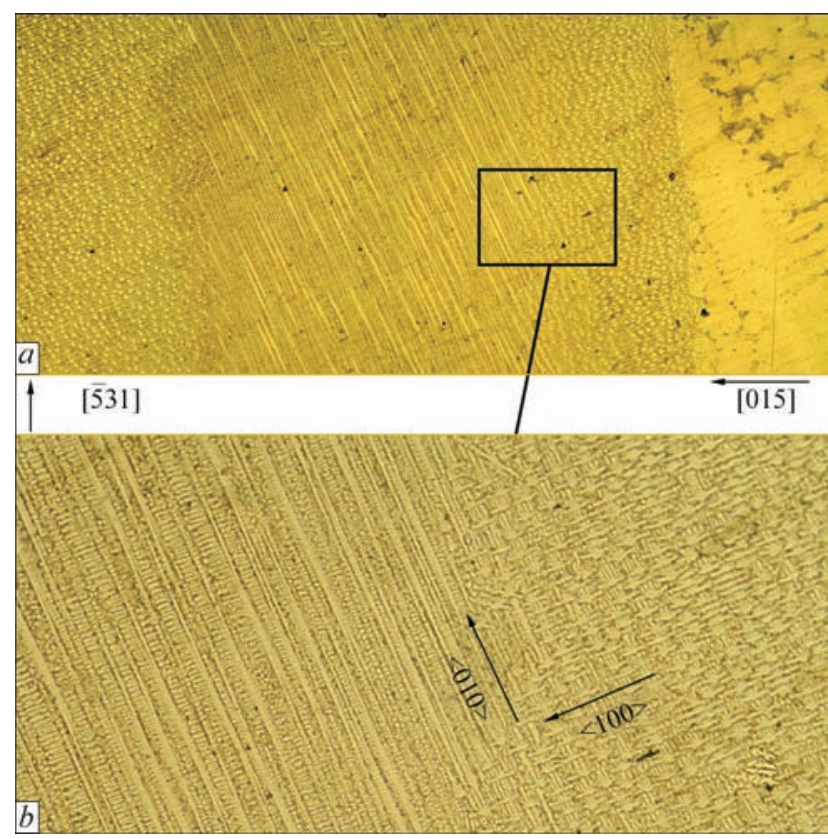

Figure 5. Microstructure of weld metal with initial asymmetric crystallographic orientation of welded joint produced with regulation of temperature-rate parameters of weld pool solidification: $a-\times 30 ; b-\times 50$

field, at which direct crystallization and formation of single-crystal structure will take place;

- providing specific $G / R$ relationship in joining the elements of different crystallographic orientation (Figure 5), thickness and various grades of welded al-
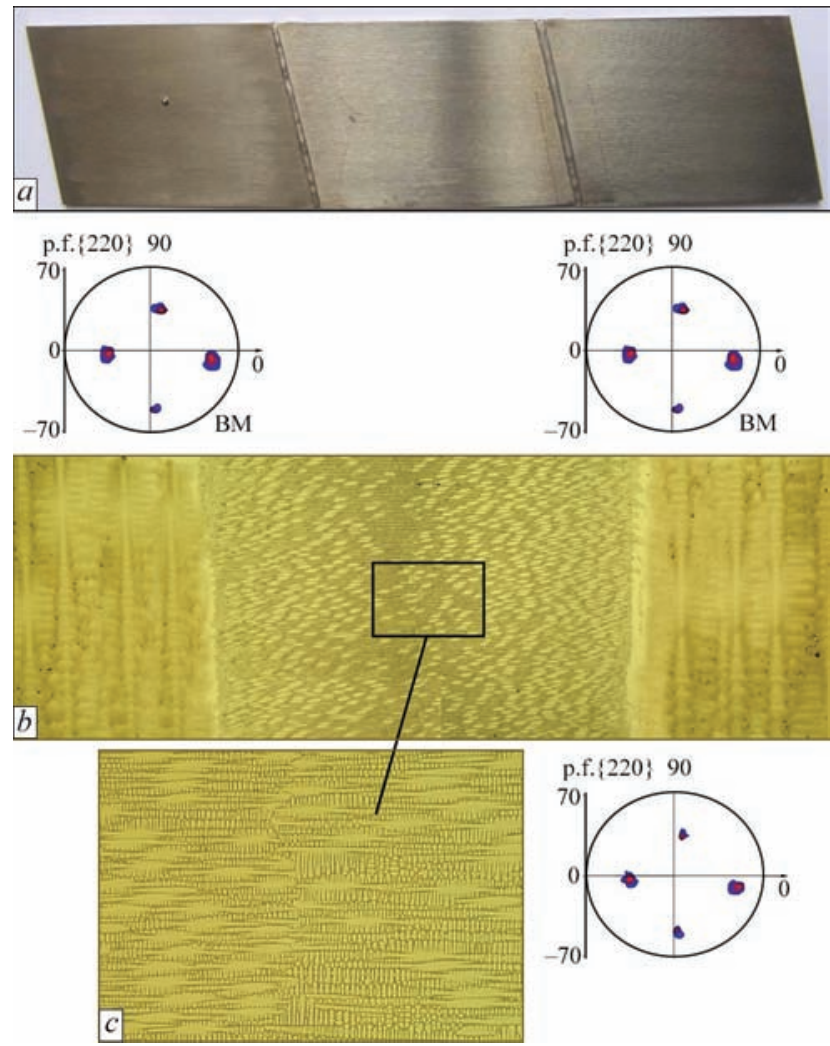

Figure 6. Example of coarsening of single-crystal of billets from ZhS32 alloy of $2 \mathrm{~mm}$ thickness using EBW. General view (a), microstrucutre $(b-\times 50 ; c-\times 100)$ and pole figures $\{220\}$ of welded joint $(b)$ loys in the joining zone due to optimum for each case relationship of the welding mode parameters.

Figures 6-9 illustrate some of the results of application of such schemes.

Figure 6 illustrates the possibility of development of the coarse single-crystals and structures by means of EBW of the billets, produced by directed solidification method. Oriented cutting of the billets and their assembly for welding provided symmetrical structure of the joint with deviation of the crystallographic orientation for not more than two degrees. Except for
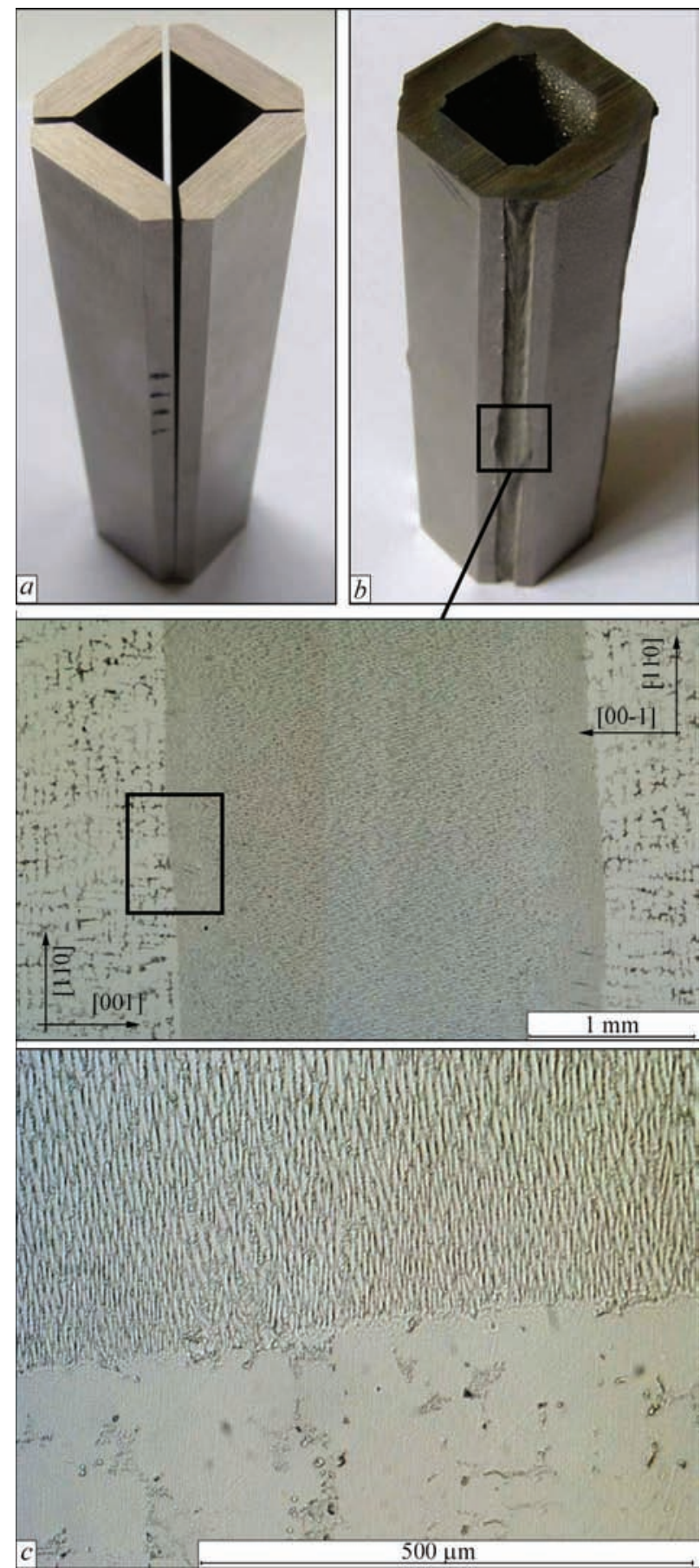

Figure 7. Variant of welded single-crystal structure of ZhS32 alloy billets made by EBW: $a$ - initial single-crystal billets of $5 \mathrm{~mm}$ thickness; $b$ - welded element; $c$ - joint microstructure 

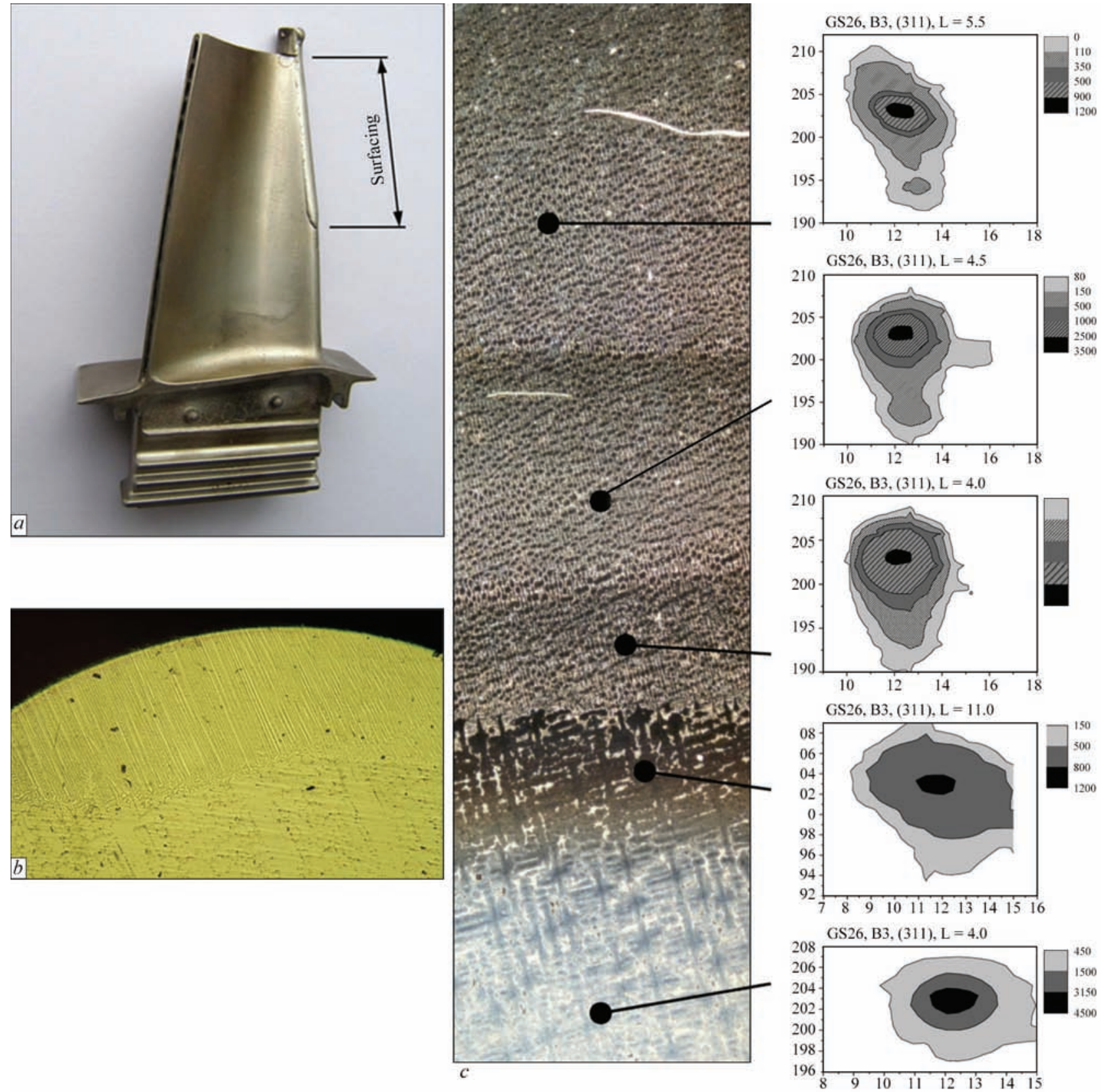

Figure 8. Appearance $(a)$ and structure $(b-\times 100 ; c-\times 50)$ of single-crystal blade from ZhS32 alloy of high-pressure turbine in aircraft GTE with leading edge repaired by singlepass $(b)$ and multi-pass $(c)$ electron beam surfacing

preserving the initial crystallographic orientation, there were developed a technology eliminating warping of longitudinal welded joint.

Figure 7 shows a single-crystal hollow billet of rectangular section, welded by electron beam of $5 \mathrm{~mm}$ thickness single-crystal plates. As it is shown by $\mathrm{X}$-ray and metallographic examinations of the joints, absence of ROG, orientation homogeneity of the weld metal and heat-affected zone with the base metal, which does not exceed five degrees, describe the single-crystal nature of welded structure in whole. High crystallographic homogeneity of the joints is reached due to set values and combinations of technological parameters, modes, EBW procedure and providing of crystallographic symmetry of butt joints in manufacture of the initial billets and their assembly. At that, it is possible to ensure fulfillment of conditions of directed crystallization, namely temperature ( $G / R$ relationship approximately $600 \mathrm{~s} \cdot{ }^{\circ} \mathrm{C} / \mathrm{mm}^{2}$ at deviation of the joint edges from high symmetry up to $20^{\circ}$ ) and orientation (formation of macroflat solidification front of pool in through penetration with the parallel fusion edges).

Figure 8 demonstrates a variant of surfacing repair of the most loaded leading edge of single-crystal blade of high-pressure turbine of ZhS32 alloy. Deposit metal inherits the initial crystallographic orientation of the blade substrate with deviation, not exceeding two degree. Height of the one deposit layer was set in the limits of $0.7-0.8 \mathrm{~mm}$. In multipass surfacing the sum 

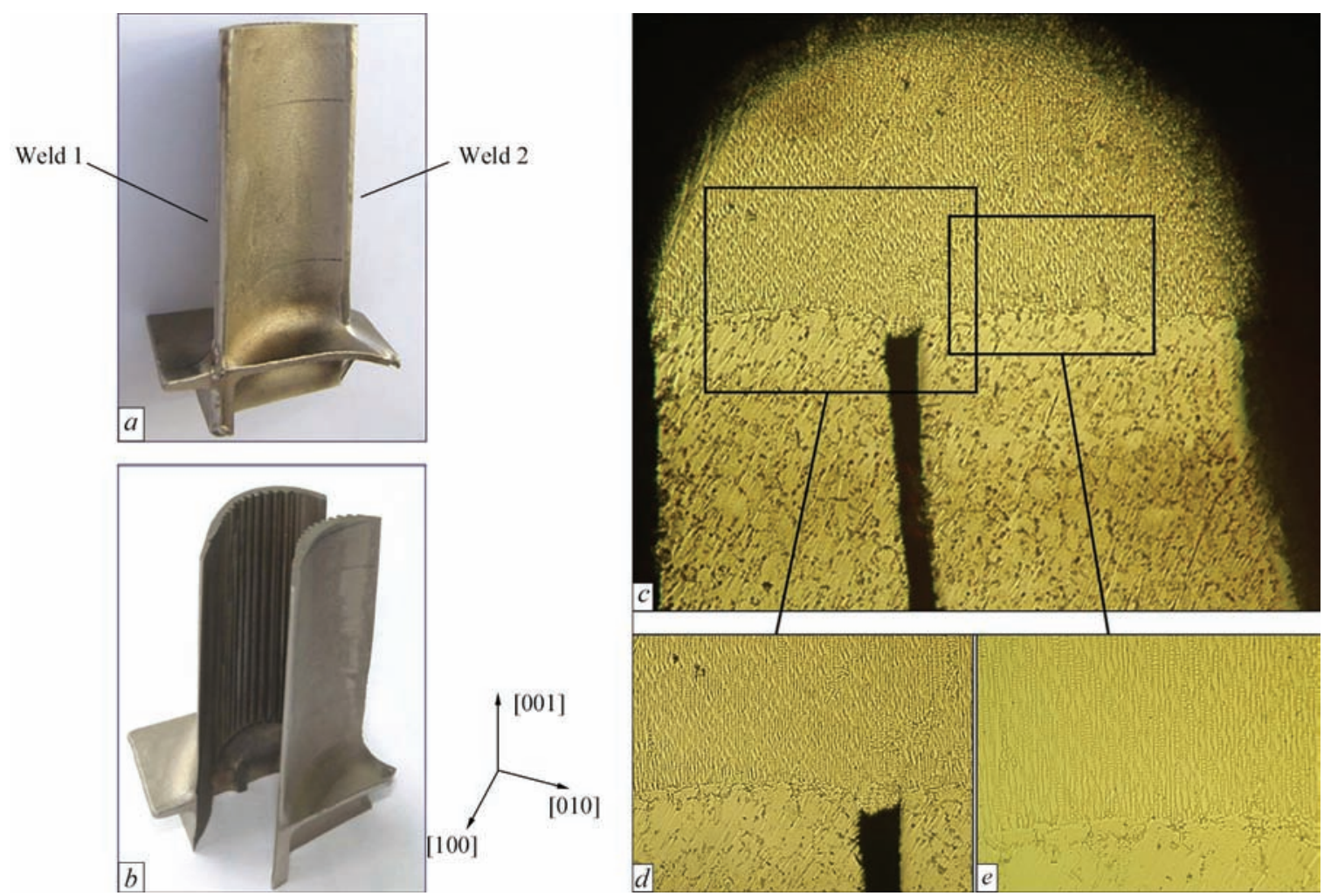

Figure 9. Prototype of all-welded single-crystal cooled blade of ZhS26 alloy (a) made by EBW of billets (b) with typical crystallographic structure, and microstructure $(c, d)$ of joint metal

height can reach $3 \mathrm{~mm}$ and more. The filler material corresponded to composition of the deposited blade. Analysis of the results of X-ray examination on shape and broadening of the isointense lines of $I_{q \perp}$ distribution indicates homogeneity of dislocation distribution and absence of ROG with formation of substructure with low-angle disorientation.

Figure 9 presents the variant of complex structure of cooled blade, produced by EBW. Such a solution in addition to simplification of production technology allows getting the optimum innovative geometry of the inner channels at lower expenses. This provides the maximum coefficient of inner blade cooling.

A basic scheme of welding technology was develop based on the results of investigations. It allows providing preservation of the single-crystal structure with disorientation of structural constituents, which does not exceed $5^{\circ}$, absence of cracks and random orientation grains. Except for known technological activities on repair of polycrystalline blades, the technology includes the following basic operations:

- determination of crystallographic orientation of the defective zone;

- optimizing the weld pool shape;

- selection of parameters of mode and procedure of electron beam welding, which provide the necessary weld pool geometry and temperature-time parameters at the solidification front.

The technology was realized in repair of blades of ZhS26 and ZhS32 alloys. The experimental works on coarsening of single-crystal billets of $1.5-5.0 \mathrm{~mm}$ thickness were carried out.

The level of mechanical properties of joints of single-crystal alloy $\mathrm{ZhS} 26$ makes:

- short-term mechanical properties of welded joints from alloy in $500-1000{ }^{\circ} \mathrm{C}$ temperature range not lower than the level of properties of initial single-crystal in the whole investigated temperature range;

- long-term strength of welded joint on the basis of $100 \mathrm{~h}$ at $900{ }^{\circ} \mathrm{C}$ reaches $\sigma_{100} \geq 240 \mathrm{MPa}$ that makes approximately $75 \%$ of the base metal.

Given results show the possibility of formation of the welded joints of complex geometry and deposits with single-crystal structure that potentially allows using fusion welding in repair and production of the single-crystal parts. Absence of grains in such a product and, respectively, high-angle boundaries, makes the grounds for high service parameters and working capacity of the welded structures.

It is obvious that application of these technologies allows:

- providing development of innovative products based on new principles with improved mechanical characteristics and service parameters.

- reduce laboriousness and production cost;

- decrease material consumption;

- provide energy saving;

- increase compatibility of the products on the international market. 
Naturally, that proposed solutions require further development from point of view of design and technological improvement together with developers and manufacturers of the products.

Taking into account outlined fundamental and applied developments it can be concluded that application of welding technologies currently can be the most reasonable, if not single in some cases, solution of the problem of development of complex geometry products of HNA with the single-crystal structure.

1. Stroganov, G.B., Chepkin, V.M. (2000) Cast high-temperature alloys for gas turbines. Moscow, ONTI MATI [in Russian].

2. Petukhov, A.N. (1993) Fatigue resistance of GTE parts. Moscow, Mashinostroenie [in Russian].

3. Kablov, E.N. (2001) Cast blades of gas-turbine engines (alloys, technology, coatings). Moscow, MISIS [in Russian].

4. Sorokin, L.I. (2004) Weldability of high-temperature nickel alloys (Review). Pt 2. Svarochn. Proizvodstvo, 10, 8-16 [in Russian].

5. Sorokin, L.I. (2004) Weldability of high-temperature nickel alloys (Review). Pt 1. Ibid., 9, 3-7 [in Russian].

6. Lippold, J., C., Cotecki, D.J. (2005) Welding metallurgy and weldability of stainless steels.Wiley Interscience. A J.Wiley@ sons inc. Publication.

7. Pollock, T.M., Murphy, W.H. (1996) The breakdown of single-crystal solidification in high refractory nickel-base alloys. Metall. Mater. Transact. A, 27A, 1081-1094.

8. Park, J.-W., Baby, S.S., Vitek, J.M. et al. (2003) Stray grain formation in single crystal Ni-base superalloy welds. J. of Applied Physics, 94(6), 4203-4209.

9. Czyrska-Filemonowicz, A., Dubiel, B., Zietara, M., Cetel, A. (2007) Development of single crystal Ni-based superalloys for advanced aircraft turbine blades. Ingynieria Materialowa, 3-4, 128-133.

10. Reed, R.C. (2006) The superalloys: Fundamentals and application. Cambridge, Cambridge University Press.

11. (2004) Aviation materials. In: Sci.-Techn. Coll.: High-rhenium high-temperature alloys, technology and equipment for production of alloys and casting of single crystal GTE blades. Moscow, VIAM [in Russian].

12. Fitzpatrick, G.A., Broughton, T. (1986) Rolls-Royse wide chord fan blade. In: Proc. of Int. Conf. on Titanium Products and Applications (San, Francisco, California, USA, October 1986).

13. Inozemtsev, A.A., Nikhamkin, M.A., Sandratsky, V.L. (2008) Principles of design of aircraft engines and power units. Vol. 2: Compressors. Combusion chambers. Afterburners. Turbines. Output devices. In: Manual for higher education institutes. Moscow, Mashinostroenie [in Russian].

14. Yushchenko, K.A., Zadery, B.A., Gakh, I.S., Karasevskaya, O.P. (2016) Formation of weld metal structure in electron beam welding of single crystals of high-temperature nickel alloys. The Paton Welding J., 8, 15-22.

15. Yushchenko, K.A., Gakh, I.S., Zadery, B.A. et al. (2013) Main theoretical backgrounds of welding of metal single crystals.
In: Physical and technical problems of modern materials science. Vol. 1. Akademperiodika, 148-176 [in Russian].

16. Yushchenko, K.A., Gakh, I.S., Zadery, B.A. et al. (2013) Influence of weld pool geometry on structure of metal of welds on high-temperature nickel alloy single-crystals. The Paton Welding J., 5, 45-50.

17. Yushchenko, K.A., Zadery, B.A., Gakh, I.S., et al. (2013) On nature of grains of random orientation in welds of single-crystal high-temperature nickel alloys. Metallofizika i Novejshie Tekhnologii, 35(10), 1347-1357 [in Russian].

18. Yushchenko, K.A., Zadery, B.A., Zvyagintseva, A.V. et al. (2009) Peculiarities of structure of metal deposited on edges of single-crystal blades made from nickel superalloys. The Paton Welding J., 8, 36-42.

19. Yushchenko, K.A., Zadery, B.A., Gakh, I.S. et al. (2009) On possibility of inheritance of single-crystal complexly-alloyed nickel alloys under nonequilibrium conditions of fusion welding. Metallofizika i Novejshie Tekhnologii, 31(4), 473-485 [in Russian].

20. Yushchenko, K.A., Zadery, B.A., Kotenko, S.S. et al. (2008) Sensitivity to cracking and structural changes in EBW of single crystals of heat-temperature nickel alloys. The Paton Welding J., 2, 6-13.

21. Yushchenko, K.A., Zadery, B.A., Karasevskaya, O.P. et al. (2006) Structural changes in crystallization process of nickel superalloys in crystallography-asymmetric location of welding pool. Metallofizika i Novejshie Tekhnologii, 28(11), 15091527 [in Russian].

22. Yushchenko, K.A., Zadery, B.A., Karasevskaya, O.P. et al. (2006) Structure of welded joints in tungsten single crystals. The Paton Welding J., 8, 29-36.

23. Yushchenko, K.A., Karasevskaya, O.P., Kotenko, S.S. et al. (2005) Inheritance of structure-oriented state of metallic materials by welded joints. Ibid., 9, 2-9.

24. Zadery, B.A., Kotenko, S.S., Polishchuk, E.P. et al. (2003) Peculiarities of crystalline structure of welded joints in single crystals. Ibid., 5, 13-20.

25. Clark, D., Bache, M.R., Whittaker, M.T. (2008) Shaped metal deposition of nickel alloy for aero engine applications. $J$. $M a$ terials Proc. Technology, 203, 439-448.

26. Ding, D., Pan, Z., Cuiuri, D., Li, H. (2015) Wire-feed additive manufacturing of components: technologies, developments and future interests. Int. J. Adv. Manuf. Technol., 81(1-4), 465-481.

27. Frazier, W.E. (2014) Metal additive manufacturing (Review). J. Mater. Eng. and Performance, 23(6), 1917-1928.

28. Brandla, E., Baufeld,?, Leyens, C., Gauitd, R. (2010) Additive manufactured Ti-6Al-4V using welding wire: Comparison of laser and arc beam deposition and evaluation with respect to aerospace materials specification. Proc. of the Laser Assisted Net Shape Engineering, 5, Pt B, 595-606.

29. (2018) Additive manufacturing: Siemens uses innovative technology to produce gas turbines. Press-Siemens Global Website, Munich, Mar 19. www.siemens.com/press/en/

30. Rockstroh, T., Abbott, D., Hix, K., Mook, J. (2013) Lessons learned from development cycle. Additive manufacturing at GE Aviation - Industrial Laser Solutions, 1-6. www.industrial-lasers.com/articles/print/volume-28/issue-6/features/additive-manufacturing-at-ge-aviation.html 


\title{
ANODE PROCESSES IN WELDING ARCS
}

\author{
I.V. KRIVTSUN \\ E.O. Paton Electric Welding Institute of the NAS of Ukraine \\ 11 Kazimir Malevich Str., 03150, Kyiv, Ukraine. E-mail: office@paton.kiev.ua
}

\begin{abstract}
The paper describes the approaches to theoretical investigation and mathematical models of physical processes running in the anode layer of high-current $\left(\sim 10^{2} \mathrm{~A}\right)$ electric arcs, burning in inert gas at atmospheric pressure. Numerical studies of anode processes in electric arcs with nonevaporating (water-cooled) anode, as well as in welding arcs (arcs with evaporating anode) were performed. It is shown that anode potential drop in the considered arcs is negative, having an essentially nonuniform distribution in the region of anode attachment of the arc. The latter circumstance largely determines the pattern of electric current flowing and energy transfer between arc plasma and anode (arc contraction on the anode or discharge distributed over anode surface). A procedure of calculation of effective anode drop in electric arcs is proposed, allowing for the above change of anode potential drop along the anode surface. 32 Ref., 1 Table, 15 Figures.
\end{abstract}

Keywords: welding arc, arc plasma, arc column, anode layer, anode potential drop, heat flow into the anode, evaporation, mathematical modeling

Fusion arc welding now is one of the main technological processes of producing permanent joints of metallic materials. Arc welding methods the most widely used in industrial production are consumable electrode welding in inert and active gases or in their mixtures (MIG/MAG), as well as inert-gas nonconsumable electrode welding (TIG), applied in fabrication of critical structures. Here we can also mention plasma welding as a variety of nonconsumable electrode welding, which allows an essential increase of penetration depth and thickness of metal being welded, respectively, due to application of a constricted arc. For effective application of electric arc as the welding heat source, it is necessary to have valid information about its thermal, electric and dynamic impact on the electrodes. In particular, at realization of the above welding methods, the anode processes have a quite important role, namely processes of interaction of arc plasma with electrode metal drop, which is the arc anode in the case of MIG/MAG welding, and with weld pool metal, which is the anode in the case of TIG and plasma welding. In the first case, the above processes determine wire melting, formation and transfer of electrode metal drops, and in the second case - penetration of the metal being welded and weld formation. As experimental determination of such important in terms of technology characteristics of the welding arc as electric current density and heat flow on the surface of the drop and the weld pool is difficult, because of high values of arc plasma temperature and molten metal surface temperature, smallness of geometrical dimensions of the arc attachment region and a number of other factors, theoretical study of anode processes,

(C) I.V. KRIVTSUN, 2018 in particular by mathematical modeling, seems to be very relevant.

There are many approaches and models for numerical study of anode processes in free-burning and constricted (plasma) arcs (see, for instance, [1-8]). In the majority of them, however, arc plasma is assumed to be single-component, i.e. containing atoms and ions of shielding or plasma-forming, most often, inert gas. Plasma of the real welding arcs, is, as a rule, multicomponent, as, alongside the gas particles, it contains atoms and ions of evaporated material of the electrodes, primarily, of the anode [9]. Thus, the multicomponent nature of arc plasma should be taken into account at construction of an adequate mathematical model of anode processes in welding arcs.

Another important characteristic of such a model should be the possibility of allowing for the interconnection of physical processes running in the anode body, on its surface and in anode layer, with the processes in arc column. It should be noted that in the majority of works on complex modeling of electric, in particular, welding arc, quite simplified models of the anode region are used [10-15], whereas in the works devoted specifically to investigation of near-electrode phenomena (see, for instance, review [16] and literature cited there), processes running in the arc column, are given insufficient attention.

This study is devoted to description of self-consistent mathematical models and numerical study of physical processes, running in near-anode plasma of high-current $\left(\sim 10^{2} \mathrm{~A}\right)$ electric arcs, burning in inert gas at atmospheric pressure, in particular welding arcs (allowing for anode material evaporation), for the conditions of MIG, TIG and plasma welding. 


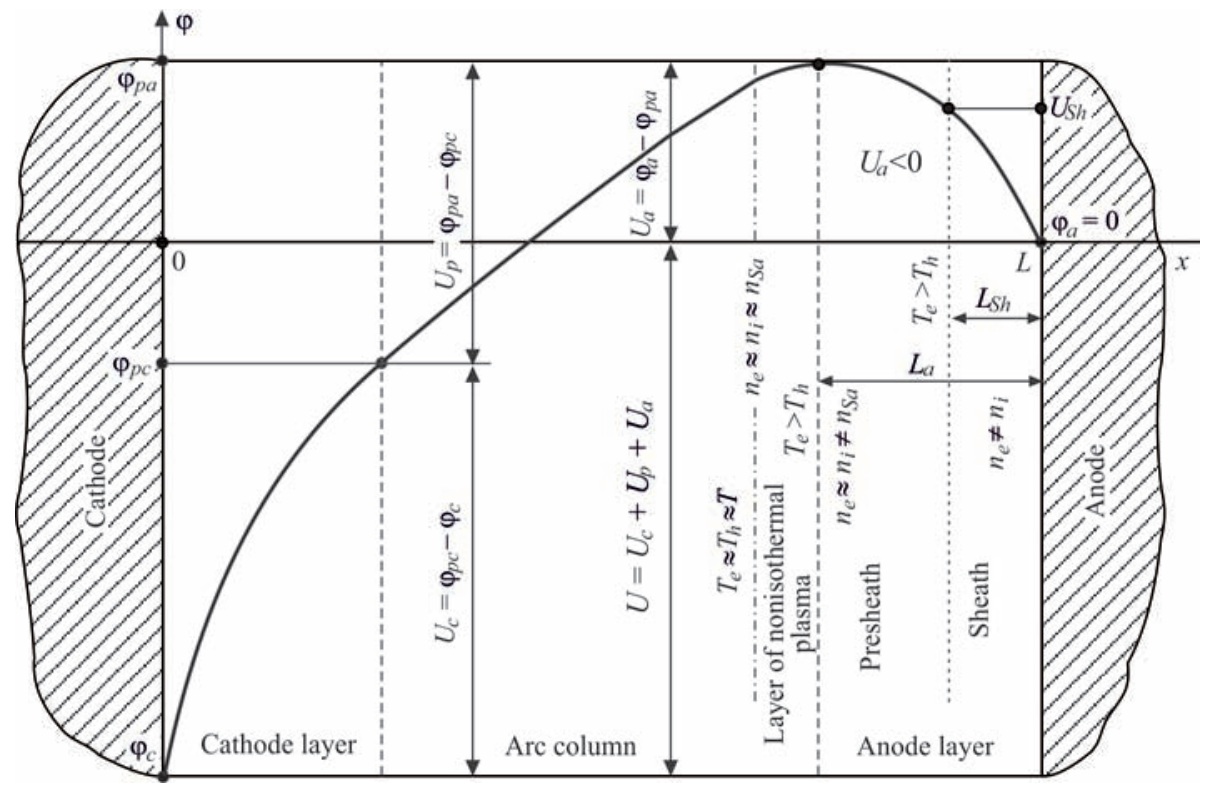

Figure 1. Distribution of electric potential along arc length: $\varphi_{c}$ — cathode surface potential; $\varphi_{p c}$ — potential of arc column plasma on the boundary with cathode layer; $L$ - arc length (for other designations see the text)

Before beginning description of the above models, we will consider the characteristic distribution of electric potential by the length of atmospheric pressure high-current arc, which is shown in Figure 1. Electric arc can be conditionally divided into three regions: near-electrode layers of nonequilibrium plasma (cathode and anode) and arc column, where plasma is in the state of local thermodynamic equilibrium [17]. Here, total arc voltage $U$ can be presented as a sum of voltage drops in cathode $U_{c}$ and anode $U_{a}$ layers, as well as in arc column $U_{p}$ :

$$
U=U_{c}+U_{p}+U_{a} .
$$

Special attention should be given to the fact that in the considered arcs, in particular, welding arcs, anode potential drop, determined as $U_{a}=\varphi_{a}-\varphi_{p a}$, where $\varphi_{a}$ is the potential of anode surface, $\varphi_{p a}$ is the potential of arc column plasma on the boundary with the anode layer, is negative [2,18-20]. Moreover, in view of the high conductivity of metallic materials, the anode surface is practically equipotential. Therefore, the anode electric potential $\varphi_{a}$ can be considered constant with good approximation, and can be selected equal, for instance, to zero, as it was done in Figure 1. Then, considering the distributions of anode temperature along its surface and near-anode plasma characteristics, determining local values of anode drop, it can be stated that the potential of arc column plasma on the boundary with anode layer also is nonuniform in the region of arc anode attachment, i.e. it depends on the coordinate along the mentioned boundary [21]. This leads to appearance of the component of electric potential gradient and, accordingly, component of current density along the anode layer boundary, that largely determines the pattern of electric current flow- ing between arc plasma and anode. The noted facts are quite nontrivial and will be considered in detail in this work.

For description of arc plasma adjacent to anode surface, we will use the approach proposed in works [1-3], in terms of which the near-anode plasma can be conditionally divided into several zones, as shown in Figure 1 on the right. The first zone directly adjacent to the anode surface is a layer of space charge (Sheath), where the condition of plasma quasineutrality is disturbed and the part of potential drop between the plasma and anode is formed. This layer can be regarded as collisionless with sufficient accuracy, as at the pressure close to the atmospheric pressure, and plasma temperature values characteristic for the considered conditions $T \sim 1 \mathrm{eV}[1,2]$, this layer thickness $L_{S h}$ commensurate with Debye radius $r_{D} \sim 10^{-8} \mathrm{~m}$, is essentially smaller than the free path of near-anode plasma particles $l \sim 10^{-7}-10^{-4} \mathrm{~m}$ (respective values of characteristic paths for atmospheric pressure Ar-plasma are given in Figure 2).

The second zone on the right in Figure 1 - the ionization layer or prelayer (Presheath), is the region of nonisothermal quasineutral multicomponent plasma, where the charged particles are generated due to plasma electrons ionizing the gas atoms, desorbing from the anode surface, and the evaporating atoms of anode material. The ions forming here are accelerated towards the anode surface by the electric field created by more mobile electrons, and recombine near this surface. Thus, the conditions of local ionization equilibrium are violated within the prelayer, i.e. concentrations of charged plasma particles $n_{e} \approx n_{i}$ differ from equilibrium concentration $n_{S a}$, calculated 
using Saha equations. In addition, a noticeable drop of near-anode plasma potential occurs here, which can be greater with its change in the space charge layer (see Figure 1).

The outer boundary of the anode layer runs at the distance from anode surface $L_{a}$, equal to several free paths of heavy plasma particles. The third zone starts behind this boundary, being the gas-dynamic region of arc plasma or the arc column, where local thermodynamic equilibrium is established. Note here that this region, in its turn, can be conditionally divided into two zones: layer of nonisothermal ionization-equilibrium plasma, the thickness of which is determined by value $l_{e a}$ (see Figure 2), and within which equalizing of the temperature of electrons $T_{e}$ and heavy particles $T_{h}$ with plasma temperature in arc column $T$ occurs, and the arc column proper (see Figure 1).

As under the considered conditions anode layer thickness $L_{a} \sim 10^{-4} \mathrm{~m}$, as a rule, is by an order of magnitude smaller than the curvature radius of the surface of the anode (drop of electrode metal or weld pool) $R \sim 10^{-3} \mathrm{~m}$, it can be considered almost flat at description of the processes, running in this layer. On the other hand, as $L_{a}$ is much smaller than the characteristic scale of the change of parameters of arc plasma in gas-dynamic region, when considering the processes in arc column, the plasma anode layer can be regarded as infinitely thin. Thus, in terms of mathematical description of physical processes occurring in near-anode plasma of the welding arc, it can be divided into two regions: anode layer of nonequilibrium plasma, including the prelayer, as well as the layer of space charge, and arc column, for which the first region actually has the role of rupture surface.

Let us first consider the model of anode layer of a high-current arc, burning in inert gas at atmospheric pressure, provided that anode metal evaporation can be neglected. In this case, near-anode plasma can be considered one-component, containing atoms $(a)$, single-charged ions (i) and electrons $(e)$ of gas. We will neglect presence of multicharged ions in near-anode plasma that is a good enough approximation up to its temperature values of $\sim 1.5 \mathrm{eV}[22]$. Moreover, we will assume that the heavy particles of plasma (atoms and ions) have common temperature $T_{h}$, which differs from electron temperature $T_{e}$ (two-temperature model).

Following paper [23], we will use one-dimensional model of the anode layer. We will direct the $x$ axis of the Cartesian coordinate system away from the anode into the plasma, and will regard the anode surface as flat and located at $x=0$. Then, the basic equations of the model can be written as follows.

Continuity equations for electrons, ions and atoms of near-anode plasma:

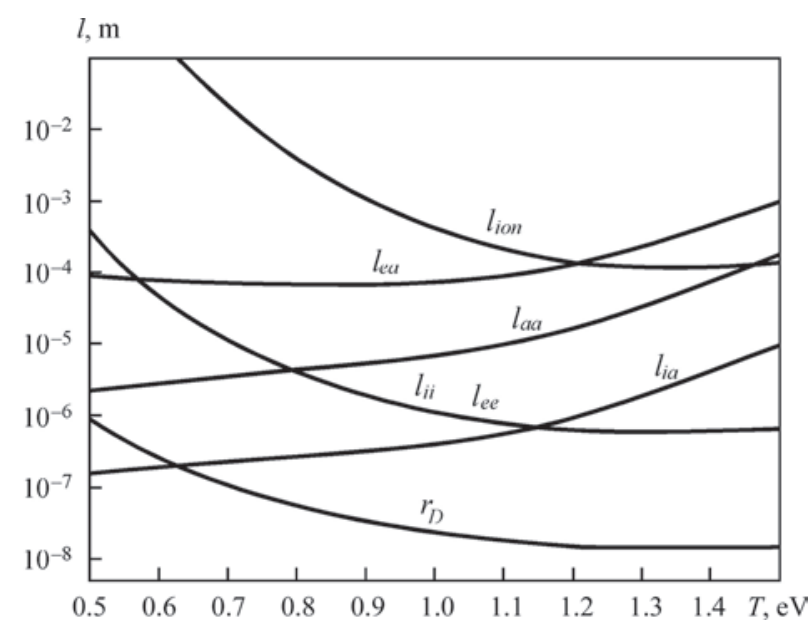

Figure 2. Characteristic lengths in equilibrium argon plasma at atmospheric pressure: $r_{D}$ — Debye radius; $l_{e e}, l_{i i}, l_{i a}, l_{a a}, l_{e a}$ - free paths relative to electron-electron, ion-ion, ion-atom, atom-atom and electron-atom collisions; $l_{\text {ion }}$ — ionization length

$$
\frac{d J_{\alpha}}{d x}=\omega_{\alpha}, \quad \alpha=e, i, a .
$$

Here $J_{\alpha}=n_{\alpha} v_{\alpha}$ is the flow density of particles of $\alpha$ species, where $n_{\alpha}$ and $v_{\alpha}$ are the concentration and velocity of the respective particles; $\omega_{\alpha}$ is the rate of change in the concentrations of plasma particles, owing to ionization-recombination reactions. As in atmospheric pressure discharges ionization occurs mainly through collision of electrons with atoms, and the dominating recombination mechanism is three-particle recombination involving an ion and two electrons, we can write

$$
\omega_{e}=\omega_{i}=-\omega_{a}=k_{i} n_{e} n_{a}-k_{r} n_{e}^{2} n_{i},
$$

where $k_{i}, k_{r}$ are the constants of the processes of ionization and recombination, respectively. Adding up continuity equations for ions and atoms, and subtracting the respective equations for ions and electrons, we get

$$
J_{i}=-J_{a} ; J_{i}-J_{e}=j_{a} / e,
$$

where $j_{a}$ is the density of electric current in the anode layer; $e$ is the elementary charge.

The first condition reflects the law of conservation of heavy particles of plasma, and the second - the continuity of electric current within the anode layer. It should be noted that relationships (4) can be used instead of any two continuity equations (2).

Equations of motion of plasma components can be written as [24]:

$$
\begin{gathered}
-\frac{d p_{\alpha}}{d x}+n_{\alpha} z_{\alpha} E+\sum_{\beta} v_{\alpha \beta} \mu_{\alpha \beta} n_{\alpha} n_{\beta}\left(v_{\beta}-v_{\alpha}\right)- \\
-R_{\alpha}^{T}=0, \quad \alpha, \beta=e, i, a .
\end{gathered}
$$

Here, $p_{\alpha}=n_{\alpha} k T_{\alpha}$ is the partial pressure of $\alpha$-component of plasma, where $k$ is the Boltzmann constant; $T_{\alpha}$ is the temperature of particles of $\alpha$ species; $z_{\alpha}$ is 
the charge of the respective particles; $E$ is the electric field intensity; $v_{\mathrm{a} \beta}, \mu_{\mathrm{a} \beta}=m_{\alpha} m_{\beta} /\left(m_{\alpha}+m_{\beta}\right)$ is the frequency of collisions with pulse transfer and reduced mass of particles of $\alpha$ and $\beta$ species, where $m_{\alpha}$ is the mass of particles of $\alpha$ species; $R_{\alpha}^{T}$ is the thermodiffusion force, defined as follows

$$
R_{\alpha}^{T}=C_{\alpha}^{(e)} n_{\alpha} k \frac{d T_{e}}{d x},
$$

where $C_{\alpha}^{(e)}$ is the coefficient of thermodiffusion of particles of $\alpha$ species. Note that the effect of thermodiffusion due to heavy particle temperature gradient is neglected.

Adding up equations (5) for all the components, we get the following relationship

$$
-\frac{d p}{d x}+e\left(n_{i}-n_{e}\right) E=0 .
$$

Here, $p=\sum_{\alpha} p_{\alpha}$ is the total pressure of plasma.

Equations of motion (5) should be complemented by Poisson equation for determination of electric field intensity

$$
\varepsilon_{0} \frac{d E}{d x}=e\left(n_{i}-n_{e}\right)
$$

where $\varepsilon_{0}$ is the electric constant. Combining equations (7) and (8), we obtain the following relationship

$$
\frac{d}{d x}\left(p-\varepsilon_{0} E^{2} / 2\right)=0,
$$

which means that total pressure in near-anode plasma, including pressure of electric field, is constant over the anode layer thickness.

Energy equations for electrons and heavy particles of plasma, neglecting convective transfer of energy by atoms and ions, have the following form:

$$
\begin{aligned}
\frac{d}{d x}\left(q_{e}+\frac{5}{2} J J^{k T_{e}}\right) & =j_{a} E+\kappa_{e h} n_{e} k\left(T_{h}-T_{e}\right)-w_{e} ; \\
\frac{d q_{h}}{d x} & =\kappa_{e h} n_{e} k\left(T_{e}-T_{h}\right) .
\end{aligned}
$$

Here, $q_{e}, q_{h}$ are the heat flows of electrons and heavy particles; $k_{e h}$ is the frequency of energy exchange between the electrons and heavy particles; $w_{e}=U_{i} \omega_{e}-$ $w_{\text {rad }}$ are the energy losses of electron component of plasma owing to nonelastic processes and radiation, where $U_{i}$ is the ionization potential of gas atoms; $w_{\text {rad }}$ are the radiation losses.

Ignoring the effect of thermodiffusion for atoms and ions, the heat flows of electrons and heavy particles of plasma can be represented in the following form:

$$
q_{e}=-\lambda \frac{d T_{e}}{d x}+k T_{e} n_{e} \sum_{\alpha} A_{\alpha}^{(e)}\left(v_{e}-v_{\alpha}\right)
$$

$$
q_{h}=-\lambda \frac{d T_{h}}{d x}
$$

where $\lambda_{e}, \lambda_{h}$ are the coefficients of heat conductivity of electrons and heavy particles, respectively; $A_{\alpha}^{(e)}$ is the kinetic coefficient.

Expressions given in $[22,23]$ can be used for calculation of the rates of ionization-recombination processes, transport and kinetic coefficients, as well as radiation energy losses, included into the equations of the described model.

Let us now go to the description of boundary conditions required for solving equations (2), (5), (8), (10), (11) in section $0 \leq x \leq \mathrm{L}_{\mathrm{a}}$. Edge conditions at $x=$ $=L_{a}$ (on the boundary of anode layer with arc column) are assigned proceeding from the taken assumption of the local thermodynamic equilibrium of plasma in arc column. Then, the concentrations of plasma particles on the above boundary can be determined from the equation of ionization equilibrium, law of partial pressures and condition of plasma quasineutrality:

$$
\begin{gathered}
k_{i} n_{e} n_{a}=k_{r} n_{e}^{2} n_{i} ; \\
p=n_{e} k T_{e}+n_{i} k T_{h}+n_{a} k T_{h} ; \\
n_{e}=n_{i} .
\end{gathered}
$$

At determination of temperatures $T_{h}, T_{e}$ on the outer boundary of the anode layer, we will assume that plasma is isothermal here, i.e. we will include the layer of nonisothermal plasma (see Figure 1) into the anode layer, and will assume that Joule heating of plasma is balanced by radiation losses of electron energy. In this case at $x=L_{a}$ we can take

$$
T_{h}=T_{e} ; j_{a} E=w_{r a d}\left(T_{e}\right) .
$$

Particle flows and electric field intensity on the outer boundary of the anode layer can be determined using relationships (4) and equations (5), allowing for the assumed uniformity of plasma in the arc column.

To assign edge conditions $x=0$ (on anode surface) we will assume that all the charged particles, reaching this surface, are absorbed by it (partially recombine, and the remaining electrons form electric current in the anode body), and electron flow due to their emission from the anode material, is absent. Then, proceeding from kinetic considerations, the electron flow at $x=0$ can be assigned as follows:

$$
J_{e}=-\frac{n_{e} \bar{v}_{e}}{4},
$$

where $\bar{v}_{e}=\sqrt{8 k T_{e} / \pi m_{e}}$ is the average velocity of thermal motion of electrons.

When assigning the edge condition for the ion flow to the anode surface, we will take into account the fact 

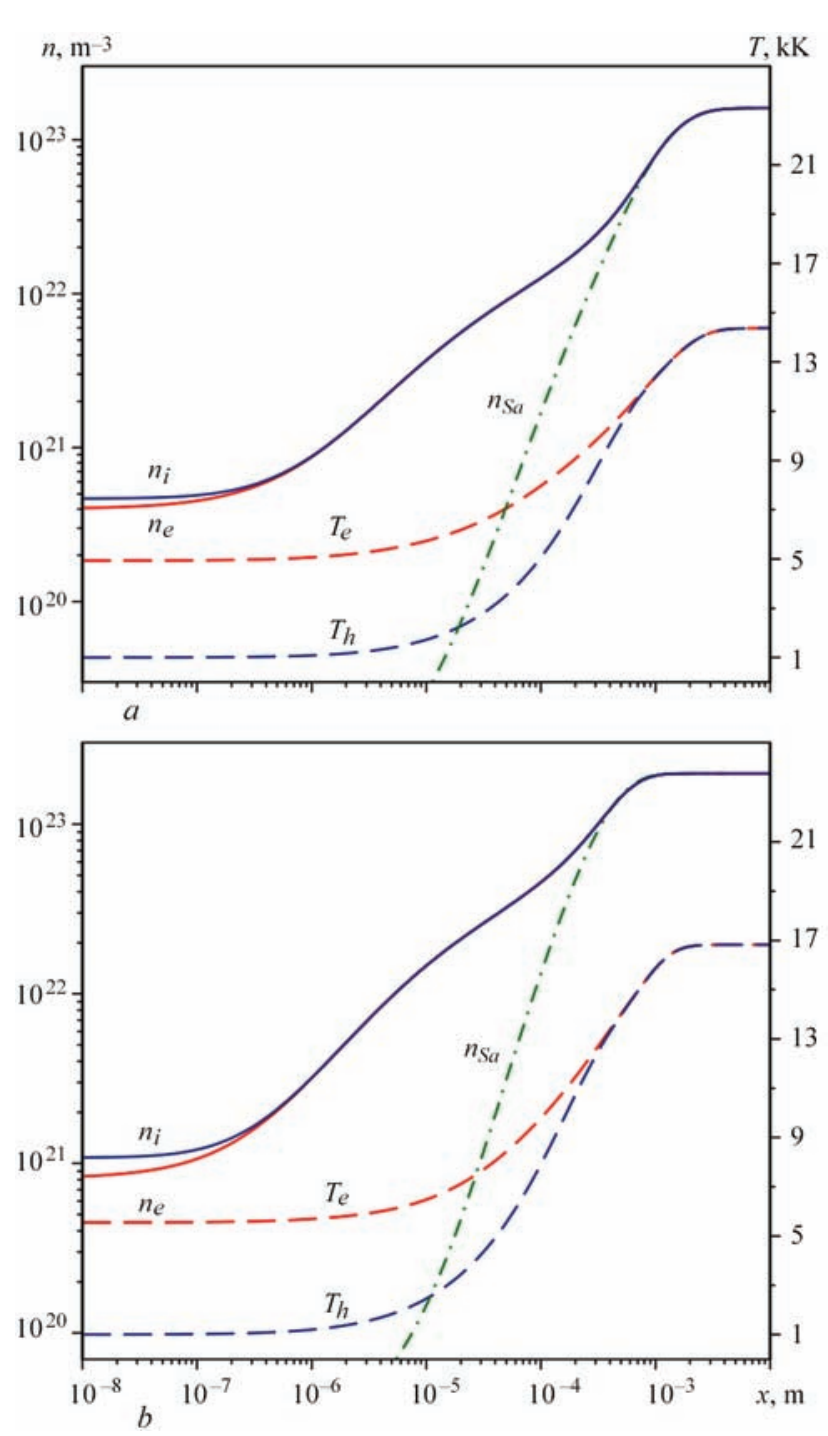

Figure 3. Distributions of the characteristics of atmospheric pressure argon arc plasma over the anode layer thickness for two values of anode current density: $a-j_{a}=700 ; b-1500 \mathrm{~A} / \mathrm{cm}^{2}$. Solid curves show concentrations of electrons $\left(n_{e}\right)$ and ions $\left(n_{i}\right)$; dashed lines are temperatures of electrons $\left(T_{e}\right)$ and heavy particles $\left(T_{h}\right)$; dash-dotted line corresponds to charged particle concentration $\left(n_{\text {Sa }}\right)$, calculated using Sakh equation

that the electron temperature near the above surface can be quite low $T \sim 0.5 \mathrm{eV}$ [22] and, according to Figure 2, the ion free path relative to collisions with atoms can turn out to be smaller than the Debye radius, i.e. thickness of the space charge layer. In this case, the space charge layer is collisional for ions, the flow of which at $x=0$ can be defined through ion mobility in an electric field:

$$
J_{i}=\frac{e E n_{i}}{v_{i a} \mu_{i a} n_{a}} .
$$

Electric field intensity near the anode surface, included into this expression, can be calculated by substituting expressions (18), (19) into the second condition (4) and using Poisson equations (8). In order to determine $T_{h}, T_{e}$ at $x=0$, we can use conditions from [23]:

$$
T_{h}=T_{s} ;
$$

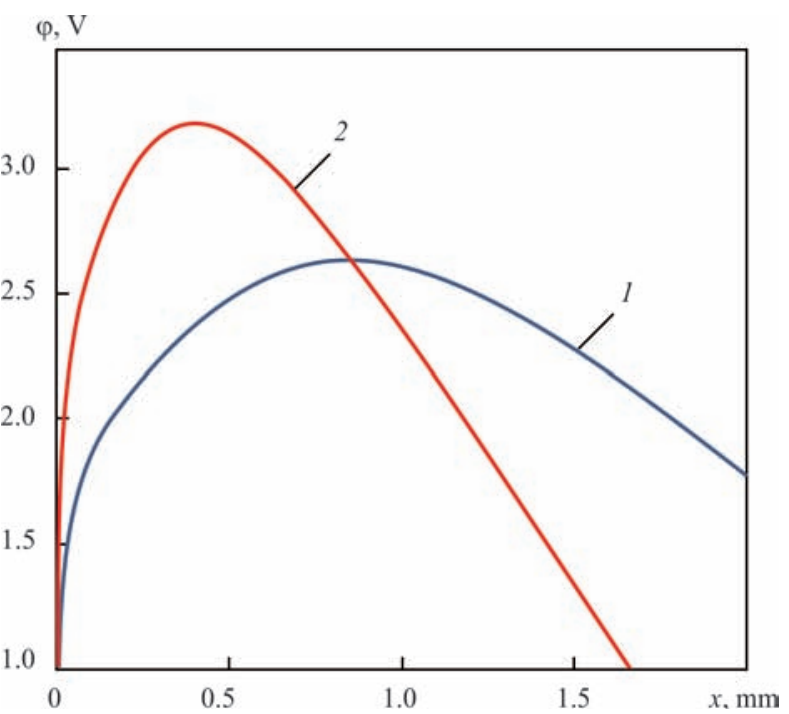

Figure 4. Distribution of electric potential over the thickness of argon arc anode layer at $j_{a}, \mathrm{~A} / \mathrm{cm}^{2}: 1-1000 ; 2-2000$

$$
-\frac{n_{e} v_{e}}{4} 2 k T_{e}=\frac{5}{2} J k T_{e}+q_{e},
$$

where $T_{s}$ is the anode surface temperature.

This completes description of the model of anode layer in an atmospheric pressure electric arc with nonevaporating anode. The model equations were solved numerically, and the solution algorithm is described in detail in [22]. Results of calculation of the characteristics of near-anode plasma of argon arc in a broad range of anode current densities $j_{a}\left(5000-2000 \mathrm{~A} / \mathrm{cm}^{2}\right)$ at pressure $p=1 \mathrm{~atm}$ and anode surface temperature $T_{\mathrm{s}}=1000 \mathrm{~K}$ are given in Figures 3-6 and in the Table.

Figure $3, a, b$ shows the distributions of arc plasma characteristics over the anode layer thickness for two $j_{a}$ values characteristic for high-current electric arcs, burning in argon at atmospheric pressure. As follows from calculated data given in these figures, the equilibrium plasma region (arc column), where $n_{e} \approx n_{i} \approx n_{S a}, T_{e} \approx T_{h}$, is located at distance $x=L_{a} \leq$ $\leq 1 \mathrm{~mm}$ from anode surface. Here, the ionization region, where $n_{e} \approx n_{i}>n_{S a}, T_{e}>T_{h}$, and the space charge layer, where $n_{i}>n_{e}$, are well resolved within the anode layer. Anode layer thickness $L_{a}$ decreases at increase of anode current density that is related to increase of plasma temperature in arc column (from $13.53 \mathrm{kK}$ at $j_{a}=500 \mathrm{~A} / \mathrm{cm}^{2}$ up to $18.04 \mathrm{kK}$ at $j_{a}=2000 \mathrm{~A} / \mathrm{cm}^{2}$ ) and respective intensification of the processes of ionization and energy exchange between plasma components. As regards electron temperature near the anode surface, it weakly depends on anode current density, and at $j_{a}$ changing from 500 to $2000 \mathrm{~A} / \mathrm{cm}^{2}$ it increases from $4.69 \mathrm{kK}$ up to $5.99 \mathrm{kK}$.

Figure 4 presents the distribution of electric potential over the anode layer thickness. Here, the anode surface potential was assumed to be zero. As follows 

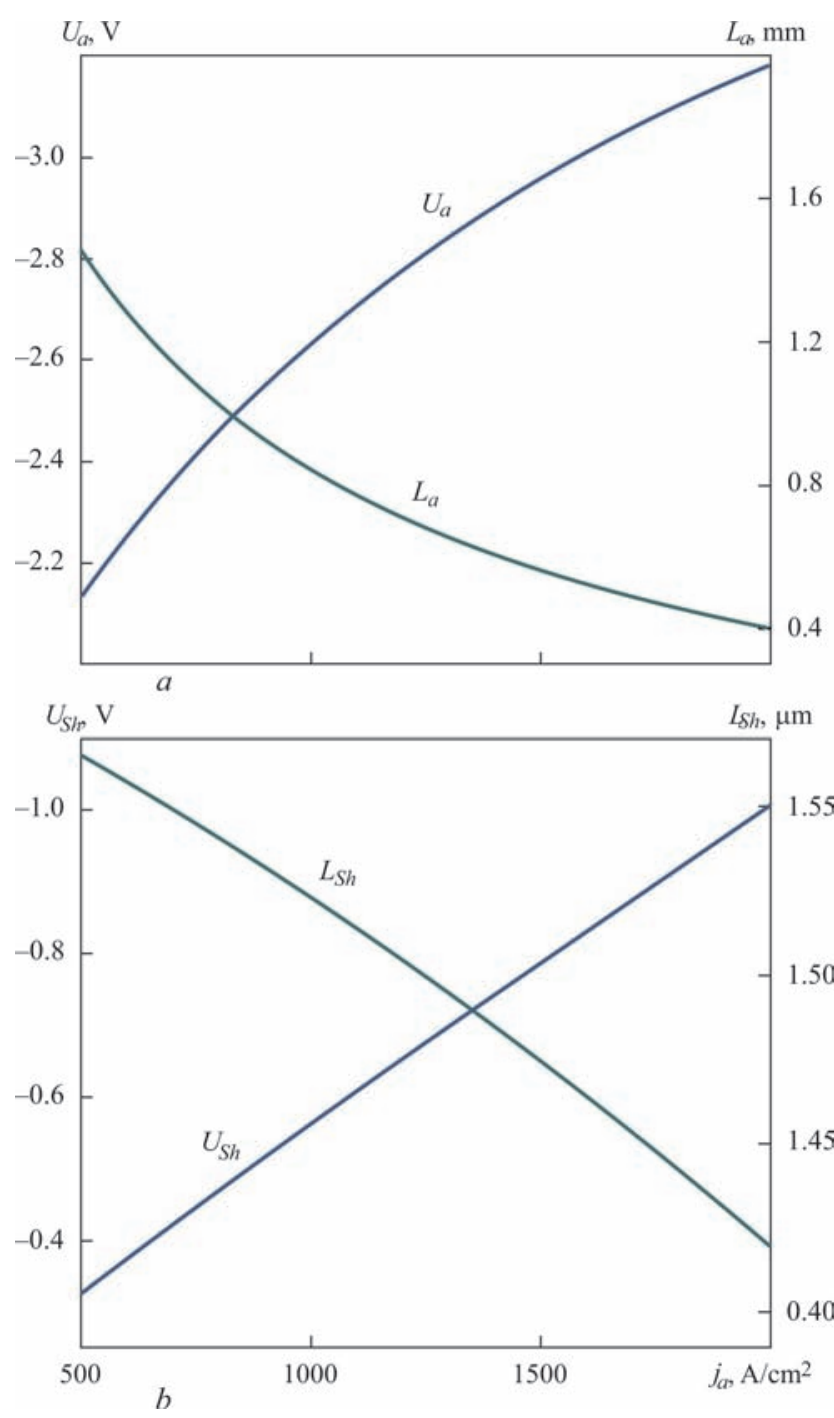

Figure 5. Anode layer thickness and anode potential drop (a); thickness of space charge layer and potential drop in this layer $(b)$ for argon arc, depending on anode current density

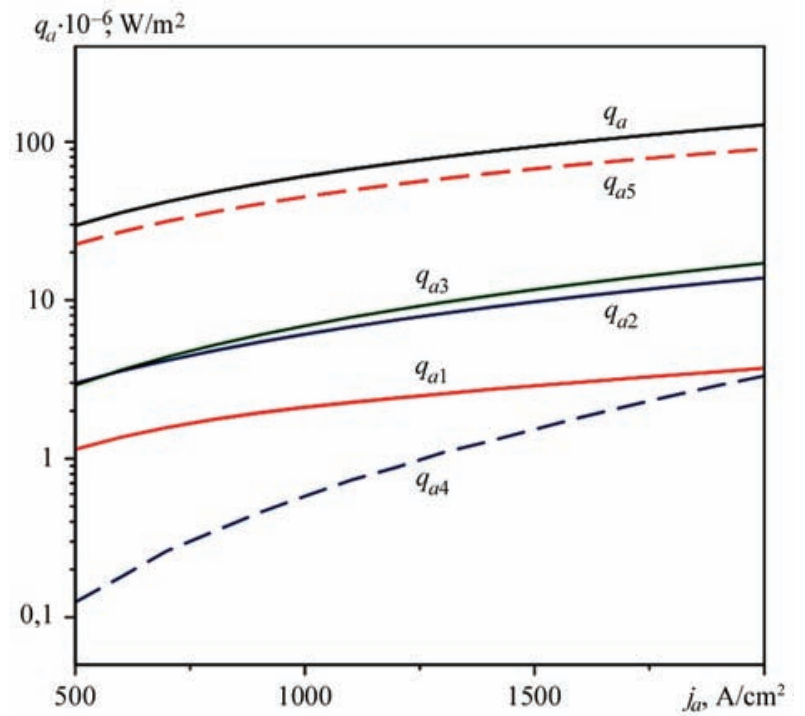

Figure 6. Total heat flow, applied to the anode by argon arc plasma, and its components, depending on anode current density from calculated data, given in this figure, distribution of electric potential in near-anode plasma is essentially nonuniform and has a maximum. In the maximum point the electric field associated with charged particles concentration gradients is equal by absolute value, and is directed opposite to the electric field, causing conduction current in the plasma. Therefore, this is exactly the point that can be regarded as anode layer boundary $x=L_{a}$ and, according to earlier accepted definition, the value of anode drop $U_{a}$ can be calculated as the difference of anode potential $\varphi_{a}=$ $=0$ and potential value $\varphi_{p a}$ on the above boundary (in maximum point). Dependencies of thus defined values $L_{a}$ and $U_{a}$ for atmospheric pressure argon arc with nonevaporating anode are given in Figure 5, a, from which it follows that anode layer thickness decreases with increase of anode current density, and anode drop increases by absolute value, remaining negative in the entire considered range of $j_{a}$, calculated $U_{a}$ values being in good agreement with those measured experimentally [18].

Figure $5, b$ gives the dependence of thickness $L_{S h}$ of space charge layer defined as the distance between anode surface and point, in which the relative charge separation $\Delta_{S h}=\left(n_{i}-n_{e}\right) / n_{e s}$, where $n_{e s}$ - electron concentration at $x=0$, is equal to $1 \%$, as well as dependence of potential drop $U_{S h}$ across the above layer on anode current density. Dependencies of $L_{S h}$ and $U_{S h}$ on $j_{a}$ keep the tendencies inherent in the respective dependencies of $L_{a}$ and $U_{a}$ (see Figure 5, a, b) with the difference that the thickness of space charge layer changes only slightly with increase of current density.

Let us now consider the heat flow $q_{a}$ contributed by arc plasma to the anode. The total heat flow can be represented as follows:

$$
q_{a}=q_{a 1}+q_{a 2}+q_{a 3}+q_{a 4}+q_{a 5} .
$$

Here, $q_{a 1}=\lambda_{e} \frac{d T_{e}}{d x}, q_{a 2}=\lambda_{h} \frac{d T_{h}}{d x}$ are the heat flows duetoheatconductivity of electronsandheavyparticles, respectively; $q_{a 3}=-\frac{5}{2} J e^{k T_{e}}-k T_{e} n_{e} \sum_{\alpha=i, a} A_{\alpha}^{(e)}\left(v_{e}-v_{\alpha}\right)$ is the heat flow due to convective energy transfer by plasma electrons; $q_{a 4}=-J_{i} U_{i}, q_{a 5}=\left(J_{i}-J_{e}\right) \varsigma_{a}$ (where $\varsigma_{a}$ is the work function of anode material) are the components of heat flow to the anode, due to recombination of ions and absorption of electrons by its surface.

Dependencies of heat flow $q_{a}$ and its components on $j_{a}$ at $\varsigma_{a}=4.5 \mathrm{eV}$ that corresponds to steel anode, are given in Figure 6. As follows from the presented dependencies, electron absorption by anode material makes the main contribution to the heat flow, and the next most important components are convective transfer of electron energy and the heat flow due to 
heat conductivity of heavy particles. As regards calculated values of $q_{a}$ and its components, they are in good agreement with the results of calculations performed in [6], and results of measurements taken by the authors of [18].

Completing description of the results of modeling the characteristics of near-anode plasma of atmospheric pressure argon arc with nonevaporating anode, we present a summary table of calculated values of anode layer thickness $L_{a}$, anode potential drop $U_{a}$, heat flow to the anode $q_{a}$ and volt equivalent of heat on the anode $V_{a}=q_{a} / j_{a}$ for atmospheric pressure argon arc at different values of anode current density $j_{a}$.

Let us now consider a simplified model of physical processes in near-anode plasma of the welding arc for the conditions of MIG, TIG or plasma welding. Under the thermal impact of the arc on electrode metal or metal being welded (anode), the surface of the drop or weld pool can be locally heated up to temperatures, comparable with metal boiling temperature $T_{B}$. As a result of anode metal evaporation, welding arc plasma, as was already mentioned, becomes multicomponent, containing, alongside the particles of shielding or plasma-forming gas also the atoms and ions of metal vapour.

At analysis of physical processes running in anode layer of multicomponent plasma of the welding arc, we will assume that on the outer boundary of this layer arc plasma is characterized by the following parameters: $n_{e}^{0}$ - electron concentration; $n_{\alpha Z}^{0}$ concentration of atoms $(Z=0)$ and ions $(Z=1)$ of shielding or plasma-forming gas. $(\alpha=g)$, atoms $(Z=$ $=0)$ and ions $(Z=1,2)$ of metal vapour $(\alpha=m)$; $Z e-$ ion charge; $T_{e}^{0}$ - electron temperature; $T_{h}^{0}-$ temperature of heavy plasma particles, assumed to be the same for all atom and ion species, but different from $T_{e}^{0}$ (two-temperature plasma model); $m_{e}$ - electron mass; $M_{\alpha}$ - masses of heavy particles (atoms and ions) of shielding gas $(\alpha=g)$ and metal being welded or electrode metal $(\alpha=m)$. As was already noted, the anode layer of welding arc plasma can be regarded as flat, that is why we will consider the values of concentrations $n_{e}^{0}, n_{\alpha Z}^{0}$, temperatures $T_{e}^{0}, T_{h}^{0}$ and normal to anode surface component of current density $j_{a} \equiv j_{a n}$ in the region of anode attachment of the arc, as local values, corresponding to a given point of anode surface and characterized by certain value of temperature $T_{\mathrm{s}}$.

We will, as we did earlier, assume that electric current transfer between arc plasma and anode is carried only by electrons and ions, coming from plasma (all the ions trapped on the anode surface, recombine there, and come back in the form of atoms, while the flow of electrons emitted by its surface, is negligibly
Main characteristics of the arc anode layer, depending on anode current density

\begin{tabular}{|c|c|c|c|c|}
\hline $\begin{array}{r}j_{a} \cdot 10^{-4}, \\
\mathrm{~A} / \mathrm{m}^{2}\end{array}$ & $\begin{array}{c}L_{a} \cdot 10^{-3}, \\
\mathrm{~m}\end{array}$ & $\begin{array}{c}U_{a}, \\
\mathrm{~V}\end{array}$ & $\begin{array}{c}q_{a} \cdot 10^{-6}, \\
\mathrm{~W} / \mathrm{m}^{2}\end{array}$ & $\begin{array}{c}V_{a}, \\
\mathrm{~V}\end{array}$ \\
\hline 500 & 1.46 & -2.13 & 21.5 & 4.30 \\
\hline 700 & 1.14 & -2.36 & 30.3 & 4.33 \\
\hline 1000 & 0.84 & -2.63 & 43.9 & 4.39 \\
\hline 1500 & 0.56 & -2.96 & 67.6 & 4.51 \\
\hline 2000 & 0.40 & -3.18 & 92.7 & 4.64 \\
\hline
\end{tabular}

small). Then, the total density of electric current between arc plasma and anode can be represented as

$$
j_{a}=j_{e}-j_{i}, \quad\left(j_{a}>0\right) .
$$

Here $j_{e}$ is the density of current of plasma electrons, reaching the anode surface; $j_{i}=\sum_{\alpha=m, g ; Z \geq 1} j_{\alpha Z}$ is the total density of ion current to anode surface (for ions of all species and charges).

Following [25] further we will approximate the electron component of arc plasma within the anode layer as collisionless (see Figure 2), and electron temperature as constant over its thickness. Moreover, as plasma potential on the anode layer boundary $\varphi_{p a}$ is higher than anode surface potential $\varphi_{a}[2,18-20]$, electrons are decelerated by the electric field, while ions are accelerated towards this surface. In this case, electron current density between the plasma and anode can be written as $[2,3]$ :

$$
j_{e}=\frac{1}{4} e n_{e}^{0} \bar{v}_{e}^{0} \exp \left(-\frac{e \varphi_{p a}}{k T_{e}^{0}}\right),
$$

where $\bar{v}_{e}^{0}=\sqrt{8 k T_{e}^{0} / \pi m_{e}}$ is the thermal velocity of electrons on the anode layer outer boundary.

Here, it should recalled that owing to high conductivity of anode metal, compared to that of near-anode plasma, its surface potential is practically constant in the region of anode attachment of the arc and is assumed to be zero (see Figure 1).

In order to determine ion currents, it is necessary to consider the processes in the ionization region, where ion generation and their acceleration towards the anode surface occur. For this purpose, we use an approach [26], which is based on the assumption that ion free path relative to Coulomb intercollisions $l_{i i}$ is much smaller than ionization length $l_{\text {ion }}$ and their free path relative to collisions with atoms $l_{i a}$ (see Figure 2). This suggests that all the ions in the prelayer are intensively maxwellized and acquire a common velocity of directional movement, the value of which on the boundary of ionization region with space charge layer in the diffusion mode of anode metal evaporation is determined by the following expression [25]: 


$$
V_{i_{S h}} \equiv V_{i}\left(L_{S h}\right)=\sqrt{\frac{\sum_{\alpha=m, g ; Z \geq 1} k\left(Z T_{e}^{0}+T_{h}^{0}\right) n_{\alpha Z}^{0}}{\sum_{\alpha=m, g ; Z \geq 1} m_{\alpha} n_{\alpha Z}^{0}}} .
$$

Selecting as the boundary of the prelayer with space charge layer such an $x=L_{S h}$, at which the condition of plasma quasineutrality is disturbed [27], we find the concentrations of charged particles on this boundary [25]:

$$
\begin{aligned}
& n_{e_{S h}} \equiv n_{e}\left(L_{S h}\right)=n_{e}^{0} \exp \left(-\frac{1}{2}\right) ; \\
& n_{\alpha Z_{S h}} \equiv n_{\alpha Z}\left(L_{S h}\right)=n_{\alpha Z}^{0} \exp \left(-\frac{1}{2}\right) \text {, } \\
& \alpha=m, g ; \quad Z \geq 1 \text {. }
\end{aligned}
$$

The ion currents to anode surface can be written as follows:

$$
j_{\alpha Z}=Z \operatorname{Zen}_{\alpha Z}^{0} \exp \left(-\frac{1}{2}\right) V_{i_{S h}}, \quad \alpha=m, g ; Z \geq 1 .
$$

Knowing the electron and ion currents to anode surface, it is easy to find from equation (23) the plasma potential $\varphi_{p a}$ relative to the specified surface

$$
\varphi_{p a}=\frac{k T_{e}^{0}}{e} \ln \left(\frac{e n_{e}^{0} \bar{v}_{e}^{0}}{4\left[j_{a}+\sum_{\alpha=m, g ; Z \geq 1} j_{\alpha Z}\right]}\right) .
$$

The anode potential drop $U_{a}$ is more often used in welding publications instead of $\varphi_{p a}$ value. It is defined as the difference of potentials between anode surface and outer boundary of anode layer of arc plasma. Considering that anode surface potential was taken to be zero at $\varphi_{p a}$ definition, we obtain $U_{a}=-\varphi_{p a}$. Thus, as was already noted, the anode potential drop is negative in welding arcs. Moreover, taking into account the respective distributions of values, included into expression (28), along the anode surface $\varphi_{p a}$ can be essentially nonuniform in the region of anode attachment of the arc, i.e. it can depend on the coordinate along anode layer boundary. This may lead to appearance of the component of electric potential gradient, and, accordingly, component of current density along the boundary of arc column with anode layer, the direction and magnitude of which determine the pattern of electric current flowing between the arc plasma and the anode (arc contraction on the anode or discharge distributed over the anode surface).

Calculation of the distributions of $j_{e}, j_{\alpha z}, \varphi_{p a}$ or $U_{a}$ values along the boundary of anode layer with arc column requires knowledge of the respective distributions of temperatures $T_{e}^{0}, T_{h}^{0}$ and concentrations $n_{e}^{0}$, $n_{\alpha Z}^{0}$ of charged particles on the mentioned boundary, as well as distributions of normal to the anode surface component of electric current density $j_{a}$. Assuming the multicomponent plasma in welding arc column to be ionization-equilibrium, the composition of such plasma in each point of the mentioned boundary can be determined using the following system of equations:

Sakh equations allowing for plasma nonideality

$$
\begin{gathered}
\frac{n_{e}^{0} n_{\alpha Z+1}^{0}}{n_{\alpha Z}^{0}}=\left(\frac{2 \pi m_{e} k T_{e}^{0}}{h^{2}}\right)^{3 / 2} \frac{2 \theta_{\alpha Z+1}}{\theta_{\alpha Z}} \times \\
\times \exp \left[-\frac{e\left(U_{\alpha Z}-\Delta U_{Z}\right)}{k T_{e}^{0}}\right], \quad \alpha=m, g ; Z \geq 0 .
\end{gathered}
$$

Here $h$ is the Planck's constant; $\theta_{\alpha z}$ are the statistical sums for heavy particles of $\alpha$ species, which are in charge state $Z ; U_{\alpha Z}$ are the ionization potentials (for transition of particles of $\alpha$ species from charge state $Z$ into $Z+1) ; \Delta U_{Z}=(e(Z+1)) / r_{D}$ are the reductions of ionization potentials, which are due to interaction of charged particles in the plasma [28], where

$$
r_{D}=\left[k T_{e}^{0} \varepsilon_{0} / e^{2}\left(n_{e}^{0}+\frac{T_{e}^{0}}{T_{h}^{0}} \sum_{\alpha=m, g ; Z \geq 1} n_{\alpha Z}^{0} Z^{2}\right)\right]^{1 / 2}
$$

is the Debye radius.

Condition of multicomponent plasma quasineutrality

$$
n_{e}^{0}=\sum_{\alpha=m, g ; Z \geq 1} n_{\alpha Z}^{0} Z .
$$

Law of partial pressures

$$
p=n_{e}^{0} k T_{e}^{0}+\sum_{Z \geq 0} n_{m Z}^{0} k T_{h}^{0}+\sum_{Z \geq 0} n_{g Z}^{0} k T_{h}^{0}-\Delta p .
$$

Here, $p$ is the plasma pressure near the anode surface; $\Delta p=\frac{1}{6} \frac{e^{2}}{r_{D}}\left(n_{e}^{0}+\sum_{\alpha=m, g ; Z \geq 0} n_{\alpha Z}^{0} Z^{2}\right) \quad$ is the pressure lowering, because of plasma nonideality [28].

In order to close the system of equations (29)-(31), one more condition is required, which determines the distribution of the concentration of heavy particles (atoms and ions) of metal vapour along the anode layer boundary. In the diffusion mode of anode metal evaporation, assuming that the rate of vapour diffusion in arc plasma is small, i.e. vapour state is close to saturation, we can select as such a condition the equality of local value of partial pressure of evaporated metal heavy particles on the above boundary to the saturated vapour pressure $p_{s}$ over the metal surface, having the respective temperature value $T_{s}$ :

$$
\sum_{Z \geq 0} n_{m Z}^{0} k T_{h}^{0}=p_{s} \equiv p_{0} \exp \left[\frac{\lambda_{v}}{k}\left(\frac{1}{T_{B}}-\frac{1}{T_{s}}\right)\right]
$$


where $p_{0}$ is the atmospheric pressure; $\lambda_{v}$ is the work function of anode metal atom; $T_{h}^{0}=T_{s}$.

As an example, Figure 7 gives the thus calculated values of anode potential drop $U_{a}$ as a function of $T_{e}^{0}, j_{a}$ and $T_{s}$ under the conditions, characteristic for TIG welding of low-carbon steel (Fe is the evaporating element) in argon. As follows from calculated data presented in this figure, the values of anode potential drop are negative, increasing by absolute value with increase of electron temperature of plasma near the anode, as well as its surface temperature (see Figure 7, $a, c)$, and somewhat decreasing with $j_{a}$ increase (see Figure 7,b). Value $U_{a}$ for the considered conditions falls in the range from -1 to $-4 \mathrm{~V}$, that correlates quite well with the experimental data of [18], and in the case of small values of anode surface temperature, when evaporation of its material can be neglected, with the results of calculations made in [22] for nonevaporating anode.

Let us now consider the processes of energy transfer in anode layer of multicomponent plasma of the welding arc. Heat flow $q_{a}$ from near-anode plasma to the surface of metal being welded can be represented as follows:

$$
q_{a}=q_{e}+q_{i},
$$

where $q_{e}, q_{i}$ are the flows of potential and kinetic energy, carried by plasma electrons and ions, respectively.

Expression for $q_{e}$ can be written in a simplified form as $[2,18]$ :

$$
q_{e}=j_{e}\left(\frac{5 k T_{e}^{0}}{2 e}+\zeta_{a}\right) .
$$

Considering the initial energy of ions on the outer boundary of space charge layer, as well as their additional acceleration in this layer, we can write for $q_{i}$

$$
q_{i}=\sum_{\alpha=m, g ; Z \geq 1} j_{\alpha Z}\left(\varphi_{S h}+\frac{M_{\alpha} V_{i_{S h}}^{2}}{2 Z e}+\frac{1}{Z} \sum_{Z^{\prime}=1}^{Z} U_{\alpha Z^{\prime}}-\zeta_{a}\right),
$$

where $\varphi_{S h} \equiv \varphi\left(L_{S h}\right)=\varphi_{p a}-\frac{1}{2} \frac{k T_{e}^{0}}{e}$ is the potential of arc plasma on the boundary of space charge layer (see Figure 1).

Expression (33) can be rewritten as

$$
q_{a}=j_{a} V_{a},
$$

where $V_{a}$ is the value of volt equivalent heat, released on the anode, which, unlike the respective value of anode drop $U_{a}$, is always positive (see Table). Considering (33)-(35) for calculating $V_{a}$ we find

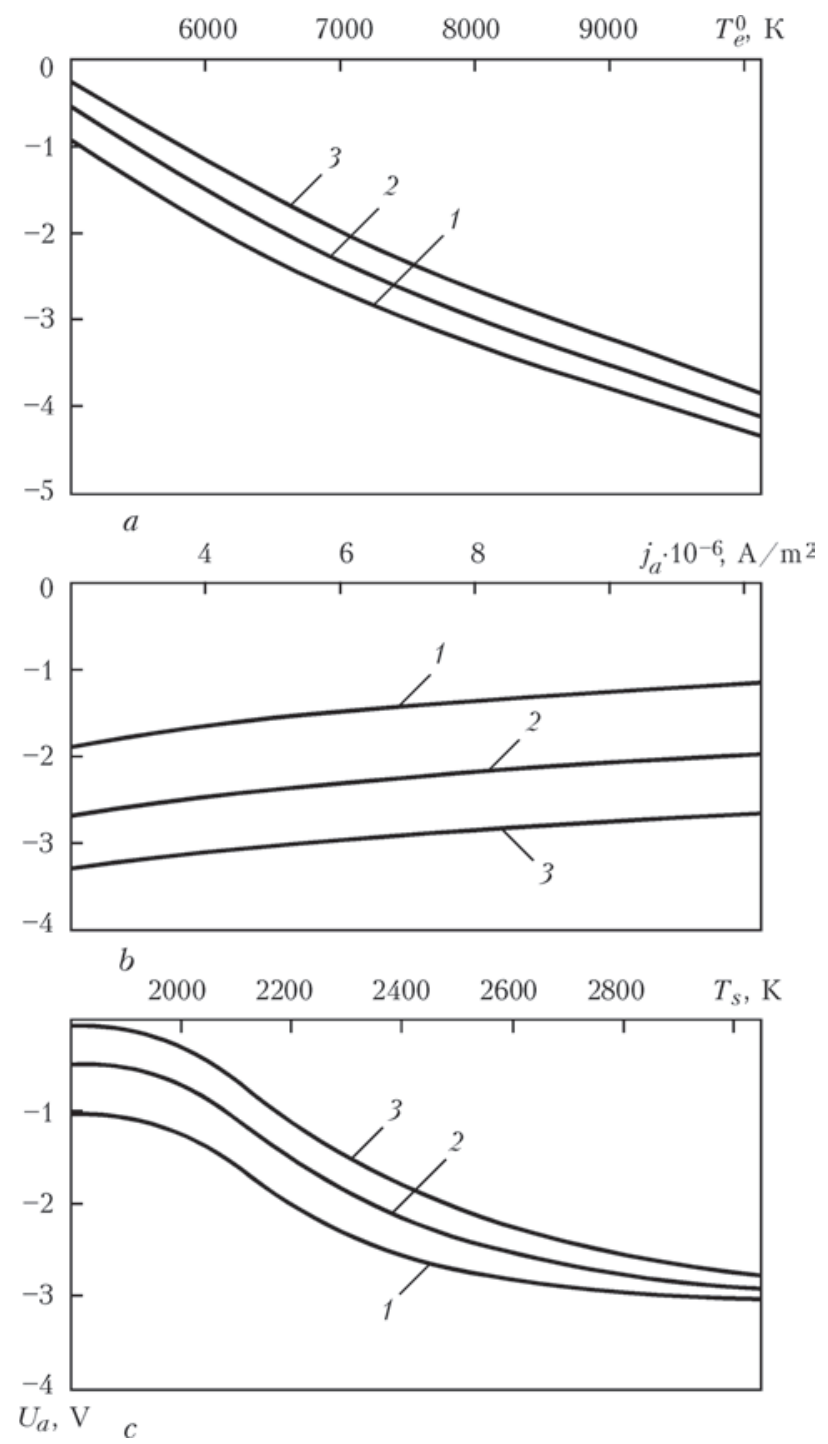

Figure 7. Dependencies of anode potential drop on electron temperature on anode layer boundary $(a)$, anode current density $(b)$ and its surface temperature $(c)$ under the conditions, characteristic for TIG welding of low-carbon steel in argon: $a-T_{\mathrm{s}}=2472 \mathrm{~K}$ $\left(1-j_{a}=2 \cdot 10^{6} ; 2-5 \cdot 10^{6} ; 3-10^{7} \mathrm{~A} / \mathrm{m}^{2}\right) ; b-T_{s}=2472 \mathrm{~K}(1-$ $\left.T_{e}^{0}=6 \cdot 10^{3} ; 2-7 \cdot 10^{3} ; 3-8 \cdot 10^{3} \mathrm{~K}\right) ; c-T_{e}^{0}=7 \cdot 10^{3} \mathrm{~K}\left(1-j_{a}=\right.$ $\left.=2 \cdot 10^{6} ; 2-5 \cdot 10^{6} ; 3-10^{7} \mathrm{~A} / \mathrm{m}^{2}\right)$

$$
\begin{gathered}
V_{a}=\varsigma_{a}+\frac{j_{e}}{j_{a}} \frac{5 k T_{e}^{0}}{2 e}+ \\
+\sum_{\alpha=m, g ; Z \geq 1} \frac{j_{\alpha Z}}{j_{a}}\left(\varphi_{S h}+\frac{M_{\alpha} V_{i_{S h}}^{2}}{2 Z e}+\frac{1}{Z} \sum_{Z^{\prime}=1}^{Z} U_{\alpha Z^{\prime}}\right) .
\end{gathered}
$$

Figure 8 shows the results of calculations of the heat flow to the anode as a function of $T_{e}^{0}, j_{a}$ and $T_{s}$ under the conditions, characteristic for TIG welding of low-carbon steel in argon. As follows from the given calculated dependencies, value $q_{a}$ increases with electron temperature in plasma anode layer, normal component of anode current density and its surface temperature, this tendency being the strongest in $q_{a}\left(T_{s}\right)$ dependence (see Figure 8, $c$ ). 

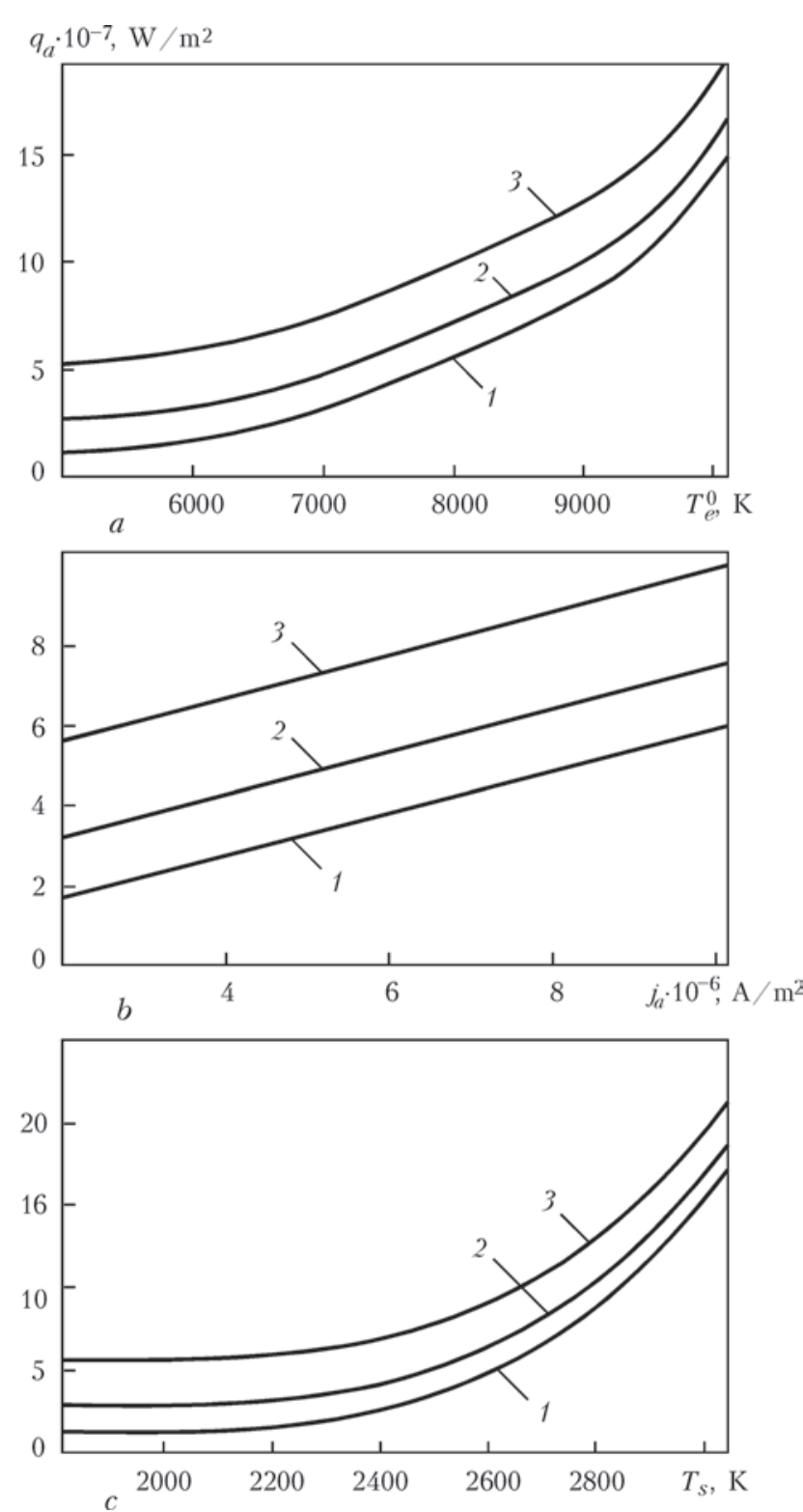

Figure 8. Dependencies of heat flow to the anode on electron temperature on anode layer boundary $(a)$, anode current density $(b)$ and its surface temperature $(c)$ under the conditions characteristic for TIG welding of low-carbon steel in argon (parameters and designations are the same as in Figure 7)

Let us now consider the distributions of near-anode plasma characteristics along the boundary of anode layer with welding arc column for the conditions of TIG welding of low-carbon steel in argon atmosphere. For calculation of the temperature and composition of multicomponent plasma on the mentioned boundary, determining the respective distributions of values $\varphi_{p a}$ or $U_{a}, j_{a}$ and $q_{a}$ over the region of anode attachment of the arc, we will use the proposed in [29] and modified in [30] self-consistent model of the processes of energy-, mass- and charge-transfer in the column and anode region of an electric arc, burning between a refractory cathode and flat evaporating anode.

We will consider two characteristic variants of thermal state of the anode, namely diffusively evap- orating anode ( $\mathrm{Fe}$ is the evaporating element) and water-cooled (nonevaporating) anode with surface temperature $T_{\mathrm{s}}=500 \mathrm{~K}$. In the first case, we assume that the anode surface temperature in the region of the arc anode attachment changes by an exponential law $T_{s}(r)=\left(T_{s 0}-T_{\infty}\right) \exp \left(-a^{2} r^{2}\right)+T_{\infty}$ and in the center of this region it reaches value $T_{s 0}$, not exceeding the temperature, at which evaporation goes into the convective mode [25]. Here, $r$ is the radial coordinate, calculated from the arc axis; $T_{\infty}=500 \mathrm{~K}$ is the surface temperature at considerable distance from the axis, and concentration rate $a$ is defined so that the radius of molten zone on steel anode surface was equal to $2.5 \mathrm{~mm}[21]$.

Calculations of spatial distributions of temperature, composition and other characteristics of near-anode arc plasma were conducted at the following parameters: arc length $L=3 \mathrm{~mm}$; arc current $I=200 \mathrm{~A}$; anode surface temperature $T_{s 0}$ in the center of arc attachment region was varied in the range from 500 up to $3065 \mathrm{~K}$. Results of calculation of $\gamma_{a}(r), T_{p a}, \varphi_{p a}(r)$, $j_{a}(r)$ and $q_{a}(r)$ distributions for different thermal states of the anode are given in Figures 9-13. Here, parameter $\gamma_{a}=n_{m} /\left(n_{g}+n_{m}\right)$, where $n_{m}, n_{g}$ are the summary concentrations of heavy particles (atoms and ions) of metal and shielding gas, characterizes metal vapour content in near-anode plasma; $T_{p a}$ is the temperature of plasma on the outer boundary of anode layer, and the other quantities were defined earlier. Curves 1-3 in the mentioned figures correspond to the following $T_{\text {s0 }}$ values: 2700,$3000 ; 3065 \mathrm{~K}$ (evaporating anode), dashed curves show the respective dependencies for an arc with nonevaporating anode at $T_{s}=500 \mathrm{~K}$.

As follows from Figure 9, maximum content of metal vapour in arc plasma is achieved on anode layer axis, increasing with $T_{s 0}$. Here, the maximum value of the rate of vapour outflow from anode surface also increases at the specified temperature rise. So, for instance, at $T_{s 0}=3000 \mathrm{~K}$ this rate can reach the value of more than $10 \mathrm{~m} / \mathrm{s}$. Such an intensive flow of relatively cold vapour, moving from anode surface into the arc column, causes local cooling down of near-anode plasma. This effect is manifested in that part of the anode layer, which is adjacent to the most heated zone of anode surface, and the stronger, the higher is the surface temperature in this zone (see Figure 10).

Despite the fact that increase of the concentration of easily ionizable (compared to $\mathrm{Ar}$ ) metal (Fe) vapour in near-anode plasma with $T_{s 0}$ rise should lead to increase of its electric conductivity $\sigma$, and, consequently, increase of electric current density, the above-noted effect of local cooling of plasma has a more essential role, causing a lowering of electric current density in paraxial zone of the region of anode 


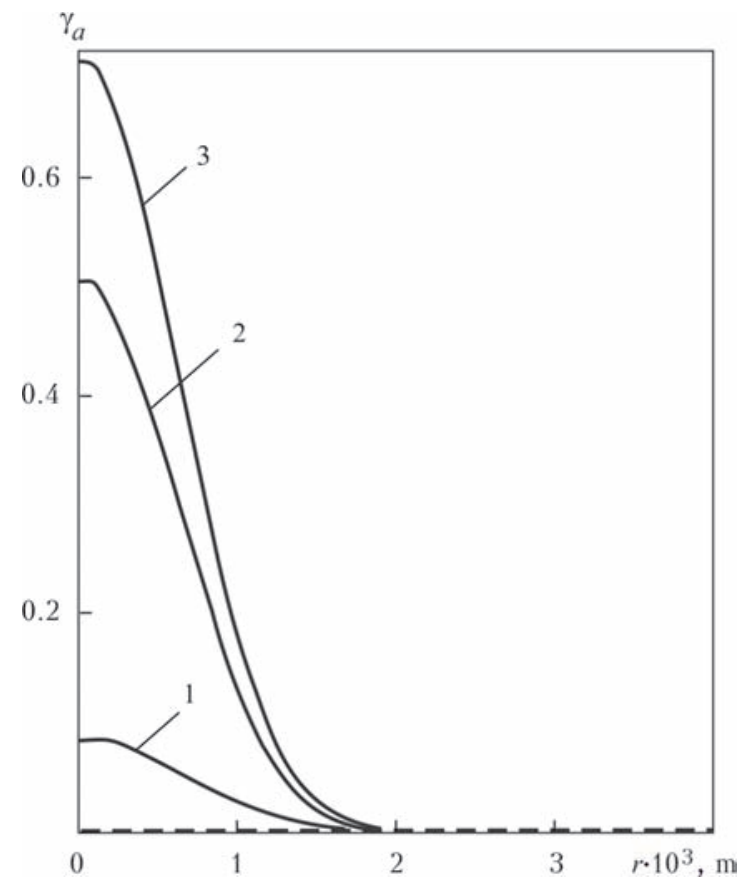

Figure 9. Radial distributions of the fraction of heavy particles of iron in multicomponent arc plasma $(\mathrm{Ar}-\mathrm{Fe})$ on the boundary of arc column with anode layer under the conditions characteristic for TIG welding of low-carbon steel in argon (for designations of curves $1-3$ see the text)

attachment of the arc with evaporating anode, as follows from Figure 11.

The cause of such behaviour of value $j_{a}(r)$ in the case of evaporating anode is the shown in Figure 12 local decrease of plasma potential $\varphi_{p a}$ in the center of the region of anode attachment of the arc, associated, according to the data in Figure 7, $a$, with the respective lowering of the temperature of near-anode plasma electrons (see Figure 10). Calculated dependencies of $\varphi_{p a}(r)$ shown in Figure 12, reveal that in case

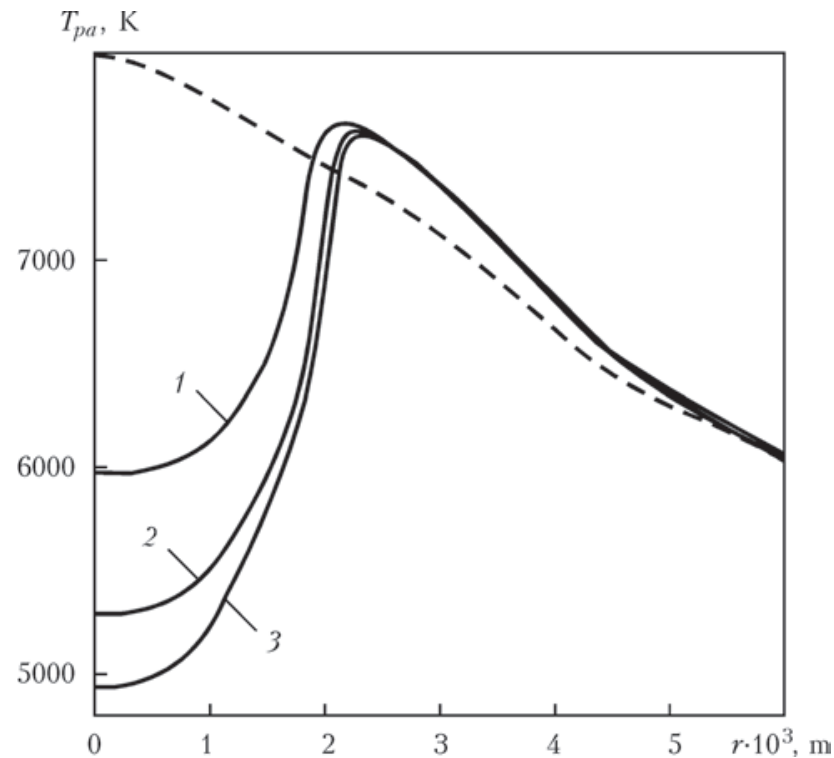

Figure 10. Radial distributions of arc column plasma temperature on the boundary with anode layer (for designations of curves 1-3 see the text)

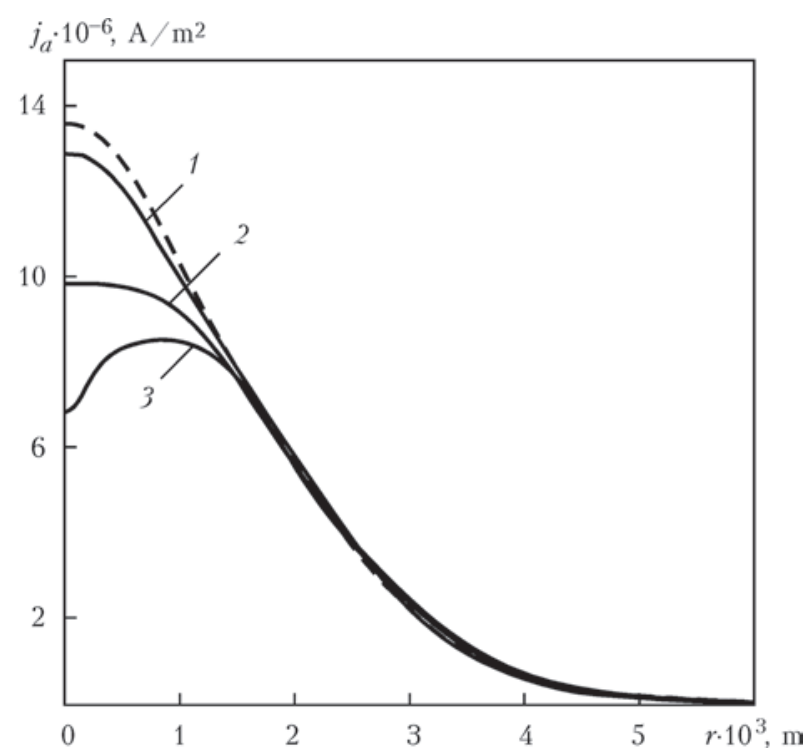

Figure 11. Radial distributions of anode current density (for designations of curves $1-3$ see the text)

of evaporation of anode metal, a radial component of electric current density $j_{a r}=-\sigma_{p a} \frac{\partial \varphi_{p a}}{\partial r}$ appears on the boundary of anode layer with arc column, where $\sigma_{p a}$ is the electric conductivity of plasma on the mentioned boundary. This component is directed towards the arc axis and increases with $T_{\text {s0 }}$ rise. It leads to an essential lowering of current density in the center of the region of anode attachment of the arc with evaporating anode relative to the arc with water-cooled (nonevaporating) anode.

Density of heat flow, contributed by the arc to the evaporating anode (see Figure 13) demonstrates similar behaviour. Considerable lowering of $q_{a}$ at high temperatures of the anode surface, is related to reduction of the convective flow of energy from the arc column, as a result of the respective change of gas-dynamic

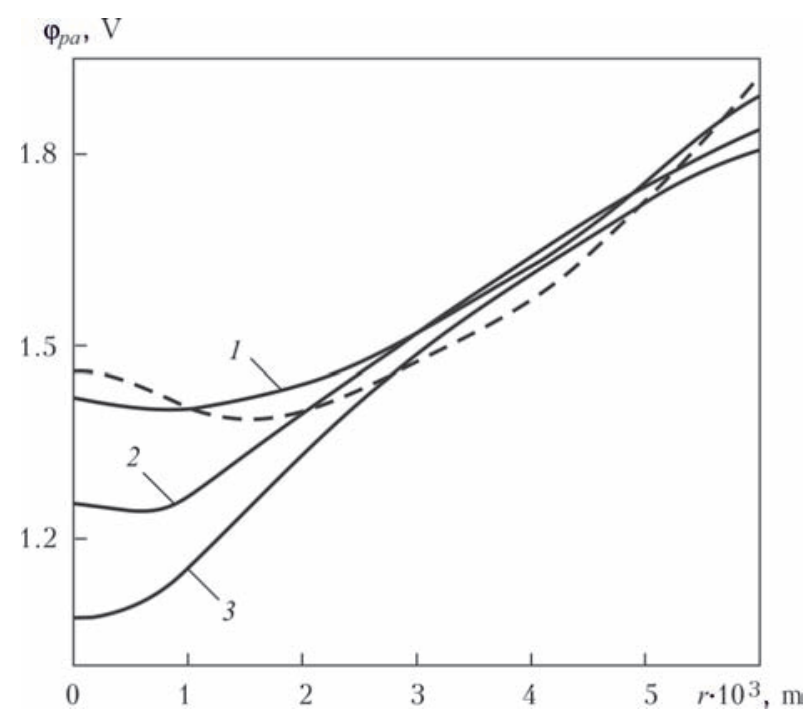

Figure 12. Radial distributions of arc plasma potential on the boundary of the anode layer with arc column (for designations of curves $1-3$ see the text) 


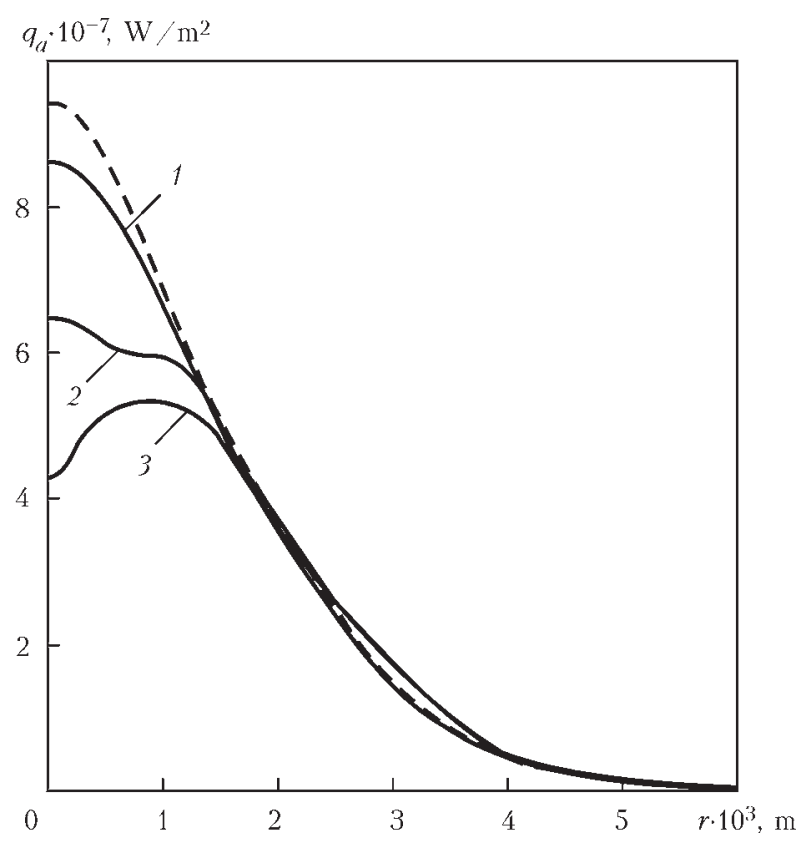

Figure 13. Radial distributions of heat flow applied by the arc to the anode (for designations of curves 1-3 see the text)

and electromagnetic situation in near-anode region of arc plasma, as well as to reduction of heat flow carried to the anode by electrons, owing to above-mentioned local reduction of $j_{a}$ (see Figure 11).

We will perform qualitative analysis of the effect of nonuniformity of distribution of $\varphi_{p a}$ or $U_{a}$ values over the region of arc anode attachment and its impact on the features of welding arc burning, as well as on the nature of penetration of the metal being welded in TIG welding and electrode metal transfer in MIG/ MAG welding.

Figure 14 schematically shows two possible distributions of electrical characteristics of arc plasma near

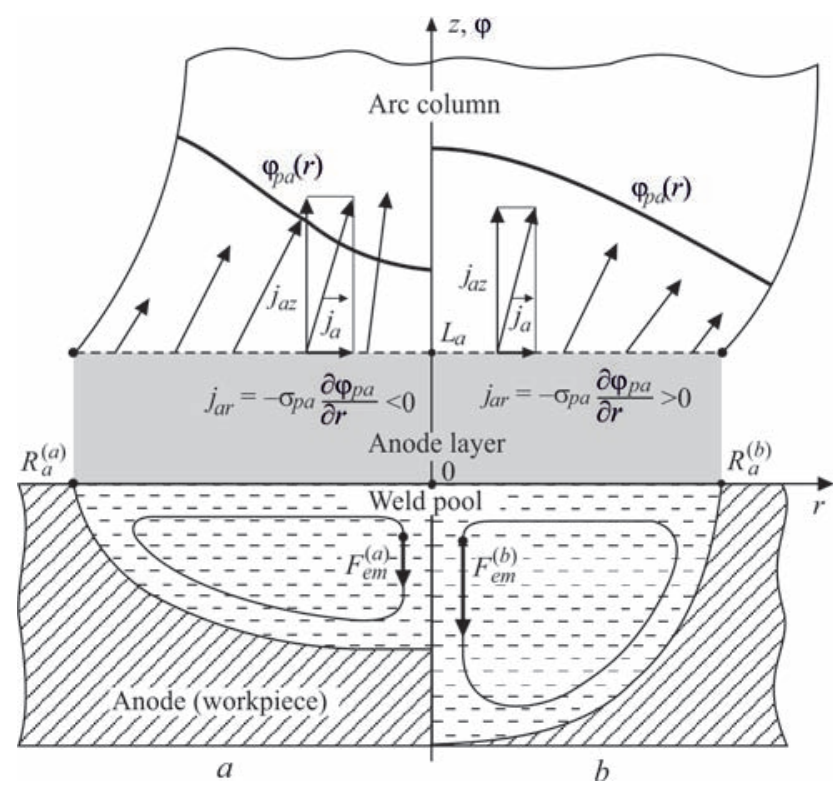

Figure 14. Schematic representation of electrical characteristics of near-anode plasma of the welding arc and process of metal penetration in TIG welding: $a$ - discharge distributed over the anode surface; $b-$ arc contraction on the anode the surface of metal being welded (anode), under the conditions, characteristic for spot TIG welding (weld pool is assumed to be axisymmetric, and its surface to be flat). Figure 14, $a$ presents a situation, when distribution of electric potential on the boundary of anode layer with arc column $\varphi_{p a}(r)$ is an increasing function of radius (distance from arc axis). In this case, the radial component of current density on the above boundary $j_{a r}=-\sigma_{p a} \frac{\partial \varphi_{p a}}{\partial r}$ is negative, and accordingly, anode current density turns out to be smaller than that in arc column, and the transverse dimension of current channel is greater, that corresponds to a discharge distributed over the anode. In the opposite case, when $\partial \varphi_{p a} / \partial r<0$ (see Figure 14, $b$ ) anode current density increases, and current channel radius becomes smaller, i.e. arc contraction on the anode $R_{a}^{(b)}<R_{a}^{(a)}$ is observed (see Figure 14, $a, b$ ). As a result, the electromagnetic force, applied to molten metal of the weld pool in its paraxial zone, turns out to be greater, than in the first case $F_{e m}^{(b)}>F_{e m}^{(a)}[31]$. This leads to a more intensive convective heat transfer from the most heated central part of pool surface to its bottom, thus increasing the depth of penetration of the metal being welded (see Figure 14, $a, b)$.

In the case of MIG/MAG welding, as shown in Figure 15, two variants of arc attachment to electrode metal drop are also possible. In the first case (see Figure $15, a$ ), when distribution of electric potential on the boundary of anode layer with arc column $\varphi_{p a}(S)$ is the increasing function of coordinate $s$, measured from top of the drop along the generatrix of its surface, the tangential component of current density on the mentioned boundary $j_{a s}=-\sigma_{p a} \frac{\partial \varphi_{p a}}{\partial s}$ is negative, and, accordingly, current density on the drop surface turns out to be smaller, than in the arc column, and area of arc anode attachment is larger than the cross-sectional area of its column, respectively. In the case, when $\partial \varphi_{\mathrm{pa}} / \partial s<0$ (see Figure 15, b), the tangential component of current density on the anode layer boundary is directed away from the drop top to arc periphery, that is manifested in arc contraction on the drop surface. As a result, $s_{a}^{(b)}<s_{a}^{(a)}$, and, accordingly, electromagnetic force applied to the drop turns out to be greater in the first case, than in the second case $F_{e m}^{(a)}>F_{e m}^{(b)}$ [32]. This leads to the drop size being smaller in the first case (see Figure 15, a) than in the second case (see Figure 15, b).

The above-noted nonuniformity of anode potential drop along the anode surface requires determination of integral (effective) value of anode drop $\left\langle U_{a}\right\rangle$, which summed up with appropriately determined 
cathode potential drop $\left\langle U_{c}>\right.$ and column voltage $\left\langle U_{p}>\right.$ should, according to (1), yield total arc voltage $U=\left\langle U_{c}\right\rangle+\left\langle U_{p}\right\rangle+\left\langle U_{a}\right\rangle$ As the potentials of the surface of metal anode and cathode can be considered constant with sufficient accuracy (because of the high electric conductivity of metal), arc voltage can be defined as the difference of potentials of the surface of anode and cathode, i.e. $U=\varphi_{a}-\varphi_{c}$ can be assumed (see Figure 1). However, such a generally accepted definition of voltage as an integral electrical characteristic of the arc discharge is not applicable for calculation of cathode and anode drops, as plasma potential on anode layer boundary $\varphi_{p a}$, as well as plasma potential on cathode layer boundary $\varphi_{p c}$ are variable along boundaries $\Gamma_{p a}$ and $\Gamma_{p c}$, separating the anode and cathode layers from the arc column.

We will introduce the concept of effective voltage drop as an integral electrical characteristic of current-conducting medium with nonisopotential boundaries [21]. As electric current density in arc column plasma is defined by expression $\vec{j}=-\sigma \nabla \varphi$, the following balance relationship follows from equation $\operatorname{div} \vec{j}=0$ :

$$
\int_{\Omega} \frac{|\vec{j}|^{2}}{\sigma} d V=-\int_{\Gamma} \varphi j_{n} d S,
$$

where $\Omega$ is the region taken up by arc column plasma; $\Gamma$ is its boundary; $j_{n}$ is the projection of current density vector on outer normal $\vec{n}$ to $\Gamma$.

We will present boundary $\Gamma$ in the following form: $\Gamma=\Gamma_{p a}+\Gamma_{p c}+\Gamma_{p e}$, where $\Gamma_{\mathrm{pe}}$ is the part of boundary $\Gamma$, through which the current does not flow $\left(\left.j_{n}\right|_{\Gamma_{\mathrm{pe}}}=0\right)$. Then, from (38) we obtain

$$
\int_{\Omega} \frac{|\vec{j}|^{2}}{\sigma} d V=\int_{\Gamma_{p a}} \varphi_{p a} j_{a n^{\prime}} d \Gamma_{p a}-\int_{\Gamma_{p c}} \varphi_{p c} j_{c n} d \Gamma_{p c},
$$

where $\vec{n}^{\prime}=-\vec{n}$. The expression in the left-hand part of (39) is the thermal power released in the arc column. In keeping with integral Joule-Lenz law, we will write:

$$
\int_{\Omega} \frac{|\vec{j}|^{2}}{\sigma} d V=I\left\langle U_{p}\right\rangle,
$$

where by $\left\langle U_{p}>\right.$ we will mean effective voltage drop in arc column,

$$
\left\langle U_{p}\right\rangle=\frac{1}{I} \int_{\Omega} \frac{|\vec{j}|^{2}}{\sigma} d V .
$$

As surfaces $\Gamma_{p a}$ and $\Gamma_{p c}$ are nonisopotential, we will introduce the concepts of effective values of potential $\Phi_{p a}$ and $\Phi_{p c}$ for them as follows:

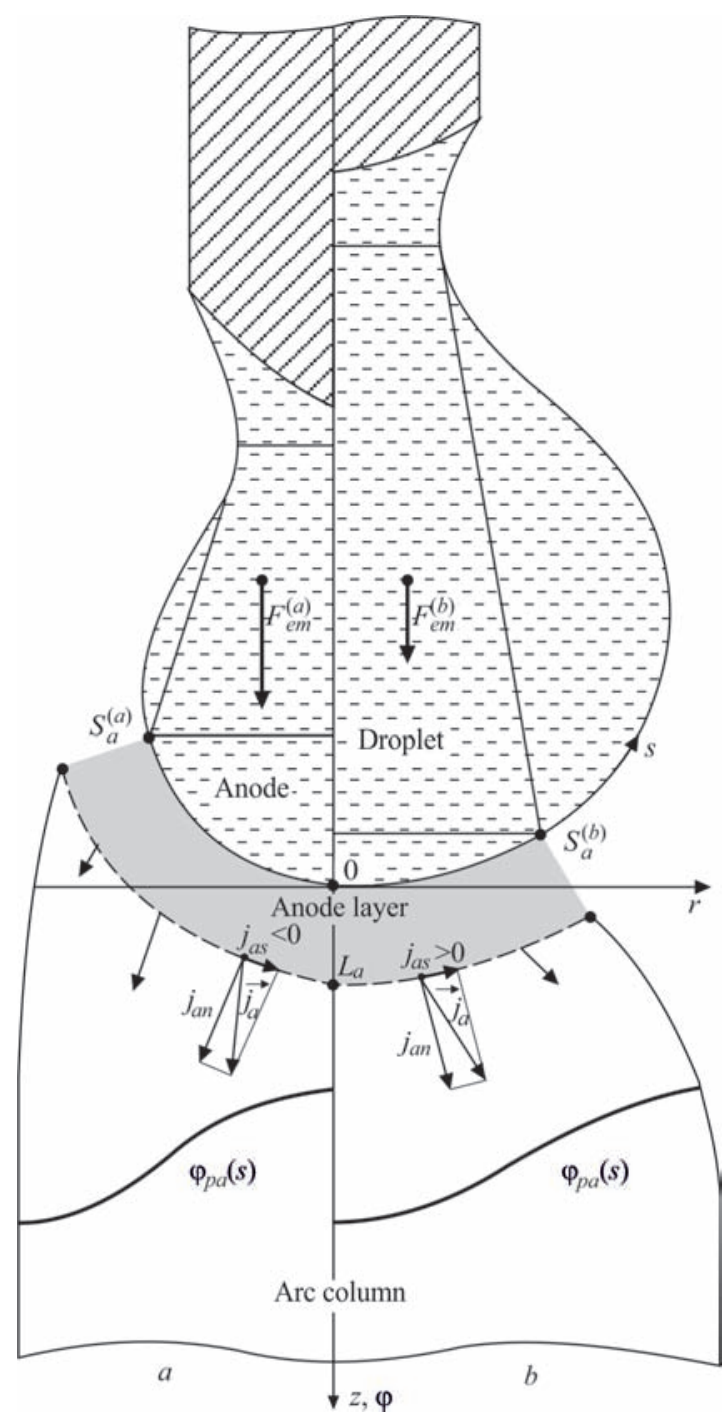

Figure 15. Schematic representation of electrical characteristics of near-anode plasma of the welding arc and process of electrode metal drop formation at MIG/MAG welding: $a$ - discharge distributed over the anode surface; $b$ - arc contraction on the anode

$$
\begin{gathered}
\Phi_{p a}=\frac{1}{I} \int_{\Gamma_{p a}} \varphi_{p a} j_{a n^{\prime}} d \Gamma_{p a} ; \\
\Phi_{p c}=\frac{1}{I} \int_{\Gamma_{p c}} \varphi_{p c} j_{c n} d \Gamma_{p c} .
\end{gathered}
$$

Then from (39) the voltage drop on arc column can be defined as the difference of effective values of potentials $\Phi_{p a}$ and $\Phi_{p c}$, i.e. $\left\langle U_{p}>=\Phi_{p a}-\Phi_{p c}\right.$ can be assumed. Using (40) we will define the effective anode $\left\langle U_{a}>\right.$ and cathode $\left\langle U_{c}\right\rangle$ drops in the following form: $\left\langle U_{a}\right\rangle=\varphi_{c}-\Phi_{p a},<U_{c}>=\Phi_{p c}-\varphi_{c}$. Within these definitions, we can obtain a standard expression for arc voltage in the form of the sum of voltage drops in separate portions of the arc discharge:

$$
U=\left\langle U_{c}>+\left\langle U_{p}\right\rangle+\left\langle U_{a}>,\right.\right.
$$

where one should bear in mind that the effective anode voltage drop is negative. 
Finally, in terms of the thus introduced effective values of voltage drops the total power balance of arc discharge components can be written similar to (41):

$$
P=\left\langle P_{c}>+<P_{p}>+<P_{a}\right\rangle,
$$

where $P=I U,\left\langle P_{c}>=I<U_{c}>,\left\langle P_{p}>=I<U_{p}>,\left\langle P_{a}>=\right.\right.\right.$ $=I<U_{a}>$.

Note here that thermal power released in the arc column and cathode layer exceeds $I U$ by value $\left|\left\langle P_{a}\right\rangle\right|$, consumed for maintaining the anode layer.

1. Nemchinsky, V.A., Perets, L.N. (1977) Near-anode layer of high-current high-pressure arc. ZhTF, 47, 1868-1875 [in Russian].

2. Dinulescu, H.A., Pfender, E. ( 1980) Analysis of the anode boundary layer of high intensity arcs. J. of Appl. Phys., 51, 3149-315.

3. Dyuzhev, G.A., Nemchinsky, V.A., Shkolnik, S.M. et al. (1983) Anode processes in high-current arc discharge. Khimiya Plazmy, 10, 169-209 [in Russian].

4. Nazarenko, I.P., Panevin, I.G. (1989) Analysis of the near-anode processes character in argon arc discharge of high pressure. Contrib. Plasma Phys., 29, 251-261.

5. Nemchinsky, V.A. (1994) Size and shape of the liquid droplet at the molten tip of an arc electrode. J. Phys. D: Appl. Phys., 27, 1433-1442.

6. Jenista, J., Heberlein, J.V.R., Pfender, E. (1997) Numerical model of the anode region of high-current electric arcs. IEEE Trans. on Plasma Sci., 25, 883-890.

7. Amakawa, T., Jenista, J., Heberlein, J. et al. (1998) Anode-boundary-layer behavior in a transferred, high intensity arc. J. Phys. D: Appl. Phys., 31, 2826-2834.

8. Tanaka, M., Ushio, M., Wu, C. S. (1999) One-dimensional analysis of the anode boundary layer in free-burning argon arcs. Ibid., 32, 605-611.

9. Murphy, A. B. (2010) The effects of metal vapour in arc welding. Ibid., 43, 434001.

10. Zhu, P., Lowke, J.J., Morrow, R. et al. (1995) Prediction of anode temperatures of free burning arcs. Ibid., 28, 1369-1376.

11. Lowke, J.J., Morrow, R., Haidar, J. (1997) A simplified unified theory of arcs and their electrodes. Ibid., 30, 2033-2042.

12. Fan, H.G., Kovacevic, R. (2004) A unified model of transport phenomena in gas metal arc welding including electrode, arc plasma and molten pool. Ibid., 37, 2531-2544.

13. Nishiyama, H., Sawada, T., Takana, H. et al. (2006) Computational simulation of arc melting process with complex interactions. ISIJ Int., 46, 705-711.

14. Hu, J., Tsai, H. L. (2007) Heat and mass transfer in gas metal arc welding. Pt 1: The arc. Int. J. Heat and Mass Transfer, 50, 833-846.
15. Masquere, M., Freton, P., Gonzalez, J.J. (2007) Theoretical study in two dimensions of the energy transfer between an electric arc and an anode material. J. Phys. D: Appl. Phys., 40, 432-446.

16. Benilov, M.S. (2008) Understanding and modelling plasma-electrode interaction in high-pressure arc discharges (Review). Ibid., 41, 144001.

17. Lancaster, J.F. (1986) The physics of welding, 2nd Edition. Pergamon Press.

18. Sanders, N.A., Pfender, E. (1984) Measurement of anode falls and anode heat transfer in atmospheric pressure high intensity arcs. J. of Appl. Phys., 55, 714-722.

19. Tanaka, M., Ushio, M. (1999) Observations of the anode boundary layer in free-burning arcs. J. Phys. D: Appl. Phys., 32, 906-912.

20. Krivtsun, I., Demchenko, V., Lesnoi, A. et al. (2010) Modelling of electromagnetic processes in system «welding arc evaporating anode». Pt1: Model of anode region. Sci. and Techn. of Welding and Joining, 10, 457-462.

21. Krivtsun, I., Demchenko, V., Krikent, I. et al. (2015) Distributed and integrated characteristics of the near-anode plasma of the welding arc in TIG and hybrid (TIG $+\mathrm{CO}_{2}$-laser) welding. Mathematical Modelling of Weld Phenomena 11 Techn. Universität Graz, Graz, Austria, 837-874.

22. Semenov, I.L., Krivtsun, I. V., Reisgen, U. (2016) Numerical study of the anode boundary layer in atmospheric pressure arc discharges. J. Phys. D: Appl. Phys., 49, 105204.

23. Almeida, N.A., Benilov, M.S., Naidis, G.V. (2008) Unified modelling of near-cathode plasma layers in high-pressure arc discharges. Ibid., 41, 245201.

24. Zhdanov, V.M. (2002) Transport processes in multicomponent plasma. London, Taylor\&Francis.

25. Krivtsun, I.V. (2001) Model of evaporation of metal in arc, laser and laser-arc welding. The Paton Welding J., 3, 2-9.

26. Baksht, F.G., Dyuzhev, G.A., Mitrofanov, N.K. et al. (1973) Probe measurements in low-temperature dense plasma at high degree of ionization. ZhTF, 43(12), 2574-2583 [in Russian].

27. Chen, F. (1967) Electrical probes: Plasma disgnostics. Ed. by R. Huddleston et al. Moscow, Mir [in Russian].

28. Griem, H.R. (1962) High-density correction in plasma spectroscopy. Phys. Rev., 128, 997-1001.

29. Krivtsun, I.V., Demchenko, V.F., Krikent, I.V. (2010) Model of the processes of heat, mass and charge transfer in the anode region and column of the welding arc with refractory cathode. The Paton Welding J., 6, 2-9.

30. Krikent, I.V., Krivtsun, I.V., Demchenko, V.F. (2014) Simulation of electric arc with refractory cathode and evaporating anode. Ibid., 9, 17-24.

31. Demchenko, V.F., Krivtsun, I.V., Krikent, I.V. et al. (2017) Force interaction of arc current with self magnetic field. Ibid., 3, 15-24.

32. Semenov, O., Demchenko, V., Krivtsun, I. et al. (2012) A dynamic model of droplet formation in GMA welding. Modelling and Simulation in Materials Sci. and Engin., 20, 045003.

Received 10.09.2018 


\title{
MAGNETOACOUSTIC DIAGNOSTICS OF HYDROGEN DAMAGE OF FERROMAGNETICS
}

\author{
Z.T. NAZARCHUK and V.R. SKALSKY \\ H.V. Karpenko Physico-Mechanical Institute of the NAS of Ukraine \\ 5 Naukova Str., Lviv, 79050, Ukraine. E-mail: pminasu@ipm.lviv.ua
}

\begin{abstract}
The experimental investigations were carried out in order to quantitatively determine a change of parameters of signals of magnetoelastic acoustic emission under effect of structural transformations and level of hydrogenation of ferromagnetic materials. It is shown that application of a phenomenon of magnetoelastic acoustic emission can make a basis for development of the new high-sensitive nondestructive technologies for detection of hydrogen accumulated by material during long-term operation of structures. 7 Ref., 9 Figures.
\end{abstract}

Keywords : nondestructive testing technologies, metallic structures, hydrogen-containing media, magnetoelastic emission, parameters of signals, increase of sensitivity

Diagnostics of state of the products and elements of structures as well as equipment operating in hydrogen-containing media is important in a majority of industry branches, in particular, aerospace, chemical, power, oil-refining, in pipeline transport and machine building. During this it is especially important to determine the places of material hydrogenation in addition to the areas of accumulation of small defects or local plastic deformations. Since their expansion can result in accelerated nucleation of microcracks, and, therefore, to failure and disastrous effects for the equipment as well as environment.

Modern methods of nondestructive testing (NDT), which are used for technical diagnostics of such objects, in particular, ultrasonic and X-ray flaw detection, require corresponding treatment of surface of object being tested, they are laborious enough, and it is difficult to use them under equipment operation conditions. A method of acoustic emission (AE) $[1,2]$ is perspective for solution of this problem. However, during its realization it is necessary to apply to the object being tested additional external mechanical loading, which locally sometimes can significantly exceed allowable optimum modes of stresses in the material, that limits application of AE-diagnostics. In order to expand these boundaries as well as increase detection efficiency of places of hydrogenation of ferromagnetic elements in the structures and products it is proposed to use the phenomenon of generation of signals of magnetoelastic acoustic emission (MAE) under effect of external magnetic field [3]. The latter, as it is known, provokes displacement of walls of magnetic domains (Barkhausen effect). These processes are the most intensive in the vicinity of separate defects or their accumulations, i.e. in the places of significant gradients of mechanical stresses. Besides, changes of material structure, level of its hydrogen degradation during operation etc. considerably effect the parameters of MAE signals.

Aim of this work is to determine quantitative indices of change of MAE parameters under effect of structure-mechanical and other physical factors during remagnetizing of ferromagnetics in the external quasi-static magnetic field and, in particular, under presence of occluded hydrogen in them.

Evaluation of effect of structural factor on MAE generation. To determine the content of carbon in material the plate samples of 240x30x2 mm size were remagnetized in solenoid. Remagnetizing frequency made $9 \mathrm{~Hz}$. MAE were selected by primary transducer (AET) with working band of frequencies $0.2-0.6 \mathrm{MHz}$ and registered by MAE-1L system. The conditions of experimental investigations hereinafter for all samples were the same. Work [4] presents the structural scheme of measurements.

Figure 1 shows a dependence of sum of MAE signals amplitude on magnetic field intensity for alloys with different content of carbon. It can be seen that the highest MAE activity is observed for steel $08 \mathrm{kp}$ (boiling), since its microstructure consists of coarse ferrite grains with insignificant inclusions of pearlite on grain boundaries. Thus, during remagnetizing of this material neither grain boundary, no presence of carbide inclusions or other phases effect steplike domain rotation.

MAE signals with somewhat smaller amplitudes are generated during steel 15 remagnetizing. It is explained by insignificant refinement of steel structure and presence of grain boundaries, along which pearl- 


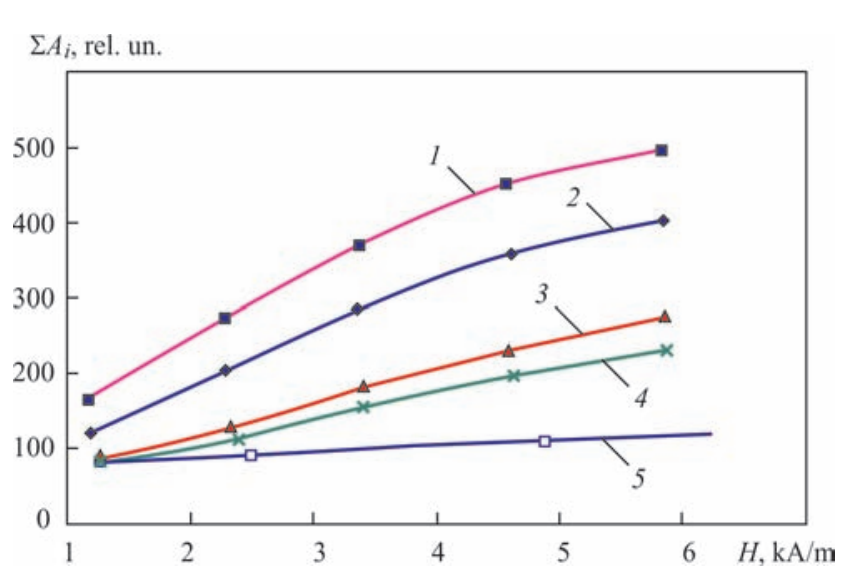

Figure 1. Dependence of sum of MAE signal amplitudes on amplitude of magnetic field intensity during remagnetizing of alloys with different content of carbon: 1 - steel 08kp (boiling); 2 steel $15 ; 3$ - steel $65 \mathrm{G} ; 4$ - U8; 5 - grey cast iron SCh10

ite inclusions are located. As it is known [3], fine grain structures and presence of phases on grain boundaries complicate rotation of domains during material remagnetizing, so activity of generation of the elastic waves, provoked by their jumps, is reduced.

Rapid reduction of activity of MAE generation was observed during remagnetizing of the samples from high-carbon steel $65 \mathrm{G}$. In this case, displacement of domain walls is blocked by presence in the microstructure of large amount of pearlite and fine carbide inclusions that are located not only along the boundaries, but in grain body solid solution.

In the case of remagnetizing of eutectoid steel of U8 grade, structure of which after full annealing consisted of fine pearlite, MAE signals with amplitudes lower than in mentioned hypoeutectoid steels were observed. Apparently, such an effect is provoked by structure refinement and presence of large number of pearlite grain boundaries, which are an obstacle for domain wall rotation. The lowest activity to MAE generation has grey cast iron, microstructure of which consists mainly of pearlite, graphite inclusions and insignificant amount of ferrite.

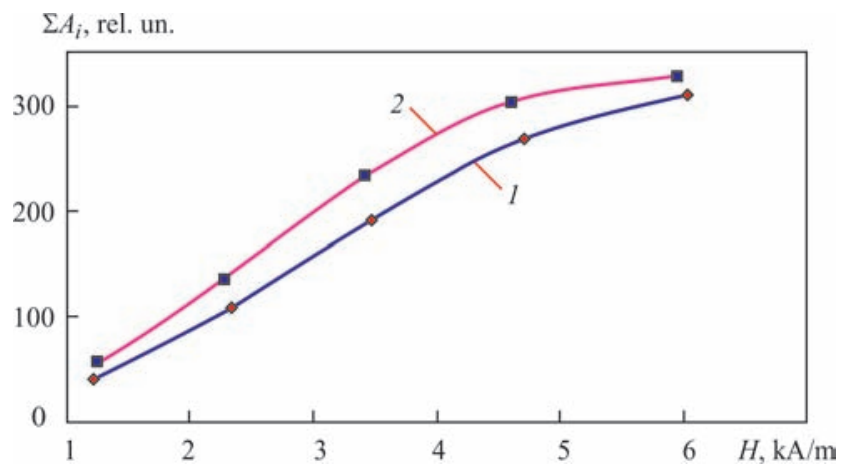

Figure 2. Dependence of sum of MAE signal amplitudes on amplitude of magnetic field intensity during remagnetizing of samples from steel 15: 1 - initial material; 2 - annealed at 900 $910{ }^{\circ} \mathrm{C}$
Effect of annealing on MAE generation. The samples of low-carbon steel 15 were remagnetized in the solenoid. They were heated to $900-910{ }^{\circ} \mathrm{C}$ temperatures with further cooling together with furnace. Such a heat treatment results in growth of ferrite grains, thinning of their walls, and coagulation of a part of pearlite phase by joining in single inclusions. This heat treatment also promotes reduction of residual stresses, provoked by plastic deformation during rolling of steel sheets. Such conditions improve displacement of domain walls during remagnetizing of steel as well as increase MAE generation activity. Figure 2 shows dependence of sum of amplitudes of MAE signals on magnetic field intensity for steel 15 subjected to heat treatment. It can be seen that a curve after full annealing of steel is characterized with higher MAE activity.

Peculiarities of MAE generation in quenching structures were investigated by remagnetization of high-carbon steel $65 \mathrm{G}$ samples in the solenoid. The samples were quenched in oil and then were subjected to low-, medium- and high-temperature tempering. Figure 3 shows the dependence of sum of MAE signal amplitudes on magnetic field intensity for the samples subjected to different heat treatment. It can be seen that rise of tempering temperature of steel increases activity of MAE generation. This is, first of all, related with relaxation of residual stresses appearing in quenching structure due to increase of volume during transfer of a lattice from face-centered into body-centered cubic one.

Low mobility of domain walls in the quenched metal (Figure 3, curve 1) is also related with presence in the structure of packages of acicular inclusions of martensite and residual austenite [5].

Heating of quenched steel to $T=80-200^{\circ} \mathrm{C}$ results in martensite decay. Transfer from tetragonal to cubic lattice takes place during this. Besides, there is decay of residual austenite, carbide transformation and

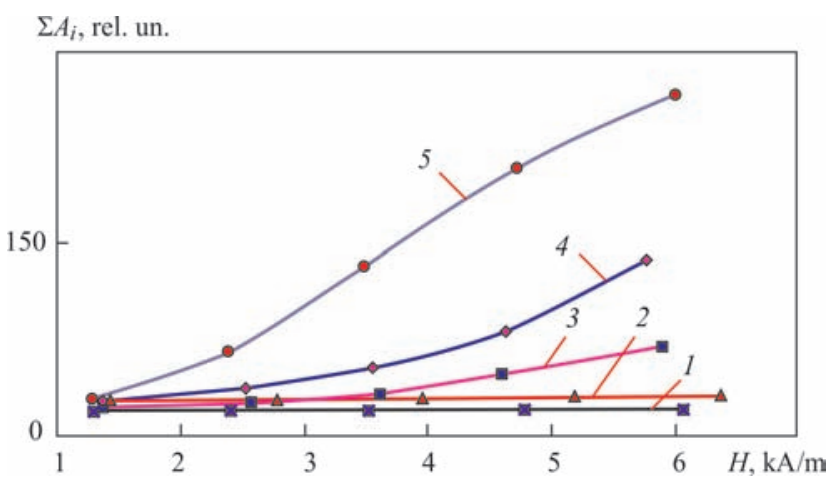

Figure 3. Dependence of sum of MAE signal amplitudes on amplitude of magnetic field intensity during remagnetizing of samples from steel 65G: 1 - quenched material; 2 - tempering at $180{ }^{\circ} \mathrm{C} ; 3$ - tempering at $350{ }^{\circ} \mathrm{C} ; 4$ - tempering at $580{ }^{\circ} \mathrm{C}$; 5 - output metal 
coagulation of carbides; smoothing of irregularity of crystallite structure of $\alpha$-solid solution; reduction of residual stresses. These processes partially improve mobility of domain wall structure that in turn rises activity of MAE generation (Figure 3, curve 2).

Medium and high tempering of steel $65 \mathrm{G}(T=$ $=300-400{ }^{\circ} \mathrm{C}$ ) generates troostite structures and sorbite under $500-600{ }^{\circ} \mathrm{C}$. These temperatures provoke the changes of structure, which are not related with phase transformations, i.e. shape and size of carbides and structure of ferrite are varied. At that, cementite crystals increase and their shape gradually approaches to spheroidal. The boundaries between lamellar ferrite crystals are removed, their size rises and shape approaches to equiaxed. This has positive effect on increase of jumps of domain walls during remagnetizing of such materials and, therefore, activity of MAE rises (Figure 3, curves 3, 4).

Thus, based on the results of experimental investigations, it is determined that steplike displacement of domain walls during metal remagnetizing significantly depends on its microstructure, and, therefore, influences on MAE generated at that. The factors complicating domain mobility are fine microstructure, presence of grain boundaries, carbides, quenching structures, nonmetallic inclusions, residual stresses, etc. Thus, increase of carbon concentration in steel results in significant changes of structure, which reduce MAE activity. Annealing of low-carbon steel promotes increase of MAE amplitude due to coarsening of ferrite grains and coagulation of pearlite inclusions.

It is shown that activity of MAE is minimum during remagnetizing of quenched steel $65 \mathrm{G}$. This is related with presence in steel structure of marten- site packages and inclusions of residual austenite. It is determined that increase of steel tempering temperature rises MAE activity. The latter is provoked by relaxation of residual stresses, increase of cementite crystals, coalescence of boundaries between lamellar crystals of ferrite, size of which rises and approaches to equiaxed by shape.

Work [3] shows negative effect of plastic deformations on MAE generation, namely the higher plastic deformation, the less index of $\Sigma A_{i}$ parameter is.

Effect of electrolytic hydrogen. The investigations were carried out in solenoid-electrolytic cell, appearance and structure of which are shown on Figure 4. Solenoid winding was coiled on PVC pipe of $50 \mathrm{~mm}$ diameter, which served as an electrolytic cell body. Solenoid base from the both sides was closed by covers with corresponding openings for pour in - pour out of electrolyte, sealing for sample alignment pin, output of sample, input of electron thermometer and anode.

Cylinder samples of $12 \mathrm{~mm}$ diameter, $260 \mathrm{~mm}$ length made of cold rolled steel 15 were examined. The latter has high content of $\alpha$-iron, which provides it with high (in comparison with pure iron) ferromagnetic properties. The lower part of the sample was completely located in the electrolytic cell volume, when the other insignificant part comes out over the upper cover of the sample for electric connection and installation of transducers of acoustic emission.

The electrolytic-cell was filled with $0.1 \mathrm{n}$. NaON solution. It is suitable electrolyte, which provides high electric conductivity, does not promote emission of by-products of electrolysis, apart of hydrogen and oxygen as well as secure the surface of steel sample from excessive corrosion during cathode current cut
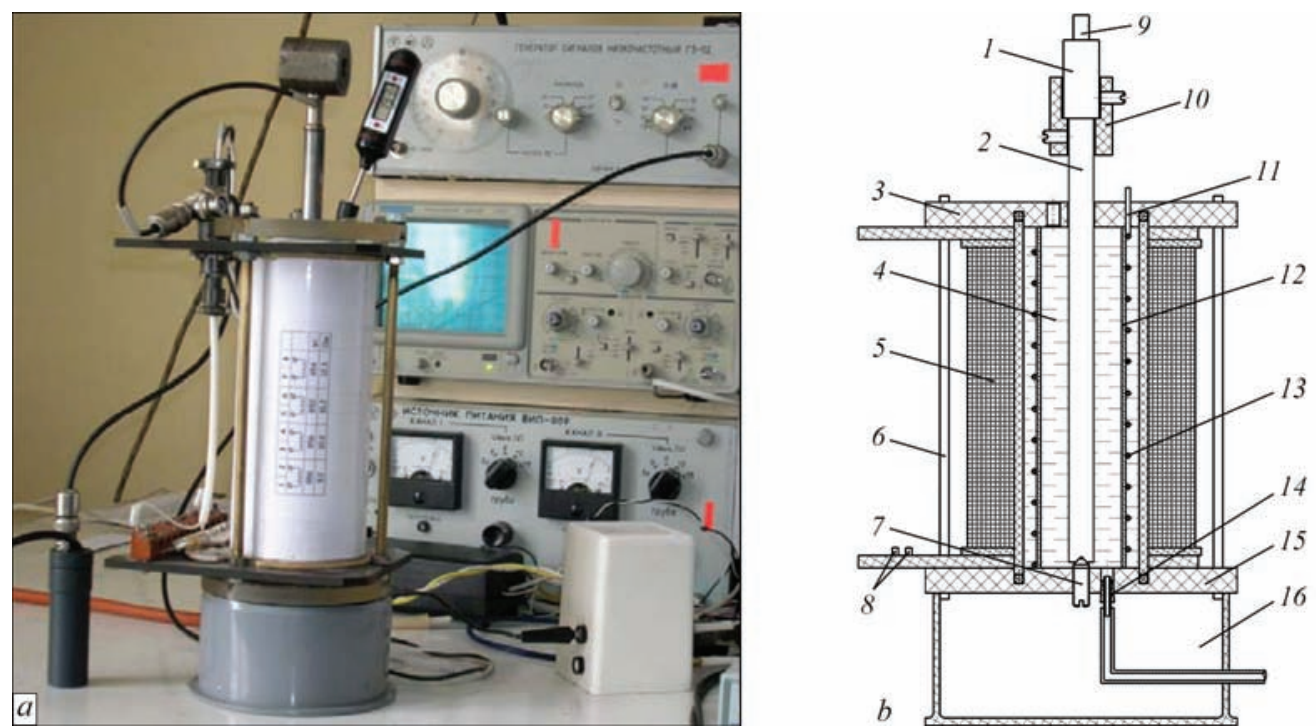

Figure 4. Appearance $(a)$ and structure $(b)$ of solenoid-electrolytic cell for investigation of effect of hydrogen absorbed by metal on parameters of MAE signals: 1 - AET; 2 - sample; 3 - upper cover; 4 - electrolyte; 5 - solenoid winding; 6 - buckle; 7 - support; 8 - terminals; 9 - high-frequency cable; 10 - nozzle; 11 - anode output; 12 - frame; 13 - platinum electrode; 14 - isolation base; 15 - lower cover; 16 - pan 
off. A platinum anode was installed coaxially with the solenoid and sample. It together with sample (cathode) through digital measurement device UT101 that operated in a mode of measurement of electrolytic cell current was connected to a stabilized current source Etalon EP.10010.1.3. An average density of current on the surface of working electrode in $0-20 \mathrm{~mA} / \mathrm{cm}^{2}$ range was provided using these devices.

An electrochemical hydrogenation of metal in comparison with a gas phase hydrogenation has an advantage that metal is not subjected to heat effect, which can promote not only relaxation of internal residual stresses (magnetic properties of ferromagnetics are sensitive to them), but change of microstructure of material: phase transformations, segregation of secondary phase, grain growth, resolidification, etc. In addition activity of the reduced hydrogen on the metal surface is easily controlled by current intensity under conditions of the electrolytic hydrogenation. Under such conditions it is not difficult to reach significant concentration of the atomic hydrogen on the metal surface and it is also easy to control insignificant activity of the reduced atomic hydrogen. It is important since solubility of hydrogen in $\alpha$-iron is very insignificant, as small and critical concentrations of hydrogen, that causes irreversible failures of microstructure (tens, or even hundredth part of ppm for pure iron [6] and not more than $1 \mathrm{ppm}$ for pipe steel [7]).

In order to establish magnetic field inside the sample, solenoid winding through low-frequency amplifier and system of current measurement was connected to digital functional generator of alternating voltage PCG10/8016, which is controlled by computer. The generator frequency can be varied in $0.01 \mathrm{~Hz}-1.0 \mathrm{MHz}$ limits. A signal was controlled using the computer oscilloscope module PCS500. Effect of different shapes of alternating supply voltage of the solenoid, namely sinusoidal, meander, saw-tooth, triangular, pulse was checked during investigations.

A broadband high sensitive AET (coefficient of conversion of elastic waves into electric signal is not less than $1.6 \cdot 10^{9} \mathrm{~V} / \mathrm{m}$ ) was connected to upper end of the sample using special holder. It converted elastic oscillations of metal surface due to propagation of MAE waves into electric signal. Nonuniformity of conversion coefficient of primary transducer made $\pm 7 \mathrm{~dB}$ in a band of operating frequencies $0.2-1.0 \mathrm{MHz}$.

MAE signal from AET through preamplifier SAA6 with amplification coefficient $40 \mathrm{~dB}$ came into an input of the information-measurement system MAE-1L. It provided amplification, processing and registration of MAE signal. MAE-1L system software designed in DELPHI medium allows setting coefficients of signal amplification, values of their discrimination thresh- old, digitizing speed and sampling volume. Through a parallel port this block is connected to computer, which saves digitized data and analyses MAE signals using MAESTAT, MS EXCEL, MATLAB, etc. programs. The information-calculation block MAE-1L is synchronized with signal of a functional generator. It means that beginning of MAE signal registration takes place under the same phase delay of the first quarter of the period, i.e. on sine curve rise.

MAE elastic waves that provoked displacement of metal surface appeared due to steplike displacement of the domain walls during steel remagnetizing in variable magnetic field, moreover, dynamics of the domain walls displacement can reflect, on the one hand, unsoundness of metal structure, and, on the other hand, energy distribution of microstructure defects. These both characteristics of metal depend on amount of absorbed hydrogen, which, in turn, depends on current and time of electrolysis and other physical-chemical parameters. Taking into account this, the dependencies of parameters of MAE signals on value of cathode current through the sample surface, magnetic field intensity and time of hydrogenation were investigated.

Two series of experiments were carried out. In the first one, the MAE signals registered by measurement system were subjected to typical post-processing using the algorithm of threshold values. It extracted from the calculations the pulses with lower levels of amplitudes in comparison with threshold ones. In the second series of experiments the registered MAE signals were subjected to other processing procedure. During it displacements of MAE-pulse values caused by a principle of measurement system operation was only removed and (for preservation of fullness of useful MAE signal) low amplitude pulses were not filtered.

For the first series of experiments Figure 5 shows a dependence of sum of amplitudes of magnetoacoustic emission $\Sigma A_{i}$ signals (in thousands of relative units) on time of electrolytic hydrogenation of the sample by cathode current $I_{\mathrm{c}}=50 \mathrm{~mA}$. As can be seen from the Figure, initial $\left(t_{\mathrm{i}}=0\right)$ values of $\Sigma A_{i}$ makes approximately 100 , and such level of MAE signal intensity lasts more than $5 \mathrm{~min}$.

Reasonable that such a time is necessary for reduction of surface oxide-hydroxide films and for penetration of sufficient amount of atomic hydrogen on such a depth that to effect $\Sigma A_{i}$ integral parameter of MAE signals. In $600 \mathrm{~s}$ after start of cathode hydrogenation the sum of $\Sigma A_{i}$ amplitudes exceeds 400 and continue to rise reaching the value $\Sigma A_{i}=500$ after $72 \mathrm{~min}$ of action of cathode current.

Change of sensitivity of $\Sigma A_{i}$ parameter of MAE signals on amplitude of magnetic field intensity $H_{a}$ 
due to electrolytic hydrogenation (Figure 6) was also evaluated.

Considerable difference in the MAE signal intensity before and after samples hydrogenation can be observed. Although, relative increase of value of $\Sigma A_{i}$ parameter due to metal hydrogenation is smaller than in Figure 5, it still makes more than $100 \%$. It indicates exceptionally high sensitivity of the MAE method before electrolytic hydrogenation and perspective of the investigations in this direction.

It is important to evaluate the change of intensity of MAE signals at increase of the cathode current applied to the sample, and, thus, with pressure of atomic hydrogen on the metal surface. $\Sigma A_{i}$ parameter from time $t_{\mathrm{i}}$ of electrolytic hydrogenation for cathode current $I_{\mathrm{c}}=150 \mathrm{~mA}$ at the same amplitude of magnetic field intensity $H_{\mathrm{a}}=8.7 \mathrm{kA} / \mathrm{m}$ that is on Figure 4 was registered. It can be seen that $300 \mathrm{~s}$ was enough for high cathode current to provide $\Sigma A_{i}$ parameter with almost its maximum value. At the same time, this parameter remained still on noise level for $I_{\mathrm{c}}=50 \mathrm{~mA}$ after $300 \mathrm{~s}$. Secondly, in the case of lower cathode current $\Sigma A_{i}$ parameter has stable tendency to insignificant rise during hydrogenation, whereas for $I_{c}=150 \mathrm{~mA}$ this parameter reached its maximum in $10 \mathrm{~min}$ of hydrogenation, after what it gradually decreases approximately by $8 \%$ till the end of experiment.

After the dependencies mentioned above were obtained, a second series of experiments was carried out with similar remagnetizing conditions, but somewhat lower levels of cathode currents and another processing of measurement results. Figure 6 presents the dependences of $\Sigma A_{i}$ parameter on amplitude of remagnetizing field intensity $H_{\mathrm{a}}$ and cathode current of hydrogenation. If obtained dependencies of $\Sigma A_{i}$ on $H_{\mathrm{a}}$ are parabolically approximated (approximation curves are not shown) than for the method of least squares the coefficients of determination within $R^{2}=$ 0.9982-0.9999 limits are obtained, that indicate functional dependence of the variables. A parabolic nature of these dependencies means that the induction level during the experiments does not reach ferromagnetic saturation. Thus, the main source of MAE elastic waves was steplike displacements of $90^{\circ}$ domain walls due to change of directions of magnetizing vectors from one axis of body-centered iron crystal to another. It is necessary to note that the peaks of parabolic approximations are located at the levels of registered noises $\Sigma A_{i}=58 \pm 1$ (rel. unit) in the limits of experimental data scatter.

As can be seen from Figure 7, the intensity of MAE signal significantly rises, in particular for the cathode currents of hydrogenation higher than $50 \mathrm{~mA}$, under conditions of electrolytic hydrogenation of the

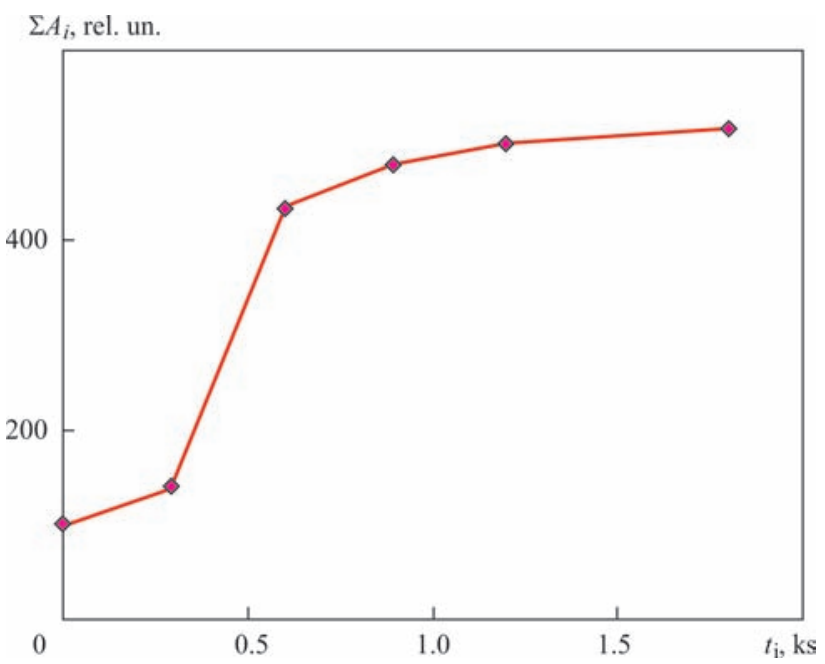

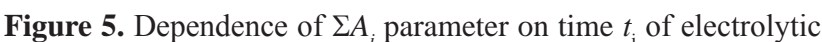
hydrogenation of sample (cathode current $I_{\mathrm{c}}=50 \mathrm{~mA}$, amplitude of magnetic field intensity $H_{\mathrm{a}}=8.7 \mathrm{kA} / \mathrm{m}$ )

sample-ferromagnetics. However, as in the previous series experiments, in these experiments increase of MAE signals intensity with the cathode current took place in $25 \rightarrow 100 \rightarrow 150 \rightarrow 50 \mathrm{~mA}$ sequence, i.e. was non-systematic, reflecting, maybe, simultaneous effect of several mutually competitive factors. Application of hydrogenation cathode current of $25 \mathrm{~mA}$ value provides only insignificant rise of MAE signal intensity above the level that is generated before cathode current switch-on, i.e. before hydrogenation process. Therefore, taking into account high reproducibility of MAE signals for initial samples (before the start of hydrogenation) this current can be considered as a boundary of method sensitivity for the cathode hydrogenation of metal.

Another series of experiments, the results of which are presented on Figure 8, was dedicated to study of time change of $\Sigma A_{i}$ parameter after switch-on of the sample hydrogenation current for different values of the cathode currents values (amplitude of remagnetizing field for all cases was similar and made

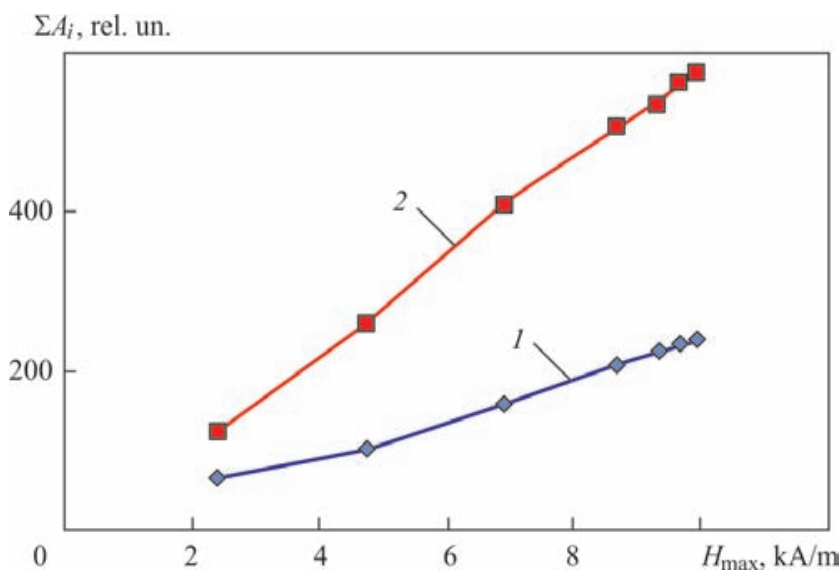

Figure 6. Dependence of $\Sigma A_{i}$ parameter on magnetic field intensity $H_{\mathrm{a}}$ for sample before (1) and after (2) of electrolytic hydrogenation by cathode current $I_{\mathrm{c}}=50 \mathrm{~mA}$ during $1800 \mathrm{~s}$ 

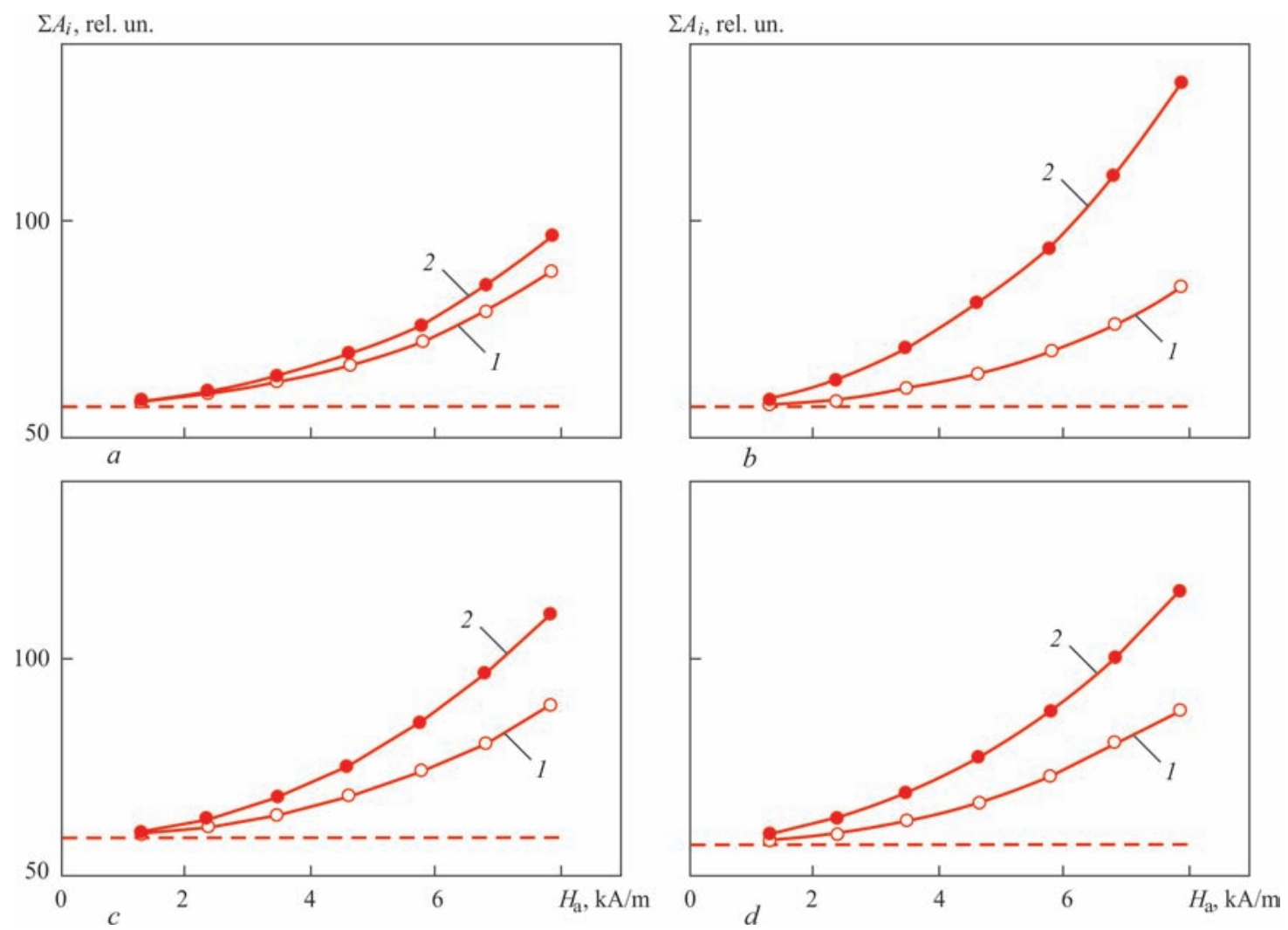

Figure 7. Effect of remagnetizing field intensity $H_{\mathrm{a}}$ on $\Sigma A_{\text {p }}$ parameter for samples to (curve 1) and after (curve 2) electrolytic reduction of 1.8 ks duration by current, $\mathrm{mA}: a-25 ; b-50 ; c-100 ; d-150$ (level of noise is shown by dashed line)

$\left.H_{\mathrm{a}}=6.8 \mathrm{kA} / \mathrm{m}\right)$. At the moment of switch-on $t=0, \Sigma A_{i}$ parameter was in the limits of 76.6-80.6 (rel. units) and already in $0.4-0.8 \mathrm{ks}$ reached its maximum value.
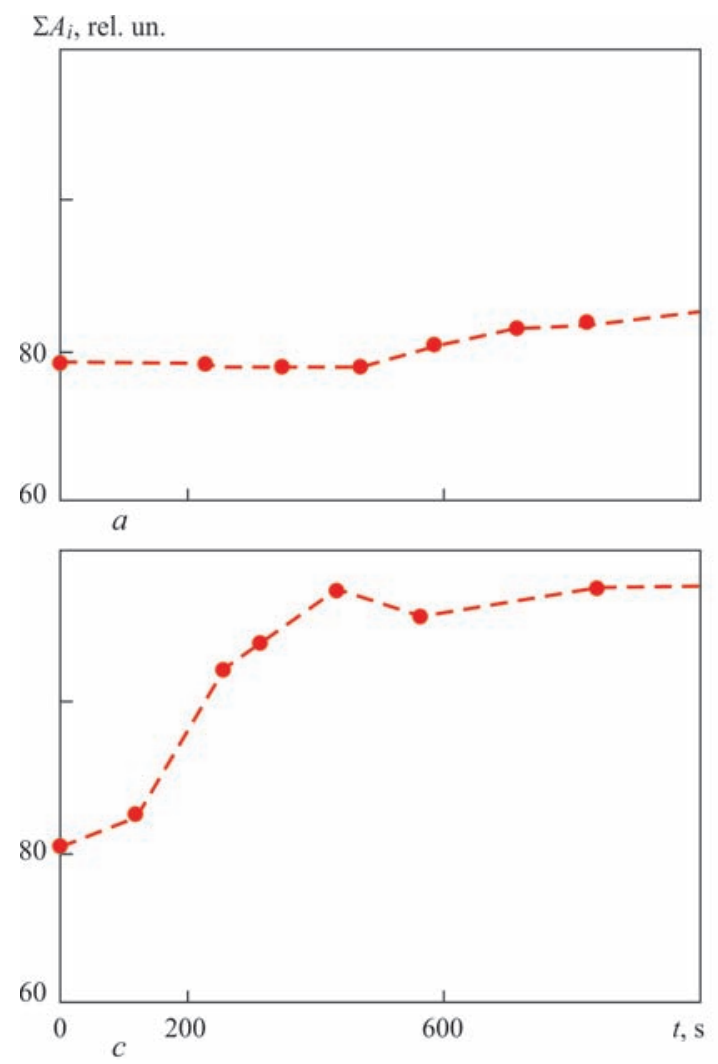

Based on obtained dependencies (Figure 8) the duration of transition process rises with the current value. It should be noted that for $25 \mathrm{~mA}$ cathode cur-
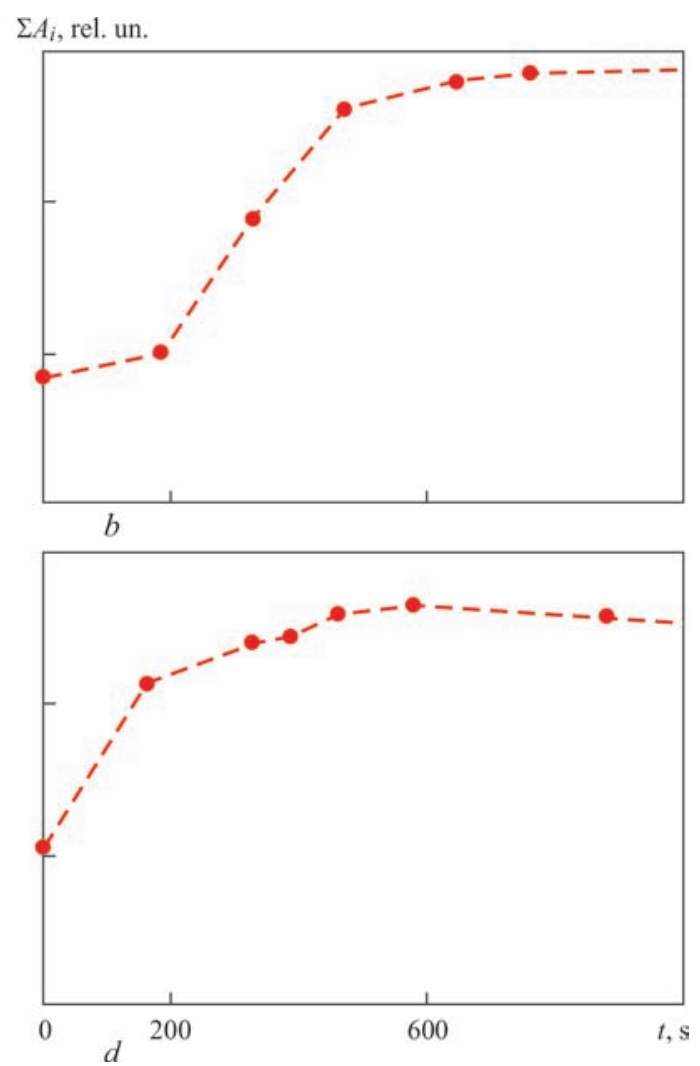

Figure 8. Time dependence of $\Sigma A_{i}$ parameter at electrolytic hydrogenation by current, mA: $a-25 ; b-50 ; c-100 ; d-150$ 
rent the level of MAE signal during the first $0.47 \mathrm{ks}$ is stable, similar to the signal, obtained at absence of cathode hydrogenation. Therefore, $\Sigma A_{i}$ gradually increases, reaching the stationary value in approximately $2.0 \mathrm{ks}$ after current switch-on. Movement of $\Sigma A_{i}$ parameter to a stationary level increases with current growth. At that it should be noted that a hydrogenation of MAE signal level for stationary reproducibility deteriorates that matches with the results given above. Moreover, for $I_{c}=150 \mathrm{~mA}$ the level of MAE signal is somewhat reduced after reaching its maximum value (in moment $t=0.58 \mathrm{ks}$ ), again confirming the results obtained above.

Delay in process of $\Sigma A_{i}$ increase, after cathode current switch-on is apparently caused by reduction of surface oxyhydroxide films and creation of the conditions of high activity of atomic hydrogen on metal surface. The time necessary for reaching the stationary level of MAE signal is considerably shorter than the time necessary for hydrogenation of volume of $12 \mathrm{~mm}$ diameter sample. It can mean, for example, that only sub-surface layers of metal are responsible for increment of MAE signal, or another phenomenon comes into action, which suppresses the signal level at metal volume hydrogenation. As previously, MAE signal significantly reduces under conditions of elastic deformation of iron through magnetocrystalline anisotropy and during plastic deformation due to increment of unsoundness of its structure.

For comparison Figure 9 shows the results of remagnetization of samples, which were hydrogenised from gas phase [3].

Comparing obtained results for electrolytic hydrogenation with data after gas phase hydrogenation it can be noted that MAE parameters during cathode hydrogenation have considerably higher sensitivity. It can be explained by a role of diffusion active hydrogen under conditions of electrolysis and its absence in the experiments with gas-like hydrogenation. Combining these two methods it is possible to separate effect of active and nonactive hydrogen on the parameters of MAE signals, but such issues require further investigation.

\section{Conclusions}

Application of phenomenon of magnetoacoustic emission can become the basis for new high-sensi-
$P \cdot 10^{-3}$, rel. un

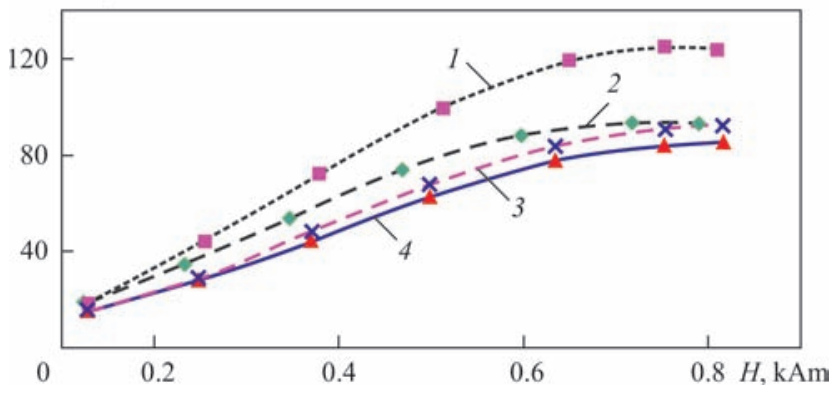

Figure 9. Dependence of power $P$ of MAE signals on change of magnetic field intensity $H$ of solenoid for samples of steel $15(d=$ $=2 \mathrm{~mm} ; f=9 \mathrm{~Hz}$ ) with different concentration of hydrogen (hydrogenation from gas phase) $1-C_{\mathrm{h}}=5.6 \mathrm{ppm} ; 2-1.85 ; 3-$ $1.1 ; 4-0.74$ (initial material)

tive nondestructive method of hydrogen detection in structural materials. At that, it refers to nonactive hydrogen that is kept in microstructure traps, as well as diffusion active one. This phenomenon allows detection of such level of hydrogenation, which do not provoke a danger at short-term effect, but can be significant factor of degradation of structural materials under long-term operation. In comparison with initial state of metal increase the MAE intensity depends on concentration of electrolytic hydrogen as well as from gas phase, absorbed by metal.

1. Nazarchuk, Z., Skalskyi, V., Serhiyenko, O. (2017) Acoustic emission. Methodology and application. Springer Int. Publish. AG.

2. Nazarchuk, Z.T., Neklyudov, I.M., Skalskyi, V.R. (2016) Method of acoustic emission in diagnostics of NPP reactor vessels. Kyiv, Naukova Dumka [in Ukrainian].

3. Nazarchuk, Z.T., Andrejkiv, O.E., Skalskyi, V.R. (2013) Estimation of hydrogen degradation of ferromagnets in magnetic field. Kyiv, Naukova Dumka [in Ukrainian].

4. Skalskyi, V., Stankevych, O., Dubytskyi, O. (2018) Estimation of effect of hydrogen on the parameters of magnetoacoustic emission signals. J. of Magnetism and Magnetic Materials, 375-385.

5. Gorkunov, E.S., Novikov, V.F., Bakharev, M.S., Yatsenko, T.A. (1997) Piezomagnetic effect of residually-magnetized state of steels 45 and 60G. In: Proc. of Regional Sci.-Tekhn. Conf. on New Materials and Technologies in Mechanical Engineering. Tyumen, TyumGNGU, 58-59 [in Russian].

6. Beck, W., Bockris, J. O’M., McBeen, J., Nanis, L. (1966) Hydrogen permeation in metals as a function of stress, temperature and dissolved hydrogen concentration. Proc. of the Roy. Soc. London A, 290, 220-235.

7. Maccagno, T.M., Ikeda-Cameron, K., Jack, T. et al. (1998) Hydrogen effects in gas transmission pipeline steels. In: Proc. of the Int. Pipeline Conf. New York, ASME Int., 1, 479-484. 


\title{
SOME TECHNOLOGICAL PECULIARITIES OF PERFORMANCE OF ASSEMBLY-WELDING WORKS IN CONSTRUCTION OF ISOTHERMAL TANKS
}

\author{
M. BELOEV ${ }^{1}$ and N. LOLOV ${ }^{2}$ \\ ${ }^{1} \mathrm{KZU}$ Holding Group \\ 12 Khisto Vakarelski Str., 1700, Sofia, Bulgaria \\ ${ }^{2}$ Technical University \\ 8 Kliment Ohridski Blvd., 1000, Sofia, Bulgaria
}

\begin{abstract}
The paper deals with the main technological features which should be taken into account during design, fabrication and mounting of tanks from $9 \% \mathrm{Ni}$ steel for liquefied natural gas storage. They include preparation for welding, selection of welding processes and consumables, modular assembly of the tank in site, application of ultrasonic peening of welded joints for stress relieving. 8 Ref., 6 Tables, 5 Figures.
\end{abstract}

Keywords : arc welding, isothermal tanks, $9 \%$ Ni steel, design, fabrication, mounting, modular assembly, ultrasonic peening

Isothermal tanks are quite extensively used for liquefied gas storage. The liquefaction allows a significant reduction of gas volume at its transition from the gaseous state into the liquid state. For instance, the natural gas volume decreases more than 600 times. Isothermal tanks are double hull, the inner tank being in direct contact with the product, stored at a low temperature. This means that special attention should be given to the material, from which it is made, as well as to performance of assembly-welding operations during its construction.

Table $1[1,2]$ gives the required temperatures of liquefaction of different gases and appropriate materials for construction of facilities for storage and transportation.

The interest to use of natural gas as an energy source is growing constantly. More and more new natural gas fields are found every year, and on the global

Table 1. Gas liquefaction temperatures and materials applicable at these temperatures

\begin{tabular}{|c|c|c|}
\hline Gas & $T,{ }^{\circ} \mathrm{C}$ & Base metal grade \\
\hline Ammonia & -33.4 & Carbon steel \\
\hline Propane & -42.1 & Deoxidized fine-grained steel \\
\hline Propylene & -47.7 & \multirow{2}{*}{ Steel with $2.25 \% \mathrm{Ni}$} \\
\hline Carbon disulphide & -50.2 & \\
\hline Carbon dioxide & -78.5 & \multirow{3}{*}{ Steel with $3.5 \% \mathrm{Ni}$} \\
\hline Acetylene & -84.0 & \\
\hline Ethane & -88.4 & \\
\hline Ethylene & -103.8 & \multirow{4}{*}{ Steel with 5-9\% Ni } \\
\hline Methane (natural) gas & -163.0 & \\
\hline Oxygen & -182.9 & \\
\hline Argon & $\begin{array}{l}-185.9 \\
\end{array}$ & \\
\hline
\end{tabular}

(c) M. BELOEV and N. LOLOV, 2018 scale 4 times more gas is produced than consumed. As the fields (countries of the Persian Gulf, Central Asia, Alaska, Arctic shelf) and the industrial centers of gas consumption (Europe, Japan, North America) are significantly removed from each other, natural gas has to be transported to very large distances, and its greater part should be stored in isothermal tanks in the liquefied state.

Materials for construction of LNG tanks should have low-temperature toughness. According to SEW 680, steels with low-temperature toughness are those which preserve the fracture energy of at least $27 \mathrm{~J}$ at impact toughness testing at temperatures below $-10^{\circ} \mathrm{C}$, and according to DIN 17280 the ultimate value of low-temperature toughness is specified at the temperature of $-60{ }^{\circ} \mathrm{C}$.

Moreover, the materials should have high strength that will allow reducing the vessel wall thickness and ensuring welding without the risk of brittle fracture. High-alloyed and special materials were applied up to now. They include austenitic welding consumables, nickel alloys, aluminium alloys, copper and copper alloys. As the scope of applications in cryogenic engineering is growing continuously, and the above-mentioned materials are very expensive, the need arose for development of less expensive low-alloyed steels. These most often are nickel steels with different content of nickel, depending on operating temperature.

As the temperature of liquefied natural gas is equal to $-163{ }^{\circ} \mathrm{C}, 9 \% \mathrm{Ni}$ steel is a suitable material for manufacturing isothermal tanks for liquefied natural gas, which provides a good combination of properties at an acceptable price. 
Table 2. Requirements to $9 \% \mathrm{Ni}$ steel to ASTM and JIS

\begin{tabular}{|c|c|c|c|c|}
\hline \multirow{2}{*}{ Standard } & \multicolumn{2}{|c|}{ ASTM } & \multicolumn{2}{|c|}{ JIS G 3127} \\
\hline & A353 & A353 (Type I) & SL9N 520 & SL9N 590 \\
\hline Maximum thickness (mm) & 50 & 50 & 50 & 100 \\
\hline Heat treatment & $\mathrm{NNT}^{* 1}$ & Improved & NNT & Improved \\
\hline $\mathrm{C}(\%)$ & \multicolumn{2}{|c|}{$\leq 0.13$} & \multicolumn{2}{|c|}{$\leq 0.12$} \\
\hline $\mathrm{Si}(\%)$ & \multicolumn{2}{|c|}{$0.15-0.40$} & \multicolumn{2}{|c|}{$\leq 0.30$} \\
\hline $\operatorname{Mn}(\%)$ & \multicolumn{2}{|c|}{$\leq 0.90$} & \multicolumn{2}{|c|}{$\leq 0.90$} \\
\hline $\mathrm{P}(\%)$ & \multicolumn{2}{|c|}{$\leq 0.035$} & \multicolumn{2}{|c|}{$\leq 0.025$} \\
\hline S (\%) & \multicolumn{2}{|c|}{$\leq 0.035$} & \multicolumn{2}{|c|}{$\leq 0.025$} \\
\hline $\mathrm{Ni}(\%)$ & \multicolumn{2}{|c|}{$8.50-9.50$} & \multicolumn{2}{|c|}{$8.50-9.50$} \\
\hline Yield limit (MPa) & $\geq 515$ & $\geq 585$ & $\geq 520$ & $\geq 590$ \\
\hline Ultimate strength (MPa) & \multicolumn{2}{|c|}{$690-825$} & \multicolumn{2}{|c|}{$690-830$} \\
\hline Relative elongation (\%) & \multicolumn{2}{|c|}{$\geq 20.0$} & \multicolumn{2}{|c|}{$\begin{array}{l}\geq 21(6 \leq t \leq 16)^{* 2} \\
\quad \geq 25(t>16)^{* 2} \\
\quad \geq 21(t>20)^{* 3}\end{array}$} \\
\hline Impact toughness at $-192{ }^{\circ} \mathrm{C}$ & \multicolumn{2}{|c|}{$\geq 34$} & $\geq 34$ & $\geq 41$ \\
\hline Lateral expansion at $-196^{\circ} \mathrm{C}$ & \multicolumn{2}{|c|}{$\geq 0.38$} & \multicolumn{2}{|c|}{-} \\
\hline \multicolumn{5}{|c|}{$\begin{array}{l}{ }^{* 1} \mathrm{NNT} \text { — two times normalized and quenched. } \\
{ }^{{ }_{2}} \text { Flat sample No.5, according to JIS Z } 2201 \text { (50 mm measuring length). } \\
{ }^{*} \text { Round sample No.4, according to JIS Z } 2201 \text { (50 mm measuring length). }\end{array}$} \\
\hline
\end{tabular}

Exceptional low-temperature properties of impact toughness are the result of producing super fine-grained structure of strong nickel ferrite. Small quantities of residual austenite, formed at tempering, improve the impact toughness after heat treatment. Alongside good toughness, resulting from structure improvement, this steel also has higher strength properties.

Table 2 [3] gives information about the chemical composition and mechanical properties of $9 \% \mathrm{Ni}$ steel. To ensure good low-temperature toughness this steel should have high purity, i.e. it should have a low content of phosphorus and sulphur, as well as balanced content of the main alloying elements.

There is a wide range of requirements and standards for design, construction, inspection and servicing of $9 \% \mathrm{Ni}$ steel tanks for liquefied natural gas. A number of the respective ASME, API, EN and JIS standards are given below.

1. ASME Sec. VIII, Div.1: Rules for Construction of Pressure Vessels; Div. 2: Alternative Rules.

2. API Standard 620: Design and Construction of Large, Welded, Low-Pressure Storage Tanks, Appendix Q: Low-pressure storage tanks for liquefied hydrocarbon gases at temperatures not lower than $-168^{\circ} \mathrm{C}$.

3. EN 14620-1 (2006): Design and manufacture of site built, vertical, cylindrical, flat-bottomed steel tanks for the storage of refrigerated, liquefied gases with operating temperatures between $0{ }^{\circ} \mathrm{C}$ and $-165^{\circ} \mathrm{C}$. Part 1: General.

4. JIS B8265 (2010): Construction of Pressure Vessels - General Principles; JIS B8267 (2008): Construction of Pressure Vessels.
Factors influencing welding of $9 \% \mathrm{Ni}$ steel. Successful welding of $9 \% \mathrm{Ni}$ steel at preservation of its low-temperature toughness depends on a number of factors, which are:

- production method and grade of supplied steel;

- base metal properties, residual magnetism, susceptibility to arc magnetic blow, heat conductivity and thermal expansion;

- welding process;

- welding heat input;

- cooling rate, dependence on preheating temperature and interpass temperature.

Preparation for welding. Nickel steels with low-temperature toughness are prone to preservation of residual magnetism that has a negative effect on their behaviour during welding, because of appearance of magnetic arc blow. Therefore, it is necessary to perform their welding in demagnetized condition. When ordering steel supply, alongside the certificate of compliance with EN 10204 «Certification of materials», it is also necessary to specify the inspection of magnetic field strength, which should not exceed the average value of $20 \cdot 10^{-4} \mathrm{~T}$. This guarantees the magnetic field not influencing the welding process, particularly in welding the tubular or cylindrical elements.

Welding processes and welding consumables. The following methods are used for welding $9 \% \mathrm{Ni}$ steel: coated electrode manual electric arc welding, fluxcored wire welding, submerged-arc welding, TIG and MIG welding. Selection of welding consumables for these steels depends on a number of factors: safety, calculation of product design and cost effectiveness. 
Product safety is determined by the following properties of welding consumable: thermal expansion coefficient, hydrogen sensitivity, hot cracking resistance, impact toughness, crack propagation, relative elongation after rupture (tensile strength) at tensile deformation, behaviour from the viewpoint of fracture mechanics.

Product design should take into account the following: yield limit, strength and impact toughness at design temperature.

Cost effectiveness requires taking into account such factors as welding methods, efficiency of welding consumable application, repairability, and welding consumable cost.

Characteristics of individual groups of welding consumables. Welding with matching consumables. This group covers welding consumables with $9 \% \mathrm{Ni}$. Welding with matching welding consumable is not recommended for critical structures, as it cannot guarantee sufficient impact toughness of weld metal.

1. Chromium-nickel austenitic welding consumables (Cr-Ni alloying type). They provide good ultimate strength and yield limit of weld metal. Weld metal has high impact toughness at temperatures above $-196^{\circ} \mathrm{C}$.

2. Nickel alloys. This variant is preferable due to greater stability of weld metal at variable temperatures.

Nickel-based welding consumables, such as Inconel (Ni-Cr alloy) and Hastalloy (Ni-Mo alloy), have almost the same linear expansion coefficient, as $9 \%$ $\mathrm{Ni}$ steel, whereas austenitic welding consumables have linear expansion coefficient, different from that of $9 \% \mathrm{Ni}$ steel. If this peculiarity is ignored, there is the risk of thermal fatigue, resulting from different thermal expansion. Nickel-based welding consumables provide a lower crack propagation rate.

Specification and properties of welding consumables. Table 3 [3] gives the requirements of AWS and JIS specifications to welding consumables for $9 \%$ Ni steel.

Coated electrodes. In keeping with AWS-A5.11 specification, the following types of coated electrodes are recommended: EniCrFe-9, EniCrMo-6 and EniMo-8, and according to JIS Z 3225: D9Ni-1 and D9Ni-2.

Flux-cored wires. Application of flux-cored wire for welding LNG tanks from $9 \% \mathrm{Ni}$ steel is limited because of stringent control of welding parameters in a narrow range to avoid hot cracking, and welding in all the positions is difficult.

TIG-welding with mechanized feed of solid wire. It is mostly used in Japan.

The main advantage of TIG welding is the possibility of automatic welding application at higher values of current and intentional magnetic deviation of the arc to produce high-quality weld metal. This welding method is two times more effective than coated-electrode welding and four times more effective that manual TIG welding. More over, this method allowed reducing defects almost to zero, and improving the welding time, total cost and weld quality.

Submerged-arc welding. In specifications for submerged-arc welding in A 5.14 AWS only the wires are regulated. Contrarily, JIS standard specifies a combination of wire and flux. It is shown in Table 4 [3].

Welding procedures. A key factor in cost-effective and sound design of a tank is reduction of production operations in site. This can be achieved through development of a modular structure, in which each individual module is manufactured in shop conditions, and transported to the assembly site for subsequent mounting. Even the domed cap of the tank for liquefied natural gas is manufactured in the plant as enlarged elements and is joined to the hull in site by its lifting by compressed air.

Welded joints made in site are shown in Figure 1. The procedures of welding individual types of joints are given in Table 5.

Figure 2 gives the examples of beveling of the edges to be welded for different welding methods and welding positions.

Recommendations for obtaining the best results. Avoiding steel magnetization. At transportation, storage and further processing, one should avoid material magnetization, which may occur at circular

Table 3. AWS and JIS specifications for welding consumables for $9 \% \mathrm{Ni}$ steel

\begin{tabular}{|l|c|l|}
\hline \multicolumn{1}{|c|}{ Process } & AWS standard & \multicolumn{1}{c|}{ Specifications for } \\
\hline Manual electric arc welding & A5.11/A5.11M:2005 & Coated electrodes from nickel and nickel alloys \\
\hline Flux-cored wire welding & A5.34/A5.34M:2007 & Flux-cored wire from nickel alloys \\
\hline $\begin{array}{l}\text { MIG welding } \\
\text { TIG welding } \\
\text { Submerged-arc welding }\end{array}$ & A5.14/A5.14M:2005 & Solid wire from nickel and nickel alloys \\
\hline Process & JIS стандарт & Specifications for \\
\hline Manual electric arc & Z 3225:1999 & Coated electrodes for 9\% Ni steel \\
\hline TIG welding & Z 3332:1999 & Rods and solid wire for TIG welding of $9 \% \mathrm{Ni}$ steel \\
\hline Submerged-arc welding & Z 33335:1999 & $\begin{array}{l}\text { Solid wire and fluxes for submerged-arc welding of } 9 \% \mathrm{Ni} \\
\text { steel }\end{array}$ \\
\hline
\end{tabular}


Table 4. Technical requirements for wire (AWS) and fluxes (JIS) for automatic welding

\begin{tabular}{|c|c|c|c|}
\hline \multirow[b]{2}{*}{ Technical requirements } & \multirow{2}{*}{$\begin{array}{c}\text { AWS A5.14 } \\
\text { Wire ERNiMo-8 }\end{array}$} & \multicolumn{2}{|c|}{ JIS Z 3333} \\
\hline & & $\begin{array}{c}\text { FS9Ni-F/YS9Ni flux } \\
\text { weld metal }\end{array}$ & $\begin{array}{l}\text { FS9Ni-H/YS9Ni flux } \\
\text { weld metal }\end{array}$ \\
\hline \multicolumn{4}{|c|}{ For chemical composition } \\
\hline $\mathrm{C}(\%)$ & $\leq 0.10$ & $\leq 0.10$ & $\leq 0.10$ \\
\hline $\mathrm{Si}(\%)$ & $\leq 0.75$ & $\leq 1.5$ & $\leq 1.5$ \\
\hline $\operatorname{Mn}(\%)$ & $\leq 1.5$ & $\leq 3.5$ & $\leq 3.5$ \\
\hline $\mathrm{Ni}(\%)$ & $\geq 60.0$ & $\geq 60.0$ & $\geq 60.0$ \\
\hline $\mathrm{Cr}(\%)$ & $0.5-3.5$ & - & - \\
\hline Мo (\%) & $17.0-20.0$ & $10.0-25.0$ & $10.0-25.0$ \\
\hline W (\%) & $2.0-4.0$ & - & - \\
\hline $\mathrm{Fe}(\%)$ & $\leq 10.0$ & $\leq 20.0$ & $\leq 20.0$ \\
\hline \multicolumn{4}{|c|}{ For mechanical composition } \\
\hline Yield limit (MPa) & - & $\geq 365$ & $\geq 365$ \\
\hline Ultimate strength (MPa) & - & $\geq 660$ & $\geq 660$ \\
\hline Relative elongation (\%) & - & $\geq 25$ & $\geq 25$ \\
\hline Impact toughness $K C V$ at $-196^{\circ} \mathrm{C}$ & - & $\begin{array}{c}\text { Average value } \geq 34 \\
\text { Each sample } \geq 27\end{array}$ & $\begin{array}{c}\text { Average value } \geq 34 \\
\text { Each sample } \geq 27\end{array}$ \\
\hline
\end{tabular}

Table 5. Welding procedures for individual joint types (see Figure 1)

\begin{tabular}{|l|l|l|l|l|}
\hline \multicolumn{1}{|c|}{ Joint number } & \multicolumn{1}{|c|}{1} & \multicolumn{1}{c|}{ 2 } & \multicolumn{1}{c|}{4} \\
\hline Element & Hull & Hull & Bottom to hull & Bottom \\
\hline Joint type & X-shaped asymmetric & X-shaped asymmetric & X-shaped asymmetric & Overlap \\
\hline Position & Vertical & Horizontal & Horizontal & Horizontal \\
\hline Welding process & $\begin{array}{l}\text { Coated electrodes with flux- } \\
\text { cored wire } \\
\text { Auto-TIG }\end{array}$ & $\begin{array}{l}\text { Submerged-arc } \\
\text { Auto-TIG }\end{array}$ & $\begin{array}{l}\text { Coated electrode } \\
\text { Auto-TIG }\end{array}$ & $\begin{array}{l}\text { Coated electrodes with flux- } \\
\text { cored wire } \\
\text { Auto-TIG }\end{array}$ \\
\hline
\end{tabular}

bending between the rolls; at transportation by magnetic cranes; at cutting with guidance along magnetic rollers; under the impact of current-conducting welding cables or at other similar impacts. Before welding, the magnetic field between the edges being welded should be controlled by a magnetometer. Values of magnetic field intensity up to $60 \cdot 10^{-4} \mathrm{~T}$ do not have any significant effect on welding.

One of the following measures can be proposed to solve the problem of magnetic blow:

- alternating current welding;

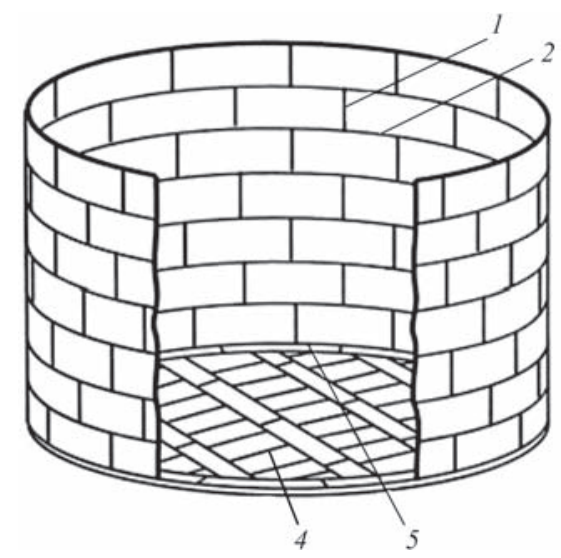

Figure 1. Typical welded joints of the hull and bottom of $9 \% \mathrm{Ni}$ steel tank made in site conditions (for description of 1-4 see Table 5)
- use of modern welding current sources, for instance square-wave source, etc.;

- deposition of a buffer layer on the edges to be welded with an electrode selected for welding;

- after occurrence of magnetization it can be reduced or eliminated by applying conductors (cables), through which alternating current runs, or using electric magnets. Thicker sheets may require application
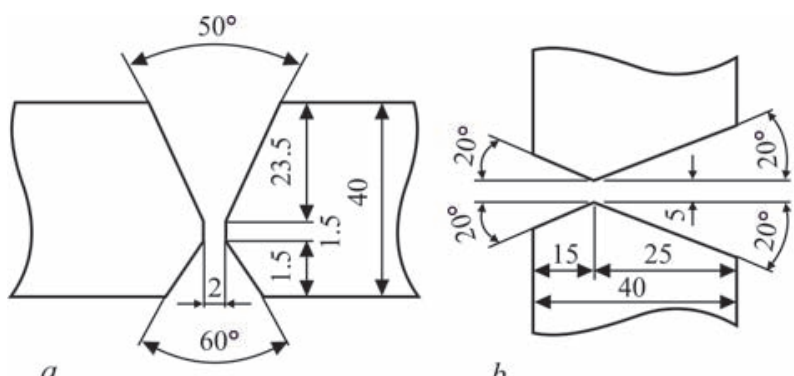

$b$
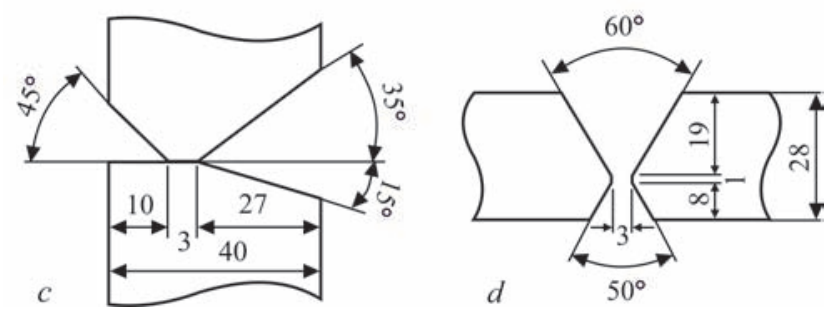

Figure 2. Typical groove for different welding methods and positions: $a-$ SMAW; $b-$ GTAW; $c-$ SAW; $d-$ FCAW 
of special demagnetization equipment, which uses gradual decrease and change of current direction. In extreme cases, residual magnetism can be eliminated by heat treatment.

Rate of energy input (heat input). Similar to other low-temperature steels, it is necessary to control the heat input for preservation of impact toughness in the HAZ metal. As a rule, steel is welded with small heat input - it is best to perform even submerged-arc welding with $1.6 \mathrm{~mm}$ wire.

It is recommended to perform welding with the heat input from 1.2 up to $2.0 \mathrm{~kJ} / \mathrm{mm}$. Irrespective of the thickness, it is not recommended to conduct welding with more than $2.0 \mathrm{~kJ} / \mathrm{mm}$ heat input. If higher heat inputs are used for submerged-arc or MIG welding, it is necessary to first make sure that the weld strength is not lower than $655 \mathrm{MPa}$. Care should also be taken at less than $13 \mathrm{~mm}$ thickness, as in welding sheets the joints cool down slower, and, in addition, the values of impact toughness for thinner sheets are initially lower.

Preheating. $9 \% \mathrm{Ni}$ steels have relatively good cold cracking resistance. Irrespective of that, it is recommended to preheat plates of more than $25 \mathrm{~mm}$ thickness up to $35^{\circ} \mathrm{C}$, and not to weld thinner sheets at temperature below the dew point. The interpass temperature should not exceed $150^{\circ} \mathrm{C}$.

Postweld heat treatment. If not specifically recommended by the manufacturer, postweld heat treatment is not performed on up to $51 \mathrm{~mm}$ thicknesses. To avoid the possible lowering of steel impact toughness during heat treatment performance, after welding it is necessary to control the temperature in the range of $551-583{ }^{\circ} \mathrm{C}$, but not higher than that of tempering with subsequent cooling at not less that $167^{\circ} \mathrm{C} / \mathrm{h}$ rate.

Hot cracking. As a rule, it concerns hot cracks in the crater. It is recommended to grind off the crater every time you stop the arc.

Base metal participation. Mechanical properties of weld metal depend on the quantity of base metal, participating in the weld metal. This most often influences lowering of mechanical properties. It is recommended to first check the effect of welding parameters, devel-

Table 6. ISO requirements to impact toughness of samples with V-shaped notch

\begin{tabular}{|c|c|c|c|}
\hline \multirow{2}{*}{ Material grade } & \multirow{2}{*}{$\begin{array}{c}\text { Testing } \\
\text { temperature, } \\
{ }^{\circ} \mathrm{C}\end{array}$} & \multicolumn{2}{|c|}{ Fracture energy ${ }^{*}, \mathrm{~J}(\mathrm{~min})$} \\
\hline & & $\begin{array}{c}\text { Along the rolling } \\
\text { direction }\end{array}$ & $\begin{array}{c}\text { Across the rolling } \\
\text { direction }\end{array}$ \\
\hline Sheets & \multirow{3}{*}{-20} & - & 27 \\
\hline Pipes & & 41 & - \\
\hline Forgings & & 41 & 27 \\
\hline
\end{tabular}

oping a procedure that would ensure the required ultimate strength and yield limit of weld metal.

Isothermal tanks for liquefied ammonia storage. It was found that stress corrosion cracking is one of the main causes for accidents in anhydrous ammonia tanks under normal service conditions [3]. These cracks propagate predominantly inside the crystalline grains and are usually observed in steels with higher strength and hardness.

Observations show that cracks are most often associated with welded joints, where HAZ hardness is higher than that of base metal and weld metal, and tensile residual stresses are in place. Performance of postweld heat treatment for relieving residual stresses reduces the cracking susceptibility [4], but it often cannot be conducted, because of large dimensions of the tanks. Moreover, heat treatment after commissioning of the facility can be ineffective, as the stress fields at the tip of the initiating cracks have quite high intensity, which supports crack propagation.

Measures required for designing tanks for ammonia storage. Factors promoting formation of stress corrosion cracking can be divided into three groups:

Tank material. From the viewpoint of material the following factors should be taken into account: material type, mechanical properties and chemical composition, microstructure, deformations and surface condition. Plates from low-carbon low-alloyed steel with nominal yield limit not higher than $355 \mathrm{MPa}$ are recommended for manufacture [5] of tanks for anhydrous ammonia. Actual yield limit should not exceed $440 \mathrm{MPa}$, and relative elongation $\delta_{5}$ should be higher than $22 \%$.

It is recommended that the chemical composition is in the following range, wt.\%: $0.18 \mathrm{C} ; 0.10-0.50 \mathrm{Si}$; $1.65 \mathrm{Mn} ; 0.030 \mathrm{P} ; 0.025 \mathrm{~S}$; $\min 0.020 \mathrm{Al} ; 0.20 \mathrm{Cr}$; $0.35 \mathrm{Cu} ; 0.08 \mathrm{Mo} ; 0.40 \mathrm{Ni} ; 0.10 \mathrm{~V}$. After normalizing the steel should have a fine-grained structure. It should be taken into account that for steels with nominal yield limit of $355 \mathrm{MPa}$ the chemical composition is selected in order to limit the maximum yield limit to $440 \mathrm{MPa}$. If alloying with nickel is required, the maximum value can be $0.85 \%$.

All the pressure vessel materials should meet the requirements to impact toughness, according to Table 6 .

Active medium. In terms of active medium, the following should be taken into account: medium type, impurities, temperature and electrochemical conditions.

Anhydrous ammonia is stored at temperature in the range from ambient temperature to $-33^{\circ} \mathrm{C}$. Crack formation during stress corrosion greatly depends on presence of oxygen and water in ammonia. In all the cases, crack formation is associated with oxygen presence. Cracking susceptibility becomes higher with 


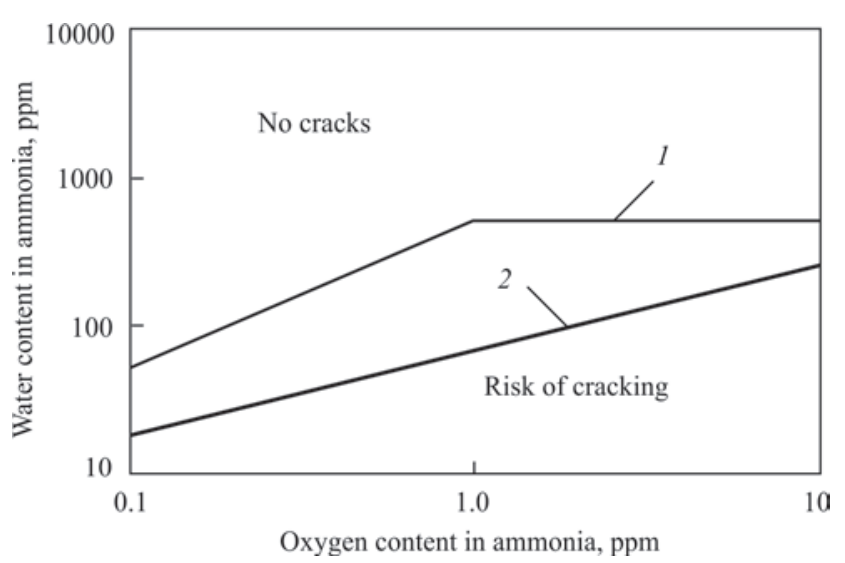

Figure 3. Relationship between water and oxygen content in ammonia and risk of crack formation during stress corrosion [6] $\left(1-18 ; 2--33^{\circ} \mathrm{C}\right)$

increase of oxygen content and decreases with water content (Figure 3). Usual practice is use of water as corrosion inhibitor.

Under normal working conditions of a cooled ammonia tank, oxygen content should be lower than $0.5 \mathrm{ppm}$, and water content should be in the range from 100 up to $1000 \mathrm{ppm}$.

Stresses. In terms of stresses, the following should be taken into account; residual stresses, resulting from the production process when building the construction, in particular welding stresses, working stresses and such kinds of stresses as static and cyclic, arising in service. Surface tensile residual stresses have a special role in stress corrosion cracking. For this reason, serious attention should be given to means for their reduction.

Features of welding technology, ensuring maximum fatigue life of welded structure. To achieve maximum fatigue life of ammonia tanks, it is necessary to comply with a number of conditions in development and introduction of welding technology, concerning selection of welding consumables and welding mode, in order to avoid stress corrosion cracking.

For manual electric arc welding, it is necessary to use coated low-hydrogen basic electrodes (diffusible hydrogen content below $8 \mathrm{ml} / 100 \mathrm{~g}$ ). It is allowed to apply only welding consumables, containing no molybdenum or vanadium. Strength of deposited material should exceed that of the base metal by a minimum value. Weld hardness, including the heat-affected zone, should not be higher than $H V 230$. This should be confirmed at testing and approval of the procedure.

Preheating temperature should be minimum, in order to maintain low welding stress and welded joint hardness. Preheating and interpass temperatures should be not lower than $100{ }^{\circ} \mathrm{C}$, and all the welding operations should be performed by a multilayer welding technique.

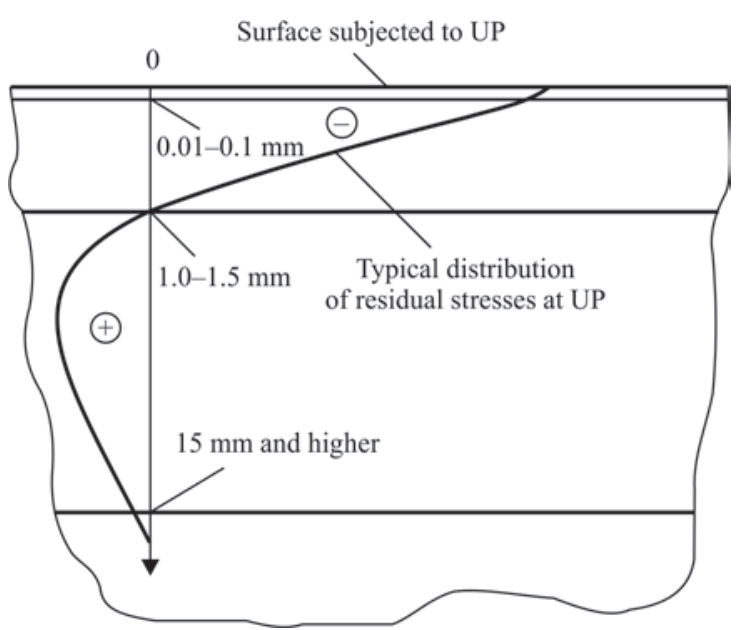

Figure 4. Scheme of the cross-section of material/item, improved by ultrasonic peening [7]

Welding defects, such as excessive weld reinforcement and points of striking (the arc) on the base metal should be eliminated by grinding off. All the butt welds and welds with full penetration of the tank wall should be made by multilayer technique.

In a specific case a suitable method for relieving residual stresses is the ability to perform their ultrasonic peening. If required, the weld surfaces should be ground.

Ultrasonic peening. Ultrasonic peening (UP) is an effective method of relieving harmful tensile residual stresses and generation of favourable compressive residual stresses in surface layers of parts or welded elements. UP method is based on a combined effect of high-frequency shocks applied by special strikers and ultrasonic oscillations in the treated material. During ultrasonic treatment the striker oscillates in a small gap between the end face of ultrasonic converter and treated sample, striking at the surface being treated.

Ultrasound has the following effects on the metal: acoustic softening (hardness lowering), acoustic strengthening, acoustic heating, etc. In the first case (acoustic softening, so-called acoustoplastic effect), acoustic radiation reduces the stress that is required for plastic deformation.

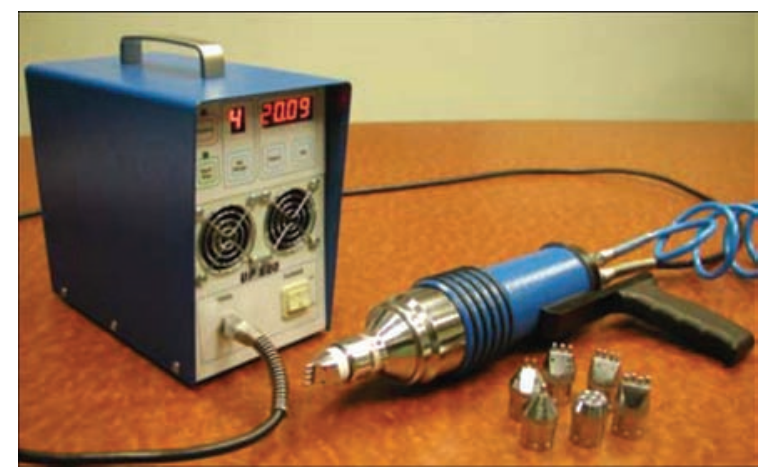

Figure 5. System for ultrasonic peening, increasing the fatigue life of welded elements and structures [8] 
On the whole, influence of ultrasound on the mechanical behaviour can be compared with the effect of material heating. The difference consists in that the acoustic softening occurs directly after the impact of ultrasonic irradiation on the metal. Moreover, ultrasonic waves with relatively small amplitude do not have any residual effects on the physical properties of metals after exposure to acoustic radiation.

At UP the ultrasonic converter (piezoelectric or magnetostriction) oscillates at a high frequency, typical frequency being $20-30 \mathrm{kHz}$. Irrespective of the technology of converter manufacturing. its end face will oscillate with $20-40 \mathrm{~mm}$ amplitude.

UP can provide an increase of resistance to stress corrosion cracking by inducing compressive residual stresses in the surface layers of metals and alloys, reduction of stress concentration in the heat-affected zone, and improvement of mechanical properties of the material surface layer. Figure 4 shows the scheme of the cross-section of material/item, treated by UP.

Figure 5 shows the configuration of the device for ultrasonic peening, which can be used for processing of both the weld HAZ and large surface areas, if required [7]. Treatment speed is about $0.4 \mathrm{~m} / \mathrm{min}$.

Control of residual stresses. After treating the structure to relieve or lower the residual stresses, it is necessary to find out the degree of this treatment effectiveness and efficiency. There are two groups of methods for residual stress measurement: destructive and nondestructive. The first group of methods (destructive) is not applicable for control of ammonia tanks.

The nondestructive group of testing methods include magnetic, ultrasonic, and X-Ray diffraction. Ultrasonic testing methods are quite promising. They are based on acoustoelastic effects, as a result of which the speed of elastic wave propagation in solids depends on mechanical stresses [7].

In conclusion it should be noted that the high operational reliability of the tanks for storage of liquefied natural gas can be ensured at comprehensive compliance with the requirements at the stages of design, manufacture, mounting and service.

1. Welding liquide natural gas tanks and vessels in $5 \%$ and $9 \%$ nickel steels. ESAB.

2. Welding LNG tanks and vessels in $5 \%$ and $9 \%$ nickel steels. ESAB.

3. (2011) Welding Today, 14(2), 1-10. Kobe, co.

4. Det Norske Veritas - Report No: 98-3111, rev. 01-15.19 Ammonia stress corrosion cracking.

5. (1992) No.33: Guidelines for the construction of pressure vessel type tanks intended for the transportation of anhydrous ammonia at ambient temperatures.

6. Alghamdi, T., Liu, S. (2014) Low transformation temperature (LTT) welding consumables for residual stress management: Consumables development and testing qualification. Welding $J ., 93(7)$.

7. Kudryavtsev, Y. (2013) Ultrasonic measurement of residual stresses in welded specimens and structures. In: Proc. of the ASME 2013 Pressure Vessels and Piping Conf. PVP2013 (July 14-18, 2013, Paris, France).

8. Kudryavtsev, Y., Kleiman J. (2013) Fatigue improvement of welded elements and structures by ultrasonic peening. In: Ibid.

Received 09.07.2018 


\title{
STRUCTURE AND PROPERTIES OF WEAR-RESISTANT ION-BEAM VACUUM COATINGS
}

\author{
M.A. ANDREEV and L.V. MARKOVA \\ SSI «Powder Metallurgy Institute» \\ 41 Platonov Str., 220005, Minsk, Belarus
}

\begin{abstract}
The work investigates the structure and properties of coatings, formed by ion-beam spraying in vacuum of composite targets based on chromium with addition of ultradisperse diamonds and nanodisperse particles of $\mathrm{Al}_{2} \mathrm{O}_{3}$ and $\mathrm{ZrO}_{2}$. It is shown that the ion-beam spraying method allows transferring target material on the product in form of coating keeping the composition and stoichiometry of the compound. A structural model of ion-beam vacuum coatings based on chromium with addition of ultradisperse diamonds $\mathrm{Al}_{2} \mathrm{O}_{3}$ and $\mathrm{ZrO}_{2}$ was developed. 1 Ref., 5 Figures.
\end{abstract}

Keywords : ion-beam vacuum coatings, nanosized particles, structure, properties

One of the relevant directions in the field of science and technology is modification of surface of the structural and tool steels in order to increase the service characteristics of machine and mechanism parts operating under excessive wear and aggressive media. Today the enterprises of machine-building and metal-working industries widely use the different methods of surface modification such as deposition of wear-resistant coatings by vacuum physical and chemical methods, different types of thermochemical treatment.

The method of ion-beam spraying is the most efficient among the physical methods of functional coating formation for deposition of multicomponent material films. Currently, one of the most perspective ways to increase the efficiency of wear-resistant coatings is addition to a spraying target a small amount (up to several percent) of nanosized particles of oxides of some metals as well as ultradisperse diamonds (UDD). Since ion-beam spraying provokes complete transfer of composition and stoichiometry of multicomponent material of spaying target into the coating, this method can be used for obtaining the thin composite coatings with additives of nanosized particles, introducing the materials mentioned above in the spraying target.

Materials and investigation procedure. The ionbeam coatings based on chromium with 1 and 5 wt.\% of $\mathrm{ZrO}_{2}$ and $\mathrm{Al}_{2} \mathrm{O}_{3}$ additives, respectively [1] as well as 1 and 5 wt.\% of UDD additives. Size of $\mathrm{ZrO}_{2}$ particles made $50 \mathrm{~nm}$, that of $\mathrm{Al}_{2} \mathrm{O}_{3}$ was $20 \mathrm{~nm}$. The time of spraying made $2 \mathrm{~h}$. Coatings were formed on St. 3 grade steel.

Investigations of surface morphology of coating surface was carried out on scanning electron micro- scope (SEM) of high resolution Mira of Tescan Company (Czech Republic), resolution of which makes $1.7 \mathrm{~nm}($ at $30 \mathrm{kV}$ ) and $3.0 \mathrm{~nm}$ (at $5 \mathrm{kV}$ ) as well as atomic-force microscope NT-206 (ODO Microtestmachines, Gomel). Resolution of AFM makes: vertical $-0.2 \mathrm{~nm}$; horizontal $-2 \mathrm{~nm}$.

Phase composition of ion-beam coatings was examined using electron backscatter diffraction (EBSD) method with add-on device to scanning electron microscope HKL. A principle of EBSD-analysis is based on formation of Kikuchi bands as a result of electron backscatter diffraction.

A value of load on indenter of Knoop type in microhardness measurement made $0.02-0.03 \mathrm{~N}$.

Adhesion strength was determined using a special block, in which Rockwell type indenter was moved over the surface with $5 \mathrm{~mm} / \mathrm{min}$ speed at smoothly increasing loading. A value of adhesion strength was determined on a value of normal load, at which coating detachment takes place on acoustic emission signal.

Wear-resistance was determined at loading on indenter $0.2 \mathrm{~N}$ and path $10 \mathrm{~m}$. The indenter in form of $3 \mathrm{~mm}$ diameter ball reciprocates over the sample surface without lubricant. A length of single pass over the sample surface made $10 \mathrm{~mm}$. A friction coefficient was determined during wear-resistance tests.

Chromium-based ion-beam coatings. Chromium is a material of increased wear resistance. The technology used for production of these coatings develops pore-free coating, which is virtually inaccessible for effect of the most severe and aggressive chemical agents. Therefore, in this case the etchant of the next composition, namely $50 \% \mathrm{HCl}+50 \% \mathrm{HF}$ was used for determination of corrosion resistance.

Structure of the ion-beam chromium coating presents itself a three-layer composition. It consists of a 

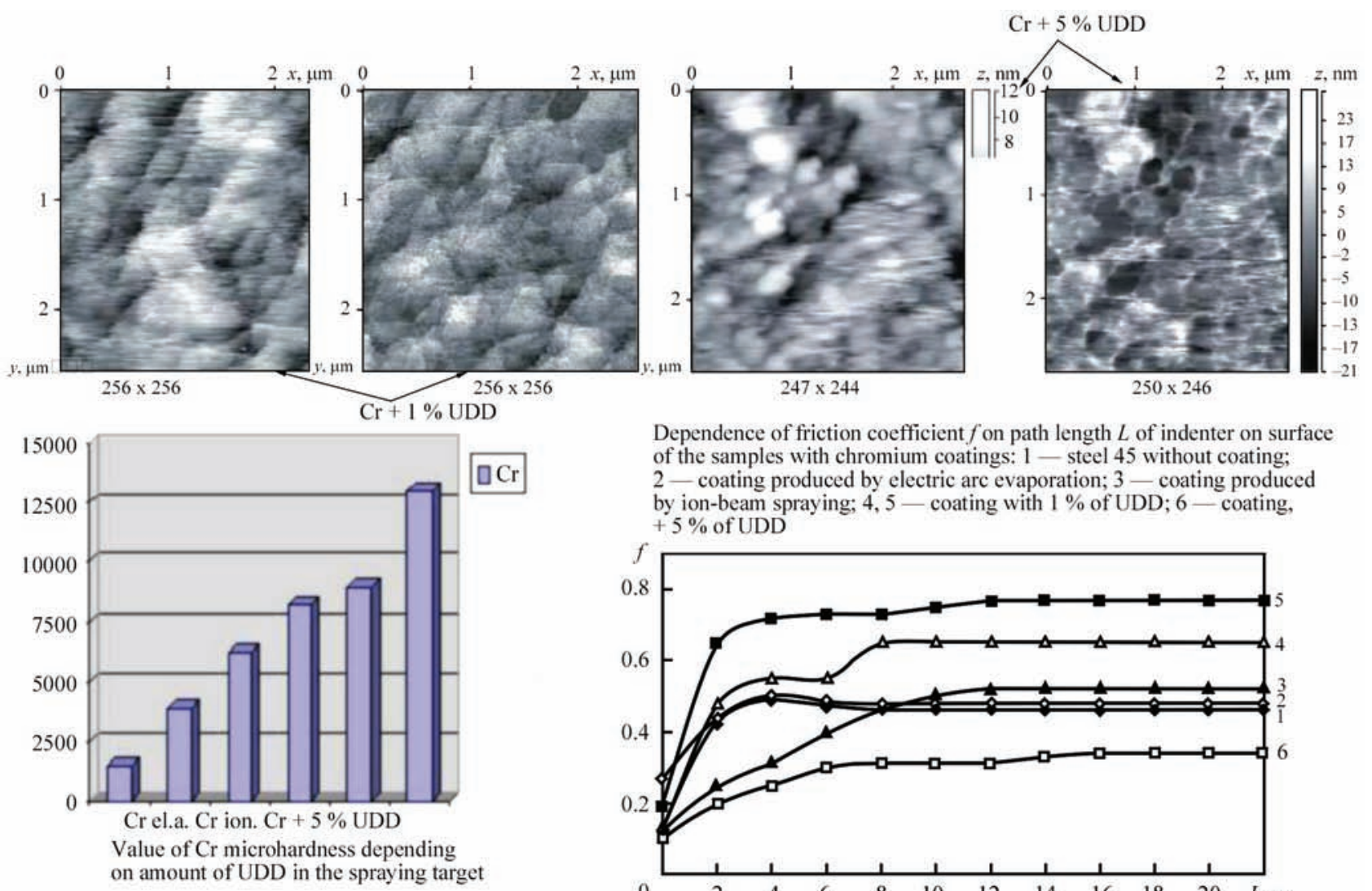

Dependence of friction coefficient $f$ on path length $L$ of indenter on surface of the samples with chromium coatings: 1 - steel 45 without coating;

2 - coating produced by electric arc evaporation; 3 - coating produced by ion-beam spraying; 4,5 - coating with $1 \%$ of UDD; 6 - coating, $+5 \%$ of UDD

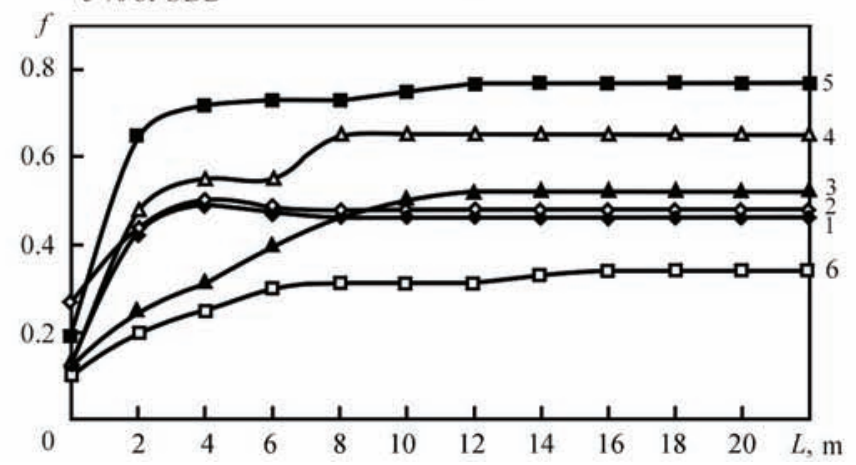

Figure 1. Microstructure, microhardness and coefficient of metal friction of Cr-based ion-beam coating with 1 and $5 \%$ of UDD additives

very thin surface layer, middle layer, having columnar branched structure, and formed under it a layer of grain structure. Addition of $1 \%$ of UDD into the spraying target increases coating resistance to etchant effect. Only some areas have got the etching pits, characterizing dislocation accumulation areas. A surface of chromium coating with $1 \%$ of UDD is virtually completely free from the single etching pits, however, a fine grain structure (Figure 1) starts its development. An analysis of coating cross-section structure showed that addition of $1 \%$ of UDD promoted absence of obvious columnarity in the cross-section structure.

Examination of morphology of the surface of ionbeam coatings based on $\mathrm{Cr}+\mathrm{UDD} 5 \%$ is much more complex. The etching pits even at very long etching appear as separate accumulations in form of sparse chains and single dots. In some instances, when etching on defect structure has reached the basis, it was possible to reveal coating cross-section structure based on chromium. Photos of the structure (Figure 1) clearly show columnar structure of the coating middle layer, moreover, this structure is laminar, but very dense. There are virtually no defects in it. Size of separate structural constituents does not exceed $10 \mathrm{~nm}$. Very fine and absolutely structureless for SEM resolution film is observed on the coating surface. It seems as the coating columnar structure grows in it.
A morphology of surface of the ion-beam chromium coating, obtained using atomic-force microscope has the following characteristics, namely grain height does not exceed $40 \mathrm{~nm}$, and it size varies in 100-150 nm limits (Figure 1).

Addition of $1 \%$ of UDD into the chromium spraying target promotes refining of the coating grain structure. An average grain size makes $70 \mathrm{~nm}$, and grain height does not exceed 15-20 nm. The grain boundaries are weakly revealed. The grain boundaries close to the coating develop more clearly when adding 5\% of UDD in the spraying chromium target. An average size of grains reduces from 40 to $50 \mathrm{~nm}$, and height does not exceed $10 \mathrm{~nm}$. In this case the grain boundary hardening using the phase contrast mode can be well seen (Figure 1).

The interesting results were obtained in microhardness measurement of the chromium ion-beam coatings. The values of microhardness of chromium ion-beam coatings with different content of UDD make:

- microhardness $16500 \mathrm{MPa}$ in the case of addition of $1 \%$ of UDD into the spraying target;

- microhardness $25900 \mathrm{MPa}$ (Figure 1) in the case with UDD $5 \%$.

It is known that the coatings, produced by ionbeam spraying method are brittle, as a rule. High hardness of the coatings can promote their cracking 
and further delamination. Therefore, the tribotechnical tests of these coatings have been carried out. The analysis of dependence of friction coefficient on a length of indenter path over the surface of samples with chromium coatings showed (Figure 1) that they can be divided into two steps.

The first step corresponds to friction surfaces running-in. At that, all samples demonstrate increase of the friction coefficient. It is explained by low roughness of the initial surface of examined coatings. During running-in the smooth initial surface is broken and equilibrium structure of coating surface layers with roughness exceeding the initial one is formed.

The second step is set wear-out. The friction coefficient at this step is not changed. The path of indenter until reaching the set wear-out can be one of the criteria for coating wear resistance evaluation. Addition of UDD into the spraying target increases the length of path before running-in, value of which reaches the maximum in the coating containing UDD $5 \%$. The minimum friction coefficient corresponds to this coating. The classical third step of change of the friction coefficient, namely breaking of the coating and friction over substrate, is absent in all examined samples after $22 \mathrm{~m}$ of path.

Thus, the examinations showed that the composite chromium coatings formed by the method of ion-beam spraying have sufficiently good wear resistance. An important index of wear-resistance is morphology of wear surface in a mode of set running-in. The largest part of the wear surface of coating, produced by spraying of powder chromium target with $1 \%$ of UDD, is free of any traces of intender impact. At the same time, on separate sections of friction paths there are sufficiently deep grooves, probably, being the result of incorporation of chipped particles of the coating.

Addition of UDD in the spraying target except for direct transfer of diamond phase provokes its partial decomposition and formation of chromium carbides. They are located along the grain boundaries and prevent emergence of the dislocation on the surface, simultaneously increasing hardness of the coating. Rise of diamond phase up to $5 \%$ allows forming a network virtually along the whole coating surface. It completely blocks the possibility of dislocation emergence on the surface. The processes of diffusion dislocation climb from the base into the coating on interphase boundaries and processes of formation of grown-in dislocations do not stop in coating formation. Formation of dislocation networks additionally strengthens the coating. It is proved by microhardness data.

In the coating with $5 \%$ of UDD $H_{\mu}=25900 \mathrm{MPa}$. However, the level of structural constituent refining affects the value of microhardness in addition to dislocation processes and formation of the network.

The ion-beam chromium-based coatings with addition of $1 \%$ of $\mathrm{ZrO}_{2}$ particles of $50 \mathrm{~nm}$ size into the spraying target are characterized with developed surface relief (Figure 2). There can be observed grain structure, moreover, grain size is varied from 50 to $200 \mathrm{~nm}$. After etching in acid mixture $\left(\mathrm{HNO}_{3}, \mathrm{HF}\right.$, $\mathrm{HCl}$ ) it can be clearly seen a columnar structure of the coating, besides, it is more dense in the upper layers and more porous in the base.

When comparing the coatings formed with addition of $5 \%$ of $\mathrm{ZrO}_{2}$ particles of the same size $(50 \mathrm{~nm})$, then in this case more dispersed structure will be observed, the grain size will refine and become more uniform (Figure 2). The grain size varies in the limits of 20-30 nm. An exposure in characteristic X-ray radiation indicates that $\mathrm{ZrO}_{2}$ is mainly located along the grain boundaries.

Spraying of the targets with $5 \%$ of $\mathrm{ZrO}_{2}$ results in grain size refinement. It can be clearly seen that in both cases zirconium dioxide is mainly located along the grain boundaries.

Measurements of coating microhardness showed: $17759 \mathrm{MPa}$ for targets with $1 \%$ of $\mathrm{ZrO}_{2}$ and 17800 $\mathrm{MPa}$ for targets with $5 \%$ of $\mathrm{ZrO}_{2}$.

Examination of the surface using atomic-force microscopy method were carried out for the samples formed of chromium targets containing $\mathrm{ZrO}_{2}$ (Figure 2) as well as the targets containing additives of aluminum oxide (Figure 3 ).

As it was shown by carried examination, spraying of chromium target with $5 \%$ of $\mathrm{ZrO}_{2}$ provides homogeneous structure with small grain size. Homogeneity of structure is proved by an image in lateral force mode. The grain size varies from 60 to $100 \mathrm{~nm}$ (Figure 2).

Decrease of content of $\mathrm{ZrO}_{2}$ to $1 \%$ results in coarsening of chromium coating grain. Grain size in the coating of the target with $\mathrm{ZrO}_{2}$ particles of $50 \mathrm{~nm}$ size varies from 50 to $200 \mathrm{~nm}$ (Figure 2). At that it has the largest inhomogeneity of surface in the lateral force mode. There are two phases in the surface structure, moreover, one of them is divided on subgrains of $40 \mathrm{~nm}$ size. Grain structure for the coating formed using chromium target containing $5 \%$ of $50 \mathrm{~nm}$ size $\mathrm{ZrO}_{2}$ particle is homogeneous and grain size varies in 50-100 nm range.

Obtained date can be explained in the following way. Grain size in the chromium ion-beam coating depends on density of nucleation centers in solidification of coating, to which $\mathrm{ZrO}_{2}$ particles are referred to among the others. 

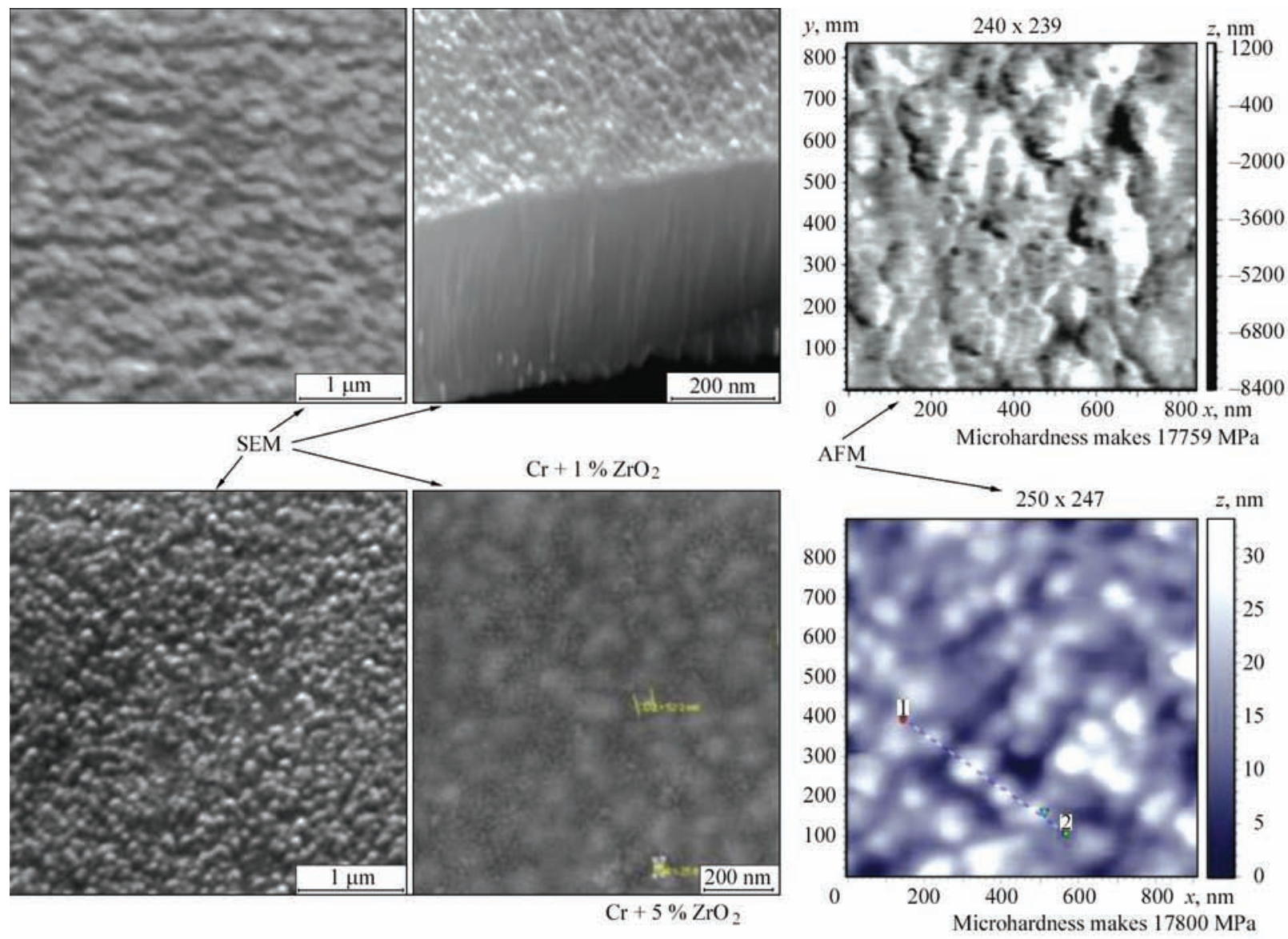

Figure 2. Microstructure of ion-beam chromium-based coating with 1 and $5 \%$ of $\mathrm{ZrO}_{2}$ additives

A size range of $\mathrm{ZrO}_{2}$ particles in $5 \%$ amount forms the optimum conditions for generation of homogeneous nanosized structure with $60-100 \mathrm{~nm}$ grain size. Content of $1 \%$ of $\mathrm{ZrO}_{2}$ oxide does not already guarantee sufficient number of nucleation centers for formation of nanosized grain in the coating.

Examination of surface of the ion-beam coating formed by spraying of chromium target with $5 \%$ of $\mathrm{Al}_{2} \mathrm{O}_{3}$ additive showed that it has grain structure. Grain size varies from 50 to $70 \mathrm{~nm}$. Etching in acid mixture revealed appearance of the coating columnar structure.

In examination of the coating formed with the help of $\mathrm{Cr}+5 \% \mathrm{Al}_{2} \mathrm{O}_{3}$ target on the field of $4 \times 4 \mu \mathrm{m}$ it is possible to determine that a relief was formed by multiple deepenings, roughness $R$ makes $9.1 \mathrm{~nm}$. On the field of $2 \times 2 \mu \mathrm{m}$ it was found that the surface was formed by deepenings of 100-400 nm diameter and separate projections of 100-200 nm diameter. In «Torsion» mode the surface has one color, therefore, it is single-phase.

Addition into the spraying chromium target of $5 \%$ of $\mathrm{Al}_{2} \mathrm{O}_{3}$ allows rising microhardness of the coating up to $29640 \mathrm{MPa}$. For these coatings the microhardness results differ by good stability.

The ion-beam chromium coatings without additives of different materials into the spraying target have microhardness values of around $7600 \mathrm{MPa}$. Ad- dition of aluminum oxides into the spraying target allows forming on the samples' surface the coatings having almost two-three times higher microhardness.

Scratch test was used to determine the adhesion strength of titanium coatings. Normal loading on indenter in scratch increases from 0 to $90 \mathrm{~N}$, indenter movement speed over the surface made 5 and $10 \mathrm{~mm} / \mathrm{min}$. Wear resistance was evaluated on wearout value, which was determined on area of a crater on profilograms across the friction pathes. The largest wear-out was registered in coatings of the target $« \mathrm{Cr}+$ $+1 \% \mathrm{ZrO}_{2}$ of $50 \mathrm{~nm}$ grain size», for which $K_{\mathrm{fr}}=0.7$ was determined. High value of wear-out and $K_{\mathrm{fr}}$ as well as low value of adhesion strength are explained by coating damage. The best results were registered for coatings formed from the target $« \mathrm{Cr}+5 \% \mathrm{ZrO}_{2}$ of $50 \mathrm{~nm}$ grain size», in this case $K_{\mathrm{fr}}=0.17$. Absence of pronounced wear craters indicates formation in the friction zone of the transferred layers.

Analysis of phase structure of the ion-beam coatings was carried out by method of electron backscatter diffraction (EBSD) with the help of add-on device to scanning electron microscope HKL. A principle of EBSD-analysis is based on formation of diffraction pattern, which is generated as a result of electron backscatter diffraction. The information embedded in the diffraction pattern contains data on symmetry of crys- 


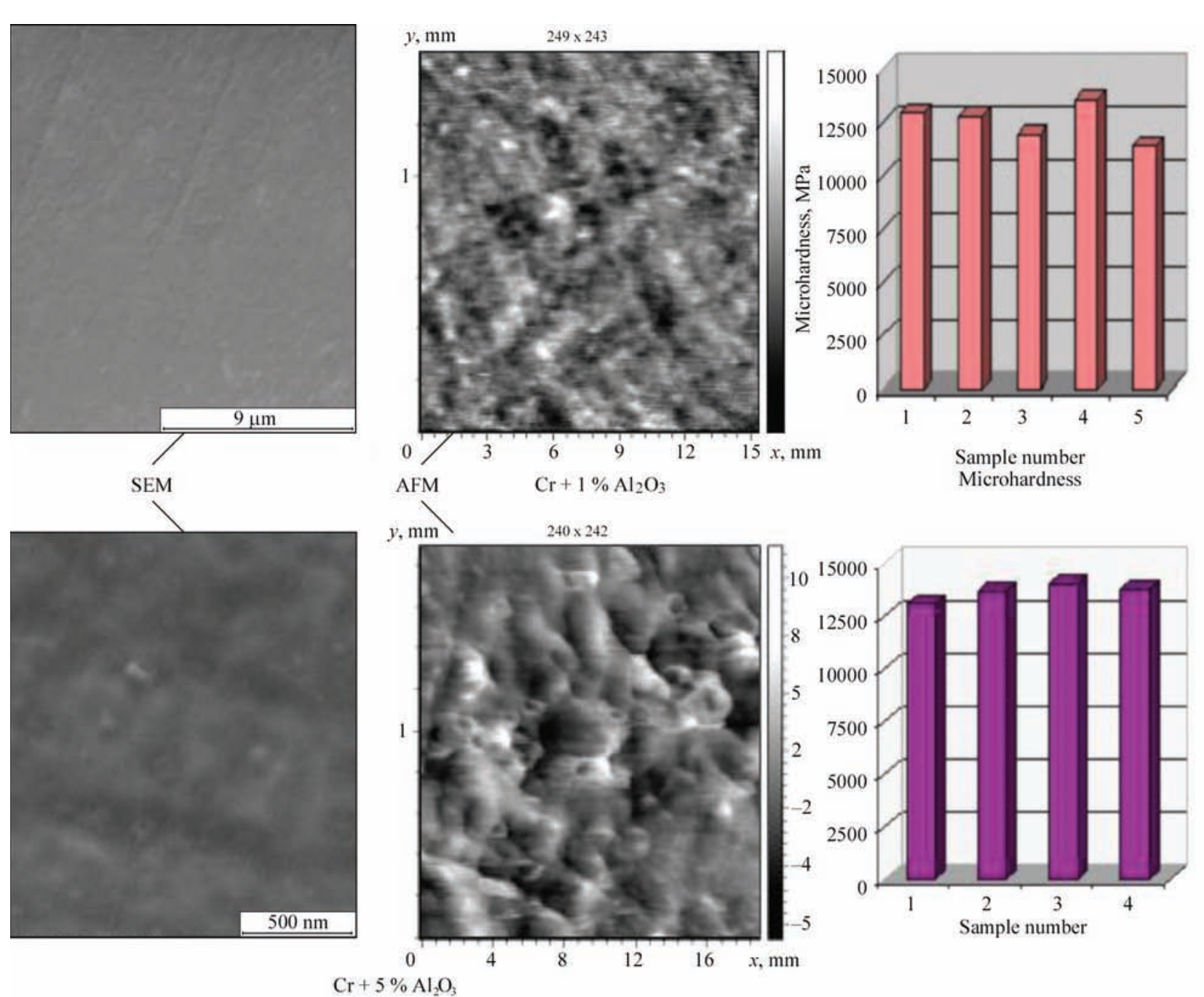

Figure 3. Microstructure and microhardness of ion-beam Cr-based coating with 1 and $5 \%$ additive of $\mathrm{Al}_{2} \mathrm{O}_{3}$

tal and its orientation. Since the angles between the planes and axes of the zone are explicitly determined by crystal symmetry and parameters of its lattice, then information taken from the diffraction pattern is used for determination of phases contained in the sample.

The results of point X-ray phase examination of the ion-beam coatings are presented in Figure 4. Kikuchi bands were assigned with Miller indices in accordance with crystalline planes, which formed them, and the intersection points of bands are marked with the symbols of zone axes. The coating structure was examined in the several points (areas) of the coating.

Collision of the particles of gas phase with the substrate surface took place on the first stage, after what the particles can firmly attach on the substrate or in some time evaporate or elastically reflect from the surface.

Following the results of X-ray phase analysis, different phases of target metal as well as nanodisperse additives can be formed in the process of formation of the ion-beam coatings.

Thus, for example, in spraying of the target with $\mathrm{ZrO}_{2}$ additives, chromium and titanium there can be formed hexagonal, cubic or tetragonal lattice.
$\mathrm{ZrO}_{2}$ crystalline lattice in the coating can be rhombic and monoclinic. $\mathrm{Al}_{2} \mathrm{O}_{3}$ forms a face-centered cubic lattice and body-centered trigonal lattice, formation of rhombic lattice is also possible.

Analysis of obtained results allows concluding that three types of crystalline lattices, namely cubic, hexagonal and body-centered cubic are formed by chromium in the process of ion-beam spraying independent on composition and amount of additives. Besides, it is determined that common cubic lattice and body-centered cubic lattice have different interplane distances $-0.288 \mathrm{~nm}$ for common cubic and $0.459 \mathrm{~nm}$ for body-centered cubic. Hexagonal lattice has $a=b=0.272 \mathrm{~nm}, c=0.443 \mathrm{~nm}$ (Figure 4).

Application of EBSD method allowed proving that $\mathrm{ZrO}_{2}$ and $\mathrm{Al}_{2} \mathrm{O}_{3}$ are transferred from the target on the coating in form of cluster crystalline structures during the process of ion-beam spraying. Thus, $\mathrm{ZrO}_{2}$ in the chromium-based coatings was found in form of three crystalline lattices, i.e. tetragonal with interplane distances $a=b=0.363 \mathrm{~nm}, c=0.520 \mathrm{~nm}$ and rhombic with similar interplane distances. $\mathrm{Al}_{2} \mathrm{O}_{3}$ in a coating composition has three crystalline lattices, name- 

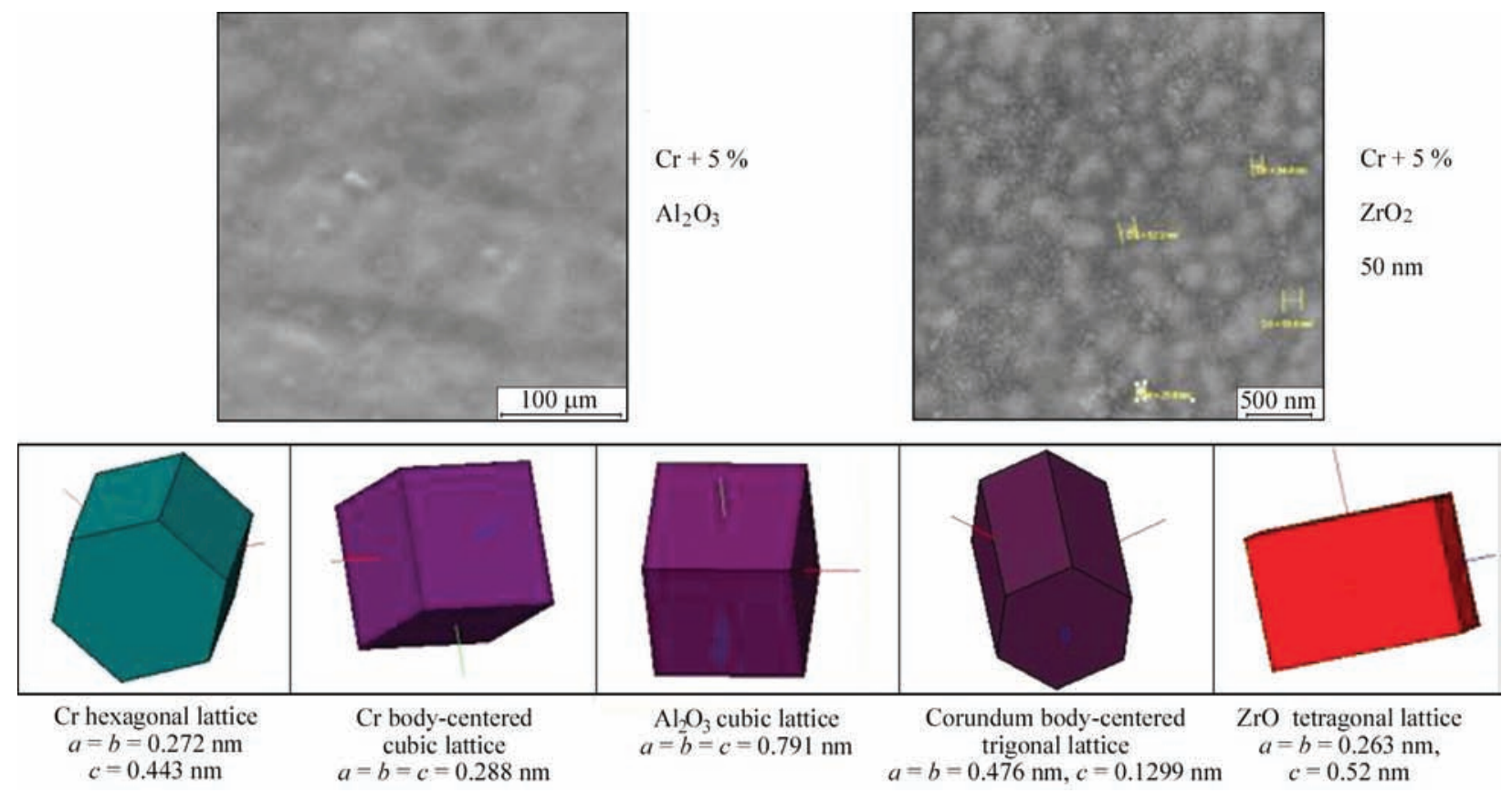

Figure 4. Morphology of surface of ion-beam coating based on $\mathrm{Cr}$ with different additives and HKL data
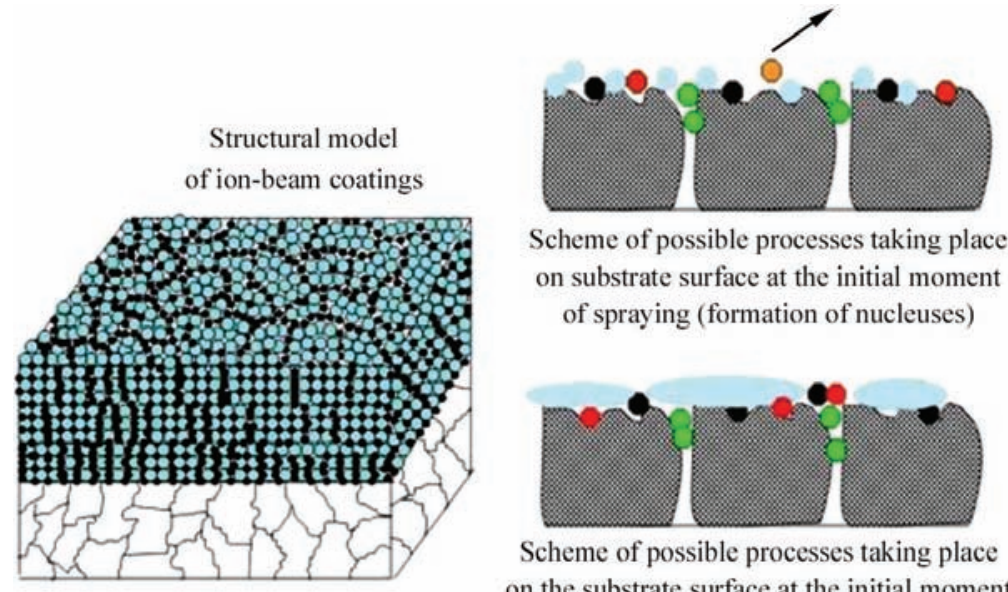

Scheme of possible processes taking place on substrate surface at the initial moment of spraying (formation of nucleuses)

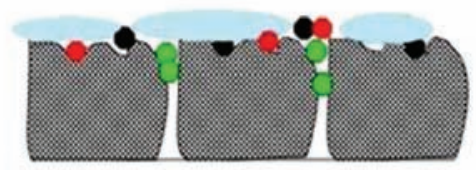

Scheme of possible processes taking place on the substrate surface at the initial moment of spraying (formation of islands)
Target base material

- Ultradisperse additives

- Chemically bonded particles

- Volumetric diffusion of particles

Elastically reflected particles

Figure 5. Structural model of ion-beam coatings formed based on chromium with UDD additives, $\mathrm{Al}_{2} \mathrm{O}_{3}$ and $\mathrm{ZrO}_{2}$

ly face-centered cubic, rhombic and body-centered trigonal lattice typical for corundum (Figure 4).

The reasons of formation of different crystalline phases in process of ion-beam coating formation require further investigation.

\section{Conclusions}

Carried investigations allowed making a conclusion that the method of solid body surface modification using coating deposition by ion-beam spraying provides the possibility in wide limits to change purposefully the surface properties of structural and tool materials.

Carried examinations of Cr-based ion-beam coatings with ultradisperse diamond additives proposed a concept of mechanism for these coatings formation. Examination of the Cr-based ion-beam coatings with addition of metal oxides verifies proposed mecha- nism. Let's in short words describe the main stages of formation of ion-beam coatings.

The process of formation of thin films independent on metal transfer method and its composition takes place in several stages (Figure 5):

- nucleation of new phase particles;

- growth of particle size without their number change;

- formation of islands and further increase of islands' size;

- coalescence of islands in the solid film.

On the first stage collision of the particles from gas phase with the substrate surface takes place after what the particles can strongly fix on the substrate or can be elastically reflected from the surface.

The substrate before coating formation is subjected to cleaning with ion beam that results in appear- 
ance of the defects on it, which determine behavior of the particles entering the surface during spraying. These are so-called dislocation tubes (areas of dislocation accumulation), located, as a rule, along the grain boundaries. Particles of the sprayed material, captured in such tubes, penetrate deep into the substrate, and in this case so-called volumetric diffusion takes place. Part of the particles is kept on the substrate due to surface attraction force, part of the particles forms chemical bond with coating material. Part of the particles can be elastically reflected from the base surface.

Growth of thin films after formation of nucleuses can develop on three possible mechanisms, namely layer-by-layer, island or mixed.

A layer-by-layer growth is a successive filling of the substrate with monocluster layers. An island growth takes place, if bonding of the particles in the islands is more than with the particles in the neighbor islands that result in prevailing upward growth of the islands. The processes of layer-by-layer and island growth can get simple physical interpretation.

In the first case, there is complete wetting of the surface. Attachment of the particles to the nucleus side edges is thermodynamically preferred up to complete filling of the first layer.

In the second case, accumulation in a drop is better for the nucleus. In the process of growth, the conditions of good wetting can be disturbed, and then change of layer-by-layer to island mode will take place.

After the islands consisting of two-four adsorbed particles on the coating surface approach to bonded particles and particles caught in the dislocation tubes, their coalescence and formation of the coarse islands take place. The next step is formation of coating monolayer. Later on this layer becomes a basis for growth of the next layers. It is determined that ionbeam coatings have mixed growth mode. It means that after some initial time of spraying (10-20 s) bonding between the particles in the islands becomes higher then between the particles of the neighbor islands. In this case islands start upward growing and columnar structure is formed. Besides, the columnar structural constituents themselves have lamination. Presence of columnar structure can promote appearance of coating nanoporosity.

Nanodisperse particles of zirconium and aluminum oxides added to the chromium spraying targets adsorb on the substrate surface. The nanodisperse particles of oxides prevent coalescence of the chromium particles into coarse structural constituents, and this, in turn, results in coating structure refinement. Part of nanodisperse particles is located over defects of crystals of sprayed materials and part over the boundaries of chromium grains. Carried examinations using EBSD method showed that in the process of formation of ion-beam coatings there is complete transfer of target material into the coating.

1. Ilyushchenko, A.F., Andreev, M.A., Markova, L.B., Koleda, V.V. (2010) Examination of influence of $\mathrm{ZrO}_{2}$ particles size in target for spraying on structure of ion-beam coatings based on titanium and chromium. In: Proc. of $9^{\text {th }}$ Int. Sci.-Techn. Conf. on New Materials and Technologies: Powder Metallurgy, Composite Materials, Protective Coatings, Welding (Minsk, Belarus, 29-30 September 2010). Minsk, SRPPMA, 204-207.

Received 10.07.2018 


\title{
ELECTRON BEAM TECHNOLOGIES OF WELDING, SURFACING, PROTOTYPING: RESULTS AND PROSPECTS
}

\author{
V.M. NESTERENKOV, K.S. KHRIPKO and V.A. MATVIICHUK \\ E.O. Paton Electric Welding Institute of the NAS of Ukraine \\ 11 Kazimir Malevich Str., 03150, Kyiv, Ukraine. E-mail: office@paton.kiev.ua
}

\begin{abstract}
For many decades the E.O. Paton Electric Welding Institute of the NAS of Ukraine has been specializing in the development of technology and equipment for electron beam welding of modern structural alloys. The electron beam equipment developed at the Institute allows solving problems of joining the elements of complex structures of different branches of industry. The examples and technical capabilities of the installations, mostly demanded by industry, are shown. A number of products are given, in the production of which both equipment and new technological processes were successfully applied, which include restoration repair of parts of gas turbine engines and the technologies of layer-by-layer manufacturing of products using the method of rapid prototyping.
\end{abstract}

Ke y w ord s : electron beam welding, equipment, repair, 3D technologies

For many decades the E.O. Paton Electric Welding Institute of the NAS of Ukraine has been specializing in the development of technology and equipment for electron beam welding of modern structural alloys. A new generation of electron beam equipment, developed at the Institute, allows solving problems of joining elements of complex structures of the aerospace industry, which occupy a leading place in the use of light and strength alloys of nonferrous metals.

The study of physical processes of melting metals in vacuum began at the Institute in 1958, when, by the initiative of the academician B.E. Paton, the first installation for electron beam welding (EBW) was created. Already within a year, the results of the E.O. Paton Electric Welding Institute turned out to be in demand by the industry and, first of all, in the production of jet engines. With the growth in the number of tasks put forward by the industry and their complexity, installations with different volumes of vacuum chambers, control systems, powerful high-voltage power sources and welding guns were required.

Today, at the E.O Paton Electric Welding Institute the installations are designing and manufacturing for EBW of all sorts of products of different industry branches. All these installations can be conditionally divided into several types according to the dimensions of welding chambers, and, accordingly, to the sizes of parts to be welded: «small» with a volume of $0.26-5.70 \mathrm{~m}^{3}$ (Figure 1), «medium» with a volume of 19-42 $\mathrm{m}^{3}$ (Figure 2) and «large» with a volume of $80-100 \mathrm{~m}^{3}$ (Figure 3). The Institute is designing and manufacturing the welding chambers of different sizes and appropriate configurations of the vacuum sys- tem, as well as configurations of the mechanism for moving the electron beam gun and a part welded for specific customer tasks, namely: the sizes and shape of welded assemblies, type and location of welded joints in the assembly. Moreover, in the latter case, except of the equipment itself, also a specific technology for welding such assemblies is being developed, i.e. the customer purchases a welding installation together with the technology for EBW of a specific list of products. The similar technical ideology is followed by well-known manufacturers of EBW equipment like Sciaky Inc. (USA), PTR Group (Germany) and Techmeta (France) [1].

Using the modern means of pumping-out, in the widely applied installations with a volume of vacuum chambers of 19-42 $\mathrm{m}^{3}$, a working vacuum of $2 \cdot 10^{4}$ Torr is achieved for $18-20 \mathrm{~min}$. If necessary, a complete set of a vacuum system is possible, providing a pressure of less than $5 \cdot 10^{5}$ Torr in a chamber for less than $20 \mathrm{~min}$.

A typical mechanical configuration of the installation provides a movable intra-chamber electron beam welding gun fixed on a precision mechanism of the multi-axial movement. This mechanism provides a linear movement of the gun, controlled by CNC, along three Cartesian coordinate axes (along the chamber $-X$, across the chamber $-Y$ and vertically $-Z$ ), as well as rotation of the gun at an angle of $0-90^{\circ}$ in the $Z-X$ plane (from vertical orientation of the gun to the horizontal one) [2].

The rotation of a part welded is usually provided by precision welding manipulators with horizontal and vertical axes of rotation. The greatest technolog- 


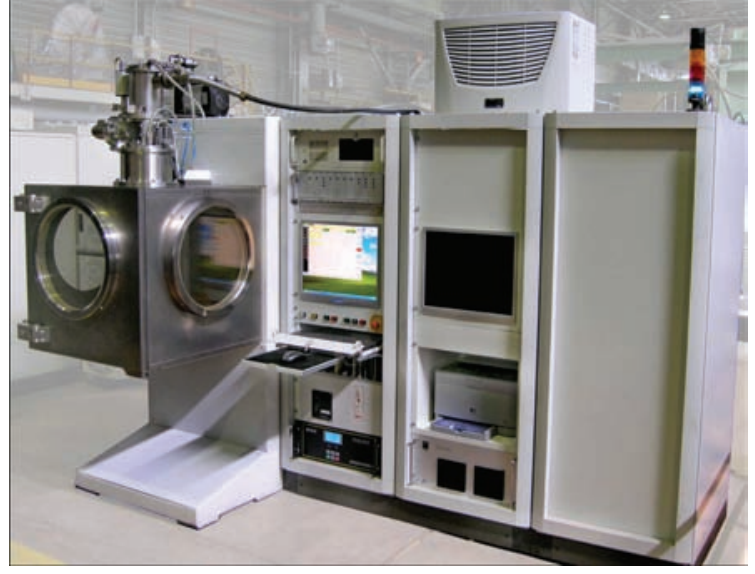

Figure 1. Appearance of small-sized electron beam installation ical flexibility is provided by welding manipulator with an inclined axis of rotation. It allows inclining the rotation axis of the faceplate in the range from $30^{\circ}$ to $+90^{\circ}$ (from vertical), which provides electron beam welding of intricate concentric sections of aircraft engines or aircraft assemblies with a varying geometry (Figure 4).

Depending on the specific purpose (the material to be welded and its thickness), the considered installations are completed with the latest high-voltage inverter welding power sources of 15, 30, 60 and $120 \mathrm{~kW}$ operating at a fixed accelerating voltage of 60 and $120 \mathrm{kV}$ [3]. The basis of the power part of the power sources is a high-voltage inverter power unit, for example, of a domestic company «Torsion» (Kharkov, Ukraine) or the companies Guth (Germany) and Technics (France). The welding source is designed in such a way that it represents actually a separate self-contained hardware complex, all interaction with which is carried out through the industrial interface (bus CAN). At the same time, the communication with the «outer world» is realized by the corresponding microcontroller units connected to the bus CAN and designed for control and diagnostics of all the welding source channels.

The welding source is quite compact in sizes and at a power of up to $30 \mathrm{~kW}$ it is usually located in one power cabinet (Figure 5). In the case of higher power, the welding source is divided into two parts and occupies two cabinets.

Together with a high-voltage welding power source, all the electron beam installations of the Institute are completed with the system of secondary emission electronic visualization RASTR-6, which is inseparably integrated into this source. Such integration is predetermined by the fact that the functioning of this system itself is directly associated with the formation of a beam of electrons irradiating the product observed. The block diagram of the classical system «RASTR-6» is shown in Figure 6 [4].

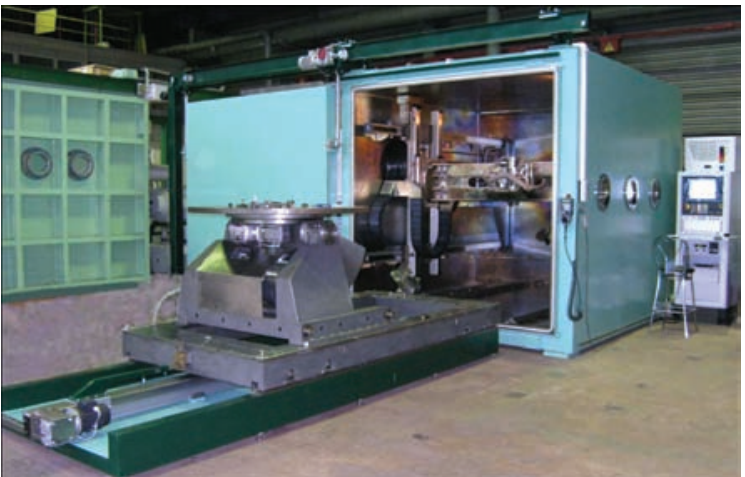

Figure 2. Appearance of medium-sized installation with a mobile intra-chamber welding gun and wheel-out working table

Approximately 3 times per second after a special command pulse, the units for setting and amplifying of the system RASTR-6 form, respectively, for a short period of time the line and frame signals of the raster scanning, supplied to the welding gun deflecting coil. Then, based on the modulation signal generated by the system RASTR 6, the microcontroller of the welding current channel of the welding power source, acting according to a special algorithm, switches off the momentary welding current and forms a short-term low-power current pulse. As a result of this interaction between the two subsystems of the installation (its welding power source and the system RASTR6), the front surface of a part welded is irradiated by a formed electronic raster. By passing along the lines of the raster of the "probing" electron beam at the point of spot bombardment of the part surface by its electrons («primary»), the secondary electrons are emitted. The power of the electron beam is chosen as sufficient in the given specific conditions, for example, depending on the distance of the welding gun to the part for the formation of the required level of such secondary-emission radiation. This short-term pulse of secondary electrons is picked up by a special passive sensor (Figure 7,a), usually located at the end of the welding gun, and which can have a different

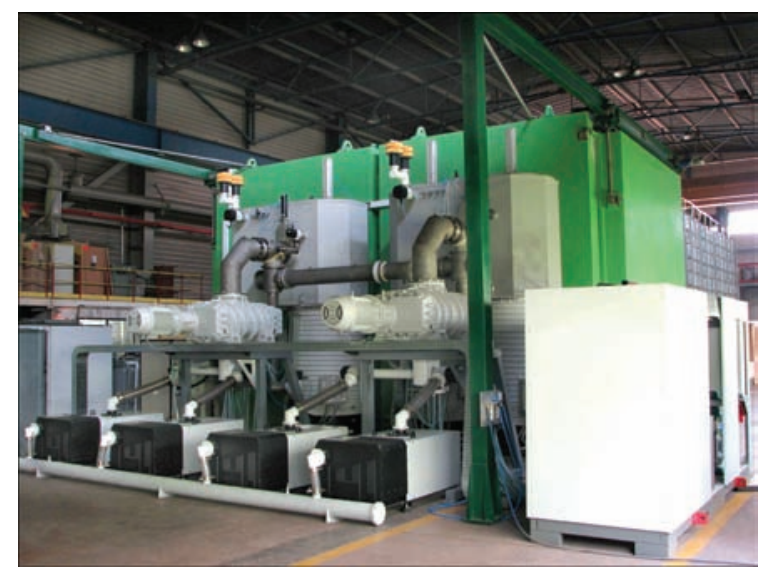

Figure 3. Electron beam installation with a vacuum chamber of $100 \mathrm{~m}^{3}$ 


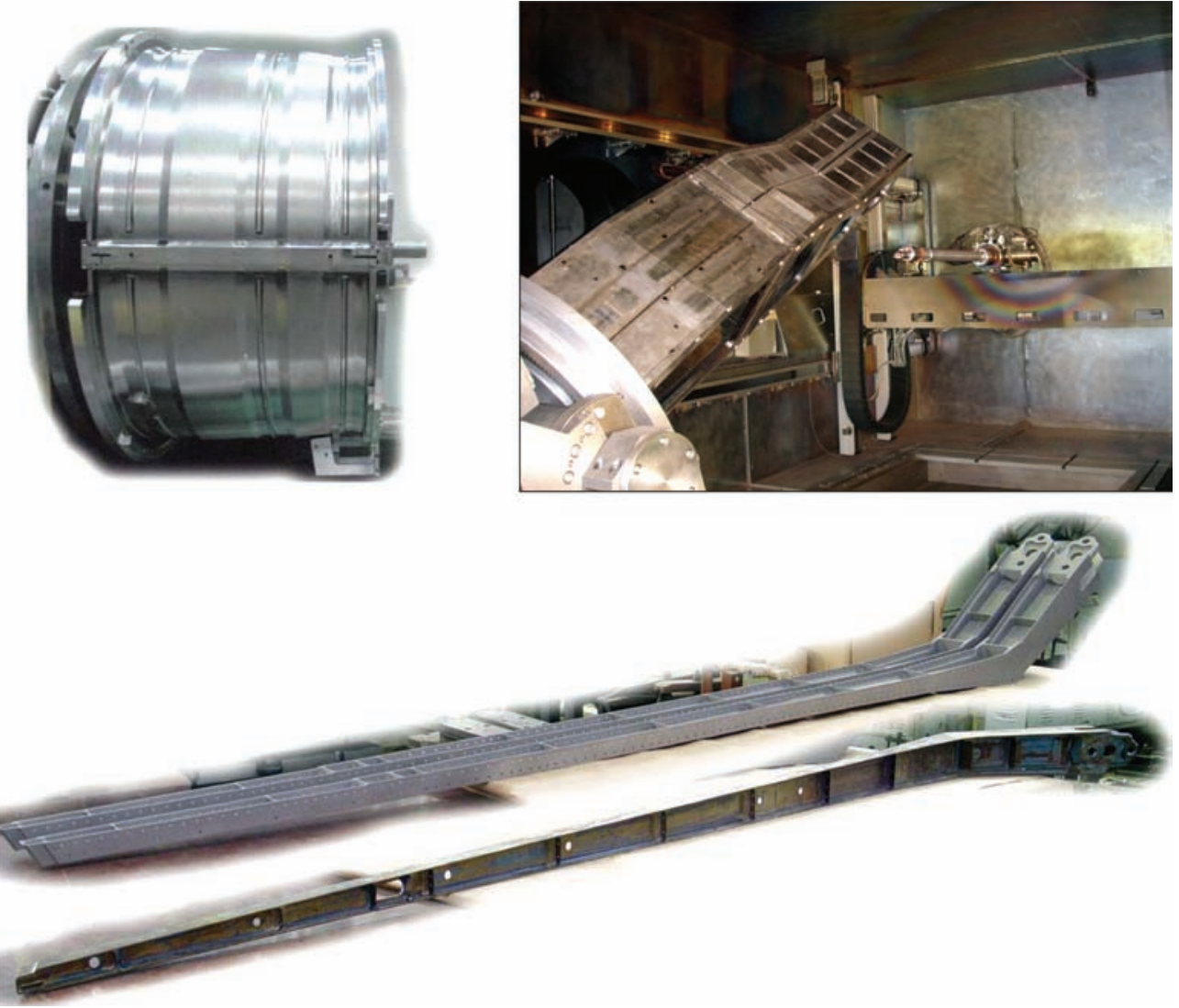

Figure 4. Examples of aircraft assemblies with intricate geometry, manufactured by EBW in the installations of medium and large overall dimensions

design performance. In the direct vicinity from this sensor a compact unit of the preliminary amplifier is located, which forms and amplifies a useful signal, the voltage of which is proportional to the current signal taken from the sensor. This already amplified signal is withdrawn from the welding chamber along

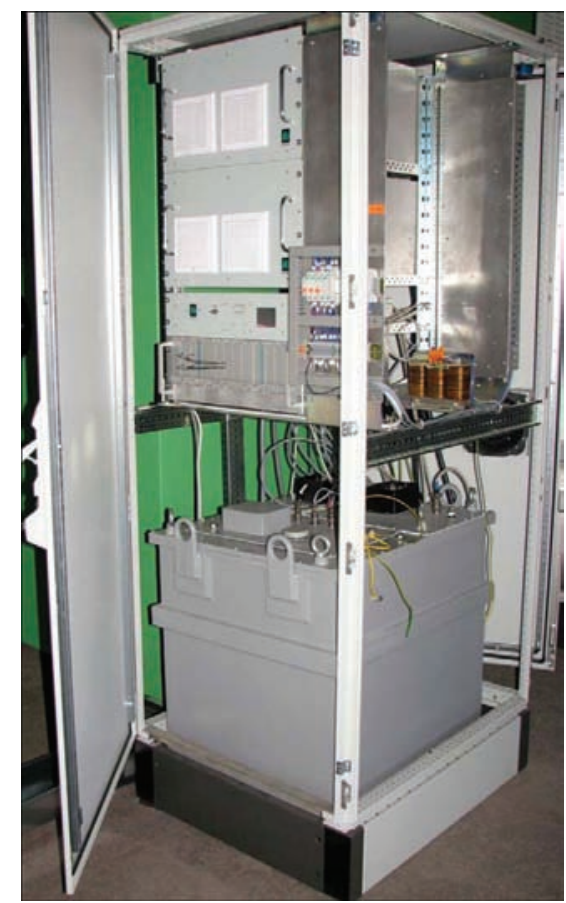

Figure 5. Appearance of inverter high-voltage power source the screened coaxial line and is supplied to the main video amplifier of the system. As a result, the signal is digitized by a specialized computer board WLCA and issued to the operator interface in the form of an image (Figure $7, b$ ), used both for the visual observation and manual guidance to the butt welded, as well as for the operation of special software algorithms, which help the user (welding operator) in making new welding programs and reproduction of existing programs for welding of repetitive standard parts. The system allows forming a fairly stable image of the welding zone, both before and after welding, as well as directly during welding.

The installations for EBW, designed and manufactured by our Institute, have a modern control system. The part of the equipment, on which the welding process directly depends, is under the continuous program control.

Here the concept of high-level program control is used, in which the user interacts with the equipment exclusively through a Windows-based graphical user interface (GUI). The interface is operated using standard tools: a keyboard and a mouse-type manipulator. The interface is self-explanatory and does not require special skills, as in the «low-level» machine programming. Each of the subsystems of the installation is served by a corresponding window graphic toolset 


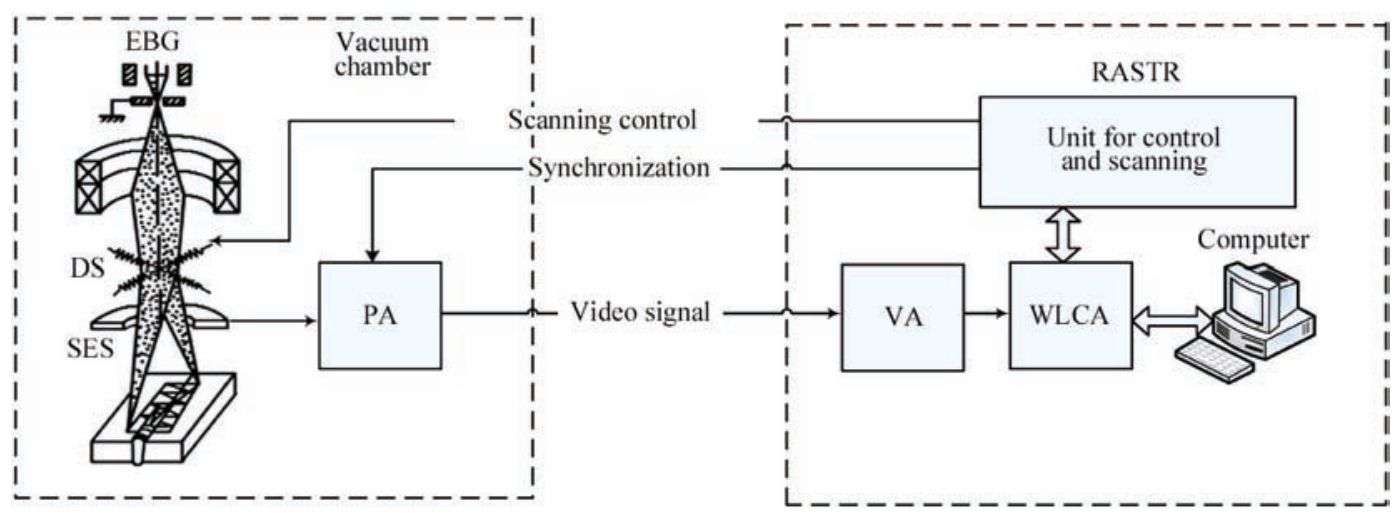

Figure 6. Block diagram of the classical system RASTR-6, where: EBG is the electron beam (welding) gun; DS is the deflecting system of the gun; SES is the secondary electron sensor; PA is the preliminary amplifier; VA is the video amplifier; WLCA is the specialized computerized visualization board

with input data checking and blocks to provide the safety of the user and equipment.

Hierarchically, the control system is divided into two software/hardware levels: upper and lower. The upper level includes all means of communication with the user, including graphical interface, means of creating and storage of welding programs, acquisition and storage of diagnostic data, administration, etc. The lower level deals with the direct performance of all the procedures set by the commands from the upper level.

Currently, we are using two basic configurations of the EBW software system for installations control. The first one involves the use of full industrial system Sinumeric 840D of Siemens Company, and the second one - the «shortened» industrial system Synamics S120 of the same Company.

In case of using the system Sinumeric 840, the main program is installed in the standard industrial computer of the upper level Sinumerik PCU-50. The upper level directly interacts with the elements of the lower level: the machine control panel MCP, the main module NCU and the connecting module Basic PN, to which the control panel of manual movement of the gun or a part is connected.

In addition, the upper level interacts with the computer system RASTR. As a result, the upper level program (in PCU-50) can display the secondary emission image of the surface of a part welded, formed by the system RASTR.

The welding movement is under the full control of the CNC in both manual mode of displacement, as well as in automatic welding. In the latter case, the text script of the prepared welding program is transmitted from the upper level to NCU, where CNC calculates the trajectories, interpolations, velocities and accelerations by each of the participating axes using its own algorithms. In automatic welding, CNC provides a complete synchronization of all axes, both mechanical and virtual, which use channels of welding and focusing currents, as well as technological scanning of the electron beam.

The use of means of numerical program control for the technological process of EBW allowed solving the complex problems of joining different critical-purpose structures. The existing possibilities of precision control of the trajectory of the electron beam movement during welding due to its mechanical movement or its electromagnetic deviation, as well as the ability to control the power of the electron beam and the shape of distribution of this power in space led to a significant expansion of technological capabilities of EBW and to a dramatic improvement in repeatability of the process of welding of the serial parts in the preliminary programmed welding mode. Below (Figures 8, 9) the examples of implementation of the developed control system during EBW of intricate products are given.

In our installations, the restoration repair of expensive parts of gas turbine engines is successfully performed [5].

Thus, during operation of aircraft gas turbine engines, one of the main causes for their early replacement is the damage of titanium fan blades and compressor as a result of foreign objects entering the engine. Usually, at negligible defects of the input and output edges of the blade airfoil without tears, the op-

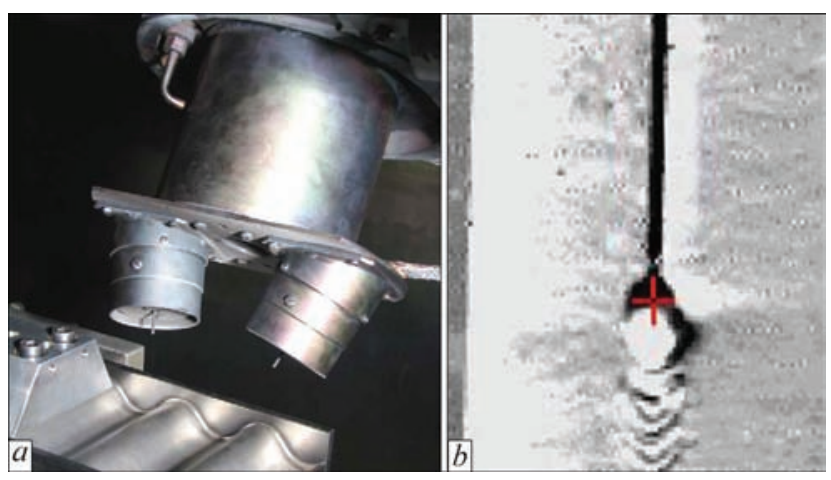

Figure 7. Secondary electron sensor of the system RASTR-6 (a) and the image formed by it in the welding zone $(b)$ 

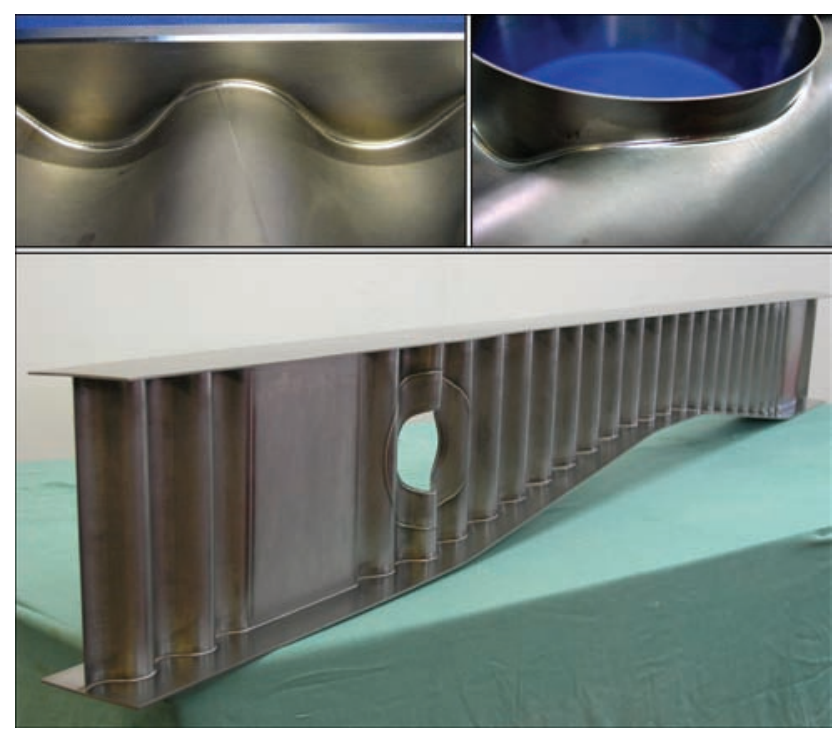

Figure 8. Appearance of welded thin-sheet aircraft structures of titanium alloys

eration of engine is admitted. A part of the defects are admitted to be repaired directly on the engine. So, for example, raised edges of the material near the nicks are dressed, the bends of the blades are eliminated by straightening. The corrected places are then polished. Sometimes it is allowed to eliminate the nicks by smooth rounding of the edge with a radius of 10 $12 \mathrm{~mm}$. The correction of local damages of the blades exceeding the admissible norms, already requires dismantling of the damaged blade and repair in the production conditions. Usually, the repair consists in mechanical removing of the defect area up to the borders of the deliberately undamaged metal of the blade with the following attachment (welding, brazing) of an insert of the same metal of the appropriate size and with a technological tolerance in thickness to obtain a required blade profile by the subsequent machining. In order to realize the repair technology using electron beam welding, a scheme of a welded joint of a repair insert and a blade airfoil was developed, regardless of whether it is a spot or extensive defect. The joint is

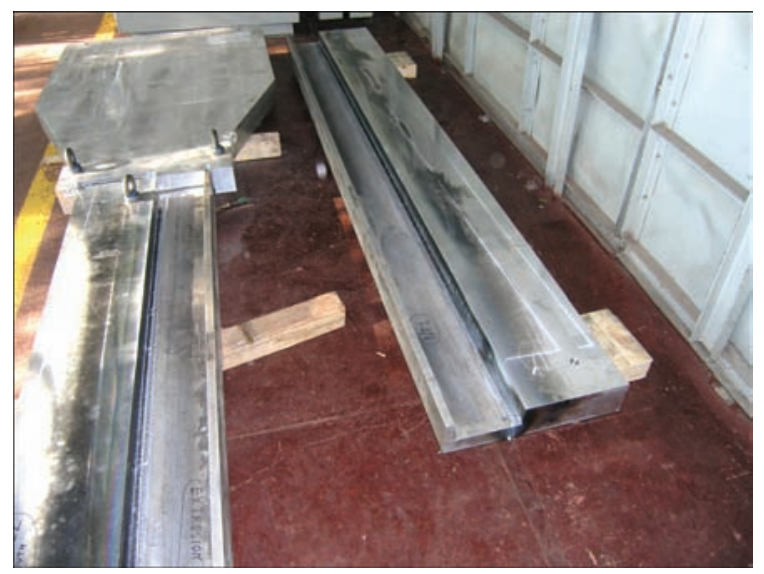

Figure 9. Appearance of welded thick-sheet aircraft structures of high-strength aluminum alloys

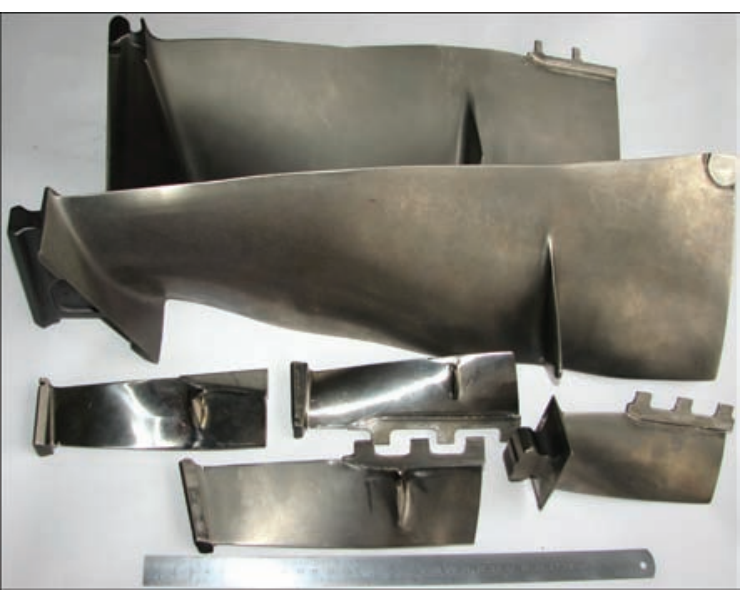

Figure 10. Examples of fan blades, repaired by electron beam

produced by a single-pass EBW, and a smooth transition from the surface of the insert to the base metal is provided both by a moderate concentration of the electron beam and also by a sufficient amount of additional metal due to the applied design of welded joint with an overhanging flange. This allows obtaining a smooth transition of metal from the insert to the surfaces of the blade airfoil, both in the places with a sufficiently large thickness of the airfoil, as well as in the thinnest places adjacent to the very edge of the airfoil.

The technology of repairing three types of blade airfoil defects was mastered: «spot» damage to the blade angle, «spot» damage of the edge of the main part of the blade airfoil and extended local defects of the edge starting from the blade angle.

The repair of both types of «spot» defects is carried out using cylindrical inserts of different diameters, depending on the size of the airfoil edge defect. The works initially were carried out on the specimens, simulating the real products. Then, the technology was successfully tested on the experimental batches of defective blades given to the SE LRZ «Motor» and the SE «Ivchenko-Progress» (Figures 10, 11).

In addition to repairing of local defects of blades, an equally important task is to replace the separate elements of the permanently assembled components of the gas turbine engine. The guiding devices from the $3^{\text {rd }}$ to the $8^{\text {th }}$ stage of stator of the high-pressure compressor of a gas turbine engine are composed of semi-rings with a set of cantilever blades brazed

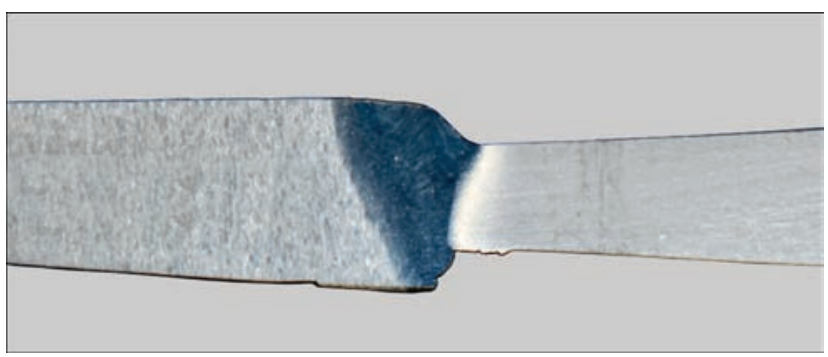

Figure 11. General view of welded joint of the blade repair shop bay 


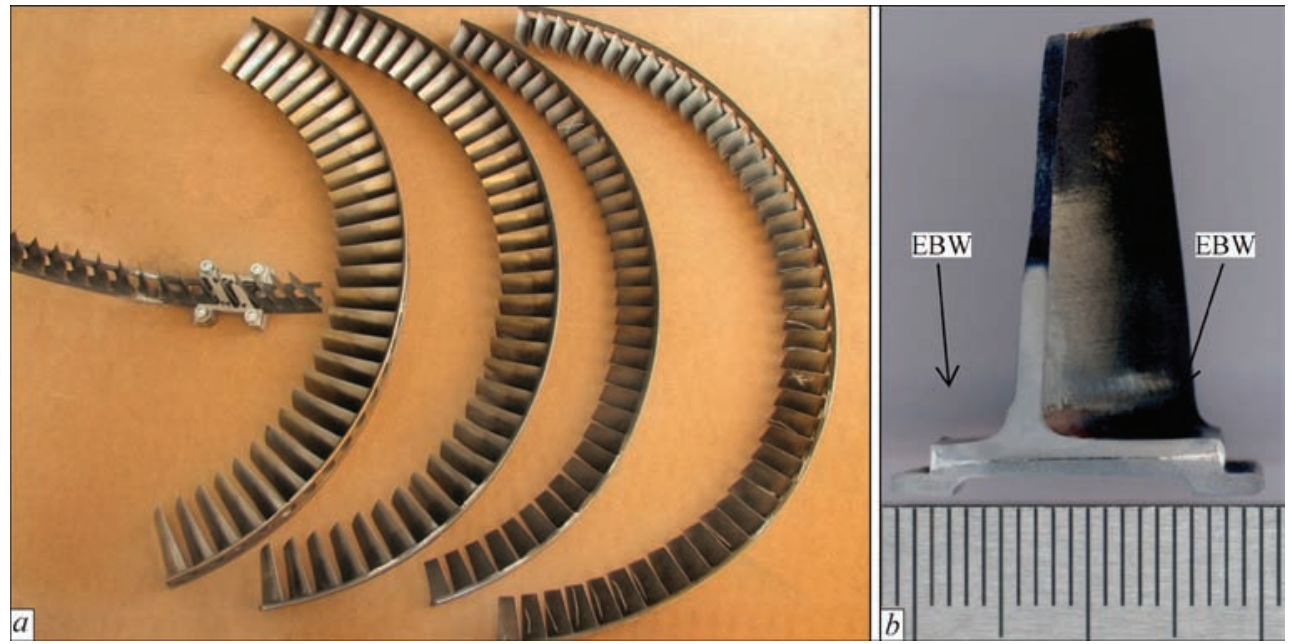

Figure 12. Scheme of assembly of the blade-donor with a semi-ring $(a)$ and the macrosection of the joint $(b)$ of the high-pressure compressor stator guiding device

into them. The blades from the $3^{\text {rd }}$ to the $6^{\text {th }}$ stage are made of the alloy EP-866 (15Kh16K5N2MFAB-sh), and the blades of the $7^{\text {th }}, 8^{\text {th }}$ stages are made of the alloy EP-718-ID (KhN45MVTYuBR-ID); the working temperature of the assemblies is $300-500{ }^{\circ} \mathrm{C}$.

During operation of such engines, there are also cases of arising nicks and cracks on the blades, as well as their tearing off because of a local lack of brazing with the semi-ring wall. For this case, the following scheme of repair is proposed. A defective blade is removed by milling from the semi-ring till the very wall, including the whole brazing alloy, maintaining the blade earlier, and on its place an undamaged blade-donor is selected. In other words, in the semiring a fully cleaned platform is made for mounting the blade-donor. It is obvious that it is impossible to repeat the initial brazing process without touching the adjacent, still serviceable blades. Therefore, a method of fixing with a local and concentrated temperature effect on the entire assembled unit is required.

The EBW method is ideally suited in this case, providing a reliable welded joint of sufficient depth with a relatively small heat input into the product welded.

A scheme of the welded joint of a blade-donor with a wall of a semi-ring of a guiding device was developed. The joining of the blade with the wall is produced by a double-sided EBW with an intermediate turnover of the product by $180^{\circ}$. The design strength of two similar welds is sufficient for reliable fixation of the blade, not inferior to the adjacent, brazed ones.

To improve the weld formation and reduce the total heat input, the EBW pulsed mode was used, which allowed producing high-quality joints of parts with local gaps in the joint of up to $0.1 \mathrm{~mm}$. Then the selected EBW modes were corrected already on the real joints of blades with the semi-ring of the guiding device. Figure 12 shows the scheme of assembly of a blade-donor with a semi-ring and macrosection of the produced joints.

The technology was successfully tested in the repair of a batch of real semi-rings of guiding devices of different stator stages of a high-pressure compressor.

The electron beam equipment and technologies developed at the Institute open up new opportunities for the rapid prototyping of parts of the specified shape and structure with preliminary predicted properties [6].

The developed technologies and equipment were initially focused on the needs of domestic enterprises. For the production it was proposed to use low-cost domestic raw materials required by the manufacturer. This approach will provide the feasibility of manufacturing parts and assemblies by rapid prototyping, coming from the needs of the consumer and in close contact with him. The developed technologies will reduce the time of implementation of new types of products, expand their range, as well as create fundamentally new types of products with preliminary predicted properties, the manufacture of which is impossible without applying 3D-printing methods.

Since installations for 3D-printing of domestic development do not exist yet, the project was started at the Institute for creating the equipment and software for realization of additive electron beam manufacturing, which is unique to Ukraine, free from imported raw materials and focused on the implementation at the aerospace and turbine-building enterprises like SE NPKG «Zorya-Mashproekt», LRZ «Motor», JSC «Motor Sich» and SE KB «Yuzhnoye» [7].

The equipment is created on the basis of the installation for electron beam welding of type SV-212M. It involves modernization of vacuum chamber, development of systems of control of drives for moving the table in vertical and the unit for powder distribution in 


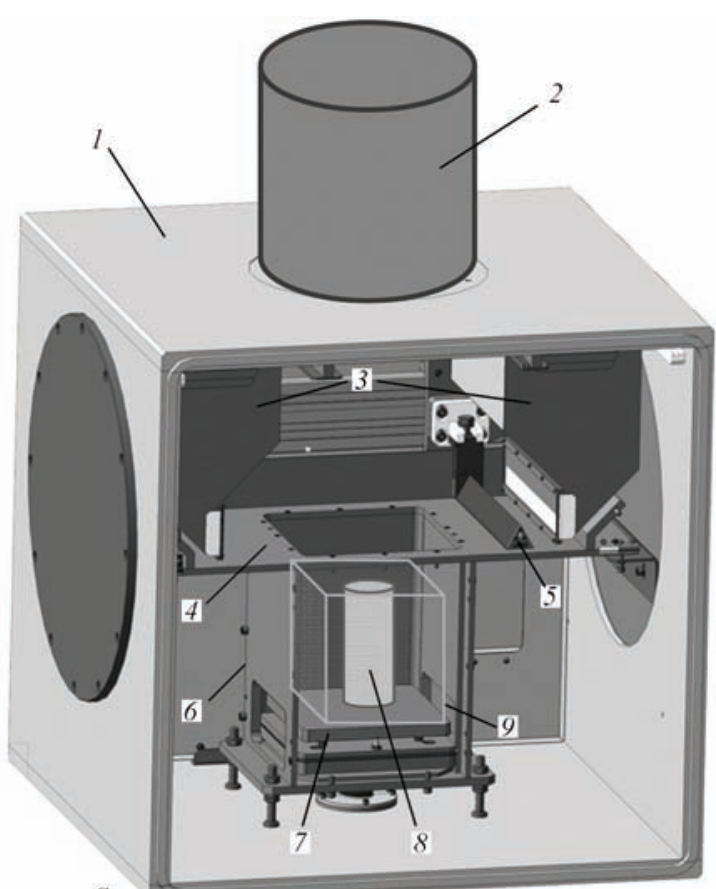

$a$

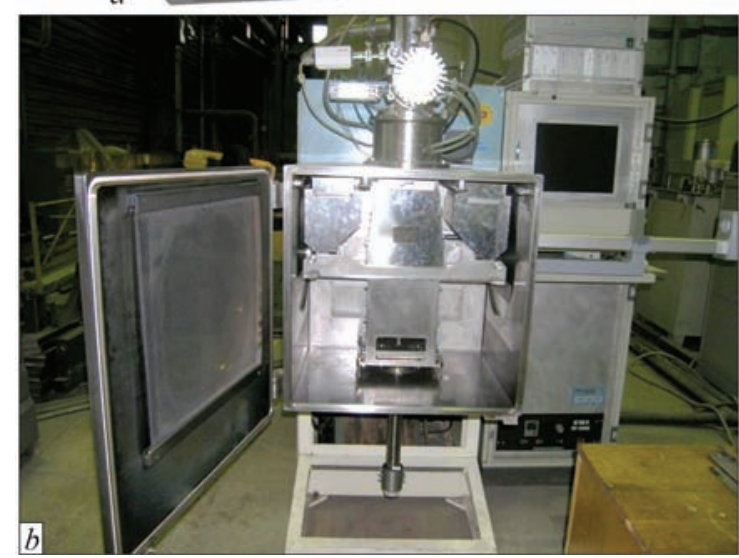

Figure 13. Scheme ( $a$ ) and industrial model $(b)$ of installation for additive manufacturing using metallic powder materials, where 1 - vacuum chamber; 2 - electron beam gun; 3 - hopper; 4 table; 5 - rack; 6 - shaft; 7 - pallet; 8 - product; 9 - powder the chamber, as well as the development of appropriate software for realizing the additive manufacturing.

The scheme of installation and its industrial model are presented in Figure 13. The process of electron beam surfacing takes place in a vacuum chamber 1 in vacuum of less than $1 \cdot 10^{-4}$ Torr. The metal powder is supplied in bulk to the working table 4 from hoppers 3 . The rack 5, moving along the table 4 , forms the layer of

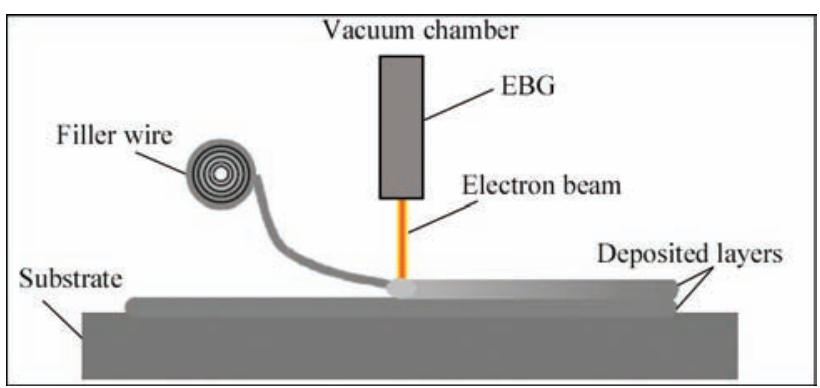

Figure 14. Scheme of layer-by-layer electron beam melting of filler wire powder of a preset thickness on the surface of the pallet 7. In the initial position, the pallet is located at the top of the shaft 6 . The focused electron beam, formed by the electron beam gun 2 melts the surface of the powder along the preset trajectory. Thus, according to the algorithm, the contours of the product and its layer are formed. Further, the pallet 7 is lowered to the preset value and the next layer of powder is applied. The process is repeated and the product is layer-by-layer deposited. At the end of the manufacturing cycle the part is removed from the vacuum chamber, cleaned from nonmelted powder 9 and machined.

The technology of layer-by-layer electron beam fusion of metals in vacuum using powder materials allows creating dense metal products of a preset shape with a high geometric accuracy. The overall dimensions of the products are $250 \times 250 \times 250 \mathrm{~mm}$, and the efficiency of electron beam surfacing according to EBM technology does not exceed $0.3 \mathrm{~kg}$ of metallic powder per hour.

The second investigated process of electron beam melting of metals is the process of melting metal wire in vacuum with the formation of successive layers (DM). To heat and melt the wire, an electron beam of the required power is used. The scheme of the DM process is shown in Figure 14.

The surfacing takes place in a vacuum chamber. The filler wire is fed into the zone of the electron beam action, where it is heated and melted. The electron beam gun and/or the substrate, on which the product is formed, are moved, forming a layer of deposited metal. The product is made by a digital model. The data of the CAD program are converted to the CNC code. The part is formed in layers: each subsequent layer of metal is deposited onto the previous one, and so it continues layer-by-layer until the product reaches the preset shape. Then, it is subjected to heat and mechanical treatment.

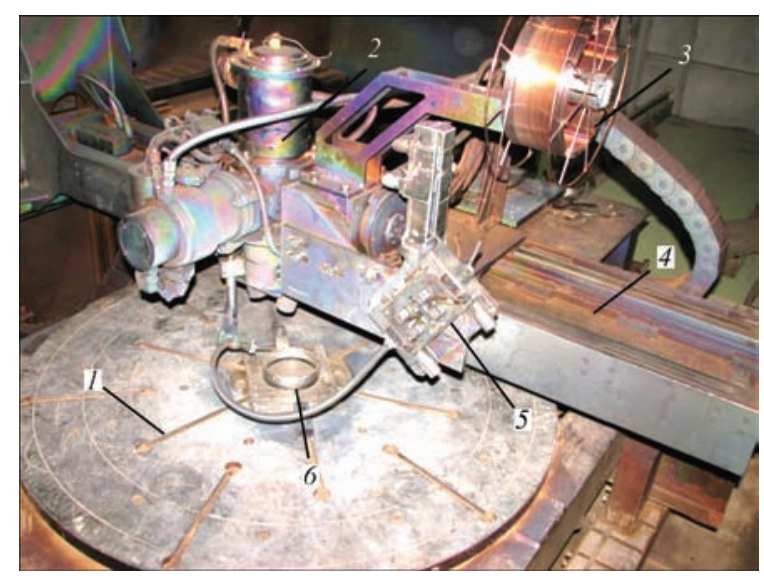

Figure 15. Arrangement of equipment for surfacing using wire in vacuum chamber 
The efficiency of electron beam surfacing according to DM technology varies from 3 to $9 \mathrm{~kg}$ of metal per hour, depending on the selected material and characteristics of the product, which makes it the fastest process of additive manufacturing.

On the basis of equipment for electron beam welding of the type KL-209, a laboratory installation was created to implement the additive DM process. In the vacuum chamber of the installation (Figure 15) the following items are located: 2 - electron beam gun (EBG) of type ELA-60, 4 - multicoordinate module for EBG movement, mechanism for feeding filler wire 5 with coil 3, rotator 1 . On the rotator a product 6 is located, produced by the DM technology.

In the laboratory equipment, a satisfactory formation of round and rectangular specimens was obtained, of which it is possible to form intricate geometric shapes in the form of a combination of rotation bodies and rectangles. The wall thickness of the specimens ranged from 6 to $10 \mathrm{~mm}$ using four types of wires.

Our Institute is in the process of continuous improvement of both the system for control of EBW equipment and the functionality of the developed equipment, taking into account the evolution of hardware and element base, as well as gained many years experience in the development of technology and equipment for EBW of various materials with different thicknesses.
In conclusion, it can be said that the above information on the manufacture of equipment for electron beam welding of assemblies and parts used in aircraft construction, according to the technologies developed at the E.O. The Paton Electric Welding Institute, as well as the installations themselves, have found application both in the domestic industry and abroad, and are the significant achievement of the Institute specialists.

1. Hamm, R.W. (2008) Reviews of accelerator science and technology. Industrial Accelerators, 1, 163-184.

2. Paton, B.E., Nazarenko, O.K., Nesterenkov, V.M. et al. (2004) Computer control of electron beam welding with multi-coordinate displacements of the gun and workpiece. The Paton Welding J., 5, 2-5.

3. Nazarenko, O.K. et al. (1987) Electron beam welding. Ed. by B.E. Paton. Kiev, Naukova Dumka [in Russian].

4. Matvejchuk, V.A. (2016) Systems for visualization of welding processes in real-time mode using noise-proof channel for transfer of secondary electron emission signal. The Paton Welding J., 5-6, 59-62.

5. Nesterenkov, V.M., Orsa, Yu.V., Khripko, K.S., Gusev, Yu.V. (2017) Restoration repair of elements and units of gas turbine engines. Ibid., 11, 13-17.

6. Nesterenkov, V.M., Matvejchuk, V.A., Rusynik, M.O. (2018) Manufacture of industrial products using electron beam technologies for 3D-printing. Ibid., 1, 24-28.

7. Nesterenkov, V.M., Matvejchuk, V.A., Rusynik, M.O., Ovchinnikov, A.V. (2017) Application of additive electron beam technologies for manufacture of parts of VT1-0 titanium alloy powders. Ibid., 3, 2-6. 


\title{
TENDENCIES OF DEVELOPMENT OF SPECIAL ELECTROMETALLURGY OF TITANIUM IN UKRAINE
}

\author{
S.V. AKHONIN \\ E.O. Paton Electric Welding Institute of the NAS of Ukraine \\ 11 Kazimir Malevich Str., 03150, Kyiv, Ukraine. E-mail: office@paton.kiev.ua
}

\begin{abstract}
The analysis was carried out on the main tendencies of development of special electrometallurgy of titanium in Ukraine. It is one of five countries, having complete cycle of titanium production from extraction of titanium-containing ores, their enrichment and production of spongy titanium to melting of titanium alloy ingots and production of virtually complete spectrum of titanium semi-products. Metallurgical processing of spongy titanium into ingots in Ukraine is based on technology of electron beam melting with cold hearth, which finds wide application in the world for melting of ingot-slabs. This technology provides guaranteed removal of refractory inclusions and the possibility to get the ingots of various cross-section per one remelting from charge materials of low price that ensures high technical-economical indices of melting process. Production of semi-products of titanium alloys from the ingots was organized at the Ukrainian enterprises. There are castings, forgings, rods, hot- and cold-rolled pipes, mechanical properties of which correspond to requirements of the standards. Today Ukraine has got a competitive for the world's markets production of high-quality ingots and ingot-slabs from titanium and alloys on its basis, which has large perspectives for further development. 8 Ref., 1 Table, 10 Figures.
\end{abstract}

Keywords : titanium, electron beam melting, ingot, quality, refractory inclusions

Titanium and alloys on its basis are unique structural materials, which allow significantly increasing service characteristics of new equipment. Due to high specific strength and good corrosion resistance, they have found wide application in rocket- and aircraft construction, power and chemical machine building, shipbuilding and production of medical equipment, including endoprostheses and implants.

Ukraine is one of five countries in the world, having complete cycle of titanium production from extraction of titanium-containing ores, their enrichment and production of spongy titanium to melting of titanium ingots and production of virtually complete spectrum of titanium semi-products, namely castings, forgins, rods, pipes and wire.

The main deposits of titanium-containing ores are concentrated in Dnepropetrovsk and Zhytomyr regions. Their enrichment is carried out at Volnogorsk Mining and Smelting and Irshanskii Mining and Processing Combines, respectively. These Combines not only completely provide with raw materials the Ukrainian producers of spongy titanium and pigmentary titanium dioxide, but supply titanium concentrates for export.

Production of spongy titanium in Ukraine at Zaporozhye Titanium \& Magnesium Combine (ZTMC) is based on technology of melting of ilmenite concentrates in ore-thermal furnaces with production of tita- nium slags containing $\mathrm{TiO}_{2}$ as in rutile concentrates. Moreover, cost of such slags is approximately 1.5 times less than rutile cost. Spongy titanium is obtained after slag chlorination in salt chlorination units by magnesium reduction of titanium tetrachloride. Now ZTMC has mastered production of the blocks of spongy titanium of 0.7 and $3.8 \mathrm{t}$ weight per cycle and melting of ingots in the electron beam unit developed at the E.O. Paton Electric Welding Institute.

Regardless the fact that technology of vacuum-arc remelting (VAR) of titanium is traditional and more widespread industrial method for production of ingots of titanium and alloys on its basis, in the recent years the technology of electron beam melting with cold hearth (EBM) has found more and more application in titanium metallurgy. The latter differs by a series of advantages in comparison with traditional method of ingot production, namely vacuum-arc remelting:

- complete elimination from a technological cycle of an operation of consumable electrode pressing, which requires special pressing equipment of large capacity or specialized welding equipment;

- possibility of production of ingots of round as well as ingot-slabs of rectangular cross-section used as billets for sheet products production;

- guaranteed removal of refractory nonmetallic inclusions in the cold hearth, increase due to this of quality of ingots' metal; 


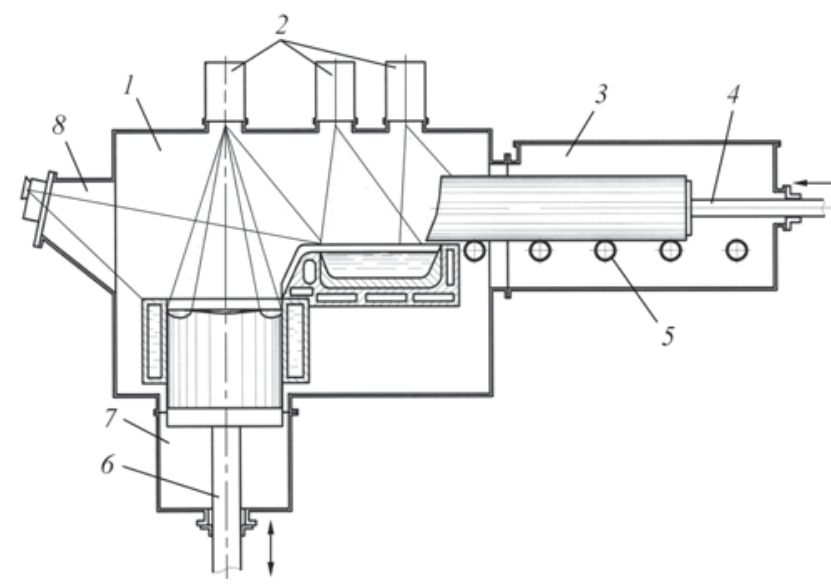

Figure 1. Scheme of unit: 1 - melting chamber; 2 - electron guns; 3 - billet chamber; 4 - billet feed mechanism; 5 - roll table; 6 - mechanism of ingot drawing; 7 - ingot chamber; 8 view system

- obtaining the structurally and chemically homogeneous ingots with equiaxial structure;

- increase of metal yield due to reduction of amount of remelts (one instead of two-three).

EBM process is carried out in high-vacuum at the electron beam unit chamber (pressure of residual gases in the melting chamber is kept in $0.01-1.00 \mathrm{~Pa}$ range) and lies in successive melting of initial charge into the cold hearth under effect of heating with electron beams, melt refining in the cold hearth and its pouring down in a intermediate copper water-cooled mould, where ingot formation takes place (Figure 1).

Up to the moment there are 32 electron beam units of megawatt class in commercial operation in China, Japan and Ukraine and three more are in construction stage. Germany and Kazakhstan each have got one electron beam unit of megawatt class for melting of titanium ingots. Such a wide application of EBM technology is caused by good quality of obtained metal as well as high technical-economical indices of the process, in particular, in melting of ingot-slabs of rectangular section. Usage of the ingot-slabs allows eliminating from the technological chain of titanium rolled stock production a capital intensive and energy-consuming operation of reforging of cylinder ingots into slabs. The mechanical properties of hot-rolled sheet produced from titanium ingot-slabs completely correspond to the requirements of domestic and foreign standards.

An important problem in production of titanium ingots and semi-products is the problem of removal from metal of refractory inclusions with increased content of interstitial impurities, namely nitrogen, oxygen and carbon stabilizing $\alpha$-phase. They consist of solid particles of $\alpha$-titanium, saturated with these impurities as well as compounds of these elements with titanium, i.e. nitrides, oxides and carbides [1]. Besides, a serious problem is also inclusions formed

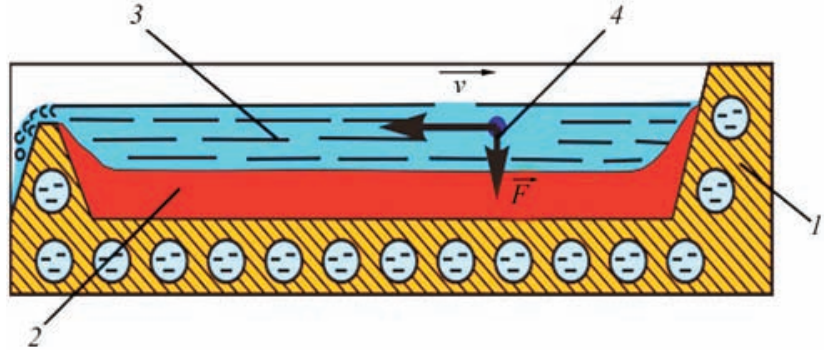

Figure 2. Scheme of process of refractory inclusion deposition: 1 - cold hearth; 2 - scull; 3 - melt; 4 - refractory inclusion

by compounds with large density and high melting temperature. A source of their formation is, as a rule, fractions of cutting tool based on carbides of refractory metals (WC, MoC, and others), which enter the metal together with charge components, most often, with chips [2]. Presence of such refractory defects in the products from titanium significantly reduces fatigue characteristics of metal.

One of the main mechanisms of removal of refractory inclusions from titanium is their gravitation settling: in process of melting the liquid metal in the cold hearth flows horizontally, whereas inclusions with higher density than density of liquid titanium go down under effect of gravity force (Figure 2), deposit on the surface of scull and freeze in it.

The investigations carried at the E.O. Paton Electric Welding Institute of the NAS of Ukraine showed that in process of EBM virtually all refractory inclusions, except for titanium dioxide, will be deposited on the bottom of cold hearth and removed from the melt. Found dependencies allow determining the dimensions of cold hearth, which will provide guaranteed removal of the refractory inclusions from titanium by means of their deposition (Figure 3).

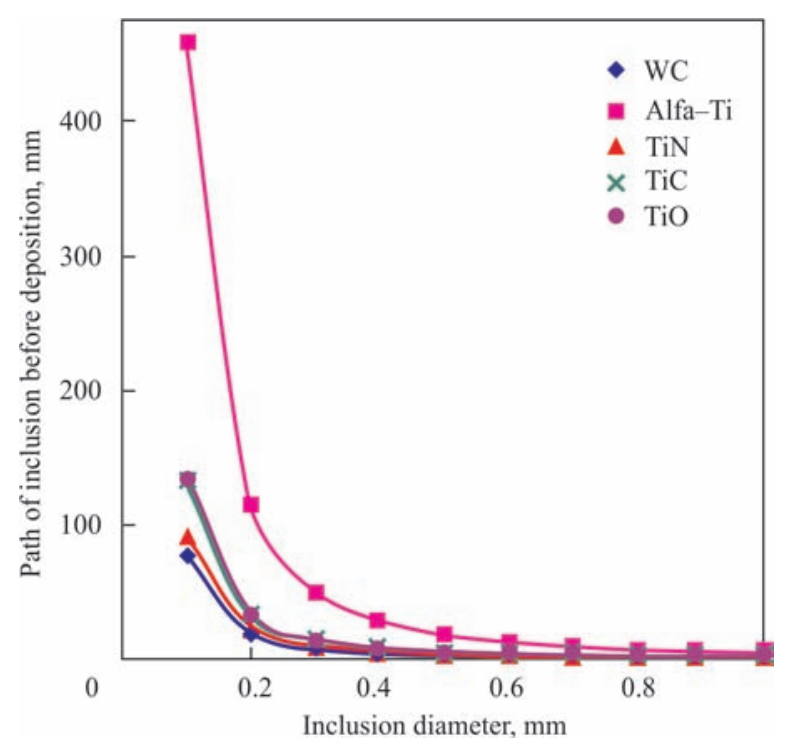

Figure 3. Dependence of path of refractory inclusion before deposition to scull surface on its size 


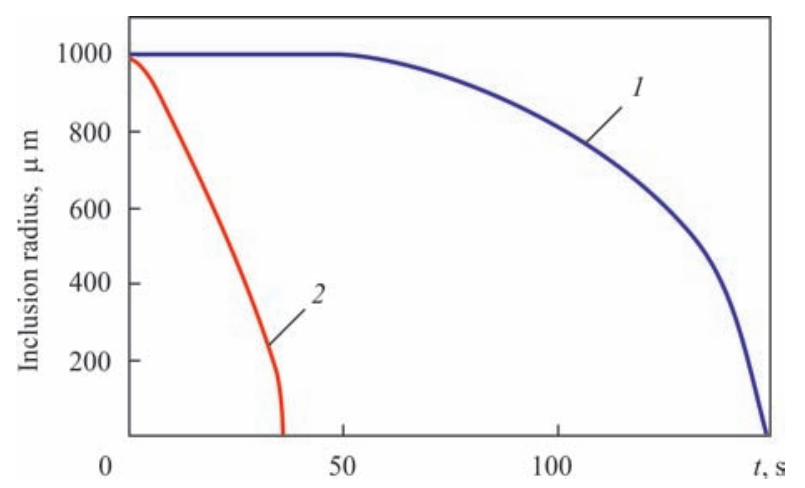

Figure 4. Dependence of radius of $\alpha$-Ti inclusion on time of staying in the melt: $1-T=1950 ; 2-2000 \mathrm{~K}$

Regardless the fact that temperature of melting of the low density refractory inclusions can significantly exceed the temperature of titanium melting, and, respectively, melt temperature, entering of such inclusions into the titanium melt provokes process of their dissolution. A mechanism of dissolution of LDI inclusions in the titanium melt was studied experimentally [3] as well as theoretically [4] and is caused by diffusion processes of interstitial impurities (nitrogen, oxygen and carbon) from inclusion volume to the melt.

Investigation of the process of dissolution of the refractory inclusions showed that the nature of dissolution of these inclusions significantly depends on melt temperature [5]. Thus, for example, $\alpha$-titanium particle dissolves virtually at constant speed (displacement speed of interphase surface makes around $28 \mu \mathrm{m} / \mathrm{s}$ ) at overheating of titanium melt by $59{ }^{\circ} \mathrm{C}$ over titanium melting temperature, whereas at overheating by $9{ }^{\circ} \mathrm{C}$, dissolution speed of such particle is considerably nonlinear, i.e. at initial stage the dimensions of inclusions remain virtually the same, and

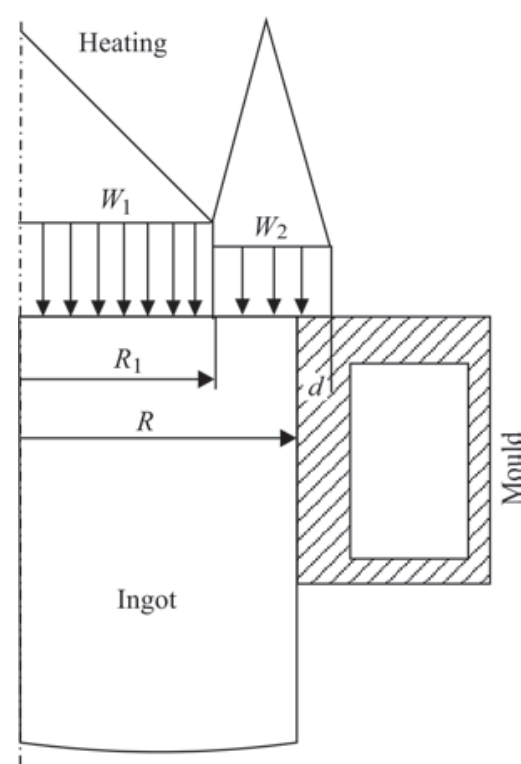

Figure 5. Scheme for modeling a process of formation of cylinder ingot in EBM then its dimensions start reducing with rising speed up to complete dissolution (Figure 4).

Also, the time of complete inclusion dissolution for solid particles of different chemical compositions and dimensions was determined. Thus, the investigation of processes of dissolution of refractory inclusions (particles of $\alpha$-titanium, nitride, carbide or titanium oxide) in liquid titanium allowed calculating the speed of their dissolution and determining the time of complete dissolution of such inclusions depending on chemical composition and initial dimensions.

EBM technology provides not only high refining from detrimental impurities and nonmetallic inclusions, but significant improvement of ingot structure. This is caused by division of the processes of melting and refining of metal in the cold hearth and solidification of metal in the mould.

E.O. Paton Electric Welding Institute of the NAS of Ukraine has developed a mathematical model of heat processes in EBM [6] for determination of dependencies of solidification of ingots from titanium alloys. This mathematical model allows getting distribution of temperatures in the ingot at any moment of time, and, respectively, configuration of liquid pool and zone of solid-liquid state of metal depending on technological parameters of electron beam melting, i.e. efficiency of process, frequency of melt pouring into the mould and power of electron beam heating.

The model considers the process of ingot formation in a copper water-cooled mould (Figure 5).

The ingot surface is heated with two electron beams, moreover power of one of them is uniformly distributed in the central zone $\left(0<r<R_{1}\right)$ and another in the periphery zone $\left(R_{1}<r<R\right)$. The controlled technological parameters in the mathematical model are the power of central and periphery beams $W_{1}$ and $W_{2}$, value of portion, frequency of pouring, melting efficiency. A heat transfer process is described by a heat conductivity equation in the cylinder system of coordinates $(r, o, z)$ for a case of axial symmetry, where axis $O Z$ matches with the ingot axis (symmetry axis), and axis $O R$ with radial direction. Start of the coordinates is set at lower end face of the ingot. The heat conductivity equation in this case has the next form:

$$
\begin{gathered}
\tilde{n} \rho \frac{\partial T}{\partial t}=\frac{1}{r} \frac{\partial}{\partial r}\left(r \lambda(T) \frac{\partial T}{\partial r}\right)+\frac{\partial}{\partial z}\left(\lambda(T) \frac{\partial T}{\partial z}\right), \\
0<r<R ; \quad 0<z<s(t) ; \quad t>0 .
\end{gathered}
$$

where $T$ is the temperature; $C$ is the specific heat capacity; $\rho$ is the material density; $\lambda$ is the heat conductivity; $s(t)$ is the current position of ingot upper end face.

Heat application due to heating with electron beams and radiation of heat on Stefan-Boltzmann law takes place in the upper end face of the ingot. A 

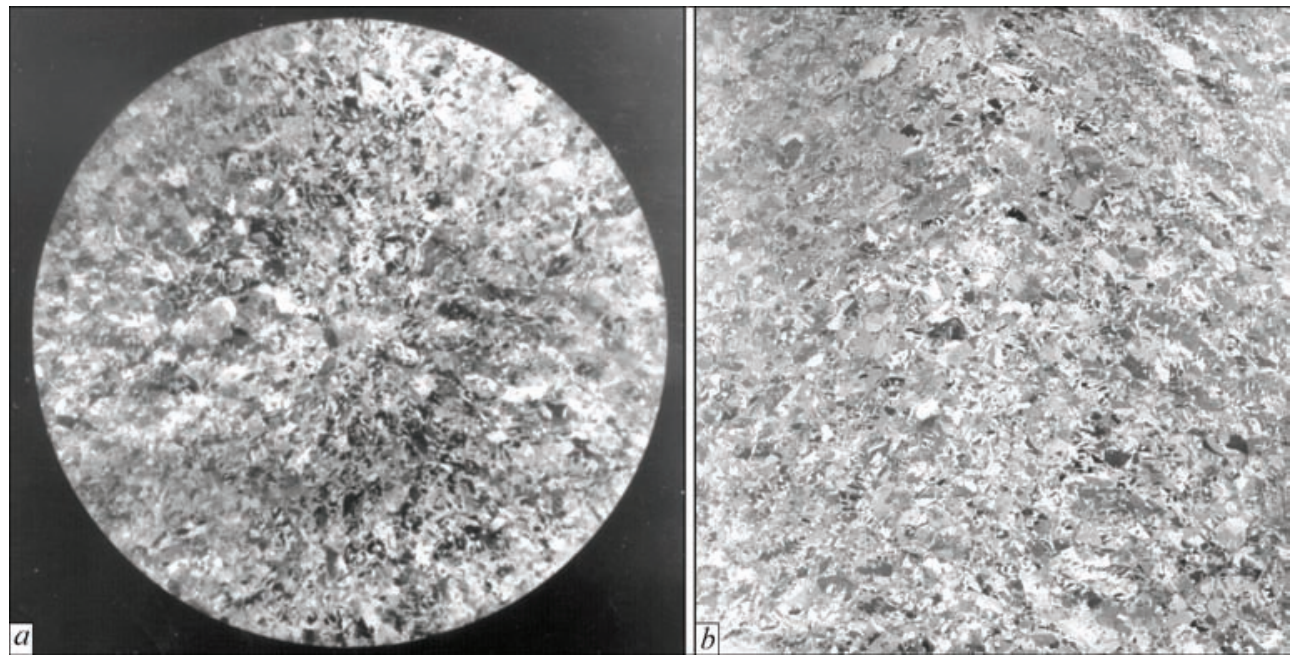

Figure 6. Macrostructure of VT6 titanium alloy ingot in cross ( $a$ ) and longitudinal (b) sections

boundary condition on upper end face of the ingot is presented in form of

$$
\left.\lambda(T) \frac{\partial T}{\partial z}\right|_{z=s(t)}=-\varepsilon \sigma\left(T^{4}-T_{\text {med }}^{4}\right)+w(r, t),
$$

at that specific power of electron beam heating $w(r, t)$ is distributed in the following way:

$$
\begin{gathered}
w(r, t)=w_{1}(t) \text { at } r<R_{1} ; \\
w(r, t)=W_{2}(t) \sin ^{2} \pi\left(\frac{r-R_{1}}{R+d-R_{1}}\right) \\
\text { at } R_{1}<r<R .
\end{gathered}
$$

On side surface of the ingot, contacting with the walls of mould and bottom plate, the heat exchange takes place on Newton-Richmann law

$$
-\left.\lambda(t) \frac{\partial T}{\partial r}\right|_{r=R}=\alpha\left(T-T_{\text {med }}\right),
$$

where $\alpha$ is the coefficient of heat transfer between the ingot and copper water-cooled wall; $T_{\text {med }}$ is the temperature of medium having heat exchange with the ingot.

On the free side surface of the ingot, the heat exchange is described by Stefan-Boltzmann law:

$$
-\left.\lambda(t) \frac{\partial T}{\partial r}\right|_{r=R}=\varepsilon \sigma\left(T^{4}-T_{\text {med }}^{4}\right) .
$$

The results of calculation on the mathematical model of heat processes in titanium ingot at EBM showed that varying a power of electron beam heating of the melt free surface in the mould and melting rate, it is possible to change the volume of liquid pool metal and shape of solidification front, thus, controlling formation of ingot structure [6]. Carried full-scale experiments on melting of titanium ingots at different technological modes showed high level of adequacy of the designed models and proved the possibility of production of ingots from titanium and its alloys with fine equiaxial structure in performance of melts with the optimum technological parameters (Figure 6).

In melting of titanium alloy ingots by EBM method there is a problem of providing a set chemical composition of metal. It is caused by the fact that in EBM the alloying elements with vapor pressure exceeding titanium vapor pressure evaporate more intensively than in VAR. Aluminum, chromium, manganese and others refer to such elements. But, first of all, it refers to aluminum, since it is an alloying element of virtually all titanium alloys.

Carried at the PWI fundamental investigations of the processes of alloy components evaporation from the melt in vacuum under conditions of heating of surface with electron beam allowed designing the mathematical models of processes of evaporation of alloy components in EBM $[6,7]$. They set the dependence of concentration of alloying elements in titanium ingot from the technological parameters of melting and concentration of these elements in consumed billet:

$$
\begin{aligned}
& \frac{\partial}{\partial t} \int_{V_{j}} \rho\left[X_{i}\right]_{j} d V=m_{j-1}\left[X_{i}\right]_{j-1}-S_{j} \pi_{j}^{i}-m_{j}\left[X_{i}\right]_{j} ; \\
& \frac{\partial}{\partial t} \int_{V_{j}} \rho[\mathrm{Ti}]_{j} d V=m_{j-1}[\mathrm{Ti}]_{j-1}-S_{j} \pi_{j}^{\mathrm{Ti}}-m_{j}[\mathrm{Ti}]_{j},
\end{aligned}
$$

where $i=1,2 \ldots n$ indicate alloying elements; $j=1$, 2,3 are the melting zones (film of liquid metal on the end face of consumable billet, pools of liquid metal in the cold hearth and in the mould); $V_{j}$ is the volume of liquid metal in $j$ th zone of melting, $\mathrm{m}^{3} ; \rho$ is the melt density, $\mathrm{kg} / \mathrm{m}^{3} ;\left[X_{i}\right]_{j}$ is the concentration of $i$ th alloying element in $j$ th zone of melting, mass fraction; $[\mathrm{Ti}]_{j}$ is the concentration of titanium in $j$ th zone of melting, mass fraction; $S_{j}$ is the area of liquid metal free surface in $j$ th zone of melting, $\mathrm{m}^{2} ; \pi_{j}^{i}$ and $\pi_{j}^{\mathrm{Ti}}$ are the specific flows of alloying elements and titanium through interphase surface into vapor phase in $j$ th zone of melting, $\mathrm{kg} /\left(\mathrm{s} \cdot \mathrm{m}^{2}\right) ; m_{j-1}$ is the mass velocity of melt coming in 


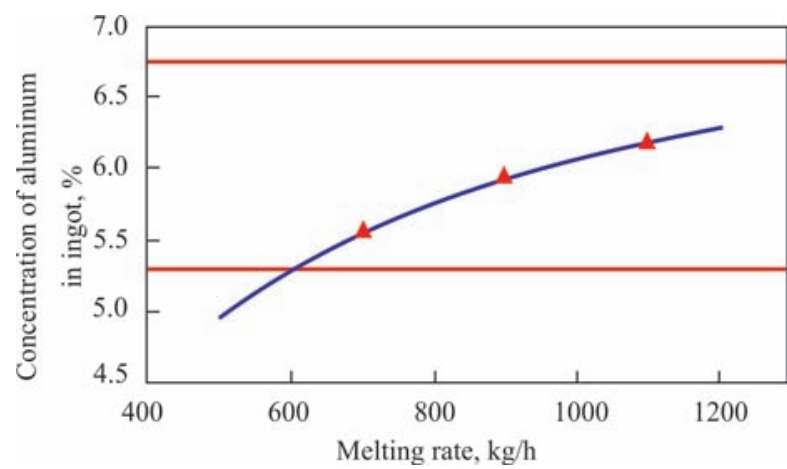

Figure 7. Dependence of aluminum content in EBM ingot of VT6 alloy on melting rate (solid line - calculation; dots - experiment)

$j$ th zone of melting, $\mathrm{kg} / \mathrm{s} ; m_{3}$ is the mass velocity of melt solidification in the mould, $\mathrm{kg} / \mathrm{s}$.

The dependencies of content of aluminum in the ingot on melting rate were plotted by the example of evaporation of aluminum from the melt of titanium alloy $\mathrm{Ti}-6 \mathrm{Al}-4 \mathrm{~V}$ (wt.\%) in vacuum using mathematical model (4). Comparison of data of experimental melts of Ti-6Al-4V titanium alloy ingots with obtained dependencies showed high accuracy of description of developed model of real process of aluminum evaporation in EBM (Figure 7).

Application of these models allows predicting compositions of melted titanium alloy ingots and providing production of the ingots with guaranteed composition. Compensation of component loss of alloys with high vapor pressure in EBM is carried out by additional alloying with these elements of initial charge. Homogeneity of distribution of alloying elements on ingot volume has great importance in melting of titanium alloy ingots. Under EBM conditions this is provided by homogeneity of burdening of consumed billet, stability of technological parameters in melting and special technological means.

Carried complex of theoretical and experimental investigations of the EBM process allowed developing a technology for production of high-quality ingots of high-strength and heat-resistant titanium alloys

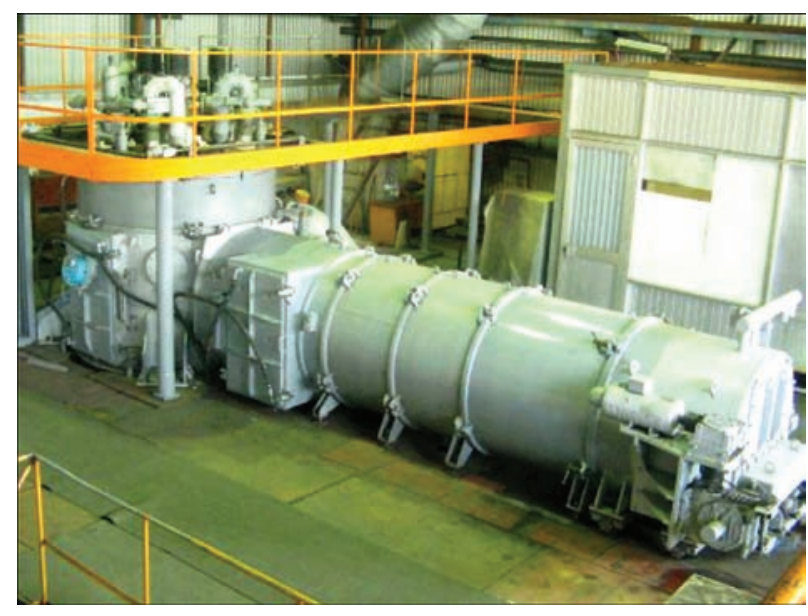

Figure 9. Appearance of commercial electron beam unit UE5812 of up to $840 \mathrm{~mm}$ diameter and design equipment for their commercial realizing (Figures 8, 9).

For evaluation of metal quality in produced ingots there were carried out an examination of composition of the samples taken on length of the ingot from upper, middle and lower parts. The results of analysis of metal composition of the produced ingots (Table) showed that distribution of alloying elements on ingots' length is uniform and correspond to grade composition. EBM titanium alloy ingots have no inhomogeneities, nonmetallic inclusions of more than $1 \mathrm{~mm}$ size as well as dense accumulations of finer inclusions. Metal structure is dense, crystalline inhomogeneity and zonal liquation are absent in the ingot.

In process of ingots production due to a series of reasons, caused by metallurgical and technological peculiarities, their surface layer turns out to be defective. In order to eliminate such defects the surface of produced ingots and cast billets are subjected to mechanical treatment, as a result of which amount of wastes can reach up to $15 \%$ of weight of treated ingot.

In order to reduce metal loss instead of mechanical treatment the PWI has developed a technology of electron beam melting of side surface of ingots of round as well as rectangular section by electron
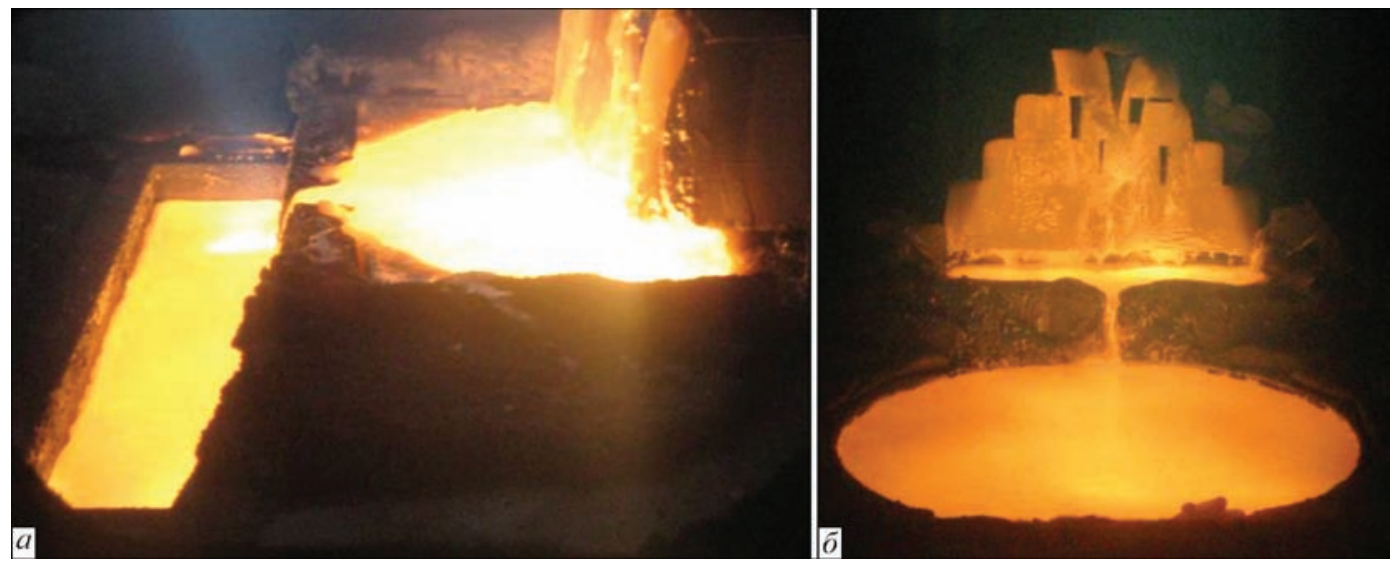

Figure 8. EBM process: $a$ - ingot-slab of VT23 titanium alloy; $b$ - VT22 titanium alloy ingot of 400 mm diameter 
Distribution of alloying elements and impurities on length of titanium alloy ingots, produced by EBM,wt.\%

\begin{tabular}{|c|c|c|c|c|c|c|c|c|c|}
\hline Alloy grade & Ingot part & $\mathrm{Al}$ & Mo & V & $\mathrm{Fe}$ & $\mathrm{Zr}$ & $\mathrm{Cr}$ & $\mathrm{O}$ & $\mathrm{N}$ \\
\hline \multirow{3}{*}{ VT23 } & Upper & 4.5 & 2.0 & 4.7 & 0.5 & 0.01 & 0.9 & 0.11 & 0.012 \\
\hline & Middle & 4.6 & 2.1 & 5.0 & 0.6 & 0.01 & 0.9 & - & - \\
\hline & Lower & 4.3 & 1.9 & 4.7 & 0.5 & 0.01 & 0.9 & - & - \\
\hline \multicolumn{2}{|c|}{ OST 190013-81 } & $4.0-6.3$ & $1.5-2.5$ & $4.0-5.0$ & $0.4-1.0$ & $<0.3$ & $0.8-1.4$ & $<0.15$ & $<0.05$ \\
\hline \multirow{3}{*}{ VT22 } & Upper & 5.1 & 4.2 & 4.9 & 0.90 & 0.01 & 1.3 & 0.11 & 0.012 \\
\hline & Middle & 5.2 & 4.5 & 4.7 & 1.0 & 0.01 & 1.4 & - & - \\
\hline & Lower & 5.0 & 4.1 & 5.0 & 1.0 & 0.01 & 1.4 & - & - \\
\hline \multicolumn{2}{|c|}{ GOST 19807-91 } & $4.4-5.7$ & $4.0-5.5$ & $4.0-5.5$ & $0.5-1.5$ & $<0.3$ & $0.5-1.5$ & $<0.15$ & $<0.05$ \\
\hline \multirow{3}{*}{ VT20 } & Upper & 6.85 & 1.60 & 2.05 & 0.10 & 1.60 & $<0.1$ & 0.08 & 0.019 \\
\hline & Middle & 6.90 & 1.63 & 2.05 & 0.11 & 1.63 & $<0.1$ & - & - \\
\hline & Lower & 6.70 & 1.63 & 2.08 & 0.11 & 1.60 & $<0.1$ & - & - \\
\hline \multicolumn{2}{|c|}{ GOST 19807-91 } & $5.5-7.0$ & $0.5-2.0$ & $0.8-2.5$ & $<0.25$ & $1.5-1.5$ & $<0.1$ & $<0.15$ & $<0.05$ \\
\hline
\end{tabular}

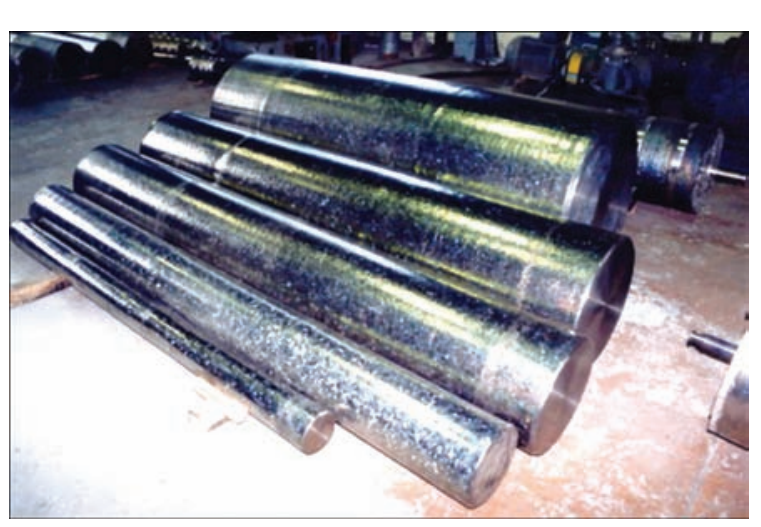

Figure 10. Appearance of titanium alloy ingots of 100-600 mm diameter with melted side surface

beams and corresponding equipment for its realizing (Figure 10). Developed technology provides effective removal of surface defects at up to $10 \mathrm{~mm}$ depth ensuring at that the quality of side surface and correspondence of composition of the melted layer at the level of requirements of standards, rising at that metal yield by $7-15 \%$ depending on section and dimensions of the ingot [8].

Check of quality of the semi-products in form of rods and forgings, produced from EBM ingots, showed that their mechanical properties correspond to all the requirements, which the industry makes to titanium alloy quality. At that the semi-products are isotropic on mechanical properties.

In cooperation with the enterprise-partners, in Ukraine there was organized a production of hot- and cold-rolled pipes from the titanium alloy ingots, made by EBM method. Their quality completely corresponds to the standard requirements.

Developed at the E.O. Paton Electric Welding Institute of the NAS of Ukraine technological processes of electron beam melting provide the possibility to obtain high-quality ingots of titanium and its alloys with uniform defect-free structure. The developed technologies allow reducing prime cost of the titanium semi-products due to application of cheaper raw materials and increase of through metal yield and, thus, rising competitiveness and expanding fields of application of titanium in different branches of industry.

Realizing the EBM technology under conditions of commercial enterprises permitted to organize in Ukraine competitive on the world markets production of high-quality ingots and ingot-slabs of titanium, which have large perspectives for further development.

1. Koshelap, A.V., Rajchenko, A.I. (1999) On possibility of structure refining of cast titanium and its alloys due to their modification with titanium nitride particles. Protsessy Litya, 3, 44-52 [in Russian].

2. Mitchel, A. (1987) The production of high-quality materials by special process. J. Vac. Technol., Jul./Aug.(4), 2672-2677.

3. Bewley, B.P., Gigliotti, M.F.X. (1977) Dissolution rate measurements of TiN in Ti-6242. Acta Mat., 45(1), 357-370.

4. Bellot, J.P., Mitchell, A. (1994) Hard-alfa particle behavior in a titanium alloy liquid pool. Light Metals, 2, 1187-1193.

5. Akhonin, S.V., Kruglenko, M.P., Kostenko, V.I. (2011) Mathematical modeling of process of dissolution of oxygen-containing refractory inclusions in titanium melt. Sovrem. Elektrometall., 1, 17-21 [in Russian].

6. Paton, B.E., Trigub, N.P., Akhonin, S.V., Zhuk, G.V. (2006) Electron beam melting of titanium. Kiev, Naukova Dumka [in Russian]

7. Akhonin, S.V., Trigub, N.P., Zamkov, V.N., Semiatin, S.L. (2003) Mathematical modeling of aluminium evaporation during electron-beam cold-hearth melting of Ti-6 Al-4V ingots. Metall. and Mater. Transact. B, 34B(August), 447-454.

8. Akhonin, S.V., Berezos, V.A., Pikulin, A.N., et al. (2014) Electron beam melting of surface of titanium alloy ingots. Sovrem. Elektrometall., 2, 21-25 [in Russian]. 


\title{
EFFECT OF MULTI-PASS FRICTION STIR PROCESSING ON THE MICROSTRUCTURE AND MECHANICAL PROPERTIES OF DUAL PHASE STEEL
}

\author{
T. KÜÇÜKÖMEROĞLU ${ }^{1}$, S.M. AKTARER ${ }^{2}$, G. İPEKOĞLU ${ }^{3}$ and G. ÇAM ${ }^{3}$ \\ ${ }^{1}$ Department of Mechanical Engineering, Karadeniz Technical University, Trabzon, Turkey. E-mail: tkomer@ktu.edu.tr \\ ${ }^{2}$ Department of Automotive Technology, Recep Tayyip Erdogan University, \\ Rize, Turkey. E-mail: semih.aktarer@erdogan.edu.tr \\ ${ }^{3}$ Department of Mechanical Engineering, Iskenderun Technical University, \\ 31200 İskenderun-Hatay, Turkey. E-mail: guven.ipekoglu@iste.edu.tr; gurel.cam@iste.edu.tr
}

\begin{abstract}
Dual phase (DP) steels have been widely used in the automotive industry due to the excellent engineering properties such as high strength and good formability. However, attempts have recently been ongoing to improve their mechanical and formability properties in order to achieve further weight savings. Mechanical and microstructural properties of DP steel can be improved by severe plastic deformation (SPD) techniques without changing their chemical compositions. Among SPD methods, friction stir processing (FSP) is a new method used to enhance the properties of plate and/or sheet types of metals. Therefore, the effect of multi-pass FSP (M-FSP) on the microstructure and mechanical performance of a DP steel (i.e., DP600) was investigated in the current study. M-FSP was applied to dual phase steel at the $4 \mathrm{~mm}$ steps. FSP resulted in a refined microstructure which brought about a considerable increase in both hardness and strength values. After FSP, islands of martensite as the secondary phase in the microstructure have been broken and disturbed by the rotational pin. The processed region consists of ferrite, bainite and martensite. The hardness value increased from $210 \mathrm{HV}_{0.2}$ to about $360 \mathrm{HV}_{0.2}$ after M-FSP. 36 Ref., 1 Table, 4 Figures.
\end{abstract}

Keywords : friction stir processing, dual-phase steel, fine grained microstructure, mechanical properties

Introduction. Dual Phase (DP) steels are widely used in the automotive industry due to their excellent mechanical properties such as high strength and good formability [1-3]. However, numerous studies have recently been conducted to improve the strength and ductility of these steels which in turn further reduce the weight and ensure safety [4-6]. The mechanical properties of DP steels can be improved by severe plastic deformation (SPD) techniques without changing their chemical compositions [7, 8]. Friction stir processing (FSP) is a novel SPD method which can be used for the improvement of properties. It can even be said that friction stir process is the most ideal method among the SPD methods when considering the processing of large scale plate or sheet type materials [9].

FSP is based on the basic principles of friction stir welding (FSW) [10], which is originally developed for joining difficult-to-weld Al-alloys [11-14]. FSP is a method of improvement of the properties of a material by way of severe, localized plastic deformation which is produced by immersing a non-consumable tool into the work piece, and rotating and traveling the tool in a stirring motion [15]. Many review papers on this process have been published until now, and thus detailed information on its principles can be obtained in Refs. [16-19]. As the FSP deforms a limited region, the multi-pass FSP, which is applied sequentially, leads to deformation of larger regions and allows material properties to be improved in large-scale dimensions. Up to now, multi-pass FSP has been applied to mostly aluminum alloys [20-25] and few magnesium alloys [26-28] and pure titanium [29]. Generally, it was reported that multi-pass FSP improves mechanical properties of cast $\mathrm{Al}$ alloys containing $\mathrm{Si}$ and achieves significant microstructural refinement [25, 30-32]. Aktarer et al. [33] reported that two-pass FSP of A112Si alloy leads to fragmentation of needle-shaped silicon plates from $27 \pm 23 \mu \mathrm{m}$ to about $2.6 \pm 2.4 \mu \mathrm{m}$, thus both strength and ductility are remarkably increased, i.e., about 1,3 and 7 times than that of base metal, respectively. Similarly, Lua et al. showed that multi-pass FSP significantly improved both strength and ductility of cast magnesium alloys such as AZ61 due to grain refinement and the elimination of cast defects [26]. Also, corrosion behavior of multi-pass friction stir processed (FSP) pure titanium was investigated by Fattah-Alhosseini et al. [29]. They found that grain refinement in the multi-pass FSPed sample led to a reduction in both corrosion and passive current densities.

Although there are many reports on multi-pass FSP (M-FSP) of non-ferrous alloys and single-pass FSP of steels [33, 34], only limited amount of studies have been undertaken systematically on the (M-FSP) 


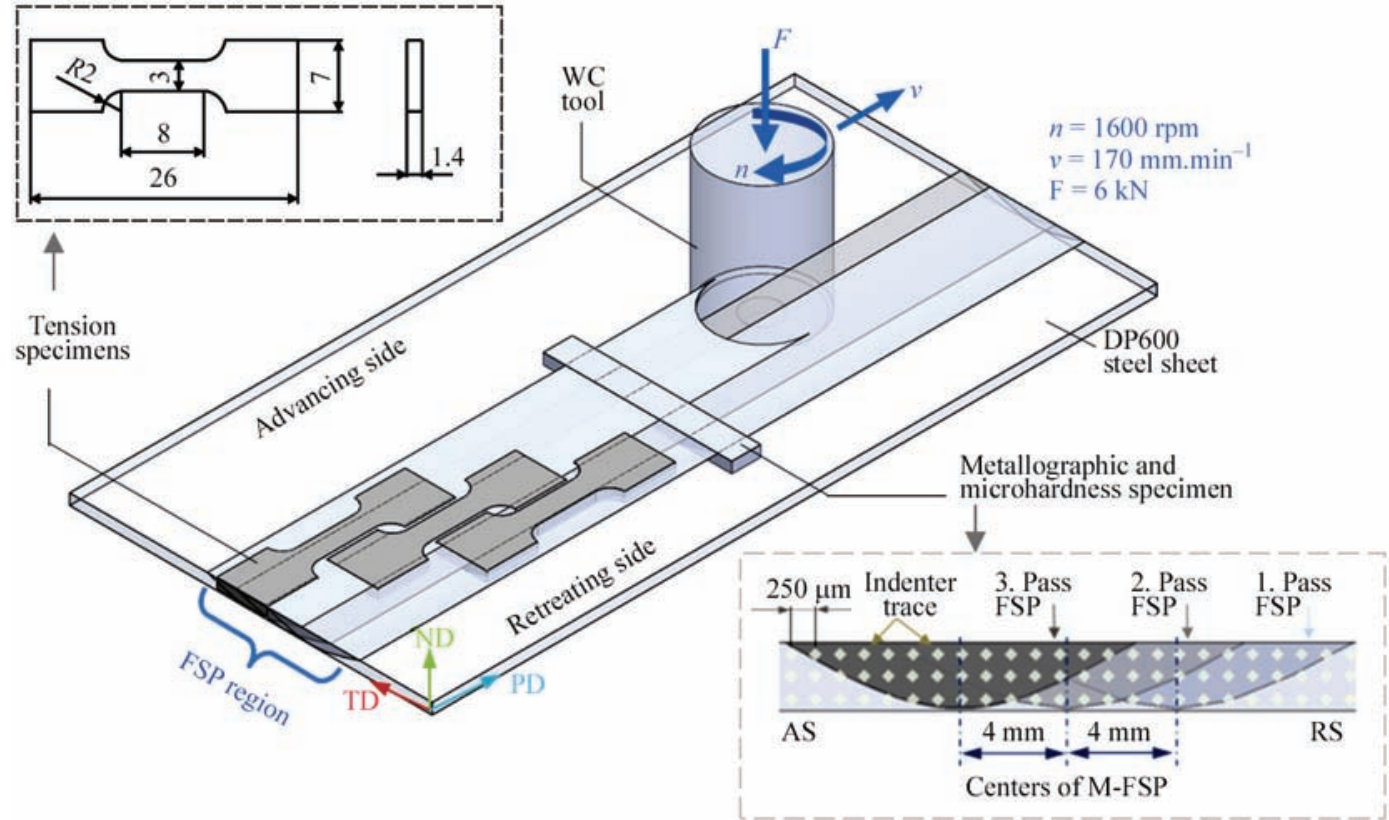

Figure 1. Schematic illustration of M-FSP technique which shows the specimens' geometries extracted from the FSPed sheet and the position from which they were extracted

of steels. Furthermore, more studies are needed for getting further improvement in mechanical properties of multi-pass friction stir process of dual phase steel. Therefore, the main purpose of this study is to determine the effect of multi-pass FSP on the microstructural evolution, microhardness and tensile properties of a dual phase steel (i.e., DP600).

Experimental procedure. Hot rolled DP600 steel with a chemical composition of $0.040 \% \mathrm{C}$, $1.436 \% \mathrm{Mn}, 0.239 \% \mathrm{Si}, 0.047 \% \mathrm{Al}, 0.035 \% \mathrm{Cu}$, $0.690 \% \mathrm{Cr}, 0.039 \% \mathrm{Ni}, 0.011 \% \mathrm{Mo}$ and balance $\mathrm{Fe}$ was used in this study. Samples with the dimensions of $200 \times 50 \mathrm{~mm} \times 1.5 \mathrm{~mm}$ were cut from the steel plate for multi-pass friction stir processing (M-FSP). M-FSP was performed with a processing tool having a flat shoulder with the diameter of $14 \mathrm{~mm}$ and a cylindrical pin with the diameter and length of $5 \mathrm{~mm}$ and $1.3 \mathrm{~mm}$, respectively. In FSP trials, a tool rotational rate of $1600 \mathrm{rpm}$ and a traverse speed of $170 \mathrm{~mm} / \mathrm{min}$ were used. The shoulder tilt angle was set at $2^{\circ}$, and the tool downforce was kept constant at $6 \mathrm{kN}$ during process. The subsequent M-FSP was shifted toward the advancing side with stepping of $4 \mathrm{~mm}$, thus a total of 3 overlapping passes were carried out on the DP600 steel sheet. Schematic illustration of M-FSP process is shown in Figure 1.

Optical microscopy $(\mathrm{OM})$ and scanning electron microscopy (SEM) were used to observe the microstructure of the samples before and after M-FSP. The metallography specimens were extracted perpendicular to the processing direction (Figure 1), polished with standard techniques and then etched in \% 2 Nital $(3 \mathrm{ml}$. $\mathrm{HNO}_{3}+97 \mathrm{ml} . \mathrm{C}_{2} \mathrm{H}_{6} \mathrm{O}$ ) for $20 \mathrm{~s}$. Mechanical properties of the base and M-FSPed samples were determined using tensile test and hardness measurements. Dog-bone shaped tensile test specimens with the gauge dimensions of $1.4 \times 3 \times 8 \mathrm{~mm}$ were extracted from the base material and M-FSPed plates by electro discharging machining (EDM) technique. These specimens were cut parallel to the process direction at three different positions inside the FSPed region as shown in Figure 1 . The positions of the tensile test specimens are representative of the center of each pass FSP region shifted to $4 \mathrm{~mm}$ steps. The tests were performed using an electro-mechanical load frame with a video type extensometer at a strain rate of $5 \cdot 10^{-4} \mathrm{~s}^{-1}$. Vickers micro-hardness tests were carried out using a load of $200 \mathrm{~g}$ and a dwell time of $10 \mathrm{~s}$. Vickers microhardness measurements were conducted throughout the cross section of the processed specimen with an interval of $250 \mu \mathrm{m}$ as illustrated in Figure 1.

Results and discussion. Microstructure. Optical micrographs showing the microstructures of DP600 steel base plates are given in Figure 2. The initial microstructure of DP600 steel sheet is consisted of elongated ferrite grains in rolling direction and dispersed martensite throughout ferrite grain boundaries. Average ferrite grain size was $6 \mu \mathrm{m}$ and the volume fraction of martensite was determined to be approximately $24 \%$. Martensite islands appear dark in optical micrograph (Figure 2,a) and they seem bright in SEM image (Figure 2, b).

An optical macrograph of M-FSPed region is given Figure 3, $a$, and the traces of the side-by-side pass are clearly visible. Final pass processed microstructure is shown in Figure 3, $b$, which is almost entirely lath martensite. This zone is seen as a darker region in macrograph. The region exhibits a similar characteristic to single-pass FSP microstructures. Since the process does not continue then there is no heat or de- 

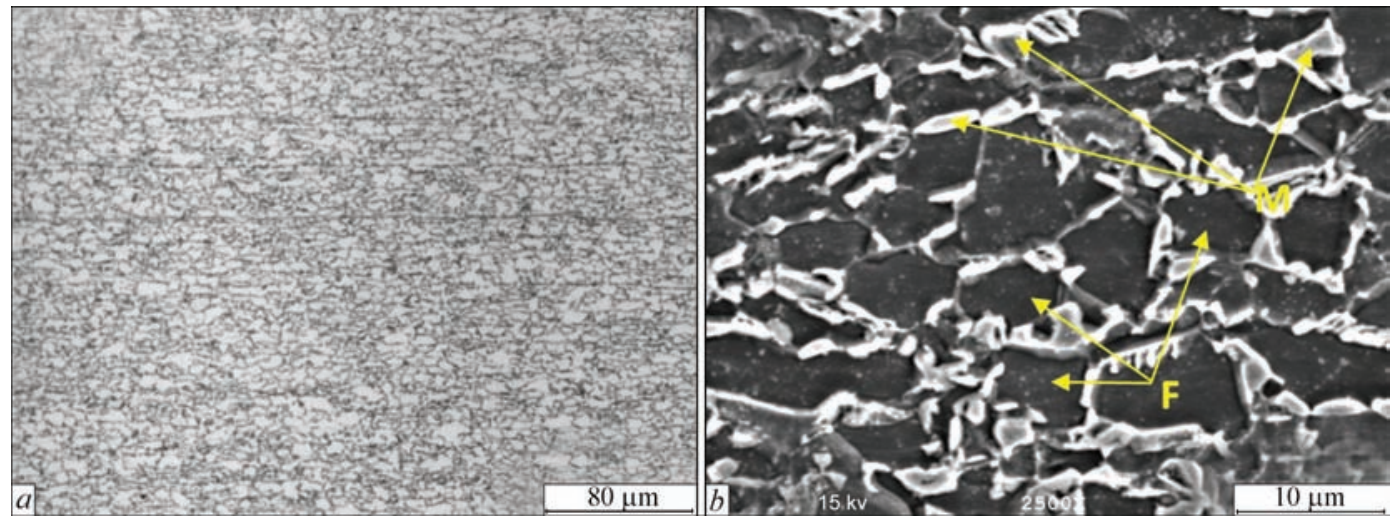

Figure 2. Micrographs showing the microstructure of DP600 steel base plate: $a$ - optical micrograph; $b$ - SEM image

formation to affect this region. However, it is a fact that the heat and deformation of the 2 nd pass process affects the 3rd processed zone and it can be separated into single pass FSP. The lath martensite formed in the microstructure shows that this region is completely austenite during the process. Therefore, it can be said that the process temperature is above the A3 temperature line. Miles et al. [35] is reported that lath martensite is observed in SZ of friction stir welded DP590 steel. The microstructure in the SZ of the second pass process is shown Figure 3, c, which also corresponds to the heat affected zone of the third process. Therefore, while microstructure of this region is fully lath martensite after the 2 nd pass process, it is trans- formed to acicular products by heat generated by the 3 rd pass process. This microstructure consists of lath martensite and dispersed acicular and globular cementite through the ferrite matrix as seen Figure 3, c. The SZ of 1st pass process is actually the heat affected zone of 2 nd pass process and its microstructure is given Figure 3, $d$. A predominantly bainitic structure is observed in this region, and the formation of various types of acicular products depends on the peak temperature, the cooling rate and the rate of deformation during the process. Kang et al. [36] reported that microstructures of bainite and martensite may form at different cooling rates in FSPed low-alloy highstrength steel. Their results clearly showed that the

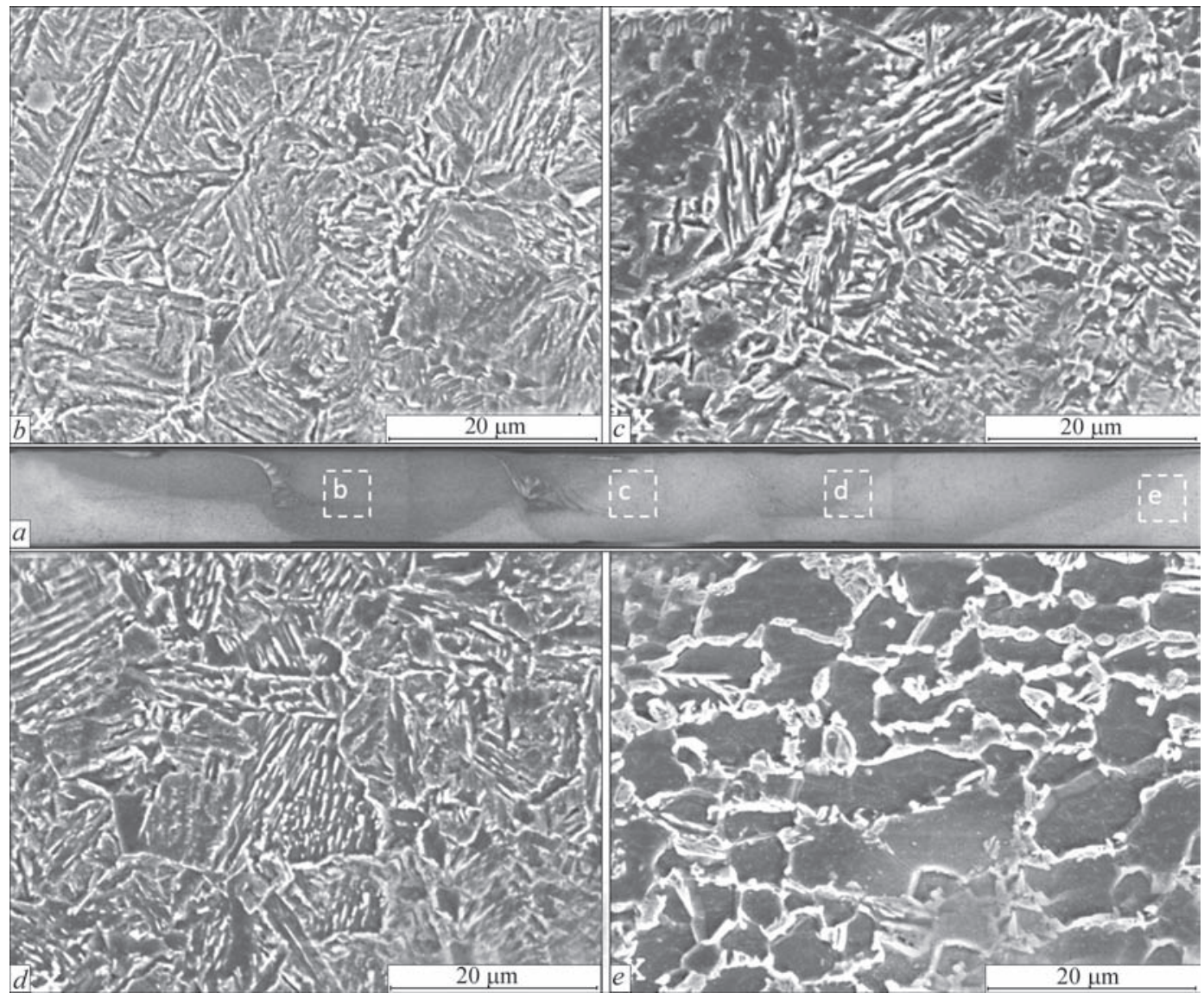

Figure 3. Optical macrograph of the cross-section of M-FSPed specimen (a), the SZ center of 3rd pass (b), the SZ center of 2nd pass $(c)$, the SZ center of 1st pass $(d)$, heat affected zone (HAZ) of 1st pass at the retracting side $(e)$ 


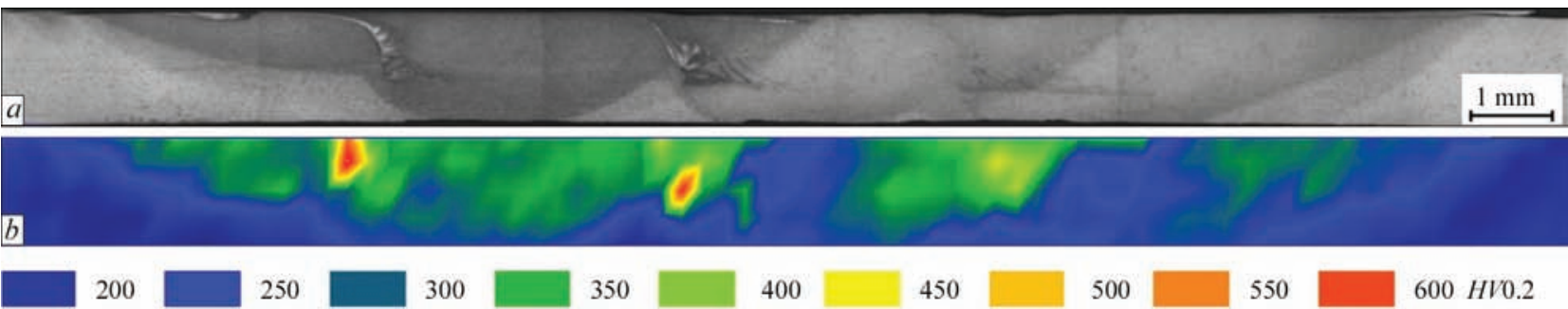

Figure 4. Optical microstructure of cross section of the M-FSPed sample extracted perpendicular to the process direction (a); a contour map showing the hardness distribution across the M-FSPed region (b)

microstructure comprises of completely bainite at a cooling rate of $30^{\circ} \mathrm{C} / \mathrm{s}$ and dominantly martensite at a cooling rate of $70^{\circ} \mathrm{C} / \mathrm{s}$.

The microstructure of HAZ of 1 st pass process at the retracting side is tempered martensite which consists of very small and uniformly dispersed cementite [37]. Figure 3(c) shows tempered martensite partially decomposing into cementite and ferrite from martensite islands in the HAZ.

Microhardness and tensile prosperities. The microhardness profile showing the hardness distribution across the M-FSPed region is given in Figure 4. The DP 600 steel base plate has a hardness of 210 $H V$. The highest hardness in the processed zone is approximately $550 \mathrm{HV}$, which is measured around the pin. The average hardness value of the stir zone corresponding to the rotating pin diameter is seen as the green region in the hardness map of $360 \mathrm{HV}$. This region corresponds to the volume of the rotating pin diameter and is also an important indication of material flow during FSP. This region has a lath martensitic microstructure with a homogeneous distribution. The stir zone of 1st pass process is affected by the heat generated by the next pass process, and the hardness drop in this zone is clearly observed with a light blue color. A similar characteristic feature is observed in the second pass process and each process is affected by the heat of the next pass process, and the hardness at the end decreases down to 250-300 HV. The microstructure of this transition region is a predominantly bainitic structure consisting of cementite and ferrite. The HAZ microstructure of retreating side is tempered martensite and this microstructure has $200 \mathrm{HV}$ hardness in a very narrow region of about $250 \mu \mathrm{m}$. Since the hardness profile represents the dark blue 200-250 HV micro hardness range in the color mapping, the hardness decrease in the tempered martensite is not clearly visible.

The tensile test results of DP 600 steel before and after M-FSP are summarized in Table. DP 600 steel base plate sample displayed a high elongation with a large strain hardening region which is a typical characteristic of dual phase steels. The yield strength and ultimate tensile strength of base plate were 412 and $615 \mathrm{MPa}$, respectively. On the other hand, the SZs of 1st pass, 2nd pass and 3rd pass processed
Main strength and ductility values of each stir zone after M-FSP

\begin{tabular}{|c|c|c|c|}
\hline Samples & $\begin{array}{c}\text { Yield strength, } \\
\mathrm{MPa}\end{array}$ & $\begin{array}{c}\text { Ultimate tensile } \\
\text { ttrength, } \\
\mathrm{MPa}\end{array}$ & $\begin{array}{c}\text { Elon-gation, } \\
\%\end{array}$ \\
\hline DP 600 & 412 & 655 & 28 \\
\hline SZ of 1. Pass & 321 & 560 & 30 \\
\hline SZ of 2. Pass & 349 & 555 & 31 \\
\hline SZ of 3. Pass & 657 & 830 & 25 \\
\hline
\end{tabular}

samples exhibited yield strength values of 321,349 and $657 \mathrm{MPa}$, respectively, and the ultimate tensile strength values of 560, 555 and $830 \mathrm{MPa}$, respectively. The low strength values exhibited by the SZs of 1 st pass and 2nd pass FSPed samples compared to that of the base plate may be attributed to the fact that the microstructures of these samples transformed from initial martensite-ferrite structure to a predominantly bainitic structure. As seen from Table 1, no significant decrease in elongation was observed after M-FSP and all the specimens exhibited similar elongation values to that of the base plate.

\section{Conclusions}

Multi-pass friction stir process (M-FSP) technique was applied to DP 600 steel and their microstructural and mechanical properties of the M-FSPed plates have been investigated. The results of this study can be summarized as follows:

- the microstructure of the M-FSPed DP600 steel consists of lath martensite, bainite and recrystallized ferrite;

- it was observed that the next pass friction stir process affects the stir zone of the previous pass friction stir processed specimen. The heat generated by the next pass FSP leads to the transformation of lath martensite (formed after the previous pass FSP) to cementite and ferrite, thus results in a reduction in hardness;

- the hardness in the processed region increased to an average of $360 \mathrm{HV}$ from $210 \mathrm{HV}$ (hardness of base plate);

- the tensile strength values in the SZ of the first pass process and the second pass process were observed to be 560 and $555 \mathrm{MPa}$, respectively, both of which were lower than that of base plate (i.e., $655 \mathrm{MPa}$ ). This may be attributed to the tempering effect experienced in this regions. On the other hand, the tensile strength of the third process stir zone in- 
creased to $830 \mathrm{MPa}$ which is due to the existence of martensitic structure in this sample.

1. Rashid, M.S. (1981) Dual phase steels. Ann. Rev. Mater. Sci., 11, 245-67.

2. Nishımoto, A., Hosoya, Y., Nakaoka, K. (1981) A new type of dual-phase steel sheet for automobile outer body panels. Transact. Iron Steel Inst. Japan; 21, 778-82.

3. Fonstein, N. (2017) 7 - Dual-phase steels. Rana R, Singh SB, editors. Automot. Steels, Woodhead Publishing, 169-216.

4. Abid, N.H., Abu Al-Rub, R.K., Palazotto, A.N. (2017) Micromechanical finite element analysis of the effects of martensite morphology on the overall mechanical behavior of dual phase steel. Int. J. Solids Struct., 104-105, 8-24.

5. Kundu, A., Field, D.P. (2016) Influence of plastic deformation heterogeneity on development of geometrically necessary dislocation density in dual phase steel. Mater. Sci. Eng. A, 667, 435-43.

6. Ashrafi, H., Shamanian, M., Emadi, R., Saeidi, N. (2017) A novel and simple technique for development of dual phase steels with excellent ductility. Ibid., 680, 197-202.

7. Son, Y Il, Lee, Y.K., Park, K.T. et al. (2005) Ultrafine grained ferrite-martensite dual phase steels fabricated via equal channel angular pressing: Microstructure and tensile properties. Acta Mater., 53, 3125-34.

8. Park. K.-T., Lee, Y.K., Shin, D.H. (2005) Fabrication of ultrafine grained ferrite/martensite dual phase steel by severe plastic deformation. ISIJ Int., 45, 750-5.

9. Ma, Z.Y. (2008) Friction stir processing technology: A review. Metall. Mater. Transact. A, 39, 642-58.

10. Mishra, R.S., Mahoney, M.W. (2007) Friction stir welding and processing. ASM Int., 368.

11. Çam, G., İpekoğlu, G., Küçükömeroğlu, T., Aktarer, S.M. (2017) Applicability of friction stir welding to steels. Journal of Achievements in Materials and Manufacturing Engineering, 80(2), 65-85.

12. Çam, G., İpekoğlu, G. (2017) Recent developments in joining of aluminium alloys. Int. J. Adv. Manuf. Technol., 91(5-8), 1851-66.

13. Çam, G., Mistıkoğlu, S. (2014) Recent developments in friction stir welding of Al-alloys. Journal of Materials Engineering and Performance, 23(6), 1936-53.

14. Çam, G. (2011) Friction stir welded structural materials: Beyond Al-alloys'. Int. Mater. Rev., 56(1), 1-48.

15. Mishra, R.S., Ma, Z.Y. (2005) Friction stir welding and processing. Mater. Sci. Eng. R Reports, 50, 1-78.

16. Padhy, G.K., Wu, C.S., Gao, S. (2018) Friction stir based welding and processing technologies — processes, parameters, microstructures and applications: A review. J. Mater. Sci. Technol., 34, 1-38.

17. Węglowski, M.S. (2018) Friction stir processing — state of the art. Arch. Civ. Mech. Eng., 18, 114-29.

18. Chaudhary, A., Dev, A.K., Goel, A. et al. (2018) The mechanical properties of different alloys in friction stir processing: A review. Mater. Today Proc., 5, 5553-62.

19. Sudhakar, M., Rao, C.H.S., Saheb, K.M. (2018) Production of surface composites by friction stir processing: A review. Ibid., 929-35.

20. Moustafa, E. (2017) Effect of multi-pass friction stir processing on mechanical properties for AA2024/ $\mathrm{Al}_{2} \mathrm{O}_{3}$ nanocomposites. Materials (Basel), 10.
21. Chen, Y., Ding, H., Malopheyev, S. et al. (2017) Influence of multi-pass friction stir processing on microstructure and mechanical properties of 7B04-O Al alloy. Transact. Nonferrous Met. Soc. China, 27, 789-96.

22. El-Rayes, M.M., El-Danaf, E.A. (2012) The influence of multi-pass friction stir processing on the microstructural and mechanical properties of aluminum Alloy 6082. J. Mater. Proc. Technol., 212, 1157-68.

23. Nakata, K., Kim, Y.G., Fujii, H. et al. (2006) Improvement of mechanical properties of aluminum die casting alloy by multipass friction stir processing. Mater. Sci. Eng. A, 437, 274-80.

24. Ramesh, K.N., Pradeep, S., Pancholi, V. (2012) Multipass friction-stir processing and its effect on mechanical properties of aluminum alloy 5086. Metall. Mater. Transact. A Phys. Metall. Mater. Sci., 43, 4311-9.

25. Singh, S.K., Immanuel, R.J., Babu, S. et al. (2016) Influence of multi-pass friction stir processing on wear behaviour and machinability of an Al-Si hypoeutectic A356 alloy. J. Mater. Proc. Technol., 236, 252-62.

26. Luo, X.C., Zhang, D.T., Zhang, W.W. et al. (2018) Tensile properties of AZ61 magnesium alloy produced by multi-pass friction stir processing: Effect of sample orientation. Mater. Sci. Eng. A, 725, 398-405.

27. Xu, N., Bao, Y. (2016) Enhanced mechanical properties of tungsten inert gas welded AZ31 magnesium alloy joint using two-pass friction stir processing with rapid cooling. Ibid., 655, 292-9.

28. Alavi Nia, A., Omidvar, H., Nourbakhsh, S.H. (2014) Effects of an overlapping multi-pass friction stir process and rapid cooling on the mechanical properties and microstructure of AZ31 magnesium alloy. Mater Des., 58, 298-304.

29. Fattah-Alhosseini, A., Attarzadeh, F.R., Vakili-Azghandi, M. (2017) Effect of multi-pass friction stir processing on the electrochemical and corrosion behavior of pure titanium in strongly acidic solutions. Metall. Mater. Transact. A Phys. Metall. Mater. Sci., 48, 403-11.

30. John Baruch, L., Raju, R., Balasubramanian, V. et al. (2016) Influence of multi-pass friction stir processing on microstructure and mechanical properties of die cast $\mathrm{Al}-7 \mathrm{Si}-3 \mathrm{Cu}$ aluminum alloy. Acta Metall. Sin., 29, 431-40. Doi:10.1007/ s40195-016-0405-2.

31. Meenia, S., Khan, M.D.F., Babu, S. et al. (2016) Particle refinement and fine-grain formation leading to enhanced mechanical behaviour in a hypo-eutectic Al-Si alloy subjected to multi-pass friction stir processing. Mater. Charact., 113, 134-43.

32. Esmaily, M., Mortazavi, N., Osikowicz, W. et al. (2016) Influence of multi-pass friction stir processing on the corrosion behavior of an Al-Mg-Si alloy. J. Electrochem. Soc., 163, C124-30.

33. Padhy, G.K., Wu, C.S., Gao, S. (2018) Friction stir based welding and processing technologies - processes, parameters, microstructures and applications: A review. J. Mater. Sci. Technol., 34, 1-38.

34. Liu, F.C., Hovanski, Y., Miles, M.P. et al. (2018) A review of friction stir welding of steels: Tool, material flow, microstructure, and properties. J. Mater. Sci. Technol., 34, 39-57.

35. Miles, M.P., Pew, J., Nelson, T.W., Li, M. (2006) Comparison of formability of friction stir welded and laser welded dual phase 590 steel sheets. Sci. Technol. Weld. Join., 11, 384-8.

36. Kang, H.C., Park, B.J., Jang, J.H. et al. (2016) Determination of the continuous cooling transformation diagram of a high strength low alloyed steel. Met. Mater. Int., 22, 949-55. 